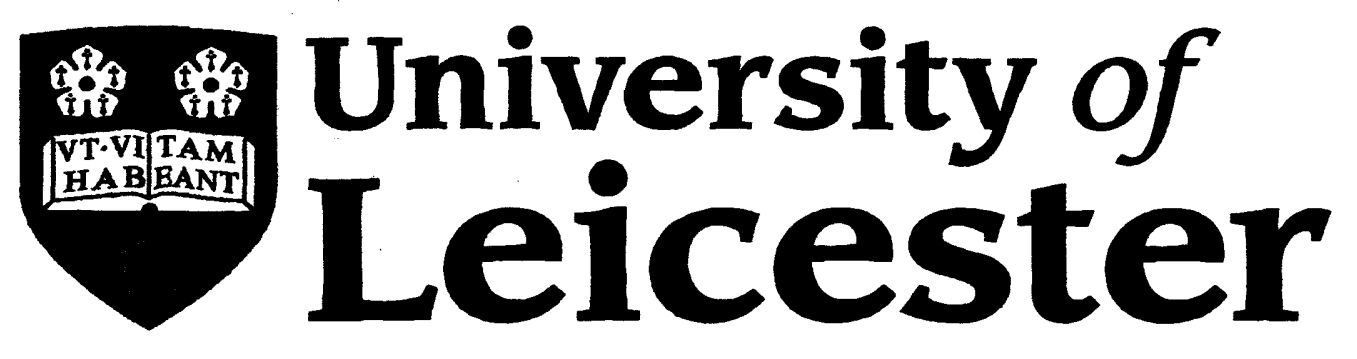

\title{
Quartz Crystal Microbalance Determination of Trace Metal lons in Solution
}

Thesis submitted for the degree of Doctor of Philosophy at the University of Leicester

by

Abdunnaser Mohamed Etorki

Department of Chemistry

Faculty of Science

University of Leicester

November 2005 
UMI Number: U220720

All rights reserved

\section{INFORMATION TO ALL USERS}

The quality of this reproduction is dependent upon the quality of the copy submitted.

In the unlikely event that the author did not send a complete manuscript and there are missing pages, these will be noted. Also, if material had to be removed, a note will indicate the deletion.

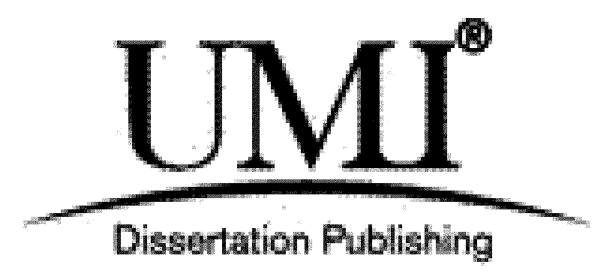

UMI U220720

Published by ProQuest LLC 2013. Copyright in the Dissertation held by the Author.

Microform Edition (c) ProQuest LLC.

All rights reserved. This work is protected against unauthorized copying under Title 17, United States Code.

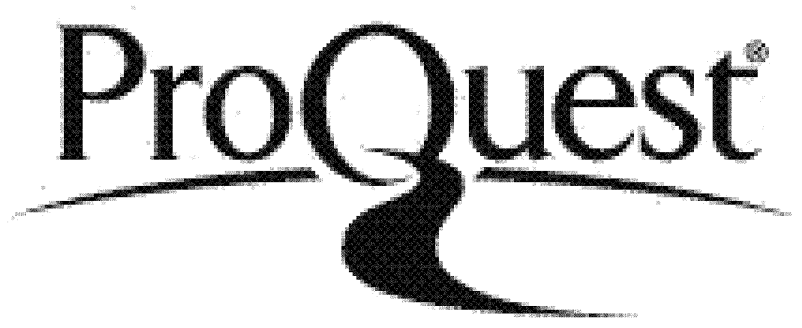

ProQuest LLC

789 East Eisenhower Parkway

P.O. Box 1346

Ann Arbor, MI 48106-1346 


\title{
Quartz Crystal Microbalance Determination of Trace Metal lons in Solution
}

\author{
By A. M. Etorki
}

This thesis describes development of a new practical acoustic wave and electrochemical sensor for detection of trace metal ions from aqueous solutions using self-assembled monolayer modified electrodes. The gold electrodes of $10 \mathrm{MHz}$ AT-cut piezoelectric quartz crystal resonators were modified with different selfassembled monolayers (SAMs) with $-\mathrm{COOH},-\mathrm{SH}$ and $-\mathrm{NH}$ free terminal functional groups. Frequency measurements were carried out on emersed and immersed crystals to determine ligand immobilisation. Cyclic voltammetry investigation of $\left[\mathrm{Fe}(\mathrm{CN})_{6}\right]^{3-14}$ redox chemistry was carried out on the SAM/Au electrodes to investigate the quality and integrity of the SAM. Analysis of this data has lead to a qualitative explanation of electron transfer process involved in the cycling.

These SAMs were employed for the detection of target metal ions such as $\mathrm{Cd}(\mathrm{II}), \mathrm{Pb}$ (II), $\mathrm{Hg}$ (II) and $\mathrm{Cu}$ (II) from single metal ion solutions, based on frequency measurements associated with the binding of different concentrations of metal ions from solution to each surface-bound monolayer. Data for emersed and immersed crystals were used to estimate the amount of metal ions bound to the SAM. Results for the mole ratio of metal ion:SAM were used to select the SAMs having the highest degree of sequestration. The uptake of metal ions from aqueous solution was followed as a function of concentration and attempts made to fit the data to the Langmuir, Temkin, Freundlich, Frumkin, El-Awady and Flory-Huggins isotherms. The relative merits of these isotherms are discussed and heterogeneous binding constants extracted. Selectivity for different metal ions was assessed by estimating the binding constant for target metal ions in single and mixed metal ion solutions. The combination of SAM selectivity and QCM sensitivity provides a potentially useful metal ion sensor in the nanomolar to micromolar range. Similarly the EQCM has been used in coulometric mode by integrating the voltammetric responses of surface-bound complexed metal ions to determine the metal ion coverage.

XPS results confirmed that SAMs are successfully immobilized on $\mathrm{Au}$ surfaces and can bind different trace metal ions from aqueous solutions with some selectivity. High resolution XPS results of the SAMs modified electrodes before and after adsorption of metal ions showed the presence of sulfur, carbon, oxygen, nitrogen and target metal ions. The M:S ratio was in good agreement with the stoichiometry extracted from QCM and EQCM measurements. 


\section{MEMORANDUM}

The work in this thesis is original except where reference or acknowledgment has been made. The project was carried out in the Department of Chemistry, University of Leicester between September 2002 and September 2005.

Abdunnaser. M. Etorki 


\section{ACKNOWLEDGEMENTS}

I would like to express my deep gratitude to my supervisor Prof. Robbert Hillman for making this study possible and for providing the support, encouragement and enthusiasm that led me through to the completion of this thesis. Thanks too to my second supervisor Dr. Wood Ward for helpful and suggestions. I would also like to thank Dr. Karl Ryder and Dr. Andrew Glidle for their assistance in XPS experiments at NCESS and for XPS data analysis. Thank you to all members of the ARH group I owe a huge debt of gratitude for putting up with me day by day over the three years. Also I would like to thank you all staff in Chemistry Department deserve a special mention including Phil, Gerry and every body else who had to put up with the fact that I wanted something every time I saw them.

I would like to acknowledge the Libyan Higher Education Department for the grant this research.

I can not end without thanking my wife, on whose constant encouragement and love I have relied throughout my time at the academy.

Last, but no means least, my heartfelt thanks goes to my parents, for allowing me the freedom to pursue my $\mathrm{PhD}$, and for reminding me daily what really matters in life. 
To my parents 


\section{Table of Contents}

\section{Chapter 1: Introduction}

1.1 Overview................................................................ 1

1.2 Modified Electrodes in Analytical Chemistry................................... 1

1.3 Self-Assembled Monolayer Modified Electrodes................................. 3

1.3.1 Modification of the Quartz Crystal Microbalance with SAMs.............. 4

1.3.2 Applications of SAMs................................................ 5

1.3.3 Advantages of SAMs for chemical sensing............................. 6

1.4 Characterisation of SAMs.................................................. 6

1.4.1 Electrochemical Techniques........................................... 7

1.4.1Non Electrochemical Techniques.......................................... 7

1.5 Immobilisation of Ligands onto SAM......................................... 9

1.5.1 Specific Terminal Functionalities........................................... 9

1.6 Binding of trace metal ions with terminal self-assembled monolayers......... 11

1.6.1 Electroactive metal ions.............................................. $\quad 11$

1.6.2 Electroinactive metal ions................................................ 17

1.7 Sensors....................................................................... 21

1.7.1 Applications............................................................. 21

1.7.2 Characterisation of Sensors............................................ 22

1.7.3 Acoustic Wave Devices............................................... 22

1.7.3.1 Thickness-Shear Mode (TSM) Device.......................... 22

1.7.3.2 Applications of the QCM in analysis of trace metal ions............ 23

1.8 Aims and Objectives...................................................... 24

1.9 References................................................................. 25

\section{Chapter 2: Theory and Techniques}

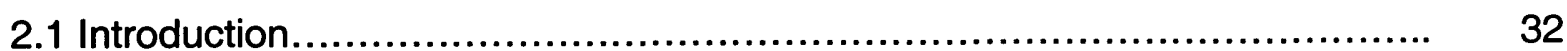

2.2 Background of the quartz crystal microbalance............................. 32

2.2.1 The Piezoelectric Effect................................................. 32

2.2.2 Modes of Vibration.................................................... $\quad 35$

2.2.3 Frequency mass relationship ....................................... $\quad 36$ 
2.2.4 The Sauerbrey equation............................................... 37

2.2.5 Sensitivity............................................................. 39

2.3 The electrochemical quartz crystal microbalance (EQCM).................... 39

2.4 Limit of mass load and boundary conditions for the (E)QCM................... 40

2.4.1 Film thickness and viscoelastic effects..................................... 40

2.4.2 Quartz Crystal-Overlayer Boundary......................................... 41

2.4.3 Uniformity.............................................................. 41

2.5 Crystal impedance analysis................................................... 42

2.6 Fundamental of Electrochemical Techniques.................................. 43

2.6.1 Electron Transfer Rates................................................. 44

2.6.1.1 Kinetically controlled current....................................... 44

2.6.2 Mass Transport............................................................. 45

2.6.2.1 Diffusion.............................................................. 45

2.6.2.2 Migration.......................................................... 46

2.6.2.3 Convection.......................................................... 46

2.6.2.4 Diffusion Limited Current.......................................... 46

2.7 Cyclic Voltammetry......................................................... 47

2.7.1 Solution Phase Voltammetry......................................... 48

2.7.2 Solid State Voltammetry.............................................. $\quad 50$

$2.8 \mathrm{X}$-ray photoelectron spectroscopy........................................ 51

2.8.1 The principle of the XPS............................................ 52

2.82 Elemental and chemical state information................................ 53

2.8.3 The basic of XPS instrumentation..................................... 54

2.9 References................................................................. 55

\section{Chapter 3: Experimental}

3.1 Introduction................................................................... 58

3.2 Quartz Crystal Microbalance (QCM) Preparations.............................. 58

3.2.1 Unpolished Crystals..................................................... 58

3.2.2 Polished Crystals....................................................... 58

3.3 Quartz Crystal Microbalance Cell.............................................. 58

3.3.1 Electrochemical Cell................................................... 59

3.3.2 Calibration of the QCM.................................................... 61

3.4 Structure of aliphatic and aromatic based thiols use to prepare SAM.......... 62

3.4.1 Chemicals............................................................... 63

3.4.2 Solvents.............................................................. $\quad 64$ 
3.5.1 SAM Solution Preparation.

3.5.2 Preparation of Surfaces. 65

3.5.3 Preparation of functionalised SAMs. 65

3.5.4 Preparation of 1000ppm of metal ions. 65

3.6 Frequency Measurements. 66

3.7 Quartz crystal impedance measurements. 66

3.8 Cyclic voltammetry measurements. 66

3.9 XPS measurements. 68

3.10 References 69

\section{Chapter 4: Characterisation of the Modification of the QCM with SAMs}

4.1 Introduction..................................................................... 70

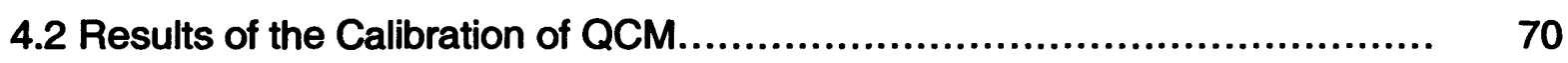

4.2.1 Discussion........................................................... $\quad 71$

4.3 Relation between $\Delta f_{\text {dry }}$ and $\Delta f_{\text {wet }}$ for SAMS ............................... 72

4.4 Quartz impedance spectroscopy for monitoring SAMs......................... 73

4.5 Cyclic voltammograms for all SAMs.......................................... 75

4.5.1 Discussion............................................................... 79

4.6 Estimate of electron transfer rate constant at SAMs.......................... 79

4.6.1 Relation between $\log \Psi$ and $\Delta E_{p}$ for some SAMs..................... 84

4.6.2 Relation between frequency change and electron transfer rate ........... $\quad 86$ constant for $\left[\mathrm{Fe}(\mathrm{CN})_{6}\right]^{-3,-4}$

4.6.3 Discussion........................................................... 88

4.7 Conclusions................................................................ 88

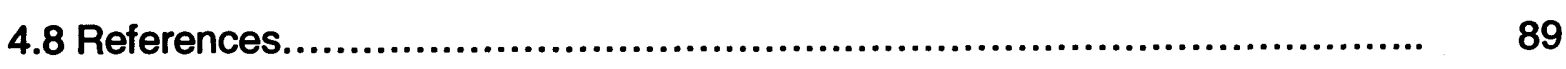

Chapter 5: QCM study of the complexation of trace metal ions with SAMs

5.1 Introduction.................................................................... 90

5.2 Complexation between 3-thiodisuccinic acid and cadmium ions............... 90

5.2.1 Strategy (stage one) .................................................. 90

5.2 .2 Results................................................................ 91

5.2.3 Strategy (stage two) ................................................. 92

5.2 .4 Results.............................................................. 92

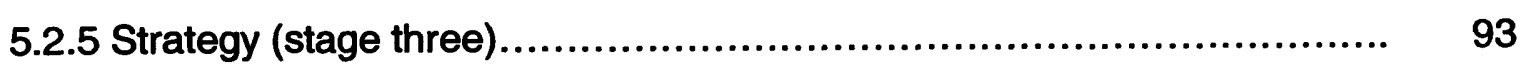

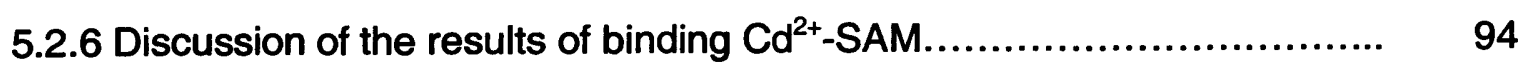


5.3 Complexation between $\mathrm{Cd}^{2+}$ and 3-thiodisuccinic acid (final stage)

5.3.1 Fitting the data to different isotherms...................................... 101

5.3 .2 Results.................................................................. 103

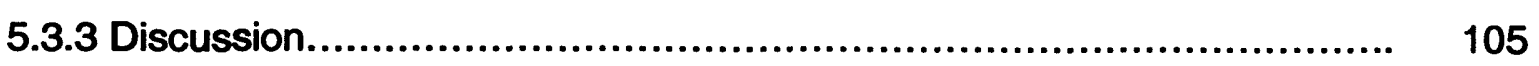

5.3.3.1 Discussion of estimated isotherm parameters........................ 105

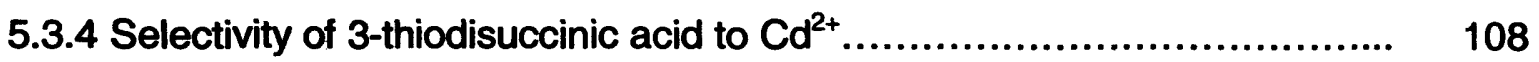

5.3.5 Interpretation............................................................... 112

5.3.6 Reproducibility and detection limit................................... 113

5.3.7 Comparison the estimated binding constants with literature............... $\quad 114$

5.4 Estimate of the equilibrium binding constant between $\mathrm{Cd}^{2+}$ and 4-

mercaptobenzoic acid........................................................ $\quad 115$

5.4.1 Comparison of the binding constants with literature data................. 118

5.5 Binding $\mathrm{Pb}^{2+}$ to different SAMs................................................. 118

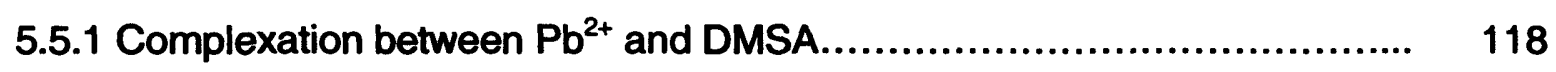

5.5.2 Fitting the data to different isotherms....................................... 119

5.5.3 Binding of $\mathrm{Cd}^{2+}$ and $\mathrm{Cu}^{2+}$ to meso-2,3-dimercaptosuccinic acid.............. 121

5.5.4 Compare of logK for $\mathrm{Pb}(\mathrm{II})$-ligand on the surface and in solution............. 123

5.6 Binding $\mathrm{Cu}(\mathrm{II}), \mathrm{Cd}(\mathrm{II})$ and $\mathrm{Pb}(\mathrm{II})$ to 3,3-thiodipropionic acid.................... 124

5.7 Binding $\mathrm{Hg}(\mathrm{II})$ to 1,6-hexanedithiol self-assembled monolayer................ 127

5.7.1 Experimental............................................................. 127

5.7.2 Results and discussion.................................................. 128

5.7.3 Effect of the pH on the complexation between $\mathrm{Hg}(\mathrm{II})$ and HDT............ 130

5.8 Analysing Mixtures of Metal lons........................................... 131

5.9 Using 3-thiodisuccinic acid to analyse mixtures of metal ions................. 131

5.9.1 Measurements at fixed concentration of $\mathrm{Cd}^{2+}$ in the presence $\mathrm{Cu}^{2+}$ or

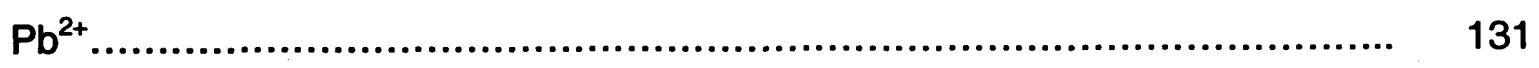

5.9.2 Methodology........................................................... 132

5.9.3 Results and Discussion................................................. 133

5.9.4 Competitive adsorption isotherms of cadmium ions in mixed metal ion solutions.......................................................... 136

5.9.4.1 Methodology...................................................... 136

5.9.4.2 Results and Discussion............................................ 136

5.9.4.3 Fitting the data to different isotherms............................... 139

5.10 Using meso-2,3-dimercaptosuccinic acid to analyse mixtures of metal ions. 
5.10.1 Measurements at fixed concentration of $\mathrm{Pb}^{2+}$, in the presence

$$
\mathrm{Cu}^{2+} \text { and } \mathrm{Cd}^{2+}
$$

5.10.2 Results and discussion................................................. $\quad 140$

5.10.3 Competitive adsorption isotherms of lead in mixed metal ion solutions.. $\quad 141$

5.10.4 Discussion.............................................................. 143

5.11 Using 1,6-hexanedithiol to analyse mixtures of metal ions.................... 143

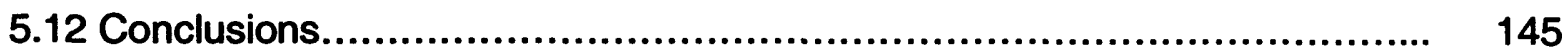

5.13 References................................................................... 146

Chapter 6: EQCM study of the complexation of trace metal ions with SAMs on gold piezoelectrodes

6.1 Introduction................................................................... 148

6.2 Complexation between 3,3-thiodipropionic acid and $\mathrm{Cu}^{2+} \ldots \ldots \ldots \ldots \ldots \ldots \ldots . . . . . . . . . . .148$

6.2.1 Experimental.............................................................. $\quad 149$

6.3 Results and Discussion..................................................... 149

6.3.1 Voltammetric response of the Au/TDP electrode to $\mathrm{Cu}$ (II) ions........... 149

6.3.1.1 Stability and influence of the sweep rate......................... 150

6.3.1.2 Effect of $\mathrm{pH}$ of preconcentration solution........................... 152

6.3.1.3 Effect of the preconcentration time................................ 154

6.3.1.4 Concentration dependence of the characteristic peak charge...... 155

6.3.1.5 Estimate of the molar ratio between $\mathrm{Cu}^{2+}$ and TDP................. 157

6.3.1.6 Incorporation mechanism of copper ions on TDP SAM electrode... 159

6.3.2 Voltammetric response of the Au/TDP electrode to $\mathrm{Pb}$ (II) ions.......... 161

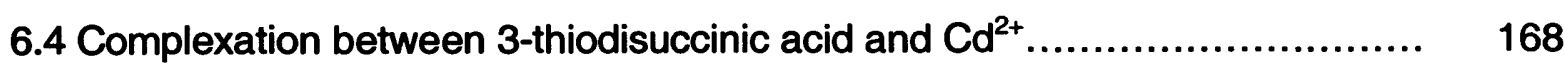

6.4.1 Experimental........................................................ 168

6.4.2 Results and discussions............................................. 168

6.4.2.1 Stability and effect of the sweep rate.................................. 169

6.4.2.2 Effect of $\mathrm{pH}$ of preconcentration solution........................... 171

6.4.2.3 Effect of the preconcentration time............................... 173

6.4.2.4 Concentration dependence of the characteristic peak charge....... 174

6.4.2.5 Estimate of the mole ratio between $\mathrm{Cd}^{2+}$ and TDS.................. 175

6.4.2.6 Renewal of TDS SAM modified Au electrode........................ 177

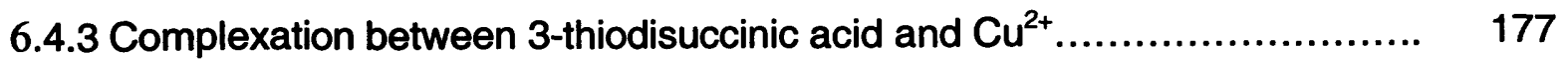

6.4.3.1 Estimate of the mole ratio between $\mathrm{Cu}^{2+}$ and TDS.................... 178

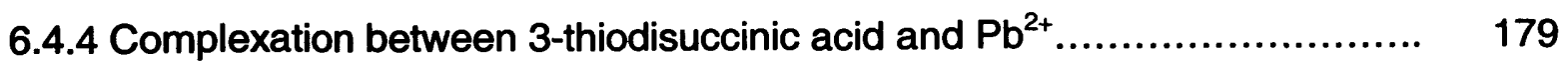


6.4.5 Interference effects on $\mathrm{Cd}(\mathrm{II})$ responses in binary mixtures.................. 180

6.5 Complexation between meso-2,3-dimercaptosuccinic acid and $\mathrm{Pb}^{2+} \ldots \ldots \ldots \ldots . \quad 181$

6.5.1 Experimental............................................................. 181

6.5.2 Results and discussion............................................. 182

6.5.2.1 Stability and effect of the sweep rate.............................. 183

6.5.2.2 Effect of $\mathrm{pH}$ of preconcentration solution.............................. 184

6.5.2.3 Concentration dependence of the characteristic peak charge....... 185

6.5.3 Complexation between meso-2,3 dimercaptosuccinic acid $\mathrm{Cu}^{2+} \ldots \ldots \ldots \ldots .188$

6.5.4 Complexation between meso-2,3 dimercaptosuccinic acid and $\mathrm{Cd}^{2+} \ldots . . \quad 189$

6.5.5 Interference effects on $\mathrm{Pb}(\mathrm{II})$ responses in binary mixtures.............. 190

6.6 Complexation between 1,4-benzenedimethanethiol and $\mathrm{Hg}^{2+} \ldots \ldots \ldots \ldots \ldots \ldots . . . . . . .192$

6.6.1 Experimental............................................................ 192

6.6.2 Results and Discussion............................................. 192

6.6.2.1 Stability and effect of the sweep rate............................... 194

6.6.2.2 Concentration dependence of the characteristic peak charge..... 194

6.7 Complexation between 2-mercaptobenzimidizole and $\mathrm{Hg}^{2+} \ldots \ldots \ldots \ldots \ldots \ldots \ldots . .196$

6.7.1 Experimental............................................................... $\quad 196$

6.7.2 Results and Discussion................................................. 196

6.7.2.1 Stability and effect of the sweep rate............................... 197

6.7.2.2 Concentration dependence of the characteristic peak charge....... 199

6.8 General Conclusions........................................................ 200

6.9 References.................................................................... 201

Chapter 7: XPS study of the complexation between trace metal ions and SAMs

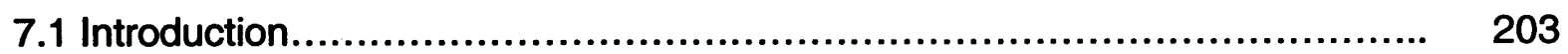

7.2 Aims and Objectives...................................................... 203

7.3 Experimental............................................................... 204

7.4 Results and Discussions................................................. 205

7.4.1 Elemental composition for SAM and SAM/M ${ }^{2+} \ldots \ldots \ldots \ldots \ldots \ldots \ldots \ldots \ldots .205$

7.4.2 XPS peak fitting......................................................... 217

7.4.2.1 Analysis using S(2p) XPS spectra............................... 217

7.4.2.2 Analysis using C(1s) XPS spectra.................................. 222

7.4.2.3 Analysis using $O(1 \mathrm{~s})$ XPS spectra................................. 225

7.4.2.4 Analysis using N(1s) XPS spectra.................................. 226

7.4.2.5 Analysis using $\mathrm{Cu}(2 \mathrm{p})$ spectra................................... 227 


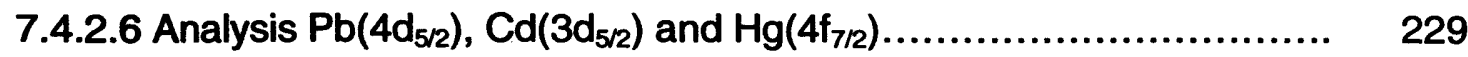

7.5 General Conclusions........................................................ 230

7.6 References............................................................... 231

Chapter 8: General Conclusions and scope for future work

8.1 Functionalization of the QCM with SAMs.................................... 233

8.2 Binding of trace metal ions to SAMs using the QCM as a gravimetric probe... 233

8.2.1 Estimate of the equilibrium binding constant between trace metal ions and aliphatic-aromatic SAMs............................................. 234

8.3 Binding of trace metal ions to SAMs using the EQCM as an

electrochemical probe.......................................................... 236

8.4 XPS study of the complexation between trace metal ions and SAMs 236

8.5 Future Prospects......................................................... 237

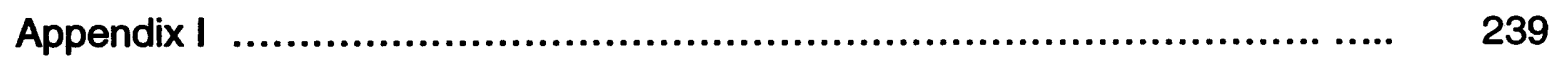

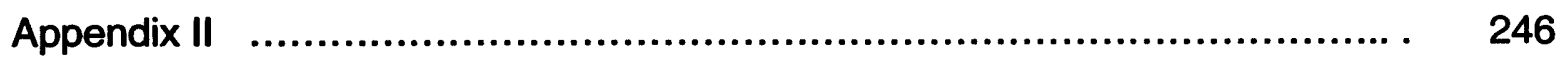




\section{Chapter 1: Introduction}

\subsection{Overview}

The development of practical sensors for the detection and quantification of trace metal ions in aqueous solutions using self-assembled monolayers immobilized on a gold electrode in a quartz crystal microbalance cell is the focus of this research. The introductory chapter will discuss the field of self-assembled monolayer modified electrodes in chemical sensors and show how successful design of these modified electrodes has relied upon utilisation of a number of characterisation techniques, including electrochemical and acoustic wave methods.

\subsection{Modified electrodes in analytical chemistry}

A modified electrode consists of an electrode with a chemical species attached to the surface. Altering the electrode in this way prevents direct contact between the metal surface and the bulk electrolyte and, as a consequence, any electrochemical reaction taking place must occur through the surface immobilised species. In doing so, the electrode will display the chemical, electrochemical and optical properties of the surface species.

The first reported modified electrode was made in 1975 by Murray et al. [1] in which tin oxide was modified with ligands such as aniline or pyridine. Since that work, a large and growing range of chemical species available for such surface attached films has been developed, resulting in an increasing variety of properties available for modified electrodes. Modified electrodes can be divided into four main categories, based on the nature of the modifying process [2]. Figure 1.1 shows the four possible routes for preparation of chemically modified electrodes. The new applications of chemically modified electrodes in analytical chemistry have been reviewed by Zen et al. [3]. 


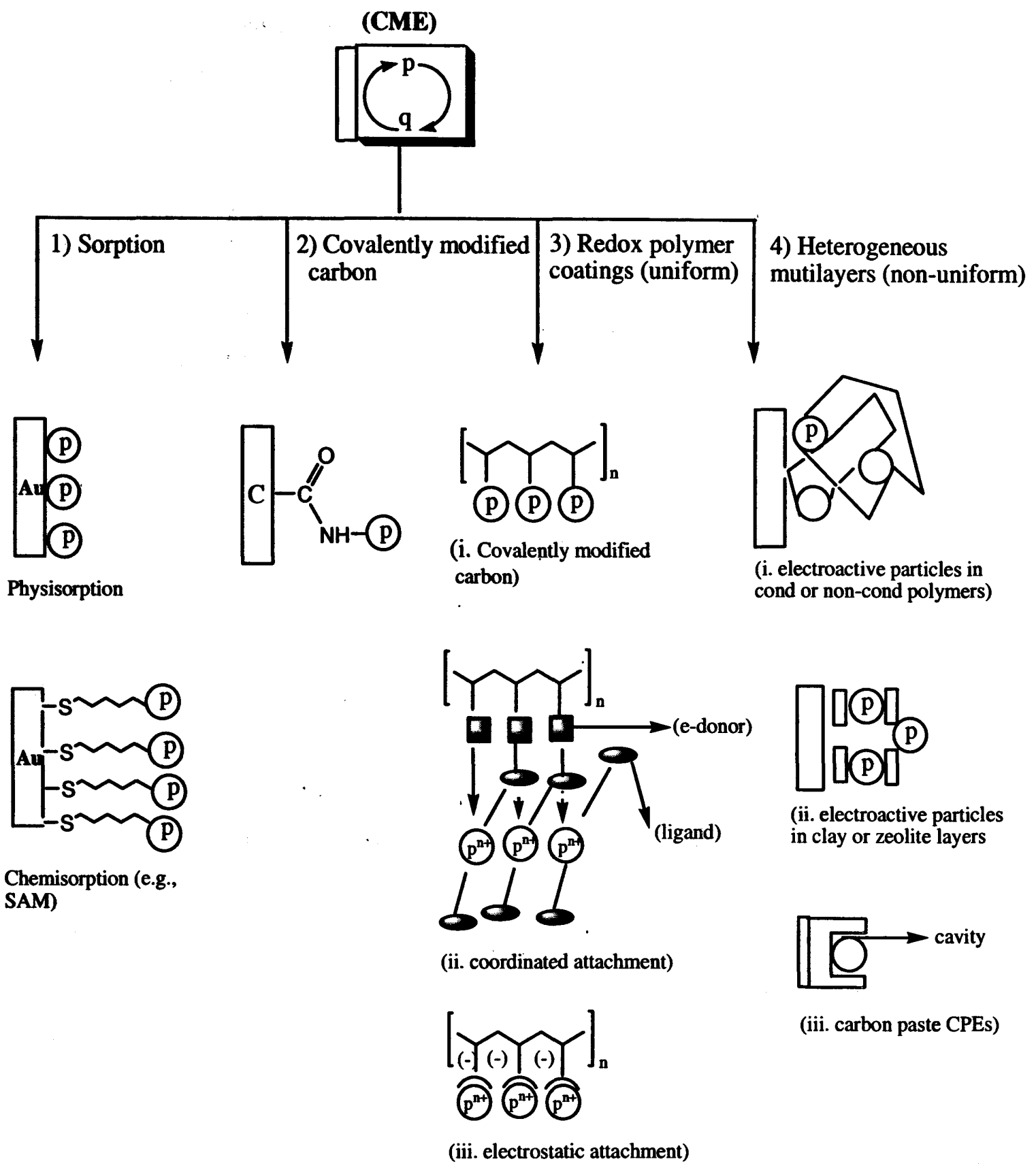

Figure 1.1: Schematic representation for various kinds of chemically modified electrodes (CME) preparation routes, The terms $\mathrm{p}$ and $\mathrm{q}$ correspond to the reduced and oxidized states, respectively of the mediator. 


\subsection{Self-assembled monolayer modified electrodes}

The modification of an electrode surface with self-assembled monolayers (SAMs) of thiols and disulfides has received considerable attention in recent years.

Self-assembled monolayers are mono-molecular layers which are spontaneously formed upon immersing a solid substrate into a solution containing amphifunctional molecules. Successful self-assembly requires a relatively strong bond between the substrate and an atom or moiety in the molecule, and additional lateral interactions between molecules in the monolayers. Self-assembled monolayers can be prepared using different types of molecules and different substrates. Widespread examples are alkyl siloxane monolayers, fatty acids on oxidic materials and alkane thiolate monolayers. All of these systems have been reviewed in great detail $[4,5$, $6,7,8,9]$. Two of the most widely studied classes of SAMs are alkyl-aromatic thiolate monolayers on gold electrodes and alkyl silane monolayers. Here we will concentrate exclusively on SAMs of functionalized alkane or aromatic thiols on gold surfaces. This type of SAM holds great promise for applications in several different areas. Gold is the most frequently used metal because it doesn't have a stable oxide under ambient conditions and therefore is the most easily used. The thiol groups chemisorb onto the gold surface via the formation a thiolate bond [10]. Research in this area began in 1983 when the first gold-alkyl thiolate monolayers were produced by Alara and Nuzzo at Bell laboratories [11]. The principle is simple: a molecule which is essentially an alkane chain, typically with 10-20 methylene units, is given a head group with a strong preferential adsorption to the substance used. Thiol (S-H) head group and $A u(111)$ substrates have been shown to work well. The thiol molecules adsorb readily from solution on the gold, creating a dense monolayer with the hydrocarbon end groups pointing outwards from the surface. Using thiol molecules with different end groups, the resulting chemical surface functionality can be varied within wide limits, as shown in Figure 1.2. 


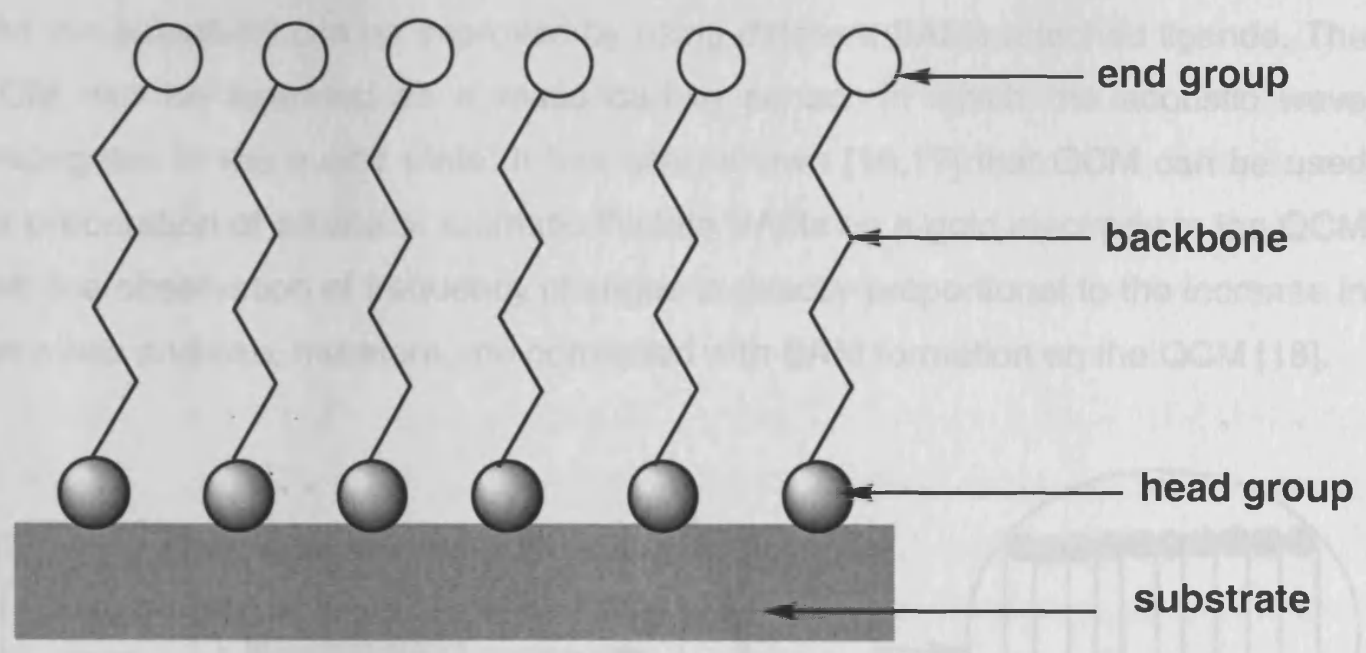

Figure 1.2: schematic of SAM. Filled circle indicates chemisorbing group and open circle endgroup (tailgroup) which can be chosen from variety of chemical functionalities.

Alternatively, it is also possible to chemically functionalize the tail groups by performing reactions after assembly of the SAM [12]. There are a number of recent reviews on self-assembled monolayers, which cover the basics of SAM formation, structure and characterization. Analytical techniques have been developed with sufficient sensitivity to allow the detailed characterization of monolayers $[13,14,15]$. These types of SAM on Au surfaces are quite stable in the potential window from 400 to $1400 \mathrm{mV}$ vs SCE. Outside of this range the thiols are oxidatively or reductively desorbed. The instability at high potentials can, however, be used to control the order of the SAMs during their formation.

\subsubsection{Modification of the Quartz Crystal Microbalance with SAMs}

Alkane or aromatic thiols can adsorb spontaneously on to a gold surface to produce highly ordered SAMs on a quartz crystal microbalance with $\mathrm{Au}$ electrodes.

Figure 1.3 shows the preparation of SAMs. The substrate is Au on quartz and is immersed into an ethanol solution of the desired thiol. Initial adsorption is fast (seconds) then an organization phase follows which should be allowed to continue for $15-24 \mathrm{~h}$ for the best results.

The QCM is a piezoelectric device that has the advantages of very small size, very high sensitivity, low cost, high measurand (frequency) stability and minimal electronic requirements. The theoretical detection limit for QCM operating $10 \mathrm{MHz}$ is around $1 \times 10^{-12} \mathrm{~g}$. Even though the QCM is not selective to metal ions in solution 
and the selectivity can be improved by using different SAMs attached ligands. The QCM can be operated as a mass-loading sensor in which the acoustic wave propagates in the quartz plate. It has been shown $[16,17]$ that QCM can be used for preparation of alkane or aromatic thiolate SAMs on a gold electrode in the QCM cell: the observation of frequency changes is directly proportional to the increase in the mass and can, therefore, be correlated with SAM formation on the QCM [18].

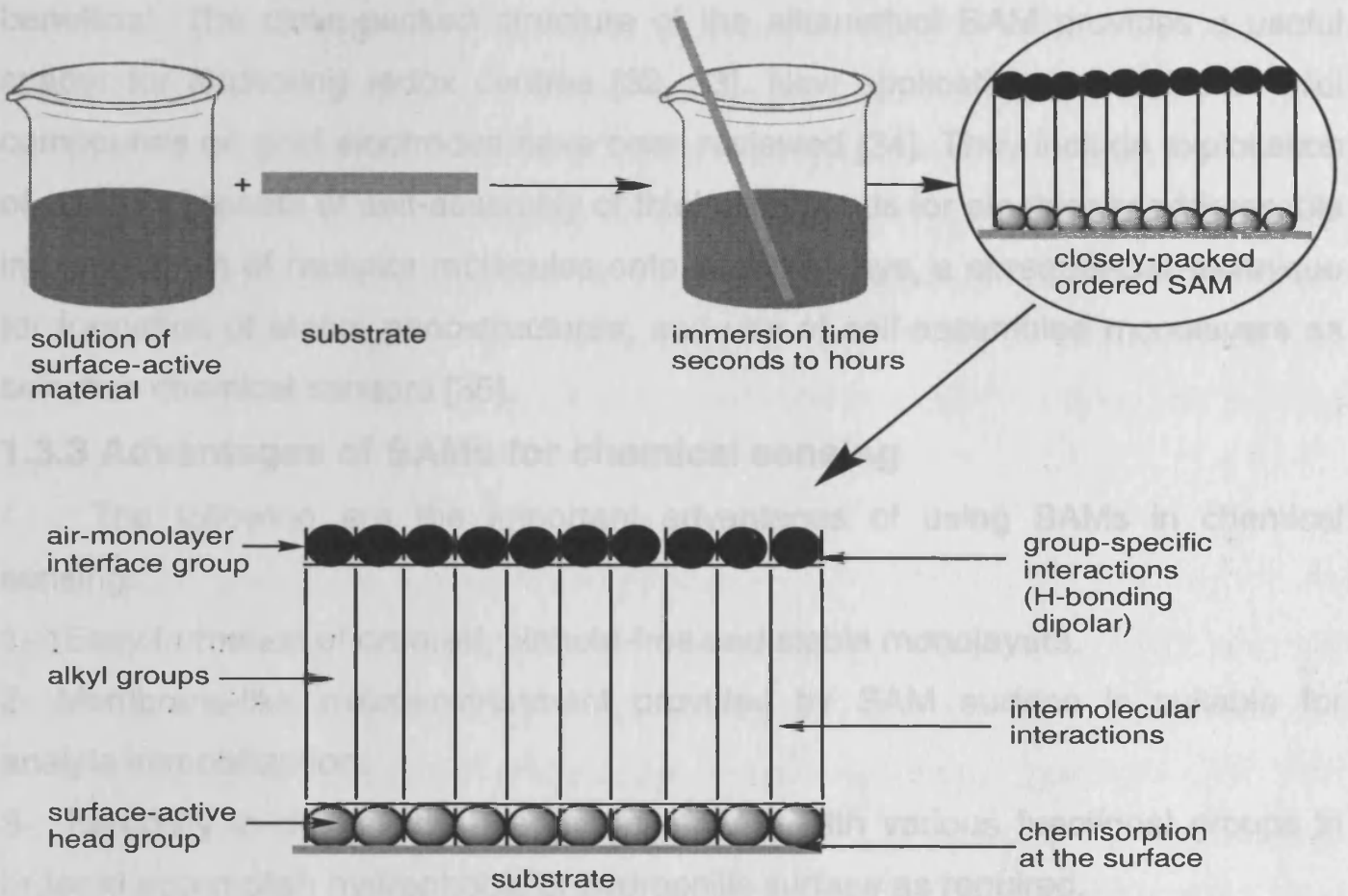

Figure 1.3: Schematic of the various processes involved in the preparation of SAMs.

Recently the quartz crystal microbalance has been especially useful for the investigation of adsorption process of SAMs [16, 19, 20,21], by measuring the mass change in real time by monitoring the change in frequency of a quartz crystal with gold electrodes.

\subsubsection{Applications self-assembled monolayers}

The most frequent application of SAMs in electroanalytical chemistry is in the development of sensors where the SAM is used to impart selectivity onto an electrode for a particular analyte. Sensors using SAMs have been used to monitor 
pH using integrated molecular systems [22], inorganic species [23] and organic molecules using both chemical and biological recognition, including enzymes, DNA, and RNA [24, 25, 26, 27]. Potential technological applications of SAMs are lubrication [28], corrosion protection [29], and photolithographical or electrical resists [30]. We can use SAMs for microarray electrodes; they offer advantages for both analytical and kinetic studies [31] and electrocatalysis. Catalysis reactions are a common area where presentation of a specific face of a molecule could be beneficial. The close-packed structure of the alkanethiol SAM provides a useful spacer for anchoring redox centres [32, 33]. New applications of SAMs of thiol compounds on gold electrodes have been reviewed [34]. They include exploitation of electrical control of self-assembly of thiol compounds for electrically addressable immobilization of receptor molecules onto sensor arrays, a spreader-bar-technique for formation of stable nanostructures, and use of self-assembled monolayers as selective chemical sensors [35].

\subsubsection{Advantages of SAMs for chemical sensing}

The following are the important advantages of using SAMs in chemical sensing:

1- Easy formation of ordered, pinhole-free and stable monolayers.

2- Membrane-like microenvironment provided by SAM surface is suitable for analyte immobilization.

3- Flexibility to design the headgroup of SAM with various functional groups in order to accomplish hydrophobic or hydrophilic surface as required.

4- Only minimum amount of analyte is needed for immobilization on SAM.

5- Reasonable stability for extended period allowing multiple reliable measurements.

\subsection{Characterisation of self-assembled monolayers}

In order to fine tune the chemical and physical properties of SAMs, it is absolutely critical that there are techniques available to characterise the films accurately. This is not as straightforward as it may as first appear. Many material characterization instruments do not have sufficient sensitivity to analyse, the small volumes or masses associated with the thin film materials. Those that do have other disadvantages such as excessive cost, extensive sample preparation, long analysis time or restrictive sample environment.

Each technique described below is particularly sensitive to different aspects of the structure, bonding or charge transfer mechanisms of surface immobilised electrode species. Methods that permit the study of modified electrodes in solution 
are termed 'in situ techniques.' In situ techniques offer the desirable prospect of studying active devices in their working environment. The relevance of ex situ techniques have also provided useful structural information on SAMs. In sections 1.4.1, 1.4.2 summary of the techniques used to study SAMs are presented.

\subsubsection{Electrochemical Techniques}

Electrochemical techniques such as cyclic voltammetry (CV) and impedance spectroscopy are widely used for SAM studies [36, 37, 38]. Electrochemistry is a useful technique to see defects in the monolayer. The two kinetics steps in SAM formation and presence of pin-hole defect free SAMs have been shown by CV studies. In impedance spectroscopy very small sinusoidal potential variations with frequencies varying between a few $\mathrm{MHz}$ and $\mathrm{kHz}$ are applied to the SAM. From the impedance spectra values for the monolayer capacitance and resistance are obtained. As in CV, these data can be related to the average thickness and packing quality of the SAM.

\subsubsection{Non Electrochemical Techniques}

Many of the non-electrochemical techniques used to characterise SAM modified electrodes are spectroscopic [39]. Spectroscopy provides direct observation of intermediates, products and adsorbates in electrochemical reactions, via their electronic structure. Vibrational spectroscopy includes IR spectroscopy and Raman scattering uses the change in polarisability associated with vibrational modes (and their anisotropy) to draw conclusions on the structure e.g. molecular chain orientation. Although vibrational spectroscopy can be used in situ, especially when the SAM in contact with an aqueous solutions, and ex situ, where the monolayer is removed from the analyte solution before analysis [40, 41, 42]. Sum frequency generation (SFG) and near-edge-X-ray absorption fine structure spectroscopy (NEXAFS) fall in a similar category, although they work in different energy ranges $[43,44]$.

$X$-ray photoelectron spectroscopy (XPS) is an excellent technique to determine the elemental analysis of the monolayer, even though the technique is limited to ex situ studies. It has been used to estimate the thickness, coverage and tilt angle as well as the orientation order [45]. A technique that is complementary to transmission spectroscopy in terms of experimental requirement and information obtained is ellipsometry. This is a reflection technique that is surface specific, giving additional 
information about thickness and refractive index before and after reaction to detect adsorption of molecules onto the SAM or binding the analyte on the SAM [46].

Diffraction-based techniques such as grazing-incidence $X$-ray diffraction (GIXD), low energy electron diffraction (LEED) and low energy atom diffraction (LEAD) $[47,48]$ have been used for diffraction from the 2D structure of SAMs. Among these techniques LEED is the oldest; it has the advantage of displaying the entire reciprocal lattice, projected in $2 \mathrm{D}$ at once. LEAD, which also provides only limited resolution, is the most surface specific and the most gentle probe. GIXD has the best resolution compared to LEED and LEAD. Information along the surface normal (e.g. layer thickness) can be obtained using X-ray with so called "rodscans", where the momentum transfer is along the surface normal; this is called specular reflection. X-ray reflectivity provides information on the electron density profile along the surface normal, i.e. film thickness, roughness and electron densities. This scattering technique is very useful also for SAM-based multilayered studies [49]. Helium atom reflectivity (HAR) also gives information on the roughness of monolayers.

Non-spectroscopic techniques include surface acoustic wave and crystal impedance. The surface acoustic wave devices are capable of detecting mass changes in the range of several nanograms $[50,51]$. They have been used to study the kinetics of monolayer adsorption and binding of molecules to SAMs $[52,53]$. The electrochemical quartz crystal microbalance (EQCM) allows one to study the transfer of ions and neutral species between the bathing electrolyte and monolayer via associated mass changes. This will be described in greater detail in chapter 2. Crystal impedance allows the study of the mechanical properties of the SAMs and will be described in detail in chapter 2 . Surface plasmon resonance (SPR) is a technique that monitors changes in the thickness of the monolayer that covers the electrode. It has mainly been used in the detection of large biomolecules such as DNA and protein immobilisation on SAM [54].

Thermally-programmed deposition (TPD) allows one to determine the thickness or coverage of monolayers. Metastable induced electron spectroscopy (MIES), nuclear magnetic resonance (NMR), neutron scattering (NS), and fluorescence spectroscopy $[55,56]$ are other techniques that have been used to study the properties of SAMs. Finally, techniques such as scanning tunnelling microscopy (STM) and atomic force microscopy (AFM) can look at the surface morphology of SAM modified electrodes [57, 58], whilst scanning electrochemical microscopy ( SECM ) provides valuable information about kinetic, charge transport and thermodynamic parameters [59]. 


\subsection{Immobilisation of Ligands onto SAM}

Since a SAM can serve as an interface layer between a gold metal surface and a species present in solution, selectivity offered by ligands can be profitably used for chemical sensing. More significantly, immobilisation of these ligands using SAMs requires only minimal amounts provided by the monolayer albeit maintaining their chemical activity. These ligands may be monodentate or polydentate, based upon optimisation of binding chemistry.

\subsubsection{Specific terminal functionalities}

Covalent attachment of ligands to the surface of the monolayer containing some free terminal groups like $-\mathrm{CH}_{3},-\mathrm{OH},-\mathrm{NO}_{2},-\mathrm{COOCH}_{3},-\mathrm{OCOC}_{6} \mathrm{H}_{5},-\mathrm{COOH},-\mathrm{OSO}_{3} \mathrm{H}$, $\mathrm{NH}_{2}$ is possible. The tail group that provides the functionality of the SAM, the stability, uniform surface structure and relative ease of varying the functionalities make SAMs particularly suitable for developing applications in the area of sensors. The self-assembled monolayers of alkane or aromatic thiols on gold are probably one of the best currently available surfaces for accomplishing the functionalization and patterning necessary for fabrication of sensors, with the advantages of flexibility and stability $[60,61,62]$. FTIR and Raman spectroscopy allow the investigation of the successful immobilisation of these ligands and can also be used to pick out functional groups $[63,64]$.

In recent years, it has been reported that many functional supramolecular systems including crown ethers, cyclodextrin derivaties, resorcinarenes and calixarenes $[65,66,67]$, can be immobilised on gold surfaces through specific chemisorption or self-assembly mechanisms. These studies are based upon optimisation of binding chemistry and molecular recognition. Over several years, many attempts with a variety of synthetic receptors have partially succeeded in fabricating functionalised electrode surfaces with selective affinity for organic species or metal ions [68]. Chemically sensitive monolayers are often prepared from receptors with long hydrophobic alkyl chains. Figure 1.4 shows some ligands using in chemical sensing. 


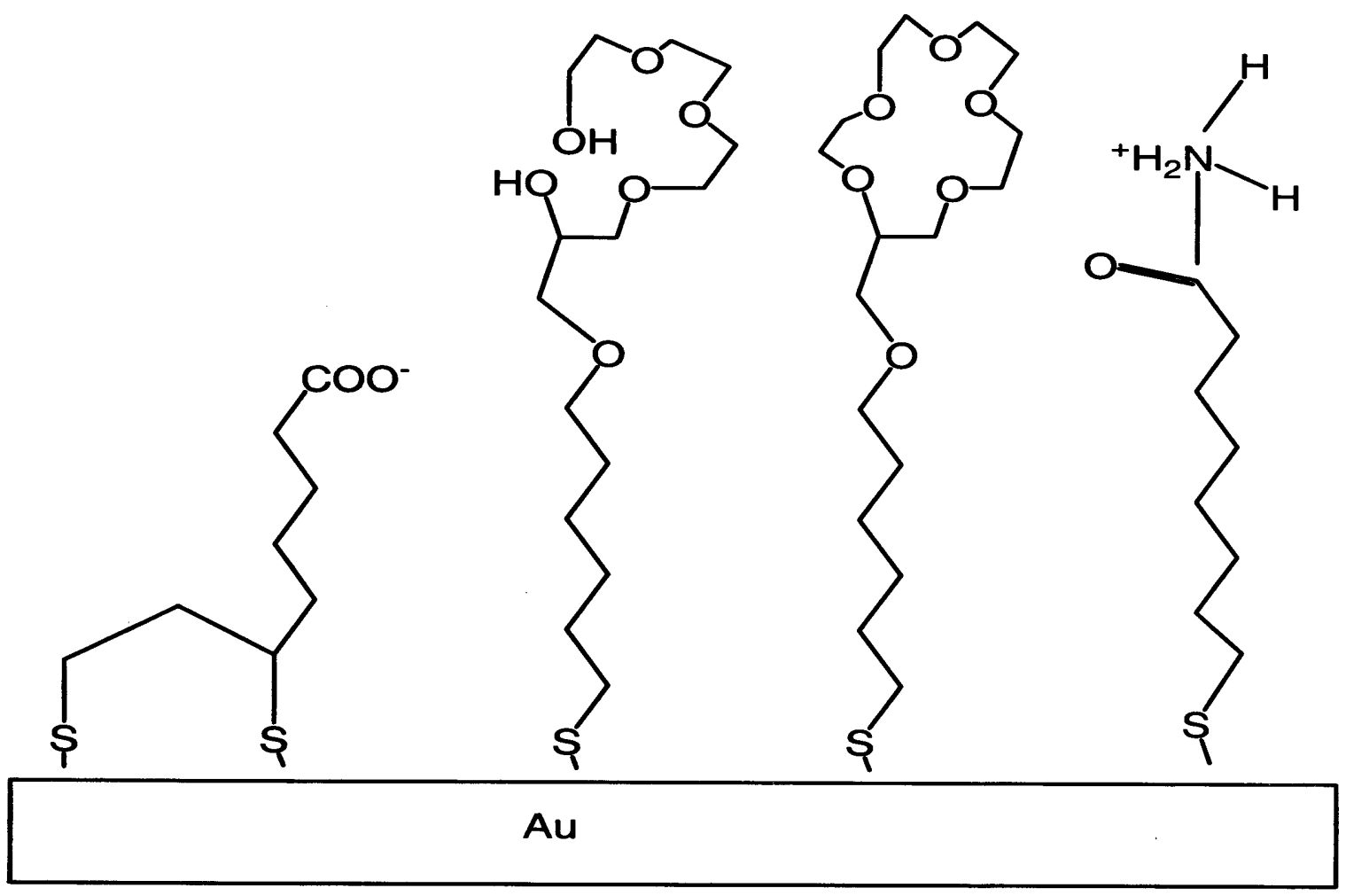

Figure 1.4: A schematic of some surface-attached ligands used to bind metal ions in solution $[65,66,67,68]$.

Recently the quartz crystal microbalance (QCM) has been used as a sensor for immobilisation of ligand functionalised SAMs on gold surfaces based on the mass change caused by the selective adsorption of these ligands [69, 70,71]. Most of the published papers concern aliphatic thiol-ligands. On the other hand, the behaviour of SAMs formed from thiols containing $\pi$ electron rich aromatic substituents may prove interesting. However, investigations of only a few such systems have been reported, including co-adsorption of short aromatic thiols along with alkane thiols $[72,73,74]$ and the influence of a polar aromatic group present in the middle of the aliphatic chain of alkane thiol [75]. Studies on SAMs of thiols containing a chain of aromatic rings reveal that a monolayer of aromatic ring containing a single benzene ring does not have a good stability. Further, the order and the stability of monolayer films is reported to increase with increasing the chain length. It has been found that thiols with fused aromatic rings can form compact and impervious monolayers despite the absence of a long methylene group. 


\subsection{Binding of Trace Metal lons with Terminal Self-Assembled Monolayers}

There have been a number of demonstrations of the applications of SAM based modifications of electrodes for the detection of both redox active and redox inactive metal ions in aqueous media. The modification of the electrode surface is designed to do one or more of pre-concentrate the metal ion, prevent fouling of the electrode, or enhance the selectivity of the interface for that metal ion. Pre-concentration implies that the metal ion partitions preferentially into the terminal-SAM or is attached to external surface, so that the analytical signal is enhanced. Preconcentration without designed binding sites depends on hydrophobic-hydrophilic SAM or electrostatic forces $[76,77]$. The ability to pre design the monolayer on a molecular basis, introducing specific functional groups and microenvironments makes the means of assembling electrodes involving SAMs an attractive method for metal ions, because the termini of SAM molecules are composed of only one type of functional group. The chelation specificity is a way to improve the selectivity, and to introduce multidentate or multi functional terminal groups $[78,79$, 80], so that the chelation discrimination between analytes and the interfering substances is enhanced. FTIR spectroscopy and the QCM [81, 82, 83] are techniques that enable the determination of metal ion binding to the terminal-SAM.

\subsubsection{Electroactive metal ions}

The first report of an ion-selective SAM was described by Rubinstein et al. [84]. They showed that a mixed SAM composed of 2,2-thiobisethyl acetoacetate (TBEA) and n-octadecylmercaptan (OM) on gold surfaces recognized selectively $\mathrm{Cu}^{2+}$ in the presence of other metal ions such as $\mathrm{Fe}^{3+}$. Selectivity was achieved by specific interactions through TBEA. Optimizing the system with a silane blocking agent, i.e. n-octadecyl trichlorosilane, instead of $O M$, enabled one to detect trace amounts of $\mathrm{Cu}^{2+}\left(10^{-7} \mathrm{M}\right), \mathrm{Pb}^{2+}\left(10^{-5} \mathrm{M}\right)$, and $\mathrm{Zn}^{2+}\left(10^{-9} \mathrm{M}\right)$, in the presence of $\mathrm{Fe}^{2+}$ or $\mathrm{Fe}^{3+}$. The first three ions readily form four-coordinate complexes, while iron cations prefer a six coordinate geometry. Highly selective ligands have been immobilized on gold electrodes and used for detection of electrochemically active ions by cyclic voltammetry. The selectivity is as much a consequence of the potential windows of the ion of interest as the selectivity of the ligand for the metal ion. Figure 1.6 shows of some ligands using for recognition of $\mathrm{Cu}^{2+}, \mathrm{Cd}^{2+}$, and $\mathrm{Cr}^{(\mathrm{VI})}$. 


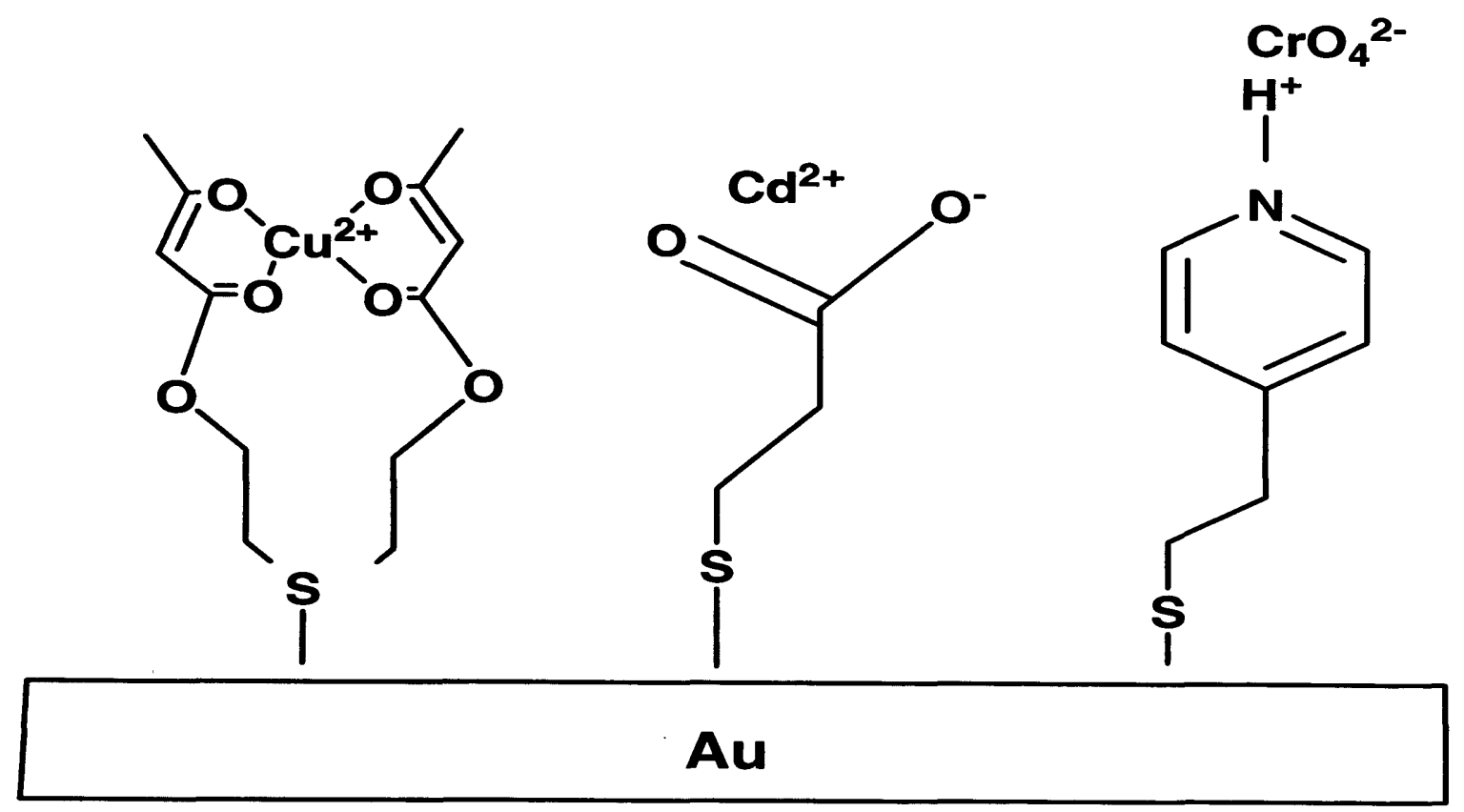

Figure 1.5: Ligands used for the voltammetric detection of metal ions.

Carboxylic acid-terminated SAMs (3,3-thiodipropionic acid) have also been used [85], to detect $\mathrm{Cu}^{2+}$ and $\mathrm{Ag}^{+}$. Although both metal ions are bound to the ligand, their different reduction potentials prevented problems of interference. The electrode interface was designed using a mixed SAM. The short chain 3,3thiodipropionic acid, which allowed close approach of the metal to the electrode, served as the recognition element while the function of decane thiol was to block direct access of the metal to the electrode, and to prevent fouling the electrode by proteins. Blocking of the electrode surface was also exploited by Stora et al. [86]. The metal-specific ligand, nitrotriacetic acid (NTA) was attached to a selfassembled monolayer of 11-mecaptoundecanoic acid. This interface was used to detect $\mathrm{Cu}^{2+}$ and $\mathrm{Ni}^{2+}$, two metals which have similar redox potentials, by monitoring a change in interfacial capacitance. Although both metals bind to the NTA, the affinity constant of copper with the ligand was approximately two orders of magnitude greater than the nickel-ligand complex. Overcoming the problem of multiple metal ions binding to the same ligand requires ligands with selectivity, which relies on more than electrostatic attraction, such as providing the appropriate number of coordinating sites and the correct size of the given metal ion [87]. Mirkhalaf et al. [88] studied the binding of heavy metal ions with ligands chemically attached to electrode surfaces. They studied dithiozone attached SAM to detect 
trace amounts of $\mathrm{Cu}^{2+}$ in solution. The characterisation of SAM-attached dithiozone was studied using cyclic voltammetry, surface and in situ FTIR spectroscopies. In contrast with other metal ions, the copper secondary complex of dithiozone showed strong Raman signals due to resonance Raman enhancement, of particular orientation effects from a comparison of the binding of pre formed complexes. Zhong [89] reported a study of the electroactivity of $\mathrm{Cu}^{2+}$ species with 11mercaptoundecanoic acid: concentrations of copper ion below $1 \mathrm{ppm}$ were detected.

The key drawback of using selective ligands is the need to design and synthesize a new ligand for each metal ion. Therefore, a simple generic synthetic strategy is required if arrays of metal ion sensors are to be developed. Gooding and Arrigan have addressed this issue by borrowing from nature by using amino acid ligands [90, 91, 92], and poly peptides [93] as selective ligands towards metal ions. A high degree of selectivity for $\mathrm{Cu}^{2+}$ with ultra low detection limits has been achieved. Glutathione and 3-mercaptopropionic acid were used to detect $\mathrm{Cu}^{2+}$. SAM modified electrodes show better sensitivity and selectivity for copper ions in solution in the range of $10 \mu \mathrm{M}$ to $0.1 \mathrm{mM}$ without pre-concentration [94]. Liu et al. [95] reported a detection limit of $0.39 \mathrm{nM}$ for $\mathrm{Cu}^{2+}$ employing Osteryoung square wave voltammetry (OSWV) at a cysteine monolayer-coated gold electrode. Detection of copper ion down to $10^{-7} \mathrm{M}$ was possible with cyclic voltammetry conducted by Arrigan and Bihan at an L-cysteine monolayer-coated gold electrode [90]. Heineman et al. [96] used underpotential deposition with 3-mercaptopropionic acid used stripping methods to obtain separte peaks in the analysis of mixtures of $\mathrm{Cu}^{2+}$ and $\mathrm{Pb}^{2+}$. The anodic peak potential shifts and the peak shape changes, indicating that the SAM changes the deposition and stripping steps of these heavy metal ions. Kim et al. [97] reported that self-assembly of a squarylium dye containing an alkanethiol on a gold surface gave high selectivity for $\mathrm{Cu}^{2+}$. Using a surface plasmon resonance sensing system, the detection limit is $10^{-12} \mathrm{M}$.

A few reports have shown the detection of low levels of $\mathrm{Cd}^{2+}$ using ligand attached SAM. Turyan and Mandler [98] developed an electrode for determining cadmium ions based on mercaptocarboxylic acid monolayers on gold electrodes. Optimisation of the parameters that govern the sensitivity and the selectivity resulted in a detection limit of $10^{-12} \mathrm{M}$ with a relatively high selectivity. The method has been verified by determining cadmium in standard solutions and it has been successfully applied to the analysis of cadmium ions in sea water. Figure 1.6 shows carboxylic acid terminated SAMs used to detect $\mathrm{Cd}^{2+}$, varying the length of alkyl chain i.e. $n=1,2,5$ and 10 of the monolayers adsorbed on gold. 
Electrochemical detection was based on cyclic voltammetry and Osteryoung square wave voltammetry to identify redox peaks. Stora and Torres [86] have reported the self-assembled nitrotriacetic acid modified thio-alkane monolayer detection of cadmium ions from solution monitored by impedance spectroscopy via a capacitance change of the blocked gold electrode. Figure 1.6 shows how the cadmium ions bind to the monolayer.
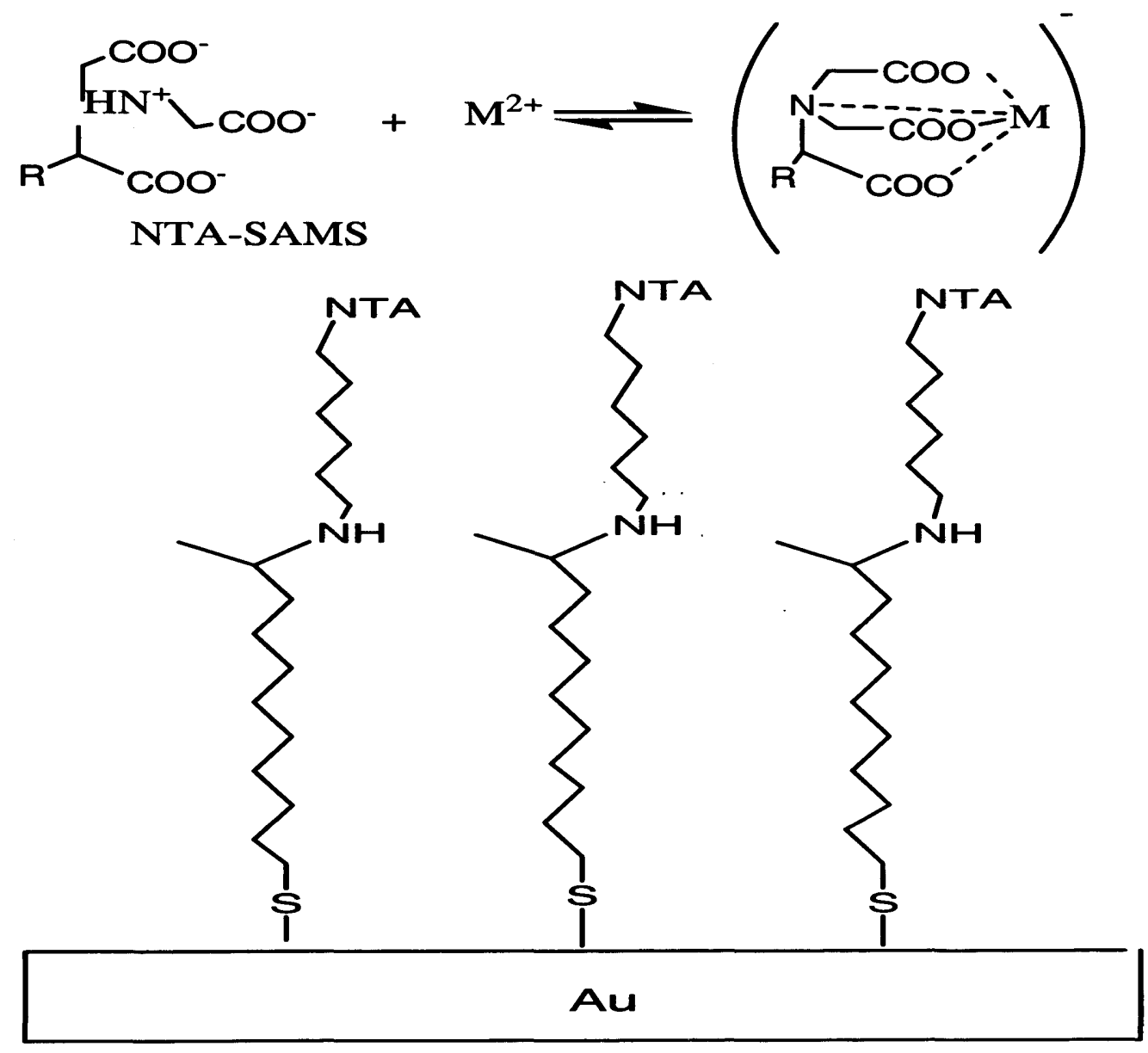

Figure 1.6: Schematic view of a thio-NTA monolayer on gold electrode and chemical equilibrium describing binding of cadmium ion to NTA groups.

Sastry et al. [99] reported that SAMs of a small aromatic bifunctional molecule, 4-carboxythiophenol, formed on gold electrodes have been used to bind cadmium ions and lead ions at the surface of the SAMs. Quartz crystal microbalance (QCM) measurements performed ex situ have been used to investigate the $\mathrm{pH}$-dependent, counter ion surface-interaction of $\mathrm{Cd}^{2+}$ and $\mathrm{Pb}^{2+}$ ions as well as the kinetics of ion binding to the SAM surface. 
Turyan and Mandler [100] have developed a selective electrode for chromium (VI) based on a self-assembled monolayer of 4-mercapto-n-alkyl pyridinium monolayers on gold surfaces. The detection limit for $\mathrm{Cr}^{\mathrm{VI}}$ was 1 part per trillion using a pyridinium monolayer and square wave voltammetry for detection. One of the major advantages in applying SAMs in electroanalytical chemistry stems from their relatively high organisation, which makes it possible to correlate the analytical performance and structure at the interface. They used XPS and FTIR to characterise monolayers at the solid-liquid interface, and applied these ligands to real sample analysis. Figure 1.5 shows how the chromate ions bind to the monolayer. They showed that the determination of $3.2 \times 10^{-10} \mathrm{M}$ is not influenced by a $10^{4}-10^{9}$ fold excess of added $\mathrm{Fe}^{3+}, \mathrm{Cu}^{2+}$ and $\mathrm{Ag}^{+}$.

Jung et al [101] reported the interaction between $\mathrm{Cr}^{\prime \prime \prime}$ and $\mathrm{Cr}^{\mathrm{VI}}$ and 16mecaptohexadecanoic acid monolayers; they studied the binding sites of chromium ions to the ligand using $X$-ray photoelectron spectroscopy. Ammonium pyrrolidine dithiocarbamate was used by Arcos et al. [102] to determine $\mathrm{Cr}^{\mathrm{Vl}}$ in aqueous media: the detection limit obtained was $1.09 \times 10^{-9} \mathrm{M}$. They used differential pulse adsorptive stripping voltammetry (DPASV) for selective determination of $\mathrm{Cr}^{\mathrm{IV}}$ in the presence of $\mathrm{Cr}^{\prime \prime \prime}$ at $\mathrm{pH}=3$. Recently Thundat et al [103] have developed micro cantilevers modified with SAMs that respond selectively to specific ion concentrations. They have reported the detection of trace amounts of $\mathrm{CrO}_{4}{ }^{2-}$ using micro cantilevers modified with SAMs of triethyl-12-mercaptododecyl ammonium bromide. It was found that a concentration of $10^{-9} \mathrm{M}$ of chromate ions can be detected using this technology in a flow cell.

Yegnaraman et al. [104] had attempted the application of 2-mercaptobenzimidazole SAM for the determination of $\mathrm{Hg}^{2+}$. The detection limit was shown to be $1 \mathrm{nM} \mathrm{Hg}^{2+}$ using anodic stripping voltammetry. Another method for detection of mercury ion was developed recently by Thundat et al. [105], this method used micro cantilevers modified with 1-dodecanethiol SAMs coated with gold electrode. The micro cantilever undergoes bending due to accumulation of $\mathrm{Hg}^{2+}$ on the gold surface. It was found that a concentration of $10^{-11} \mathrm{M}$ of mercury ions can be detected. Other cations such as $\mathrm{Pb}^{2+}, \mathrm{Zn}^{2+}, \mathrm{Ni}^{2+}, \mathrm{Cd}^{2+}$ and $\mathrm{Cu}^{2+}$ have little or no effect on the determination. He et al. [106] have reported a novel calix-4arene derivative containing benzothiazole coated on glassy carbon electrode and then applied this to the recognition of mercury ions. Cyclic and square wave voltammetry results showed that the modified electrode selectively recognizes mercury ions in aqueous media. This modified electrode does not present any significant interference from alkali, alkaline earth and transition metal ions except 
for $\mathrm{Pb}^{2+}, \mathrm{Ag}^{+}$, and $\mathrm{Cu}^{2+}$ ions. They used the SAM modified electrodes on gold to detect lead ions in solution.

Arrigan et al. [107], have reported that a SAM of 3-mercaptopropane sulfonate (MPS) on polycrystalline gold electrodes can be used to determine $\mathrm{Pb}^{2+}$ ions using the underpotential deposition current. $1 \mu \mathrm{M}$ of lead ions could be detected in aqueous media using square wave voltammetry. This work has implications for analytical applications for monolayer coated gold electrodes. The covalent attachment of 1,5-bis(4-carboxyphenyl)thiocarbazone a useful ligand for the extraction and spectrophotometric determination of $\mathrm{Pb}^{2+}$ on gold electrode surface has been discussed [108]. Although, the geometry of dithizonate complexes is still controversial, it is known that dithizone in solution forms two kinds of complexes with lead ions, primary and secondary. The former contains a $2: 1$ ligand to metal ion ratio whereas the latter has a $1: 1$ ratio. Secondary complexes are preferentially formed at high metal to ligand concentration ratios at high values of $\mathrm{pH}$.

Turyan and Mandler [109] studied monolayers of mercaptodecanesulfonic acid. A gold electrode modified with 10-mercapto-1-decanesulfonic acid allowed the selective detection of $\mathrm{Fe}^{2+}$ in the presence of a range of organic species which interfere with $\mathrm{Fe}^{2+}$ oxidation at a bare gold electrode. Both $\mathrm{Fe}^{2+}$ and $\mathrm{Fe}^{3+}$ have been detected in aqueous systems, the latter being in the $\mathrm{nM}$ range for detection.

Silver ions can be accumulated from aqueous solution using SAM modified electrodes. Zheng et al. [110], have reported that $\mathrm{Ag}^{+}$can be detected chemically at a DL-dithiothreitol (DTT) SAM modified gold electrode with a potentiodynamic control function; it exhibits a pair of redox waves at about $0.23 \mathrm{~V}$. When this $\mathrm{Au} /$ DTT electrode was further modified with dodecylmercaptan, more silver ions can be accumulated and the peak grows. Conditions, such as $\mathrm{pH}$ and supporting electrolyte, were optimized for $\mathrm{Ag}^{+}$determination: the dynamic range is $0.6-2.4 \mu \mathrm{M}$ using cyclic voltammetry as the detection technique.

There are few studies on $\mathrm{Ni}^{2+}, \mathrm{Zn}^{2+}, \mathrm{Co}^{2+}$ and $\mathrm{Mn}^{2+}$ determination using SAM modified electrodes e.g. Kalinina et al. [111], reported that the complexation of N,N-dicetyl-1,4,7,10-tetraaza-cyclododecane (dicetyl cyclen) with transition metal ions in such as $\mathrm{Ni}^{2+}, \mathrm{Zn}^{2+}, \mathrm{Ag}^{+}$and $\mathrm{Mn}^{2+}$, and their mixtures. It was established that the selectivity of the interaction of the monolayer composed of this ligand with transition metal ions is determined by the subphase $\mathrm{pH}$ value. It was disclosed that, in the acidic region of subphase $\mathrm{pH}$ values, dicetyl cyclen in the monolayer accumulated predominantly the $\mathrm{Ni}^{2+}$ ions from solutions containing $\mathrm{Cu}^{2+}$ and $\mathrm{Ni}^{2+}$ 
ions. Caneto et al. [112] have reported that dioctadecyl dithiocarbamate (DODTC) forms stable dense and compact monolayers at the water-air interface. The DODTC molecule adopts an interfacial conformation with the dithiocarbamate group almost parallel to the interface. When $\mathrm{Ni}^{2+}$ is present in the subphase, this specific orientation of the polar heads groups favours the formation of $1: 2$ complexes between the nickel ion and two molecules of DODTC.

Most of the published reports study aliphatic thiols to detect trace metal ions in solution. On the other hand, aromatic thiols have been used in a few reports to detect trace metal ions in solution. Figure 1.7 shows how one can use the aromatic SAM ligands to detect $\mathrm{Hg}^{2+}$ in aqueous media.

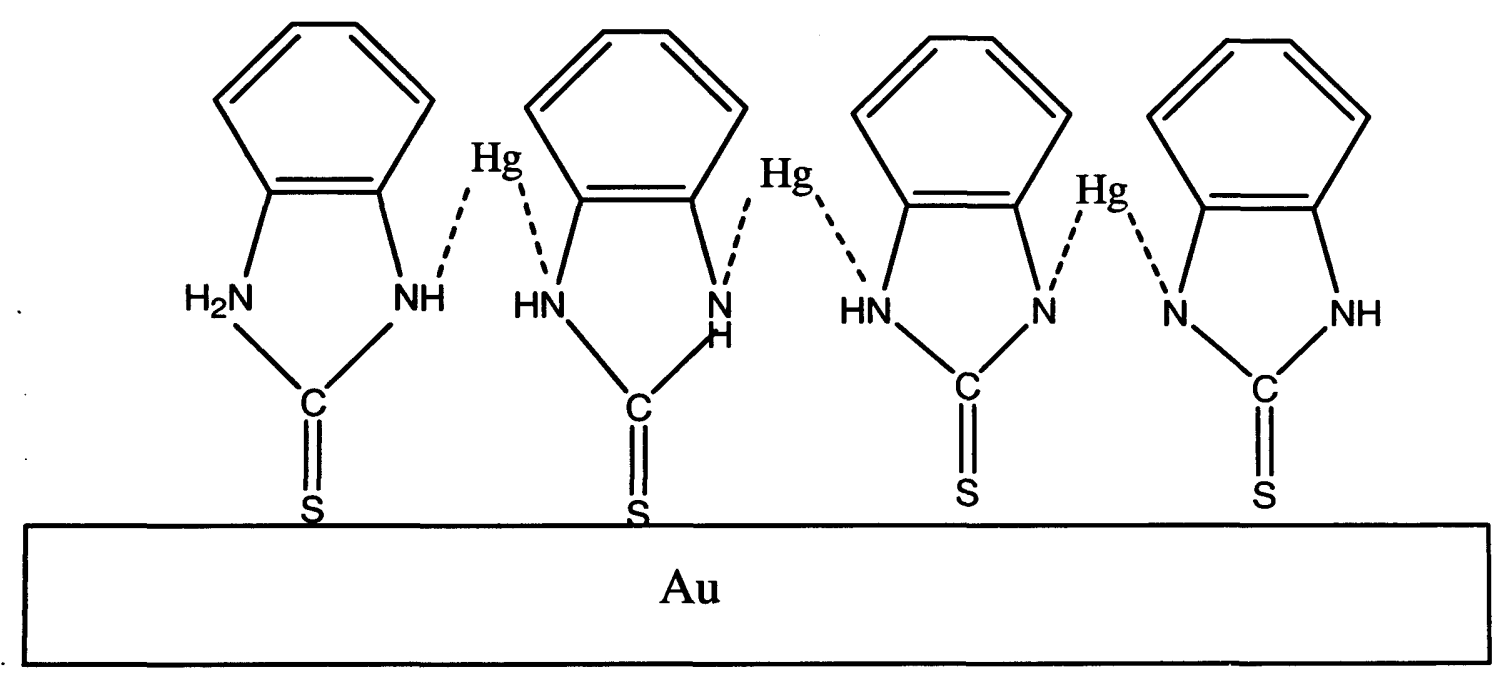

Figure 1.7: A schematic for formation of the self-assembled monolayer of 2-mercaptobenzimidazole (MBI) on $\mathrm{Au}$ and non-electrolytic pre-concentration of $\mathrm{Hg}$ at a $\mathrm{Au} / \mathrm{MBI}$ electrode.

\subsubsection{Electroinactive metals}

Crown ethers are well known compounds that are able to complex cations from aqueous environments. An important factor that influences the selectivity of the receptor is the relation between the cation diameter and diameter of the cavity. In addition to the geometric fitting of host and guest, the desolvation energy of the metal ion also has a significant effect on the stability of the complex. The functionalization of several crown ethers with an alkanethiol substituent to detect alkali and alkaline metal ions has been studied. Some exceedingly clever approaches have been employed in the detection of non-redox active metal ions using several functionalizations of several crown ethers with SAMs. 
Flink et al. [113] and Yang et al. [114], have described synthetic methods to prepare aliphatic alkanethiols terminated in crown ethers. The binding of the sodium ions with 12-crown-4 or potassium ions with 15-crown-5 as the crown ether was transduced using $\mathrm{Ru}\left(\mathrm{NH}_{3}\right)_{6}{ }^{2+/ 3+}$ as a reporter ion. In the absence of the metal ion the reporter can penetrate the SAM and show redox activity. Once the metal ion is bound, the interface has a net positive charge and the selectivity of the interface for a metal ion can be manipulated by altering the density of the crown ethers in the recognition interface. Diluting the receptor surface concentration by increasing the concentration of a dilute thiol prevents the sandwich complex forming with a concomitant reduction in the selectivity for $\mathrm{Na}^{+}$and $\mathrm{K}^{+}$. Mass sensitive devices (e.g. quartz crystal microbalance), cyclic voltammetry and impedance spectroscopy have been especially successfully applied [115,116]. Figure 1.8 shows complexation of potassium ions using SAM-attached crown ethers.

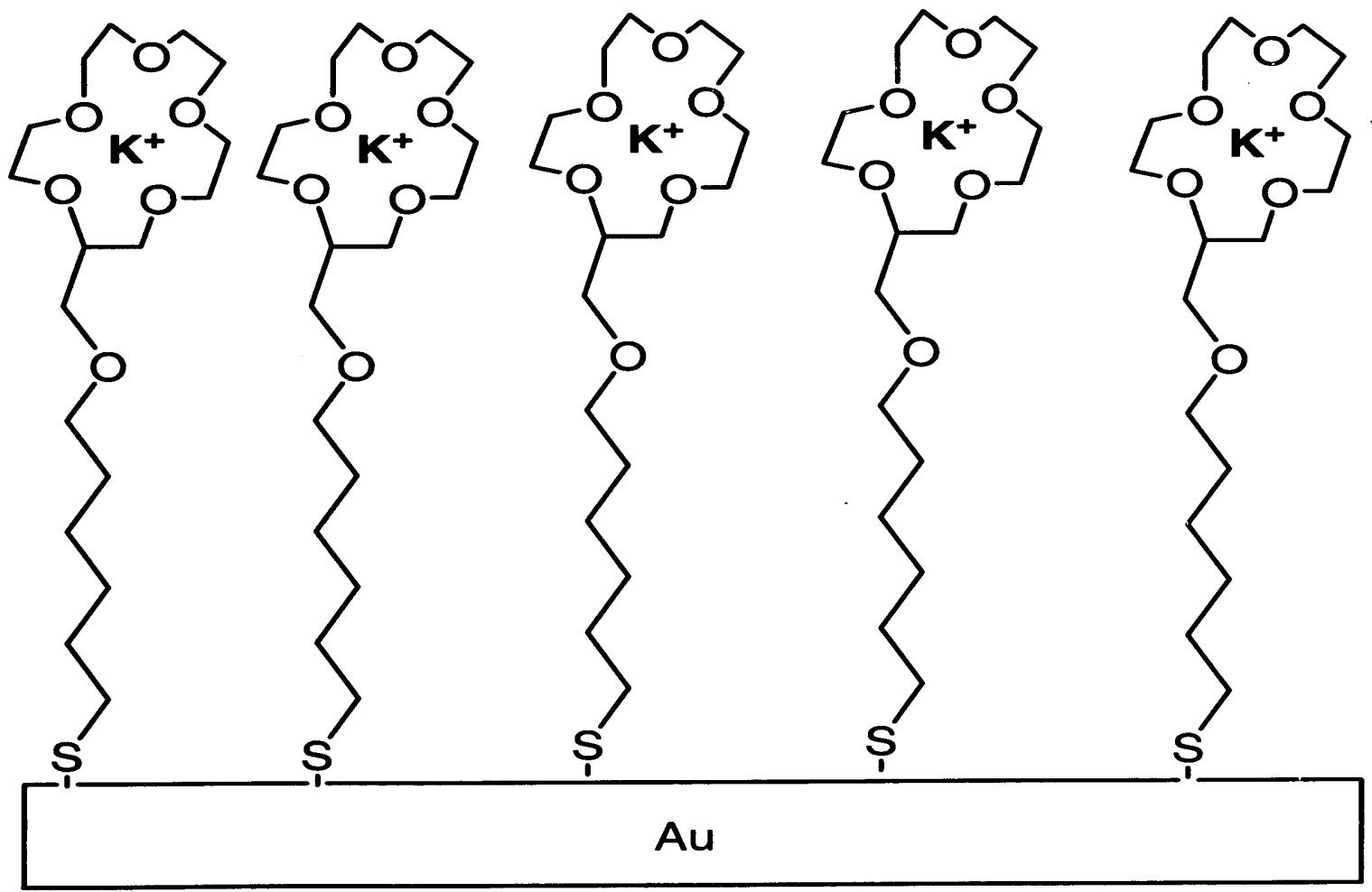

Figure 1.8: Complexation of potassium ions using SAM-attached crown ethers.

Bandlyopadhyyay et al. [117] used bis-thioacetic acid derivatives of oligo-ethylene glycols as ligands for detection of $\mathrm{Na}^{+}$and $\mathrm{K}^{+}$. The oligo-ethylene glycol acted as linear crown ether. An oligo-ethylene with three ether oxygens and two ester 
groups was shown to be selective for sodium ions while four ether oxygens and two ester groups was selective for potassium ions. $\mathrm{Ru}\left(\mathrm{NH}_{3}\right)_{6}{ }^{+2 /+3}$ was used as the reporter ion and the increase in charge transfer resistance with high metal ions concentrations was used to transducer the recognition. A coupling of redox species with a ligand receptor in the same molecule has been used to detect inactive metal ions by Moore et al. [118] and Liu et al [119]. In both cases tetrathiafulvalene (TTF) based SAMs, were synthesized with crown ethers attached. The binding of metal ions to the SAM causes a change in the redox properties of the TTF. Normally TTF exhibits two one-electron redox couples. In the example by Moore et al. [118] the crown was selective for silver ions. Responses were also observed for $\mathrm{Li}^{+}, \mathrm{K}^{+}, \mathrm{Na}^{+}$ and $\mathrm{Ba}^{++}$but to a lesser extent than $\mathrm{Ag}^{+}$. An interesting aspect of the molecule fabricated by Liu et al. [119] is that each TTF has two thioacetic acid moieties attached, thus providing four attachment points to the gold. These SAMs have excellent long-term stability for determination of $\mathrm{K}^{+}, \mathrm{Na}^{+}$and $\mathrm{Li}^{+}$.

Kimura et al. [120] have reported SAMs having a helical peptide with crown ether units and successful electrochemical detection of alkali metals such as potassium ions, the detection limit is about $10^{-5} \mathrm{M}$. Two types of helical peptide self-assembled monolayers are prepared by connecting either $\mathrm{N}$-terminal or $\mathrm{C}$-terminal to a gold surface. The recognition of cations in these helical peptide SAMs is detected by electrochemical measurements such as cyclic voltammetry and impedance spectroscopy.

The incorporation of more than one functionality in the same molecule has also been demonstrated by Chung et al. [121] where the redox reporter used to transduce metal binding is also the receptor ligand. A redox active calixarene was synthesized with two thiol moieties to allow self-assembly formation. The calix[4]arene was selective to $\mathrm{Ba}^{2+}$. The complexation of barium ions with calix[4]arene produced an electroactive species with a reduction potential anodic of the barium-free calixarene. Therefore, the relative ratio of the two peaks in squarewave voltammograms represents the proportion of calixarenes that have a $\mathrm{Ba}^{2+}$ guest. The calixarene based sensor also responded to $\mathrm{Sr}^{2+}$ and $\mathrm{Ca}^{2+}$ but the selectivity coefficient for $\mathrm{Ba}^{2+}$ over $\mathrm{Sr}^{2+}$ was 100 and for $\mathrm{Ba}^{2+}$ over $\mathrm{Ca}^{2+}$ was $>500$. The metal ions $\left(\mathrm{Ba}^{2+}, \mathrm{Ca}^{2+}, \mathrm{Sr}^{2+}\right)$ could be removed from the host using EDTA to regenerate the sensing surface.

A variety of other approaches where SAM modified electrodes were used for the detection of electroinactive metal ions have been developed such as ionchannel mimetic sensors. Umezawa et al [122] have reported chemical modification of gold electrodes with phosphoric acid ester attached to the gold 
surface by thioethyl groups and designed to mimic ion-channel membranes. The selectivities of such sensors toward alkaline earth metals and trivalent ions such as $\mathrm{La}^{3+}$ and $\mathrm{Al}^{3+}$ are described. The sensor responded to $\mathrm{Ca}^{2+}$ and trivalent cations $\mathrm{La}^{3+}$ and $\mathrm{Al}^{3+}$ at concentrations as low as $10^{-7} \mathrm{M}$. Cyclic voltammograms of $\mathrm{Fe}(\mathrm{CN})_{6}{ }^{3-}$ as electroactive marker were measured in the presence of various types of analyte cations.

A SAM-ion selective membrane hybrid approach by Fibbioli et al. [123] is particularly novel. In the development of solid-state ion selective electrodes the potential stability is often poor because of poorly defined redox reactions at the metal surface and the formation of a thin aqueous layer between the metal and the membrane. Echegyen et al. [124] used cyclic and acyclic polyether self-assembled monolayers on gold electrodes to detect sodium and potassium ions at ppm levels. Impedance spectroscopy data for these SAMs fit the Langmuir isotherm and allow determination of ion association constants of sodium and potassium ions.

Electrochemical impedance spectroscopy has also been used by Jorefors et al. [125] to evaluate the change in interfacial capacitance upon calcium and magnesium coordination to a phosphate modified SAM electrode. It was also shown that the interaction must take place close to the gold surface, for example, by using a short alkane.thiol. Recently Ji et al. [126] have reported a new sensor concept based on an ion-selective SAM modified microcantilever which can detect caesium ion concentrations in situ in the range $10^{-11}$ to $10^{-7} \mathrm{M}$ and shown its potential for use in developing a new family of real time in situ metal ion sensors with high selectivity and sensitivity for chemical and biological applications. Figure 1.8 shows complexation of potassium ions using SAM-attached crown ethers. 


\subsection{Sensors}

\section{Sensor}

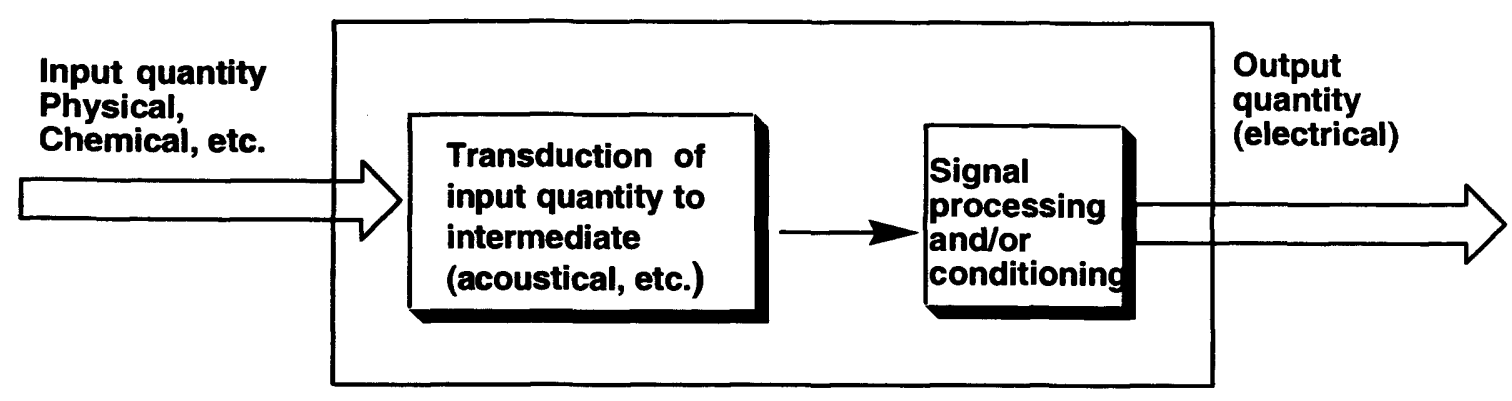

Figure 1.9: Schematic diagram of a sensor that produces an electrical output in response to the presence of an input quantity.

Sensors are analytical devices than are capable of monitoring chemical or physical changes within a specific environment. In the majority of sensors, the output signal is electrical. The range of input quantities is extremely large, ranging from physical quantities e.g., mechanical properties of thin films, to chemical and biological quantities, e.g., concentration and identities of unknown species in air and liquid media. Figure 1.9 shows the general mechanism for a typical sensor.

\subsubsection{Applications}

Sensors have an important role to play in today's world. For example, sensors can be used to warn of the presence of a toxic substance or combined with other signals to provide a physician with information on which to base a medical decision. Sensors can be classified into different types depending on the mechanism of the analyte recognition. Electrochemical, optical, thermal and mass sensors can be easily identified depending on the type of signal used for gathering information about the analyte. It would be impossible to cover the range of these sensors in this literature as they number well into the thousands. For the purpose of this study a selective overview covers electrochemical sensors and acoustic wave sensors capable of analysing low levels of trace ions in solution, including electrochemical sensors using SAMs. Self-assembled monolayer modified electrodes are superb candidates for application as electrochemical sensors [127]. Acoustic wave sensors are described in section 1.7.3. For further information on sensors and their application, the reader is referred to the literature [128]. 


\subsubsection{Characterisation of Sensors}

Different sensors will require different specification depending on what the user wishes to monitor. These can be divided in terms of three parameters:

(1) Sensitivity: this is a measure of the magnitude of output signal produced in response to an input quantity of given magnitude.

(2) Resolution: this is a measure of the minimum change of input quantity to which they can respond.

(3) Selectivity: this characterises the degree to which they can distinguish one input quantity from another. It can be defined via the ratio of the responses to two different species present at the same concentration.

\subsubsection{Acoustic Wave Devices [129, 130, 131]}

Acoustic wave (AW) devices have been used since the mid-1960's and are ideally suited to thin film characterisation due their extreme sensitivity to thin film properties. Not only are they cheap to produce and small, but they can monitor a whole range of physical properties (e.g. mass, viscoelasticity, density, conductivity, etc.) for many different chemical systems. Another advantage of these devices is that they can be used in situ during an electrochemical experiment. This makes acoustic wave devices very attractive and versatile tools to use.

Although bulk properties of most materials are either known or relatively easy to determine, it is not realistic to assume that thin film materials will possess the same characteristics. AW devices are therefore uniquely suited to the direct characterisation of thin film materials.

There have been a number of different types of acoustic wave devices that have been used for sensor applications and material characterisation. They all consist of a piezoelectric substrate.

\subsubsection{Thickness-Shear Mode (TSM) Device}

The thick-shear mode (TSM) device is the most commonly used acoustic wave sensor amongst electrochemists for the study of chemically modified electrodes [132]. In this field, it is commonly referred to as the quartz crystal microbalance (QCM) and this term will be used throughout this report.

The QCM is a piezoelectric device that is commonly used as a sensitive gravimetric monitor. It is sensitive enough to monitor mass changes as low as $1 \mathrm{ng} \mathrm{cm}^{-2}$ that translate into hundredths of a monolayer of atoms and average film thickness of 
hundredths of nanometers. An in-depth theoretical description of the QCM will be found in Chapter 2.

Electrochemical systems on the QCM generally involve the exchange of ions and small molecules between a surface film and its bathing medium. This is widely used as the basis of a sensor. This strategy has been applied for self-assembled monolayers immobilised on electrode surfaces and exposed to liquid media. The QCM allows one to quantify the uptake of the target species.

\subsubsection{Applications of the QCM in analysis of trace metal ions}

The applications of QCM for mass sensing in liquids has been reviewed $[133,134,135,136,137,138]$. Gomes et al $[139,140141]$ reported sensors based on QCM have been applied to metal ion analyses in solution using electrodeposiotion, adsorption and stripping methods. Figure 1.10 shows the mechanisms involved in metal ion sensing. on the QCM. Price et al [142] used piezoelectric quartz resonators coated with chemically engineered copolymer resins for the selective detection of lithium, potassium and sodium ions in aqueous solutions. The detection limit was estimated at $0.4 \mathrm{ppm}$ for potassium ions.

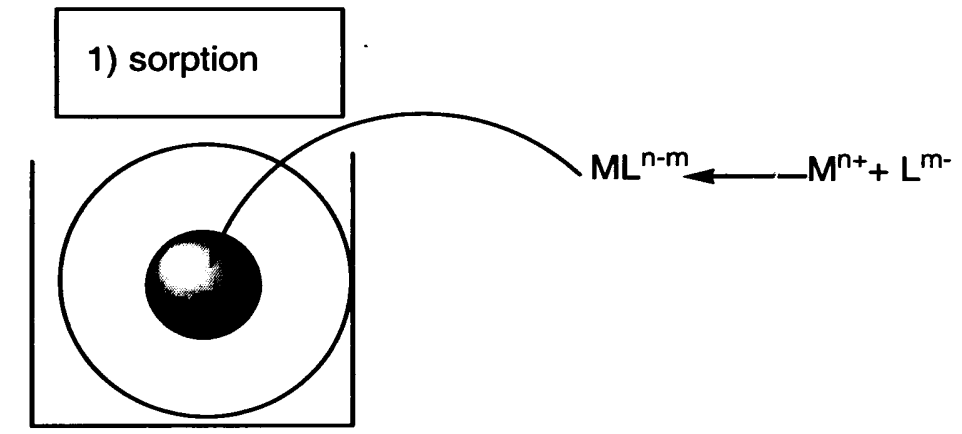

coated crystal use or uncoated crystal is also possible

\section{2-a) electrdeposition}

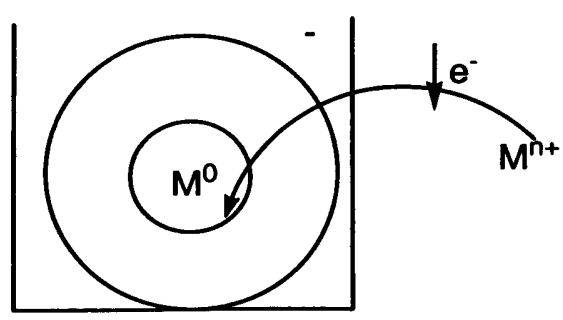

negative $\delta F$ negative $\delta F$

2-b) stripping

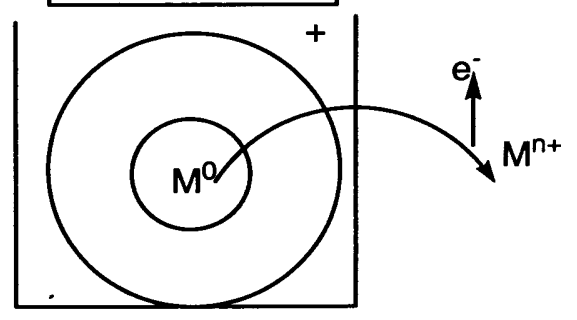

positive $\delta F$

Figure 1.10: Schematic representation of the mechanisms involved in metal ion sensing ( $M=$ Metal ion, $L=$ Ligand). 
Matthew et al. [143] described a gold surface on the QCM modified by attachment of silica particles derivatised with $\mathrm{N}$-[3-trimethoxysilyl propyl] ethylenediaminetriacetic acid. The device was employed to detect trace metal ions such as $\mathrm{Pb}^{2+}$ and $\mathrm{Ag}^{+}$from aqueous solutions. Recently Port et al. [144] used the QCM as a sensor for the detection of metal cations in particular actinide metal ions, combined with ligands having a high affinity towards cations of specific valence state. I will make new combination of SAM modified gold electrodes on QCM surfaces to detect trace metal ions in solution. One objective of our research is to combine the selectivity, conferred by the molecular recognition properties of SAMs with sensitivity and capability of the QCM. We exploit this by modifying the gold electrode (on a $10 \mathrm{MHz}$ AT-cut piezoelectric quartz crystal resonator) with different structures of SAMs.

\subsection{Aims and Objectives}

Heavy metals such as cadmium, lead, mercury, chromium, arsenic and antimony pose a real threat to living systems. The development of sensitive probes and techniques for their determination is of great important and interest to varying fields.

Currently several methods are commonly employed for trace metal analysis e.g. atomic absorption spectroscopy, inductively-coupled plasma-optical emission spectroscopy, electrochemical methods and liquid chromatography with electrochemical or fluorescence detectors. These techniques are both expensive and bulky. In my study I will use the quartz crystal microbalance modified with selfassembled monolayers attached with ligands to determine trace metal ions in solution and develop electrochemical - acoustic wave sensors capable of analysing aqueous solutions for low levels of trace metal ions in aqueous media of environmental relevance.

The method should be capable of detecting trace metal ions at (ppb-ppm) levels and should offer the possibility of determing both electroactive and electroinactive ions. If possible, this method should, through appropriate chemical variation, have the capability to select individual species in mixtures and obtain total levels of metal ions in mixtures. It is based upon a pre-concentration.strategy involving surface immobilised ligands and combined with trace metal ions (self-assembled monolayers with specific terminal functionalities) towards binding trace metal ions in aqueous solution. Selectivity for different metal ions or groups of metal ions will be based upon appropriate choice of ligand. For electroactive ions, detection will be coulometric and gravimetric based upon integration of voltammetric responses of 
surface-bound complexed metal ions. For electroinactive ions detection will be gravimetric, based on quartz crystal microbalance determination of the increase in surface-attached mass associated with complexation of the metal ions by surface bound ligands. One can use this method for both electroactive and electroinactive metal ions.

I will divide my work into three parts:

1) Surface functionalization: selected ligands will (after additional functionalization if necessary) be bound to the surfaces of gold electrodes. I will use self-assembled monolayer attached ligands bound to the gold surface on the QCM cell producing a mass change of the QCM-monolayer composite resonator (QCM-SAM-ligand).

2) Metal ion complexation and determination: functionalised electrodes mounted on quartz crystal resonators will be exposed to metal ion solutions (single and mixed ions) and their capabilities for electrochemical and acoustic wave detection determined. The selectivity of the SAM modified electrodes towards target metal ions will be studied by estimating the binding constants in single and mixed ion solutions after fitting the data to different isotherms.

3) We will use X-ray photoelectron spectroscopy (XPS) to probe the nature of the adsorbed metal ion, its coordination environment, oxidation state, bonding state of the $S$ atoms of the SAM and that of the underlying SAM as a function of electrode potential. We hope to learn more about the preconcentration equilibria and metal speciation at SAMs exposed to dilute aqueous solutions of trace metal ions.

\subsection{References}

1) P. R. Moses, L. Wier, and R. W. Murray, Anal. Chem., 47, (1975), 1882.

2) R. W. Murray, Anal. Chem, 59, (1987), 379A.

3) J. M. Zen, A. S. Kumar and D. M.Tasi, Electroanalysis., 15, (2003), 1073.

4) A. Ulman, Chem. Rev, 96, (1996), 1533.

5) H. O. Finklea, self-assembled monolayers on electrodes: Encyclopedia of Analytical Chemistry, John Wiely and Sons Ltd, Chichester 1997.

6) A. R. Bishop and R. G. Nuzzo, Curr Opin Colloid Interface Sci., 1, (1996), 127.

7) F. Schreiber, Prog. Surf. Sci., 65, (2000), 151.

8) C. M. Knobler and D. K. Schwartz, Curr. Opin colloid interfaces., 4, (1999), 46.

9) J. J. Gooding, F. Meanrns, W. Yang and J. Liu, Electroanalysis.,15 , (2003), 81.

10) G. E. Poirier, Chem. Rev., 97, (1997), 1117.

11) R. G. Nuzzo and D. L. Allara, J. Am. Chem. Soc., 105, (1983), 4481. 
12) C. D. Bain, J. Evall and G. M. Whitesides, J. Am. Chem. Soc., 111, (1989), 7155.

13) L. H. Dubois and R. G. Nuzzo, Annu. Rev. Phys. Chem., 43, (1992), 437.

14) A. Ulman, Thin Films: Self-Assembled Monolayers of Thiols, 1998, Vol 24.

15) N. Sandhyarai and T. Pradeep, Int. Reviews in Physical. Chemistry, 22, (2003),221.

16) T. Kawaguchi, H. Yasuda and K. Shimazu, Langmuir., 16, (2000), 9830.

17) L. T. You, J.Y. Gui, S. Dong and E. Wang, Electroanalysis., 11, (1999), 1112.

18) C. D. Bain, E. B. Troughton, Y. T. Tao, J. Evall, G. M. Whitesides and R. G. Nuzzo, J. Am. Chem. Soc., 111, (1989), 321.

19) T. A. P. Rocha, M. T. S. R. Gomes, A. C. Duarte and J. A. B. P. Oliveira, Anal. Commun, 35, (1998), 415.

20) S. Dazhong, K. Qi, Z. Xiaoli, W. Lizeng and M. Chengsong, Anal. Commun., 34, (1997), 97.

21) T. W. Schneider and D. A. Buttry, J. Am. Chem. Soc.,115, (1993), 12391.

22) J.J.Hickman, D.Ofer, P.E. Laibinis and M.S.Wrighton, Science., 252, (1991), 688.

23) J. Park, S. Kang, T. D. Ching, S. Kyachang and H. Kim, Microchemical . Journal., 68, (2001), 109.

24) N. K. Chaki and K.Vijayamohanan, Biosensors and Bioelectronics., 17, (2002), 1.

25) T. Wink, S.G.V.Zuilen, A.Bult and W. P. V.Bennekom, Analyst., 122, (1979), 43A.

26) J.J. Gooding and D. B. Hibbert, Trends Anal. Chem., 18, (1999), 525.

27) M. Aslanoglu. A. Houlton and B. R. Horrocks, Analyst., 123, (1998), 753.

28) I. Rubinstein, J. Rishpon, E. Subatani, A. Redondo and S. Gottesfeld, J. Am. Chem. Soc., 112, (1990), 6135.

29) A. Kumar, H. A.Biebuyck, N. L.Abbott and G. M. Whitesides, J. Am. Chem. Soc., 114, (1992), 9188.

30) Z.Wang, M. J.Cook, A. M.Nygard and D. A.Russell, Langmuir, 19, (2003), 3779.

31) E. Sabatani and I. Rubinstein, J. Phys. Chem, 91, (1987), 6663.

32) S. Berchmans, V. Yegnaraman and G. P. Rao, J. Solid State Electrochem., 3, (1998), 52.

33) A. E. Kaifer, Israel J. Chem., 36, (1996), 389.

34) V. M. Mirsky, Trends Anal. Chem., 21, (2002), 439. 
35) V. M.Mirsky, M.Vasjari, I.Novotny, V.Rehacek, V.Tvarozek and O. S.Wolfbeis, Nanotechnoligy., 13, (2002), 175.

36) P. Diao, D. Jiang, X. Cui, D. P. Gu, R. Tong, B. Zhong, J. Electroanal. Chem., 464, (1999), 61.

37) Y. F. Xing, S. J. Oshear and S. F. Y. Li, J. Electroanal. Chem., 542, (2003), 7.

38) T. M. Nahir and E. F. Bowden, ElectroChim Acta., 39, (1996), 2347.

39) C. D. Bain and P. H. Greene, Curr Opin Colloid Interface Sci., 6, (2002), 313.

40) H. Hoffmann, U.Mayer, H. Brunner and A. Krischanitz, Vibrational Spetroscopy., 8, (1995), 151.

41) A. Michota and J. Bukowska, J. Raman Spectrosc., 34, (2003), 21.

42) N. Sandhyarani, K. V. G. K. Murty and T. Pradeep, J. Raman Spectrosc., 29, (1998), 359.

43) O. Dannenberger, M. Buck and M. Gruze, J. Phys. Chem., 103, (1999), 2202.

44) J. Heeg, U. Schubert and F. Kuchenmeister, Fresenius J Anal Chem., 365, (1999), 272.

45) R. Brito, R. Tremont, O. Feliciano and C. R. Cabrera, J. Electroanal. Chem., 540, (2003), 53.

46) M. D. Porter, T. B. Bright, D. L. Allara and C. E. D. Chidsey, J. Am. Chem.; 109, (1987), 3559.

47) L. H. Dubois, B. R. Zegarski and R. G. Nuzzo, J. Phys. Chem., 98, (1993), 687.

48) C. E. D. Chidsey, G. Y. Liu, P. Rowntree and G. Scoles, J. Chem. Phys., 91, (1989), 4421.

49) I. M. Tidswell, B. M. Ocka, P. S. Pershan, S. R. Wasserman, G. M. Whitesides and J. D. Axe, Phys. Rev. B., 41, (1990), 1111.

50) R. Luclum, C. Behling and P. Hauptmann, Sensors and Actuators B, 65, (2000), 277.

51) D. S. Ballantine and J. H. Wohltjen, Anal Chem., 61, (1989), 704A.

52) H. J. Kim, S. Kwak, B. I. Seo, E. R. Kim and H. Lee, Thin Solid Films., 191, (1989), 327.

53) X. Y. Xiao and S. G. Sun, ElectroChim Acta., 45, (2000), 2897.

54) T. T. Ehler, N. Malmberg, K. Carron, B. P. Sullivan and L. J. Noe, J. Phys.Chem.B., 101, (1997), 3174.

55) G. Kataby, T. Prozoorov, Y. Koltypin, H. Cohen, C. N. Sukenik, A. Uiman and A. Gedanken, Langmuir., 13, (1997), 6151

56) S. Mitra, B. Nair, T. Paadeep, P. S. Gogal and R. Mukhopadhyay, J. Phys. Chem. B., 106, (2002), 3960. 
57) R. E. Palmer and Q. Guo, Phys. Chem. Chem. Phys., 4, (2002), 4275.

58) C. A. Alives, E. L. Smith and M. D. Porter, J. Am. Chem. Soc.,114, (1992),

59) U. Kleineberg, A. Brechling, M. Sundermann and U. Heinzmann, Adv. Funct. Maret.,11, (2001), 208.

60) S. Flink, F. C. J. M. van Veggel and D. N. Reinhoudt, Adv. Mater., 2000, 12, 1315.

61) V. Chechik, R. M. Crooks and C. J. M. Stirling, Adv. Mater., 12, (2000), 1161.

62) T. P. Sullivar and W. T. S. Huck, Eur. J. Org Chem., 17, (2003).

63) A. Zhang, J. Qin, J. Gu and Z. Lu, Thin Solid Films., 375, (2000), 242.

64) N. Kanayama, T. Kanbara and H. Kitano, J. Phys. Chem. B., 104, (2000), 271.

65) S.Flink, F.C.J.M. van Veggel and D. N. Reinhoudt, Sensors Update, 1, (2000), 3.

66) C.Marenco, C.J.M.Stirling and J.Yarwood, J. Raman Spectrosc, 32, (2001), 183.

67) J. Rickert, T. Weiss and W. Gopel, Sensors and Actuators B, 31, (1996), 45.

68) K. Hayashi, A. Hozumi, N. Saito, H. Sugimura and O. Takai, Surface Science., 1, (2003).

69) D. H. Kim, J. Noh, M. Hara and H. Lee, Bull. Korean Chem Soc., 22, (2001), 276.

70) D. Shen, X. W. X. Liu, Q. Kang and S. Cher, Microchemical Journal., 63, (1999), 322.

71) R. D. Vaughan, C. K. O'Sullivan and G. G. Guilbaut, Fresenius J Anal Chem., 364, (1999), 54.

72) Q. Jin, J. A. Rodriguez, C. Z. Li, Y. Darici and N. J. Tao, Surface Science., 425, (1999), 101.

73) J. Scharf, H. H. Strehblow, B. Zeysing and A. Terfort, J Solid State Electrochem., 5, (2001), 396.

74) N. Garg, E. C. Molina and T. R. Lee, Langmuir., 18, (2002), 2717.

75) S. D. Evans , E. Urankar, A. Ulman and N. Ferrisn, J. Am. Chem. Soc.,113, (1991), 4121.

76) H. Shen, J. E. Mark, C. J. Seliskar, H. B. Mark and J. W. R. Heineman, J Solid State Electrochem., 1, (1997), 148.

77) A. Badia, R. B. Lennox and L. Reven, Acc. Chem. Res., 33, (2000), 475.

78) M. J. Esplandia, H. Hagenstrom and D. M. Kolb, Langmuir., 17, (2001), 828.

79) C. R. Raj and T. Ohaska, Electroanalysis., 14, (2002), 679.

80) I. Markovich and D. Mandler, Analyst., 126, (2001), 1850.

81) C. E. Jordan, P. L. Freg, S. Kornguth, R. M. Corn, Langmuir., 14, (1998), 2761. 
82) M. Martins, C. Freire and A. R. Hillman, Chem Commun, (2003), 434.

83) T. Stefan, D. Mandler and D. A. Scherson, Langmuir.,18, (2002), 6976.

84) S. Steinberg and I. Rubinstein, Langmuir., 8, (1992), 1183.

85) T. Nagaoka, Z. Chen, H. Okuno, M. Nakayama and K. Ogura, Analytical Sciences., 15, (1999), 857.

86) T. Stora, R. Hovius, Z. Dienes, M. Pachoud and H. Vogel, Langmuir, 13, (1997), 5211.

87) I. Rubinstein, S. Steinberg, Y. Tor, A. Shanzer and J. Sagiv, Nature, 332, (1988), 426.

88) F. Mirkhalaf and D. J. Schiffrin, J. Chem. Soc., Faraday Trans., 94, (1998), 1321.

89) L. Israel, N. N. Kariuki, L. Han, M. M. Maye, J. Luo and C. J. Zhong, J. Electroanal. Chem., 517, (2001), 69.

90) D. W. M. Arrigan and L. L. Bihan, Analyst., 124, (1999), 1645.

91) W. Yang, D. Jaramillo, J. J. Gooding, D. B. Hibbert, R. Zhang, C. D. Willett and K. J. Fisher, Chem. Commun., (2001), 1982.

92) W. Yang, J. J. Gooding and D. B. Hibbert, Analyst., 126, (2001), 1573.

93) J. J. Gooding, D. B. Hibbert and W. Yang, Sensors., 1, (2001), 75.

94) B. Zeng, X. Ding and F. Zhao, Electroanalysis., 14, (2002), 651.

95) A. C. Liu, D. C. Chen, C. Clin, H. H. Chou and C. H. Chen, Anal. Chem., 71, (1999), 1549.

96) H. Shen, J. E. Mark, C. J. Seliskar, H. B. M. Jr and W. R. Heineman, J Solid State Electrochem., 1, (1997), 241.

97) S. H. Kim, S. K. Han, J. H. kim, M. B. Lee, K. N. Koh and S. W. Kang, Dyes and Pigments.,44, (2000), 55.

98) I. Turyan and D. Mandler, Anal. Chem., 66, (1994), 58.

99) M. Sastry, V. Patil and K. S. Mayya, Thin solid films., 307, (1997), 280.

100) I. Turyan and D. Mandler, Anal. Chem., 96, (1997), 894.

101) D. R. Juny and A. W. Czanderna, J. Vac. Sci. Technol., A 13, (1995), 1337.

102) O. Dominguez, M. A. Alonso, M. J. Acros, Electroanalysis., 14, (2002), 1083.

103) H. F. Ji, T. Thundat, R. Dabestani, G. M. Brown, P. F. Britt and P. V. Bonnesen, Anal. Chem., 73, (2001), 1572.

104) S. Berchmans, S. Arivukkodi and V. Yegnaraman, Electrochem. Commun., 2, (2000), 226.

105) X. Xu, T. G. Thundat, G. M. Brown and H. F. Ji, Anal. Chem., 74, (2002), 3611.

106) J. Lu, X. He, X. Zheng, Q. Wan and Z. Zhang, Talanta., 59, (2003), 553. 
107) D. W. M. Arrigan, L. L. Bihan and M. J. Pickup, Analyst., 124, (1999), 1797.

108) F. Mirkhalaf. D.Whittaker and D. J. Schiffrin, J. Electroanal. Chem. 452, (1998), 203.

109) I. Turyan, M. Atiya and D. Mandler, Electroanalysis., 13, (2001), 653.

110) B. Zheng, X. Ding, D. Pan and F. Zhao, Talanta., 59, (2003), 501.

111) M. A. Kalinina, V. V. Arslanov and S. Z. Vatsadze, Colloid Journal., 65, (2003), 177.

112) C. Caneto, G. Caminati, D. Berti, M. Puggell and G. Gabrielli, I L Nuovo Cimento, 16 D, (1994), 1579.

113) S. Flink, B. A. Boukamp, A. van den Berg, F. C. J. M. van Veggel and D. N. Reinhoudt, J. Am. Chem. Soc, 120, (1998), 4652.

114) X. Yang, N. Kumar, J. J. Gooding, D. B. Hibbert, Org. Prep. Proc. Intern., 31, (1999), 425.

115) A. C. Ion, J. C. Moutel, A. Pailleret, A. Popescu, E. S. Aman, E. Siebert and E. M. Ungureanu, J. Electroanal. Chem., 464, (1999), 24.

116) J. J. Xu, H. Q. Fang and H. Y. Chen, J. Electroanal. Chem., 426, (1997), 139.

117) K. Bandyopadhyay, S. G. Liu, H. Y. Liu and L. Echegoyen, Chem. Eur. J, 6, (2000), 4385.

118) A. J. Moore, L. M. Goldenberg, M. R. Bryce, M. C. Petty, A. P. Monkman, C. Marenco, J. Yarwood, M. Joyce and S. N. Port, Adv. Mater., 10, (1998), 395.

119) H. Y. Liu, S. G. Liu and L. Echegoyen, Chem. Commun., (1999)., 1493.

120) Y. Miura, S. Kimura, S. Kobagashi, Y. Imanishi and J. Umemura, Biopolymers and Peptide Science., 55, (2000), 391.

121) T. D. Chung, J. Park, J. Kim, H. Lim, M. J. Choi, J. R. Kim, S. K. Chang and H. Kim, Anal. Chem., 73, (2001), 3975.

122) M. Takaya and P. Biihlmannand Y. Umezawa, Mikrochim. Acta, 132, (1999), 55.

123) M. Fibbioli, K. Banyopadhyay, S. G. Liu, L. Echegoyen, O. Enger, F. Diederich, P. Buhlmann and E. Pretsch, Chem. Commun., (2000), 339.

124) M. A. Herranz, B. Colonna and L. Echegyen, PNAS., 90, (2002), 5040.

125) J. Ekeroth, P. Konradsson, F. B. Jorefors, I. Lundstrom and B. Liedberg, Anal. Chem., 74, (2002), 1979.

126) H. F. Ji, E. Finot, R. Dabestani, T. Thundat, G. M. Brown and P. F. Britt, Chem. Commun., (2000), 457.

127) D. Mandler and I. Turyan, Electroanalysis., 8, (1996), 207. 
128) D. S. Ballantine, R. M. White, S. J. Martin, A. J. Ricco, E. T. Zeller, G. C. Frye and $\mathrm{H}$. Wohltjen, Acoustic Wave Sensors: Theory, Design and Physico Chemical Applications; Academic Press, 1997.

129) J. W. Grate, S. J. Martin and R. M. White, Anal. Chem., 65, 1993, A 940.

130) J. W. Grate and G. C. Frye, Sensors Update., 2, (1996), 37.

131) E. Benes, M. Groschl, W. Burger and M. Schmid, Sensors and Actuators, A : Physical., 48, (1995), 1.

132) Z. Ali, J. Therm. Anal. Cal., 55, (1999), 397.

133) J. Auge, P. Hauptmann, F. Eichelbaum and S. Rosler, Sensors and Actutators B., 18-19, (1994), 518.

134) C. K. O'Sullivan and G. G. Guilbault, Biosensors and Bioelectronics., 14, (1999), 663.

135) H. D. Liess, A. Knezevic, M. Rother and J. Muenz, Faraday. Discuss., 107, (1997), 107, 1.

136) T. Nomura and M. Lijima, Analyticachimica Acta., 131, (1981), 102.

137) C. Eickes, J. Rosenmund, S. Wasle, K. Doblhofer, K. Wang and K. G. Weil, Electrochimica Acta., 45, (2000), 3623.

138) L. Daikhin, E. Gileadi, G. Katz, V. Tsionsky, M. Urbakh and D. Zagidulin, Anal. Chem., 74, (2002), 554.

139) M. T. S. R. Gomes, IEEE Sensors Journal., 1, (2001), 109.

140) M. T. S. R. Gomes, A. Alexandre, F. Silva, A. C. Duarte and J. A. B. P. Sensors and Actuators B., 48, (1998), 383

141) M. T. S. R. Gomes, K. S. Tavares and J. A. B. P. Oliveira, Fresenius J Anal Chem., 369, (2001), 616.

142) P. L. Drake and G. J. Price, Polym Int., 49, (2000), 926.

143) M. T. Cox, L. M. R. Hill and L. R. Gahan, Analyst., 124, (1999), 859.

144) B. C. Port, S. N. Shackleford and S. G. David, Britsh Nuclear Fuels PLC, (2002), 44. 


\subsection{Introduction}

The quartz crystal microbalance is powerful experimental tool in interfacial electrochemistry. This technique has been used to develop the understanding of thin film deposition and redox chemistry.

The QCM, more correctly referred to as a thickness shear mode resonator (TSM), can be used as a gravimetric probe in solution or as the working electrode in an electrochemical cell in which either potential or current controlled experiments can be carried out. A significant amount of information can be extracted from the QCM's responses which can be quantitatively interpreted. The sensitivity of this technique allows detection of submonolayers, monolayers and small mass change on a nanogram scale.

In this chapter established theory is introduced. This includes fundamental principles of the QCM, which we used to immobilize self-assembled monolayer ligands and bind trace metal ions from solution, followed by the crystal impedance technique (or network analysis), which is a relatively new method for the study of mechanical behaviour for the SAM on gold surfaces.

Finally, a summary of the principles of some of electrochemical techniques to study the electrochemical characterization of self-assembled monolayers on gold electrodes is presented.

\subsection{Background to the Quartz Crystal Microbalance}

\subsubsection{Piezoelectric Quartz Crystal Resonators}

Piezoelectricity was first described in 1880 by Pierre and Jacques Curie and the term describes the generation of electrical charges on opposing surfaces of a solid material upon deformation (torsion, pressure, bending, etc.) along an appropriate direction. Conversely, mechanical deformation of material induced by an external electric field is called the converse piezoelectric effect. The converse effect was predicated by Lippmann and is the basis of the QCM [1]. Piezoelectricity occurs in the 21 classes of crystals that do not possess a centre of symmetry. Alpha quartz is a widely used piezoelectric material owing to its water insolubility and resistance to temperature, up to $573^{\circ} \mathrm{C}$, with no loss of piezoelectric properties. Alpha quartz provides the unique combination of mechanical, electrical, chemical and thermal properties. Shortly after this discovery, scientists verified the converse piezoelectric effect in which a potential applied across one of these crystals produced a 
corresponding deformation. Sandwiching a thin quartz wafer between two metal electrodes allow the application of an external electrical potential that produces internal mechanical stress resulting in deformation of the crystal.

Figure 2.1 shows how the application of an electric field across the quartz induces reorientation of dipoles resulting in shear deformation. If an alternating electric field is induced perpendicular to the surface of crystal, this deformation will oscillate at the frequency of the applied field. When the crystal thickness is an odd multiple of half the acoustic wavelength, a standing wave is set up and the crystal resonates. The piezoelectric effect is entirely explained using crystal symmetry arguments; distortion of an asymmetric crystal lattice with an applied stress rearranges the electric dipole moments responsible for the detected voltage change, see Figure 2.2. The Curies experimentally verified the converse piezoelectric effect, whereby application of a potential different across crystal faces causes deformation of the crystal lattice resulting in mechanical strain at the surfaces.
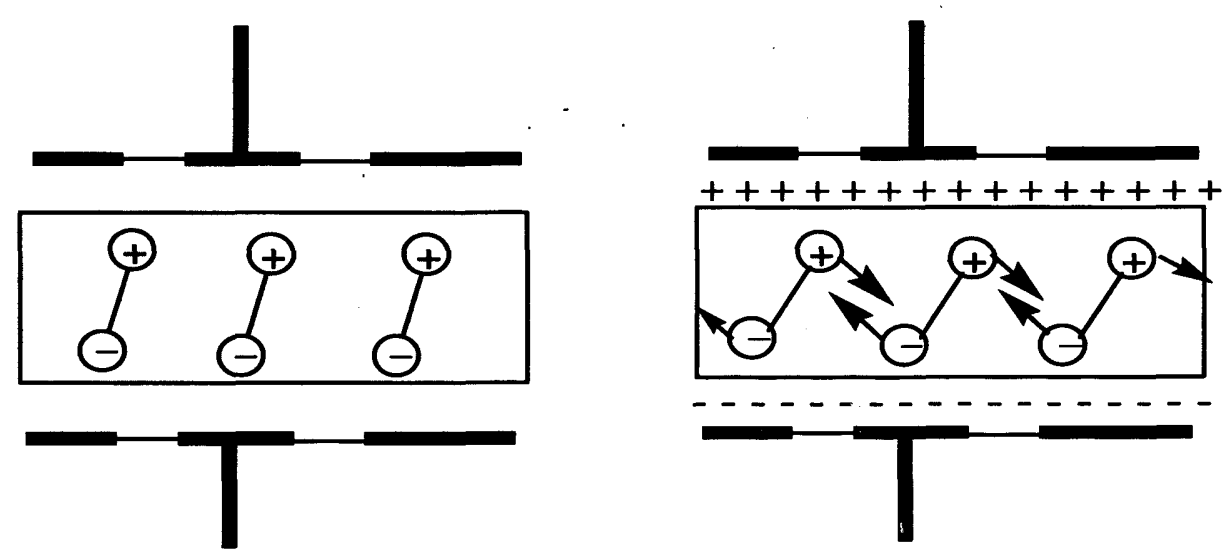

Figure 2.1: Schematic representation of converse piezoelectric effect for shear motion.

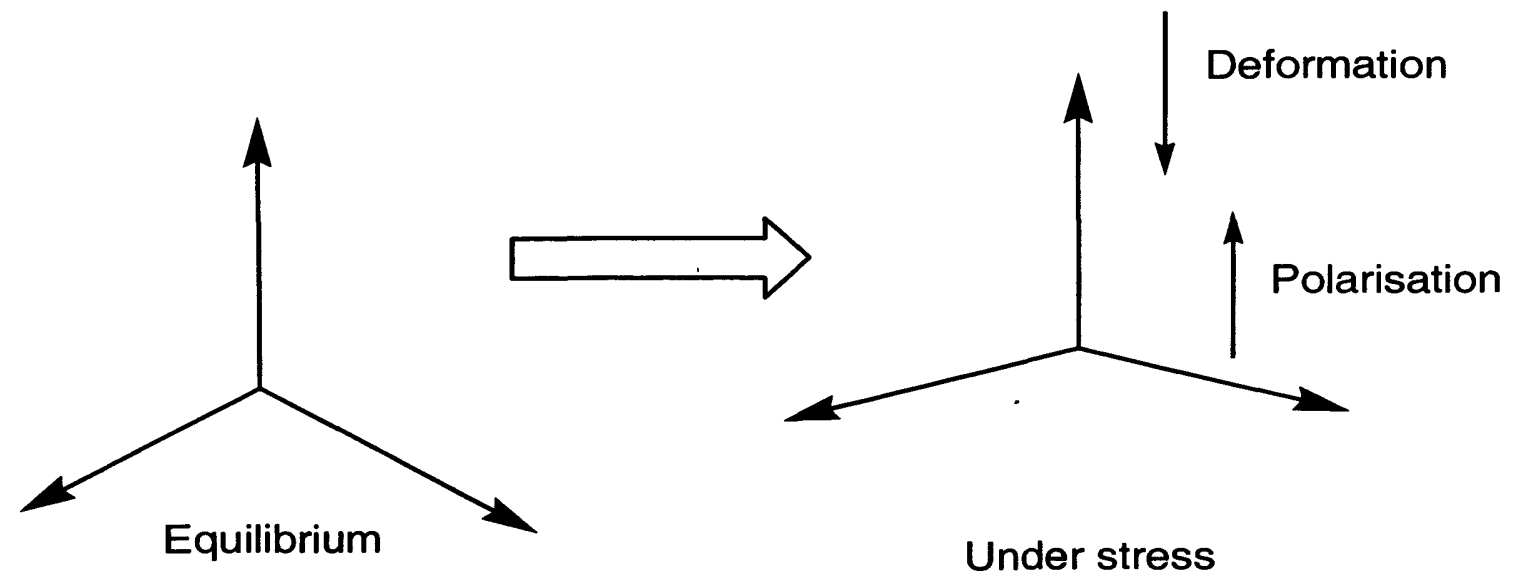

Figure 2.2: Stress induced displacement of centres of gravity leading to formation of electric field. 
The converse piezoelectric effect is exploited in quartz thickness shear mode resonators and it is the fundamental basis of the quartz crystal microbalance methodology [2]. A voltage with a sinusoidally alternating polarity causes an alternating deformation in the crystal, with opposite potential polarities giving rise to crystal deformations equal in magnitude but opposite in direction. This deformation is typically $10-100 \mathrm{~nm}$, for AT-cut crystals operating in the frequency range of $1-10$ $\mathrm{MHz},[3]$. Quartz is the most popular piezoelectric material used to date because of its high frequency stability, high sensitivity, cheapness and chemical inertness. A range of electrode materials has been used, the most popular being gold, due to the ease with which it can be evaporated onto quartz. Other electrode materials include copper, platinum and nickel. The electrode configuration used throughout my work is shown in Figure 2.3. The quartz is cut to a thickness of around $0.17 \mathrm{~mm}$ in order for it to resonate at frequency of $10 \mathrm{MHz}$.

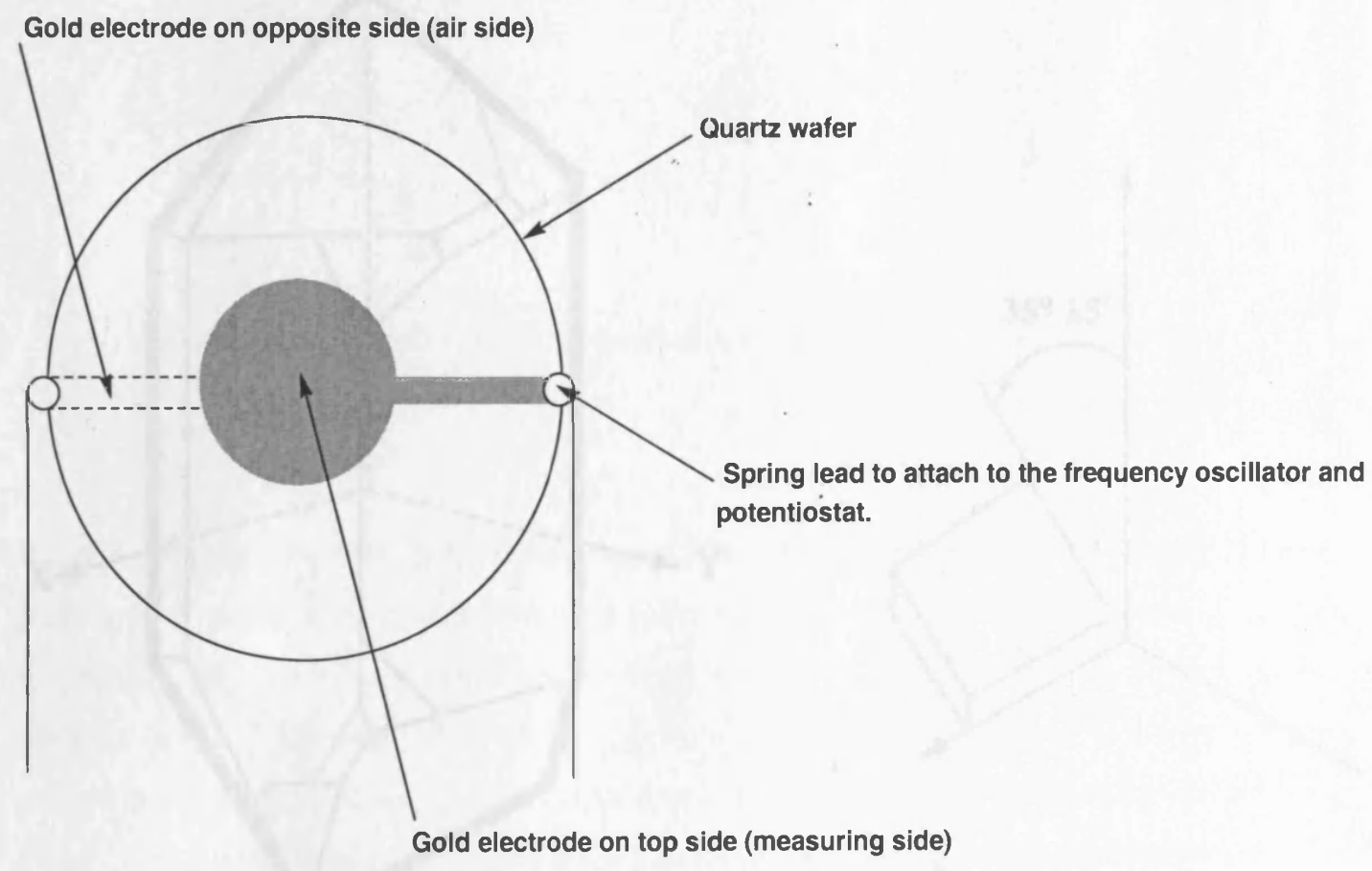

Figure 2.3: Electrode design for the quartz crystal microbalance. 


\subsubsection{Modes of vibration}

When an oscillating potential is applied across a slab of piezoelectric quartz crystal (cut from a single crystal), the quartz can be made to vibrate, with the maximum amplitude of vibration occurring at the mechanical resonance frequency of the crystal. For quartz there are many modes of vibration (for example, longitudinal (extensional), lateral (flexural and shear), and torsional (twist)) depending upon the orientation of the crystal lattice in the electric field. Ideally one would want the quartz crystal resonator to oscillate in only one mode, with all other modes suppressed [2].
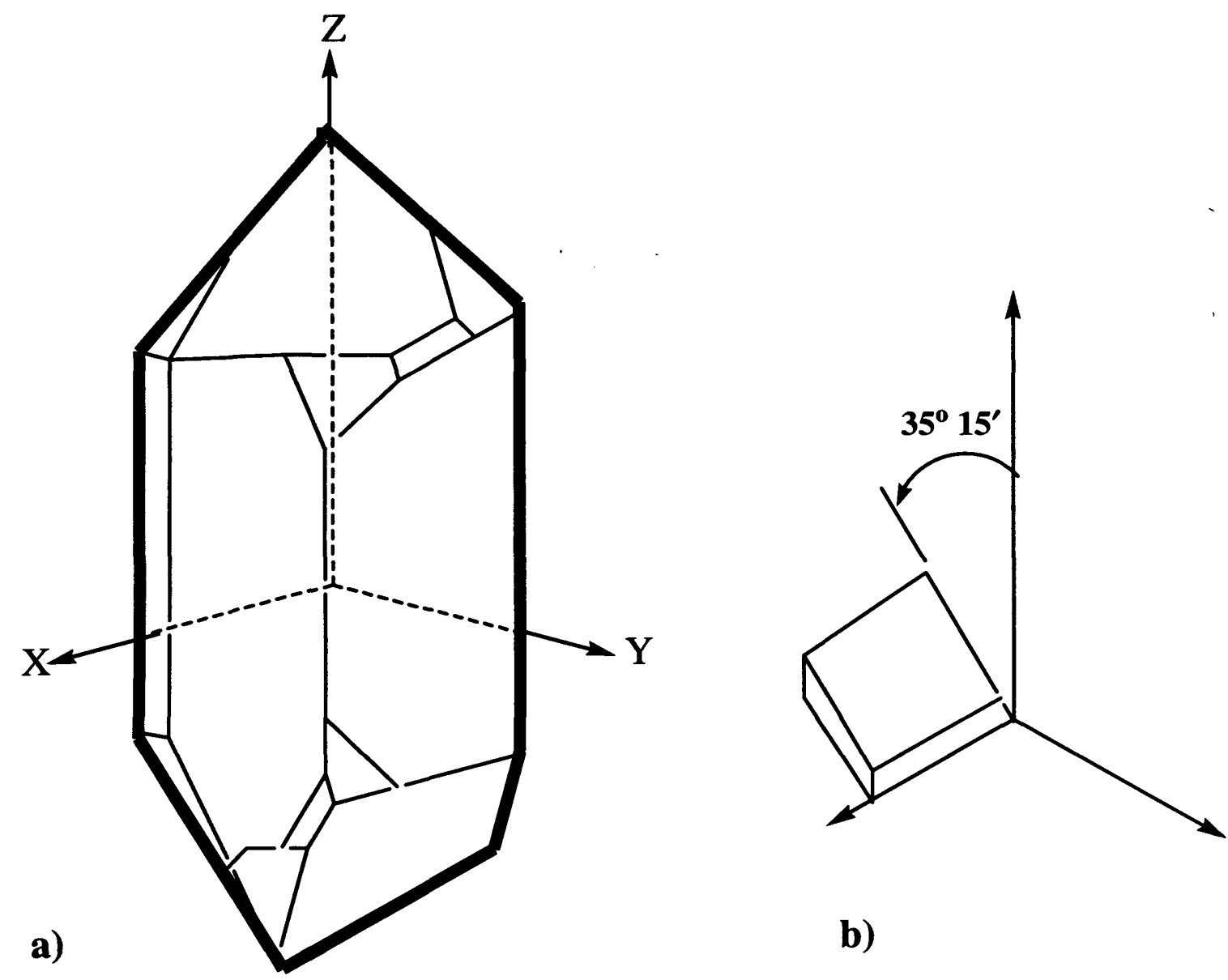

Figure 2.4: a) Assignment of axes to a quartz crystal. b) Cutting angle of AT-cut quartz crystal plate. 
Figure 2.4a shows the assignment of axes to a quartz crystal in its perfect natural form. The properties of the crystal (temperature stability; response to surface stress) are dependent on the precise crystallographic angle at which the slab of crystal is cut. Wafers cut at $35^{\circ} 15^{\prime}$ and $49^{\circ} 00^{\prime}$ with respect to the crystallographic axes, yield quartz vibrating in the thickness shear mode and are known as the ATand BT-cuts respectively. The cut used for the majority of studies is "AT" $\left(35^{\circ} 15^{\prime}\right.$ shown in Figure 2.4b) in which a thickness-shear mode (TSM) oscillation occurs. The thickness shear vibration in the fundamental mode is illustrated in Figure 2.5.

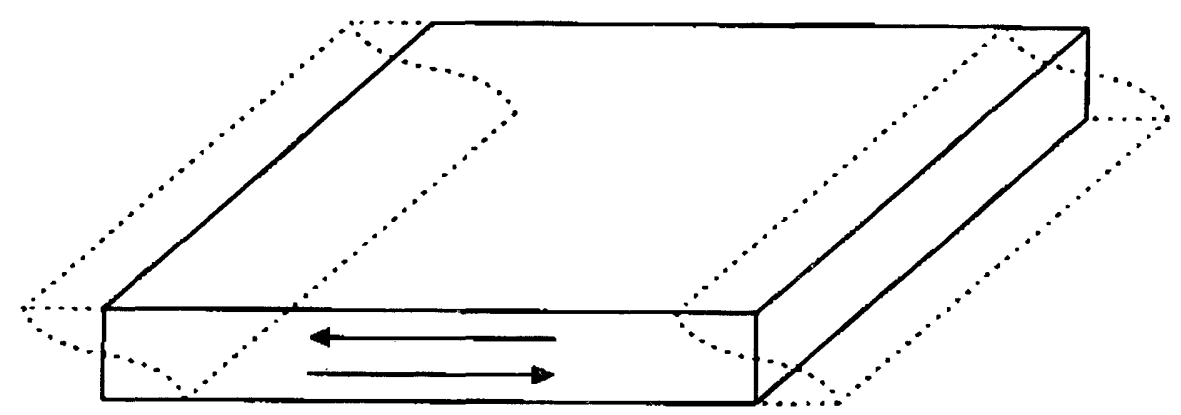

Figure 2.5: Thickness-shear mode oscillation for an AT-cut quartz crystal

AT-cut crystals are the most widely used due to their sensitivity to attached mass and lack of sensitivity to temperature variation around room temperature. It should be noted that many papers in the literature $[4,5,6]$ refer to the AT-cut quartz crystals as a TSM resonator more general instead of the QCM. This is a more sensible name as it directly describes the mode of oscillation. The term QCM for a TSM oscillator is more widely accepted and will be used in my experiments.

\subsubsection{Frequency-mass relationship}

Thickness shear mode oscillators have a natural vibration frequency known as the resonant or fundamental frequency, which is analogous to the resonant frequency of a mass on a spring or to a vibrating string. Lord Rayleigh first described the phenomenon of waves which occur on the surface of solids [7] White and Voltmer developed the interdigital transducer, which allowed the generation of surface 
waves in piezoelectric solids. It is this theory which has been developed for mass sensing using thickness shear mode oscillators, such as the quartz crystal microbalance (QCM). The QCM applies thickness shear mode resonators to exploit the frequency-mass relationship by exciting and measuring the crystal resonant frequency using a sinusoidally alternating potential. The fundamental theory describing the relationship between frequency change and mass change was derived by Sauerbrey [6].

\subsubsection{The Sauerbrey equation}

In 1959 Sauerbery provided the first mathematical treatment of mass sensitivity of the AT-cut quartz oscillator [6]. By analytically solving the one-dimensional equation of motion, he showed that an ideal foreign mass layer results in a frequency decrease $\Delta f$ that is proportional to the deposited mass $\Delta M$ if the resonator is operated in air or vacuum. He showed that a sufficiently small change in mass at the crystal surface can be treated as a change in mass of the crystal itself [6].

a)
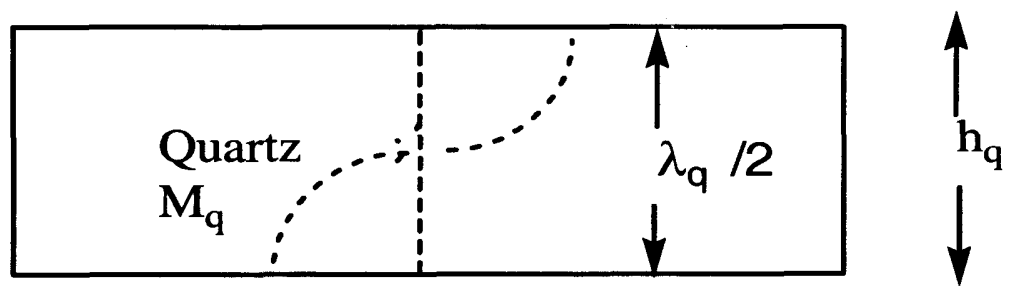

b)

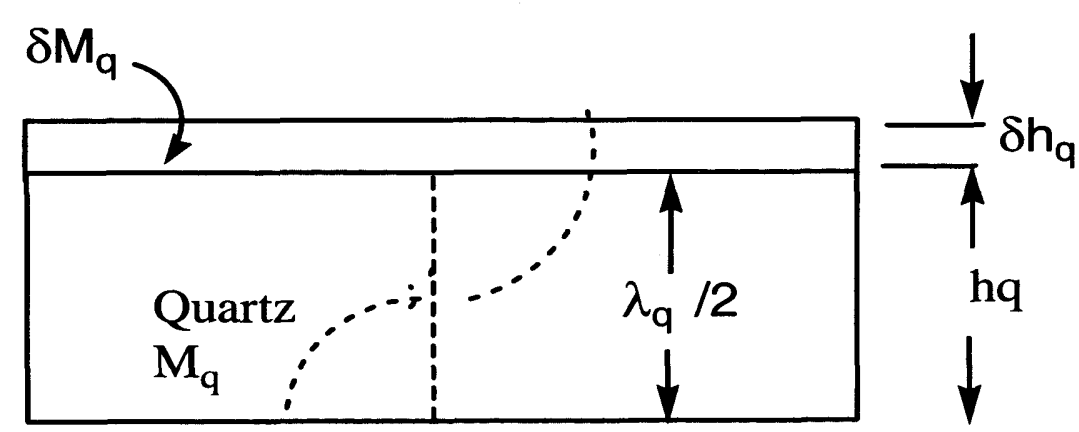

Figure 2.6: Schematic representation of a thickness shear mode (TSM) resonator. (a) At resonance the wavelength, $\lambda_{q}$, is related to the thickness of the quartz plate, $h_{q}$ (b) An increase in $h_{q}$ results in an increase in $\lambda_{q}$ (a decrease in the resonant frequency). 
The resonant frequency, $f_{o}$, of an AT-cut quartz resonator with a mass $M_{q}$ and a thickness $h_{q}$ is given by:

$$
f_{o}=\frac{\mathrm{V}_{\mathrm{q}}}{\lambda}=\frac{\mathrm{V}_{\mathrm{q}}}{2 \mathrm{~h}_{\mathrm{q}}}
$$

where $\lambda$ is the wavelength (equal to $2 h_{q}$ ) and $V_{q}$ is the speed of the wave in the quartz. It follows that a change in the thickness of the quartz will affect its resonant frequency, see Figure 2.6.

$$
\frac{\Delta f_{o}}{f_{o}}=-\frac{\Delta \mathrm{h}_{\mathrm{q}}}{\mathrm{hq}}
$$

A relative increase in the crystal thickness will cause a decrease in the resonant frequency. This can also be expressed in terms of the crystal mass:

$$
\frac{\Delta f_{o}}{f_{o}}=\frac{\Delta \mathrm{M}_{\mathrm{q}}}{\mathrm{M}_{\mathrm{q}}}
$$

Combining Equations (2.1) and (2.2) yields:

$$
\Delta f_{o}=-\left(\frac{2 f_{o}^{2}}{\rho_{\mathrm{q}} \mathrm{V}_{\mathrm{q}}}\right) \frac{\Delta \mathrm{M}_{\mathrm{q}}}{\mathrm{A}}
$$

where $A$ is the piezoelectrically active area $\left(\mathrm{cm}^{2}\right), \rho_{\mathrm{q}}$ is the density of the quartz $\left(2.648 \mathrm{~g} \mathrm{~cm}^{-3}\right)$ and $\mathrm{Vq}$ is the acoustic velocity of quartz $\left(3.34 \times 10^{5} \mathrm{~cm} \mathrm{~s}^{-1}\right)$. Substituting numerical values for $\rho_{q}$ and $V_{q}$ into Equation 2.4 yields: 


$$
\Delta f_{o}=-2.26 \times 10^{-6} f_{o}^{2} \frac{\Delta \mathrm{M}_{\mathrm{q}}}{\mathrm{A}}
$$

Sauerbrey assumed that small, rigid uniformly distributed masses deposited on the oscillator surface could be treated as a mass change of the oscillator itself. Equation 2.5 can then be written in a more generalised form:

$$
\Delta f=-2.26 \times 10^{-6} f_{o}^{2} \frac{\Delta \mathrm{M}}{\mathrm{A}}
$$

The Sauerbrey Equation (2.6) has been used very successfully. Throughout the 1970 's the QCM was predominantly used as a thickness monitor for deposition of solids from the gas phase in which the Sauerbrey equation was a good approximation for mass determination, for example, monitoring the deposition of metals. Since then, Equation (2.6) has been exploited to determine mass changes in many different systems. For example, coating the surface of the QCM with a chemically sensitive film allowed for the detection of trace constituents in the gas phase [7]. Also, the electrochemical quartz crystal microbalance (EQCM) is a widely used technique where the interpretation is based on the equation derived by Sauerbrey $[8,9]$. This will be described in the next section.

\subsubsection{Sensitivity}

The great sensitivity of the QCM can be seen from Sauerbrey Equation (2.6). For an AT-cut quartz crystal with fundamental frequency of $10 \mathrm{MHz}$, a decrease in frequency of $1 \mathrm{~Hz}$ would correspond to an overall mass of 1.1ng. (for an electrode area in the region of $0.25 \mathrm{~cm}^{-2}$ ). This is equivalent in monolayer terms to $10^{-9} \mathrm{~mol}$ $\mathrm{cm}^{-2}$. For $\mathrm{a}+/-1 \mathrm{~Hz}$ resonance frequency change, the QCM would be sensitive to mass changes as small as $5 \%$ of a monolayer.

The Sauerbrey equation only applies for thin rigid films (no damping of oscillations). A modification is necessary if the attached film is non-rigid since a film would no longer oscillate synchronously with the crystal, and $\Delta f$ would differ from the value predicted by [10].

\subsection{The electrochemical quartz crystal microbalance (EQCM)}

The EQCM method is very sensitive to the smallest changes in mass on the crystal. Initially it was believed that the addition of a liquid to one side of the quartz crystal would result in excessive energy loss to the solution from viscous effects, to the extent that the crystal would cease to oscillate. This was first proved incorrect 
by Nomura in 1980 [11] who demonstrated that the QCM had potential applications for chemical sensors in the liquid phase. Since realising that the QCM could be used in liquid media, the EQCM has become a widely used technique amongst electrochemists.

The EQCM involves using one of the electrodes on the QCM as the working electrode in a conventional three electrode electrochemical cell. It is a very sensitive in-situ technique that monitors changes in the mass at the working electrode via associated frequency change. The nanogram sensitivity and the short data acquisition time of the EQCM technique have allowed the study of electrochemically driven processes in terms of the overall reaction (under thermodynamic control) and of their mechanism (under kinetic control).

In 1985 it was discovered that the resonant frequency shift, upon immersion of the QCM in a liquid, is proportional to the square root of the liquid density-viscosity product. The Sauerbrey equation was modified and it was demonstrated that the QCM could be used as a sensitive mass detector in situ $[12,13]$. Since then the QCM has been an invaluable tool to researchers in the study of liquid properties and solid-liquid interfaces $[14,15]$. The EQCM has been used to study: reduction of metal ions [16, 17], detection of anions [18], characterisation of polymers [19], deposition and dissolution of metal oxides-hydroxides [20], adsorption of proteins and enzymes on self-assembled monolayers [21], adsorption of self-assembled monolayers on different metals $[22,23]$, and to study the binding of an analyte to the monolayer [24]. The EQCM was also used to remove heavy metals from aqueous solutions by cation-exchange between modified electrode and its bathing electrolyte [25].

\subsection{Limit of Mass Load and Boundary Conditions for the (E)QCM}

So far the interpretation of (E)QCM data has revolved around the Sauerbrey equation (Equation 2.6). Several assumptions are made in the derivation of this equation, and if these assumptions are not valid, erroneous results will be obtained. The following sections outline the problems that must be overcome if the Sauerbrey approximation is to be used.

\subsubsection{Film thickness and viscoelastic effects}

The viscoelastic nature of the film will become a significant part of the response of the quartz crystal, thereby requiring a more complete model. The obvious limitation for the Sauerbrey equation is the fact that films must be thin and rigid. The 
Sauerbrey analysis was found to be valid for up to a $20 \%$ difference in the frequency between the perturbed and unperturbed quartz crystal [26]. For greater loadings the energy loss due to viscous drag in the overlayer becomes appreciable and the (E)QCM response is no longer solely a function of film mass change, but is also a function of the film viscoelastic characteristics, represented by shear modulus.

Miller and Bolef [27] in 1968 were the first people to identify the failure of the Sauerbrey equation by studying the QCM with an elastic overlayer. They obtained complicated results that were simplified by Lu and Lewis [26] who recognised that knowledge of not only the overlayer density, but also the shear modulus was required.

\subsubsection{Quartz Crystal-Overlayer Boundary}

In deriving Equation 2.6, Sauerbrey assumes that the density and acoustic velocity of the quartz crystal and the overlayer are identical, i.e. that the additional mass layer is treated as an extension of the quartz. In doing so, there is no accounting for the quartz-overlayer boundary [28].

\subsubsection{Uniformity}

The overlayer must be of uniform composition and thickness over the piezoelectrically active area of the quartz. The reason for this is that there is a sensitivity gradient across the electrode area. There will be maximum amplitude of oscillation (highest sensitivity) in the centre of the electrodes and almost zero oscillation (least sensitivity) at the edge [29]. The Sauerbrey approximation does not take this sensitivity gradient into account. This increases the importance of uniform mass attachment over the whole of the electrode. QCM radial sensitivity has also been investigated when the resonator is in contact with a liquid, under electrochemical conditions. Ward et al electrodeposited copper [30] and Gabrielli et al electrodeposited silver [31], at known positions on the resonator surface and correlated EQCM frequency measurement and electrochemical charge to obtain the measured frequency shift for a mass of known radial position. However, as long as the film roughness is not longer than the acoustic wavelength and uniformities are randomly distributed, the Sauerbrey equation will yield an average value of areal mass density of the deposited mass, provided that the departure from uniformity is not severe. 


\subsection{Crystal Impedance Analysis}

Upon deviation from the rigidity constraint of a TSM resonator, a crystal impedance technique is employed for thin film characterisation. The technique involves the detection of the entire crystal impedance spectrum in the region of crystal resonance.

Analysis of modified electrode responses show that, up to a point, electrode performance improves with increasing film thickness [32], e.g., immobilisation of more mediators can increase reaction flux, thereby increasing sensor sensitivity. Under such circumstances, the film may no longer be rigidly coupled to the underlying electrode. If this is the case then the crystal response is not only a function of mass on the electrode surface (Sauerbrey, Equation 2.6), but also a function of the film's viscoelastic characteristics. It is therefore extremely important to be able to recognise whether a given film is (non-)rigid if reliable results are to be obtained. Crystal impedance is a very useful technique that can determine the extent of departure from rigidity for a film. The technique involves using a network analyser in which impedance measurements are made across a specified frequency range in the vicinity of crystal resonance. This results in an admittance spectrum (reciprocal of impedance) around crystal resonance, i.e. it determines the shape, not merely the position, of the crystal resonance. Schematics. of typical spectra are shown in Figure 2.7.

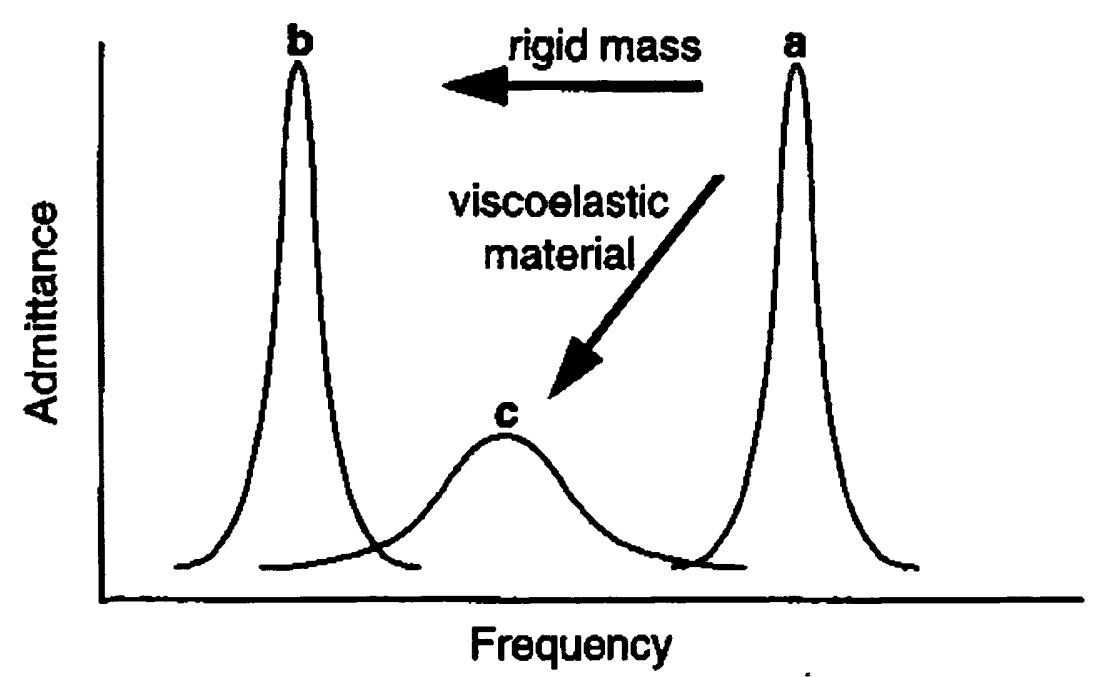

Figure 2.7: Crystal impedance response for (a) unperturbed QCM; (b) thin rigid mass layer; (c) viscoelastic layer. 
Studying the shape as well as the position of resonance allows one to extract qualitative and quantitative information on the viscoelastic properties of surface perturbations on the QCM.

Quantitative information about the viscoelastic properties of thin films can be extracted from the shape of the crystal impedance response. For example, a rigid film is characterised as having a large admittance and small peak width at half height, whereas a non-rigid film is characterised as having a lower admittance and broader peak width at half height. More specifically, if the crystal impedance response is compared to that of the unperturbed crystal, it is possible to tell directly whether or not a film on the surface of the crystal is rigid.

Figure 2.7 shows the responses for; (a) unperturbed QCM; (b) mass changes only (rigid film); (c) for the same mass change as (b), but the film is viscoelastic. The addition of a rigid mass does not change the shape of the spectra, but merely the position on the frequency axis. However, the response to a viscoelastic film is clearly different. Here the shape of the crystal impedance response is changed from that for a more damped response. There is still a decrease in the frequency at maximum admittance, however, it is different than for a rigid film (hence the Sauerbrey equation becomes invalid).

Previous work has been carried out using the crystal impedance technique for many systems. For example, studying film-coated crystals in air [33] and also for crystals in solution [34]. For a crystal immersed in liquid, the liquid phase parameters (viscosity, density and dielectric constant) influence the crystal resonance. The presence of a liquid reduces the quality factor of the crystal resonance markedly. Applying a film to the crystal will again reduce the quality factor of the admittance spectra depending upon its viscoelastic characteristics [35].

Identification and interpretation of viscoelastically-controlled responses are presently significant limitations in the implementation of QCM-based sensors. Of these two, the more complex issue is data interpretation for non-rigid systems, i.e. where film viscoelasticity is a significant factor.

\subsection{Fundamentals of Electrochemical Techniques}

This section briefly describes the principles of the electrochemical techniques such as cyclic voltammetry (CV), which can be used to characterise self-assembled monolayer films. 


\subsubsection{Electron Transfer Rates}

For the electrode reaction:

$$
\mathrm{O}+\mathrm{ne}^{-} \underset{\mathrm{k}_{\mathrm{b}}^{\prime}}{\stackrel{\mathrm{k}_{\mathrm{f}}^{\prime}}{\rightleftharpoons}} \mathrm{R}
$$

The equilibrium potential of the working electrode (fast electron transfer) is defined as:

$$
E=E_{o}+\frac{R T}{n F} \ln \left(\frac{C^{s}}{C^{s}}\right) \quad \text { Nernst Equation }
$$

This is known as the Nernst Equation and relates the potential generated by an electrochemical cell to the surface concentrations, $C^{s}$ and $C_{R}^{s}$ of the chemical species, ' $O$ ' and ' $R$ ', involved in the cell reaction and to the standard electrode potential, $E^{\circ}$. $E^{O}$ for the O/R couple is defined with respect to the potential of the standard hydrogen electrode; $R$ is the gas constant; $T$ is the absolute temperature; $F$ is the Faraday; $k_{f}$ ' and $k_{b}$ ' are the rate coefficients of the forward and reverse reaction respectively.

\subsubsection{Kinetically Controlled Current}

If the reaction is not at equilibrium, and the current is limited by the rate of electron transfer, then the reaction rate is dependent upon reaction kinetics. This situation could occur at (1) small overpotentials, where electron transfer is slow (relative to diffusion), or (2) high diffusion rates, due to stirring of the solution. Using Equation 2.7 , the net reaction flux, $j_{\text {net, }}$ can be represented in terms of the forward and backward fluxes:

$$
\mathrm{j}_{\text {net }}=\mathrm{j}_{\mathrm{f}}-\mathrm{j}_{\mathrm{b}}
$$

where:

$\mathrm{i}=\mathrm{nFA}\left(\mathrm{j}_{\mathrm{f}}-\mathrm{j}_{\mathrm{b}}\right)=\mathrm{nFA}\left(\mathrm{k}_{\mathrm{f}}^{\prime} \mathrm{c}^{\mathrm{s}}{ }_{\mathrm{O}}-\mathrm{k}_{\mathrm{b}}^{\prime} \mathrm{c}_{\mathrm{R}}{ }_{\mathrm{R}}\right)$ 
where $A$ is the electrode area.

It can be seen that the current response is dependent upon the rate constants and the surface concentration. However, the current generally responds exponentially to overpotential according to the Butler-Volmer equation. This relates the current to overpotential at any point and can be used to obtain kinetic data:

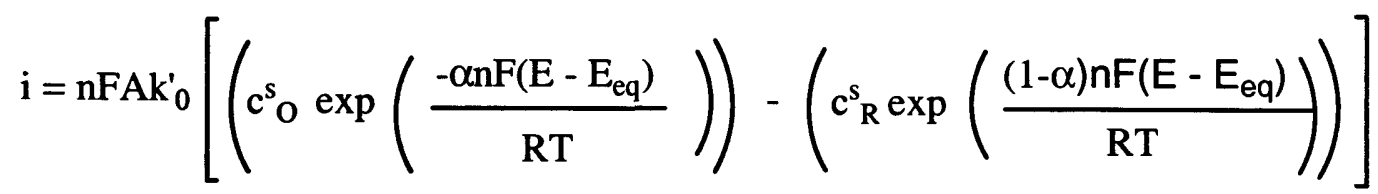

\section{Butler-Volmer Equation}

where $\alpha$ is the transfer coefficient for the reaction and $\mathrm{k}_{0}{ }^{\prime}$ is the standard rate constant, equal to the values of $k_{f}$ and $k_{b}{ }^{\prime}$ at $E=E^{o}$.

The reaction will proceed in only one direction if (a) a high overpotential is applied and (b) the reaction vessel is well stirred. In this situation one of the exponential terms in Equation 2.11 becomes negligible compared to the other. For example, if a large negative overpotential is applied, Equation 2.11 may be simplified to the Tafel equation:

$$
\ln i=\ln \left(\mathrm{nFAk}_{0}^{\prime} \mathrm{c}_{\mathrm{O}}^{\mathrm{s}}\right)-\frac{\alpha \mathrm{nF}\left(\mathrm{E}-\mathrm{E}_{\mathrm{eq}}\right)}{\mathrm{RT}}
$$

Similarly, one can simplify the Butler-Volmer equation assuming a large positive overpotential. From this, both the transfer coefficient, $\alpha$, and the standard rate constant, $\mathrm{k}_{0}$ ' can be determined by means of a plot of Ini vs. $\mathrm{E}$ (see equation 2.12).

\subsubsection{Mass Transport}

There are three modes of mass transport to be considered: diffusion, migration and convection. Commonly, mass transport is controlled by diffusion in electrochemical systems.

\subsubsection{Diffusion}

Diffusion is the movement of charged or neutral species down a concentration gradient and must occur whenever there is a chemical change at a surface. 


\subsubsection{Migration}

Migration is the movement of charged species under the influence of an electric potential gradient. It is the mechanism by which charge is carried through the electrolyte. Migration of electractive solution species can be neglected if there is a large excess of an inert electrolyte in solution. This has the effect of reducing the transport number of the redox species (most of the charge is carried by the inert electrolyte).

\subsubsection{Convection}

Convection is the movement of species due to mechanical forces that may be natural (thermal agitation) or forced (e.g. rotating disc electrode). In the case of forced convection the flow may be laminar or turbulent depending on the force. If the flow is laminar, then it can be modelled mathematically. The effects of natural convection can be ignored on short timescales, typically less than a few seconds.

\subsubsection{Diffusion Limited Current}

At high overpotentials, electron transfer rates will be very high, thus making the concentration of species $\mathrm{O}$ at electrode surface deplete to zero. This species has to diffuse from the bulk to the surface in order for the electron transfer process to take place and thus diffusion will be the rate limiting step. The current is proportional to the concentration gradient at the surface:

$$
i=n F A D\left(\frac{d c}{d x}\right)_{x=0} \quad \text { Fick's First Law }
$$

where $D$ is the diffusion coefficient and $x$ is the distance from the electrode surface. Similarly, the Cottrell equation describes the limiting diffusion current response as a function of time:

$\mathrm{i}=\mathrm{nFAc}_{\text {bulk }}\left(\frac{\mathrm{D}}{\pi \mathrm{t}}\right)^{1 / 2}$ Cottrell Equation

A plot of $i$ versus $t^{-1 / 2}$ therefore yields the diffusion coefficient, $D$, via the slope. 


\subsection{Cyclic voltammetry}

A simple potential waveform that is often used in electrochemical experiments is the linear wave form, i.e. the potential is continuously changed as a linear function of time. The rate of change of potential with time is referred to as the scan rate. Cyclic voltammetry (CV) is arguably the most widely used technique for the characterisation of redox systems [36]. It can provide qualitative information about the number of oxidation states and their stability, as well as the rate of heterogeneous electron transfer reactions [37,38]. It can also be used in combination with simulation software to calculate rates of homogenous and heterogeneous reactions. Cyclic voltammetry is a popular method used to investigate self-assembled monolayer modified electrodes because it is easy to implement and instantly provides a wealth of information. In addition, cyclic voltammetry is a powerful tool for the determination of formal redox potentials, detection of chemical reactions that precede or follow the electrochemical reaction and evaluation of electron transfer kinetics. A standard electrochemical cell consists of three electrodes immersed in electrolyte solution: the working electrode (WE), counter electrode (CE), and referençe electrode (RE).

Cyclic voltammetry involves applying an external triangular voltage to the electrochemical cell, sweeping through a potential range and reversing the direction of the sweep in a cyclic fashion (see Figure 2.8). The resulting current (i) is monitored as a function of applied potential $(E)$ to give the $i-E$ curve which is called a cyclic voltammogram [39]. The current flows between the working electrode and counter electrode (ideally the electronic circuitry ensures that no current will flow through the reference). The potential is controlled relative to the reference electrode which is placed as close to the working electrode as possible to reduce ohmic (iR) potential drop.

Integration of the current with respect to time yields the charge, a quantity that is directly related to amount of electroactive species at the surface in the case of adsorbed species, according to Faraday's Law. 


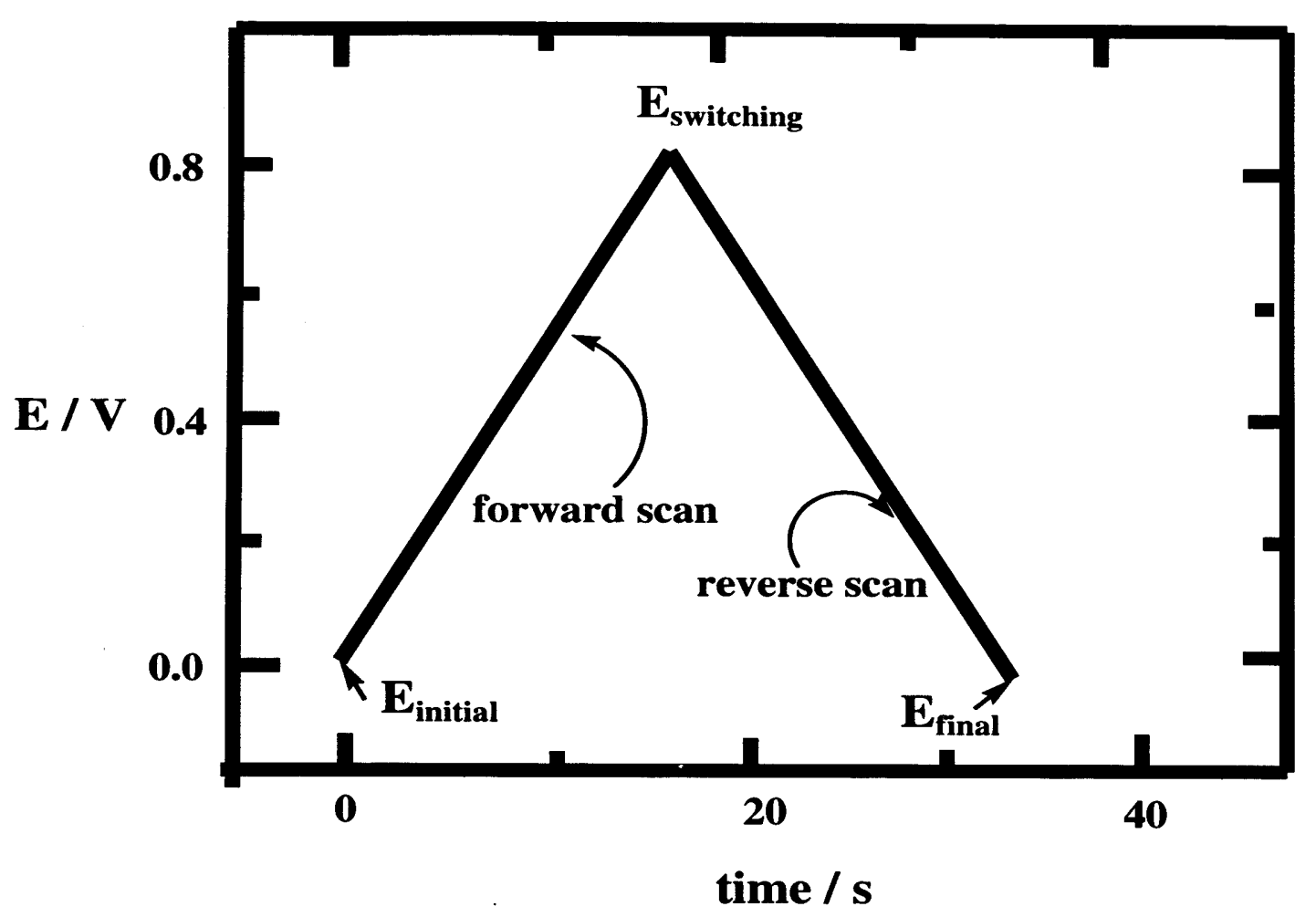

2.8: A schematic showing waveform applied in cyclic voltammetry.

\subsubsection{Solution Phase Voltammetry}

Figure 2.9 shows a solution phase voltammogram. This process could be for example the oxidation of ferrocene $\left[\mathrm{Fe}\left(\mathrm{C}_{5} \mathrm{H}_{5}\right)_{2}\right]^{0}$ in organic solvent. In this case, both the oxidised and reduced forms of the $\left[\mathrm{Fe}\left(\mathrm{C}_{5} \mathrm{H}_{5}\right)_{2}\right]^{0 /+}$ redox couple are soluble in the solvent. Initially no current is passed since the applied potential is not great enough to induce electron transfer (point ' $a$ ' in figure 2.9). The potential is then initially swept in a positive direction in order to oxidise the starting compound $\left[\mathrm{Fe}\left(\mathrm{C}_{5} \mathrm{H}_{5}\right)_{2}\right]^{0}$, to $\left[\mathrm{Fe}\left(\mathrm{C}_{5} \mathrm{H}_{5}\right)_{2}\right]^{+}$. Note in figure 2.9 , the positive direction on the current scale is oxidation current

The oxidation current increases rapidly ( $b$ to $d$ ) until the concentration of $\left[\mathrm{Fe}\left(\mathrm{C}_{5} \mathrm{H}_{5}\right)\right]^{0}$ at the electrode surface is significantly diminished. This causes the current to peak at a maximum value $E_{p}{ }^{0 x}$ and then decay ( $d$ to $g$ ) as controlled by the rate of diffusion of ferrocene from the bulk. The scan direction is switched at about $\quad 0.8 \mathrm{~V}$ (in this case) and then the potential is swept in a negative direction in order to reduce the newly formed Fe[( $\left.\left.\mathrm{C}_{5} \mathrm{H}_{5}\right)_{2}\right]^{+}$. 


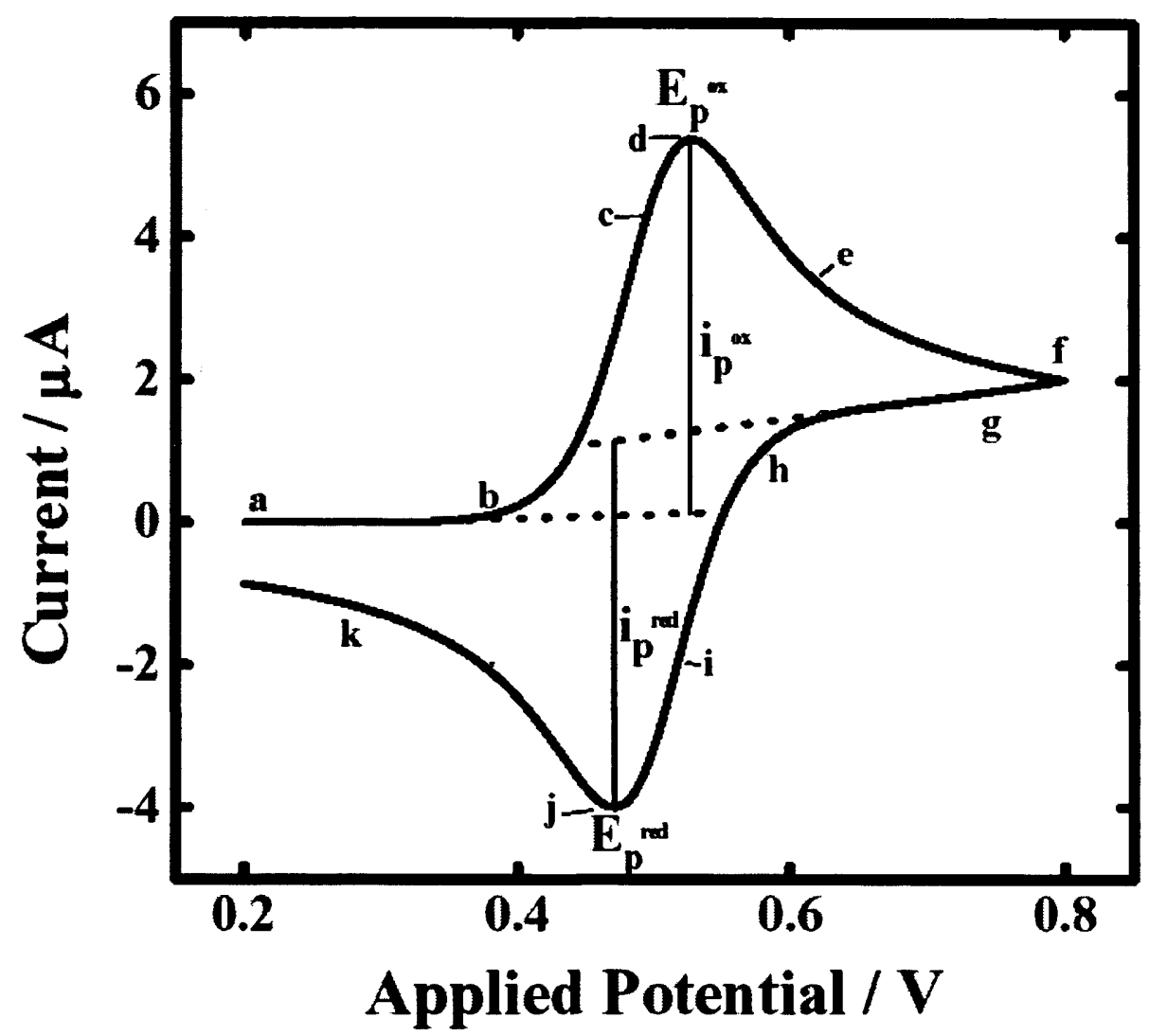

Figure 2.9: Cyclic voltammogram of $0.5 \mathrm{mM}$ ferrocene in acetonitrile. Scan rate $100 \mathrm{mV} \mathrm{s}^{-1}$. Reproduced from ref [40].

Oxidation current is still flowing even though the potential is now being swept in a negative direction. It is not until the potential is negative enough to reduce the $\left[\mathrm{Fe}\left(\mathrm{C}_{5} \mathrm{H}_{5}\right)_{2}\right]^{+}$that reduction currents are observed.

Again the current rapidly increases ( $i$ to $k$ ) to give a maximum value of $E_{p}^{\text {red }}$ as the concentration of $\left[\mathrm{Fe}\left(\mathrm{C}_{5} \mathrm{H}_{5}\right)_{2}\right]^{+}$is depleted at the electrode surface and diffusion control gives rise to current decay after a maximum value ( $\mathrm{j}$ to $\mathrm{k}$ ).

The formal reduction potential $E^{0}$ is approximately half way between the peak potentials $\left(E_{p}{ }^{o x}+E_{p}{ }^{\text {red }}\right) / 2$. The separation between peak potentials $\left(E_{p}{ }^{\text {ox }}\right.$ and $\left.E_{p}{ }^{\text {red }}\right)$ for a reversible couple is given by:

$$
\Delta E_{p}=E_{p}^{o x}-E_{p}^{\text {red }}=\frac{0.059}{n} \quad\left(\text { at } 25^{\circ} \mathrm{C}\right)
$$

Thus, the peak separation potential can be used to determine the number of 
electrons transferred, and as a criterion for a Nernstian behaviour. Accordingly, a fast one electron process exhibits a $\Delta E_{p}$ of bout $59 \mathrm{mV}$. Both cathodic and anodic peak potentials are independent of the scan rate. The variation of peak separation potential with scan rate $(v)$ can be used to estimate the kinetic parameter $\psi$. The kinetic parameter $\left.\psi=k^{\prime} /[\pi \mathrm{DnvF} / \mathrm{RT})\right]^{-1 / 2}[41]$, where the symbols have their usual meaning, accounts for heterogeneous standard rate constant $k$ ' of an uncomplicated quasi-reversible electrochemical reaction and for the scan rate $v$ used in performance cyclic voltammetry experiments. On passing from large $\psi$ values $(\psi=7)$ to sufficiently small ones $(\psi=0.1)$ the shape of the cyclic voltammograms markedly various showing a dependence of the peak potential separations on the value of $(\psi)$. The application of this method to the experimental $\Delta \mathrm{E}_{\mathrm{p}}$ data relative to the one electron reduction of ferricyanide at different structure of SAMs on gold electrodes will be studied in chapter four.

\subsubsection{Solid State Voltammetry}

For a film attached to an electrode surface, the current is confined to the surface, therefore no diffusion has to occur to / from the bulk of the solution. Here the maximum current is given by:

$$
I_{p}=\frac{\left(n^{2} F^{2} \Gamma v\right)}{4 R T}
$$

$\Gamma=$ surface coverage of redox centres in $\mathrm{mol} \mathrm{cm}$

Equation (2.16) is derived on the basis of the Langmuir isotherm which assumes an ideal situation where there no interactions between the sites. An ideal system is basically one which is dilute, but in the case of a film attached to the surface of an electrode, the system is highly concentrated.

Interactions between sites in the film, whether repulsive or attractive, will lead to a voltammogram which is either broadened down or sharpened up relative to the potential axis. In this situation, the current does vary linearly with scan rate, however the calculated ideal proportionality constant, $\left(n^{2} F^{2} \Gamma\right.$ / 4RT), will not be correct. A value of $\Gamma$, therefore cannot be obtained from the slope of the curve. 
$\Gamma$ has to be obtained by calculating the area below the peak of the voltammogram. This will give the amount of charge passed, $Q$ and hence a value of $\Gamma$, using equation (2.16).

$$
\mathrm{Q}=\mathrm{nFA \Gamma}
$$

where $Q$ is the total charge passed. For low scan rates, the experimental timescale is sufficiently long for all of the redox sites in the film to be oxidised and reduced. Therefore the total charge passed, $Q$, should be constant at these low scan rates.

\subsection{X-ray Photoelectron Spectroscopy}

XPS spectroscopy has its roots in nuclear physics research (Rutherford 1914), although it first attracted major interest in 1964 when Siegbahn and his research group demonstrated that chemical-state information could be obtained by measuring the kinetic energy of electrons emitted by a sample irradiated with soft $X$-ray beams [42]. X-rays can be used to excite strongly bound core orbital electrons, causing atoms to eject them at high speed [43]. During an XPS experiment these electrons are counted and their respective kinetic energies are determined. Since then, enormous progress has been made, both by instrument manufacturers and researchers, to improve the quality of spectrometers, standards of operation and procedures for quantification. XPS is in many cases a nondestructive technique, can be applied to insulating samples and combines high sensitivity with chemical state identification. XPS is well suited to the quantitative investigation of surface thickness in the nanometre range. XPS is therefore an excellent method to determine the elemental analysis of a substance. The atomic sensitivity is about $0.1 \%$ and it is able to detect all the elements except $\mathrm{H}$ and $\mathrm{He}$. The latest instruments with parallel data acquisition allow the realisation of XPS surface chemical state microscopy with a lateral resolution of ca $9 \mu \mathrm{m}$. By means of angular-resolved data acquisition, in depth composition profiles can be obtained without sputtering and a recent spectrometer has been produced with a lensdetector combination that allows parallel acquisition of angle-resolved data without the need for tilting the sample. 


\subsubsection{The Principle of XPS}

X-ray photoelectron spectroscopy (XPS) is based on the photoelectric effect: photons of an adequate energy, hv, from an X-ray beam ionise atoms in the sample resulting in the emission of core-level electrons. Photo-ionization comprises: (1) photon absorption, (2) electron emission, (3) displacement of the electron within the solid and (4) escape of the electron from the solid into the vacuum of the spectrometer. The atomic processes of photoelectron emission are schematically illustrated in the diagram of the energy levels of Figure 2.10.

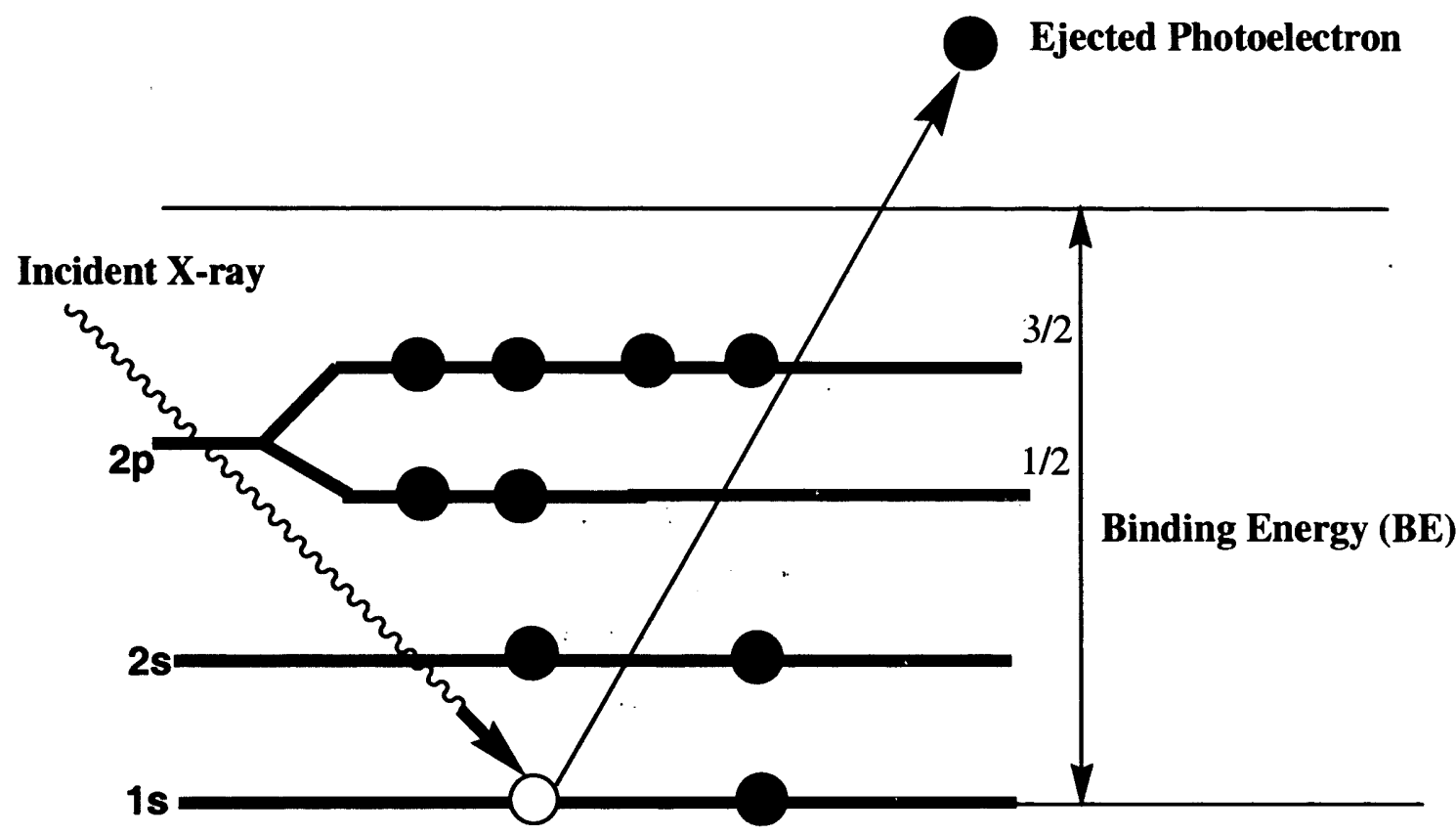

BE = X-ray Energy - Photoelectron Kinetic Energy

Figure 2.10: The XPS emission process for a model atom. An incoming photon caused the ejection of the photoelectron. The simultaneous two-electron coulombic rearrangement results in a final state with two electron vacancies.

According to the principle of conservation of energy, the sum of the energy of the initial state, $E^{i}$ plus the photon energy, $h v$, is equal to the sum of the energy of the final $E^{f}$ state plus the kinetic energy of the emitted photoelectron, KE. Rearranging, one obtains the binding energy, $\mathrm{BE}$, of the photoelectron using this equation (2.17).

$$
B E=h v-K E=E^{f}-E^{i}
$$


The difference in energy of the final and initial state is the difference between the energy of the incident beam minus the kinetic energy of the emitted photoelectron. Foe each element, there will be a characteristic binding energy associated with each core atomic orbital i.e. each element will give rise to a characteristic set of peaks in the photoelectron spectrum at kinetic energies determined by the photon energy and the respective binding energies [44]. The presence of peaks at particular energies therefore indicates the presence of a specific element in the sample under study. Furthermore, the intensity of the peaks or the area under the peaks is related to the concentration of the element within the sampled region. Thus, the technique provides a quantitative analysis of the surface composition and is sometimes known by alternative acronym, ESCA (electron spectroscopy for chemical analysis.

\subsubsection{Elemental and chemical state information}

In 1958, Siegbahn's group in Uppsala University (Sweden) [45] showed that copper (I) oxide could be distinguished from copper (II) oxide by XPS. The difference in binding energy between the same atom, either in two chemically different sites in the same compound or in two different compounds, is referred to as the chemical shift. The chemical shift results from a change in the electrostatic screening of the core electrons when the valence electrons are attracted or repelled in the emitting atom. A description of chemical shift theory is given in the literature [46].The oxidation states of an element can also be distinguished because of the different electrostatic environments surrounding electrons bound to different atoms. For example, carbon in a very electronegative environment (i.e. an ester carboxyl group) will eject a $1 \mathrm{~s}$ electron that appears at a higher binding energy than a carbon in a less electronegative environment (i.e an aliphatic group). Generally the electrons of atoms with high electronegative substituent groups exhibit higher binding energies than the same atoms bound to groups with lower electronegativity. From the analytical point of view, it is very useful to compare the measured binding energy for the unknown element with the binding energy, measured under the same experimental conditions as a reference compound. Tables of peak positions may be found in the literature and a database produced by Wagner may be obtained from NIST [47]. Qualitative elemental analysis of sample surfaces relies upon the comparison of measured binding energy values with those for reference materials. Quantitative analysis is based on the fact that the ionization cross section of a core electron is essentially independent of the valence state of the element. Hence, the area under the peaks will always be 
proportional to the number of atoms within the detected volume. However, it is not always possible to identify the chemical state of an element based on binding energy alone. Other features of the XPS spectra have to be examined, such as the presence of shake-up satellites or the combination with the chemical shift obtained from the X-ray-excited Auger lines in a two dimensional Wagner plot [48].

\subsubsection{The basic of XPS Instrumentation}

A block diagram of an X-ray photoelectron spectrometer is shown in Figure 2.11. It consists of an ultra-high vacuum chamber equipped with an $X$-ray source, usually MgK $\alpha(1235 \mathrm{eV})$ or AlK $\alpha(1486.6 \mathrm{eV})$, which can be monochromatic. Two types of $X$-ray illumination are used in commercial XPS instruments. In the first, the X-ray beam is formed into a small spot (an X-ray probe) on the sample surface; in the other, the sample is flooded with $X$-rays. The irradiation specimen ejects photoelectrons and a position of these is collected by a lens system which focuses them into an energy analyzer. Electrons selected by the analyser are then collected and registered by an electron detector. The electron energy analyser is the heart of the system; its task is to measure the electron energy spectrum. The $\mathrm{X}$-ray source, specimen, lens, analyser and detector are mounted in an ultra-high vacuum (UHV) chamber. Electronics include the necessary power supplies and the control. The user interface is usually a computer system. The output is in the form of an electron energy spectrum or map of the distribution of chemical elements (or their chemical state) from part of the sample surface. 


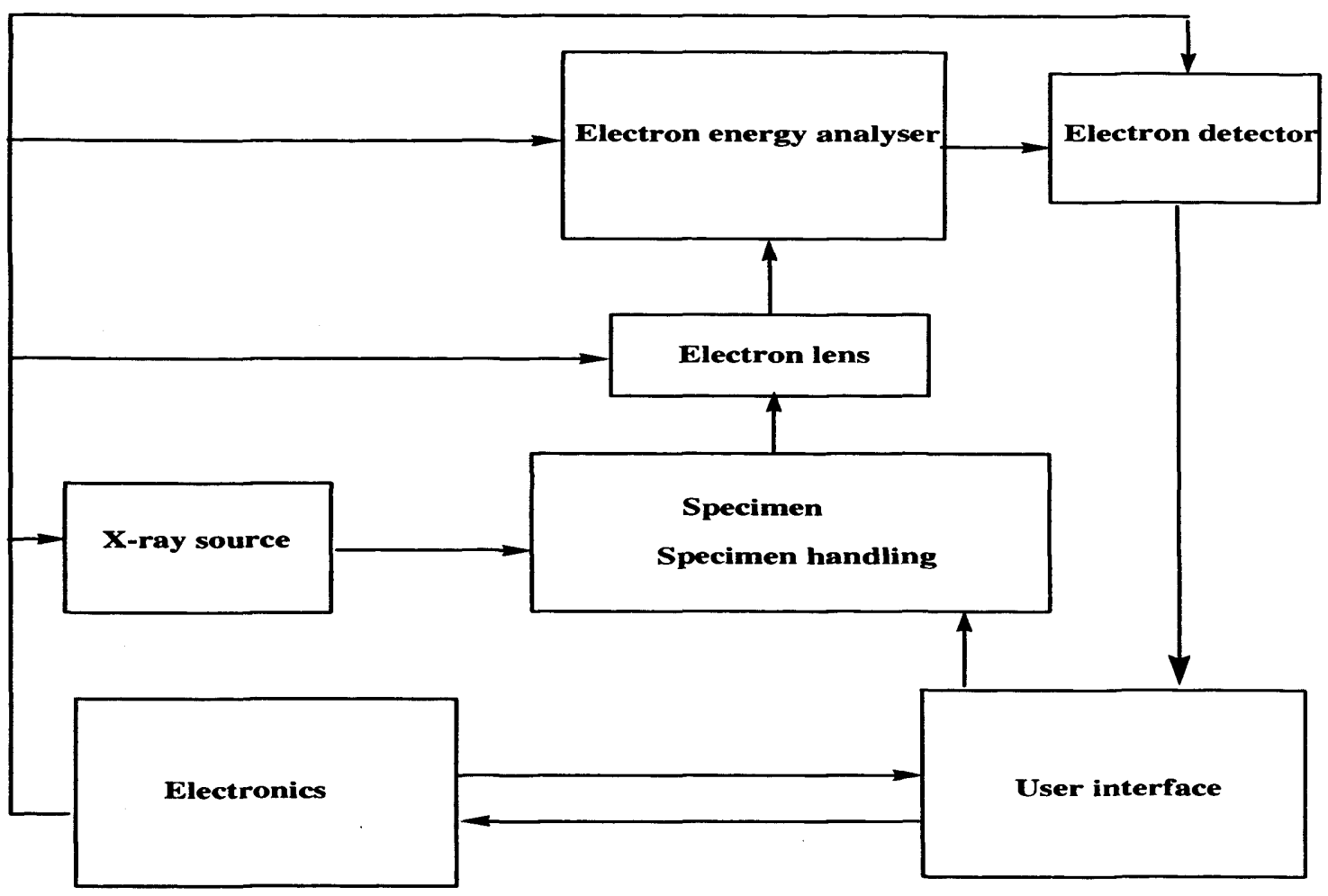

Figure 2.11: Block diagram of the essential components of an XPS instrument.

\subsection{References}

1) A. Janshoff, J. Gutenberg and C. Steinem, Sensors update., 9, (2001), 313.

2) C. Lu and A. W. Czanderna (Eds), Applications of Piezoelectric Quartz Crystal Microbalances, Elsevier, Amsterdam, (1984).

3) D.A.Buttry, Applications of the Quartz crystal Microbalance to Electrochemistry, from Electroanalytical Chemistry: A series of Advances in Electroanalytical Chemistry, edited by Allen Bard, Marcel Dekker, Vol 17, (1991).

4) M. S. Yang and M. Thompson, Anal. Chim. Acta., 282, (1993), 505.

5) M. S. Yang and M. Thompson, Anal. Chem., 65, (1993), 1158.

6) G. Sauerbrey, Zeitschrift fur Physik., 155, (1959), 206.

7) J. Hlavay and G. G. Guilbault, Anal. Chem., 49, (1977), 1890.

8) R. Schumacher, G. Borges and K. K. Kanazawa, Surf. Sci., 163, (1985), L 621.

9) K. Shin and C. S. Kim, Materials Science and Engineering., C23, (2003), 407.

10) W. H. Kao, V. J. Weibel and M. J. Root, J. Electrochem. Soc., 139, (1992), 1223.

11) T. Nomura and A. Minemura, Nippon Kagaku Kaishi., (1980), 1261.

12) S. Bruckenstein and M. Shay, Electrochim. Acta., 30, (1985), 1295.

13) K. K. Kanazawa and J, G. Gordon, Anal. Chim. Acta., 175, (1985), 99. 
14) D. Z. Shen, L. H. Nie and S. Z. Yao, J. Electroanal. Chem., 367, (1994), 31.

15) A. R. Hillman, The Elecrochemical Quartz Crystal Microbalance, from Encyclopedia of Electrochemistry: Instrumentation and Electroanalytical Chemistry, Vol 3, edited by P. R. Unwin, (2003).

16) T. Nomura, M. Kumaggai and A. Sato, Anal. Chim. Acta., 343, (1997), 209.

17) T. Nomura, M. Isawa, H. Matsuzawa and Y. Shibukawa, Anal. Chim. Acta., 286, (1994), 205.

18) S. Z. Yao, P. Chen and W. Z. Wei, Food Chemistry., 67, (1999), 311.

19) M. Skompska and A. R. Hillman, J. Electroanal. Chem., 433, (1997), 127.

20) Y. B. Mo, E. Hwang and D. A. Scherson, J. Electrochem. Soc., 143, (1996), 37.

21) Y. Okahata and H. Ebato, Trends Anal. Chem., 11, (1992), 344.

22) J. Rickert, T. Weiss, W. Kraas, G. Jung and W. Gopel, Biosensors and Bioelectronics., 11, (1996), 591.

23) R. M. Crooks, H. C. Yang, L. J. M. Ellistrem, R. C. Thomas and A. J. Ricco, Faraday Discuss., 107, (1997).

24) J. M. Kim, J. Y. Park, S. H. Song, B. J. Lee, H. Muramastsu and S. M. Chang, Sensors and Actuators B., 76, (2001), 74.

25) M. Hepel, Z. Xingmin, R. Stephenson and S. Perkins, Microchemical Journal., 56, (1997), 79.

26) C. S. Lu and O. Lewis, J. Appl. Phys., 43, (1972), 4385.

27) J. G. Miller and D. L. Bolef, J. Appl. Phys., 39, (1968), 4589.

28) K. K. Kanazawa and J. G. Gordon, Anal. Chem., 57, (1985), 1770.

29) M. D. Ward and E. J. Delawski, Anal. Chem., 63, (1991), 886.

30) K. Wagner, J. W. Strojek and K. Koziel, Anal. Chim. Acta., 455, (2002), 69.

31) C. Cabrielli, M. Keddam and R. Torresi, J. Electrochem. Soc., 138, (1991), 2657.

32) W. J. Albery and A. R. Hillman, J. Electroanal. Chem. Interfac. Electrochem., 170, (1984), 27.

33) S. J. Martin and G. C. Frye, (1991), IEEE Ultra Son Symp, New York, 393.

34) Z. A. Shana, H. Zong, F. Josse and D. C. Jeutter, J. Electroanal. Chem., 379, (1994), 21.

35) S. J. Martin, V. E. Granstaff and G. C. Frye, Anal. Chem., 63, (1991), 2272.

36) D. K. Gosser, Cyclic Voltammetry: Simulation and Analysis of Reaction Mechanisms, Wiley-VCH, New York, 1993.

37) F.Scholz, Electroanalytical Methods "Guide to Experiments and Applications", Springer, Berlin, 2002. 
38) P.T.Kissinger and W. R. Heineman, Laboratory Techniques in Electroanalytical Chemistry, Macel Dekker, Inc, New York, (1997).

39) P. T. Kissinger and W. R. Heineman, J. Chem. Edu., 60, (1983), 702.

40) M. Rudoph, D. Reddy and S. W. Feldberg, Anal. Chem., 66, (1994), 589 A.

41) I.Lavagnini, R.Antiochia and F.Magno, Electroanalysis, 16, (2004), 505.

42) S.Hagstrom, C.Nordling and K.Siegbahn, Z. Phys., 178, (1964), 439.

43) J.F.Watta and J.Wolstenholme, An Introduction to surfaces Analysis by XPS and AES., Wiely, New York, (2003).

44) E.Sokolowski, C.Nordling and K.Siegbahn, Phys. Rev., 110, (1958), 776.

45) J.F.Modulder, W.F.Stickle, P.E.Sobol and K.D.Bomben, Handbook of X-ray Photoelectron Spectroscopy, Perkin-Elmer, Physical electronics Divison, Eden Prairie, MN 55344, (1992

46) D.Briggs and J.T.Grant, Surface Analysis by Auger and X-ray Photoelectron Spectroscopy., Surface Spectra and IM Publications, Manchester and Chichester, UK, (2003).

47) NIST Standard Reference Database 20, Version 3.3, Web version, (http://srdata.nist.gov/xps/.

48) G.Moretti, F. Filippone and M.Satta, Surf.Interface. Anal., 31, (2001), 249. 


\section{Chapter 3: Experimental}

\subsection{Introduction}

This chapter describes the experimental aspects of quartz crystal microbalance, cyclic voltammetry and X-ray photoelectron spectroscopy techniques and gives the general methodology of the experiments undertaken. It describes the preparation of different kinds of self-assembled monolayers, some aliphatic and others aromatic, and then use of the QCM to examine the rate and extent of self-assembly.

\subsection{Quartz crystal Microbalance (QCM) preparations}

The quartz crystals that are used are alpha quartz crystals (AT-cut), which give nearly zero temperature coefficients (the proportionality constant that relates the oscillating frequency in a vacuum to the temperature). Two types of QCMs were used: polished and unpolished. The preparation will be described in sections 3.2.1 and 3.2.2.

\subsubsection{Unpolished Crystals}

Unpolished $10 \mathrm{MHz}$ AT-cut quartz crystals were used as supplied from International Crystal Manufacturing (ICM) Co., Oklahoma City, USA. They consist of a thin slice of quartz (approximately $0.16 \mathrm{~mm}$ thick) sandwiched between two gold keyhole shaped electrodes as shown in figure (2.3). The quartz has a $5 \mu \mathrm{m}$ alumina abrasive finish, and the thickness of gold electrodes is ca. $250 \mathrm{~nm}$. There is no underlayer between the gold and the quartz. The piezoelectric and electrochemical active areas are $0.21 \mathrm{~cm}^{2}$ and $0.23 \mathrm{~cm}^{2}$ respectively. The electroactive area is slightly larger due to the contacting tab (see Figure 2.3).

\subsubsection{Polished Crystals}

Polished $10 \mathrm{MHz}$ AT-cut quartz crystals were used as supplied from (ICM as above), and have a smaller than $1 \mu \mathrm{M}$ alumina abrasive finish. The piezoelectrically and electrochemically active areas are $0.21 \mathrm{~cm}^{2}$ and $0.23 \mathrm{~cm}^{2}$ respectively.

\subsection{Quartz Crystal Microbalance Cell}

The electrodes were arranged in a standard microbalance cell as shown in Figure (3.1). The quartz crystal was attached onto the bottom of the cell using Dow Corning 3145 RTV silicon rubber adhesive (sealant). This is a non corrosive, room 
temperature curing silicone rubber, used to glue the crystal to the cell bases. The adhesive takes 24 hours to dry. Extreme care was taken not to make contact between the gold on the electrode and the sealant applied. The adhesive should not be in contact with the centre circular part of the electrode. This had to be kept from impinging on the gold electrode, as its presence there would severely damp the crystals oscillation and seriously reduce the mass sensing abilities of the QCM.

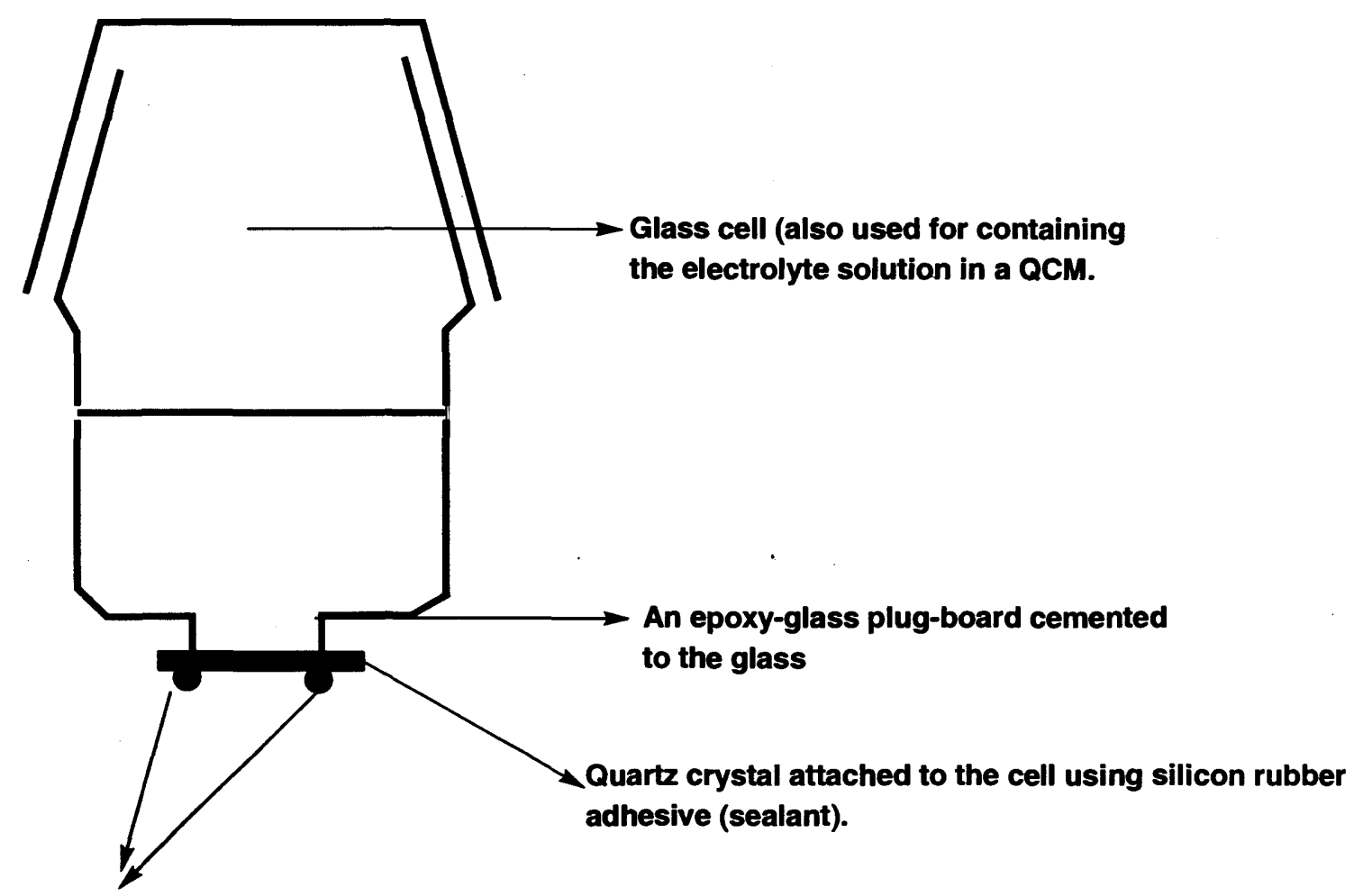

Plug jacks enabling the cell to be connected to the network analyser or the QCM circuitry.

Figure 3.1: Schematic illustration of the quartz crystal microbalance cell used to immobilise SAMs on Au quartz crystal.

\subsubsection{Electrochemical Cell}

The electrochemical cell consists of three electrodes as shown in the Figure (3.2).In this cell kinetic measurement is made by current measurements at the working electrode (quartz crystal). The potential at this electrode is controlled with respect to a reference electrode. The reason we use a third electrode is because in a two electrode cell the reference electrode necessarily passes an equal and opposite current to the working electrode. So, by definition, it is therefore not at 
equilibrium. The third electrode (counter electrode) acts to correct this problem by providing a source/sink for the electrons involved in the working electrode reaction. In general the potential is controlled between the working and reference electrodes, not the counter electrode. The current flows only between the working and the counter electrodes, not the reference electrode. In my experiments the working electrode was one of the gold electrodes of the QCM.

The counter electrode was platinum gauze with a surface area greater than that of the working electrode. The reference electrode was a saturated calomel electrode (SCE). This was prepared as described in Sawyer and Roberts [1]. The reference electrode has a standard potential of $+0.244 \mathrm{~V}$ against a hydrogen electrode (NHE).

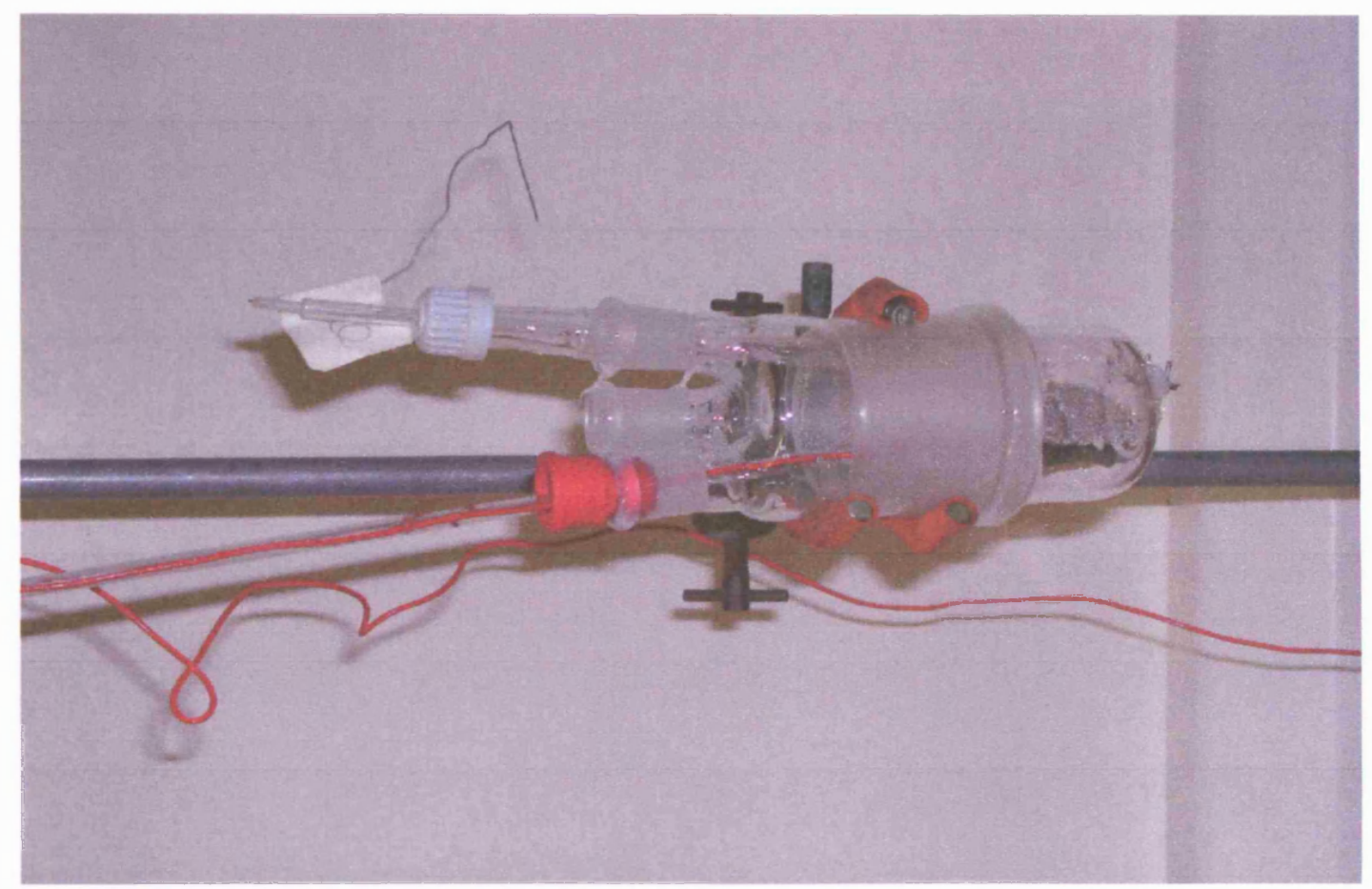

Figure 3.2: Schematic illustration of the electrochemical cell used to make cyclic voltammetry for un-modified and modified Au quartz crystal with SAMs in $5 \mathrm{mM} \mathrm{K}_{3} \mathrm{Fe}(\mathrm{CN})_{6} /$ $1 \mathrm{M} \mathrm{KCl}$. 


\subsubsection{Calibration of the QCM [2, 3, 4]}

The crystals were mounted into the cell and small amounts of pure solvents were introduced into the cell.

The output frequency for the crystal for in pure solvent was recorded after it established a stable frequency. All organic solvents were special grade chemicals. The experiment was prepared in five steps:

(1) Measure the frequency of the clean dry crystal (crystal in air $f_{1}$ ).

(2) Fill the microbalance cell with $20 \mathrm{ml}$ of organic solvent.

(3) Measure the frequency of wet crystal $\left(f_{2}\right)$.

(4) The frequency difference was measured $(\Delta f)$ several times and the mean value was calculated to give the frequency shift caused by the organic solvent. 


\section{4 structures of aliphatic and aromatic based thiols use to prepare}

\section{SAM}

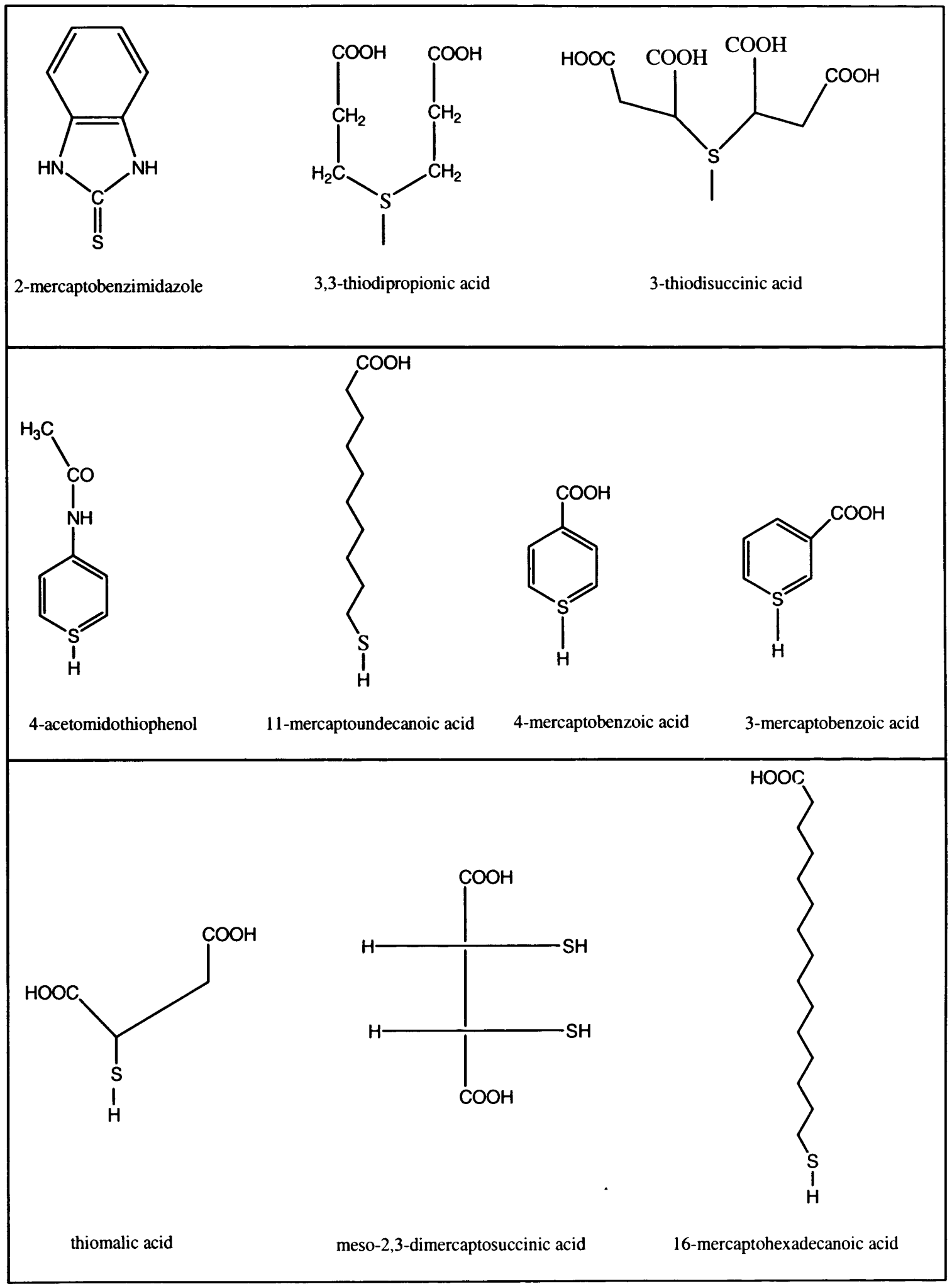

Figure 3.3: The chemical structure of the thiols and sulfides used. 


\subsubsection{Chemicals}

The chemicals used are listed in Table 3.1 below. They were used as received.

\begin{tabular}{|c|c|c|}
\hline Chemical & Supplier & purity \\
\hline 2-mercaptobenzimidazole & Aldrich & $98 \%$ \\
\hline 3,3-thiodipropionic acid & Lancaster & $99 \%$ \\
\hline 3-thiodisuccinic acid & Lancaster & $98 \%$ \\
\hline 4-acetomidothiophenol & Aldrich & $90 \%$ \\
\hline 11-mercaptoundecanoic acid & Aldrich & $95 \%$ \\
\hline 4-mercaptobenzoic acid & Aldrich & $97 \%$ \\
\hline 3-mercaptobenzoic acid & Aldrich & $95 \%$ \\
\hline Thiomalic acid & Aldrich & $97 \%$ \\
\hline Meso-2,3-dimercaptosuccinic acid & Fluka & $97 \%$ \\
\hline 16-mercaptohexadecanoic acid & Aldrich & $90 \%$ \\
\hline 1,6-hexanedithiol & Aldrich & $96 \%$ \\
\hline 1,4-benzendimethanethiol & Aldrich & $98 \%$ \\
\hline Potassium ferricyanide(III) & Aldrich & A.C.S reagent grade \\
\hline Potassium Chloride & Aldrich. & A.C.S reagent grade \\
\hline Potassium Nitrate & Aldrich & $99 \%$ \\
\hline
\end{tabular}

Table 3.1: list of the chemicals used in this study. 


\subsubsection{Solvents and Reagents}

All of the solvents used in the experiments are listed in Table 3.2 below; no further purification was used.

\begin{tabular}{|c|c|c|}
\hline Solvent & Supplier & purity \\
\hline Ethanol & Merck & Absolute Ethanol (99.8\%) \\
\hline Methanol & Aldrich & Biotech grade solvent $(99.93 \%)$ \\
\hline Chloroform & Fisher & $99 \%$ \\
\hline Toluene & Fisher & $99 \%$ \\
\hline Acetonitrile & Fisher & $99.98 \%$ \\
\hline Cyclohexene & Fisher & $99 \%$ \\
\hline Hexane & Fisher & $99 \%$ \\
\hline Acetone & Aldrich & A.C.S reagent $(99.5 \%)$ \\
\hline Hydrogen peroxide & Aldrich & A.C.S reagent ( $30.32 w t \%)$ \\
\hline Sulphuric acid & Aldrich & analytical reagent grade $(97 \%)$ \\
\hline Nitric acid & Aldrich & analytical reagent grade $(70 \%)$ \\
\hline Hydrochloric acid & Aldrich & analytical reagent grade $(37 \%)$ \\
\hline Sodium hydroxide & Aldrich & $99 \%$ \\
\hline $\begin{array}{l}\text { Ethylenediaminetetra acetic } \\
\text { acid }\end{array}$ & Aldrich & $98 \%$ \\
\hline
\end{tabular}

Table 3.2: solvents and reagents used in this study. 


\subsection{Preparation of Self-assembled Monolayers}

\subsubsection{SAM Solution Preparation}

All SAM solutions were prepared by dissolving the thiol in absolute ethanol at room temperature. The concentration of all the thiols was $10 \mathrm{mM}$ in all experiments.

\subsubsection{Preparation of Surfaces}

The crystals were cleaned in a fresh Piranha solution $\left(30 \% \mathrm{H}_{2} \mathrm{O}_{2}: 98 \% \mathrm{H}_{2} \mathrm{SO}_{4}, 1\right.$ : 3 by volume) at room temperature for $1-3$ minutes $[5,6,7]$ rinsed thoroughly by Millipore Milli-Q deionised water, dried under a stream of argon, rinsed again with absolute ethanol to remove any remaining water and dried under a stream of argon. Cleaned crystals were cycled in $5 \mathrm{mM} \mathrm{K} \mathrm{K}_{3} \mathrm{Fe}(\mathrm{CN})_{6}+1 \mathrm{M} \mathrm{KCl}$ aqueous solution from -0.4 to $+0.6 \mathrm{~V}$ vs. SCE until a stable voltammogram was obtained. After the cycling, the crystals were rinsed with absolute ethanol and dried under argon to prepare the clean surface. In order to allow SAMs formation, the crystals were used immediately after the cleaning process.

\subsubsection{Preparation of Functionalized SAMs}

Before preparing the modification solution, absolute ethanol was purged with argon for $30 \mathrm{~min}$ to remove any oxygen from the solution. $25 \mathrm{ml}$ of $1 \mathrm{mM}$ modification solutions were transferred to the quartz crystal microbalance cell (see figure 3.1). Self-assembled monolayers were prepared by immersing the quartz crystals (clean crystals) in $25 \mathrm{ml}$ of $10 \mathrm{mM}$ ethanolic modification solutions for $24-48$ hours to form a full monolayer on the surface. After the functionalization, the solutions were removed from the QCM cell; the crystals were rinsed with absolute ethanol to remove any excess on the surface and blown dry with argon. All the modifications were done at room temperature.

\subsubsection{Preparation of 1000ppm of cadmium, lead, copper and mercury ions}

1000ppm solution of cadmium ions was prepared by dissolving $2.744 \mathrm{~g}$ of cadmium nitrate (99.9\%, Aldrich), $3.660 \mathrm{~g}$ of copper nitrate (99.9\%, Aldrich), $1.598 \mathrm{~g}$ of lead nitrate $(99.9 \%$, Aldrich) and $1.63 \mathrm{~g}$ of mercury nitrate $(99.9 \%$, Aldrich) in ultra pure deionised water and diluted to $1000 \mathrm{ml}$. From this solution you can prepare a range of concentrations of cadmium ions $(1 \mu \mathrm{M}$ to $10 \mathrm{mM})$. The measurements were first made for single metal ion solution (non competitive binding) of $\mathrm{Cd}(\mathrm{II}), \mathrm{Pb}$ (II), $\mathrm{Cu}$ (II) and $\mathrm{Hg}(\mathrm{II})$ at the natural $\mathrm{pH}$ 's of stock solutions $(\mathrm{pH} 6.3,4.8,5.4$ and 5.5, 
respectively). In all dilute solutions, the $\mathrm{pH}$ of the solution was adjusted with $0.1 \mathrm{M}$ $\mathrm{KOH}$ or $0.1 \mathrm{M} \mathrm{HNO}_{3}$ after added ultra pure water to keep all the dilute solutions at same $\mathrm{pH}$. These procedures were done before QCM and EQCM measurements. The effect of ligand protonation state was explored separately by adjusting the solution $\mathrm{pH}$ (with $0.1 \mathrm{M} \mathrm{KOH}$ or $0.1 \mathrm{M} \mathrm{HNO}_{3}$ ). The $\mathrm{pH}$ was measured with a $\mathrm{pH}$ meter.

\subsection{Frequency Measurements}

All frequency measurements were carried out using EQCM 2 (6/96). The oscillating frequency of the QCM was measured with Hewlett Packard 5313A universal counter. as can be seen in Figure 3.4. The sequence of actions was:

1) Measure the frequency for the clean crystal (crystal in air $f_{\text {dry }}$ ). $f_{\text {dry }}$ is the frequency shift for the emersed crystal (Au / air).

2) Measure the frequency for the (clean crystal in thiol solution, $f_{\text {wet }}$ ). $f_{\text {wet }}$ is the frequency shift for the immersed crystal ( $\mathrm{Au} / \mathrm{RSH}, \mathrm{EtOH}$ ).

3) Measure the frequency for the wet crystal again after $24 \mathrm{~h}$ exposure to the modification solution of SAM (Au / RSH, EtOH) to give $\left(f_{\text {wet-SAM }}\right)$.

4) Measure the frequency for the dry crystal again after $24 \mathrm{~h}$ and after rinsed with absolute ethanol, promptly dried with argon $(\mathrm{Au} / \mathrm{RSH})$ to give $\left(f_{\text {dry-SAM }}\right)$.

5) $\Delta f_{\text {dry }}$ for the $S A M=f_{\text {AU-RSH-24h-air }-f_{\text {dry }}}$

6) $\Delta f_{\text {wet }}$ for the SAM $=f_{\text {Au-RSH-EtOH-24h }}-f_{\text {wet }}$

All the frequency shift measurements and modification were obtained at room temperature (typically $20^{\circ} \mathrm{C}$ ).

\subsection{Quartz Crystal Impedance measurements}

Quartz crystal impedance measurements were carried out at a series of frequencies close to resonance frequency of the quartz crystal using a Hewlett Packard HP8751A network analyser operating in reflectance mode utilising a HP8512A transmission/ reflection test unit. In order to fully characterise the SAM films, crystal impedance measurements were taken for (a) uncoated crystal in air (Au / air), (b) un coated crystal in thiol solution (Au / RSH, EtOH), (c) coated crystal with SAM in ethanolic solution after $24 \mathrm{~h}$ (Au / SAM $24 \mathrm{~h} / \mathrm{EtOH}$ ) and (d) coated crystal with SAM in air after $24 \mathrm{~h}$ (Au / SAM $24 \mathrm{~h}$ air). 


\subsection{Cyclic Voltammetry measurements}

All electrochemical measurements were carried out using a PGSTAT20 potentiostat (Ecochemie, Holland) see Figure 3.5. The working electrodes were gold quartz crystals of the EQCM. The counter electrode was Pt gauze and the potentials were quoted vs. a SCE reference electrode (see Figures 3.1 and 3.2). CV was performed from -0.4 to $0.6 \mathrm{~V}$ vs. SCE using the SAM modified electrodes in supporting electrolyte solution which contained $5 \mathrm{mM} \mathrm{K}_{3} \mathrm{Fe}(\mathrm{CN})_{6} / 1 \mathrm{M} \mathrm{KCl}$ to investigate the quality and integrity of the SAM on the gold surface. The electrochemical readings were taken at the scan rates of $5,10,50,100 \mathrm{mV} \mathrm{s}^{-1}$. All experiments were performed under ambient conditions. Solutions were purged with argon for 20 minutes before measurements and blanketed with argon during the measurements to prevent $\mathrm{O}_{2}$ redissolution. All cyclic voltammograms were obtained at room temperature (typically $20^{\circ} \mathrm{C}$ ).

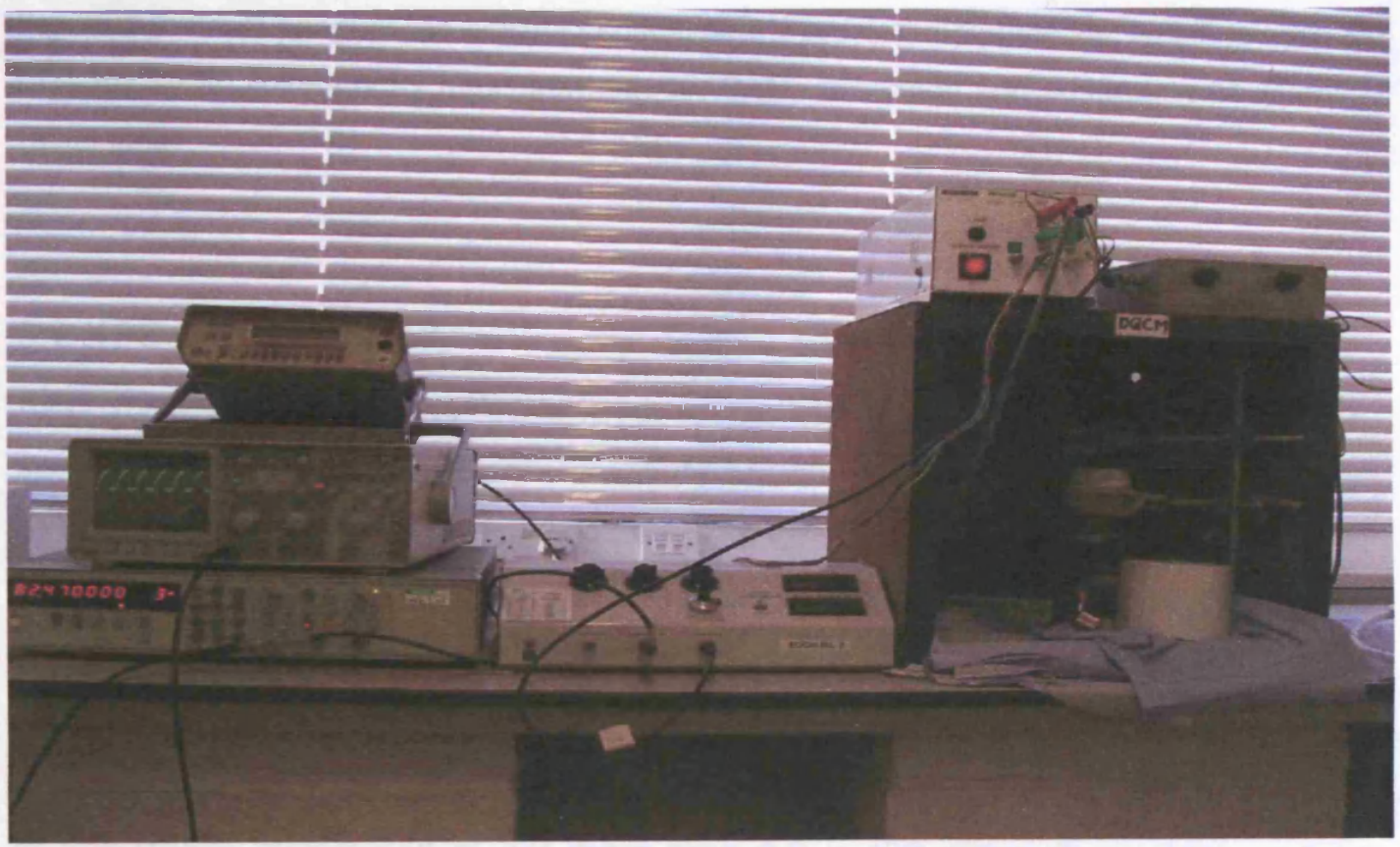

Figure 3.4: Front panel of the quartz crystal microbalance, which is used to take the frequency measurements for SAMs formation and adsorption of different metal ions. 


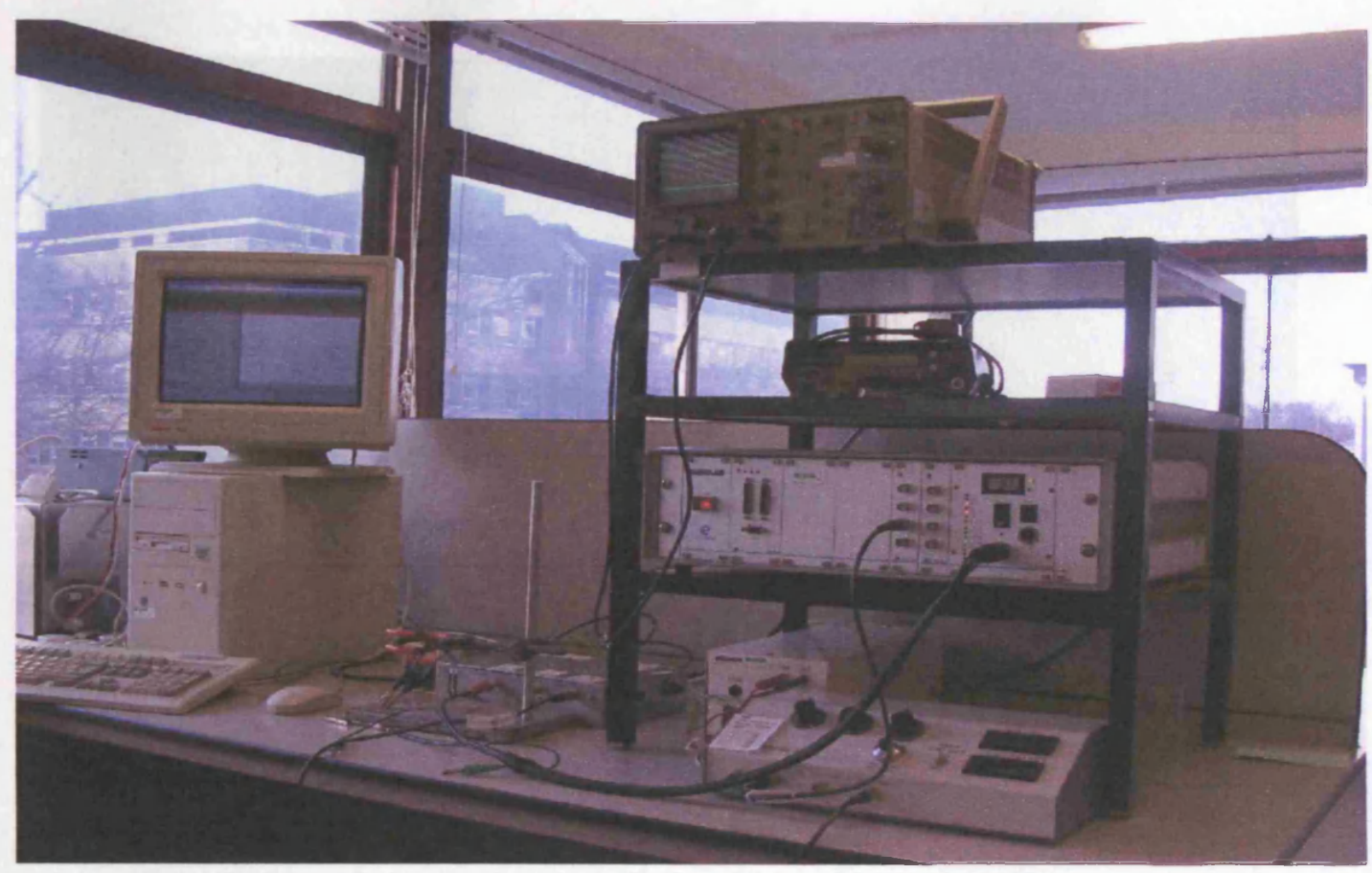

Figure 3.5: Front panel of the electrochemical quartz crystal microbalance, which is used to take the cyclic voltammetry measurements for SAMs formation and adsorption of different metal ions.

\subsection{XPS Measurements}

XPS measurements were performed on a Scienta ESCA 300 photoelectron spectrometer (RUSTI / NCESS Daresbury Laboratory, UK) using monochromated Al $\mathrm{Ka}(1486.7 \mathrm{eV})$ radiation, a slit width of $0.8 \mathrm{~mm}$, take off angle of $10^{\circ}$ and a flood gun energy of either 1.0 or $2.0 \mathrm{eV}$. A monochromated Al Ka rotating anode $\mathrm{X}$-ray source was operated at $14 \mathrm{kV}$ and $20 \mathrm{~mA}$. Photoelectrons were detected by a hemispherical analyzer and a two dimensional position sensitive detector (microchannel plates with CCD camera). The base pressure in the analysis chamber was maintained at not more than $2.0 \times 10^{-9}$ mbar. Each SAM sample studied was mounted on a stub using double sided adhesive tape and then placed in the ultra-high vacuum analysis chamber of the spectrometer. The spectra were referenced to the $A u 4 f_{7 / 2}$ line positioned at $84.0 \mathrm{eV}$. Figure 3.6 shows the picture of the Scienta ESCA300 spectrometer at NCESS. The ESCA300 photoelectron spectrometer is capable of high intensity, high energy resolution and high spatial resolution. 


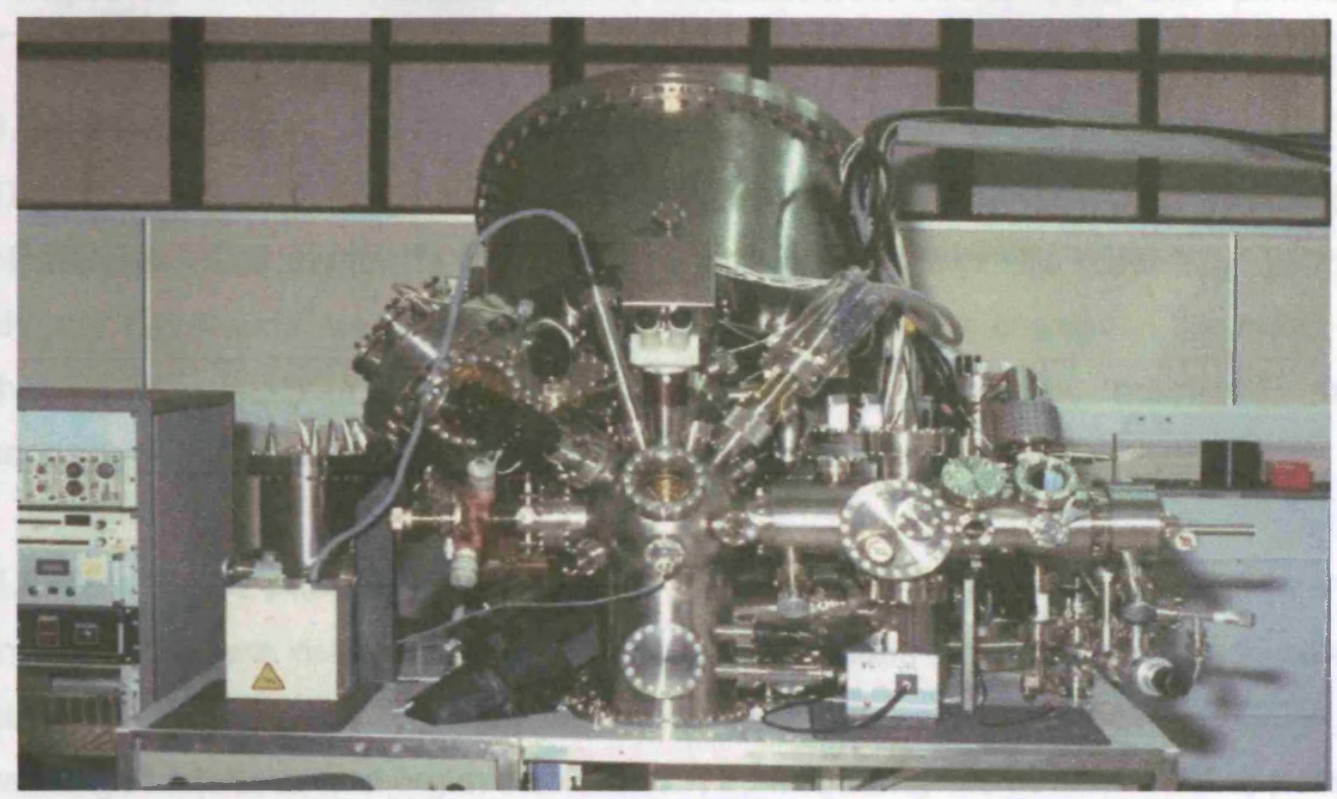

Figure 3.6: Picture of the front panel of the ESCA300 photoelectron spectrometer at NCESS.

The Au films were deposited on the clean SF10 microscope slides in a homemade vacuum chamber using Ti binder layers. SAMs before and after adsorption of different metal ions were prepared on to gold microscope slides after cutting the gold slides into pieces $1.5 \times 1.5 \mathrm{~cm}^{2}$.

\subsection{References}

1) D. T. Sawyer and J. L. Roberts (Ed.), Experimental Electrochemistry for Chemists, Wiley, New York, 1986.

2) T. Nomura and M. Okuhara, Analyticachimica Acta., 142, (1982), 281.

3) G. Mchale, M. I. Newton, M. K. Banerjee and S. M. Rowan, Faraday Disuss., 107, (1997).

4) L. Dailkhin and M. Urbakh, Faraday Disuss., 107, (1997).

5) B. D. Spangler and B. J. Tyler, Anal. Chim. Acta., 399, (1999), 51.

6) T. Baunach and D. M. Kolb, Anal Bioanal Chem., 373-374, (2002), 743.

7) E. Huang, F. Zhopu and L. Deng, Langmuir., 16, (2000), 3272. 


\subsection{Introduction}

The primary objective of this work was to have successful surface functionalization of a clean QCM crystal to form a QCM-monolayer composite resonator. In this chapter the results are presented in two stages: firstly, the change in the mass of the QCM composite resonator interpreted (using Sauerbrey equation), and secondly the use of cyclic voltammetry to characterise the monolayer quality.

\subsection{Results of calibration of QCM}

In this study we measured the frequency of crystals immersed in modification solution before and after SAM immobilisation. We need to check the QCM is working in aqueous and organic solutions. The frequency shift of the QCM depends on the viscosity and density of the solution, as described in chapter two (section 2.3). The frequency decrease for two different quartz crystals (polished and unpolished) immersed in various organic solvents are shown in Table 4.1. It is apparent that the responses of the unpolished crystals are significantly greater than polished crystals.

\begin{tabular}{|l|l|l|l|l|l|l|l|}
\hline Solvent & $\begin{array}{c}(\eta) \\
\left(\mathbf{c P}^{*}\right)\end{array}$ & $\begin{array}{l}(\rho) \\
\left(\mathrm{g} / \mathbf{c m}^{3}\right)\end{array}$ & $\begin{array}{c}-\Delta f \\
\text { polished } \\
(\mathrm{Hz})\end{array}$ & $\begin{array}{c}-\Delta f \\
\text { unpolished } \\
(\mathbf{H z})\end{array}$ & $\begin{array}{l}-\Delta f / \rho \\
\text { polished } \\
\mathbf{s}^{-1} \mathbf{g}^{-1} \mathbf{c m}^{3}\end{array}$ & $\begin{array}{c}-\Delta f / \rho \\
\text { unpolished } \\
\mathbf{s}^{-1} \mathbf{g}^{-1} \mathbf{c m}^{3}\end{array}$ & $(\eta / \rho)^{1 / 2}$ \\
\hline Chloroform & 0.538 & 1.498 & 2696 & 5953 & 1799 & 3973 & 0.059 \\
\hline Ethanol & 1.078 & 0.785 & 2862 & 5035 & 3645 & 6413 & 0.117 \\
\hline Methanol & 0.542 & 0.786 & 2133 & 4217 & 2711 & 5361 & 0.083 \\
\hline Toluene & 0.552 & 0.862 & 2189 & 4010 & 2538 & 4649 & 0.080 \\
\hline Acetonitrile & 0.344 & 0.777 & 3141 & 3717 & 4043 & 4785 & 0.066 \\
\hline Cyclohexene & 0.93 & 0.773 & 2254 & 5176 & 2912 & 6688 & 0.010 \\
\hline Hexane & 0.305 & 0.654 & $\#$ & 4486 & $\#$ & 6852 & 0.068 \\
\hline Acetone & 0.302 & 0.784 & $\#$ & 4000 & $\#$ & 5099 & 0.063 \\
\hline Water & 0.010 & 0.998 & 2762 & 5637 & 2766 & 5647 & 0.010 \\
\hline
\end{tabular}

Table 4.1: Frequency shifts of the crystal between air and liquids at $20^{\circ} \mathrm{C}$.

$\mathrm{CP}=10^{-2} \mathrm{~g} \mathrm{~cm}^{-1} \mathrm{~s}^{-1} \quad \rho=$ density at $20^{\circ} \mathrm{C} \quad \eta=$ viscosity at $20^{\circ} \mathrm{C}$. \# No reading taken. 


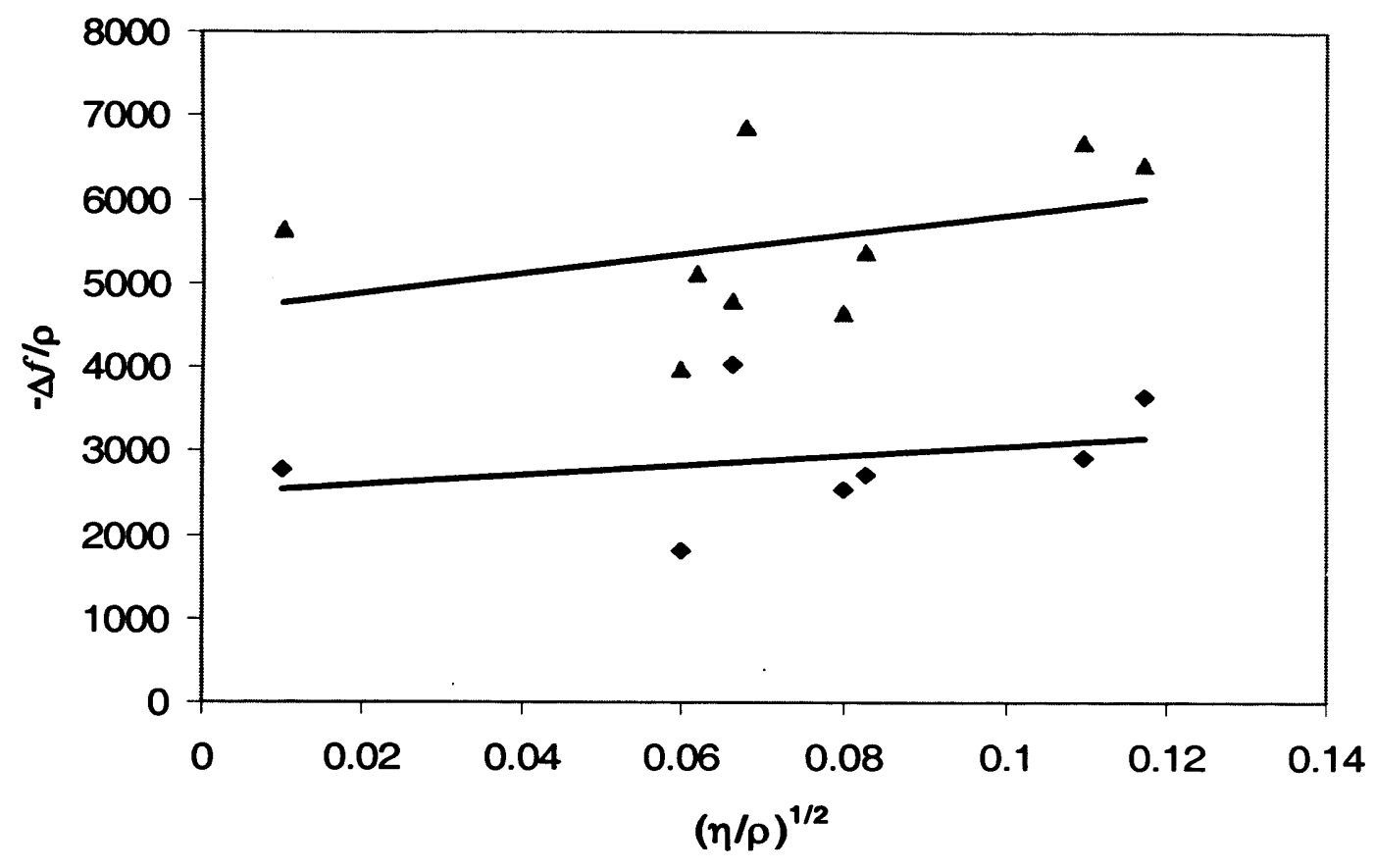

Figure 4.1: Normalised frequency shifts for different solvents at polished and unpolished $\mathbf{A}$ crystals. Lines are least squares fits.

\subsubsection{Discussion}

The QCM frequency shifts (Figure 4.1) show that the frequency shifts variation with solvent density and viscosity. For example, although acetone, ethanol and methanol have almost the same density, the frequency shifts were proportional to the square root of their viscosities. In contrast, methanol and chloroform have similar viscosities, and their different frequency shifts depended on their densities. The crystal did not oscillate in liquids of high density and viscosity such as nitrobenzene and carbon tetrachloride. The frequency shift results on polished and unpolished crystals for organic solvents are parallel. In agreement with theory [1] the groove depth influences the resonant frequency and shows that, as a groove depth increases, the resonant frequency change increases as well. This can be viewed as resulting from the deeper grooves trapping more liquid. One you can see from analysing the intercepts in (Figure 4.1), estimate the effective height of the grooves (roughness of the surface). The effect height of the grooves on unpolished and polished crystals was $131 \mathrm{~nm}$ and $40 \mathrm{~nm}$, respectively. 


\subsection{Relation between $\Delta f_{\text {dry }}$ and $\Delta f_{\text {wet }}$ for SAMs}

In this section we will present data obtained from the QCM frequency measurements of dry crystals with SAMs and wet crystals immersed in the modification solution of SAMs after $48 \mathrm{~h}$. The resonant frequency was dramatically decreased during the self-assembly process of the different SAMs as can be seen in table 4.2 .

\begin{tabular}{|lll|c|c|}
\hline \multicolumn{2}{|c}{ SAM } & & $-\Delta f_{\text {dry }}$ (Hz) & $-\Delta f_{\text {wet }}(\mathrm{Hz})$ \\
\hline (1) & 2-mercaptobenzimidazole & (MBI) & 2302 & 2435 \\
\hline (2) & 3,3-thiodipropionic acid & (TDP) & 267 & 139 \\
\hline (3) & 3-thiodisuccinic acid & (TDS) & 206 & 174 \\
\hline (4) & 4-acetomidothiophenol & (AMTP) & 2131 & 116 \\
\hline (5) & 11-mercaptoundecanoic acid & (MUA) & 452 & 590 \\
\hline (6) & 4-mercaptobenzoic acid & (MBA) & 1840 & 453 \\
\hline (7) & 3-mercaptobenzoic acid & (3-MBA) & 1642 & 525 \\
\hline (8) & thiomalic acid & (TMA) & 190 & 256 \\
\hline (9) & meso-2,3-dimercaptosuccinic acid & (DMSA) & 190 & 60 \\
\hline (10) & 16-mercaptohexadecanoic acid & (MHA) & 457 & 258 \\
\hline (11) & 1,6-hexanedithiol & (HDT) & 68 & 81 \\
\hline (12) & 1,4-benzenedimethanethiol & (BDMT) & 110 & 130 \\
\hline
\end{tabular}

Table 4.2: Showing the frequency change measurements on crystals after $48 \mathrm{~h}$ for $\Delta f_{\text {dry-SAM }}=f_{\text {dry-SAM after } 24 \mathrm{~h}}-f_{\text {dry crystal }}$ and $\Delta f_{\text {wet-SAM }}=f_{\text {wet-SAM after } 24 \mathrm{~h}}-f_{\text {wet crystal }}$.

Figure 4.2 shows the relation between frequency shifts on dry and wet crystals for the formation of SAMs. It shows that for the aliphatic SAMs the range of frequency shifts is from $150-500 \mathrm{~Hz}$ and for aromatic SAMs is from $1600-2500 \mathrm{~Hz}$. Generally, the resonant frequency shift for long chain alkane thiols is bigger than short chain alkane thiols and the resonant frequency shift for aroamatic thiols is bigger than alkane thiols. 


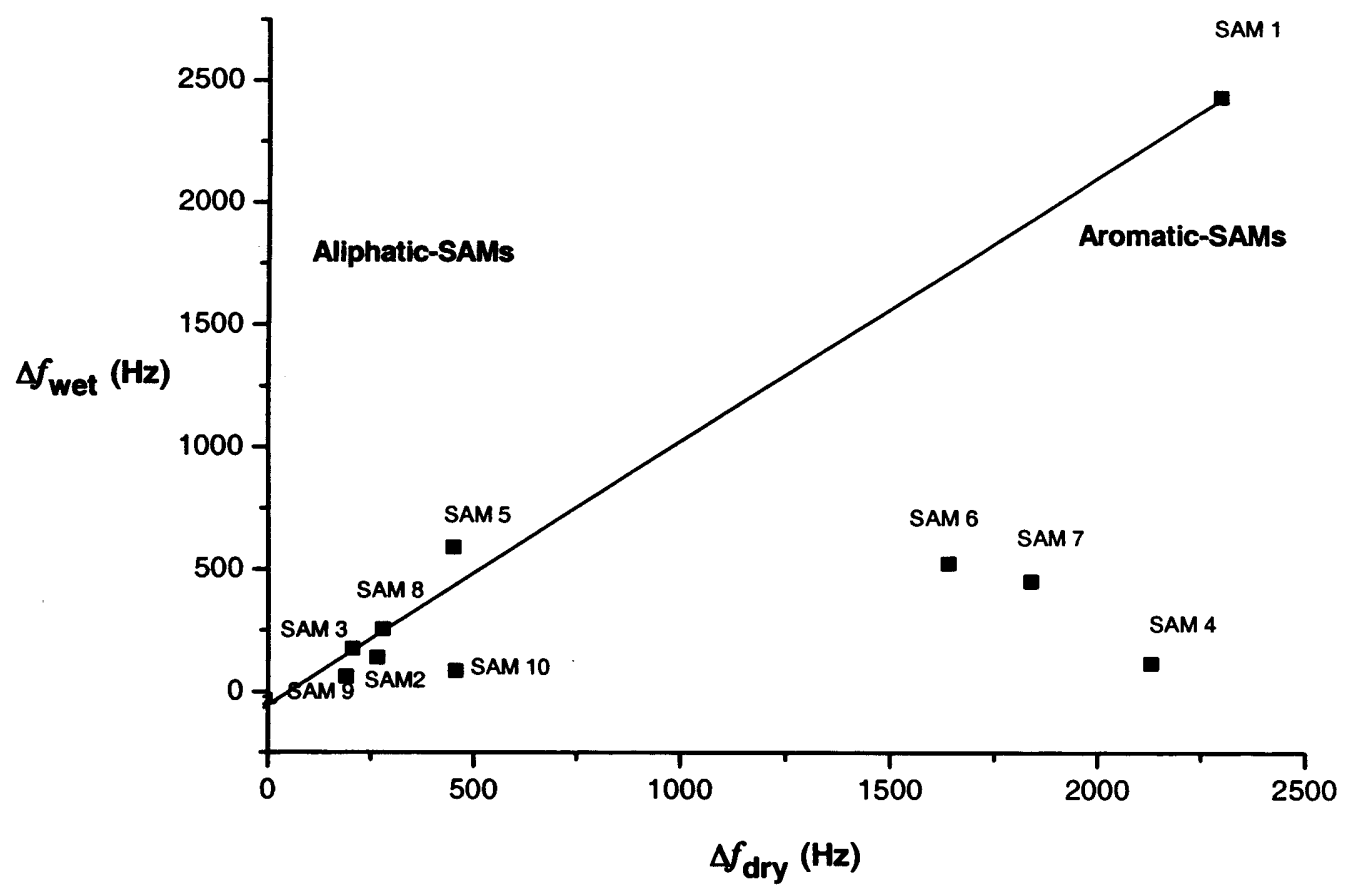

Figure 4.2: Shows the relation between dry crystals of modification SAMs) and wet crystals at $T=22 C^{\circ}$. The number of each SAM species refers to table 4.2 (from SAM 1 to SAM 10).

\subsection{Quartz Crystal Impedance Spectroscopy for Monitoring SAMs}

Establishing the rigidity of a SAM requires the plotting of the admittance vs. the frequency of the applied signal. A rigid SAM will give no change in the shape of the signal, simply a change in the position of the signal on the frequency axis. In comparison, the signal due to a non-rigid SAM will show a broadening or flattening along with a position change. The results of crystal impedance spectra for 3thiodisuccinic acid, 4-mercaptobenzoic acid and 4-acetomidothiophenol are shown in Figures 4.3 to 4.5. It was found that 3-thiodisuccinic acid forms an acoustically thin layer. Where acoustically thin indicates that the film thickness $(h)$ is much smaller than the decay length $(f)$ of the acoustic wave launched by the crystal. Typically, $\mathrm{h} \approx 1 \mathrm{~nm}$ and $f \approx 100-500 \mathrm{~nm}$, so $\mathrm{h} \ll f$. The results from the frequency shift using quartz crystal impedance also show that SAMs such as 4mercaptobenzoic acid and 4-acetomidothiophenol have a bigger frequency shift than SAMs such as 3-thiodisuccinic acid. The results of frequency shift for the immobilization of SAM on emersed and immersed crystals from $1 \mathrm{mM}$ of modification solutions indicate that SAM has been successfully immobilized on gold electrode. 


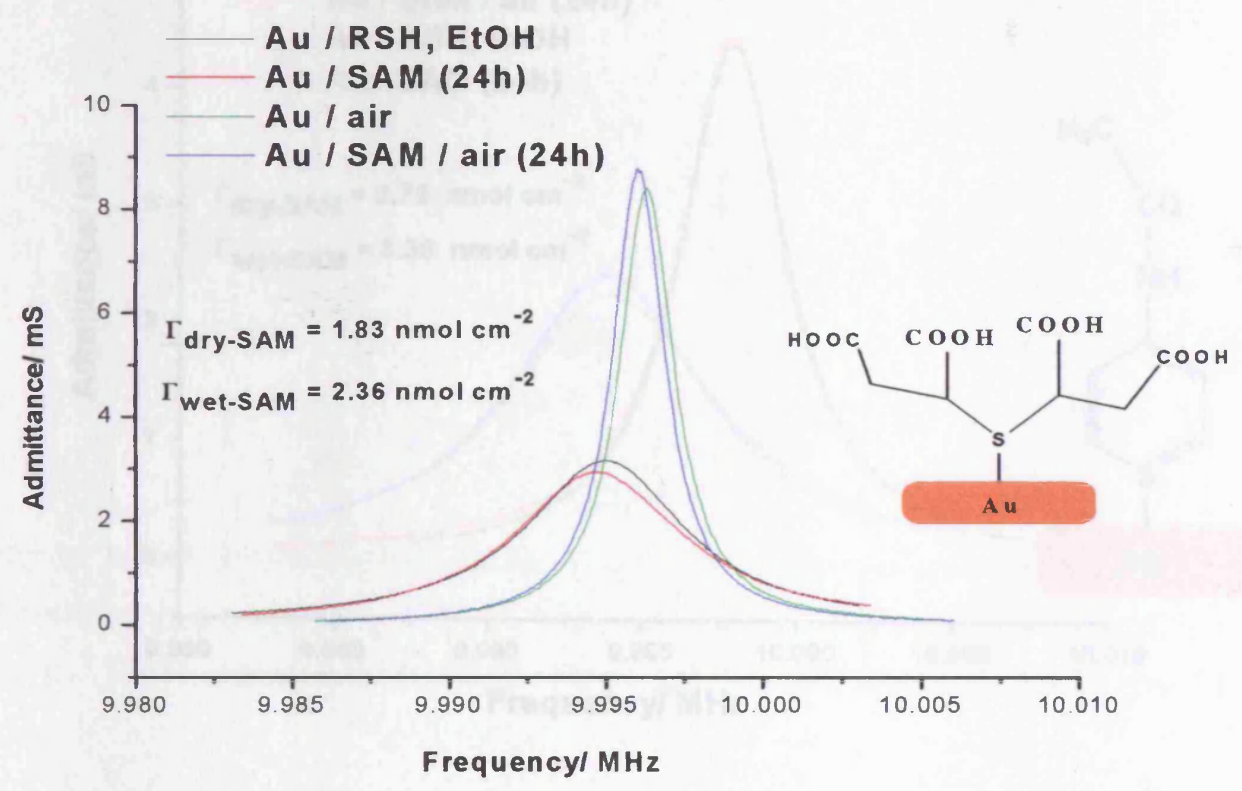

Figure 4.3: Crystal impedance spectra for a bare gold electrode and gold electrode modified with 3-thiodisuccinic acid after $24 \mathrm{~h}$ on immersed and emersed crystals). SAM formed in $10 \mathrm{mM}$ thiol ethanol solution.

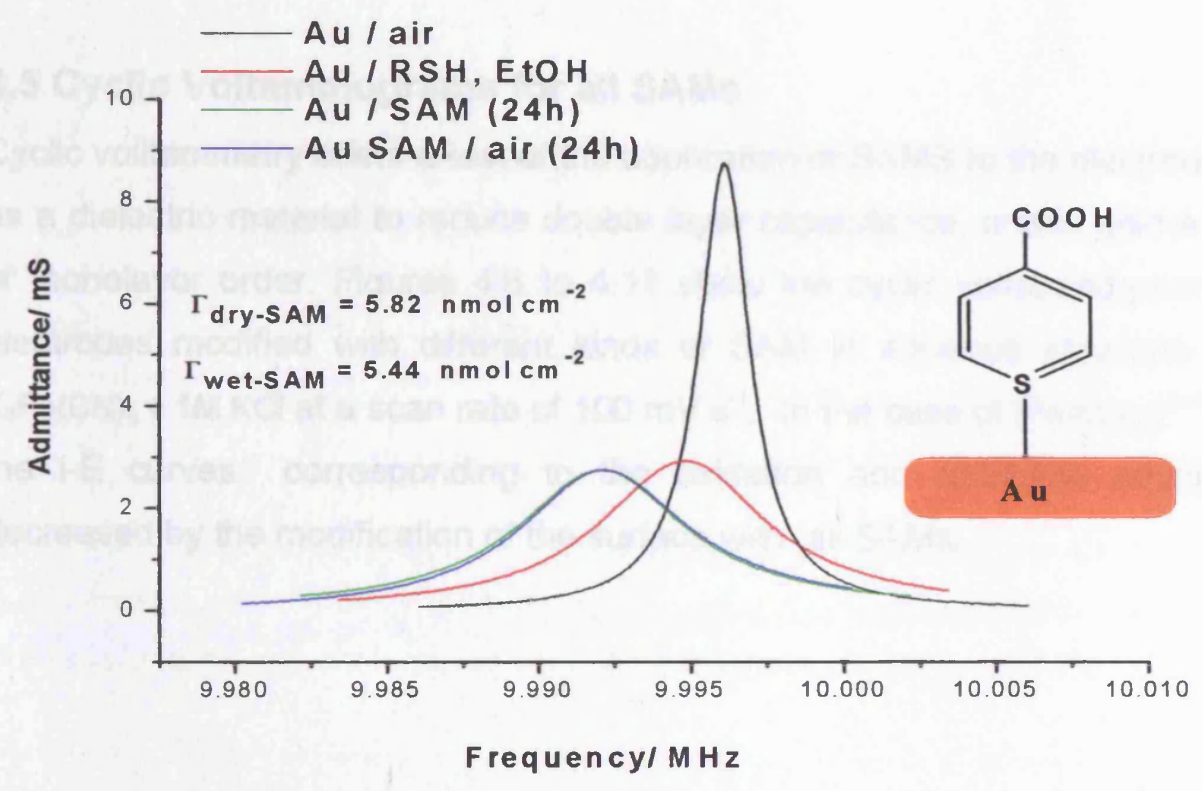

Figure 4.4: Crystal impedance spectra for a bare gold electrode and gold electrode modified with 4-mercaptobenzoic acid after $24 \mathrm{~h}$ on immersed and emersed crystals). SAM formed in $10 \mathrm{mM}$ thiol ethanol solution. 


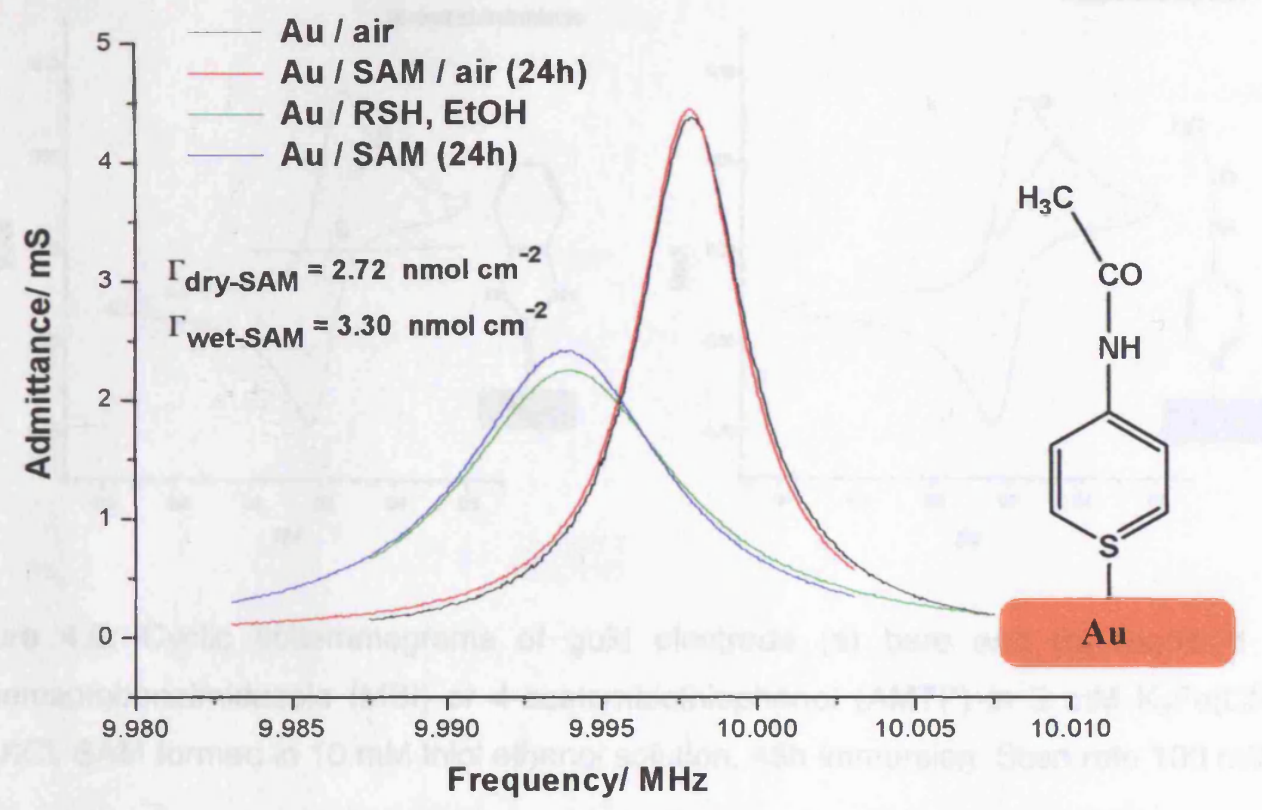

Figure 4.5: Crystal impedance spectra for a bare gold electrode and gold electrode modified with 4-acetomidothiophenol after $24 \mathrm{~h}$ on immersed and emersed crystals). SAM formed in $10 \mathrm{mM}$ thiol ethanol solution.

\subsection{Cyclic Voltammograms for all SAMs}

Cyclic volltammetry offers a test of the application of SAMS to the electrode surface as a dielectric material to reduce double layer capacitance, and is also a measure of monolayer order. Figures 4.6 to 4.11 show the cyclic voltammograms of gold electrodes modified with different kinds of SAM in aqueous solutions of $5 \mathrm{mM}$ $\mathrm{K}_{3} \mathrm{Fe}(\mathrm{CN})_{6}+1 \mathrm{M} \mathrm{KCl}$ at a scan rate of $100 \mathrm{mV} \mathrm{s}^{-1}$. In the case of $\left[\mathrm{Fe}(\mathrm{CN})_{6}\right]^{3-14}$ solution, the $\mathrm{i}-\mathrm{E}$ curves corresponding to the oxidation and reduction reactions was decreased by the modification of the surface with all SAMs. 

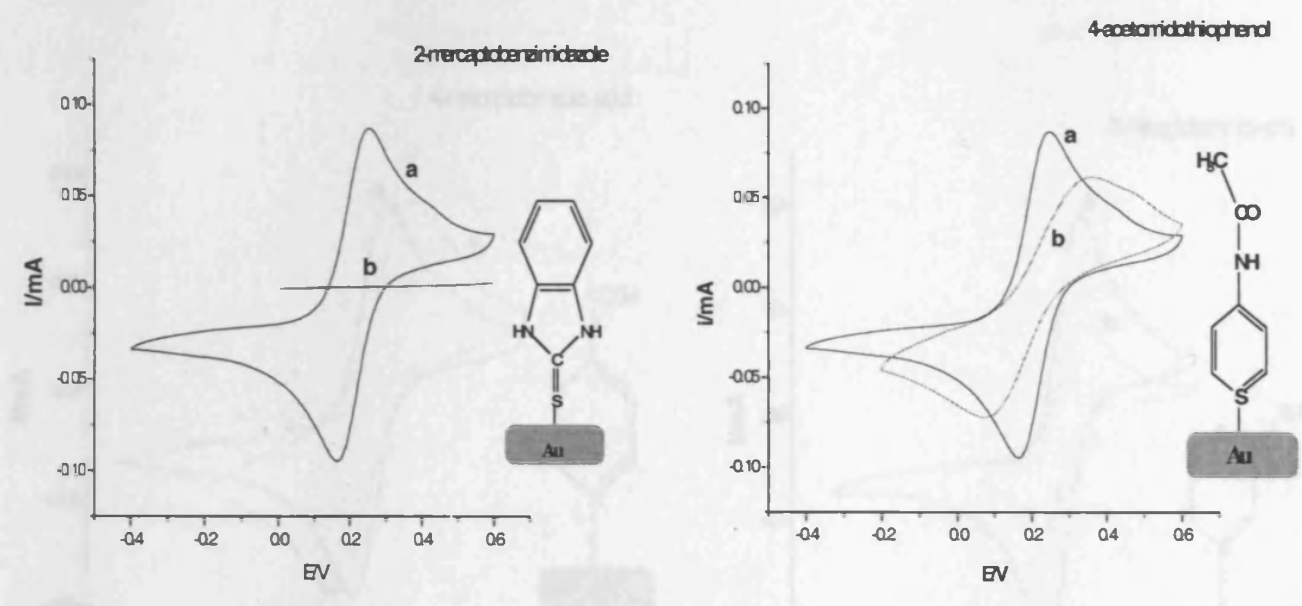

Figure 4.6: Cyclic voltammograms of gold electrode (a) bare and (b) modified with 2-mercaptobenzimidazole (MBI) or 4-acetomidothiophenol (AMTP) in $5 \mathrm{mM} \mathrm{K}_{3} \mathrm{Fe}(\mathrm{CN})_{6}+$ $1 \mathrm{M} \mathrm{KCl}$. SAM formed in $10 \mathrm{mM}$ thiol ethanol solution, $48 \mathrm{~h}$ immersion. Scan rate $100 \mathrm{mV} \mathrm{s}^{-1}$.
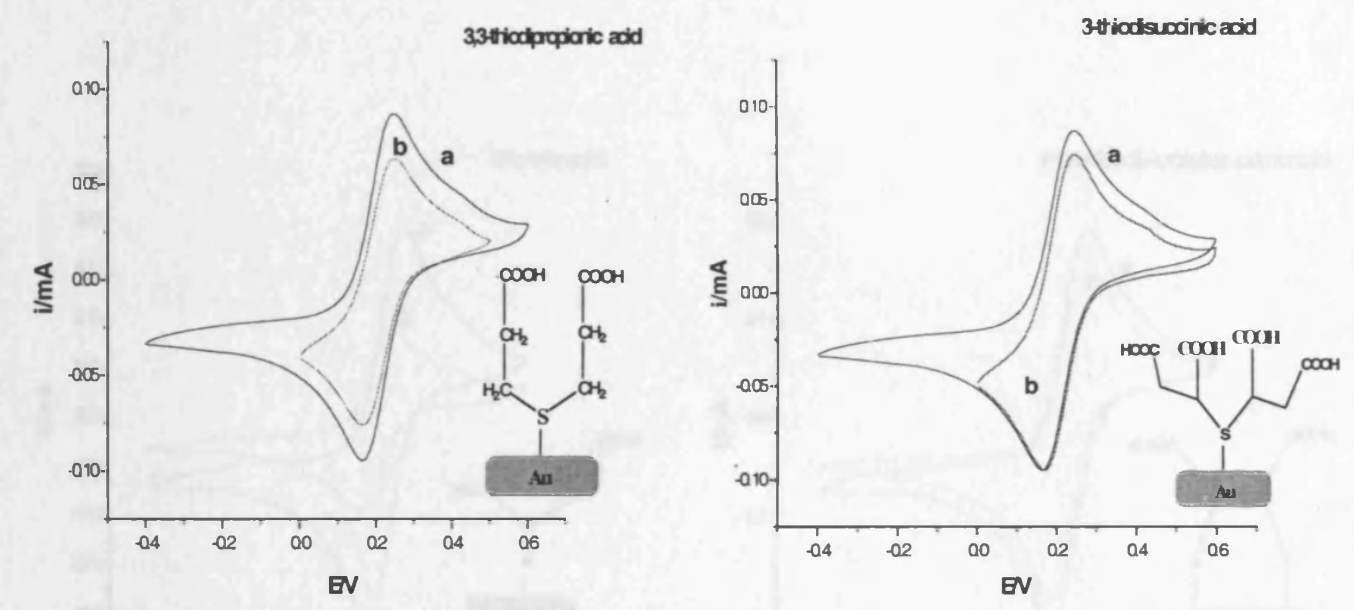

Figure 4.7: Cyclic voltammograms of gold electrode (a) bare and (b) modified with 3,3thiodipropionic acid (TDP) or 3-thiodisuccinic acid (TDS) in $5 \mathrm{mM} \mathrm{K}_{3} \mathrm{Fe}(\mathrm{CN})_{6}+1 \mathrm{M} \mathrm{KCl}$. SAM formed in $10 \mathrm{mM}$ thiol ethanol solution, $48 \mathrm{~h}$ immersion. Scan rate $100 \mathrm{mV} \mathrm{s}^{-1}$. 

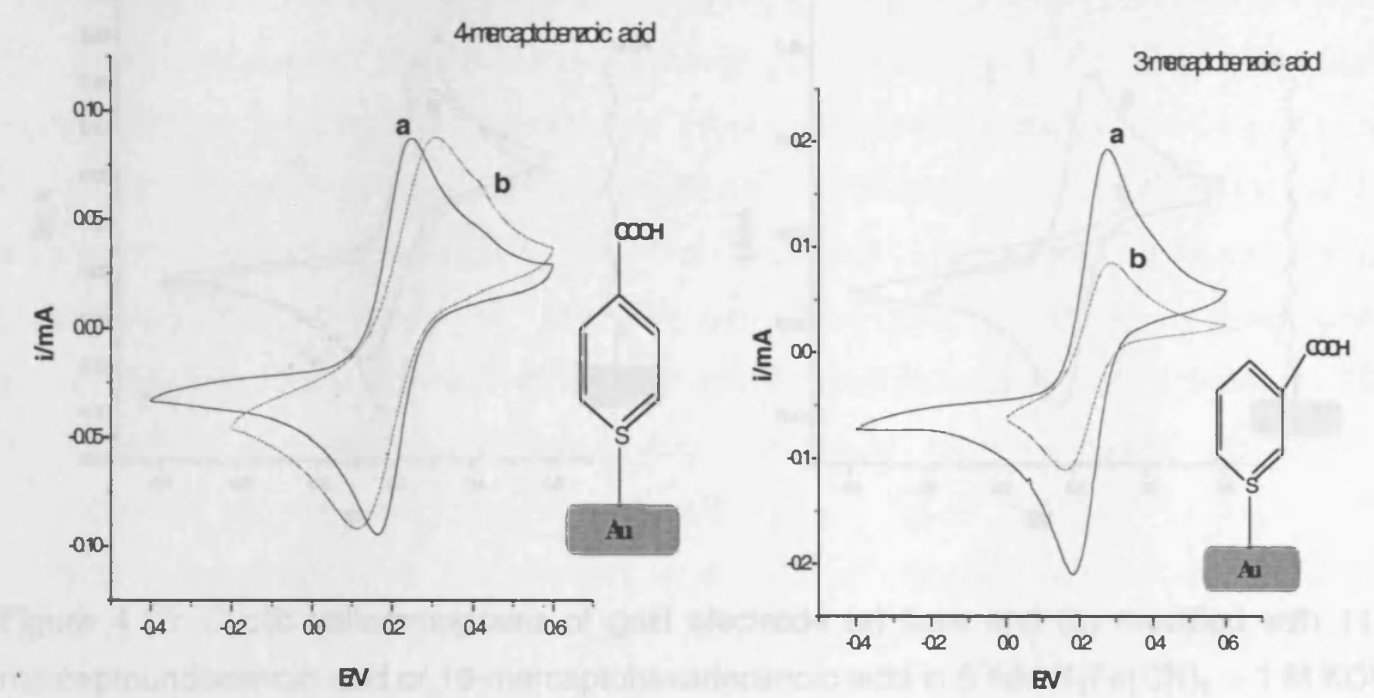

Figure 4.8: Cyclic voltammograms of gold electrode (a) bare and (b) modified with 4mercaptobenzoic acid or 3-mercaptobenzoic acid in $5 \mathrm{mM} \mathrm{K}_{3} \mathrm{Fe}(\mathrm{CN})_{6}+1 \mathrm{M} \mathrm{KCl}$. SAM formed in $10 \mathrm{mM}$ thiol ethanol solution, $48 \mathrm{~h}$ immersion. Scan rate $100 \mathrm{mV} \mathrm{s}^{-1}$.
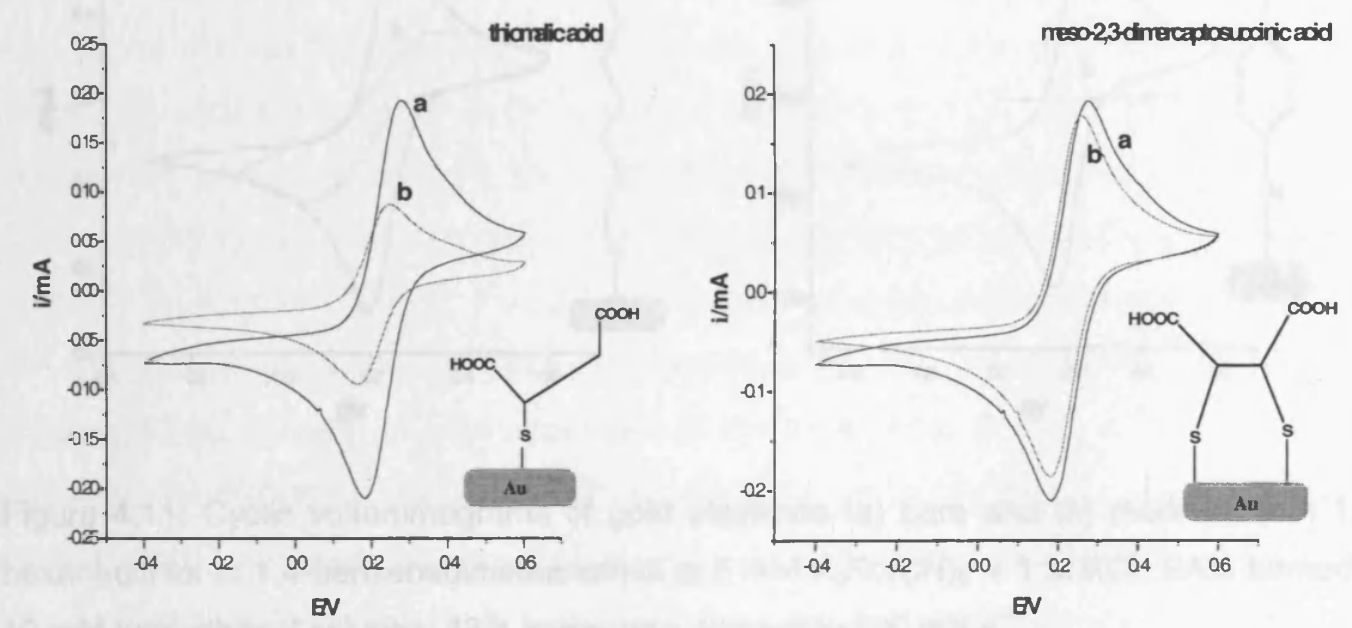

Figure 4.9: Cyclic voltammograms of gold electrode (a) bare and (b) modified with thiomalic acid (TMA) or meso-2,3-dimercaptosuccinc acid (DMSA) in $5 \mathrm{mM} \mathrm{K}_{3} \mathrm{Fe}(\mathrm{CN})_{6}+1 \mathrm{M} \mathrm{KCl}$. $\mathrm{SAM}$ formed in $10 \mathrm{mM}$ thiol ethanol solution, $48 \mathrm{~h}$ immersion. Scan rate $100 \mathrm{mV} \mathrm{s}^{-1}$. 

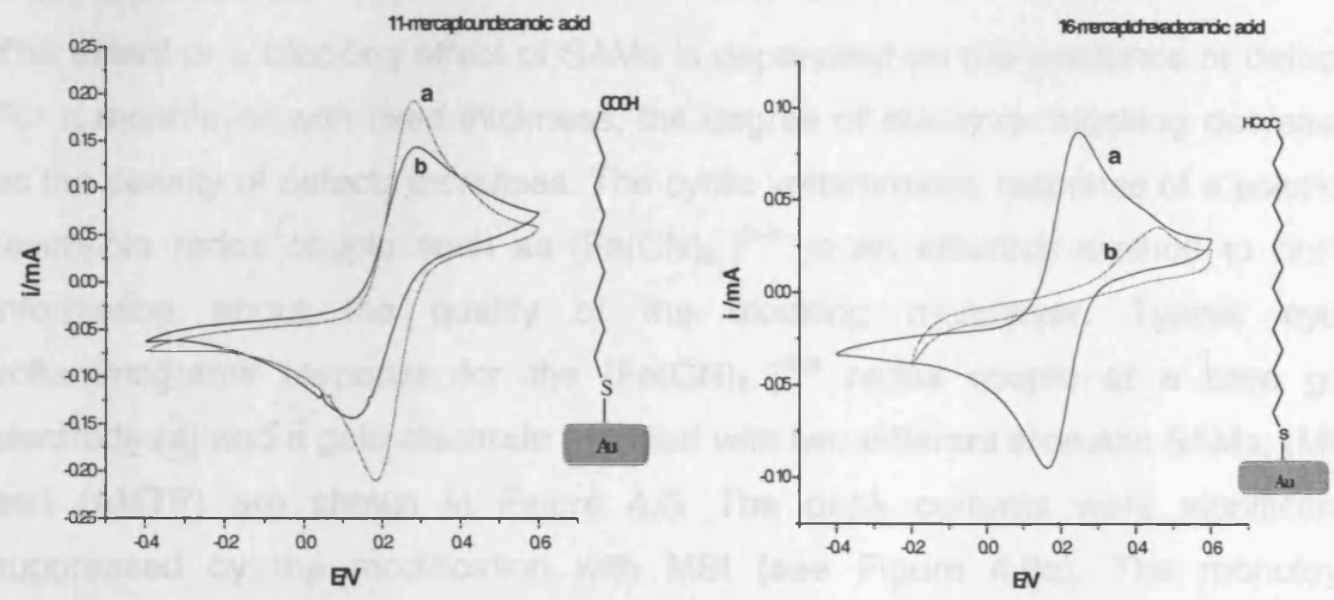

Figure 4.10: Cyclic voltammograms of gold electrode (a) bare and (b) modified with 11mercaptoundecanoic acid or 16-mercaptohexadecanoic acid in $5 \mathrm{mM} \mathrm{K}_{3} \mathrm{Fe}(\mathrm{CN})_{6}+1 \mathrm{M} \mathrm{KCl}$. SAM formed in $10 \mathrm{mM}$ thiol ethanol-toluene solution, $48 \mathrm{~h}$ immersion. Scan rate $100 \mathrm{mV} \mathrm{s}^{-1}$.
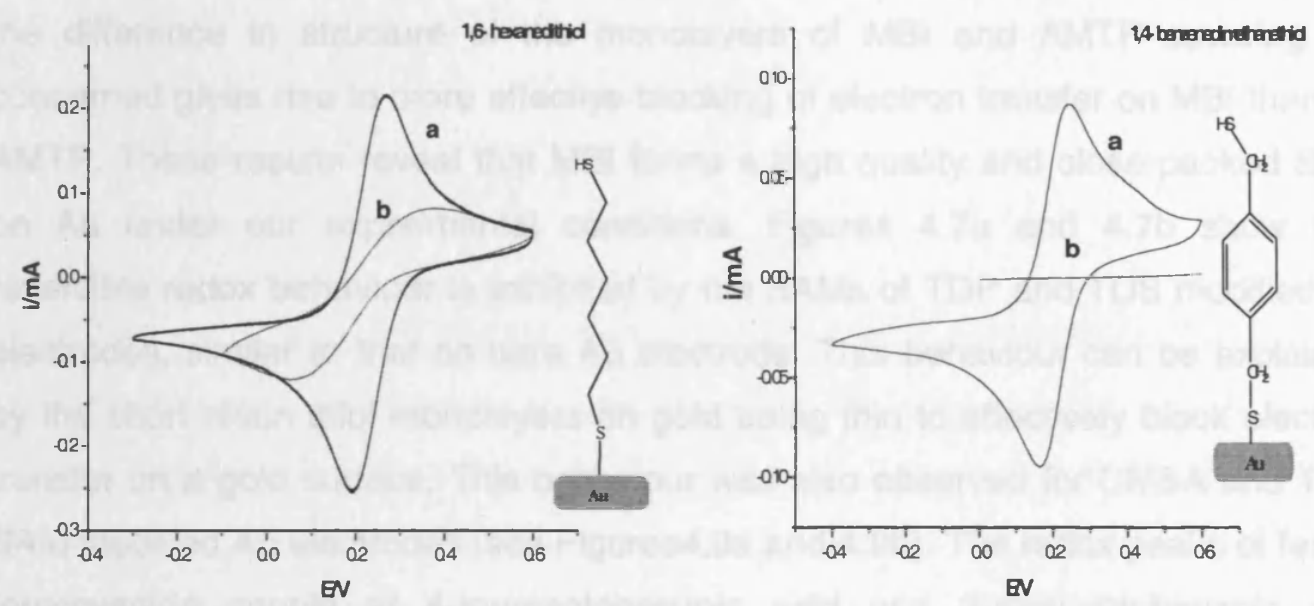

Figure 4.11: Cyclic voltammograms of gold electrode (a) bare and (b) modified with 1,6hexanedithiol or 1,4-benzenedimethanethiol in $5 \mathrm{mM} \mathrm{K}_{3} \mathrm{Fe}(\mathrm{CN})_{6}+1 \mathrm{M} \mathrm{KCl}$. SAM formed in $10 \mathrm{mM}$ thiol ethanol solution, $48 \mathrm{~h}$ immersion. Scan rate $100 \mathrm{mV} \mathrm{s}^{-1}$. 


\subsubsection{Discussion}

The extent of a blocking effect of SAMs is dependent on the existence of defects. For a monolayer with fixed thickness, the degree of electrode blocking decreases as the density of defects increases. The cyclic voltammetric response of a solutionreversible redox couple such as $\left[\mathrm{Fe}(\mathrm{CN})_{6}\right]^{3-14-}$ is an effective method to obtain information about the quality of the blocking monolayer. Typical cyclic voltammograms response for the $\left[\mathrm{Fe}(\mathrm{CN})_{6}\right]^{3-14}$ redox couple at a bare gold electrode (a) and a gold electrode modified with two different aromatic SAMs, (MBI) and (AMTP) are shown in Figure 4.6. The peak currents were significantly suppressed by the modification with $\mathrm{MBI}$ (see Figure 4.6a). The monolayer formation tends to limit the closest approach of the redox molecules to the electrode surface, decreasing the rate of electron transfer. The redox peaks of ferro I ferriccyanide couple at the AMTP SAM modified Au electrode were smaller than those for the bare $\mathrm{Au}$ electrode. Figure $4.6 \mathrm{~b}$ shows that the redox behaviour is exhibited by the $\left[\mathrm{Fe}(\mathrm{CN})_{6}\right]^{-3,-4}$ system on Au/AMTP, but not similar for $\mathrm{Au} / \mathrm{MBI}$, for example, the shape, the peak current and the peak potential difference. However the difference in structure of the monolayers of $\mathrm{MBI}$ and AMTP covering $\mathrm{Au}$ concerned gives rise to more effective blocking of electron transfer on $\mathrm{MBI}$ than on AMTP. These results reveal that MBI forms a high quality and close packed SAM on $\mathrm{Au}$ under our experimental conditions. Figures $4.7 \mathrm{a}$ and $4.7 \mathrm{~b}$ show that reversible redox behaviour is exhibited by the SAMs of TDP and TDS modified Au electrodes, similar to that on bare Au electrode. This behaviour can be explained by the short chain thiol monolayers on gold being thin to effectively block electron transfer on a gold surface. This behaviour was also observed for DMSA and TMA SAM modified Au electrodes (see Figures4.9a and 4.9b). The redox peaks of ferro / ferriccyanide couple at 4-mercaptobenzoic acid and 3-mercaptobenzoic acid modified Au electrodes are different, as can be seen in Figures $4.8 \mathrm{a}$ and $4.8 \mathrm{~b}$. The results again suggest that electron transfer for the ferro / ferricyanide couple was more effectively blocked by a SAM of 3-mercaptobenzoic acid than a SAM of 4mercaptobenzoic acid. The comparison between two alkyl chains for two different SAMs is shown in Figures 4.10a and 4.10b. 16-mercaptohexadecanoic acid forms a more dense and compact monolayer than 11-mercaptoundecanoic acid. This result is consistent with earlier studies of the good blocking properties of long-chain alkylthiol monolayers [2,3]. Figure 4.11 shows that a SAM of 1,4benzenedimethanethiol (BDMT) on an Au electrode exhibits better blocking properties than does 1,6-hexanedithiol (HDT). BDMT molecules can form more well-organized monolayers on gold compared with aliphatic 1,6-hexanedithiol 
molecules. These findings are in agreement with earlier reports, claiming that 18-24 hours of immersion of the electrode in thiol solution is enough to form a monolayer with almost $100 \%$ coverage and few defects in the SAM [4].

\subsection{Estimation of Electron Transfer rate constant at SAMs}

The electron transfer for $\left[\mathrm{Fe}(\mathrm{CN})_{6}\right]^{-3,-4}$ through self-assembled monolayer of several thiols, with $-\mathrm{COOH},-\mathrm{NH}$ and $-\mathrm{SH}$ groups in the end, on a gold surface was investigated using cyclic voltammetry. From the cyclic voltammograms, one can estimate the value of $\Delta E_{p}$ for all the SAMs. For a reversible (rapid) process, the separation between peak potentials $\left(\Delta E_{p}=E_{p a}{ }^{o x}-E_{p c}{ }^{\text {red }}\right)$ and the numbers of electrons transferred in the electrode reaction $(n)$ are related by:

$\Delta E_{p}=E_{p a}{ }^{o x}-E_{p c}{ }^{\text {red }}=0.0591 / n$ (at $25^{\circ} \mathrm{C}$ ). Slow electron transfer (irreversibility) or uncompensated resistance (IR drop) will increase the peak potential separation $\left(\Delta E_{p}\right)$. (See chapter 2 section 2.6 for more details). By using the equation:

$$
\psi=\frac{\left(\frac{D_{O}}{D_{R}}\right)^{\alpha / 2} k^{\prime}}{\left[D_{O} \pi v(n F / R T)\right]^{1 / 2}}
$$

Where we assume $\alpha=0.5$ and $D_{O}, D_{R}$ are the diffusion coefficients $\left(\mathrm{cm}^{2} \mathrm{~s}^{-1}\right)$, of the oxidised and reduced species in solution, $v$ is the scan rate $\left(V s^{-1}\right), n$ is the electron stoichiometry, $F$ is Faraday, $T$ is the temperature, $R$ is the gas constant and $k$ ' is the electron transfer rate constant. We use $\psi$ to determine the electron transfer kinetics.

Cyclic voltammograms of an aqueous solution $5 \mathrm{mM} \mathrm{K}_{3} \mathrm{Fe}(\mathrm{CN})_{6}+1 \mathrm{M} \mathrm{KCl}$ for SAM modified gold electrodes at scan rates $10,20,50$ and $100 \mathrm{mV} \mathrm{s}^{-1}$ were taken and the peak potential separations estimated for all SAMs. We find that $D_{\mathcal{O}} / D_{R}=1$, since $\quad i_{p, a} / i_{p, c}=1$ at bare Au. Thus equation (4.1) becomes:

$$
\psi=\frac{k^{\prime}}{\left[D_{O} \pi(n F / R T)\right]^{1 / 2}} \cdot \frac{1}{[v]^{1 / 2}}
$$

Since $\psi$ can be estimated from $\Delta \mathrm{E}_{\mathrm{p}}$ (based on the established theory [192]), one can use equation (4.2) to estimate the electron transfer rate constant at all SAMs. 
Typical theoretical results are shown in table 4.3. However, for $\alpha=0.5$ the $\left(\Delta \mathrm{E}_{\mathrm{p}}\right)$ values are nearly independent of $\alpha$ and depend only on $\psi$. This method is very useful in estimating $\mathrm{k}^{\prime}$ for quasi-reversible reactions by determining the variation of $\left(\Delta E_{p}\right)$ with $v$, and from this variation, $\psi$. The shift of $\left(\Delta E_{p}\right)$ with $\psi$ is shown in table 4.3 and Figure 4.12. Comparison of experimental data with this table can be used to estimate the electron transfer rate constant for $\left[\mathrm{Fe}(\mathrm{CN})_{6}\right]^{3-14-}$ at different SAMs on gold electrodes.

\begin{tabular}{|l|l|}
\hline$\Psi$ & $\Delta \mathrm{E}_{\mathrm{p}} \mathbf{m V}$ \\
\hline 20 & 61 \\
\hline 7 & 63 \\
\hline 6 & 64 \\
\hline 5 & 65 \\
\hline 4 & 66 \\
\hline 3 & 68 \\
\hline 2 & 72 \\
\hline 1 & 84 \\
\hline 0.75 & 92 \\
\hline 0.50 & 105 \\
\hline 0.35 & 121 \\
\hline 0.25 & 141 \\
\hline 0.1 & 212 \\
\hline
\end{tabular}

Table 4.3: shows the relation between the peak potential separation and kinetic parameter. Taken from reference $[5,6,7]$, for $n=1$. 


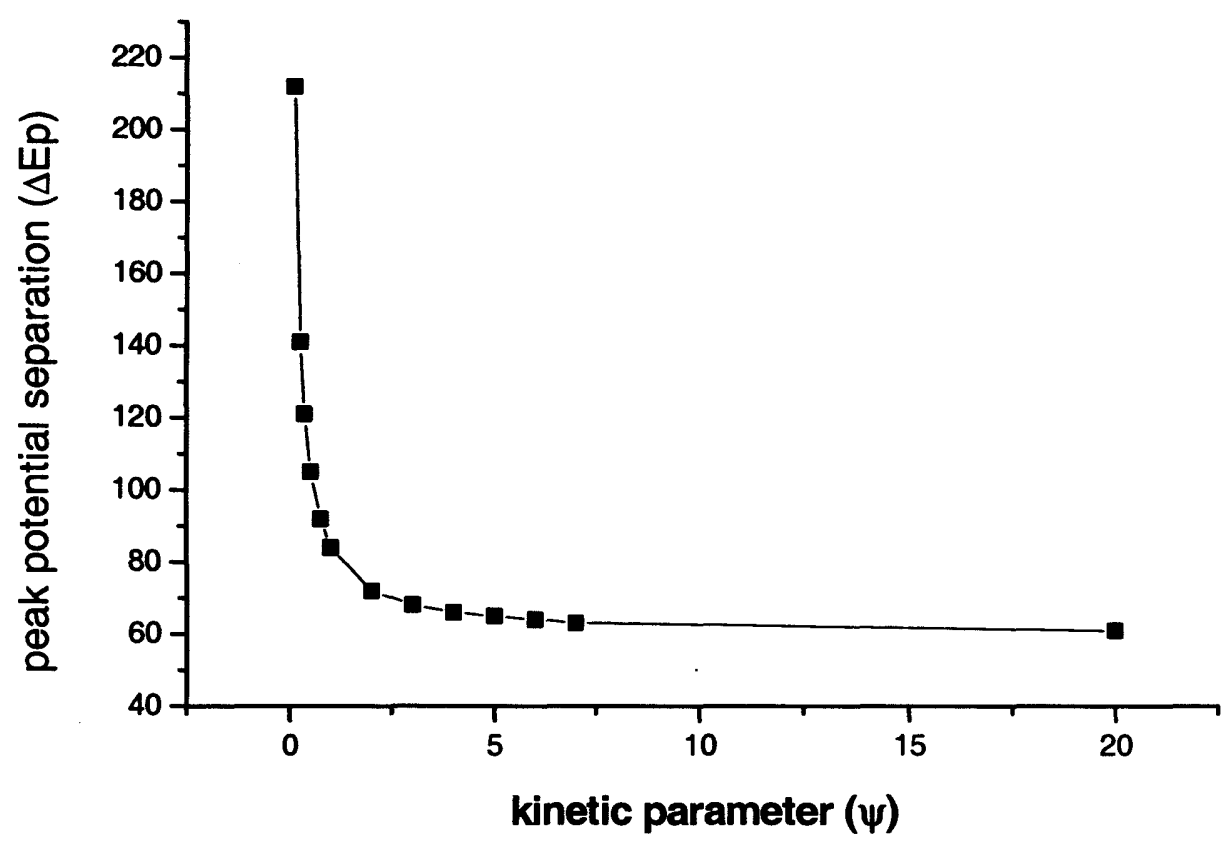

Figure 4.12: Relation between peak potential separation and kinetic parameter $(\psi)$.

The results of $k^{\prime}$ for $\left[\mathrm{Fe}(\mathrm{CN})_{6}\right]^{3-14-}$ at different kinds of SAM-modified Au electrodes are presented in tables 4.4 and 4.5 . 


\begin{tabular}{|c|c|c|c|c|}
\hline Name of SAM & $v\left(m V s^{-1}\right)$ & $\Delta E p(m V)$ & $\psi$ & $k^{\prime}\left(\mathrm{cm} \mathrm{s}^{-1}\right)$ \\
\hline \multirow[t]{4}{*}{ (1)2-mercaptobenzimidazole } & 100 & 606 & 0.053 & 0.0004 \\
\hline & 50 & 570 & 0.062 & 0.0004 \\
\hline & 20 & 540 & 0.083 & 0.0004 \\
\hline & 10 & 533 & 0.095 & 0.0004 \\
\hline \multirow[t]{5}{*}{ (2) 3,3 thiodipropionic acid } & 100 & 88 & 0.944 & 0.0053 \\
\hline & 50 & 86 & 0.926 & 0.0056 \\
\hline & 20 & 78 & 1.319 & 0.0050 \\
\hline & 10 & 76 & 1.712 & 0.0046 \\
\hline & 5 & 63 & 7.074 & 0.0135 \\
\hline \multirow[t]{4}{*}{ (3) 3-thiodisuccinic acid } & 100 & $\overline{66}$ & 2.797 & 0.0239 \\
\hline & 50 & 76 & 1.531 & 0.0092 \\
\hline & 20 & 76 & 1.531 & 0.0058 \\
\hline & 10 & 63 & 4.011 & 0.0010 \\
\hline \multirow[t]{4}{*}{ (4) 4-acetomidothiophenol } & 100 & 303 & 0.053 & 0.0009 \\
\hline & 50 & 269 & 0.053 & 0.0006 \\
\hline & 20 & 264 & 0.053 & 0.0005 \\
\hline & 10 & 242 & 0.053 & 0.0002 \\
\hline \multirow[t]{4}{*}{ (5) 11-mercaptoyndecanoic acid } & 100 & 150 & 0.234 & 0.0030 \\
\hline & 50 & 120 & 0.362 & 0.0033 \\
\hline & 20 & 90 & 0.873 & 0.0034 \\
\hline & 10 & 90 & 1.29 & 0.0034 \\
\hline \multirow[t]{3}{*}{ (6) 4-mercaptobenzoic acid } & 100 & 100 & 0.612 & 0.0052 \\
\hline & 50 & 100 & 0.613 & 0.0037 \\
\hline & 20 & 100 & 0.613 & 0.0023 \\
\hline \multirow[t]{4}{*}{ (7) 3-mercaptobenzoic acid } & 100 & 127 & 0.362 & 0.0032 \\
\hline & 50 & 113 & 0.447 & 0.0027 \\
\hline & 20 & 88 & 0.969 & 0.0037 \\
\hline & 10 & 80 & 1.138 & 0.0030 \\
\hline
\end{tabular}

Table 4.4: Results of the electron transfer rate constant for $\left[\mathrm{Fe}(\mathrm{CN})_{6}\right]^{3-14-}$ at different SAMs modified Au electrodes. 


\begin{tabular}{|l|c|c|c|c|}
\hline Name of SAM & $v\left(\mathrm{mV} \mathrm{s}^{-1}\right)$ & $\Delta \mathrm{Ep}(\mathrm{mV})$ & $\Psi$ & $\mathbf{k}^{\prime}\left(\mathrm{cm} \mathrm{s}^{-1}\right)$ \\
\hline (8) thiomalic acid & 100 & 200 & 0.167 & 0.0009 \\
\hline & 50 & 188 & 0.170 & 0.0007 \\
\hline & 20 & 180 & 0.170 & 0.0006 \\
\hline & 10 & 163 & 0.170 & 0.0004 \\
\hline & 100 & 70 & 2.255 & 0.0193 \\
\hline & 50 & 70 & 2.255 & 0.0136 \\
\hline (10) 16-meso-2,3-dimercaptosuccinic acid & 20 & 65 & 4.872 & 0.0101 \\
\hline & 10 & 60 & 4.872 & 0.0101 \\
\hline & 100 & 181 & 0.181 & 0.0015 \\
\hline & 50 & 164 & 0.202 & 0.0012 \\
\hline & 20 & 135 & 0.298 & 0.0014 \\
\hline & 10 & 125 & 0.403 & 0.0011 \\
\hline
\end{tabular}

Table 4.5: Results of the electron transfer rate constant for $\left[\mathrm{Fe}(\mathrm{CN})_{6}\right]^{3-14-}$ at different SAMs modified Au electrodes.

\subsubsection{Relation between $\log \psi$ and $\Delta \mathrm{E}_{\mathrm{p}}$ for some $\mathrm{SAMs}$}

Figures 4.13 to 4.15 show how can compare the theoretical peak potential separation results from Figure 4.12 with experimental results at SAMs. 


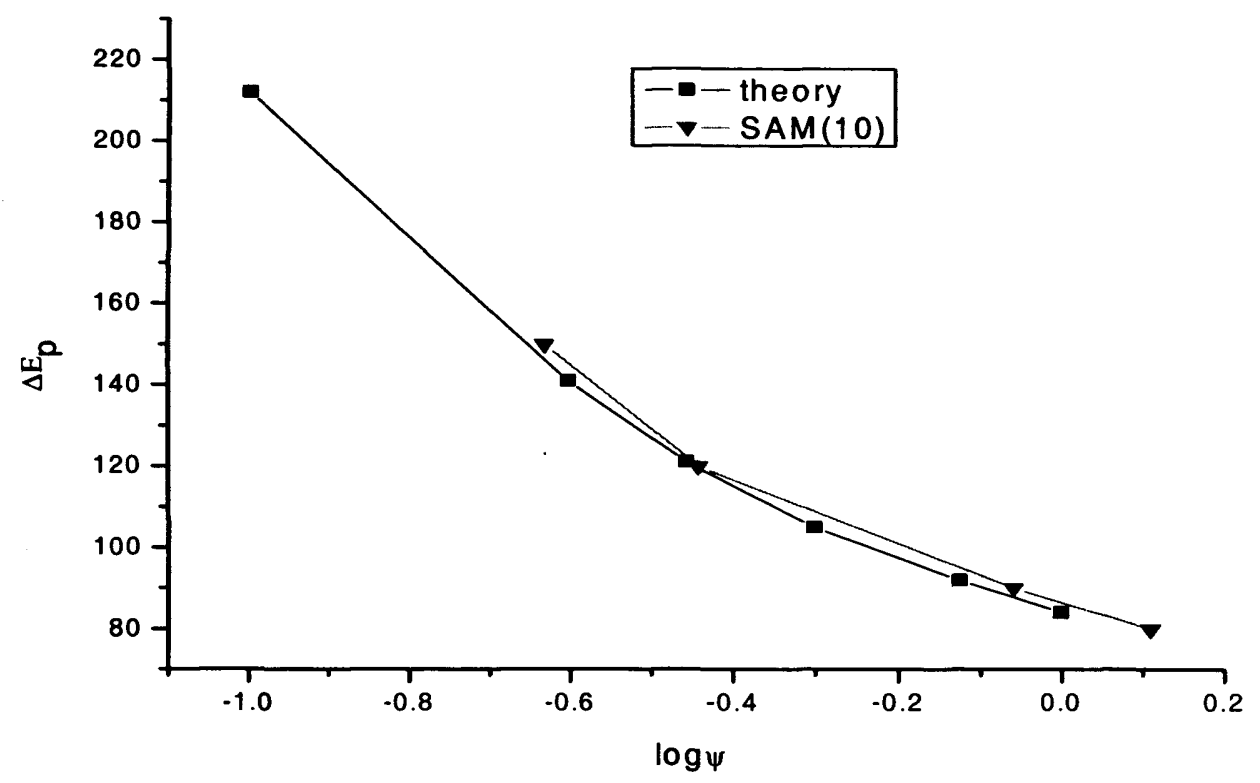

Figure 4.13: Relation between peak potential separation and kinetic parameter for 16mercaptohexadecanoic acid and theoretical cyclic voltammogram $[5,6,7]$. The value of $k^{\prime}$ is $0.001 \mathrm{~cm} \mathrm{~s}^{-1}$.

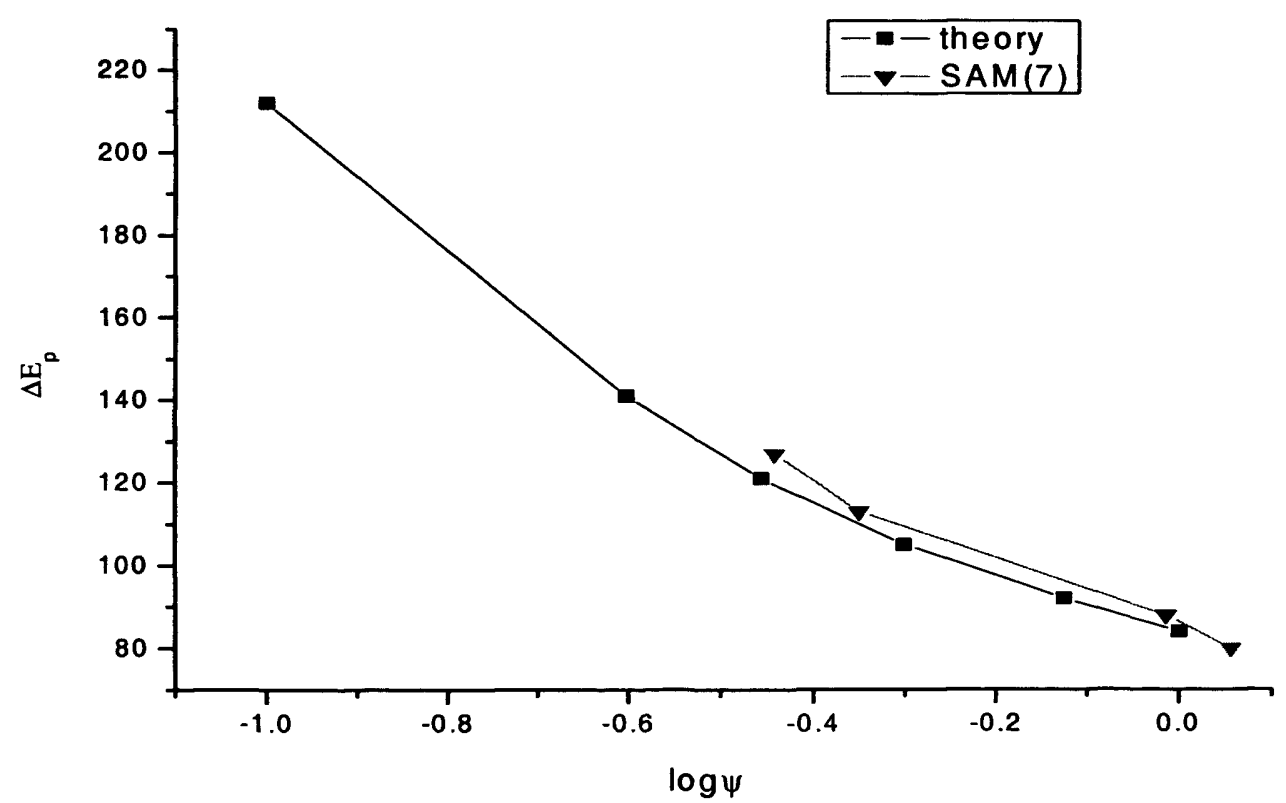

Figure 4.14: Relation between peak potential separation and kinetic parameter for 3mercaptobenzoic acid and theoretical cyclic voltammogram $[5,6,7]$. The value of $k$ ' is 0.003 $\mathrm{cm} \mathrm{s}^{-1}$. 


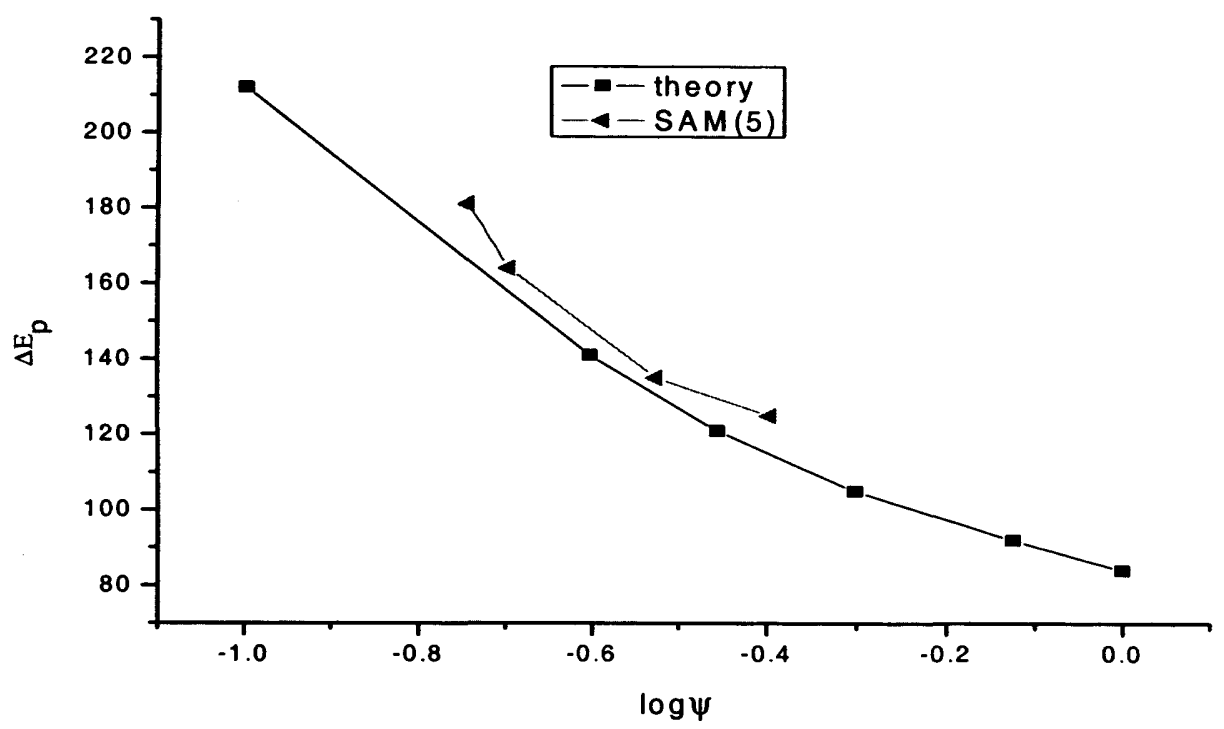

Figure 4.15: Relation between peak potential separation and kinetic parameter for11mercaptoundecanoic acid and theoretical cyclic voltammogram [5,6,7]. The value of $k$ ' is $0.0032 \mathrm{~cm} \mathrm{~s}^{-1}$.

\subsubsection{Relation between frequency change and electron transfer rate constant for $\left[\mathrm{Fe}(\mathrm{CN})_{6}\right]^{3 / 4}$ at SAMs}

One can explore whether there is a relationship between the electron transfer rate constant and frequency shifts associated with SAM formation (simplistically, adsorbate coverage). Figures 4.16 and 4.17 represent the difference between dry and wet crystal for the formation of different structures of SAMs. 


\section{Aliphatic SAMs}

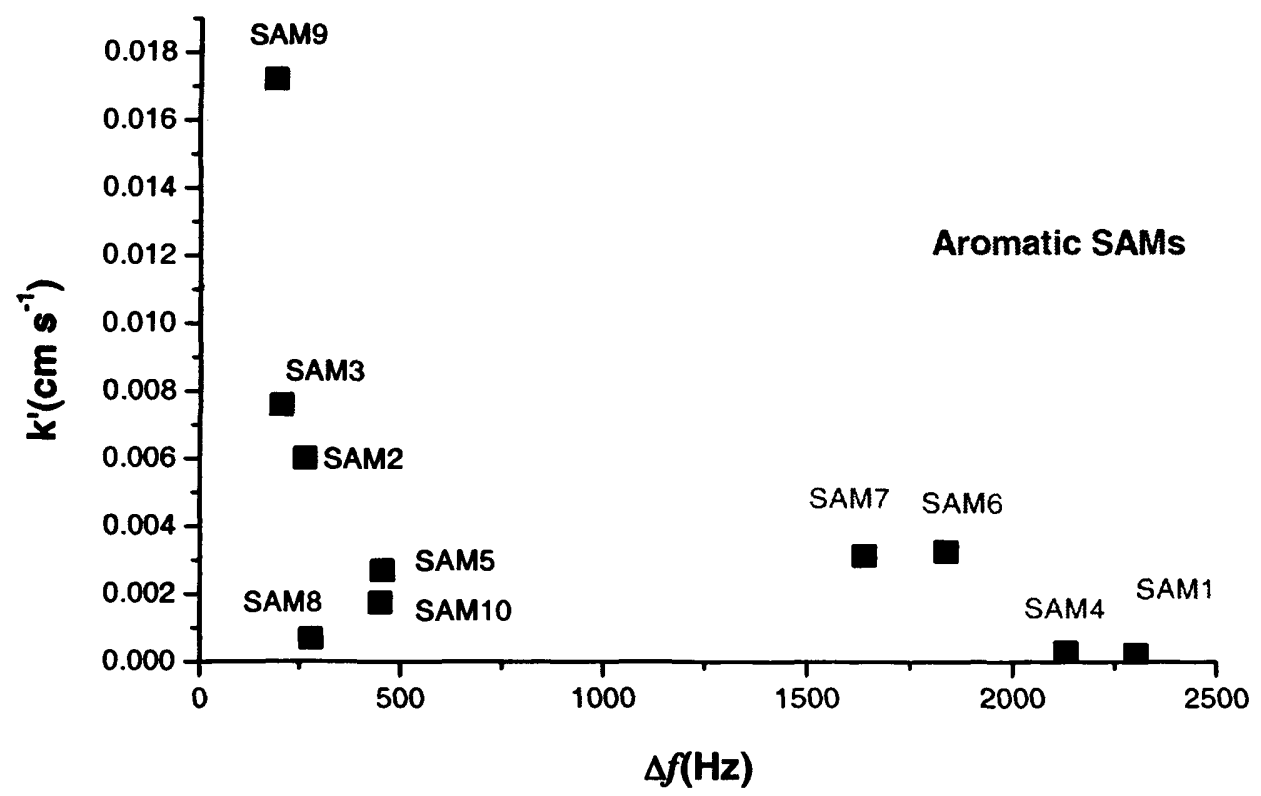

Figure 4.16: Relation between the frequency changes for SAMs on dry crystal and the electron transfer rate constant for the $\left[\mathrm{Fe}(\mathrm{CN})_{6}\right]^{3-14-}$ couple. The number of each SAM species refers to table 4.4 .

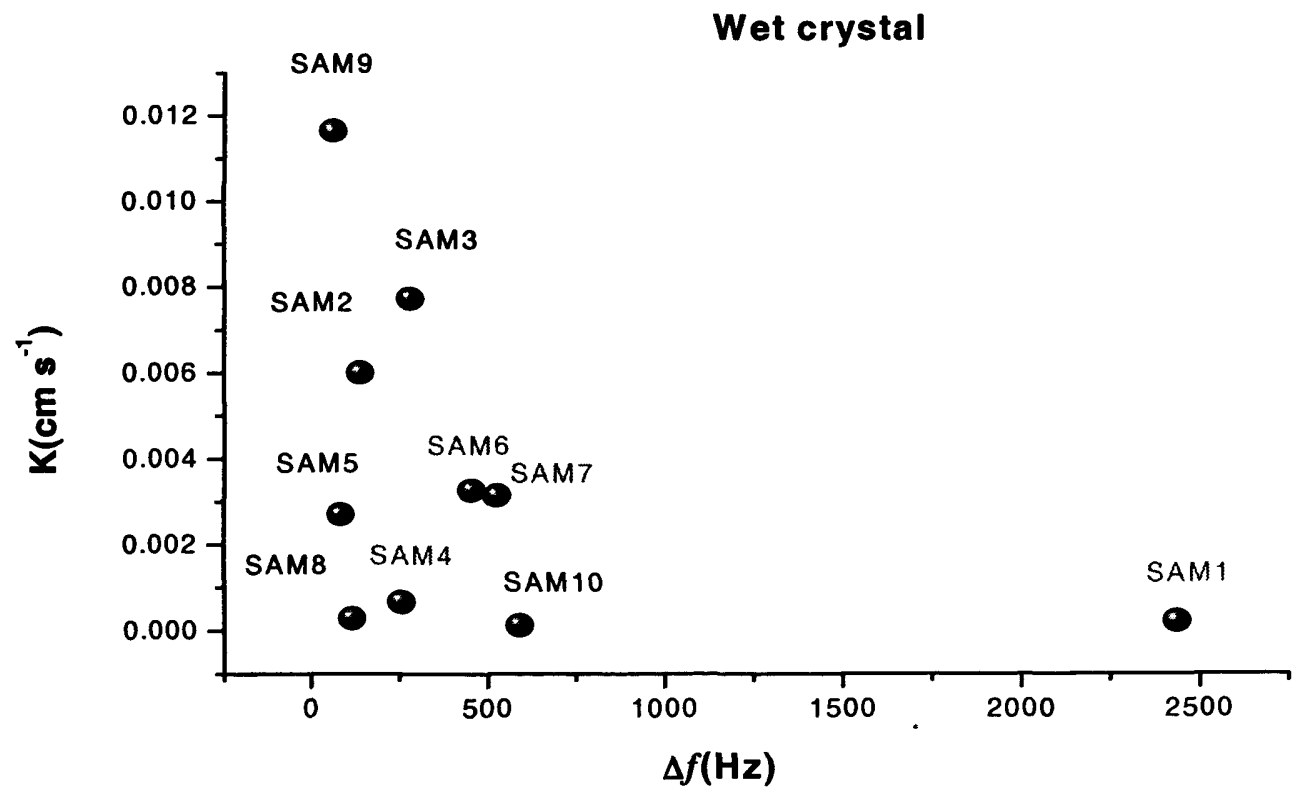

Figure 4.17: Relation between the frequency changes for SAMs on wet crystals and the electron transfer rate constant for the $\left[\mathrm{Fe}(\mathrm{CN})_{6}\right]^{3 / 4-}$ couple. The number of each SAM species refers to table 4.4 . 


\subsubsection{Discussion}

The overall rate of the redox reaction is controlled by mass transfer effects for the bare electrode, as indicated by the shape of the cyclic voltammogram, whereas for the SAM covered gold electrode, the current is significantly decreased (figures 4.3 to 4.8) and the rate of the process is determined by electron transfer (table 4.3). The monolayer retards the electron transfer rate, by increasing the separation between the gold surface and solution reactant. The electron transfer rate constant from table 4.3 depends on the structure of the monolayer. Cyclic voltammograms also allow one to judge the quality of the SAM, particularly regarding pinhole defects e.g. figure 4.7. Obviously, to study the kinetics of electron tunnelling through a SAM, a compact nearly perfect insulating film on the electrode must be ensured. A decrease in the number of the defects can be confirmed by checking the low values obtained for $\mathrm{k}^{\prime}$ for $\left[\mathrm{Fe}(\mathrm{CN})_{6}\right]^{3 / 4-}$. couple at different structure of SAMs. The value of the $k^{\prime}$ tends to decrease with increasing the carbon chain length of the thiol, because thiols with long chains form closely packed SAMs [8]. This behaviour is not observed for the aromatic SAMs, probably because the aromatic chain should have an important effect to favor the rate of electron transfer. Due to the repulsive force between the redox couple and surface, the value of $k$ ' is smaller because the electron transfer is more difficult. The relation between the electron transfer rate constant for $\left[\mathrm{Fe}(\mathrm{CN})_{6}\right]^{3 / 44}$ at SAMs and frequency shifts on dry and wet crystals was also studied (Figures 4.13 and 4.14). It was shown that the frequency shifts for aromatic SAMs are bigger than aliphatic SAMs. The values of $k^{\prime}$ for $\left.\mathrm{Fe}(\mathrm{CN})_{6}\right]^{3-14-}$ couple at aromatic SAMs are $0.0036 \mathrm{~cm} \mathrm{~s}^{-1}$ for 4mercaptobenzoic acid (6) and 3-mercaptobenzoic $\operatorname{acid}(7), 0.0002 \mathrm{~cm} \mathrm{~s}^{-1}$ for 2mercaptobenzimidazole (1) and $0.0009 \mathrm{~cm} \mathrm{~s}^{-1}$ for 4-acetomidothiophenol(4).

\subsection{Conclusions}

The experimental results clearly demonstrated that the characterization of SAMs using cyclic voltammetry and quartz crystal microbalance measurements is possible. Cyclic voltammetry provides important insights to the nature of the SAM attachment and surface coverage of the SAM. The quartz crystal microbalance (QCM) is a useful monitor of the adsorption of SAMs. The relation between $\Delta f_{\text {dry }}$ and $\Delta f_{\text {wet }}$ was linear for most SAMs studied. The relation between the electron transfer rate constant for $\left[\mathrm{Fe}(\mathrm{CN})_{6}\right]^{3 / 4}$ at $\mathrm{SAMs}$ and frequency shifts on dry and wet crystals shown that aromatic SAMs have similar electron transfer constants and bigger frequency shifts $(\Delta f)$ than aliphatic SAMs. From simple film thickness 
arguments this is slightly surprising. It can be explained by the greater conductivity of the aromatic (cf. aliphatic) chain. The electron transfer constant also tends to decrease with increase of the alkyl carbon chain length, because there is an increase in the blocking of the electron transfer for longer chains. Long chain aliphatic and aromatic thiols can form more organised monolayers on gold than short chain thiols due to extremely strong lateral van der Waals interactions and $\pi$ electron rich aromatic substituents.

\subsection{References}

1) H. D. Liess, A. Knezevic, M. Rother and J. Muenz, Faraday. Discuss., 107, (1997), 107, 1.

2) S.Berchmans, V.Yegnaraman and G.P.Rao, J. Solid State Electrochem., 3, (1998), 52.

3) R.B.Rolando, T.O.Feliciano and C.R.Cabrera, J. Electroanal. Chem., 540, (2003), 53.

4) U.K.Sur, R.Subramanian and V.Lakshminarayanan, J. Colloid Interface. Sci., 266, (2003), 175.

5) A. J. Bard and L. R. Faulkner, Electrochemical Methods: Fundamentals and Applications, John Wiley and Sons, New York, 198.

6) F.Scholz, Electroanalytical Methods "Guide to Experiments and Applications", Springer, Berlin, 2002, 51, 243, 261 and 301.

7) I.Lavagnini, R.Antiochia and F.Magno, Electroanalysis., 16, (2004), 505.

8) R.K.Mendes, R.S.Freire, C.P.Fonseca, S.Neves and L.T.Kubota, J.Braz. Chem. Soc., 15, (2004), 849. 


\section{Chapter 5: QCM study of the complexation of trace metal}

\section{ions with SAMs}

\subsection{Introduction}

This chapter describes the use of SAMs to detect trace metal ions from aqueous solutions using in situ and ex situ QCM measurements. The primary objectives of my work were to have successful immobilization of SAMs on the gold electrode of the QCM and to characterise these monolayers using cyclic voltammetry, as described in chapter 4 . The results of the third objective of binding trace metal ions to monolayers are presented in four stages. In the first and second stages, I describe the use of different concentrations of trace metal ions to bind to different kinds of SAMs. The third stage is selection of the most effective ligands using the Sauerbrey equation to measure the mass of ligand immobilized on the gold electrode and the mass of trace metal ions binding to these monolayers (QCM as a gravimetric probe). From these one can determine binding stoichometry. The final stage involves use of a range of concentrations of trace metal ions from (10 $\mu \mathrm{M}$ to $10 \mathrm{mM}$ ) with the best ligands, to obtain isotherms and estimate the values of binding constants. For each metal ion, uptake behaviour was investigated by determining uptake as a function of concentration, fitting to different isotherms, and estimating the heterogeneous binding constant for metal ions alone and mixed ion solutions; this provides selectivity information of the ligand for target metal ions.

\subsection{Complexation between 3-thiodisuccinic acid and cadmium ions}

\subsubsection{Strategy (Stage one)}

SAMs were prepared as described in sections 3.5 and 3.6. In stage one, selfassembled monolayer-attached ligands bound to the gold surface on the QCM cell produce a mass change of the QCM-SAM-ligand to QCM-SAM-ligand-Cd(II) composite resonator. Cadmium ion binding to SAMs was determined at $\mathrm{pH} 6.3$, and the following were measured:

(1) Frequency change for emersed crystal with SAM ( $\left.\Delta f_{\text {dry-SAM }}\right)$.

(2) Frequency change for immersed crystal with $S A M+8 \mathrm{ml}$ of deionised water in microbalance cell ( $\Delta f_{\text {wet-SAM }}$ ).

(3) Frequency change for immersed crystal with $S A M+8 \mathrm{ml}$ of deionised water + $2 \mathrm{ml} 1000 \mathrm{ppm}$ of cadmium solution ( $\Delta f_{\text {wet-SAM-Cd(II) }}$ ). 
(4) After drying the crystal in procedure 3, the frequency change for the emersed crystal with SAM-Cd(II) ( $\left.\Delta f_{\text {dry-SAM-Cd(II) }}\right)$. The following were then calculated

(1) $\Delta f_{\text {dry-Co(II) }}=\Delta f_{\text {dry-SAM-Co(II) }}-\Delta f_{\text {dry-SAM }}$.

(2) $\Delta f_{\text {wet-Co(II) }}=\Delta f_{\text {wet-SAM-Co(II) }}-\Delta f_{\text {wet-SAM }}$.

Measurements were made at room temperature, typically $20^{\circ} \mathrm{C}$.

\subsubsection{Results}

The results of stage one are presented in Table 5.1.

\begin{tabular}{|c|c|c|c|c|c|c|c|c|c|c|}
\hline & \multicolumn{5}{|c|}{ Emersed crystal } & \multicolumn{5}{|c|}{ Immersed crystal } \\
\hline SAM & $\begin{array}{l}-\Delta f \\
\text { SAM } \\
(\mathbf{H z})\end{array}$ & $\begin{array}{l}\Delta \mathbf{m} \\
\text { SAM } \\
\text { (ng) }\end{array}$ & $\begin{array}{l}-\Delta f \\
\operatorname{Co}(\mathbf{I I}) \\
(\mathrm{Hz})\end{array}$ & $\begin{array}{l}\Delta \mathbf{m} \\
\operatorname{cod}(1) \\
(\mathrm{ng})\end{array}$ & $\mathfrak{l}$ & $\begin{array}{l}-\Delta f \\
\text { SAM } \\
(\mathrm{Hz})\end{array}$ & $\begin{array}{l}\Delta \mathrm{m} \\
\text { SAM } \\
\text { (ng) }\end{array}$ & $\begin{array}{l}-\Delta f \\
\operatorname{Cd}(I I) \\
(\mathbf{H z})\end{array}$ & $\begin{array}{l}\Delta \mathrm{m} \\
\operatorname{Co}(\mathrm{II}) \\
(\mathbf{n g})\end{array}$ & $\mathfrak{l}$ \\
\hline MBI (1) & 1500 & 1650 & 163 & 179 & 0.14 & 1191 & 1310 & 138 & 152 & 0.15 \\
\hline TDP (2) & 1030 & 1133 & 650 & 715 & 1.00 & 797 & 877 & 370 & 407 & 0.74 \\
\hline TDS (3) & 32 & 35 & 25 & 27.5 & 0.93 & 131 & 144 & 77 & 85 & 1.39 \\
\hline AMTP (4) & 219 & 241 & 192 & 211 & 1.30 & 50 & 55 & 221 & 243 & 6.58 \\
\hline MUA (5) & 110 & 121 & 310 & 341 & 1.35 & 47 & 52 & 104 & 114 & 1.07 \\
\hline MBA (6) & 350 & 385 & 270 & 297 & 1.05 & 371 & 408 & 285 & 313 & 1.05 \\
\hline $\begin{array}{l}\text { 3-MBA } \\
\text { (7) }\end{array}$ & 119 & 131 & 87 & 96 & 1.43 & 130 & 143 & 170 & 187 & 1.00 \\
\hline TMA (8) & 72 & 79 & 49 & 54 & 1.29 & 54 & 56 & 52 & 57 & 0.90 \\
\hline DMSA (9) & 47 & 52 & 36 & 40 & 1.24 & 113 & 124 & 76 & 87 & 1.09 \\
\hline MHA (10) & 105 & 115 & 174 & 192 & 1.33 & 99 & 109 & 165 & 182 & 4.23 \\
\hline
\end{tabular}

Table 5.1: Results of frequency shifts of binding SAMs with cadmium ions, the concentration of cadmium ions was 200ppm. $\imath$ is the mole ratio between the cadmium ions and SAM. The number of each SAM species refers to table 4.2. 


\subsubsection{Strategy (Stage two)}

In this stage the concentration of cadmium ions was changed from 200ppm to $800 \mathrm{ppm}$. The frequency shifts was measured for SAM covered dry quartz crystal + $2 \mathrm{ml}$ deionised water $+10 \mathrm{ml}$ of cadmium solution (1000ppm).

\subsubsection{Results}

The results of the stage two procedures are presented in Table 5.2. This table compares the results of binding cadmium ions to SAMs on polished and unpolished crystals. 


\begin{tabular}{|c|c|c|c|c|c|c|c|c|c|c|}
\hline & \multicolumn{5}{|c|}{ Emersed crystal } & \multicolumn{5}{|c|}{ Immersed crystal } \\
\hline SAM & $\begin{array}{l}-\Delta f \\
\text { SAM } \\
(\mathrm{Hz})\end{array}$ & $\begin{array}{l}\Delta \mathbf{m} \\
\text { saM } \\
\text { (ng) }\end{array}$ & $\begin{array}{l}-\Delta f \\
\operatorname{Cod}(I I) \\
(H z)\end{array}$ & $\begin{array}{l}\Delta \mathbf{m} \\
\operatorname{cof}(1) \\
\text { (ng) }\end{array}$ & $\mathbf{l}$ & $\begin{array}{l}-\Delta f \\
\text { SAM } \\
(\mathrm{Hz})\end{array}$ & $\begin{array}{l}\Delta \mathrm{m} \\
\text { SAM } \\
\text { (ng) }\end{array}$ & $\begin{array}{l}-\Delta f \\
\operatorname{Cd}(I I) \\
(\mathbf{H z})\end{array}$ & $\begin{array}{l}\Delta \mathrm{m} \\
\operatorname{Co(II)} \\
\text { (ng) }\end{array}$ & $\mathbf{l}$ \\
\hline $\begin{array}{c}1 \\
\text { unpolished }\end{array}$ & 1651 & 1816 & 220 & 242 & 0.17 & 1406 & 1547 & 353 & 388 & 0.33 \\
\hline $\begin{array}{c}2 \\
\text { unpolished }\end{array}$ & 504 & 554 & 176 & 194 & 0.52 & 705 & 775.5 & 230 & 253 & 0.49 \\
\hline $\begin{array}{c}2 \\
\text { polished }\end{array}$ & 563 & 619 & 180 & 198 & 0.50 & 860 & 946 & 234 & 257 & 0.47 \\
\hline $\begin{array}{c}3 \\
\text { unpolished }\end{array}$ & 102 & 112 & 66 & 73 & 1.53 & 161 & 177 & 98 & 108 & 1.44 \\
\hline $\begin{array}{c}3 \\
\text { polished }\end{array}$ & 265 & 291.5 & 120 & 132 & 1.07 & 245 & 269.5 & 147 & 162 & 1.42 \\
\hline $\begin{array}{c}4 \\
\text { unpolished }\end{array}$ & 105 & 115.5 & 68 & 75 & 0.96 & 109 & 120 & 53 & 58 & 0.92 \\
\hline $\begin{array}{c}4 \\
\text { polished }\end{array}$ & 112 & 123 & 61 & 67 & 0.89 & 135 & 148.5 & 97 & 107 & 1.06 \\
\hline $\begin{array}{c}5 \\
\text { unpolished }\end{array}$ & 320 & 352 & 165 & 182 & 0.98 & 352 & 387 & 181 & 199 & 1.71 \\
\hline $\begin{array}{c}6 \\
\text { unpolished }\end{array}$ & 760 & 836 & 581 & 639 & 1.04 & 800 & 880 & 522 & 547 & 0.98 \\
\hline $\begin{array}{c}7 \\
\text { unpolished }\end{array}$ & 128 & 141 & 107 & 118 & 1.14 & 110 & 121 & 98 & 108 & 1.22 \\
\hline $\begin{array}{c}8 \\
\text { unpolished }\end{array}$ & 45 & 49.5 & 38 & 42 & 1.12 & 51 & 56 & 44 & 48 & 1.15 \\
\hline $\begin{array}{c}9 \\
\text { unpolished }\end{array}$ & 60 & 66 & 44 & 48 & 1.13 & 38 & 42 & 22 & 24 & 0.93 \\
\hline $\begin{array}{c}10 \\
\text { unpolished }\end{array}$ & 92 & 101 & 47 & 52 & 1.32 & 69 & 76 & 52 & 57 & 1.39 \\
\hline
\end{tabular}

Table 5.2: Results of frequency shifts of binding SAMs with cadmium ions. The concentration of cadmium ions was 800 ppm. $\imath$ is the mole ratio between the cadmium ions and SAM. The number of each SAM species refers to table 4.2.

\subsubsection{Strategy (Stage three)}

In this stage I will compare the stoichiometry of the cadmium ion to SAM from stage one and two. 


\begin{tabular}{|c|c|c|c|c|}
\hline SAM & $\begin{array}{l}\text { I (emersed crystal) } \\
\text { stage one }\end{array}$ & $\begin{array}{l}\text { I (emersed crystal) } \\
\text { stage two }\end{array}$ & $\begin{array}{l}\text { (immersed crystal) } \\
\text { stage one }\end{array}$ & $\begin{array}{c}\text { (1 immersed crystal) } \\
\text { stage two }\end{array}$ \\
\hline (1) MBA & 0.14 & 0.17 & 0.15 & 0.33 \\
\hline (2) TDP & 1.00 & 0.51 & 0.73 & 0.49 \\
\hline (3) TDS & 0.93 & 1.53 & 1.39 & 1.44 \\
\hline (4) AMTP & 1.30 & 0.96 & 6.55 & 0.92 \\
\hline (5) MUA & 1.34 & 0.98 & 1.07 & 1.71 \\
\hline (6) MBA & 1.05 & 1.04 & 1.05 & 0.98 \\
\hline (7) 3-MBA & 1.43 & 1.14 & 1.00 & 1.22 \\
\hline (8) TMA & 1.28 & 1.12 & 0.90 & 1.15 \\
\hline (9) MDSA & 1.24 & 1.13 & 1.09 & 0.93 \\
\hline (10) MHA & 1.33 & 1.32 & 1.23 & 1.39 \\
\hline
\end{tabular}

Table 5.3: Comparison of the mole ratio ( 2 ) of cadmium ions to SAM from stages one and two(see the sections 5.2.1 and 5.2.3), used to select the best $\mathrm{Cd}(\mathrm{II})$-ligand binding.

\subsubsection{Discussion the results of Cd(II)-SAM binding}

The comparison was made between the results of stage one and two to select the best binding between the cadmium ions and SAMs. It was found that, SAM 3, SAM 5, SAM 6, SAM 8, SAM 9 and SAM 10 have the same mole ratio one with cadmium ions on emersed and immersed crystals. See Table 5.3 and Figures 5.1 and 5.2 for more details. One can see that the relation between frequency changes for binding cadmium ions to SAMs as determined for dry (emersed) and wet (immersed) crystals is linear and the slope of the line is nearly one at higher concentration of cadmium ions (stage two) and 0.45 at low concentrations of cadmium ions (stage one) for all SAMs. This result suggests that at higher concentration of cadmium ions the saturation with cadmium ions for all SAMs occurred. In stage four a range 
of concentrations of cadmium ions is used to explore the effect of binding these concentrations to the selected SAMs. It was found that all of these monolayers are aliphatic thiols, except for SAM 6, which is aromatic. The results for binding cadmium ions to different SAMs on polished and unpolished crystals show that the absolute amounts of ligand and metal ion bound to the surface change, but their ratio ( $\mathrm{l}$, the binding stoichiometry) is un changed.

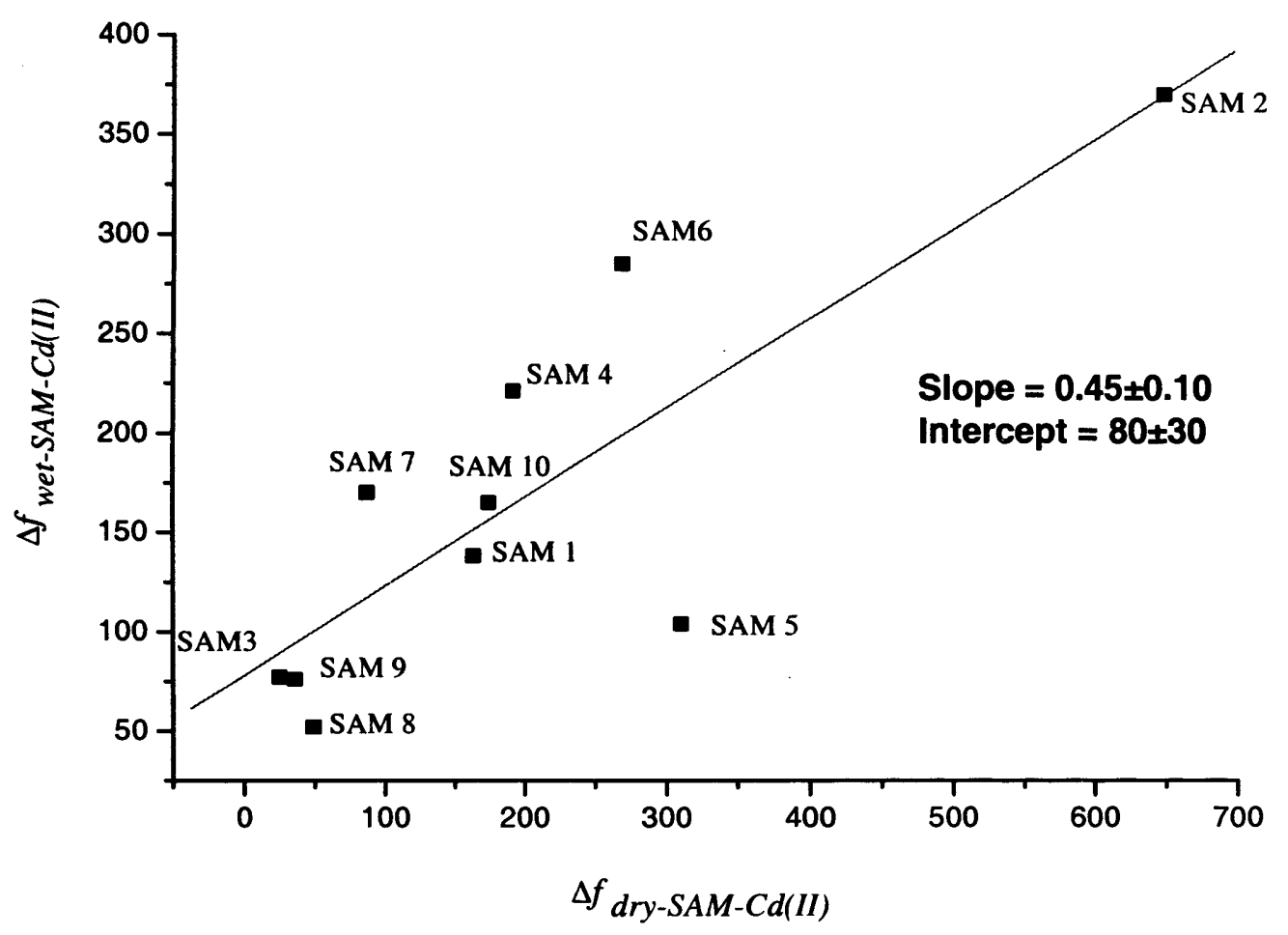

Figure 5.1: Relationship between frequency shifts $(\mathrm{Hz})$ for binding 200ppm of $\mathrm{Cd}(\mathrm{II})$ on dry (emersed) and wet (immersed) Au-SAM crystals. The results from stage one (see table 5.1). Line is least squares, slope $=0.45 \pm 0.10$. 


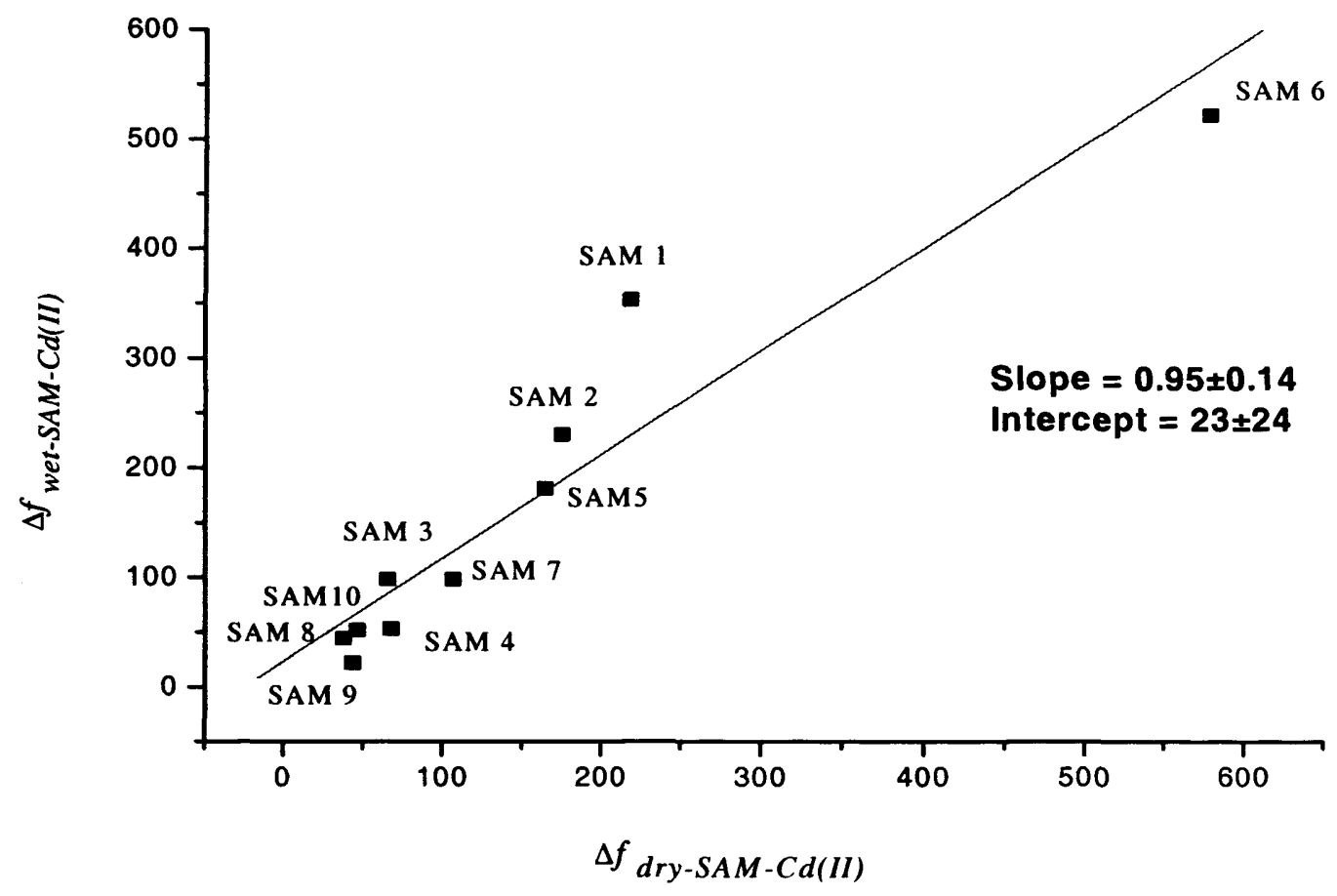

Figure 5.2: Relationship between frequency shifts $(\mathrm{Hz})$ for binding $800 \mathrm{ppm}$ of $\mathrm{Cd}(\mathrm{II})$ on dry (emersed) and wet (immersed) Au-SAM crystals. The results from stage two (see table 5.2). Line is least squares, slope $=0.95 \pm 0.14$.

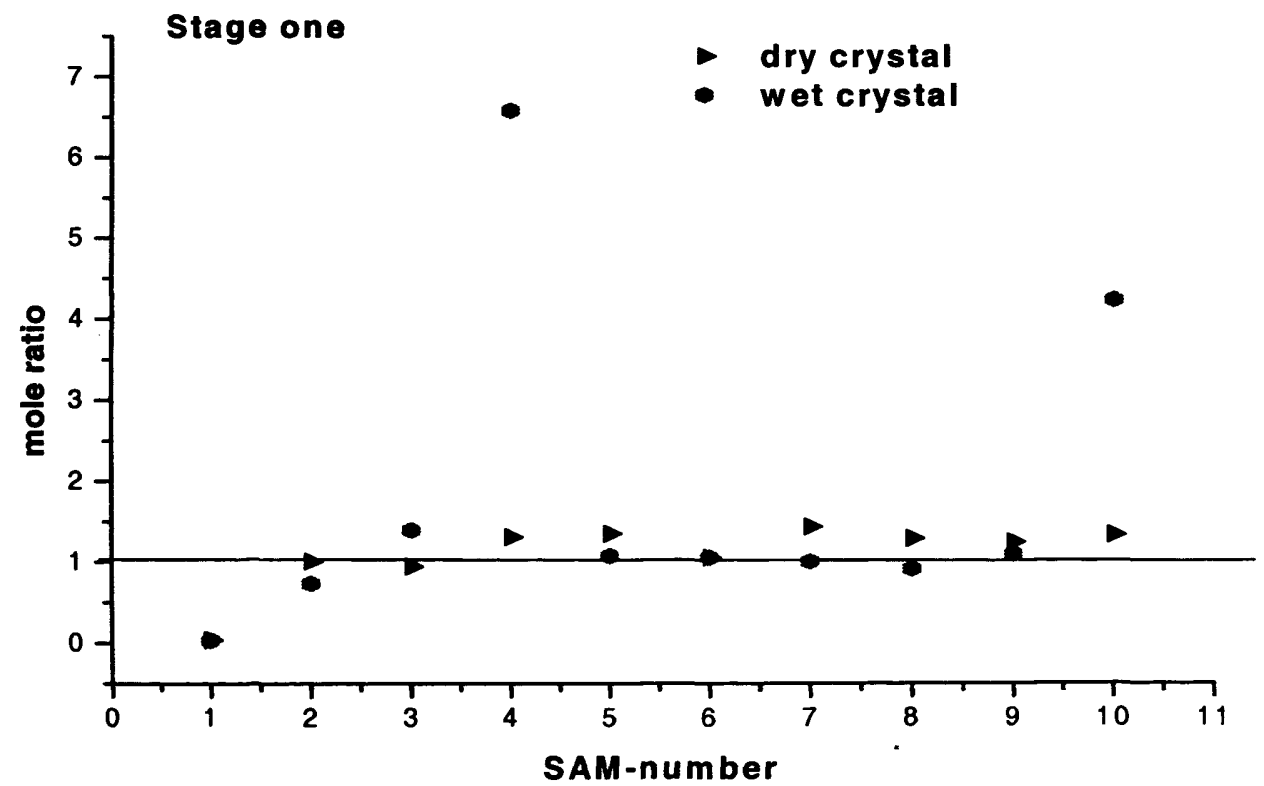

Figure 5.3: Selection of the best binding stoichometry between different structures of self-assembled monolayers and $\mathrm{Cd}^{2+}$ ions on dry (emersed) and wet (immersed) crystals; the number of each SAM species refers to table 5.3. 


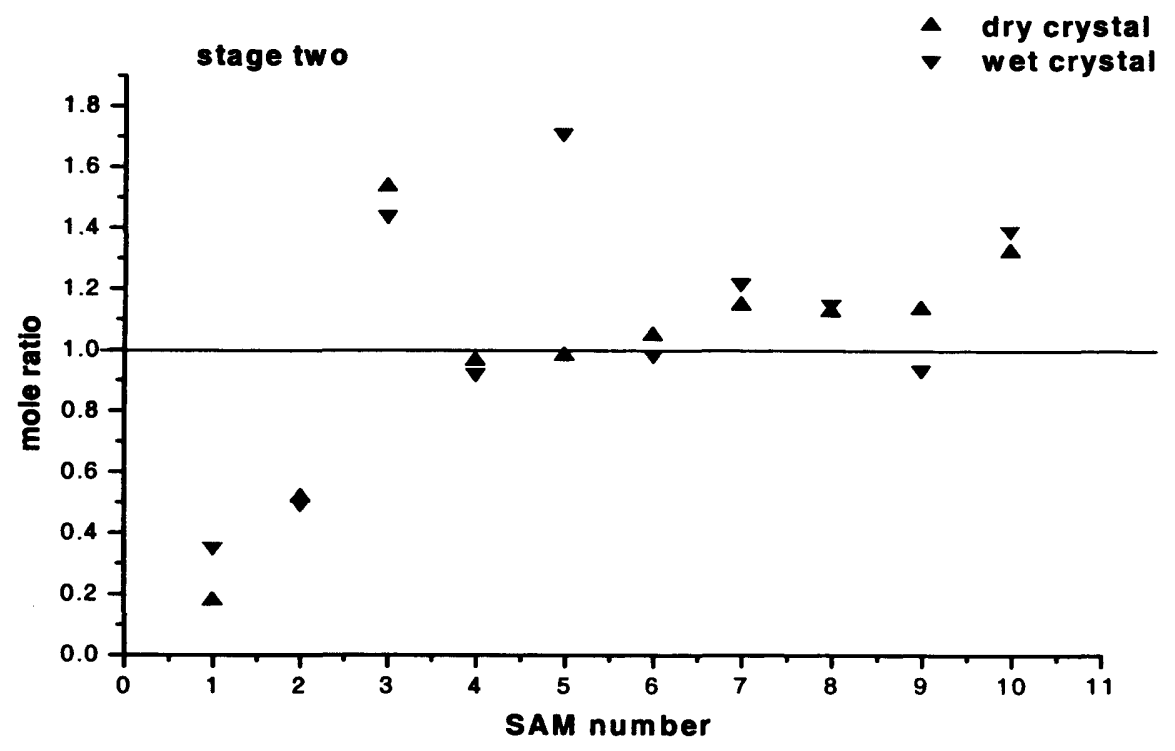

Figure 5.4: Selection of the best binding stoichometry between different structures of self-assembled monolayers and $\mathrm{Cd}^{2+}$ ions on dry (emersed) and wet (immersed) crystals; the number of each SAM species refers to table 5.3.

\subsection{Complexation between $\mathrm{Cd}^{2+}$ and 3-thiodisuccinic acid (Final stage)}

From the survey for binding 200ppm and 800ppm to different kinds of SAMs, it was found that 3-thiodisuccinic acid TDS has the same mole ratio SAM-Cd(II) on emersed and immersed crystals for 200ppm and 800ppm of cadmium ions. A series of aqueous solutions of $\mathrm{Cd}$ (II) at concentrations from $10 \mu \mathrm{M}$ to $10 \mathrm{mM}$ were exposed to the surface of the SAM at $\mathrm{pH}=6.3$ and the resulting frequency shifts recorded for emersed and immersed crystals. The same crystal was employed for the series of experiments with solutions of $\mathrm{Cd}(\mathrm{II})$ and typically duplicate or triplicate analyses were undertaken for each concentration. Tables 5.4 and 5.5 show the results of frequency shift with change in concentration of cadmium ions on emersed and immersed crystals. A plot of $\Delta f$ vs. $C d(I I)$ concentration on emersed and immersed crystals indicates that, at the higher concentrations, saturation of the cadmium ion coordination sites had occurred as shown in Figure 5.5. The mole ratio between $\mathrm{Cd}(\mathrm{II})$ and 3-thiodisuccinic acid for each concentration of $\mathrm{Cd}(\mathrm{II})$ is also shown in Tables (5.4 and 5.5). Reactions at low concentration were typically left for approximately $20 \mathrm{~min}$ and at higher concentrations for about $10 \mathrm{~min}$, to ensure equilibration between the surfaces bound 3-thiodisuccinic acid and solution of cadmium ions. Every experiment for binding different concentrations of cadmium ions was repeated three times with different coverages of 3-thiodisuccinic acid to estimate the binding constant after fitting the data to different isotherms. 


\begin{tabular}{|c|c|c|c|c|c|}
\hline $\begin{array}{c}\text { Concentration } \\
(\mu \mathrm{M})\end{array}$ & $\begin{array}{c}-\Delta \mathbf{f}_{\text {SAM-Cd(II) }} \\
(\mathbf{H z})\end{array}$ & $\begin{array}{c}\Delta \mathbf{m}_{\text {SAM-Cd(II) }} \\
(\mathbf{n g})\end{array}$ & $\begin{array}{c}\Gamma_{\text {Cod(I) }} \\
\mathbf{n} \text { moles }\end{array}$ & $\begin{array}{c}\Gamma_{\text {SAM }} \\
\text { n moles }\end{array}$ & I \\
\hline 10 & 24 & 26.4 & 0.23 & 0.42 & $\mathbf{0 . 5 6}$ \\
\hline 20 & 28 & 30.8 & 0.27 & 0.42 & $\mathbf{0 . 6 5}$ \\
\hline 50 & 36 & 39.6 & 0.35 & 0.42 & $\mathbf{0 . 8 4}$ \\
\hline 70 & 39 & 42.9 & 0.38 & 0.42 & $\mathbf{0 . 9 0}$ \\
\hline 100 & 41 & 45.0 & 0.40 & 0.42 & $\mathbf{0 . 9 5}$ \\
\hline 200 & 42 & 46.2 & 0.41 & 0.42 & $\mathbf{0 . 9 7}$ \\
\hline 500 & 45 & 49.5 & 0.44 & 0.42 & 1.04 \\
\hline 700 & 48 & 53.0 & 0.47 & 0.42 & 1.12 \\
\hline 1000 & 49 & 54.0 & 0.48 & 0.42 & 1.14 \\
\hline 5000 & 55 & 60.5 & 0.53 & 0.42 & 1.28 \\
\hline 7000 & 61 & 67.1 & 0.59 & 0.42 & 1.42 \\
\hline 8000 & 67 & 74.0 & 0.65 & 0.42 & 1.56 \\
\hline 9000 & 67 & 74.0 & 0.65 & 0.42 & 1.56 \\
\hline
\end{tabular}

Table 5.4: Frequency shift on dry (emersed) crystals for binding different concentrations of cadmium ions with 3-thiodisuccinic acid and change in stoichiometry with $\mathrm{Cd}(\mathrm{II})$ solution concentration $(\mathrm{pH}=6.3)$.

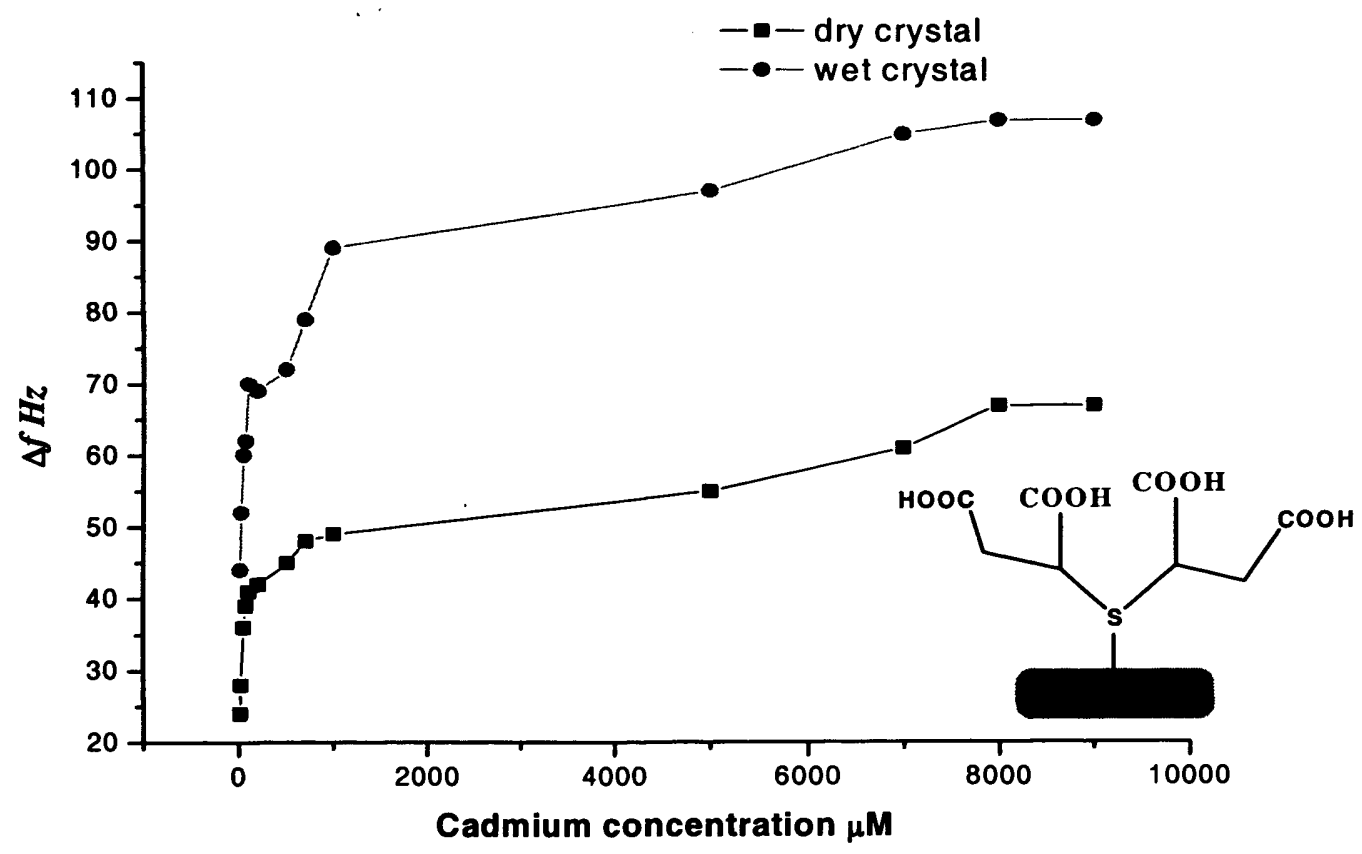

Figure 5.5: Adsorption isotherm for the reaction of an aqueous solution of $\mathrm{Cd}(\mathrm{II})$ with 3thiodisuccinic acid on dry (emersed) and wet (immersed) crystals. 


\begin{tabular}{|l|c|c|c|c|c|}
\hline $\begin{array}{c}\text { Concentration } \\
(\mu \mathrm{M})\end{array}$ & $\begin{array}{c}-\Delta \mathbf{f}_{\text {SAM-Cd(II) }} \\
(\mathbf{H z})\end{array}$ & $\begin{array}{c}\Delta \mathbf{m}_{\text {SAM-Cd(II) }} \\
(\mathbf{n g})\end{array}$ & $\begin{array}{c}\Gamma_{\text {Cd(II) }} \\
n \text { moles }\end{array}$ & $\begin{array}{c}\Gamma_{\text {SAM }} \\
n \text { moles }\end{array}$ & \\
\hline 10 & 44 & 48.4 & 0.43 & 0.69 & $\mathbf{0 . 6 2}$ \\
\hline 20 & 52 & 57.2 & 0.50 & 0.69 & $\mathbf{0 . 7 3}$ \\
\hline 50 & 60 & 66.0 & 0.58 & 0.69 & $\mathbf{0 . 8 5}$ \\
\hline 70 & 62 & 68.2 & 0.60 & 0.69 & $\mathbf{0 . 8 8}$ \\
\hline 100 & 70 & 77.0 & 0.68 & 0.69 & $\mathbf{0 . 9 9}$ \\
\hline 200 & 69 & 76.0 & 0.67 & 0.69 & $\mathbf{0 . 9 8}$ \\
\hline 500 & 72 & 79.2 & 0.70 & 0.69 & 1.02 \\
\hline 700 & 79 & 87.0 & 0.77 & 0.69 & 1.12 \\
\hline 1000 & 89 & 98.0 & 0.87 & 0.69 & 1.26 \\
\hline 5000 & 97 & 107 & 0.94 & 0.69 & 1.37 \\
\hline 7000 & 105 & 115.5 & 1.03 & 0.69 & 1.49 \\
\hline 8000 & 107 & 118 & 1.05 & 0.69 & 1.52 \\
\hline 9000 & 109 & 120 & 1.07 & 0.69 & 1.55 \\
\hline
\end{tabular}

Table 5.5: Frequency shifts on wet (immersed) crystal for binding different concentrations of cadmium ions with 3-thiodisuccinic acid and change in stoichiometry with $\mathrm{Cd}(\mathrm{II})$ solution concentration $(\mathrm{pH}=6.3)$.

In order to examine the reproducibility of cadmium complexation with the surface bound carboxylate monolayer, the experiment was repeated three times with different surface coverage of the monolayer on emersed and immersed crystals, (Figures 5.6 and 5.7 show the results for repeat the experiment three times on emersed and immersed crystal). 


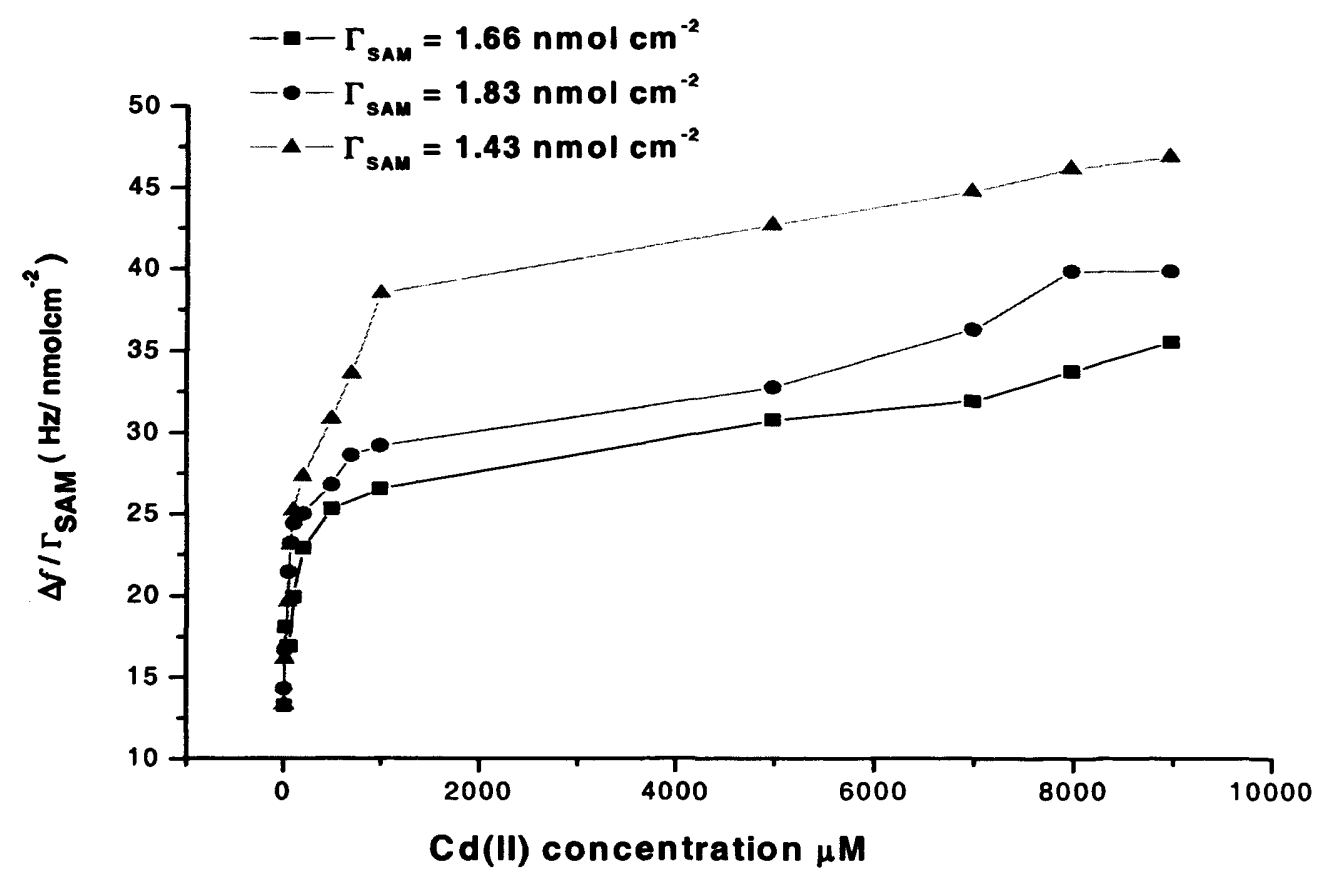

Figure 5.6: Adsorption isotherms for the reaction of an aqueous solution of $\mathrm{Cd}(\mathrm{II})$ with 3thiodisuccinic acid on emersed crystal at different surface coverages of 3- thiodisuccinic acid

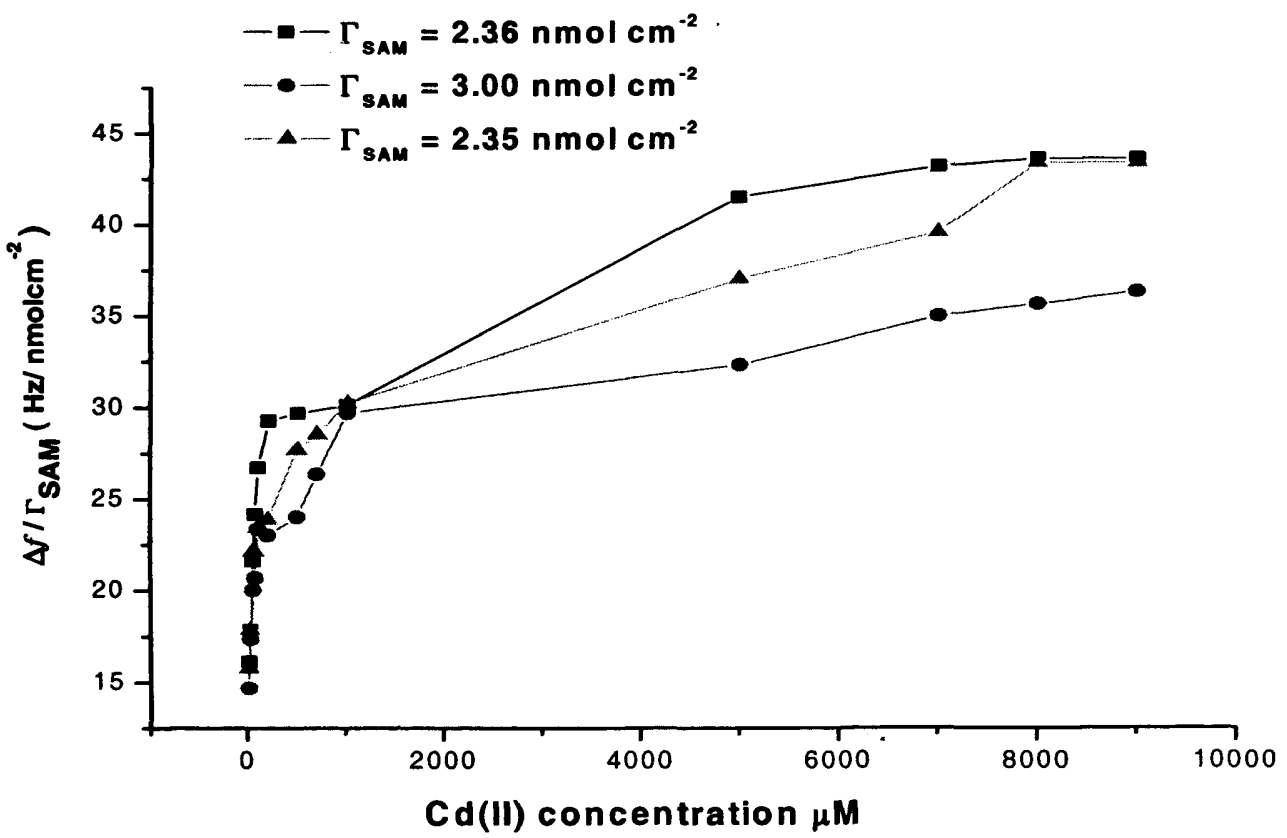

Figure 5.7: Adsorption isotherms for the reaction of an aqueous solution of $\mathrm{Cd}(\mathrm{II})$ with 3thiodisuccinic acid on immersed crystal at different surface coverages of 3- thiodisuccinic acid. 


\subsubsection{Fitting the data to the different isotherms}

The uptake of cadmium ion by 3-thiodisuccinic acid increases with increase in concentration of cadmium ion and reaches a plateau at higher concentrations.. In the context of the QCM, coverage can be calculated from:

$$
\theta=\frac{\Delta f}{\Delta f_{\infty}}
$$

where $\Delta f$ and $\Delta f_{\infty}$ are obtained from the experimental data (tables 5.4 and 5.5), $\Delta f_{\infty}$ is the limiting frequency shift at high concentrations of cadmium ions and $(\theta)$ is the fractional surface coverage of sites. The relation between fractional surface coverage of sites and the solution concentration is listed in Table (5.6). This data was used to determine the binding constant, $K_{C}$, for SAM-Cd(II) using six adsorption isotherms: Langmuir, Temkin, Freundlich, Frumkin, El-Awady and FloryHuggins. The mathematical expressions for these models are described in Table (5.7). The parameters of these models were estimated with regression by minimizing the sum of squares residuals. This was done for both dry (emersed) and wet (immersed) crystal responses.

\begin{tabular}{|c|c|c|c|c|}
\hline $1 / C \mu M^{-1}$ & $\begin{array}{c}\theta=\Delta f / \Delta f_{\infty} \\
\text { dry crystal }\end{array}$ & $\begin{array}{c}1 / \boldsymbol{\theta} \\
\text { dry crystal }\end{array}$ & $\begin{array}{c}\theta=\Delta f / \Delta f_{\infty} \\
\text { wet crystal }\end{array}$ & $\begin{array}{c}\mathbf{1} / \boldsymbol{\theta} \\
\text { wet crystal }\end{array}$ \\
\hline 0.1 & 0.490 & 2.04 & 0.557 & 1.79 \\
\hline 0.05 & 0.571 & 1.75 & 0.658 & 1.52 \\
\hline 0.02 & 0.735 & 1.36 & 0.759 & 1.32 \\
\hline 0.014 & 0.796 & 1.26 & 0.784 & 1.27 \\
\hline 0.01 & 0.837 & 1.19 & 0.886 & 1.13 \\
\hline 0.005 & 0.857 & 1.17 & 0.873 & 1.15 \\
\hline 0.002 & 0.918 & 1.09 & 0.911 & 1.09 \\
\hline 0.0014 & 0.979 & 1.02 & 1.00 & 1.00 \\
\hline 0.001 & 1.00 & 1.00 & 1.00 & 1.00 \\
\hline
\end{tabular}

Table 5.6: Relationship between $1 / \theta$ and concentration of $\mathrm{Cd}^{2+}$ for dry and wet quartz-Au-SAM crystals. 


\begin{tabular}{|c|c|c|c|c|}
\hline Isotherm & Equation & 'Model' & $\begin{array}{c}\text { Fitting } \\
\text { parameters }\end{array}$ & ref \\
\hline Langmuir(L) & $1 / \theta=1+1 / K_{L} C$ & $\begin{array}{l}\text { uniform surface } \\
\text { no interactions }\end{array}$ & $\mathbf{K}_{\mathbf{L}}$ & 2 \\
\hline Temkin (T) & $\theta=1 / f \ln K_{T}+1 / f \ln C$ & $\begin{array}{l}\text { Interactions, } \\
\text { uniform surface }\end{array}$ & $\mathbf{K}_{\mathbf{T}}, \mathbf{f}$ & 3 \\
\hline Freundlich(F) & $\ln \theta=1 / n \ln C+\ln K_{F}$ & $\begin{array}{l}0<\mathrm{n}<1 \text {, no } \\
\text { interactions, } \\
\text { logarithm, } \\
\text { rough surface }\end{array}$ & $\mathbf{K}_{\mathbf{F}}, \mathbf{n}$ & 4 \\
\hline Frumkin (Fr) & $f \theta=\ln [(1-\theta) / C \theta]+\ln K_{F R}$ & interactions & $\mathbf{K}_{\mathrm{Fr}}, \mathbf{f}$ & 5 \\
\hline El-Awady(El) & $\ln [(\theta / 1-\theta)]=\ln K_{E 1}+f \ln C$ & $\begin{array}{l}\text { Interactions. } \\
\text { mono and multi } \\
\text { layers, active } \\
\text { sites }\end{array}$ & $K_{E l}, f$ & 6 \\
\hline $\begin{array}{l}\text { Flory-Huggins } \\
\text { (FH) }\end{array}$ & $\begin{array}{l}\ln (\theta / C)=\ln f K_{F H}+f \\
\ln (1-\theta)\end{array}$ & $\begin{array}{l}\text { Interactions. } \\
\text { mono and multi } \\
\text { layers, active } \\
\text { sites }\end{array}$ & $\mathbf{K}_{\mathrm{FH}}, \mathbf{f}$ & 7 \\
\hline
\end{tabular}

Table 5.7: The linearized mathematical models of different kind of isotherms used to fit the experimental data, where $\mathbf{K}$ is the equilibrium binding constant, $\mathbf{C}$ is the concentration of cadmium ions; $f$ is the molecular interaction constant and $\theta$ is the fractional surface coverage of sites (estimated by dividing every frequency shift for every concentration by the maximum frequency change for saturation with cadmium ions). 


\subsubsection{Results}

The results of fitting the experimental data to these isotherms are presented in Figures (5.8) and (5.9).
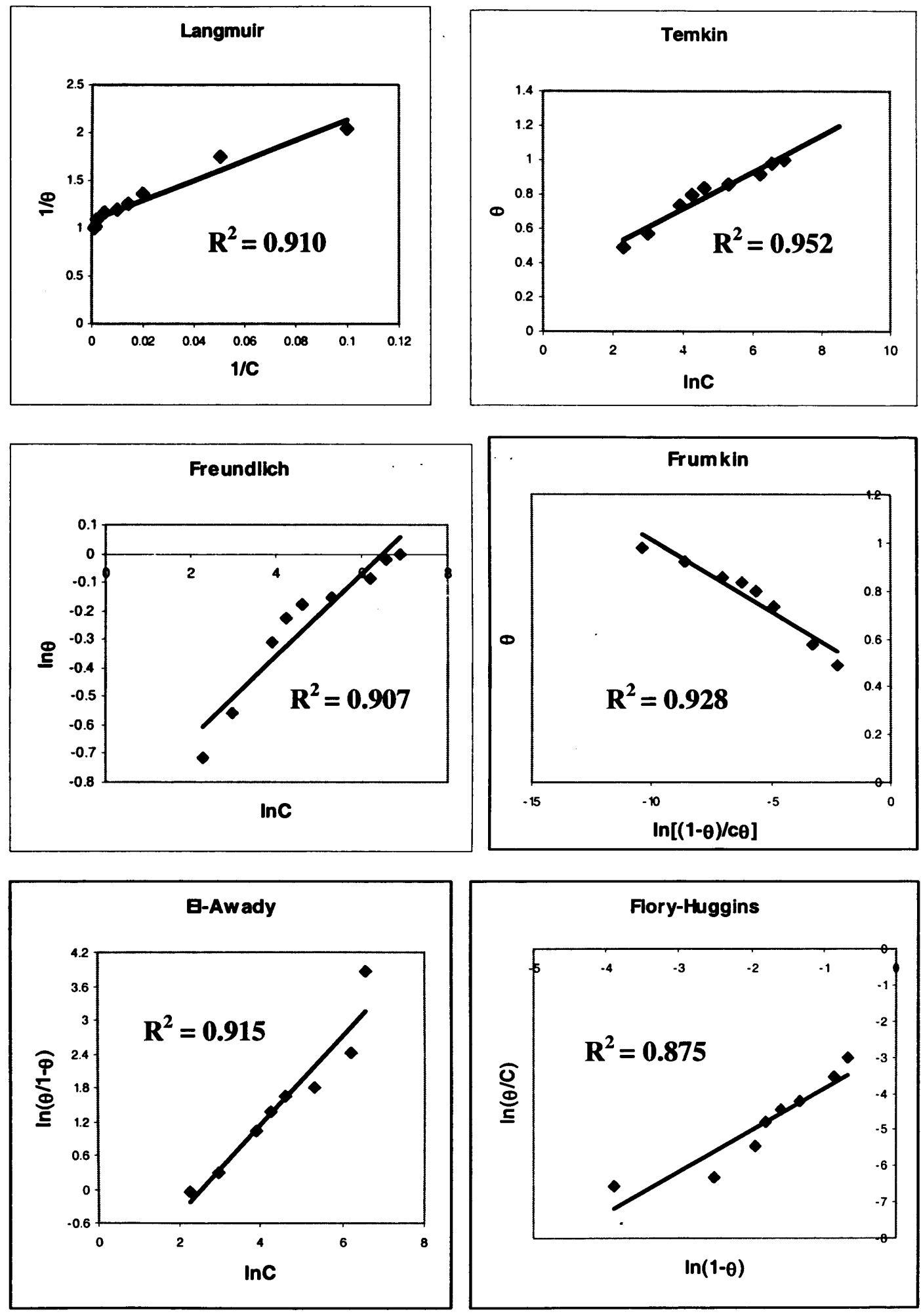

Figure 5.8: Plots of Langmuir, Temkin, Freundlich, Frumkin, El-Awady and Flory-Huggins isotherms to binding between $\mathrm{Cd}^{2+}$ and 3-thiodisuccinic acid on a dry (emersed) crystal. The line represents the linear least squares fit to the experimental data (points). 

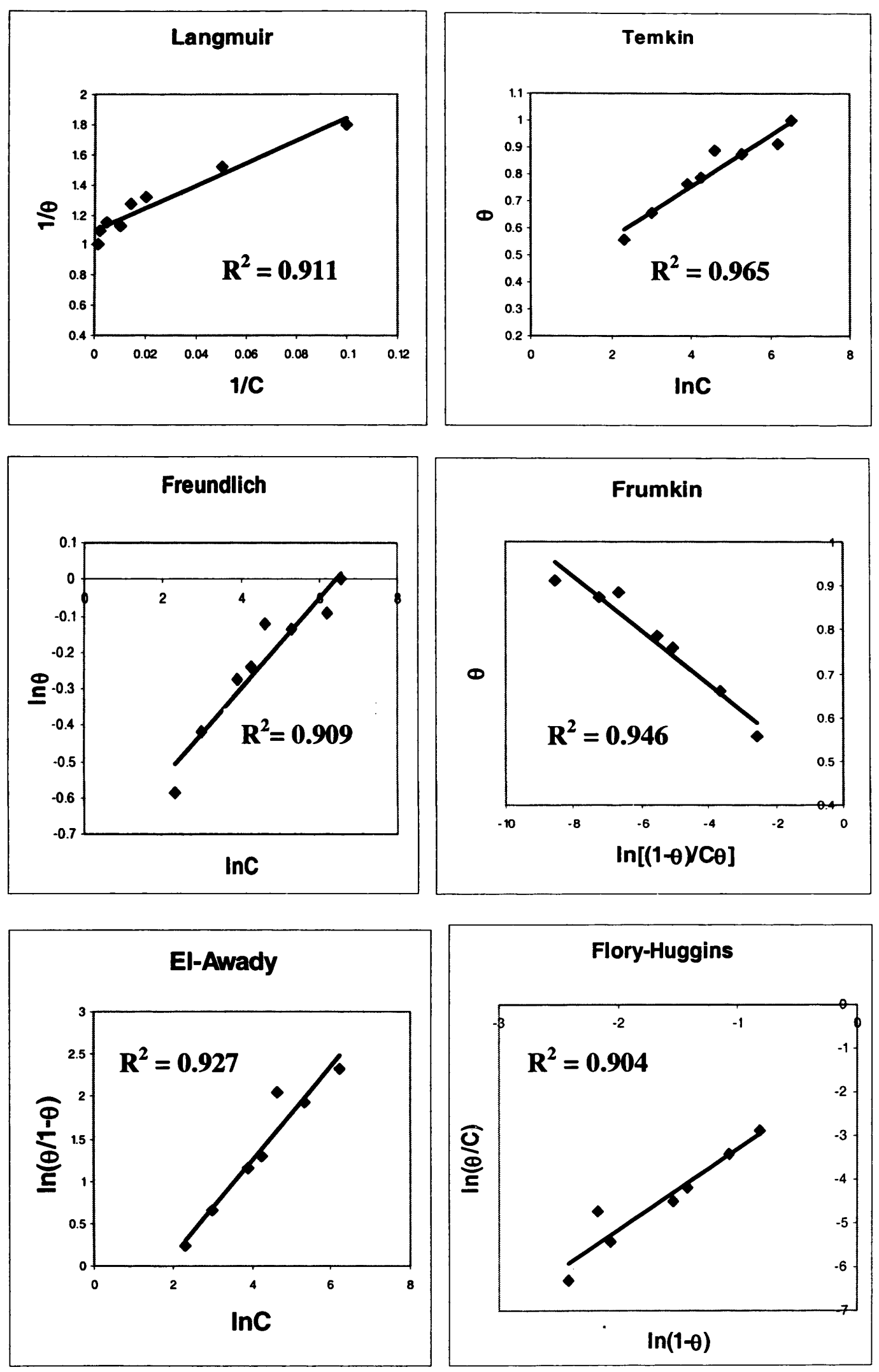

Figure 5.9: Plots of Langmuir, Temkin, Freundlich, Frumkin, El-Awady and Flory-Huggins isotherms to binding between $\mathrm{Cd}^{2+}$ and 3-thiodisuccinic acid on a wet (immersed) crystal. The line represents the linear least squares fit to the experimental data (points). 


\subsubsection{Discussion}

The adsorption isotherm is a measure of the relationship between the concentration of cadmium ions and the frequency shifts with the binding of cadmium ions to the monolayer. By fitting the experimental adsorption isotherm, it is possible to estimate the binding properties of the system using the corresponding fitting coefficients. The results from these isotherms showed that at higher $\mathrm{Cd}^{2+}$ concentrations the adsorption of $\mathrm{Cd}^{2+}$ tends to saturation at approximately $2 \mathrm{mM}$, as shown in Figure 5.5. In order to decide which type of isotherm fits better the adsorption experimental data, the values of $R^{2}$ (goodness of fit criterion) computed by linear regression for three independent isotherms were shown in Figures 5.8 and 5.9. The results showed that the experimental data fit very well to Temkin and Frumkin isotherms on emersed and immersed crystals. From these adsorption isotherms the relative ordering from best to worst isotherm is Temkin $>$ Frumkin> ElAwady > Langmuir $>$ Freundlich $>$ Flory-Huggins.

\subsubsection{Discussion of estimated isotherm parameters}

The values of binding constant $(K)$ and molecular interaction constant $(f)$ for Temkin, Freundlich, El-Awady, and Flory-Huggins isotherms were calculated from the slopes and intercepts these isotherms. The mean values of $K$ and $f$ for the reaction of cadmium ions with 3,3-thiodisuccinic acid were calculated from three independent experimental binding isotherms with different surface coverage of the 3-thiodisuccinic acid. The results are given in Tables 5.8 and 5.9. All isotherms except the Langmuir isotherm include terms that account for interactions between sites. The mean values of $\mathrm{K}$ from the Temkin isotherm are $5.1( \pm 2.8) \times 10^{6} \mathrm{M}^{-1}$ on a dry (emersed) crystal and 3.8( \pm 1.1$) \times 10^{6} \mathrm{M}^{-1}$ on a wet (immersed) crystal with a change in a mole ratio between $\mathrm{Cd}^{2+}$ and SAM from $1.22 \pm 0.16$ at the plateau state to $1.68 \pm 0.25$ at higher concentration for a dry (emersed) crystal and $1.26 \pm 0.03$ at the plateau state to $1.74 \pm 0.17$ at higher concentration for a wet (immersed) crystal. The binding constant of adsorption, $K$, is related to the standard free energy of adsorption $\Delta \mathbf{G}^{\circ}$ ads by the following equation: $\Delta \mathbf{G}^{\circ}$ ads $=-\mathbf{R T} \operatorname{InK}$, where $\mathbf{R}$ is the universal gas constant (8.314 Joule $\mathrm{mol}^{-1} \mathrm{~K}^{-1}$ ) and $\mathrm{T}$ is absolute temperature. Figure 5.10 shows the comparison between $\Delta \mathrm{G}^{\circ}$ ads on dry (emersed) and wet (immersed) crystals for all isotherms. 


\begin{tabular}{|l|l|l|l|l|l|l|}
\hline $\begin{array}{l}\text { SAM- } \\
\text { 3- } \\
\text { Cd(II) }\end{array}$ & $\ln K_{L}$ & $\ln K_{T}$ & $\ln K_{F}$ & $\ln K_{F r}$ & $\ln K_{E 1}$ & $\ln K_{F H}$ \\
\hline $\begin{array}{l}\text { Dry } \\
n=3\end{array}$ & $11.4 \pm 0.4$ & $15.6 \pm 1.2$ & $12 . .9 \pm 0.2$ & $16.3 \pm 0.1$ & $11.8 \pm 0.2$ & $11.4 \pm 0.08$ \\
\hline $\begin{array}{l}\text { Wet } \\
n=3\end{array}$ & $11.7 \pm 0.3$ & $15.1 \pm 0.3$ & $13.2 \pm 0.04$ & $16.2 \pm 0.005$ & $12.1 \pm 1.07$ & $11.8 \pm 0.7$ \\
\hline
\end{tabular}

Table 5.8: The values of InK for binding $\mathrm{Cd}^{2+}$ to 3,3-thiodisuccinic acid monolayer estimated from the slope and intercept (figures 5.8 and 5.9) using six kinds of isotherms on dry (emersed) and wet (immersed) crystals. The values are represented as a mean \pm standard deviation of three independent experimental binding isotherms.

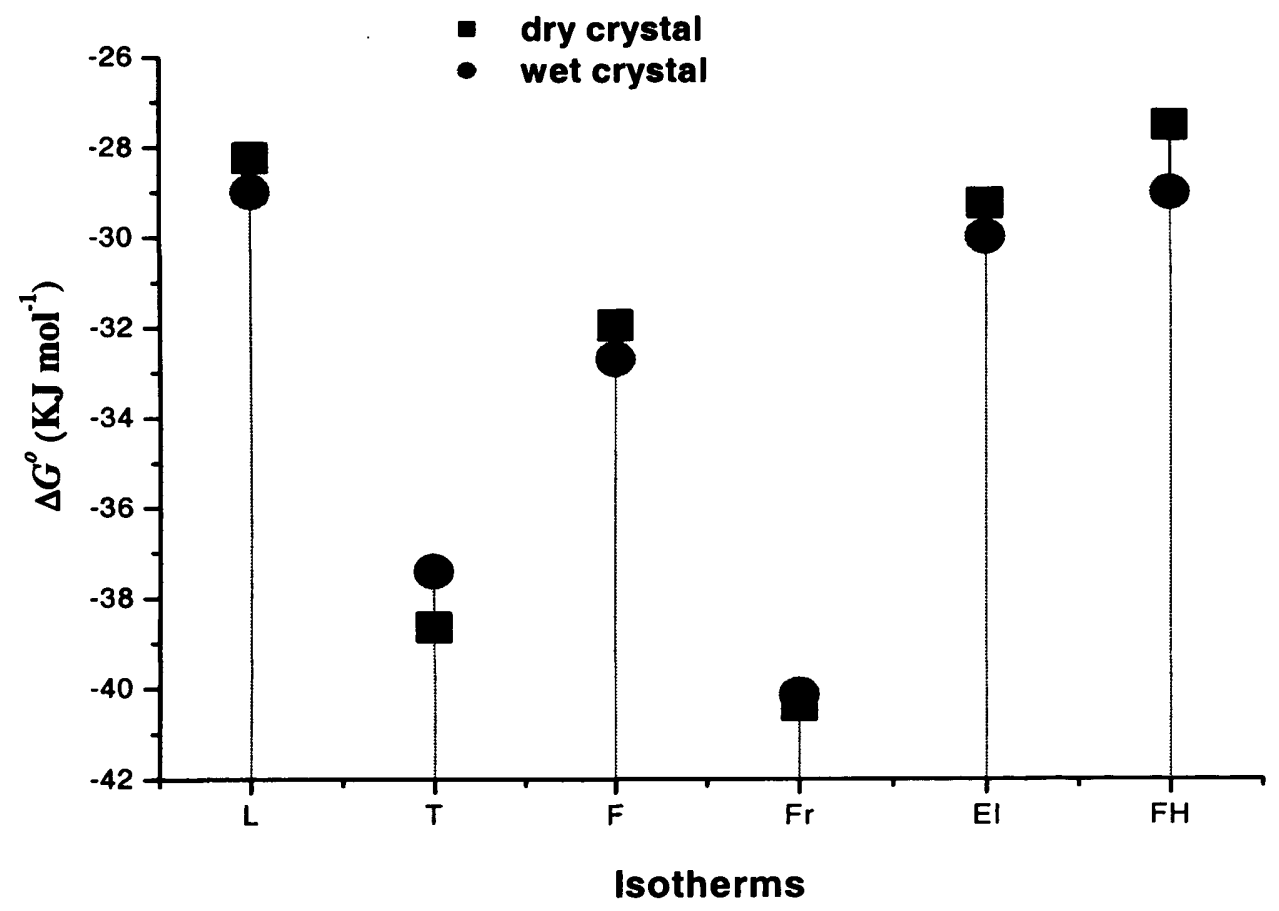

Figure 5.10: Comparison for the $\Delta G^{\circ}$ values on dry (emersed) and wet (immersed) crystals for binding $\mathrm{Cd}^{2+}$ to 3-thiodisuccinic acid using six kinds of isotherms. 


\begin{tabular}{|l|c|c|c|c|c|}
\hline $\begin{array}{l}\text { SAM- } \\
\text { Cd(II) }\end{array}$ & $\mathbf{f}_{\text {T }}$ & $\mathbf{f}_{\mathbf{F}}$ & $\mathbf{f}_{\text {Fr }}$ & $\mathbf{f}_{\text {El }}$ & $\mathbf{f}_{\text {FH }}$ \\
\hline $\begin{array}{l}\text { Dry } \\
n=3\end{array}$ & $8.6 \pm 1.6$ & $6.3 \pm 1.5$ & $-0.07 \pm 0.02$ & $1.3 \pm 0.1$ & $0.83 \pm 0.05$ \\
\hline $\begin{array}{l}\text { Wet } \\
n=3\end{array}$ & $8.8 \pm 0.2$ & $6.9 \pm 0.5$ & $-0.06 \pm 0.001$ & $1.4 \pm 0.5$ & $0.83 \pm 0.05$ \\
\hline
\end{tabular}

Table 5.9: The values of $(f)$ for binding $\mathrm{Cd}^{2+}$ to 3,3-thiodisuccinic acid monolayer estimated From the slope and intercept (figures 5.8 and 5.9) using five kinds of isotherms on dry (emersed) and wet (immersed) crystals. The values are represented as a mean \pm standard deviation of three independent experimental binding isotherms.

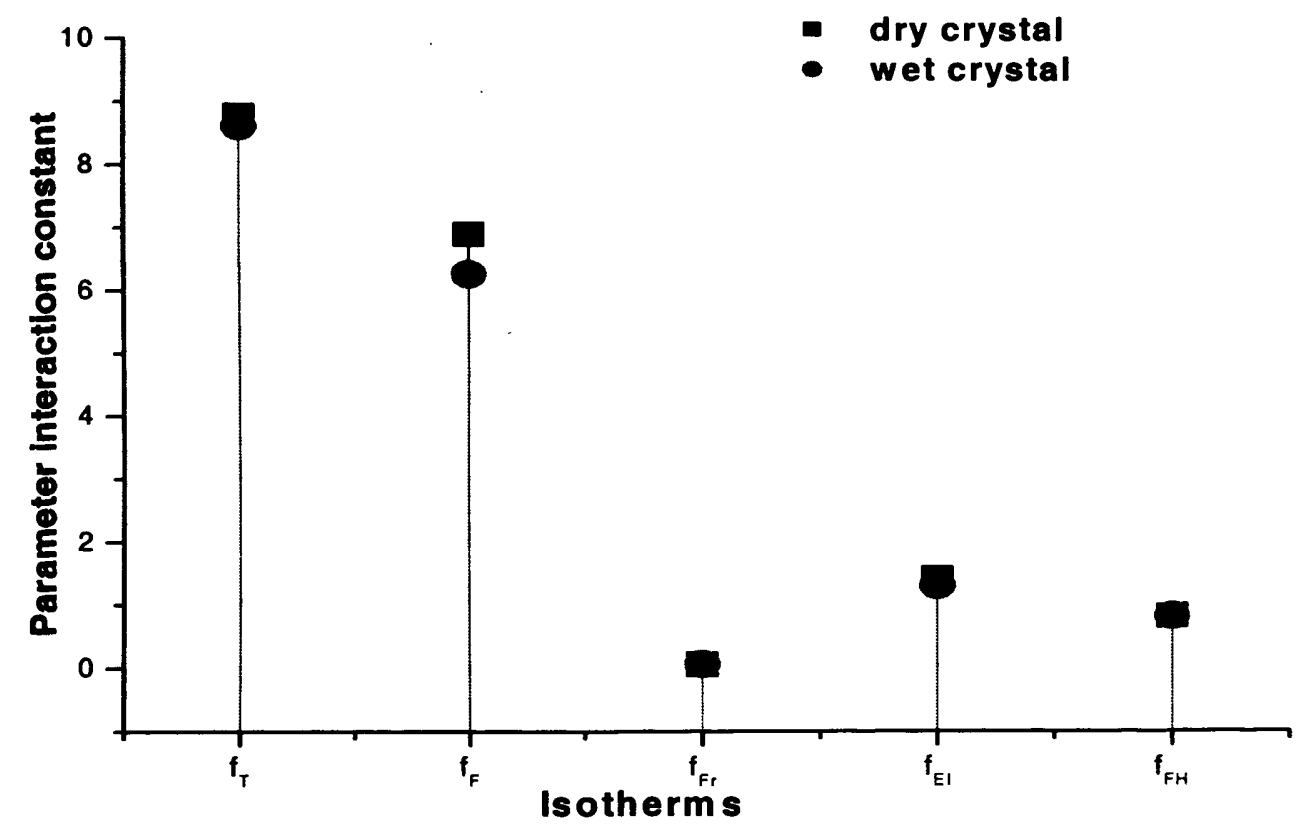

Figure 5.11: Comparison of the f values on dry and wet crystal using five kinds of isotherms on dry (emersed) and wet (immersed) crystals.

The thermodynamics of adsorption can provide valuable clues about the mechanism of adsorption, for example the negative value of $\Delta \mathrm{G}^{\circ}$ ads indicates that the adsorption of cadmium ions to the ligand is chemisorption because the adsorption free energy is more negative than $25 \mathrm{~kJ} \mathrm{~mol}^{-1}$ for all isotherms. It was 
found that the Temkin and Frumkin adsorption isotherms have more negative values of free energy than other isotherms because the high values of binding constant requires high values of interaction constants to get the same coverage of binding sites for cadmium ions to the ligand. Figure 5.11 shows the comparison between molecular interaction constants for all isotherms. The variations in observed $f$ values for Temkin, Freundlich, Frumkin, El-Awady and Flory-Huggins isotherms represented molecular repulsion between adsorption sites. The value of $f$ is related to the adsorption intensity of the metal ion on the monolayer. The value of $f>1$ (for Temkin and Freundlich) indicates that a stronger repulsion between adsorbed and adsorbing species. The magnitude of the molecular interaction constant is related to the magnitude of the repulsion experienced by cadmium ions during the adsorption. Colulombic and steric effects influence the f-values. The negative values of $f$ in the Frumkin adsorption on the dry and wet crystal because for the high coulombic repulsion between cadmium ions during the adsorption. Under the Frumkin adsorption conditions, the relation between free energy of interaction and value of $f$ can be determined from the equation: $f=\Delta \mathbf{G} / \mathbf{R T}$ [8]. From this equation the free energy can be estimated for the adsorption of cadmium ions to SAM. It should be noted a value of $f$ equal to zero corresponds to the Langmuir isotherm. The values of $f$ calculated on dry and wet crystals were $-0.07 \pm 0.02$ and $0.06 \pm 0.001$, respectively. In the Temkin and Freundlich isotherms the values of $f$ in the range 6.25 to 8.77 indicate that the repulsion between cadmium ions at adsorption sites is bigger than deduced from the Frumkin isotherm. The Temkin isotherm is based on the assumption that free energy of adsorption is a function of the surface coverage. The Temkin isotherm assumes that the adsorption is characterised by a uniform distribution of binding energies, up to some maximum binding energy $\left(\Delta G_{\max }\right)$. In the terms of El-Awady and Flory-Huggins isotherms, the values of $f$ are approximately one. Langmuir isotherm is described by a spike at a particular binding energy.

\subsubsection{Selectivity of 3-thiodisuccinic acid to $\mathrm{Cd}^{2+}$}

One objectives is to design a new selective sensor for heavy metal ions such as $\mathrm{Cd}^{2+}, \mathrm{Pb}^{2+}$ and $\mathrm{Cu}^{2+}$. The sensing strategy of the ligand (3-thiodisuccinic acid) was examined in single solutions of $\mathrm{Cd}^{2+}, \mathrm{Pb}^{2+}$ and $\mathrm{Cu}^{2+}$. The ability of the ligand to uptake selectively of these metal ions in aqueous solutions was studied by estimate the binding constant for each metal ion. 
In section 5.3.3, the binding constant between $\mathrm{Cd}^{2+}$ and the SAM was determined. In this section, the same surface coverage of the ligand was used to bind a range of concentrations $(10 \mu \mathrm{M}$ to $10 \mathrm{mM})$ of $\mathrm{Cd}^{2+}, \mathrm{Pb}^{2+}$ and $\mathrm{Cu}^{2++}$. The experimental adsorption data for the three metal ions was fitted to isotherms used in section 5.3.1. The results of adsorption isotherms are presented in Figures 5.12 and 5.13 . The same crystal was used for two types of measurements: dry-to-dry (emersed) and wet-to-wet (immersed). The maximum surface coverage of the ligand, estimated on a emersed and immersed crystal was $1.43 \times 10^{-9}$ and $2.35 \times 10^{-9} \mathrm{~mol}$ $\mathrm{cm}^{-2}$, respectively. The maximum adsorption of cadmium ions occurs at $\mathrm{pH}=6.3$, lead ions at $\mathrm{pH}=4.8$ and copper ions occurs at $\mathrm{pH}=5.4$.

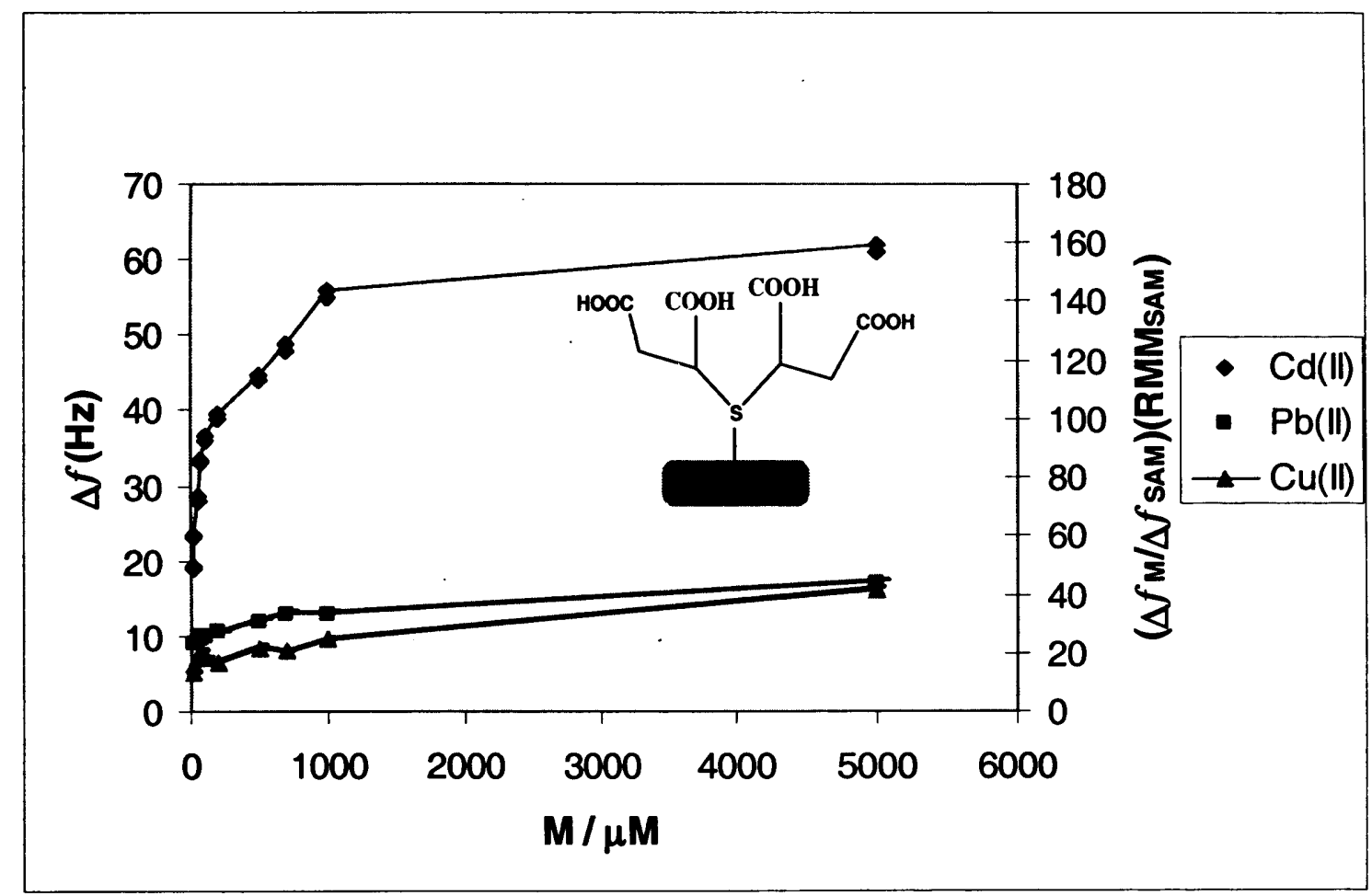

Figure 5.12: The binding isotherms of $\mathrm{Cd}^{2+}, \mathrm{Pb}^{2+}$ and $\mathrm{Cu}^{2+}$ to 3-thiodisuccinic acid estimated on a dry crystal (emersed crystal). 


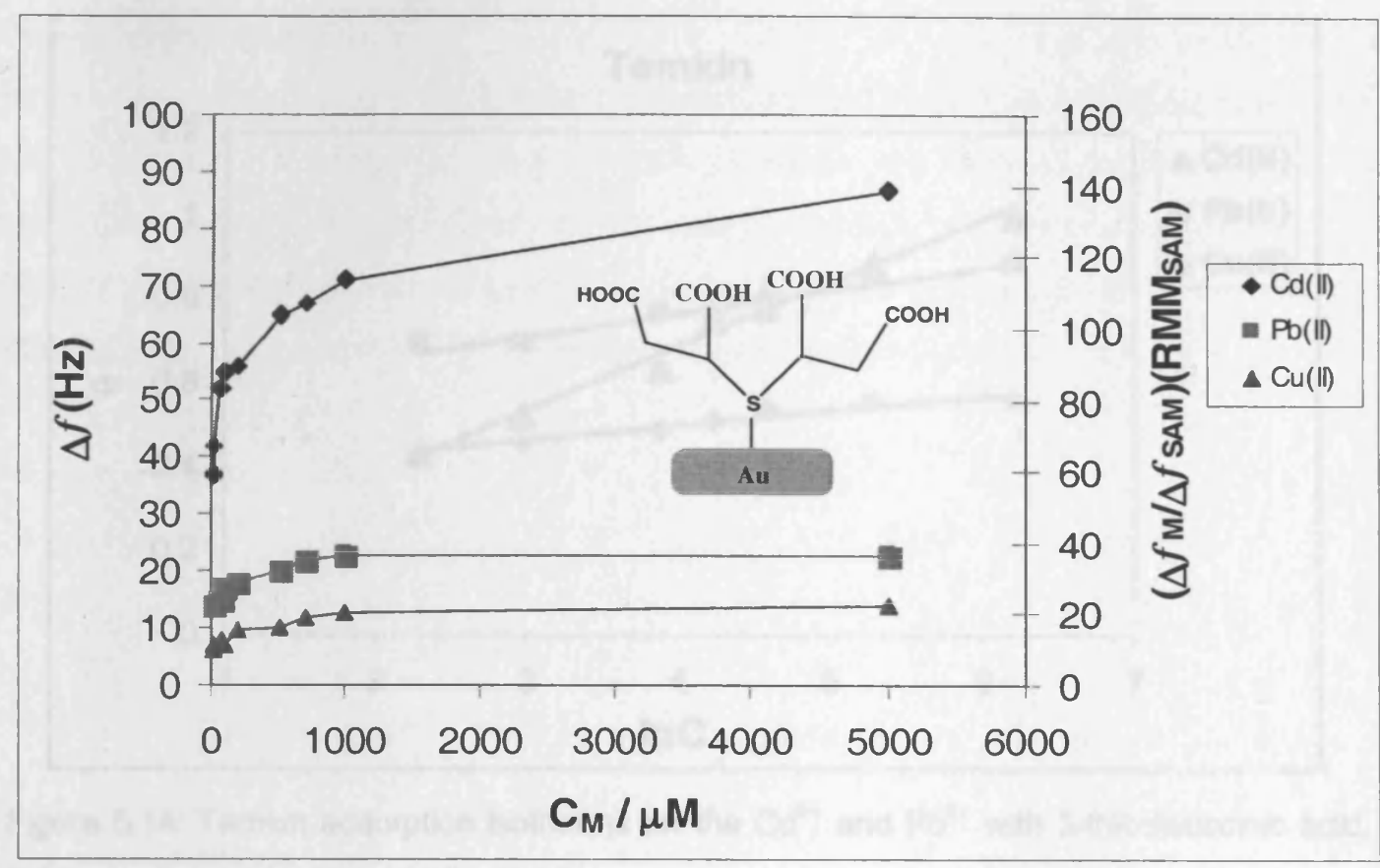

Figure 5.13: The binding isotherms of $\mathrm{Cd}^{2+}, \mathrm{Pb}^{2+}$ and $\mathrm{Cu}^{2+}$ to 3-thiodisuccinic acid estimated on a wet crystal (immersed crystal).

As can be seen, the single metal binding adsorption isotherm of $\mathrm{Cd}^{2+}$ increased more steeply than $\mathrm{Cu}^{2+}$ and $\mathrm{Pb}^{2+}$, as estimated on emersed and immersed crystals. These isotherms indicate that the ligand has high selectivity toward $\mathrm{Cd}^{2+}$ and low selectivity to $\mathrm{Pb}^{2+}$ and $\mathrm{Cu}^{2+}$. The SAM has little binding affinity for $\mathrm{Pb}^{2+}$ and $\mathrm{Cu}^{2+}$. The experimental adsorption data was fitted to six kinds of isotherms, as described in section 5.3.1. From these isotherms one can extract the binding constants for lead and copper ions. Temkin and Frumkin isotherms were used to describe the adsorption of these metal ions on the ligand (see section 5.31). Figure 5.14 shows linear regression of Temkin adsorption isotherm for cadmium, lead and copper. The values of binding constants are listed in Table 5.10. The estimated binding constants between these metal ions and the ligand follow the order $\mathrm{Cd}^{2+}>>\mathrm{Pb}^{2+}>$ $\mathrm{Cu}^{2+}$. Furthermore, the selectivity of the ligand to $\mathrm{Cd}^{2+}$ is examined by the mole ratio between the SAM and $\mathrm{Cd}^{2+}$. It was found that the mole ratio changed from $(1: 1)$ at low concentrations of cadmium ions to (1:2) at higher concentrations of cadmium ions. The mole ratio between the SAM modified $\mathrm{Au}$ electrode and $\mathrm{Pb}^{2+}$ or $\mathrm{Cu}^{2+}$ is about one at low and high concentrations of these metal ions. 


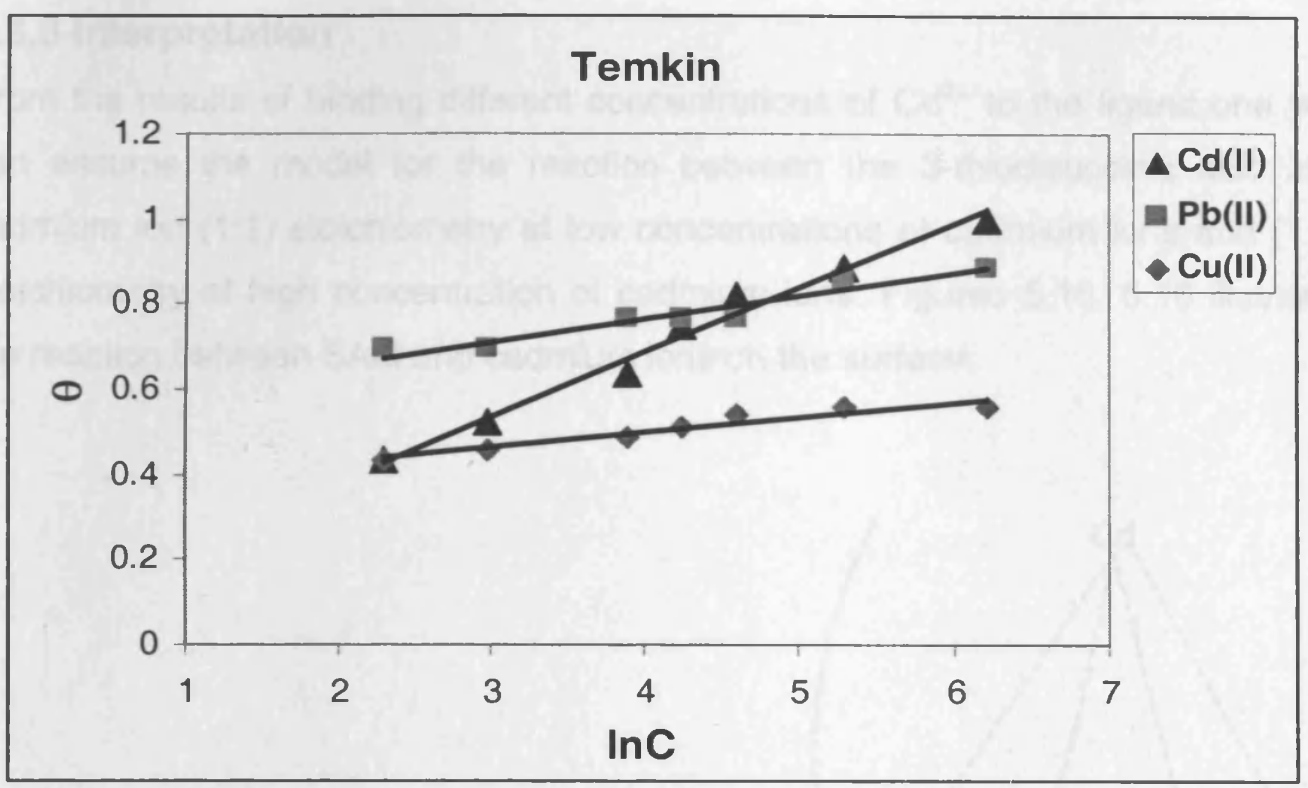

Figure 5.14: Temkin adsorption isotherms for the $\mathrm{Cd}^{2+}$ and $\mathrm{Pb}^{2+}$ with 3-thiodisuccinic acid, determined on a dry (emersed) crystal.

\begin{tabular}{|c|c|c|c|c|c|c|}
\hline & & & & & & \\
crystal & $I$ & & & & \\
\hline Dry & $15.6 \pm 0.2$ & $-0.07 \pm 0.02$ & $13.7 \pm 0.2$ & $-0.05 \pm 0.01$ & $12.4 \pm 0.6$ & $-0.09 \pm 0.04$ \\
\hline wet & $15.1 \pm 0.04$ & $-0.06 \pm 0.001$ & $13.6 \pm 0.4$ & $-0.04 \pm 0.01$ & $12.2 \pm 0.1$ & $-0.09 \pm 0.06$ \\
\hline
\end{tabular}

Table 5.10: Extracted Temkin binding and interaction constants for binding $\mathrm{Cd}^{2+}, \mathrm{Pb}^{2+}$ and $\mathrm{Cu}^{2+}$ to 3-thiodisuccinic acid on a dry (emersed) and wet (immersed) crystals.

The results from Table 5.10 show that, a very good agreement between the data extracted on dry (emersed) and wet (immersed) crystals. The binding constant for cadmium ions estimated on dry (emersed) crystal is about seven times bigger than lead ions and about twenty times bigger than copper ions. 


\subsubsection{Interpretation}

From the results of binding different concentrations of $\mathrm{Cd}^{2+}$ to the ligand one you can assume the model for the reaction between the 3-thiodisuccinic acid and cadmium ion (1:1) stoichiometry at low concentrations of cadmium ions and (1:2) stoichiometry at high concentration of cadmium ions. Figures 5.15, 5.16 illustrate the reaction between SAM and cadmium ions on the surface.

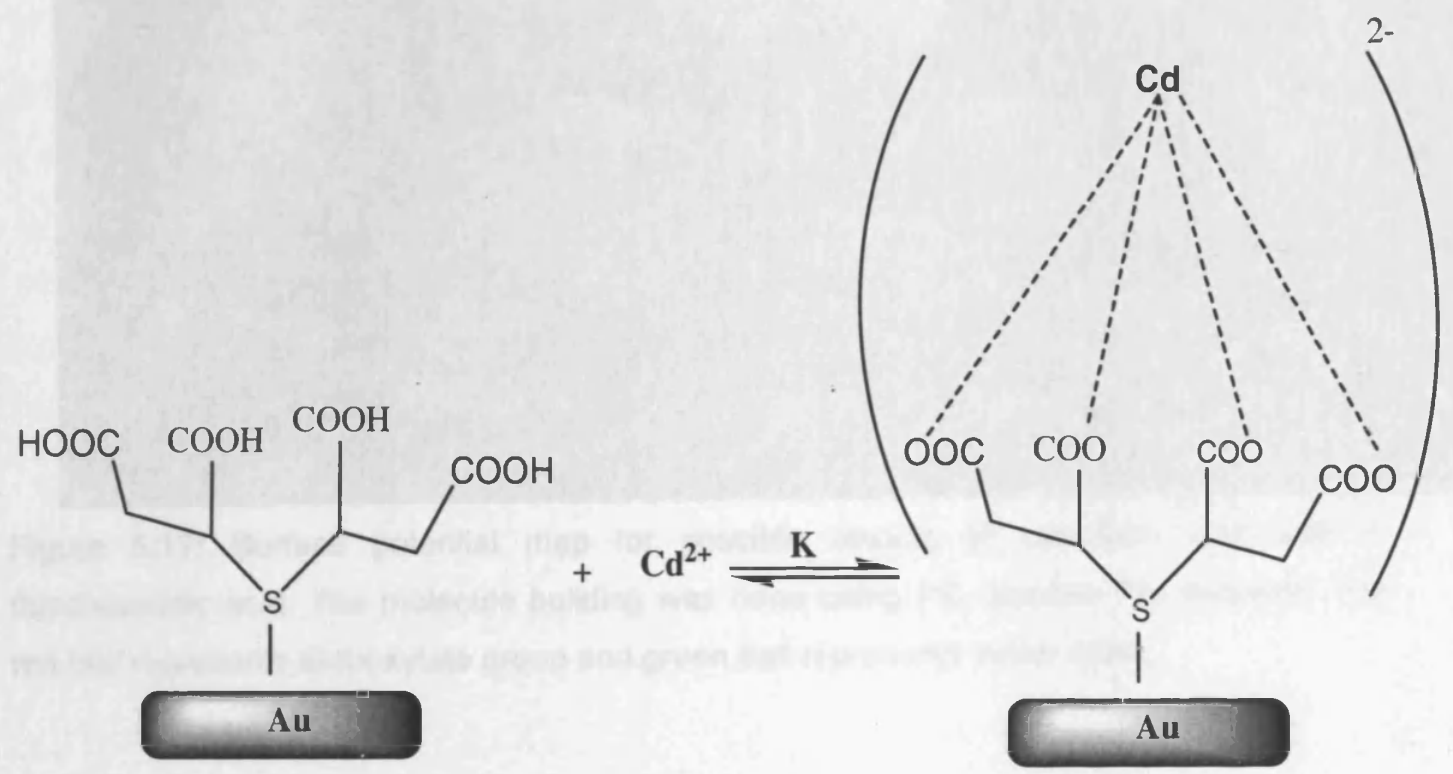

Figure 5.15: Schematic illustration of the complexation between cadmium ions and 3thiodisuccinic acid (1:1) stoichiometry; seen at low $\left[\mathrm{Cd}^{2+}\right]$.

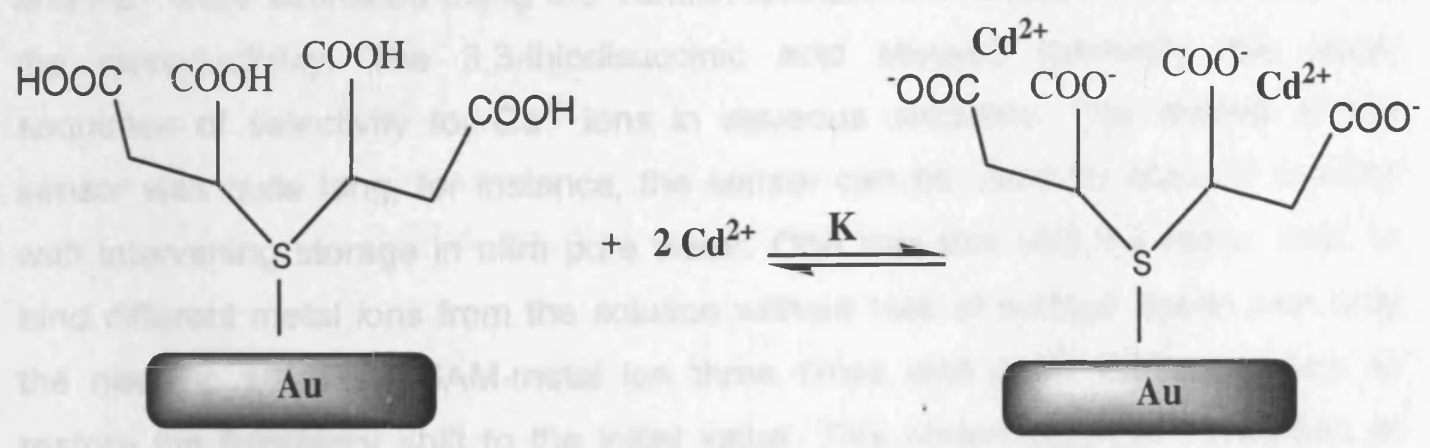

Figure 5.16: Schematic illustration of the complexation between cadmium ions and 3thiodisuccinic acid (2:1) stoichiometry; seen at high $\left[\mathrm{Cd}^{2+}\right]$. 


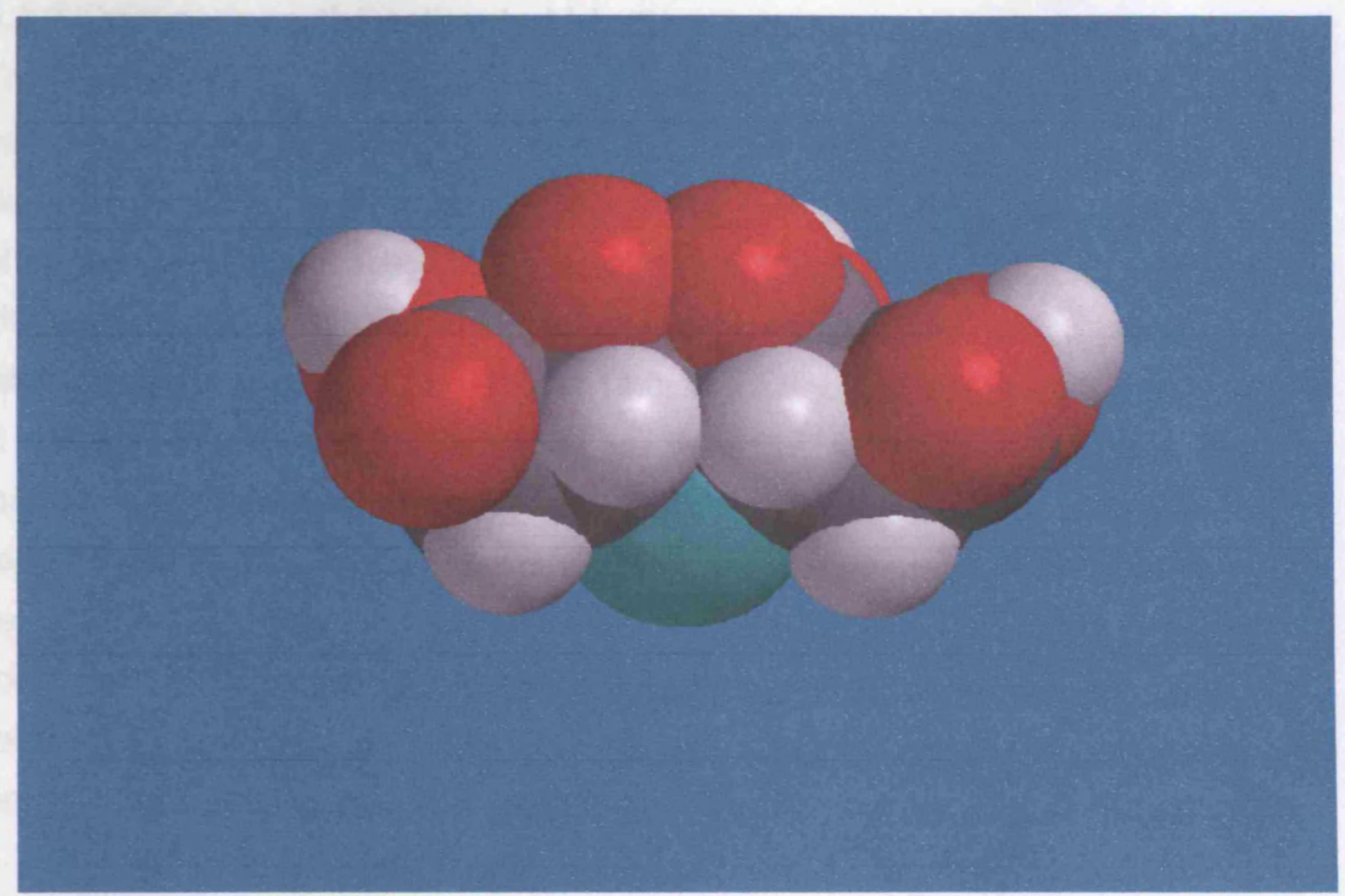

Figure 5.17: Surface potential map for possible binding of cadmium ions with 3thiodisuccinic acid. The molecule building was done using PC Spartan Pro software. The red ball represents carboxylate group and green ball represents sulfur atom.

\subsubsection{Reproducibility and limit of detection}

SAM 3,3-thiodisuccinic acid on the gold electrode has good reproducibility and stability to metal ion detections both at the same and various film coverages of the ligand. The equilibrium binding constants and interaction constants for $\mathrm{Cd}^{2+}, \mathrm{Cu}^{2+}$ and $\mathrm{Pb}^{2+}$ were estimated using the Temkin isotherm on different films to examine the reproducibility. The 3,3-thiodisuccinic acid showed generally the same sequence of selectivity for $\mathrm{Cd}^{2+}$ ions in aqueous solutions. The lifetime of the sensor was quite long, for instance, the sensor can be used for about 2 months with intervening storage in ultra pure water. One can also use the same SAM to bind different metal ions from the solution without loss of surface ligand with only the need to wash the SAM-metal ion three times with 0.1M EDTA solution to restore the frequency shift to the initial value. This shows that the adsorption of metal ions was reversible. The detection limit $(3 \times \mathrm{SD})$ was determined from the standard deviation of ten repeated determinations of the cadmium concentration at low levels of cadmium $(1$ to $10 \mu \mathrm{M})$. The limit of detection in this condition was found to be $1.5 \mu \mathrm{M}$. 


\subsubsection{Comparison the estimated binding constants with literature}

The estimated binding constants pK between the 3,3-thiodisuccinic acid on the surface and $\mathrm{Cd}^{2+}, \mathrm{Pb}^{2+}$ and $\mathrm{Cu}^{2+}$ was $(7.05,5.9$ and 5.4$)$ respectively. In the literature we found the binding constant $\mathrm{pK}$ for lead ions with 1,2phthalyidenetrathiotetraacetic acid in solution is 6.0 and the $\mathrm{pK}$ value for $\mathrm{Cu}^{2+}$, with ditartronic acid [9] is 4.39. The $\mathrm{pK}$ value for $\mathrm{Cd}^{2+}$ with 2,2dithiobis[ethyliminodiacetic acid] [10] is 14.0. We found that the pK values for $\mathrm{Cd}^{2+}$ in solution and surface have bigger values than $\mathrm{Pb}^{2+}$ and $\mathrm{Cu}^{2+}$. These data show that although the surface-bound ligand does not have the same overwhelming selectivity as its solution, probably due to geometrical constrains, it still sufficient to be of practical utility. Figure 5.18 shows the comparison between the binding constants for $\mathrm{Cd}(\mathrm{II}), \mathrm{Pb}$ (II) and $\mathrm{Cu}$ (II) with tetracarboxylate ligands in aqueous solutions reported from literature data with that extracted from binding these metal ions to SAM of 3-thiodisuccinic acid on the surface.

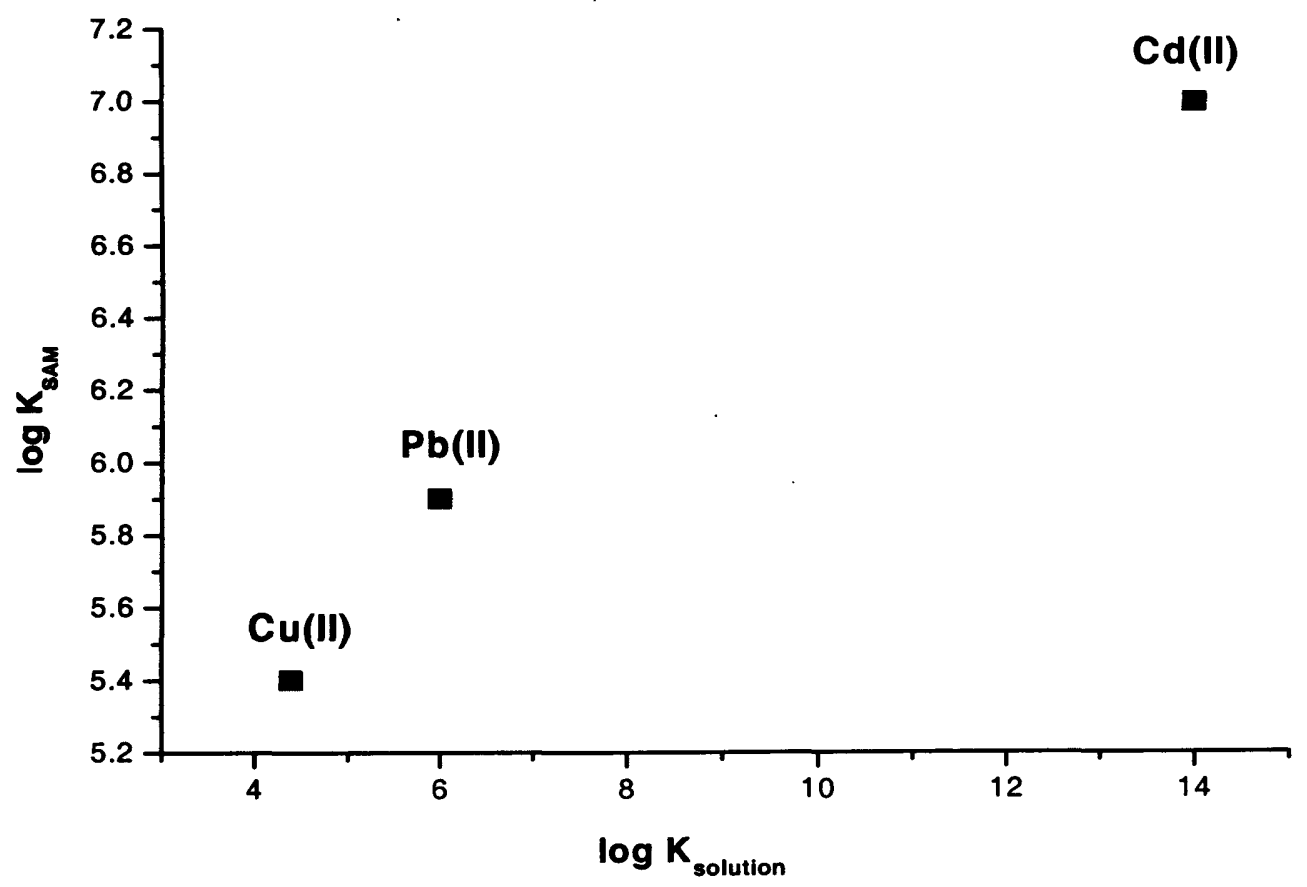

Figure 5.18: Comparison between the binding constants for $\mathrm{Cd}(\mathrm{II}), \mathrm{Pb}(\mathrm{II})$ and $\mathrm{Cu}(\mathrm{II})$ with tetra carboxylate ligands reported from the literature data and that extracted from binding these metal ions to SAM of 3-thiodisuccinic acid modified Au electrode. 
The important question we need to answer here; are the binding constants reported from literature data (solution values) a good guide to binding constants extracted here (surface values). The answer for that, the absolute values are different, but trends are maintained in both cases and the solution binding constant values is very good guide for surface binding constants for the of 3-thiodisuccinic acid SAM modified Au electrode.

\subsection{Estimate of the equilibrium binding constant between $\mathrm{Cd}^{2+}, \mathrm{Pb}^{2+}$ and $\mathrm{Cu}^{2+}$ with 4-mercaptobenzoic acid}

The aromatic self-assembled monolayer 4-mercaptobenzoic acid was studied for binding cadmium ions from aqueous solutions. It was found that from the survey in stage three in section 5.2.5, the ligand has the same stoichiometry for binding $\mathrm{Cd}^{2+}$ on dry (emersed) and wet (immersed) crystal. Furthermore, to compare the binding constant of cadmium ions with aliphatic SAM (3-thiodisuccinic acid) which was estimated in section 5.3.3 with aromatic SAM (4-mercaptobenzoic acid), the same procedure was used as in section 5.3, for the binding of cadmium ions to SAM at $\mathrm{pH}=6.3$. The $\mathrm{pK}_{\mathrm{A}}$ value of benzoic acid, which is the lowest of the molecules used in this study, is about 4.2, which indicate that all the carboxylic acid groups are expected to be completely ionized at $\mathrm{pH}=6.3$. The equilibrium mass loading due to SAM of 4-mercaptobenzoic acid formation is about $979 \mathrm{ng} / \mathrm{cm}^{2}$ on a dry (emersed) crystal and $838 \mathrm{ng} / \mathrm{cm}^{2}$ on a wet (immersed) crystal, which correspond to a molecular area per molecule of $2.61 \mathrm{~A}^{2}$ on a dry (emersed) crystal and $3.05 \mathrm{~A}^{2}$ on a wet (immersed) crystal. Due the strong binding of the thiol group with the Au quartz crystal surface, the surface of the SAM is expected to be rich with carboxylic acid functionalities. There are good fits of the experimental results to the Langmuir, ElAwady, Flory-Huggins and Freundlich isotherms and excellent fits to the Temkin and Frumkin isotherms. It can be seen that from these results the reaction between cadmium ions and SAM has 1:1 stoichiometry. Again here the experiment was repeated three times from three independent experimental isotherms with different surface coverages of the ligand on a dry (emersed) and wet (immersed) crystals to examine the reproducibility of the ligand binding to cadmium ions. The surface of 4mercaptobenzoic acid was used to bind other metal ions such as $\mathrm{Pb}^{2+}$ and $\mathrm{Cu}^{2+}$. The results of binding isotherms ( Figures 5.19 and 5.20) for $\mathrm{Pb}^{2+}$ and $\mathrm{Cu}^{2+}$ show that the ligand has similar selectivity for both ions. The experimental data for $\mathrm{Pb}^{2+}$ and $\mathrm{Cu}^{2+}$ were better fitted to the Frumkin isotherm than to the other isotherms, whether judged by emersed or immersed crystal data. 


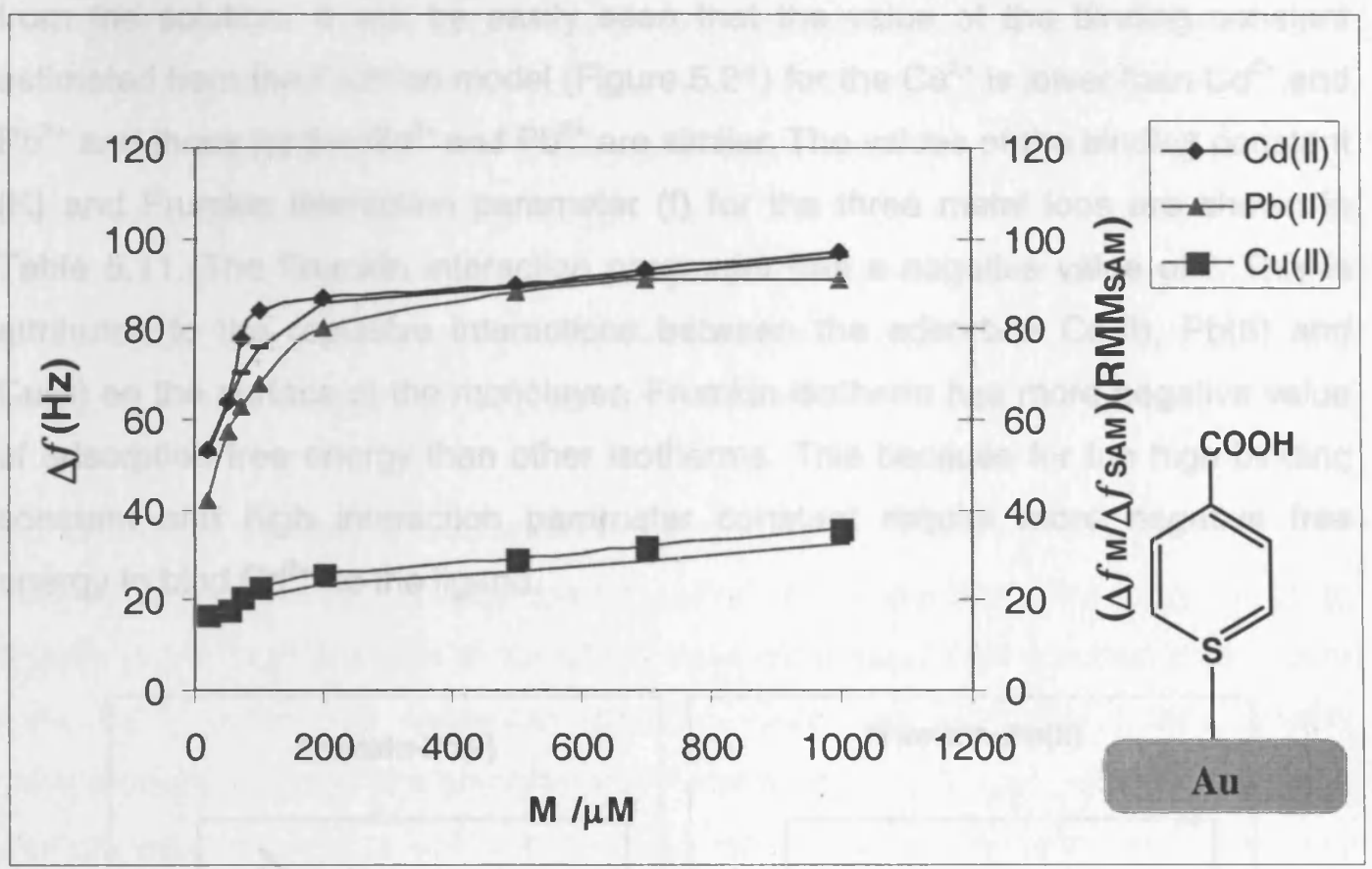

Figure 5.19: The binding isotherms for the binding $\mathrm{Cd}^{2+}, \mathrm{Pb}^{2+}$ and $\mathrm{Cu}^{2+}$ to 4mercaptobenzoic acid on a dry (emersed) crystal; the surface coverage of the ligand was $5.26 \mathrm{nmol} \mathrm{cm}^{-2}$.

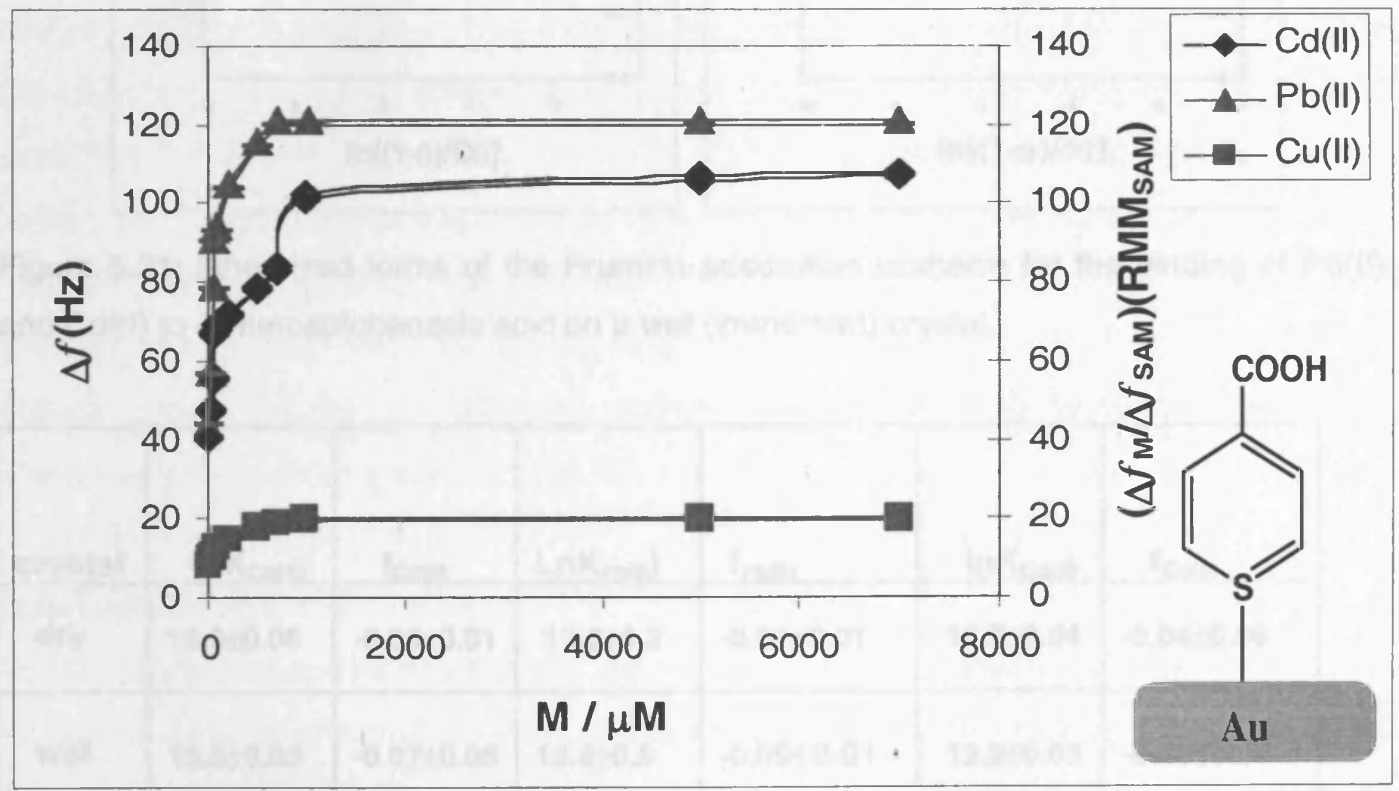

Figure 5.20: The binding isotherms for the binding $\mathrm{Cd}^{2+}, \mathrm{Pb}^{2+}$ and $\mathrm{Cu}^{2+}$ to 4mercaptobenzoic acid on a wet (immersed) crystal; the surface coverage of the ligand was $4.09 \mathrm{nmol} \mathrm{cm}^{-2}$. 
The adsorption isotherm indicates $\mathrm{Pb}^{2+}$ but not $\mathrm{Cu}^{2+}$ interference in $\mathrm{Cd}^{2+}$ uptake from the solution. It can be easily seen that the value of the binding constant estimated from the Frumkin model (Figure 5.21) for the $\mathrm{Cu}^{2+}$ is lower than $\mathrm{Cd}^{2+}$ and $\mathrm{Pb}^{2+}$ and those for the $\mathrm{Cd}^{2+}$ and $\mathrm{Pb}^{2+}$ are similar. The values of the binding constant $(K)$ and Frumkin interaction parameter $(f)$ for the three metal ions are shown in Table 5.11. The Frumkin interaction parameter has a negative value of $f$. This is attributed to the repulsive interactions between the adsorbed $\mathrm{Cd}(\mathrm{II}), \mathrm{Pb}(\mathrm{II})$ and $\mathrm{Cu}$ (II) on the surface of the monolayer. Frumkin isotherm has more negative value of adsorption free energy than other isotherms. This because for the high binding constant and high interaction parameter constant require more negative free energy to bind $\mathrm{Cd}^{2+}$ to the ligand.
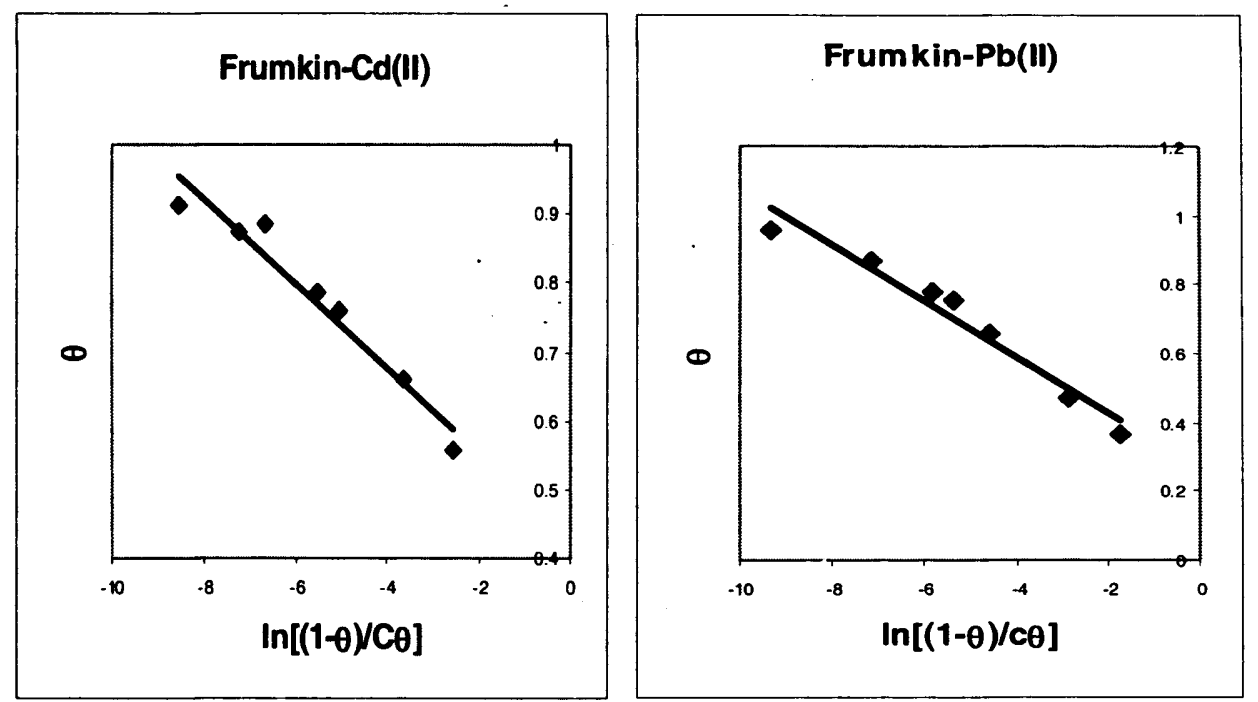

Figure 5.21: Linearized forms of the Frumkin adsorption isotherm for the binding of $\mathrm{Pb}$ (II) and $\mathrm{Cd}(\mathrm{II})$ to 4-mercaptobenzoic acid on a wet (immersed) crystal.

\begin{tabular}{|c|c|c|c|c|c|c|}
\hline crystal & InK $_{\mathrm{Cd}(11)}$ & $f_{\mathrm{Cd}(11)}$ & LnK & & & \\
\hline dry & $13.9 \pm 0.06$ & $-0.05 \pm 0.01$ & $13.5 \pm 0.2$ & $-0.08 \pm 0.01$ & $12.7 \pm 0.04$ & $-0.04 \pm 0.06$ \\
\hline wet & $13.6 \pm 0.05$ & $-0.07 \pm 0.05$ & $13.4 \pm 0.5$ & $-0.09 \pm 0.01$ & $12.9 \pm 0.03$ & $-0.05 \pm 0.09$ \\
\hline
\end{tabular}

Table 5.11: Equilibrium binding constants and Frumkin interaction parameters for metal ion binding to 4-mercaptobenzoic acid on dry (emersed) and wet (immersed) crystals. Parameters estimated from the linearized form of the Frumkin adsorption isotherm. 


\subsubsection{Comparison of the binding constants with literature data}

The estimated analogous values for binding constant for the $\mathrm{Cd}^{2+}$ and $\mathrm{Pb}^{2+}$ ions with 4-mercaptobenzoic acid is 5.9 and 5.8 respectively and is in fair agreement with that reported by Sastry et al [11] ( $\mathrm{pK}=5.30)$ and Roser et al [12] ( $\mathrm{pK}=5.35)$. Roser and Lovell mention that the $\mathrm{pK}$ value obtained for $\mathrm{Cd}^{2+}$ and $\mathrm{Pb}^{2+}$ ions in Langmiur- Blodgett films agrees with the equilibrium binding constant obtained from $\mathrm{X}$-ray reflectivity studies performed on Langmiur monolayers at the air-water interface. The equilibrium binding constant for $\mathrm{Cu}^{2+}$ has not been reported.

\subsection{Binding of $\mathrm{Pb}^{2+}$ to different SAMs}

We have shown in previous sections that self-assembled monolayers of 3thiodisuccinic acid are able to selectivity bind cadmium ions over lead and copper ions from contacting aqueous solution. Here we use the quartz crystal microbalance to study the complexation between $\mathrm{Pb}^{2+}$ and $\mathrm{Cu}^{2+}$ and different selfassembled monolayers. In the first stage, we compare the mole ratio of lead or copper ions and to surface-bound ligand for the two different concentrations of lead ions, 200 and 800 ppm, respectively.

The results of binding lead ions from aqueous solutions to different self-assembled monolayers for the two stages on emersed and immersed crystals show that SAMs of 4-mercaptobenzoic acid and meso-2,3-dimercaptosuccinic acid have the same stoichiometry (1:1). The aromatic self-assembled monolayer of 4-mercaptobenzoic acid has the same mole ratio $(1: 1)$ with $\mathrm{Pb}^{2+}$ on emersed and immersed crystal, which was described in section 5.4. The self-assembled monolayer of meso-2,3dimercaptosuccinic acid has a mole ratio (1:1) to lead ions and contains two carboxylate groups with the ability to bind lead ions from the solution. For this reason, the binding of different concentrations of lead ions to meso-2,3dimercaptodisuccinic acid was studied.

\subsubsection{Complexation between $\mathrm{Pb}^{2+}$ and DMSA}

In this study a range of concentrations of lead ions in aqueous solution was bound to the surface of meso-2,3-dimercaptosuccinic acid (DMSA) after this ligand was immobilized on the gold surface of a quartz crystal microbalance. The range of concentrations was from $10 \mu \mathrm{M}$ to $10 \mathrm{mM}$ and the same immobilized selfassembled monolayer on the crystal at $\mathrm{pH}=4.8$ was used to measure the frequency shifts after binding lead ions. The experimental adsorption isotherms were repeated three times for each of three different surface coverages of the 
ligand, to examine the reproducibility. The results of frequency shifts for each concentration binding to the monolayer on dry (emersed) and wet (immersed) crystals are shown in Figure 5.22.

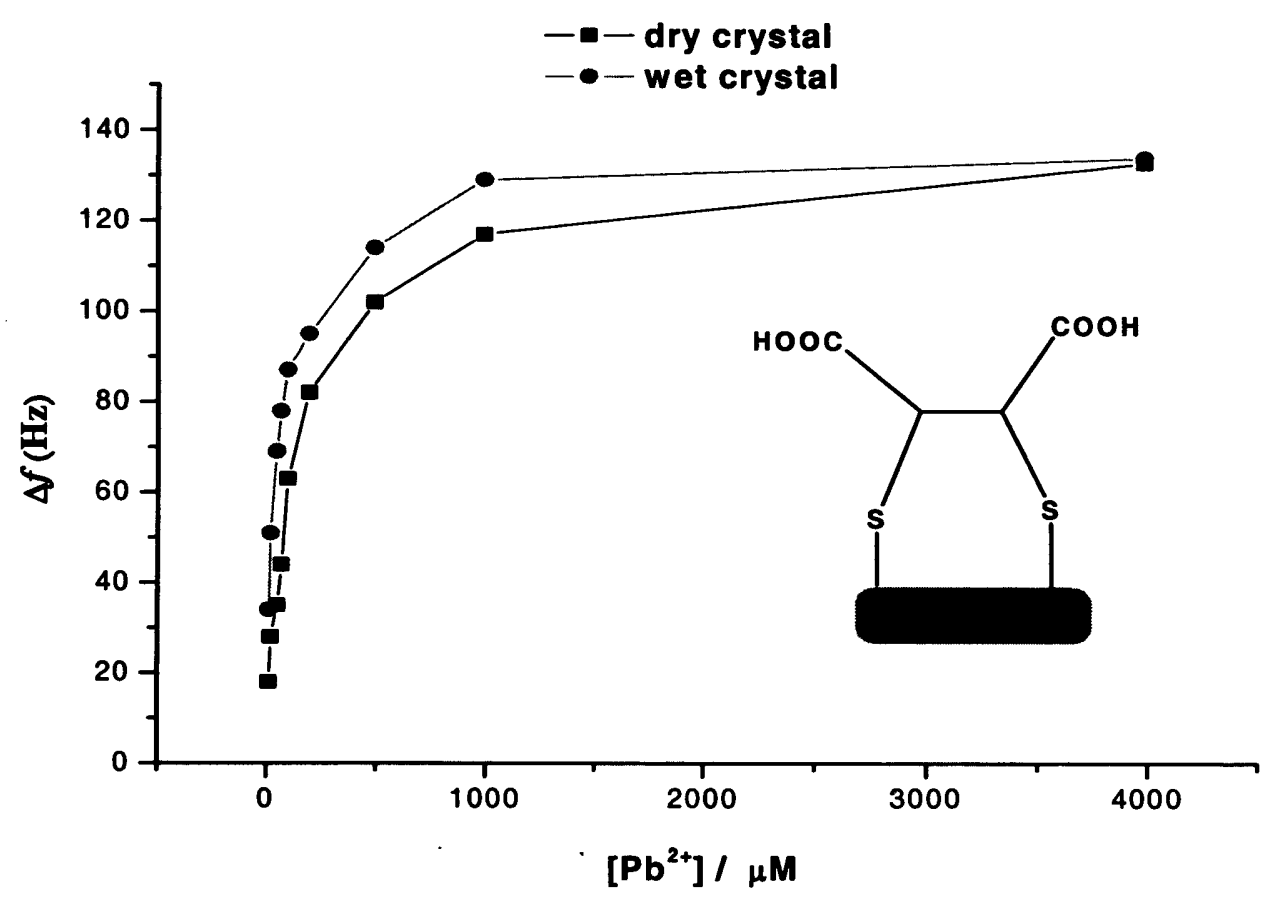

Figure 5.22: Adsorption isotherm for the reaction of aqueous solutions of $\mathrm{Pb}(\mathrm{II})$ with meso2,3-dimercaptosuccinic (DMSA SAM modified Au) on dry (emersed) and wet (immersed) crystals.

\subsubsection{Fitting the Data to Different Isotherms}

The experimental adsorption data for binding lead ions to the monolayer could be fitted to the Frumkin isotherm. In terms of the correlation constant $\left(R^{2}\right)$, the validity of different isotherms for the present experimental data follows the order: Frumkin $>$ El-Awady $>$ Temkin $>$ Langmuir $>$ Freundlich $>$ Flory-Huggins on a dry crystal and Frumkin > Langmuir > Temkin > El-Awady > Freundlich > FloryHuggins on a wet crystal. Clearly, the Frumkin isotherm is the best one and the Flory-Huggins is the worst one. From the slope and intercept of the linear regression of these isotherms one can estimate the equilibrium binding constant and interaction constant; data are presented in Table (5.12). 


\begin{tabular}{|l|l|l|l|l|l|l|}
\hline $\begin{array}{l}\text { SAM- } \\
\text { Pb(II) }\end{array}$ & $\operatorname{lnK}_{\mathrm{L}}$ & $\ln _{\mathrm{T}}$ & $\ln \mathrm{K}_{\mathrm{F}}$ & $\ln \mathrm{K}_{\mathrm{Fr}}$ & $\operatorname{LnK}_{\mathrm{El}}$ & $\ln \mathrm{K}_{\mathrm{FH}}$ \\
\hline $\begin{array}{l}\text { Dry } \\
\mathbf{n = 3}\end{array}$ & $10.8 \pm 1.7$ & $12.9 \pm 1.2$ & $12.2 \pm 0.9$ & $12.9 \pm 1.1$ & $11.3 \pm 1.7$ & $10.7 \pm 1.8$ \\
\hline $\begin{array}{l}\text { Wet } \\
\mathbf{n = 3}\end{array}$ & $10.9 \pm 0.6$ & $12.8 \pm 1.4$ & $12.7 \pm 0.4$ & $13.2 \pm 0.5$ & $11.8 \pm 1.6$ & $10.9 \pm 1.2$ \\
\hline
\end{tabular}

Table 5.12: The values of InK for binding $\mathrm{Pb}^{2+}$ to meso-2,3-dimercaptosuccinic acid (DMSA SAM modified Au electrode) estimated from the slope and intercept (figures 5.45 and 5.46) using six isotherms on dry (emersed) and wet (immersed) crystals. The values are represented as a mean \pm standard deviation of three independent experimental binding isotherms.

It was found that the mean value of the binding constant using the Frumkin isotherm on dry (emersed) and wet (immersed) crystals was, respectively, $5.0( \pm$ $0.4) \times 10^{5} \mathrm{M}^{-1}$ and $7.0 \pm(0.1) \times 10^{5} \mathrm{M}^{-1}$ at $\mathrm{pH}=4.8$. The mole ratio between the meso2,3-dimercaptosuccinic acid and $\mathrm{Pb}^{2+}$ was $0.98 \pm 0.05$ at the plateau state and $1.28 \pm 0.04$ at higher concentrations of $\mathrm{Pb}^{2+}$ for the dry (emersed) crystal, and $0.88 \pm$ 0.03 at plateau state and $1.04 \pm 0.02$ at higher concentrations for the wet (immersed) crystal. This indicates that the reaction between the carboxylate groups on the surface and $\mathrm{Pb}^{2+}$ is close to $(1: 1)$. The mean values of the Frumkin interaction constant on dry (emersed) and wet (immersed) crystals are $-0.07 \pm 0.02$ and $-0.07 \pm 0.01$, respectively. The negative values of the interaction constants indicate repulsive interaction between lead ions adsorbed on the surface of the monolayer. The complexation of $\mathrm{Pb}^{2+}$ from aqueous solutions to the meso-2,3dimercaptosuccinic acid on the surface of the gold via modification of the surface with SAM at $\mathrm{pH}=4.8$ is shown in Figure 5.23. 

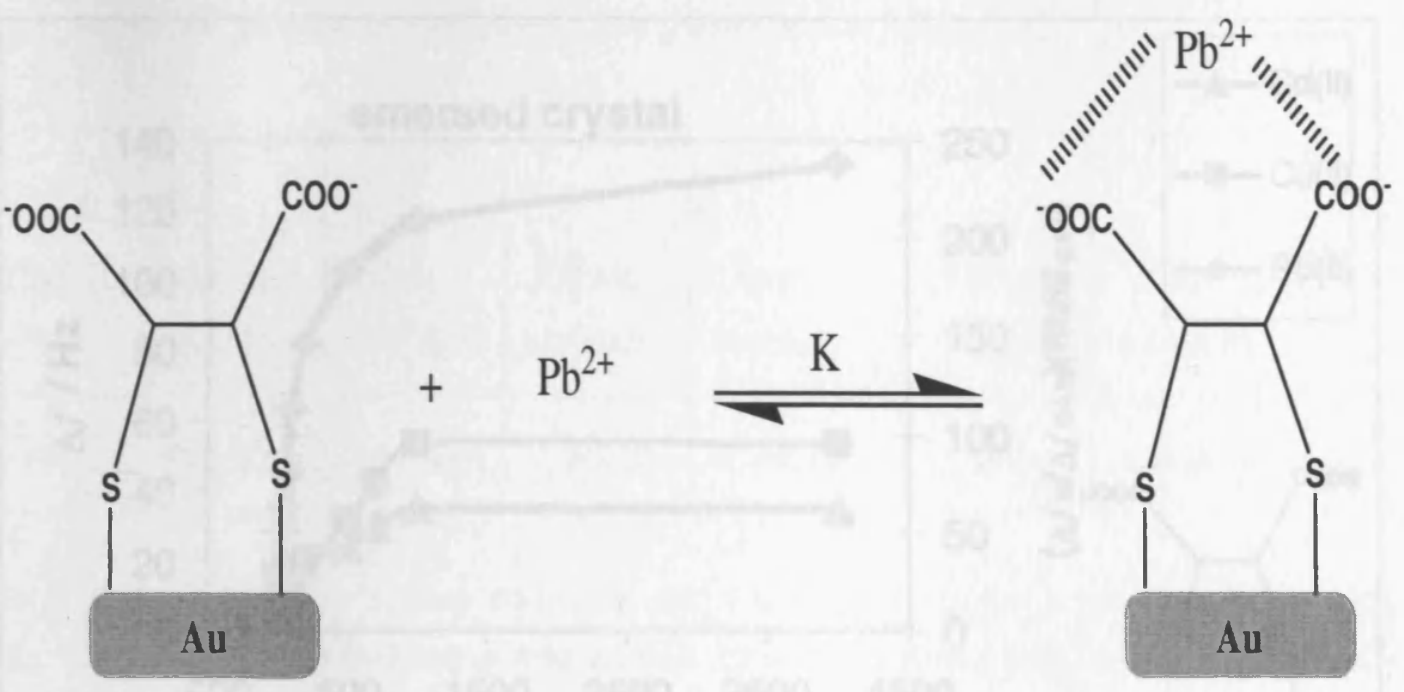

Figure 5.23: Schematic representation of binding of $\mathrm{Pb}^{2+}$ with meso-2,3-dimercaptosuccinic acid (DMSA SAM modified Au electrode) at $\mathrm{pH}=4.8$.

\subsubsection{Binding of $\mathrm{Cd}(\mathrm{II})$ and $\mathrm{Cu}(\mathrm{II})$ to meso-2,3-dimercaptosuccinic acid}

The ability of the surface bound carboxylate groups to bind $\mathrm{Cd}^{2+}$ and $\mathrm{Cu}^{2+}$ from aqueous solutions was examined by using the same ligand (meso-2,3dimercaptosuccinic acid). The ligand was bound to other metal ions such as $\mathrm{Cd}^{2+}$ and $\mathrm{Pb}^{2+}$ to examine the selectivity of the ligand to $\mathrm{Pb}(\mathrm{II})$. We use the same method and interpretation as in section 5.51. The same coverage of the ligand was used to bind $\mathrm{Cd}(\mathrm{II})$ and $\mathrm{Cu}(\mathrm{II})$ from single ion solutions. The adsorption isotherms are presented in Figures 5.24 and 5.25. The frequency shift results of binding these metal ions show that the binding of $\mathrm{Pb}(\mathrm{II})$ to the ligand is stronger than of $\mathrm{Cd}(\mathrm{II})$ and $\mathrm{Cu}(\mathrm{II}))$. The adsorption of these metal ions followed the same sequence $\mathrm{Pb}$ (II) $>\mathrm{Cu}(\mathrm{II})>\mathrm{Cd}(\mathrm{II})$ on emersed and immersed crystals. 


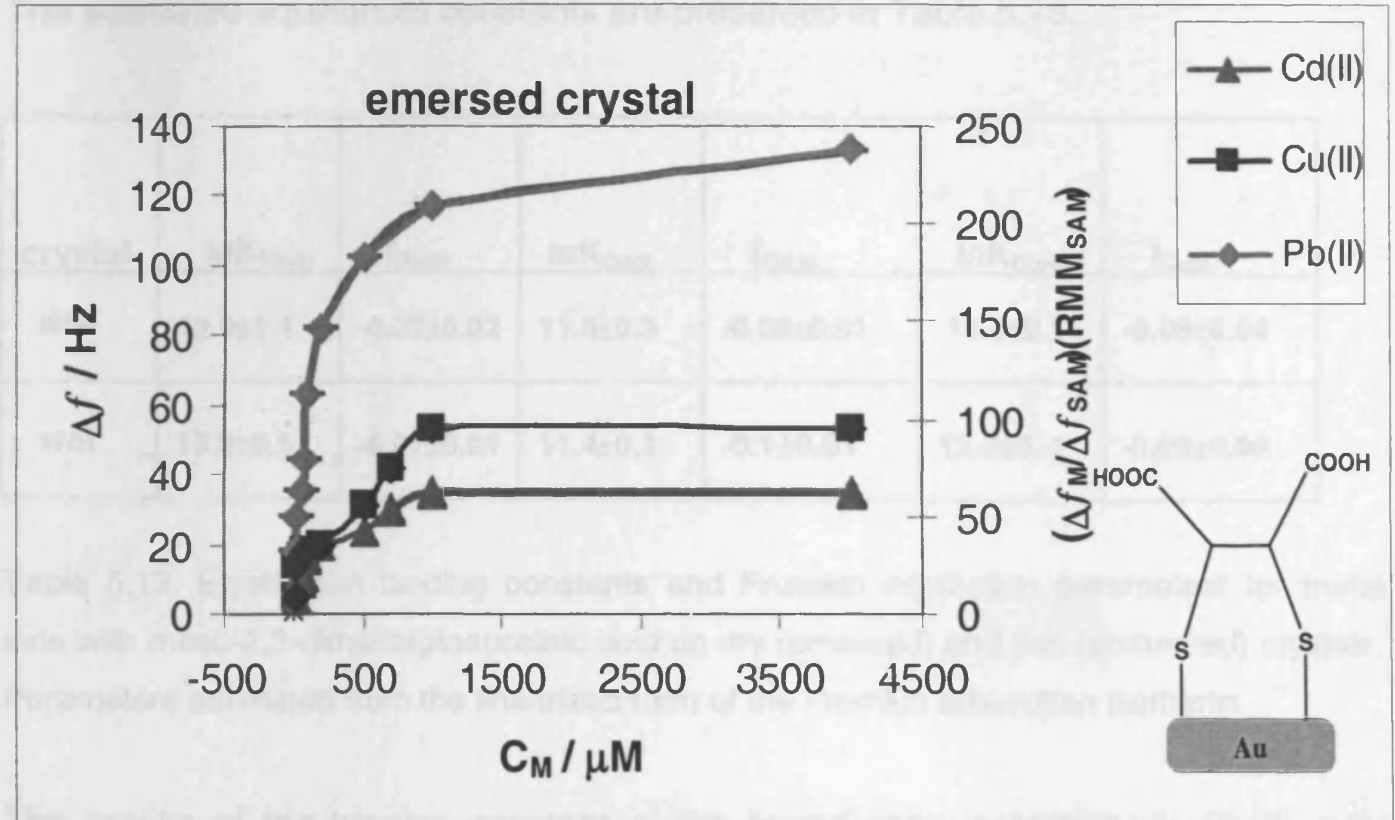

Figure 5.24: Binding isotherms for $\mathrm{Pb}^{2+}, \mathrm{Cu}^{2+}$ and $\mathrm{Cd}^{2+}$ to meso-2,3-dimercaptosuccinic acid (DMSA SAM modified Au electrode) on emersed crystals. The surface coverage of the DMSA was $2.39 \mathrm{nmol} \mathrm{cm}^{-2}$.

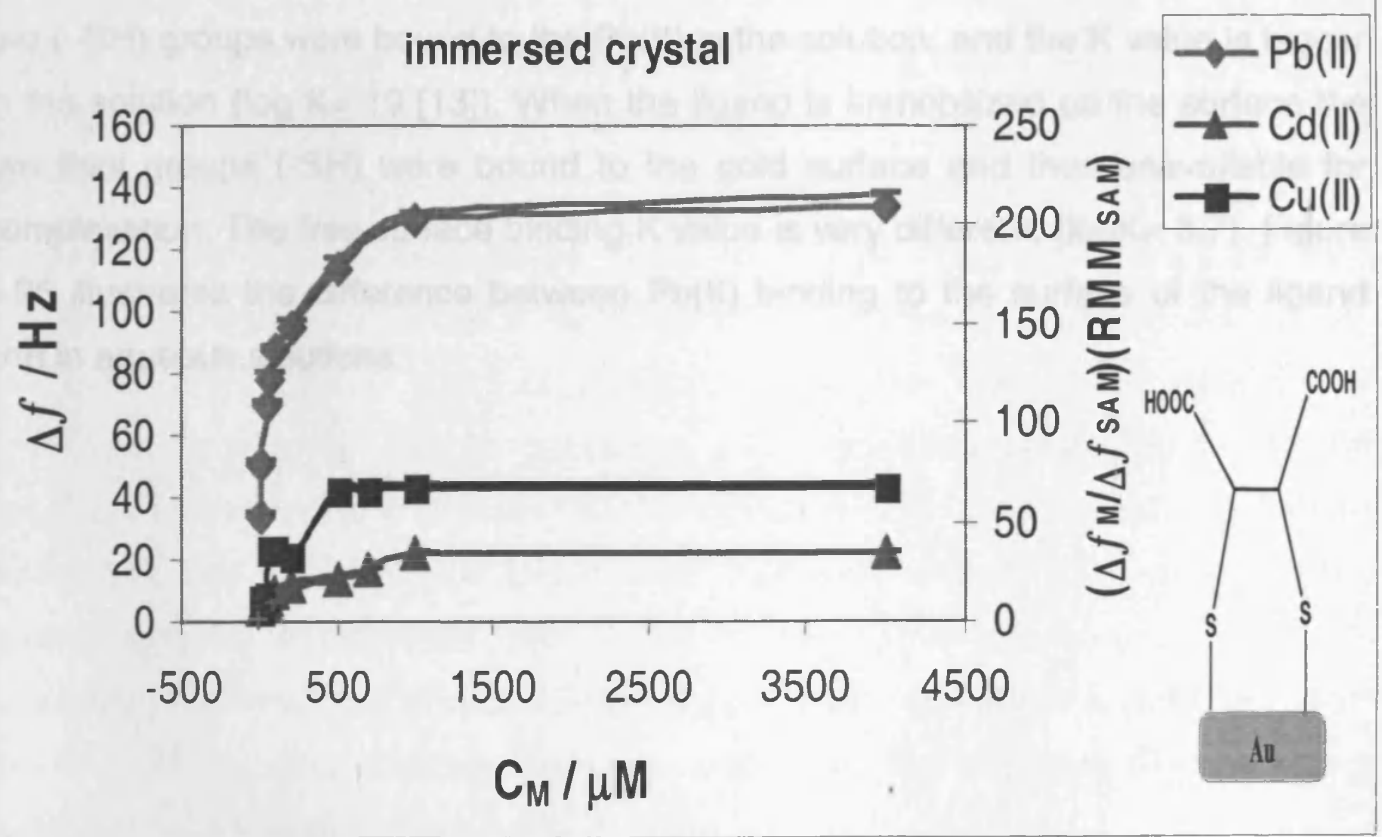

Figure 5.25: Binding isotherms for $\mathrm{Pb}^{2+}, \mathrm{Cu}^{2+}$ and $\mathrm{Cd}^{2+}$ to meso-2,3-dimercaptosuccinic acid (DMSA SAM modified Au electrode) on immersed crystals. The surface coverage of the DMSA was $2.96 \mathrm{nmol} \mathrm{cm}^{-2}$. 
The estimated equilibrium constants are presented in Table 5.13.

\begin{tabular}{|c|c|c|c|c|c|c|}
\hline crystal & In & & & & & \\
\hline dry & $12.9 \pm 1.1$ & $-0.07 \pm 0.02$ & $11.5 \pm 0.3$ & $-0.08 \pm 0.01$ & $11.9 \pm 0.1$ & $-0.08 \pm 0.04$ \\
\hline wet & $13.2 \pm 0.5$ & $-0.07 \pm 0.01$ & $11.4 \pm 0.3$ & $-0.1 \pm 0.01$ & $12.2 \pm 0.1$ & $-0.09 \pm 0.06$ \\
\hline
\end{tabular}

Table 5.13: Equilibrium binding constants and Frumkin interaction parameters for metal ions with meso-2,3-dimercaptosuccinic acid on dry (emersed) and wet (immersed) crystals. Parameters estimated from the linearized form of the Frumkin adsorption isotherm.

The results of the binding constant of the ligand show selectivity to $\mathrm{Pb}(\mathrm{II})$ over $\mathrm{Cd}(\mathrm{II})$ and $\mathrm{Cu}(\mathrm{II})$.

\subsubsection{Compare the logK for $\mathrm{Pb}(\mathrm{II})$-ligand on the surface and in solution}

In making comparison between binding $\mathrm{Pb}$ (II) to 2,3-dimercaptosuccinic acid on the surface and in aqueous solutions, we found that from the literature survey that, the two $(-\mathrm{SH})$ groups were bound to the $\mathrm{Pb}(\mathrm{II})$ in the solution, and the $\mathrm{K}$ value is bigger in the solution $(\log K=19$ [13]). When the ligand is immobilized on the surface the two thiol groups (-SH) were bound to the gold surface and thus unavailable for complexation. The free surface binding $K$ value is very different, $(\log K=5.7)$. Figure 5.26 illustrates the difference between $\mathrm{Pb}$ (II) binding to the surface of the ligand and in aqueous solutions. 


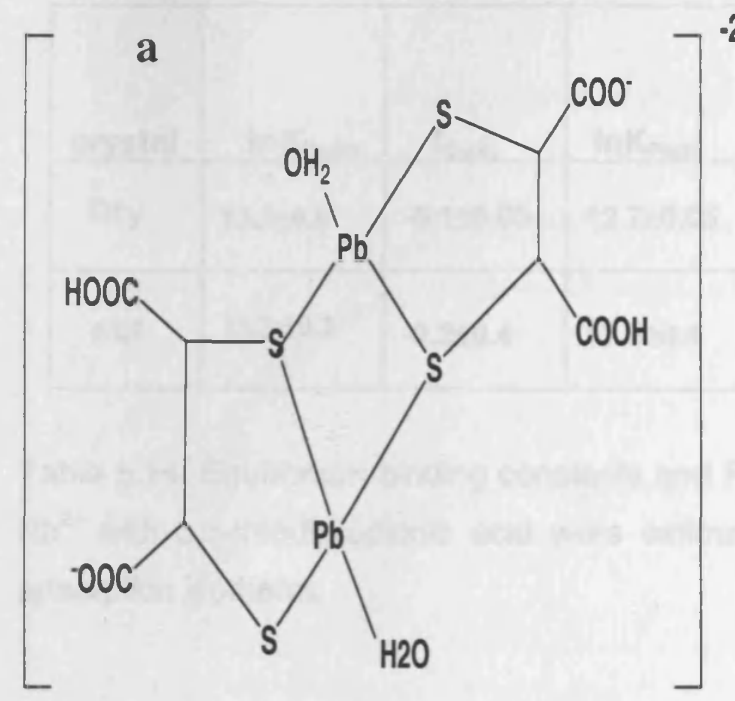

b

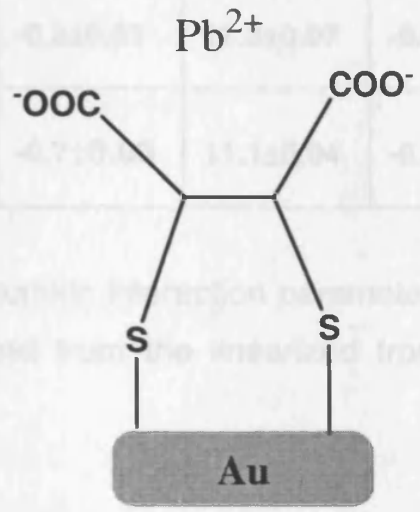

Figure 5.26: The comparison between binding $\mathrm{Pb}(\mathrm{II})$ to the meso-2,3-dimercaptosuccinic acid (a) in aqueous solution and (b) when the ligand is immobilized on the surface of the gold electrode.

\subsection{Binding $\mathrm{Cu}(\mathrm{II}), \mathrm{Cd}(\mathrm{II})$ and $\mathrm{Pb}(\mathrm{II})$ to 3,3-thiodipropionic acid}

The binding of copper, cadmium and lead ions to 3,3-thiodisuccinic acid was studied using the same methodology used for binding these metal ions to other ligands. The experimental adsorption isotherms were determined for the same coverage of the monolayer at $\mathrm{pH}=6.3$ for cadmium, $\mathrm{pH}=4.8$ for lead and $\mathrm{pH}=5.4$ for copper in the concentration range $10 \mu \mathrm{M}$ to $10 \mathrm{mM}$. The results of adsorption isotherms of three metals to the same ligand on emersed and immersed crystals are given in Figures 5.27 and 5.28, these isotherms show that the copper and lead adsorption isotherms steeply increased with increased concentration of the solution. The results also indicate that this ligand has low selectivity amongst these metal ions. The Frumkin and Temkin linear regression models (see section 5.3.1) fit very well to experimental data based on the correlation factor $R^{2}$ for both emersed and immersed crystals. From these linear regression models one can estimate the binding constant for each metal ion and compare these binding constants for these metal ions listed in Table 5.14. 


\begin{tabular}{|c|c|c|c|c|c|c|}
\hline crystal & $\ln K_{\text {Cu(II) }}$ & $f_{\text {Cu(II) }}$ & $\ln K_{\mathrm{Pb}(11)}$ & $\mathbf{f}_{\mathrm{Pb}(11)}$ & $\ln K_{\mathrm{Cd}(11)}$ & $f_{C d(11)}$ \\
\hline Dry & $13.3 \pm 0.8$ & $-0.1 \pm 0.03$ & $12.7 \pm 0.05$ & $-0.3 \pm 0.01$ & $11.3 \pm 0.07$ & $-0.08 \pm 0.04$ \\
\hline wet & $13.3 \pm 0.2$ & $-0.2 \pm 0.4$ & $12.9 \pm 0.4$ & $-0.7 \pm 0.06$ & $11.1 \pm 0.04$ & $-0.06 \pm 0.03$ \\
\hline
\end{tabular}

Table 5.14: Equilibrium binding constants and Frumkin interaction parameters for $\mathrm{Cu}^{2+}$ and $\mathrm{Pb}^{2+}$ with 3,3-thiodipropionic acid were estimated from the linearized from the Frumkin adsorption isotherm.

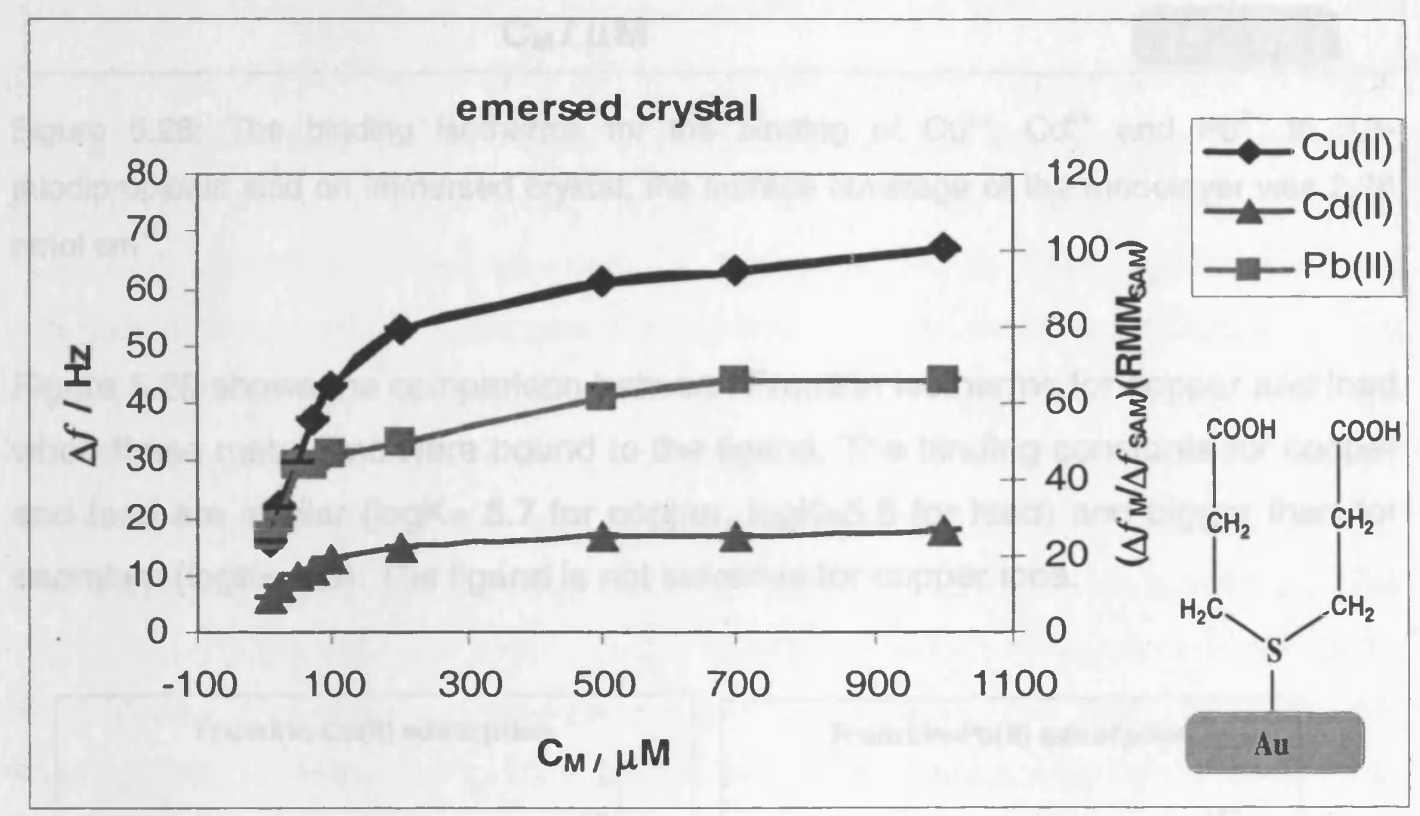

Figure 5.27: The binding isotherms for the binding $\mathrm{Cu}^{2+}, \mathrm{Cd}^{2+}$ and $\mathrm{Pb}^{2+}$ to 3,3thiodipropionic acid on emersed crystal, the surface coverage of the monolayer was 3.16 $\mathrm{nmol} \mathrm{cm}{ }^{-2}$. 


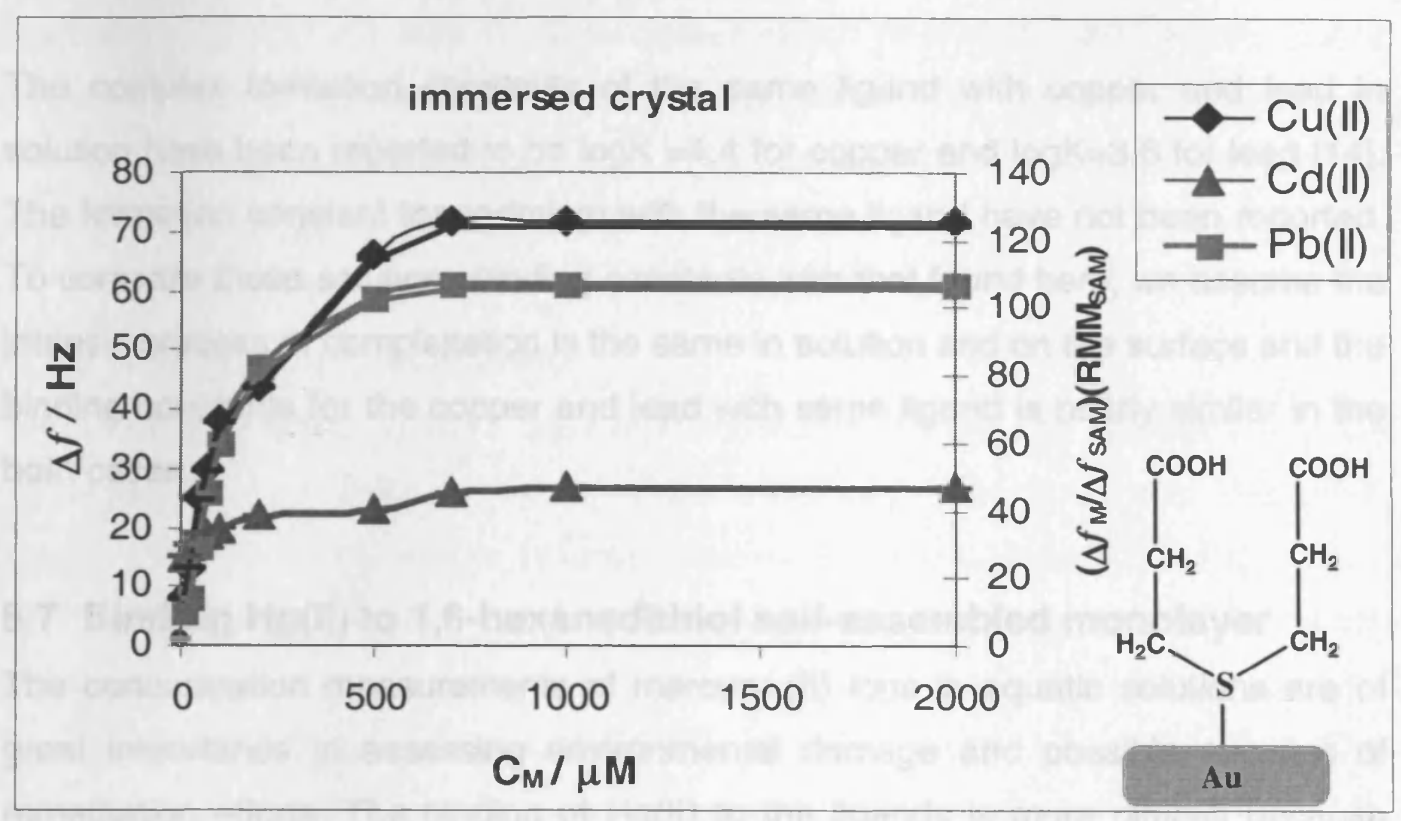

Figure 5.28: The binding isotherms for the binding of $\mathrm{Cu}^{2+}, \mathrm{Cd}^{2+}$ and $\mathrm{Pb}^{2+}$ to 3,3thiodipropionic acid on immersed crystal, the surface coverage of the monolayer was 2.28 $\mathrm{nmol} \mathrm{cm}^{-2}$.

Figure 5.29 shows the comparison between Frumkin isotherms for copper and lead when these metal ions were bound to the ligand. The binding constants for copper and lead are similar (logK= 5.7 for copper, logK=5.5 for lead) and bigger than for cadmium (logK= 4.8). The ligand is not selective for copper ions.
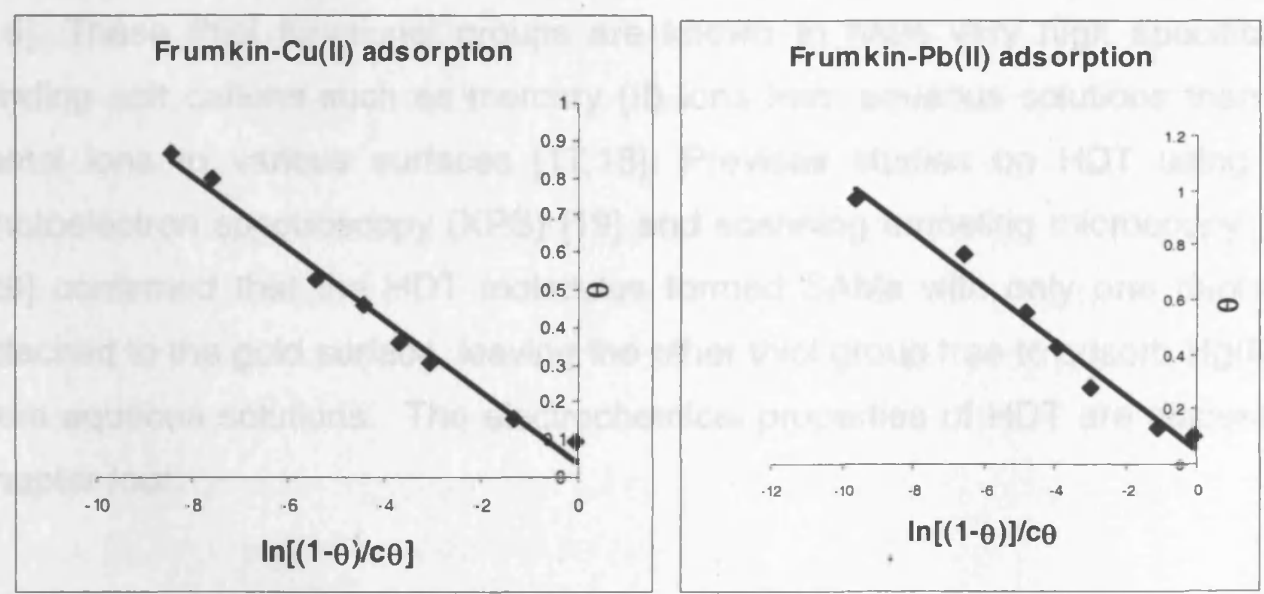

Figure 5.29: Comparison between Frumkin isotherms for the uptake of $\mathrm{Cu}^{2+}$ and $\mathrm{Pb}^{2+}$ with 3,3-thiodipropionic acid on immersed crystal; the surface coverage of the ligand was 3.16 $\mathrm{nmol} \mathrm{cm}{ }^{-2}$. 
The complex formation constants of the same ligand with copper and lead in solution have been reported to be logK $=4.4$ for copper and $\log K=3.6$ for lead [14]. The formation constant for cadmium with the same ligand have not been reported. To compare these solutions binding constants with that found here, we assume the intrinsic process of complexation is the same in solution and on the surface and the binding constants for the copper and lead with same ligand is nearly similar in the both cases.

\subsection{Binding $\mathrm{Hg}(\mathrm{II})$ to 1,6-hexanedithiol self-assembled monolayer}

The concentration measurements of mercury (II) ions in aquatic solutions are of great importance in assessing environmental damage and possible success of remediation efforts. The binding of $\mathrm{Hg}$ (II) to the ligands is more difficult because $\mathrm{Hg}$ (II) can penetrate the monolayer to react with the gold and produce a mercurygold amalgam. The mole ratio was not reproducible. The surface plasmon resonance (SPR) technique was used by Zare et al [15] to detect $\mathrm{Hg}(\mathrm{II})$ ions using 1,6-hexanedithiol (HDT). Changes in the SPR signal allowed quantification of the concentration of $\mathrm{Hg}(\mathrm{II})$ in solution from $1.0 \mathrm{nM}$ to $1.0 \mathrm{mM}$, this method also allowed selective detection of $\mathrm{Hg}(\mathrm{II})$ ions in the presence of interfering ions. In this section we will use the same ligand to adsorb $\mathrm{Hg}$ (II) using the $\mathrm{QCM}$ as a sensor to monitor the associated changes in mass at a HDT SAM modified Au electrode. The mercapto group (-SH) is known to react with heavy metal ions to form mercaptide [16]. These thiol functional groups are known to have very high specificity for binding soft cations such as mercury (II) ions from aqueous solutions than other metal ions to various surfaces [17,18]. Previous studies on HDT using $X$-ray photoelectron spectroscopy (XPS) [19] and scanning tunneling microscopy (STM) [20] confirmed that the HDT molecules formed SAMs with only one thiol group attached to the gold surface, leaving the other thiol group free to adsorb $\mathrm{Hg}$ (II) ions from aqueous solutions. The electrochemical properties of HDT are discussed in chapter four.

\subsubsection{Experimental}

The experimental procedures were described in section (5.2.1). A stock solution of $1 \mathrm{mM} \mathrm{Hg}\left(\mathrm{NO}_{3}\right)_{2}$ was used to prepare all concentrations of $\mathrm{Hg}(\mathrm{II})$ ions. $\mathrm{Hg}$ (II) at concentrations from $1.0 \mathrm{nM}$ to $1.0 \mathrm{mM}$ at $\mathrm{pH}=4.5$ was bound to the surface of $1,6-$ hexanedithiol (HDT) modified Au. The preconcentration time for low concentrations 
was about 20-25 $\mathrm{min}$ and at high concentration was about 10-15 min. Every frequency shift measurement for every concentration was repeated four times on the same surface coverage of the HDT on emersed and immersed crystal, to examine reproducibility.

\subsubsection{Results and discussion}

The binding of $\mathrm{Hg}(\mathrm{II})$ to HDT SAM modified Au electrode was studied using the same methodology used for binding these metal ions to other ligands. The same ligand was also examined for binding other metal ions such as $\mathrm{Pb}^{2+}, \mathrm{Cd}^{2+}, \mathrm{Cu}^{2+}$, $\mathrm{Zn}^{2+}, \mathrm{Ni}^{2+}$ and $\mathrm{Ag}^{+}$. The experimental adsorption isotherms were determined for the same coverage of the monolayer with $\mathrm{pH}=5.5$ for $\mathrm{Hg}(\mathrm{II}), \mathrm{pH}=4.8$ for lead, $\mathrm{pH}=5.4$ for copper, $\mathrm{pH}=6.5$ for cadmium, $\mathrm{pH}=5.0$ for zinc, $\mathrm{pH}=6.0$ for silver and $\mathrm{pH}=5.5$ for nickel. The range of concentrations for all metal ions was from $1 \mathrm{nM}$ to $1 \mathrm{mM}$. The results of adsorption isotherms of $\mathrm{Hg}^{2+}, \mathrm{Pb}^{2+}, \mathrm{Cd}^{2+}, \mathrm{Cu}^{2+}, \mathrm{Zn}^{+2}, \mathrm{Ni}^{2+}$ and $\mathrm{Ag}^{+}$to the same ligand on the dry (emersed) and wet (immersed) crystals are shown in Figures (5.30 and 5.31). The frequency shifts were proportional to $\mathrm{Hg}(\mathrm{II})$ concentration up to $10 \mu \mathrm{M}$, and steady from $50 \mu \mathrm{M}$. The saturation surface of $\mathrm{Hg}$ (II) ions at $100 \mu \mathrm{M}$ for dry (emersed) and wet (immersed) crystals was $2.86 \pm 0.05$ and $3.33 \pm 0.12 \mathrm{nmol} \mathrm{cm}{ }^{-2}(n=4)$, respectively. The surface coverage of the HDT after $18 \mathrm{~h}$ modification time estimated on emersed and immersed crystal was 2.48 and $2.60 \mathrm{nmol} \mathrm{cm}^{-2}$, respectively. The mole ratio between the saturation surface coverage of $\mathrm{Hg}(\mathrm{II})$ ions and $\mathrm{HDT}$ was $1.15 \pm 0.04$ and $1.28 \pm 0.06$, respectively. The adsorption isotherms show that $\mathrm{Hg}(\mathrm{II})$ adsorption isotherms steeply increased with increased concentration of the solution until the plateau state at $1 \mathrm{mM}$ of $\mathrm{Hg}$ (II). For other metal ions, the adsorption isotherms are less steep. The experimental adsorption data for binding different concentrations of lead ions to the monolayer were fitted to different isotherms (see section 5.31). We found that the Temkin fits very well to experimental data for all metal ions, based on a high correlation factor $\left(R^{2}\right)$ for both dry and wet crystals. From Temkin linear regression models one can estimate the binding constant for each metal ion and compared these binding constants. The results are listed in Table 5.15. 


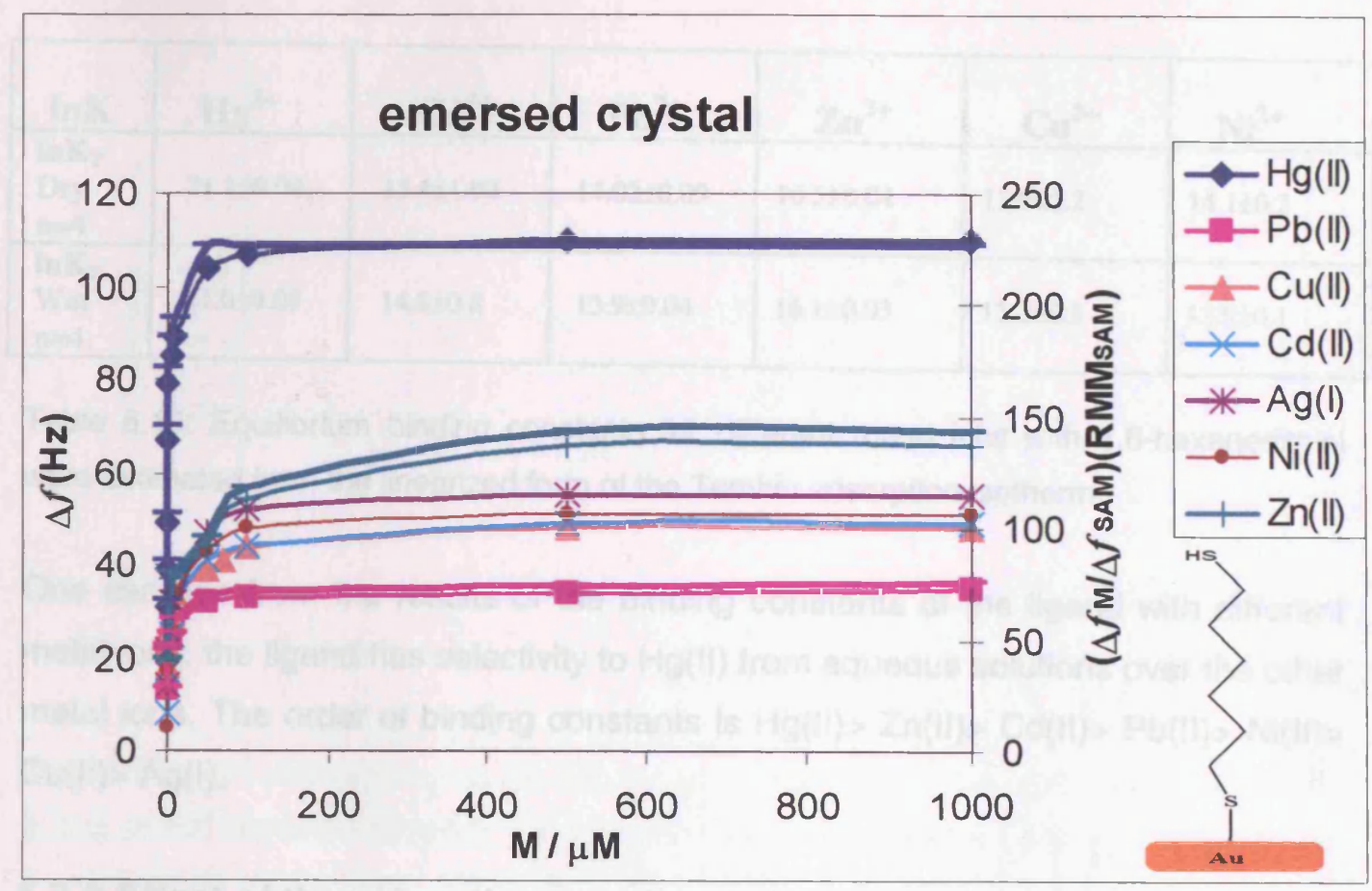

Figure 5.30: The binding isotherms for the binding $\mathrm{Hg}^{2+}, \mathrm{Cd}^{2+}, \mathrm{Pb}^{2+}, \mathrm{Cu}^{2+}, \mathrm{Ni}^{2+}, \mathrm{Zn}^{2+}$ and $\mathrm{Ag}^{+}$ to 1,6-hexanedithiol SAM on emersed crystal. The surface coverage of the monolayer was $3.14 \mathrm{nmol} \mathrm{cm}^{-2}$.

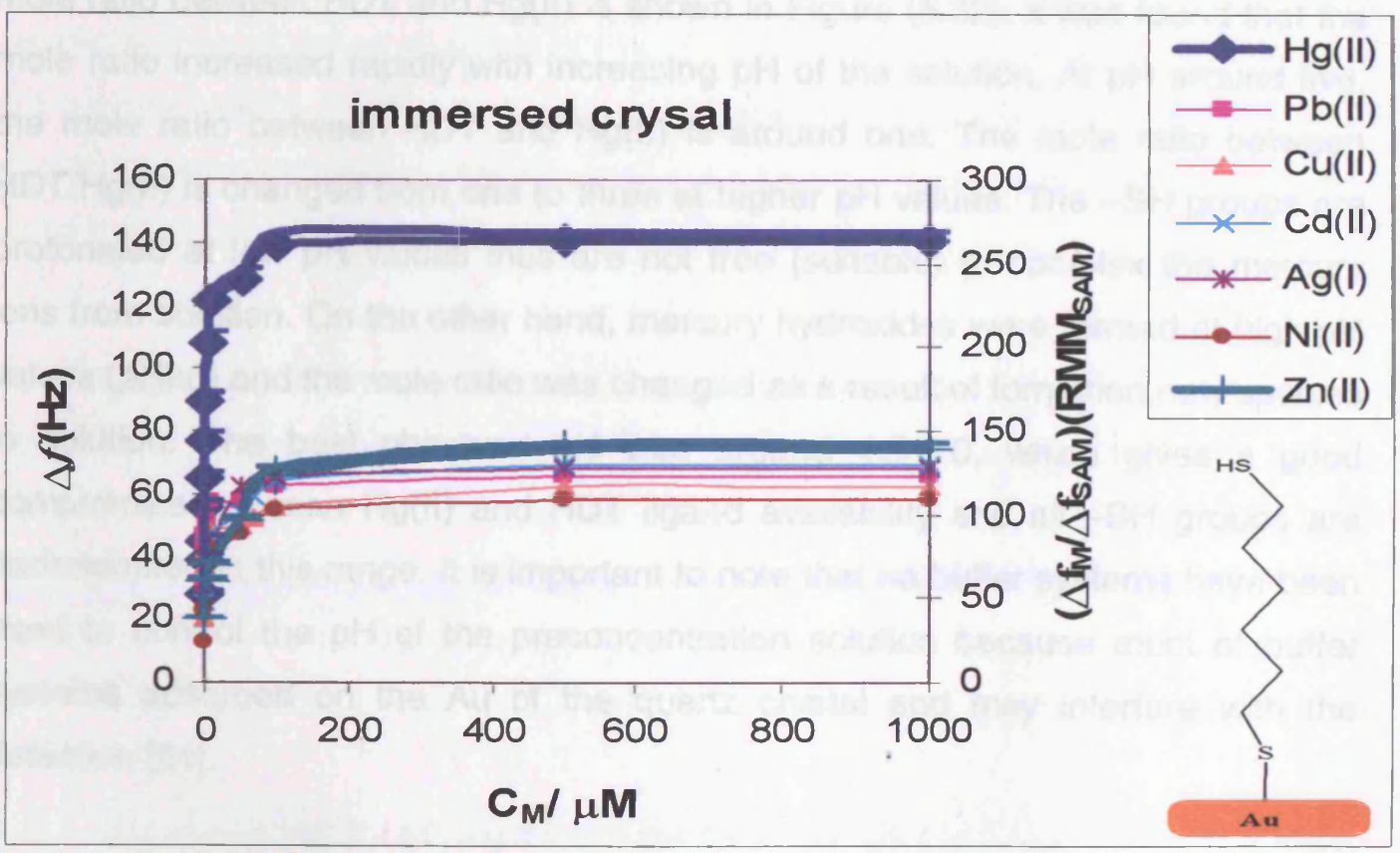

Figure 5.31: The binding isotherms for the binding $\mathrm{Hg}^{2+}, \mathrm{Cd}^{2+}, \mathrm{Pb}^{2+}, \mathrm{Cu}^{2+}, \mathrm{Ni}^{2+}, \mathrm{Zn}^{2+}$ and $\mathrm{Ag}^{+}$ to1,6-hexanedithol SAM on immersed crystal. The surface coverage of the monolayer was $3.33 \mathrm{nmol} \mathrm{cm}^{-2}$. 


\begin{tabular}{|c|c|c|c|c|c|c|}
\hline $\ln \mathbf{K}$ & $\mathbf{H g}^{2+}$ & $\mathbf{C d}^{2+}$ & $\mathbf{P b}^{2+}$ & $\mathrm{Zn}^{2+}$ & $\mathrm{Cu}^{2+}$ & $\mathrm{Ni}^{2+}$ \\
\hline $\begin{array}{l}\ln \mathbf{K}_{\mathbf{T}} \\
\text { Dry } \\
\mathrm{n}=4\end{array}$ & $21.1 \pm 0.04$ & $15.4 \pm 1.00$ & $14.02 \pm 0.09$ & $16.5 \pm 0.04$ & $13.0 \pm 0.2$ & $14.1 \pm 0.2$ \\
\hline $\begin{array}{l}\text { InK } \\
\text { Wet } \\
\mathrm{n}=4\end{array}$ & $21.0 \pm 0.09$ & $14.8 \pm 0.8$ & $13.9 \pm 0.04$ & $16.1 \pm 0.03$ & $13.2 \pm 0.3$ & $13.9 \pm 0.1$ \\
\hline
\end{tabular}

Table 5.15: Equilibrium binding constants for different metal ions with 1,6-hexanedithiol were estimated from the linearized form of the Temkin adsorption isotherm.

One can see from the results of the binding constants of the ligand with different metal ions, the ligand has selectivity to $\mathrm{Hg}$ (II) from aqueous solutions over the other metal ions. The order of binding constants is $\mathrm{Hg}(\mathrm{II})>\mathrm{Zn}(\mathrm{II})>\mathrm{Cd}(\mathrm{II})>\mathrm{Pb}(\mathrm{II})>\mathrm{Ni}(\mathrm{II})>$ $\mathrm{Cu}(\mathrm{II})>\mathrm{Ag}(\mathrm{I})$.

\subsubsection{Effect of the pH on the complexation between $\mathrm{Hg}(\mathrm{II})$ and HDT}

The effect of the $\mathrm{pH}$ on the complexation between $\mathrm{Hg}(\mathrm{II})$ ions and HDT was investigated using the procedures in section 5.3. The $\mathrm{pH}$ of the solutions was adjusted with $0.1 \mathrm{M} \mathrm{KOH}$ or $0.1 \mathrm{M} \mathrm{HNO}_{3}$. The relationship between the $\mathrm{pH}$ and the mole ratio between $\mathrm{HDT}$ and $\mathrm{Hg}(\mathrm{II})$ is shown in Figure (5.32). It was found that the mole ratio increased rapidly with increasing $\mathrm{pH}$ of the solution. At $\mathrm{pH}$ around five, the mole ratio between HDT and $\mathrm{Hg}(\mathrm{II})$ is around one. The mole ratio between $\mathrm{HDT} / \mathrm{Hg}(\mathrm{II})$ is changed from one to three at higher $\mathrm{pH}$ values. The $-\mathrm{SH}$ groups are protonated at low $\mathrm{pH}$ values thus are not free (suitable) to complex the mercury ions from solution. On the other hand, mercury hydroxides were formed at high $\mathrm{pH}$ values $(\mathrm{pH} \geq 6)$ and the mole ratio was changed as a result of formation new species in solution. The best observed $\mathrm{pH}$ was around 4.5-5.0, which gives a good compromise between $\mathrm{Hg}$ (II) and HDT ligand availability and all - $\mathrm{SH}$ groups are deprotonated in this range. It is important to note that no buffer systems have been used to control the $\mathrm{pH}$ of the preconcentration solution because most of buffer systems adsorbed on the Au of the quartz crystal and may interfere with the detection [21]. 


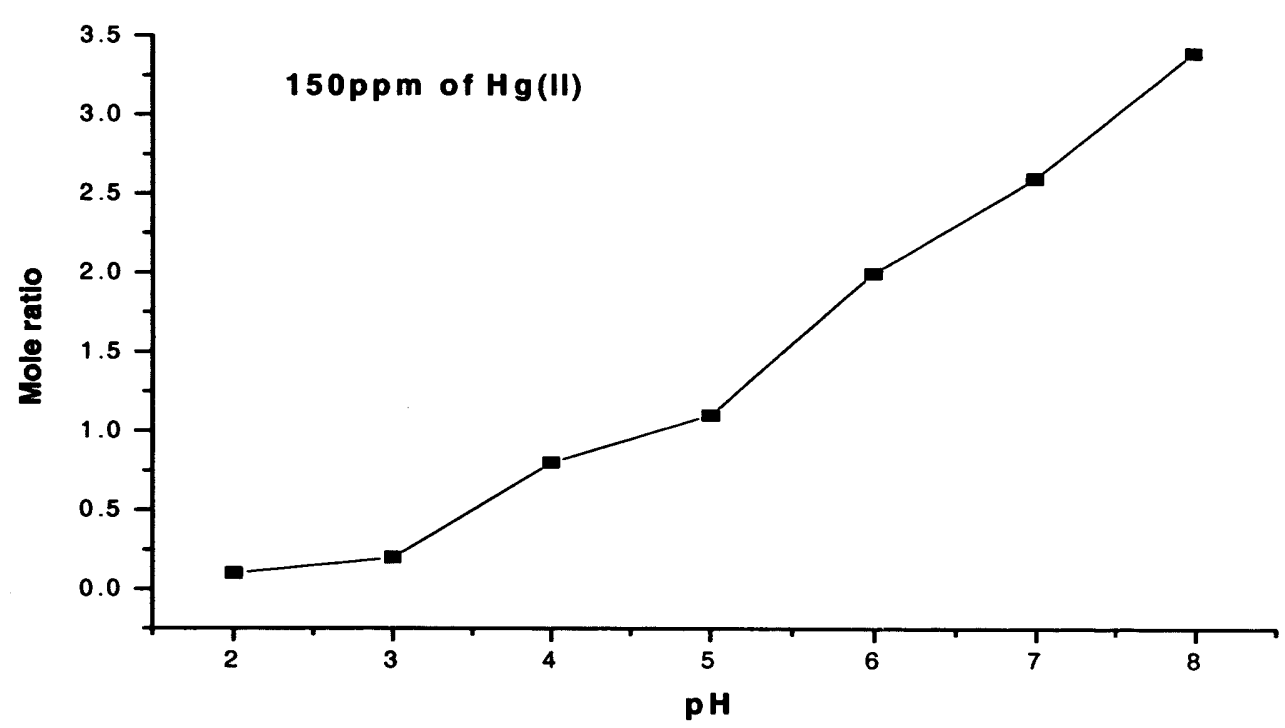

Figure 5.32: $\mathrm{pH}$ dependence on the adsorption of $\mathrm{Hg}(\mathrm{II})$ ions onto HDT SAM modified Au quartz crystal (immersed crystal). The concentration of the $\mathrm{Hg}$ (II) was $150 \mathrm{ppm}$.

\subsection{Analysing Mixtures of Metal lons}

The specific binding of metal ions to the self-assembled monolayer receptor site on the gold electrode plays an important role in the chemical speciation of metal ions. In the previous sections (from 5.3 to 5.7), the QCM frequency measurements were carried out for solutions of single $\mathrm{Cd}^{2+}, \mathrm{Cu}^{2+}, \mathrm{Pb}^{2+}$ and $\mathrm{Hg}^{2+}$ ions. In this section we will expose ligands to binary mixtures of metal ions, to explore interference effects. The frequency measurements for analysing binary mixtures of metal ions were done for both dry (emersed) and wet (immersed) crystals.

\subsection{Using 3-thiodisuccinic acid to analyse mixtures of metal ions}

The results for binding $\mathrm{Cd}^{2+}, \mathrm{Pb}^{2+}$ and $\mathrm{Cu}^{2+}$ to this ligand show that it is selective to $\mathrm{Cd}^{2+}$. The explorative of this effect for binary mixtures of metal ions will be in two stages: in first stage the concentration of cadmium ions was fixed, the interfering ion concentration was varied and in second stage the concentration of cadmium ions was varied (from $1 \mathrm{mM}$ to $10 \mathrm{mM}$ ) at constant interfering ion concentration.

\subsubsection{Measurements at fixed concentration of $\mathrm{Cd}^{2+}$, in the presence $\mathrm{Cu}^{2+}$ or $\mathrm{Pb}^{2+}$}

In this study the concentration of cadmium ions in binary mixtures of $\left(\mathrm{Cd}^{2+}, \mathrm{Pb}^{2+}\right)$ and $\left(\mathrm{Cd}^{2+}, \mathrm{Cu}^{2+}\right)$ was fixed and the concentration of copper or lead was varied as listed in Table 5.16. 


\begin{tabular}{|c|c|c|c|}
\hline Binary mixtures & $\begin{array}{l}\text { Initial } \\
\text { concentration of } \\
\text { Copper ions }\end{array}$ & $\begin{array}{l}\text { Initial } \\
\text { concentration of } \\
\text { Cadmium ions }\end{array}$ & $\begin{array}{l}\text { Final concentration of } \\
\mathrm{Cu}(\mathrm{II}) \text { and } \mathrm{Cd}(\mathrm{II}) \text { in } \\
\text { mixtures }\end{array}$ \\
\hline $1-\left(\mathrm{Cu}^{2+}, \mathrm{Cd}^{2+}\right)^{*}$ & $9 \mathrm{ml} \mathrm{Cu}(I I) 10 \mathrm{mM}$ & $1 \mathrm{ml} \mathrm{Cd}(I I) 1 \mathrm{mM}$ & $9 \mathrm{mMCu}(\mathrm{II}), 0.1 \mathrm{mMCd}(\mathrm{II})$ \\
\hline 2- $\left(\mathrm{Cu}^{2+}, \mathrm{Cd}^{2+}\right)^{*}$ & $1 \mathrm{ml} \mathrm{Cu}(I I) 10 \mathrm{mM}$ & $\begin{array}{l}1 \mathrm{ml} \mathrm{Cd}(\mathrm{II}) 1 \mathrm{mM}+ \\
8 \mathrm{ml}(\mathrm{D} . \mathrm{I} . \mathrm{W})^{\star \star}\end{array}$ & $1 \mathrm{mM} \mathrm{Cu}(I I), 0.1 \mathrm{mMCd}(I I)$ \\
\hline 3- $\left(\mathrm{Cu}^{2+}, \mathrm{Cd}^{2+}\right)^{*}$ & $9 \mathrm{ml} \mathrm{Cu}(\mathrm{II}) 0.1 \mathrm{mM}$ & $1 \mathrm{ml} \mathrm{Cd}(I I) 1 \mathrm{mM}$ & $0.1 \mathrm{mMCu}(\mathrm{II}), 0.1 \mathrm{mMCd}(\mathrm{II})$ \\
\hline
\end{tabular}

Table 5.16: Preparation of binary mixtures of metal ions using for study the effect of copper or lead in determination of cadmium using 3-thiodisuccinic acid monolayer. ${ }^{*}$ the mixture $\left(\mathrm{Cu}^{2+}, \mathrm{Cd}^{2+}\right)$ was changed with $\left(\mathrm{Pb}^{2+}, \mathrm{Cd}^{2+}\right)$ and ${ }^{* *}$ ultra pure deionised water was used in preparation of both binary mixtures.

\subsubsection{Methodology}

The following were measured:

1) Frequency shift for dry (emersed) crystal with SAM ( $\left.\Delta f_{\text {dry-SAM }}\right)$.

2) Frequency shift for wet (immersed) crystal with $S A M+2 \mathrm{ml}$ of deionised water $\left(\Delta f_{\text {wet-SAM }}\right)$.

3) Frequency shift for wet (immersed) crystal with SAM $+2 \mathrm{ml}$ deionised water + $9 \mathrm{ml} \mathrm{Cu}(\mathrm{II}) 10 \mathrm{mM}+1 \mathrm{ml} \mathrm{Cd}(\mathrm{II}) 1 \mathrm{mM}$.

4) The crystal was dried carefully and the frequency shift for the dry (emersed) crystal was measured.

5) The following the calculated:

a) $\Delta f_{\text {dry-Ca(II) }}=\Delta f_{\text {dry-SAM-Ca(II) }}-\Delta f_{\text {dry-SAM }}$

b) $\Delta f_{\text {wet-Ca(II) }}=\Delta f_{\text {wet-SAM-Ca(II) }}-\Delta f_{\text {wet-SAM }}$

6) The concentration of copper in binary mixture ( $\mathrm{Cu}(\mathrm{II}), \mathrm{Cd}(\mathrm{II}))$ was changed from $9 \mathrm{mM}$ to $1 \mathrm{mM}$ and $0.1 \mathrm{mM}$ using procedures (1) to (5). 
7) The same procedures were used for binary mixture of ( $\mathrm{Pb}(\mathrm{II}), \mathrm{Cd}(\mathrm{II}))$.

In order to assess the extent of selective uptake of cadmium ions from solution three replicates, nominally identical but using different films were used. In each case three measurements were also made for each film. Procedures (1) to (6) were repeated to examine the reproducibility of the SAM towards cadmium ions and the modified crystals was washed with EDTA three times between the steps to remove adsorbed cadmium ions on the surface of the ligand as judged by return to initial frequency; typically three washes were adequate.

\subsubsection{Results and Discussion}

The frequency shifts were taken after 10-20 min to permit a successful adsorption of cadmium ions without shaking or stirring the solution because for the strong adsorption of cadmium ions on the surface of the ligand. The results of stage one are presented in Table 5.17. For the two results of binary mixtures, the frequency shifts for both emersed and immersed crystals indicated that copper and lead have no effects on the detection of cadmium ions. Figure 5.33 compares the ability of three different surface coverages of the ligand to bind three different mixtures. 


\begin{tabular}{|c|c|c|c|c|}
\hline Binary mixtures (1) & $\begin{array}{l}\Delta f \text { dry- } \\
\text { SAM- } \\
\text { Cd(II) }\end{array}$ & $\begin{array}{l}\Delta f \text { wet- } \\
\text { SAM- } \\
\text { Cd(II) }\end{array}$ & $\begin{array}{l}\Delta f \text { dry- } \\
\text { SAM }\end{array}$ & $\begin{array}{l}\Delta f \text { wet- } \\
\text { SAM }\end{array}$ \\
\hline $9 \mathrm{mMCu}(\mathrm{II})+0.1 \mathrm{mM} \mathrm{Cd}(\mathrm{II})$ & $53 \pm 2.5$ & $67 \pm 4.0$ & 138 & 161 \\
\hline 9mMCu(II)+0.1 mM Cd(II) & $54 \pm 2.5$ & $72 \pm 3.6$ & 154 & 180 \\
\hline $9 \mathrm{mMCu}(\mathrm{II})+0.1 \mathrm{mM} \mathrm{Cd}(\mathrm{II})$ & $47 \pm 2.5$ & $66 \pm 2.5$ & 126 & 161 \\
\hline $1 \mathrm{mMCu}(\mathrm{II})+0.1 \mathrm{mM} \mathrm{Cd}(\mathrm{II})$ & $42 \pm 1.5$ & $59 \pm 2.0$ & 138 & 161 \\
\hline $1 \mathrm{mMCu}(\mathrm{II})+0.1 \mathrm{mM} \mathrm{Cd}(\mathrm{II})$ & $45 \pm 4.0$ & $70 \pm 1.5$ & 154 & 180 \\
\hline $1 \mathrm{mMCu}(\mathrm{II})+0.1 \mathrm{mM} \mathrm{Cd}(\mathrm{II})$ & $40 \pm 2.0$ & $64 \pm 3.0$ & 126 & 161 \\
\hline $0.1 \mathrm{mMCu}(\mathrm{II})+0.1 \mathrm{mM} \mathrm{Cd}(\mathrm{II})$ & $43 \pm 1.0$ & $58 \pm 0.6$ & 138 & 161 \\
\hline $0.1 \mathrm{mMCu}(\mathrm{II})+0.1 \mathrm{mM} \mathrm{Cd}(\mathrm{II})$ & $43 \pm 2.5$ & $61 \pm 2.5$ & 154 & 180 \\
\hline $0.1 \mathrm{mMCu}(\mathrm{II})+0.1 \mathrm{mM} \mathrm{Cd}(\mathrm{II})$ & $41 \pm 2.5$ & $61 \pm 4.3$ & 126 & 161 \\
\hline Binary mixtures (2) & $\begin{array}{l}\Delta f \text { dry- } \\
\text { SAM- } \\
\text { Cd(II) }\end{array}$ & $\begin{array}{l}\Delta f \text { wet- } \\
\text { SAM- } \\
\text { Cd(II) }\end{array}$ & $\begin{array}{l}\Delta f \text { dry- } \\
\text { SAM }\end{array}$ & $\begin{array}{l}\Delta f \text { wet- } \\
\text { SAM }\end{array}$ \\
\hline 9mMPb(II)+0.1 mM Cd(II) & $42 \pm 1.5$ & $58 \pm 1.0$ & 138 & 161 \\
\hline 9mMPb(II)+0.1mM Cd(II) & $43 \pm 3.6$ & $59 \pm 4.0$ & 154 & 180 \\
\hline 9mMPb(II)+0.1 mM Cd(II) & $47 \pm 4.0$ & $67 \pm 1.10$ & 126 & 161 \\
\hline $1 \mathrm{mMPb}(\mathrm{II})+0.1 \mathrm{mM} \mathrm{Cd}(\mathrm{II})$ & $39 \pm 1.5$ & $57 \pm 4.0$ & 138 & 161 \\
\hline $1 \mathrm{mMPb}(\mathrm{II})+0.1 \mathrm{mM} \mathrm{Cd}(\mathrm{II})$ & $47 \pm 2.6$ & $67 \pm 3.0$ & 154 & 180 \\
\hline $1 \mathrm{mMPb}(\mathrm{II})+0.1 \mathrm{mM} \mathrm{Cd}(\mathrm{II})$ & $43 \pm 4.0$ & $67 \pm 4.0$ & 126 & 161 \\
\hline $0.1 \mathrm{mMPb}(\mathrm{II})+0.1 \mathrm{mM} \mathrm{Cd}(\mathrm{II})$ & $43 \pm 2.5$ & $65 \pm 3.0$ & 138 & 161 \\
\hline $0.1 \mathrm{mMPb}(\mathrm{II})+0.1 \mathrm{mM} \mathrm{Cd}(\mathrm{II})$ & $43 \pm 3.0$ & $62 \pm 2.0$ & 154 & 180 \\
\hline 0.1mMPb(II)+0.1mM Cd(II) & $45 \pm 4.0$ & $66 \pm 2.0$ & 126 & 161 \\
\hline
\end{tabular}

Table 5.17: the frequency shift for the binary mixtures of cadmium, copper and cadmium, lead on dry (emersed) and wet (immersed) crystal with different concentrations of copper and lead. The concentration of cadmium is fixed at $0.1 \mathrm{mM}$. The frequency shifts are presented as a mean \pm standard deviation for three independent experiments on each individual film (state the ligand). 

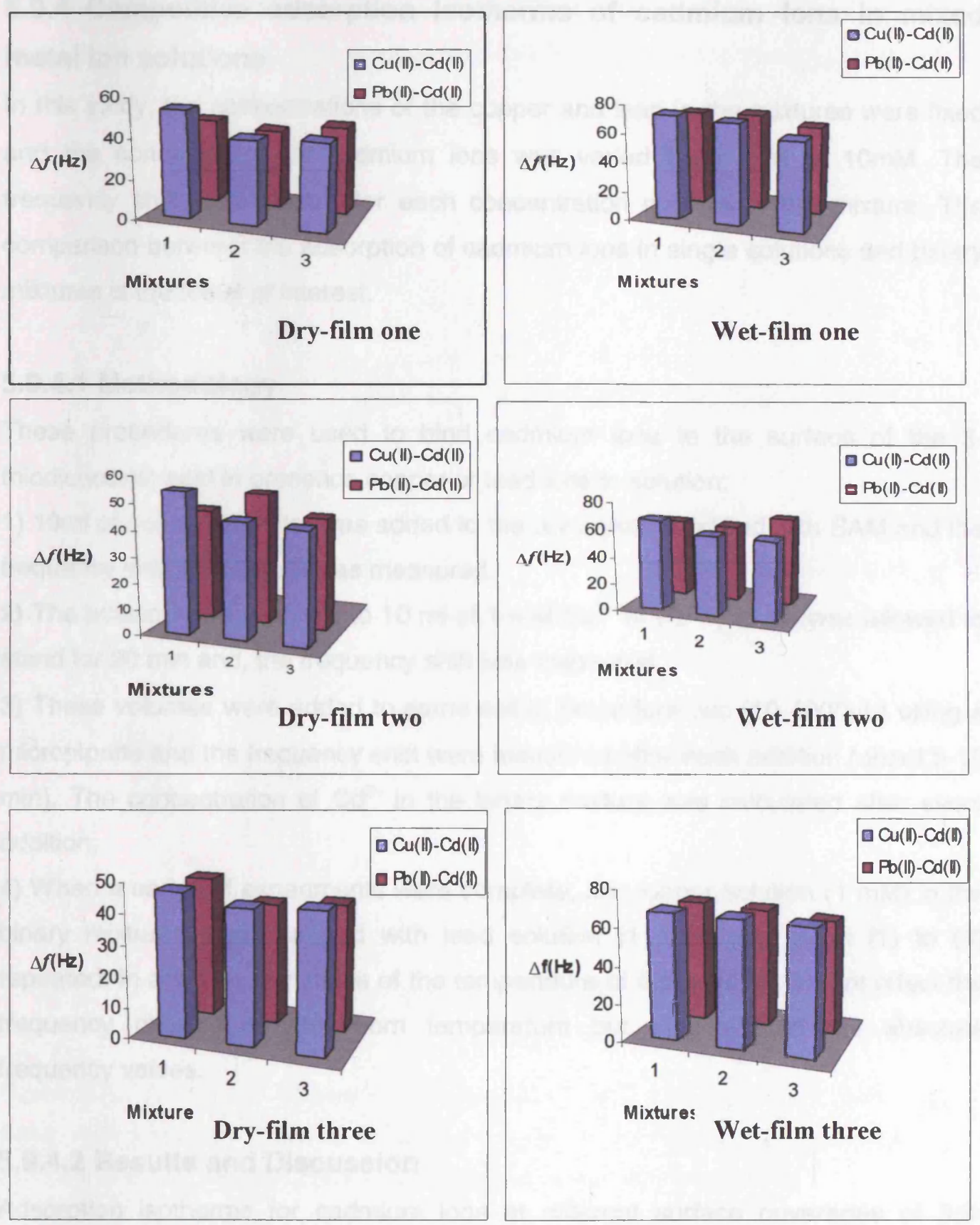

Figure 5.33: Comparison between electrodes with three different surface coverages of 3,3thiodisuccinic acid to bind cadmium in presence of three different concentrations of $\mathrm{Cu}^{2+}$ and $\mathrm{Pb}^{2+}$ in the binary mixtures on dry and wet crystals. The number of mixtures $(1,2$, and 3) refers to the table (5.16). The crystals were incubated first with mixture $\left(\mathrm{Cu}^{2+}, \mathrm{Cd}^{2+}\right)$ and then with $\left(\mathrm{Pb}^{2+}, \mathrm{Cd}^{2+}\right)$. 


\subsubsection{Competitive adsorption isotherms of cadmium ions in mixed metal ion solutions}

In this study, the concentrations of the copper and lead in the mixtures were fixed and the concentration of cadmium ions was varied from $1 \mu \mathrm{M}$ to $10 \mathrm{mM}$. The frequency shift was taken after each concentration change in the mixture. The comparison between the adsorption of cadmium ions in single solutions and binary mixtures is the focus of interest.

\subsubsection{Methodology}

These procedures were used to bind cadmium ions to the surface of the 3thiodisuccinic acid in presence copper or lead ions in solution:

1) $10 \mathrm{ml}$ of deionised water was added to the dry crystal modified with SAM and the frequency shift equilibrium was measured.

2) The solution was changed to $10 \mathrm{ml}$ of $1 \mathrm{mM} \mathrm{Cu}^{2+}$ or $\mathrm{Pb}^{2+}$, which was allowed to stand for $20 \mathrm{~min}$ and, the frequency shift was measured.

3) These volumes were added to same cell in procedure two $(10-4000 \mu \mathrm{L})$ using a micropipette and the frequency shift were measured after each addition (about 5-10 min). The concentration of $\mathrm{Cd}^{2+}$ in the binary mixture was calculated after every addition.

4) When a series of experiments were complete, the copper solution ( $1 \mathrm{mM}$ ) in the binary mixtures was changed with lead solution ( $1 \mathrm{mM}$ ) and steps (1) to (3) repeated. In addition, variations of the temperature of the solution did not affect the frequency change around room temperature but they altered the absolute frequency values.

\subsubsection{Results and Discussion}

Adsorption isotherms for cadmium ions at different surface coverages of 3,3thiodisuccinic acid in mixtures of $\left(\mathrm{Cd}^{2+}, \mathrm{Cu}^{2+}\right)$ and $\left(\mathrm{Cd}^{2+}, \mathrm{Pb}^{2+}\right)$ are shown in Figures (5.34 and 5.35). These adsorption isotherms can be compared with the single cadmium solution adsorption isotherms, which are given in Figure (5.36). A strong selectivity of the ligand for cadmium is seen. Thus, every addition of cadmium ions to the fixed copper or lead solution ( $1 \mathrm{mM}$ ), followed by adsorption of cadmium ions to the binding sites of the ligand and the copper or lead ions is lost from the surface because these ions have a smaller binding constants than cadmium ions to the ligand as described as in section 5.3. From these experimental adsorption isotherms, it can be concluded that the copper and lead in binary mixtures do not 
influence adsorption of cadmium ions from these solutions because the adsorption isotherm for single cadmium solution and the binary mixtures are located nearly in the same range of the frequency change.

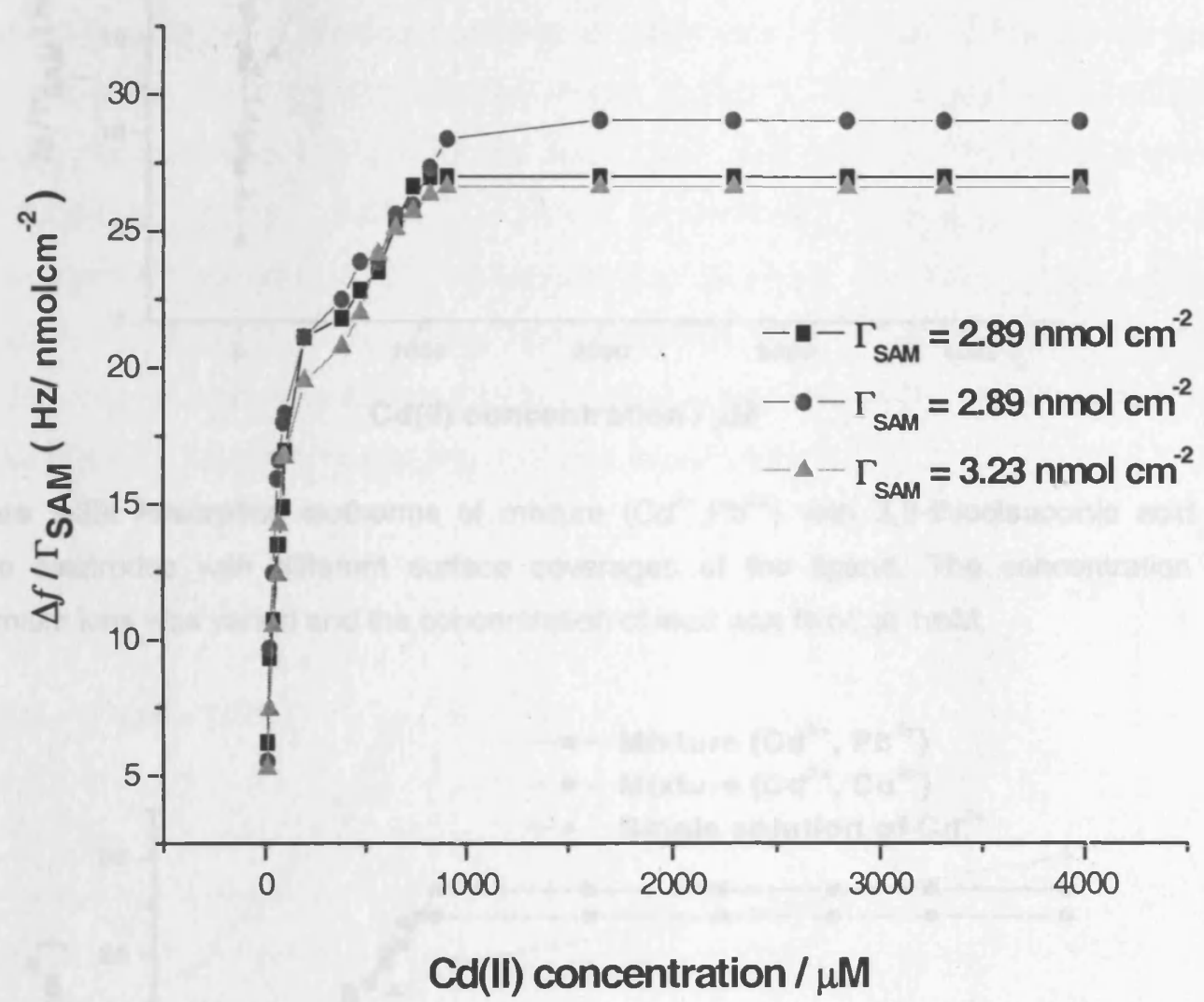

Figure 5.34: Adsorption isotherms of mixture $\left(\mathrm{Cd}^{2+}, \mathrm{Cu}^{2+}\right)$ with 3,3-thiodisuccinic acid at three electrodes with different surface coverages of the ligand. The concentration of cadmium ions was varied and the concentration of copper was fixed at $1 \mathrm{mM}$. 


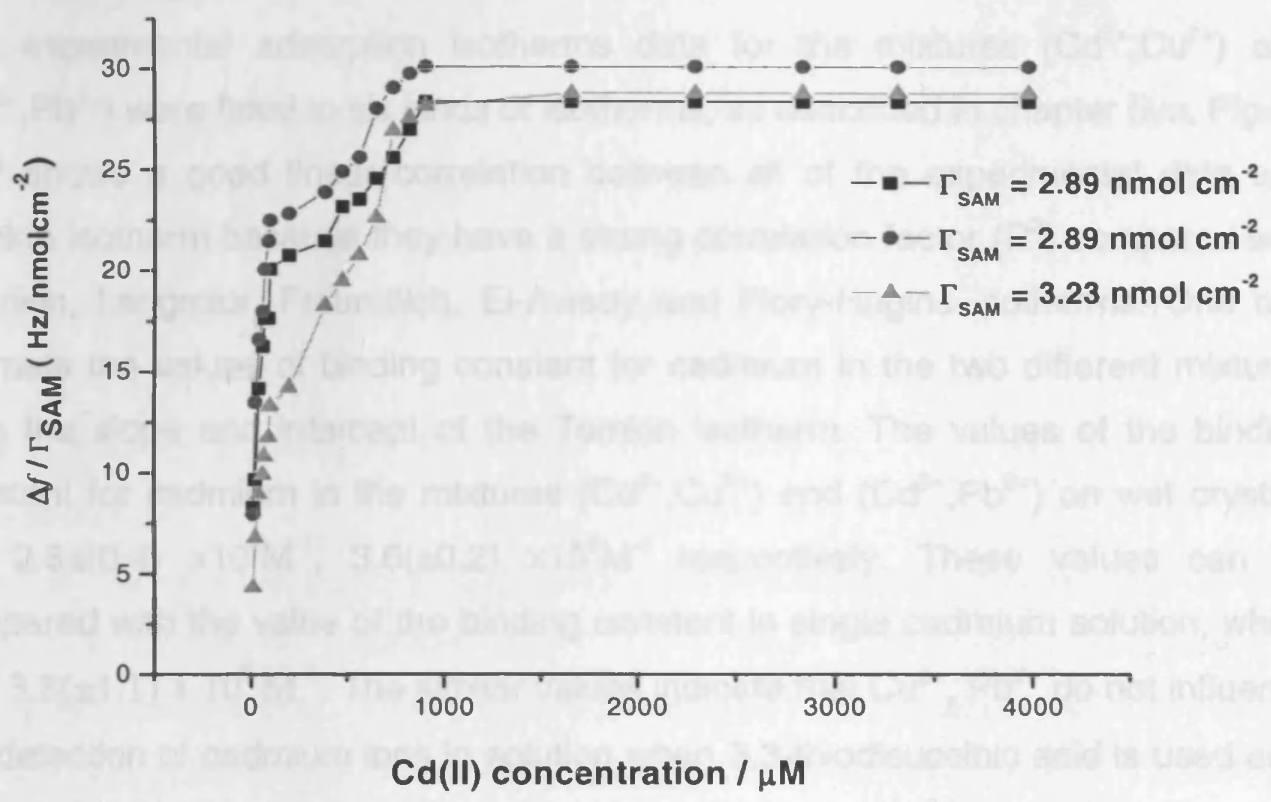

Figure 5.35: Adsorption isotherms of mixture $\left(\mathrm{Cd}^{2+}, \mathrm{Pb}^{2+}\right)$ with 3,3-thiodisuccinic acid at three electrodes with different surface coverages of the ligand. The concentration of cadmium ions was varied and the concentration of lead was fixed at $1 \mathrm{mM}$.

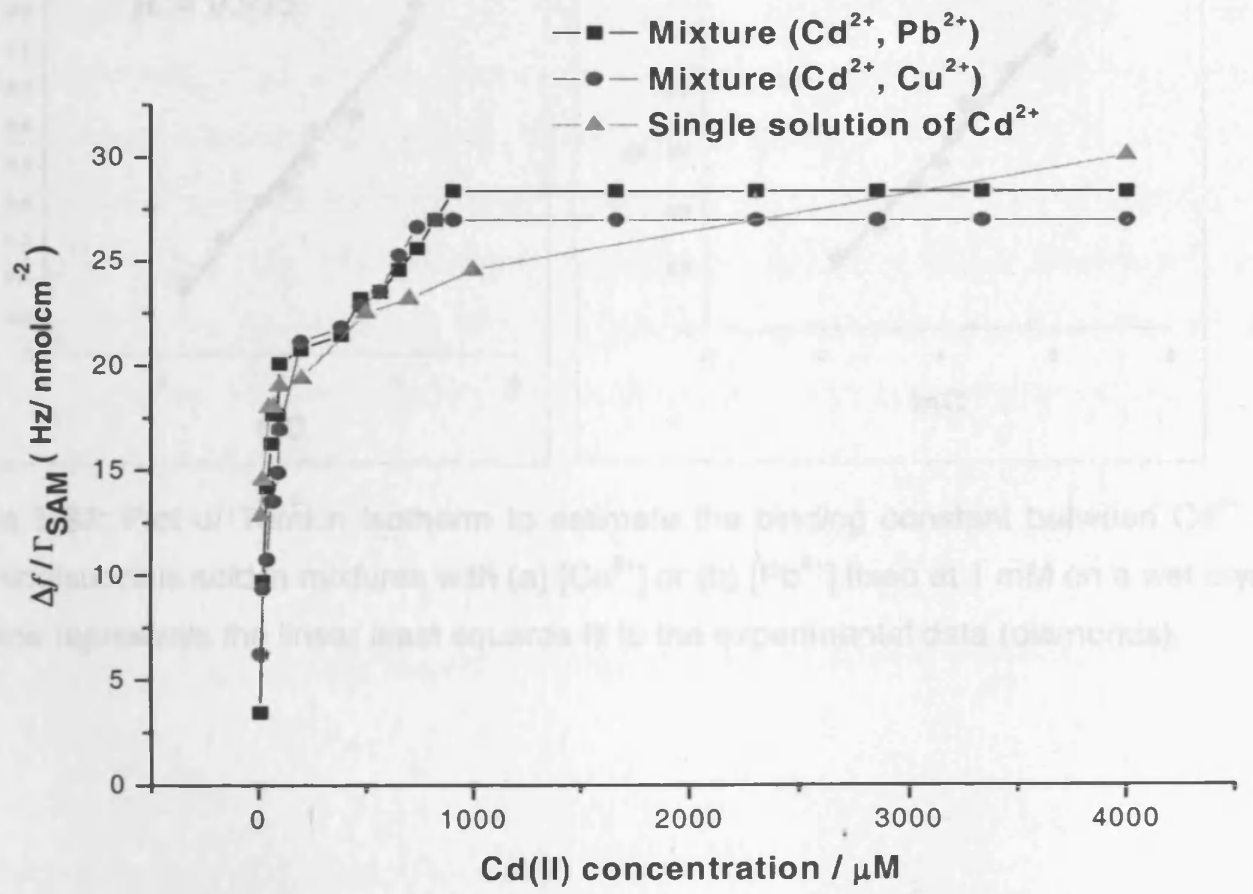

Figure 5.36: Comparison between experimental adsorption isotherms for cadmium ions from single metal solution and mixtures of $\left(\mathrm{Cd}^{2+}, \mathrm{Cu}^{2+}\right)$ and $\left(\mathrm{Cd}^{2+}, \mathrm{Pb}^{2+}\right)$ with 3,3thiodisuccinic acid, where the concentrations of $\mathrm{Cu}^{2+}$ and $\mathrm{Pb}^{2+}$ are fixed at $1 \mathrm{mM}$. The surface coverage of the ligand was $2.89 \mathrm{nmol} \mathrm{cm}^{-2}$. 


\subsubsection{Fitting the data to different isotherms}

The experimental adsorption isotherms data for the mixtures $\left(\mathrm{Cd}^{2+}, \mathrm{Cu}^{2+}\right)$ and $\left(\mathrm{Cd}^{2+}, \mathrm{Pb}^{2+}\right)$ were fitted to six kinds of isotherms, as described in chapter five. Figure 5.37 shows a good linear correlation between all of the experimental data and Temkin isotherm because they have a strong correlation factor $\left(R^{2}\right)$ compared with Frumkin, Langmiur, Freundlich, El-Awady and Flory-Hugins isotherms. One can estimate the values of binding constant for cadmium in the two different mixtures from the slope and intercept of the Temkin isotherm. The values of the binding constant for cadmium in the mixtures $\left(\mathrm{Cd}^{2+}, \mathrm{Cu}^{2+}\right)$ and $\left(\mathrm{Cd}^{2+}, \mathrm{Pb}^{2+}\right)$ on wet crystals are $2.8 \pm(0.4) \times 10^{6} \mathrm{M}^{-1}, 3.6( \pm 0.2) \times 10^{6} \mathrm{M}^{-1}$ respectively. These values can be compared with the value of the binding constant in single cadmium solution, which was 3.8( \pm 1.1$) \times 10^{6} \mathrm{M}^{-1}$. The similar values indicate that $\mathrm{Cu}^{2+}, \mathrm{Pb}^{2+}$ do not influence the detection of cadmium ions in solution when 3,3-thiodisuccinic acid is used as a surface ligand. This is because the ligand is selective for $\mathrm{Cd}^{2+}$.
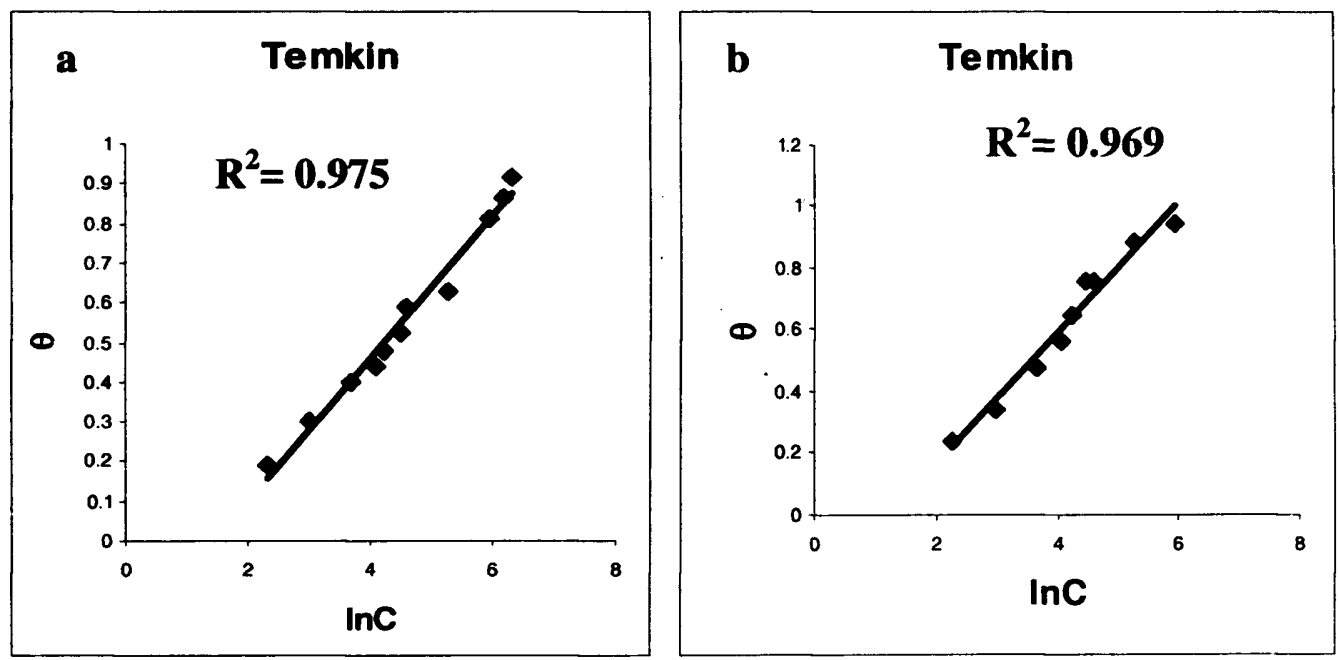

Figure 5.37: Plot of Temkin isotherm to estimate the binding constant between $\mathrm{Cd}^{2+}$ and 3,3-thiodisuccinic acid in mixtures with (a) $\left[\mathrm{Cu}^{2+}\right]$ or (b) $\left[\mathrm{Pb}^{2+}\right]$ fixed at $1 \mathrm{mM}$ on a wet crystal. The line represents the linear least squares fit to the experimental data (diamonds). 


\subsection{Using meso-2,3-dimercaptosuccinic acid to analyse mixtures of metal ions}

SAMs of meso-2,3-dimercaptosuccinicacid were studied in section 5.5 and shown to have selectivity towards lead ions in solution. The competitive effect of the various metal ions by this ligand is now studied by comparison between metal adsorption from a single metal ion solution and binary solutions.

\subsubsection{Measurements at fixed concentration of $\mathrm{Pb}^{2+}$, in the presence $\mathrm{Cu}^{2+}$ and $\mathrm{Cd}^{2+}$}

Here we will use the same methodology and procedures in sections 6.2 to bind lead ions in binary mixtures $\left(\mathrm{Cu}^{2+}, \mathrm{Pb}^{2+}\right)$ and $\left(\mathrm{Cd}^{2+}, \mathrm{Pb}^{2+}\right)$ to meso-2,3dimercaptosuccinic acid. The concentration of lead ions is fixed in the two mixtures and the concentration of copper and cadmium is varied as shown in Table 5.18. Metal solutions used in this study were in the form of their nitrate salts.

\subsubsection{Results and discussion}

In order to assess the extent of selective uptake of lead ions from binary mixtures, three independent experiments, with different surface coverages of the monolayer were performed. Figure 5.38 compares the frequency shifts for the three binary mixtures on dry (emersed) and wet (immersed) crystals.

The frequency shift for binding lead ions from a single metal ion solution to the ligand was similar to the results for binding lead ions from binary mixtures, indicating that the ligand has a stronger ability to bind lead ions from the solution than cadmium or copper ions. No influence of cadmium and copper on the uptake of lead ions was observed. 

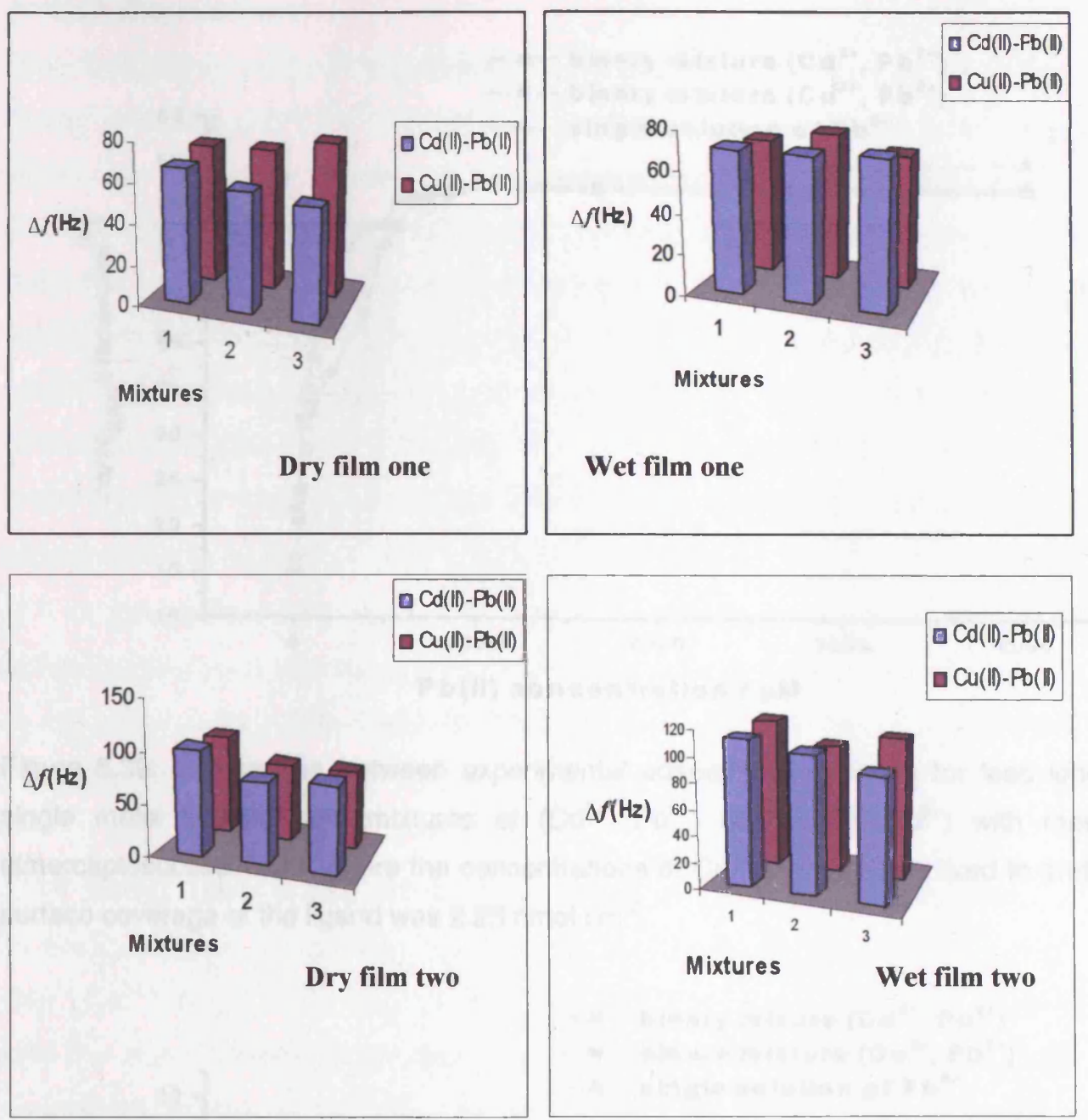

Figure 5.38: Comparison between electrodes with two different surface coverages of meso2,3-dimercaptosuccinic acid to bind lead ions in presence of three different concentrations of $\mathrm{Cd}^{2+}$ and $\mathrm{Cu}^{2+}$ in the binary mixtures on dry and wet crystals. The number of mixtures (1, 2 , and 3) refers to the table (5.16). The crystals were incubated first with a $\left(\mathrm{Cu}^{2+}, \mathrm{Pb}^{2+}\right)$ mixture and then with $\left(\mathrm{Cd}^{2+}, \mathrm{Pb}^{2+}\right)$.

\subsubsection{Competitive adsorption isotherms of lead in mixed metal ion solutions}

The concentration of lead ions in binary mixtures $\left(\mathrm{Pb}^{2+}, \mathrm{Cu}^{2+}\right)$ and $\left(\mathrm{Pb}^{2+}, \mathrm{Cd}^{2+}\right)$ was varied to different concentrations from $10 \mu \mathrm{M}$ to $10 \mathrm{mM}$ and the concentrations of copper and cadmium were fixed at $1 \mathrm{mM}$ (as in section (6.3.1)). Adsorption isotherms of lead in the two binary mixtures and in the single metal ion solution are shown in Figures 5.39 and 5.40. 


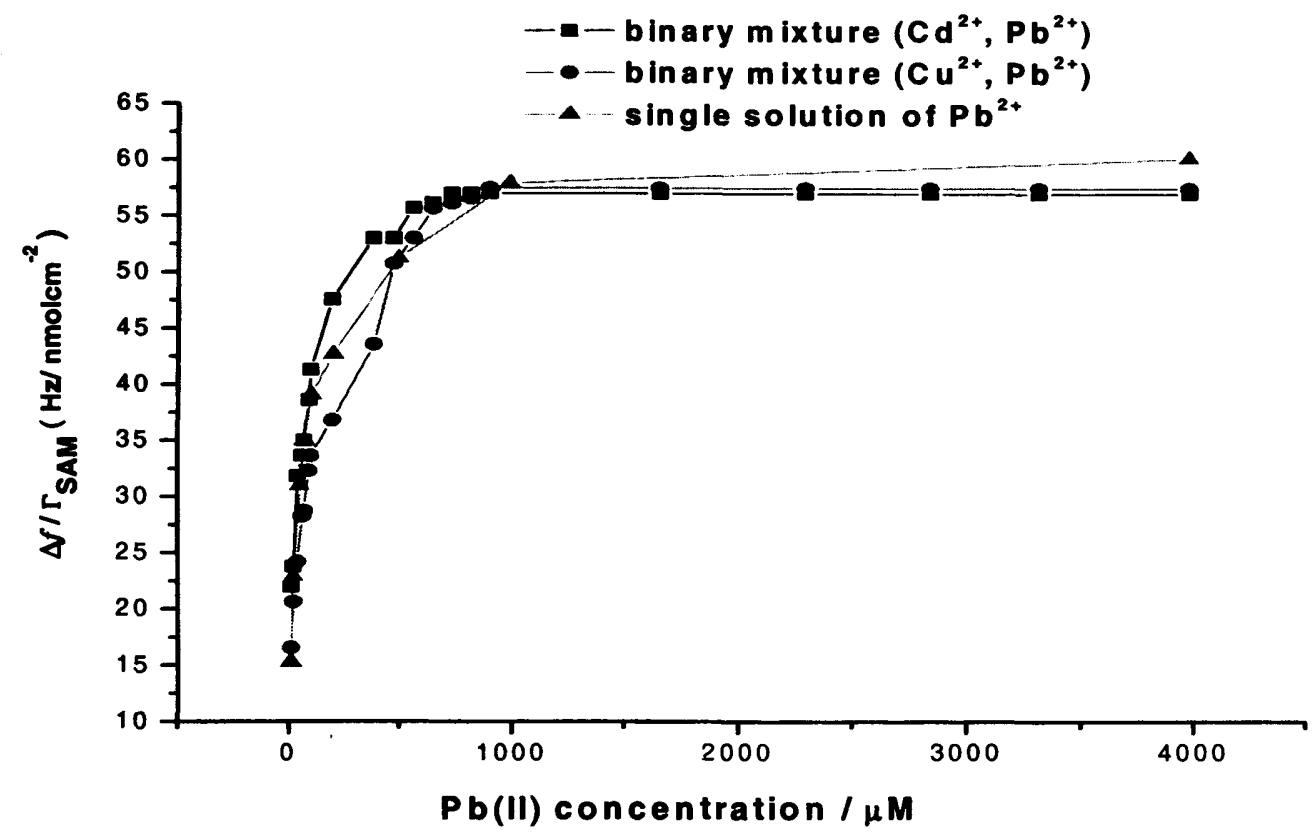

Figure 5.39: Comparison between experimental adsorption isotherms for lead ions from single metal solution and mixtures of $\left(\mathrm{Cd}^{2+}, \mathrm{Pb}^{2+}\right)$ and $\left(\mathrm{Cu}^{2+}, \mathrm{Pb}^{2+}\right)$ with meso-2,3dimercaptosuccinic acid, where the concentrations of $\mathrm{Cu}^{2+}$ and $\mathrm{Cd}^{2+}$ are fixed to $1 \mathrm{mM}$. The surface coverage of the ligand was $2.23 \mathrm{nmol} \mathrm{cm}^{-2}$.

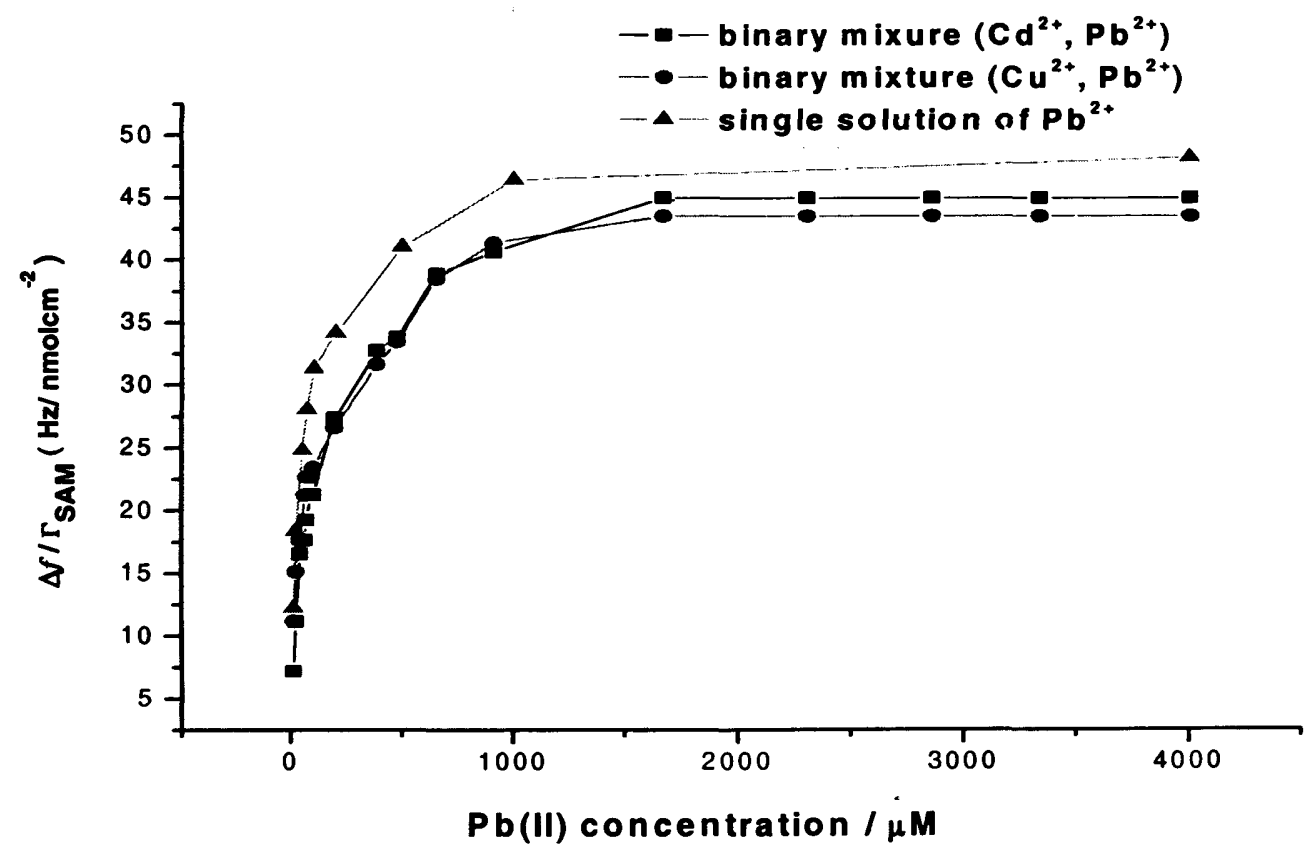

Figure 5.40: Comparison between experimental adsorption isotherms for lead ions from single metal solution and mixtures of $\left(\mathrm{Cd}^{2+}, \mathrm{Pb}^{2+}\right)$ and $\left(\mathrm{Cu}^{2+}, \mathrm{Pb}^{2+}\right)$ with meso-2,3dimercaptosuccinic acid, where the concentrations of $\mathrm{Cu}^{2+}$ and $\mathrm{Cd}^{2+}$ are fixed to $1 \mathrm{mM}$. The surface coverage of ligand was $2.78 \mathrm{nmol} \mathrm{cm}^{-2}$ 


\subsubsection{Discussion}

The frequency shifts in response to concentration changes of lead ions in the binary mixtures $\left(\mathrm{Cu}^{2+}, \mathrm{Pb}^{2+}\right)$ and $\left(\mathrm{Cd}^{2+}, \mathrm{Pb}^{2+}\right)$ are essentially the in same as single $\mathrm{Pb}$ (II) ion solution, indicating that copper and cadmium do not influence the lead determination with meso-2,3-dimercaptosuccinic acid. These results were fitted to different isotherms and we found that Frumkin and Temkin isotherms fit very well to experimental data. The experimental data fitted very well to Frumkin isotherm, which has a strong correlation factor $0.996-0.997$ for the two binary mixtures. The binding constant for lead in the two binary mixtures was calculated from the slope and intercept. It was found that the mean values of binding constant for lead in the binary mixtures were $\left(\mathrm{Cu}^{2+}, \mathrm{Pb}^{2+}\right): 6.0( \pm 0.2) \times 10^{5} \mathrm{M}^{-1}$, and $\left(\mathrm{Cd}^{2+}, \mathrm{Pb}^{2+}\right) 7.0( \pm 0.6) \times 10^{5}$ $\mathrm{M}^{-1}$. In comparison, the values for lead ion solution was $7.3( \pm 0.6) \times 10^{5} \mathrm{M}^{-1}$. We conclude that lead ions have a much stronger affinity for the ligand than do copper or cadmium ions: that there is little influence of copper and cadmium in the detection of lead ions.

\subsection{Using 1,6-hexanedithiol to analyse mixtures of metal ions}

In the first stage, the concentration of mercury ions in binary mixtures $\left(\mathrm{Hg}^{2+}, \mathrm{Cu}^{2+}\right)$, $\left(\mathrm{Hg}^{2+}, \mathrm{Cd}^{2+}\right),\left(\mathrm{Hg}^{2+}, \mathrm{Zn}^{2+}\right),\left(\mathrm{Hg}^{2+}, \mathrm{Ni}^{2+}\right),\left(\mathrm{Hg}^{2+}, \mathrm{Pb}^{2+}\right)$ and $\left(\mathrm{Hg}^{2+}, \mathrm{Ag}^{+}\right)$was fixed at $1 \mathrm{mM}$ and the concentration of interfering ions was varied from $0.1 \mathrm{mM}$ to $9 \mathrm{mM}$. In the second stage the concentration of $\mathrm{Hg}$ (II) was varied from $1 \mu \mathrm{M}$ to $1 \mathrm{mM}$, with the concentrations of interfering ions fixed at $1 \mathrm{mM}$. The results of the two stages are shown in Figures 5.41 and 5.42. The results show that the frequency shifts for $\mathrm{Hg}$ (II) in binary mixtures in both cases are essentially the same range as the frequency shifts for single mercury ion solutions. Thus, these interfering ions have no significant influence on the $\mathrm{Hg}$ (II) determination with 1,6-hexanedithiol SAM modified Au electrode. 


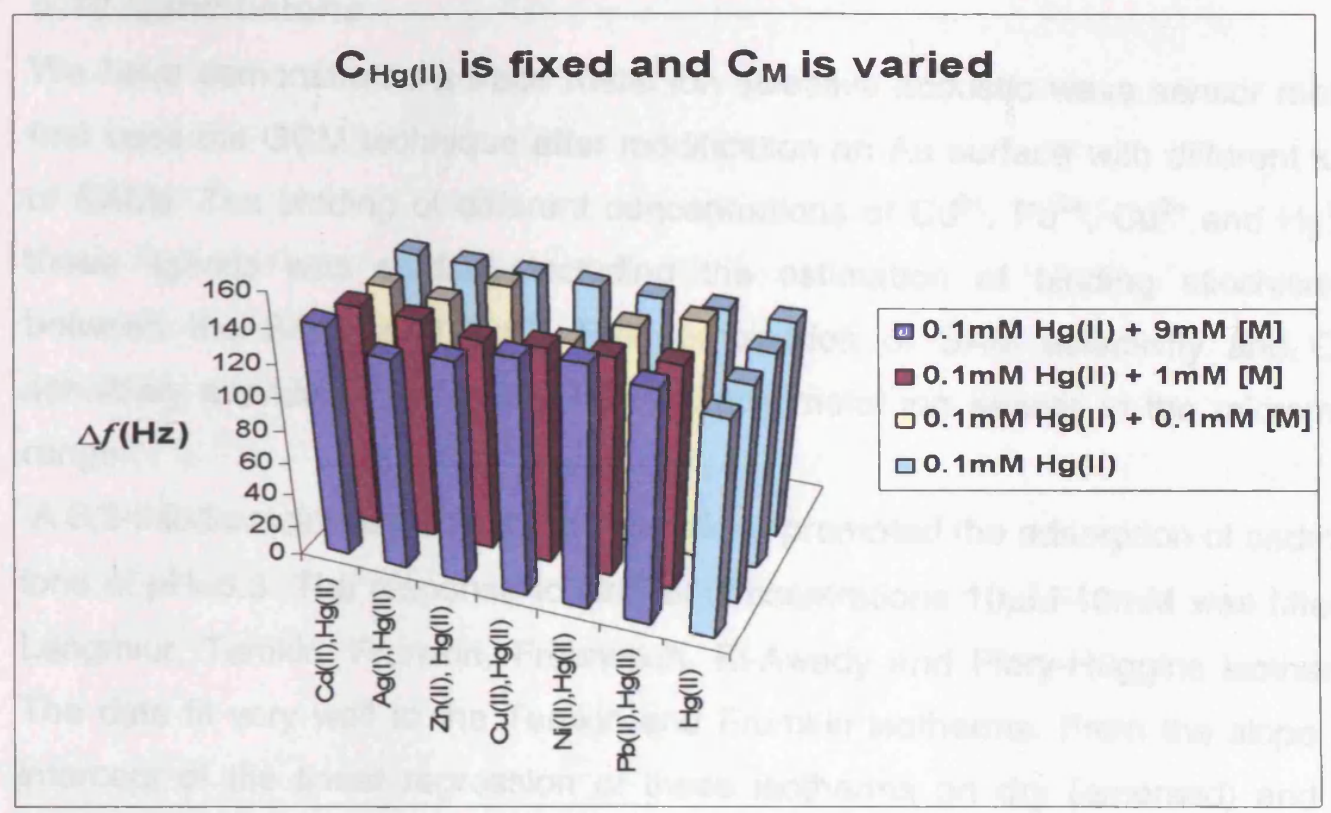

Figure 5.41: Comparison between the adsorption $\mathrm{Hg}(\mathrm{II})$ ions from single ion solutions and binary mixtures with interfering ions. The concentration of interfering metal ions was varied from $0.1 \mathrm{mM}$ to $9 \mathrm{mM}$ and the concentration of $\mathrm{Hg}(\mathrm{II})$ was fixed at $0.1 \mathrm{mM}$ in all cases. The same surface coverage of the ligand was used, $1.66 \mathrm{nmol} \mathrm{cm}$.

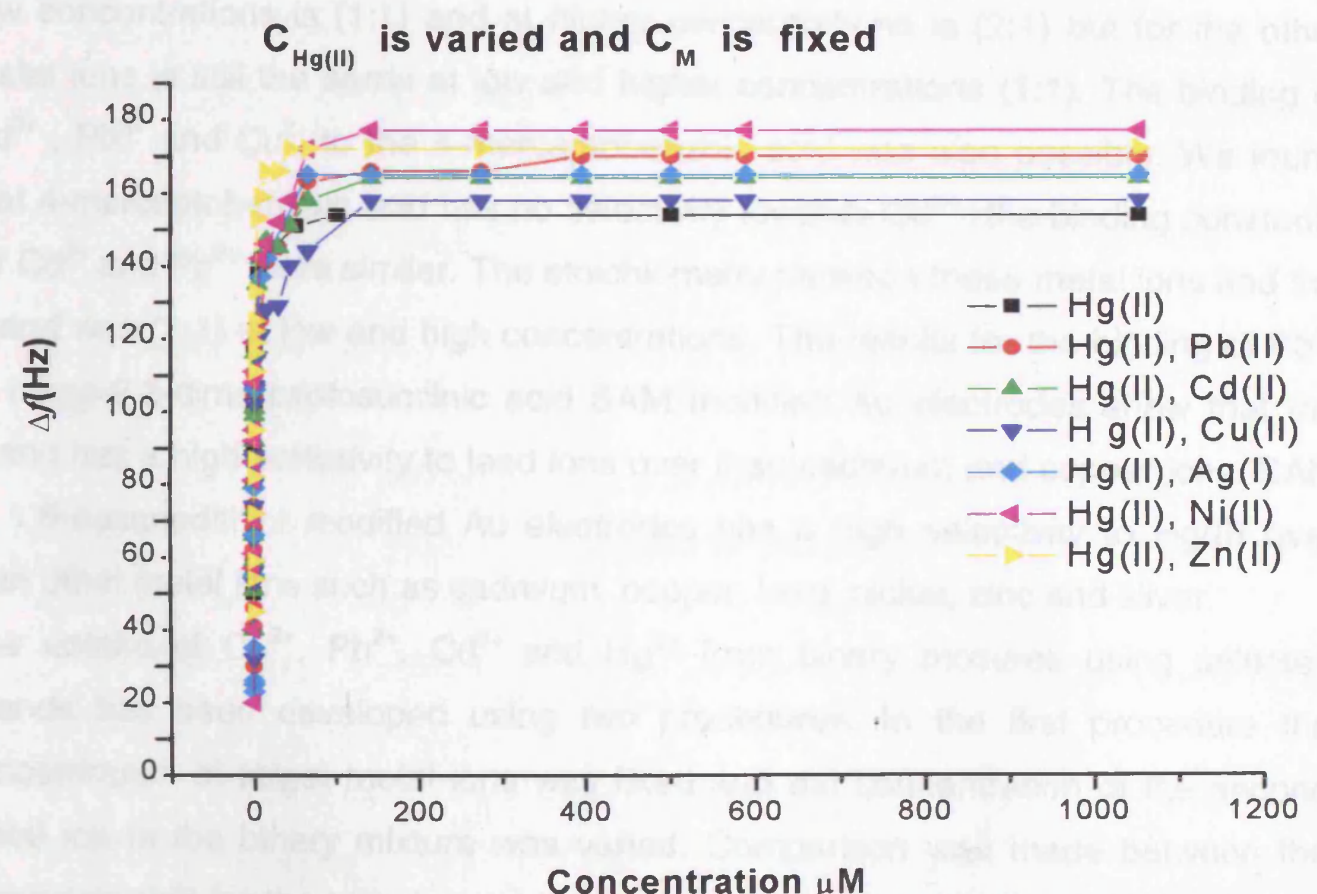

Figure 5.42: Comparison between experimental adsorption isotherms for mercury ions from single metal solution and binary mixtures with 1,6-hexanedithiol, where the concentrations of interfering ions were fixed to $1 \mathrm{mM}$. The surface coverage of ligand was $1.70 \mathrm{nmol} \mathrm{cm}$. 


\subsection{Conclusions}

We have demonstrated a trace metal ion selective acoustic wave sensor method that uses the QCM technique after modification an Au surface with different kinds of SAMs. The binding of different concentrations of $\mathrm{Cd}^{2+}, \mathrm{Pb}^{2+}, \mathrm{Cu}^{2+}$ and $\mathrm{Hg}^{2+}$ to these ligands was studied, including the estimation of binding stiochiometry between the SAMs and $\mathrm{Cd}^{2+}$. The combination of SAM selectivity and QCM sensitivity provides a potentially useful trace metal ion sensor in the micromolar range.

A 3,3-thiodisuccinic acid modified Au surface promoted the adsorption of cadmium ions at $\mathrm{pH}=6.3$. The response to $\mathrm{Cd}^{2+}$ at concentrations $10 \mu \mathrm{M}-10 \mathrm{mM}$ was fitted to Langmiur, Temkin, Frumkin, Freundlich, El-Awady and Flory-Huggins isotherms. The data fit very well to the Temkin and Frumkin isotherms. From the slope and intercept of the linear regression of these isotherms on dry (emersed) and wet (immersed) crystals, it was possible to estimate the binding constant between the $\mathrm{Cd}^{2+}$ and the ligand. To study the selectivity and sensitivity of the ligand, other metal ions such as $\mathrm{Pb}^{2+}$ and $\mathrm{Cu}^{2+}$ were bound to the monolayer and we used the methodology was used for binding $\mathrm{Cd}^{2+}$. It was found that the ligand has a strong selectivity to $\mathrm{Cd}^{2+}$ and the adsorption of these metal ions to the ligand followed the sequence $\mathrm{Cd}^{2+}>\mathrm{Pb}^{2+}>\mathrm{Cu}^{2+}$. The stoichiometry between the $\mathrm{Cd}^{2+}$ and the ligand at low concentrations is (1:1) and at higher concentrations is (2:1) but for the other metal ions is still the same at low and higher concentrations $(1: 1)$. The binding of $\mathrm{Cd}^{2+}, \mathrm{Pb}^{2+}$ and $\mathrm{Cu}^{2+}$ to the 4-mercaptobenzoic acid was also possible. We found that 4-mercaptobenzoic acid has no selectivity towards $\mathrm{Cd}^{2+}:$ the binding constants for $\mathrm{Cd}^{2+}$ and $\mathrm{Pb}^{2+}$ were similar. The stoichiometry between these metal ions and the ligand was (1:1) at low and high concentrations. The results for the binding of $\mathrm{Pb}^{2+}$ to meso-2,3-dimercaptosuccinic acid SAM modified Au electrodes show that the ligand has a high selectivity to lead ions over than cadmium and copper ions. SAM of 1,6-hexanedithiol modified $\mathrm{Au}$ electrodes has a high selectivity to $\mathrm{Hg}(\mathrm{II})$ over than other metal ions such as cadmium, copper, lead, nickel, zinc and silver.

The uptake of $\mathrm{Cu}^{2+}, \mathrm{Pb}^{2+}, \mathrm{Cd}^{2+}$ and $\mathrm{Hg}^{2+}$ from binary mixtures using selected ligands has been developed using two procedures. In the first procedure the concentration of target metal ions was fixed and the concentration of the second metal ion in the binary mixture was varied. Comparison was made between the frequency shift for the target metal ions in the single ion solution and in the binary mixture. It was found that the frequency shift for the target metal ion in single metal ions was essentially the same as in the binary mixtures. One can conclude that the 
second metal ion has no significant influence on the determination of target metal ion. In the second procedure the concentration of target metal ion in binary mixture was varied with the interfering metal ion concentration fixed. A plot of the concentration change ( $\mathrm{X}$-axis) with frequency shift ( $\mathrm{Y}$-axis) for the target metal ion yielded adsorption isotherm similar to the single metal ion solution. The adsorption isotherm of the target metal ion was fitted to six kinds of isotherms and it was found that the Frumkin and Temkin isotherms fitted the experimental data best. When comparing the values of binding constant for target metal ions in single solution to the binary mixture, it is clear that the values are similar for $\mathrm{Cd}^{2+}$ adsorbing to the 3,3-thiodisuccinic acid, $\mathrm{Pb}^{2+}$ to meso-2,3-dimercaptosuccinic acid and $\mathrm{Hg}^{2+}$ to 1,6hexanedithiol in binary mixtures.

\subsection{References}

1) W.Durnie, R.D.Marco, A.Jefferson and B. Kinsella, J. Electrochem. Soc., 146, (1999), 1751.

2) R.I .Masel, Principles of Adsorption and Reaction on Solid Surfaces, John Wiley and Sons, New York, 1996.

3) R. D. Johnson and F. H. Arnold, Biochimica. Biopysica. Acta.,1247, (1995), 293.

4) L. Lei, X. Su, Q. Xie, J. He and S. Yao, Mikrochim. Acta., 134, (2000), 63.

5) L. Novotny, T. Navratil, S. Sander and P. Basova, Electroanalysis., 15, (2003), 1687.

6) S. S. Abdelrehim, H. H. Hassan and M. A. Amin, Corrosion Science., 46, (2004), 5.

7) S. S. Abdelrehim, M. A. M. Ibrahim and K. F. Khaled, J. Appl. Electrochem., 29, (1999), 593.

8) J. H. Chun, K. H. Ra and N. Y. Kim, J. Electrochem. Soc., 149, (2002), 325.

9) E. Martell and R. M. Smith, Critical Stability Constants, Vol. 3, Other Organic Ligands, Plenum Press, New York, (1977), p. 167 and 171.

10) L. G. Sillen and A. E. Martell, Stability Constants of Metal Ion Complexes, The Chemical Socity, London, (1971).

11) M. Sastry, V. Patil and K. S. Mayya, J. Phys. Chem. B.,101, (1997), 1167.

12) S. J. Roser and M. R. Lovell, J. Chem. Soc. Faraday Trans., 91, (1995), 1783.

13) . D. Perrin, Stability Constants of Metal Ion Complexes, Pt. B. Organic Ligands, Oxford, Pergamon, (1979). p.205. 
14) T.Nagaoka, Z.Chen, H.Okuno, M.Nakayama and K.Ogura, Analytical Sciences., 15, (1999), 857.

15) S.Chah, J.Yi and R.N.Zare, Sensors and Actuators B., 99, (2004), 216.

16) T.Kang, J.Moon, S.Oh, S.Hong, S.Chah and J.Yi, Chem.Commun., (2005), 2360.

17) A.Bibby and L.Mercier, Chem. Mater.,14, (2002), 1591.

18) J.Brown, L.Mercier and T.J.Pinnavaia, Chem. Commun., (1999), 69.

19) W.Deng, L.Yang, D.Fujita, H.Nejoh and C.Bai, Appl. Phys. A., 71, (2000), 639.

20) M.J.Esplandiu, Probe Microscopy., 2, (2001), 89.

21) C.Mizuno, S.Bao, T.Hinoue and T.Nomura, Analytical Sciences., 21, (2005), 281. 


\section{Chapter 6: EQCM study of the complexation of trace metal ions with SAMs on gold piezoelectrodes}

\subsection{Introduction}

The previous chapter utilised the quartz crystal microbalance (QCM) technique as a gravimetric probe of surface composition resulting from metal ion binding. This is achieved by monitoring the resonant frequency of a gold coated quartz crystal device upon which the SAM is deposited. The idea here is to use electrochemical control with the EQCM [1] to add selectivity (for specific metal ions) to the sensitivity provided by the QCM. Coulometric data enables one to determine the metal ion coverage (by integrating the voltammetric responses of surface-bound complexed metal ions). The electrochemical technique used in this chapter is cyclic voltammetry (CV) which gives information about surface redox reactions of adsorbed species [2]. The trace metal ions will be coordinated, reduced and then re-oxidized on SAM modified Au electrodes. The influence of the preconcentration time, $\mathrm{pH}, \mathrm{pK}_{\mathrm{a}}$ of the terminal group of the SAM, the role of interferants and the influence of repetitive redox cycles on the metal ion binding will be studied. The reproducibility of the cyclic voltammograms on the same and different surface coverage of the ligand after adsorption of metal ions will be studied.

The same ligands in chapter five will be examined for selectivity towards target metal ions. We will compare the surface coverage of the metal ions as determined by gravimetric and coulometric techniques. The ligands used are 3,3thiodipropionic acid, 3-mercaptosuccinic acid, meso-2,3-dimercaptosuccinic acid, 2-mercaptobenzimidazole and 1,4-benzenedimethanethiol. The free terminal groups are $(-\mathrm{COOH}),(-\mathrm{NH})$ and $(-\mathrm{SH})$.

\subsection{Complexation between 3,3-thiodipropionic acid and $\mathrm{Cu}^{2+}$}

3,3-thiodipropionic acid (TDP) was examined before in chapter five for acoustic wave sensing of binding of $\mathrm{Cu}^{2+}, \mathrm{Pb}^{2+}$ and $\mathrm{Cd}^{2+}$. In this section we will examine the electrochemical responses for binding these metals to the TDP. The extent of ion binding is determined by integration of the charge required for reduction/oxidation of the bound ions when the modified electrode is exposed to copper-free aqueous solutions. 


\subsubsection{Experimental}

The experimental instrumentation and procedures are described in chapter 3 . Selfassembled monolayers of TDP were prepared on Au piezoelectrodes of the QCM. The frequency change was measured for immobilization of TDP on immersed and emersed crystals using the procedures described in the experimental chapter, section (4.3). The accumulation of metal ions on TDP modified electrodes (preconcentration step) was carried out under open circuit conditions to avoid the participation of reducible interferants. The TDP SAM modified electrode was dipped into $10 \mathrm{ml}$ of $1 \mathrm{mM} \mathrm{Cu}(\mathrm{NO} 3)_{2}+0.1 \mathrm{M} \mathrm{KNO}_{3}, \mathrm{pH}=5.5$ for different accumulation times without stirring. The TDP modified electrodes, incorporating copper ions, were rinsed thoroughly with ultra pure water. Then cyclic voltammetry (CV) experiments were conducted immediately in copper-free solution $0.1 \mathrm{M} \mathrm{KNO}_{3}$. The cyclic voltammograms were run over the potential range 0.5 to $-0.5 \mathrm{~V}$ (S.C.E) at different scan rates $\left(200,100,50,20\right.$ and $\left.10 \mathrm{mV} \mathrm{s}^{-1}\right)$. Deaeration was done by nitrogen gas, which is purged through the fresh blank solution for $15 \mathrm{~min}$ before every measurement and blown above the solution during the measurement.

\subsection{Results and Discussion}

The voltammetric studies of the Au/TDP modified electrode were discussed in chapter 4, section (4.4). The surface coverage of the TDP modified electrode can be estimated from the frequency changes of the QCM as described in chapter 3 , section (3.6). Experiments were repeated three to five times to examine the reproducibility of voltammetric responses. Pure potassium nitrate was investigated as a supporting electrolyte for all metal ions detections because it contained less heavy metal impurities than other electrolytes and the $\mathrm{pH}$ of the $\mathrm{KNO}_{3}$ is suitable for metal ion accumulations.

\subsubsection{Voltammetric response of the Au/TDP electrode to $\mathrm{Cu}(\mathrm{II})$ ions}

Analytical optimization of TDP modified electrode response relies on several analytical conditions. These conditions include surface coverage of the monolayer (modification time), $\mathrm{pH}$ of the preconcentraion solutions, the pKa of the SAM (terminal carboxylic acid groups), effect of the $K_{s p}$, preconcentration time in the $\mathrm{Cu}$ (II) solution at open circuit, stability and influence of the scan rate on the complexation between $\mathrm{Cu}(\mathrm{II})$ and TDP and parameters of cyclic voltammetry. All of these analytical conditions will be discussed in the following sections. 
Figure (6.1) presents the typical cyclic voltammogram of a Au/TDP electrode obtained in a copper-free solution (before preconcentration step) (curve b) and after a preconcentration step in $1 \mathrm{mM} \mathrm{Cu}^{2+}$ solution for $20 \mathrm{~min}$ (curve c). Successful binding of $\mathrm{Cu}(\mathrm{II})$ in the SAM modified electrode surface is clearly evidence by the presence of a pair of peaks $\left(E_{p, a}=0.230 \mathrm{~V}, E_{p, c}=0.156 \mathrm{~V}\right)$ for the redox chemistry of $\mathrm{Cu}^{2+} / \mathrm{Cu}^{0}$. The anodic peak was larger than the cathodic peak, which showed that an irreversible process occurred during the cycling. The difference in cathodic and anodic peak shapes and heights indicates that this is not nearly a simple electron transfer process.

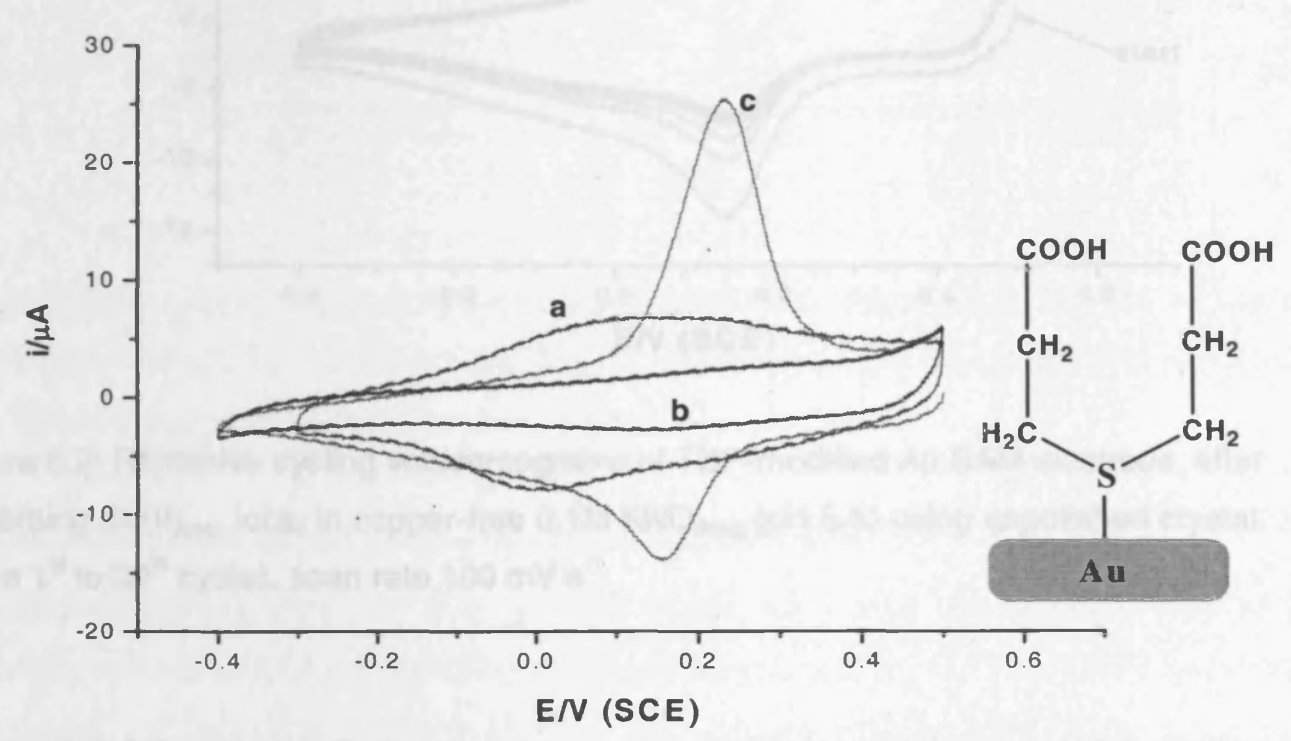

Figure 6.1: Cyclic voltammogram of TDP modified Au SAM electrode (a) Au in Cu(II)-free $\mathrm{KNO}_{3 \text { (aq) }}$ having been exposed to $1 \mathrm{mM} \mathrm{Cu}(\mathrm{II})_{(\text {(aq), }}$ (b) SAM on $\mathrm{Au}$ in $\mathrm{Cu}(\mathrm{II})$-free $\mathrm{KNO}_{3 \text { (aq) }}$, (c) $\mathrm{SAM}$ on $\mathrm{Au}$ in $\mathrm{Cu}(\mathrm{II})$-free $\mathrm{KNO}_{3}(\mathrm{aq})$ having been exposed to $1 \mathrm{mM}$ of $\mathrm{Cu}(\mathrm{II})_{(\mathrm{aq})}$. Scan rate $100 \mathrm{mV} \mathrm{s}^{-1}$

Control experiments, under the same conditions, demonstrated that $\mathrm{Cu}$ (II) ions do not adsorb onto a bare gold electrode surface (curve a). Thus, the voltammetric response of the TDP/Au electrode to $\mathrm{Cu}(\mathrm{II})$ should be ascribed to the interaction between TDP SAM and Cu(II) ions.

\subsubsection{Stability and influence of the sweep rate}

Repetitive sweep cyclic voltammograms for the reduction/ oxidation of $\mathrm{Cu}^{2+}$ complexed at the TDP modified Au SAM electrode after adsorbing $\mathrm{Cu}$ (II) ions from 
copper free nitrate solutions are shown in figure 6.2. The $\mathrm{Cu}^{2+} / \mathrm{Cu}^{0}$ redox reaction is observed over twenty cycles.

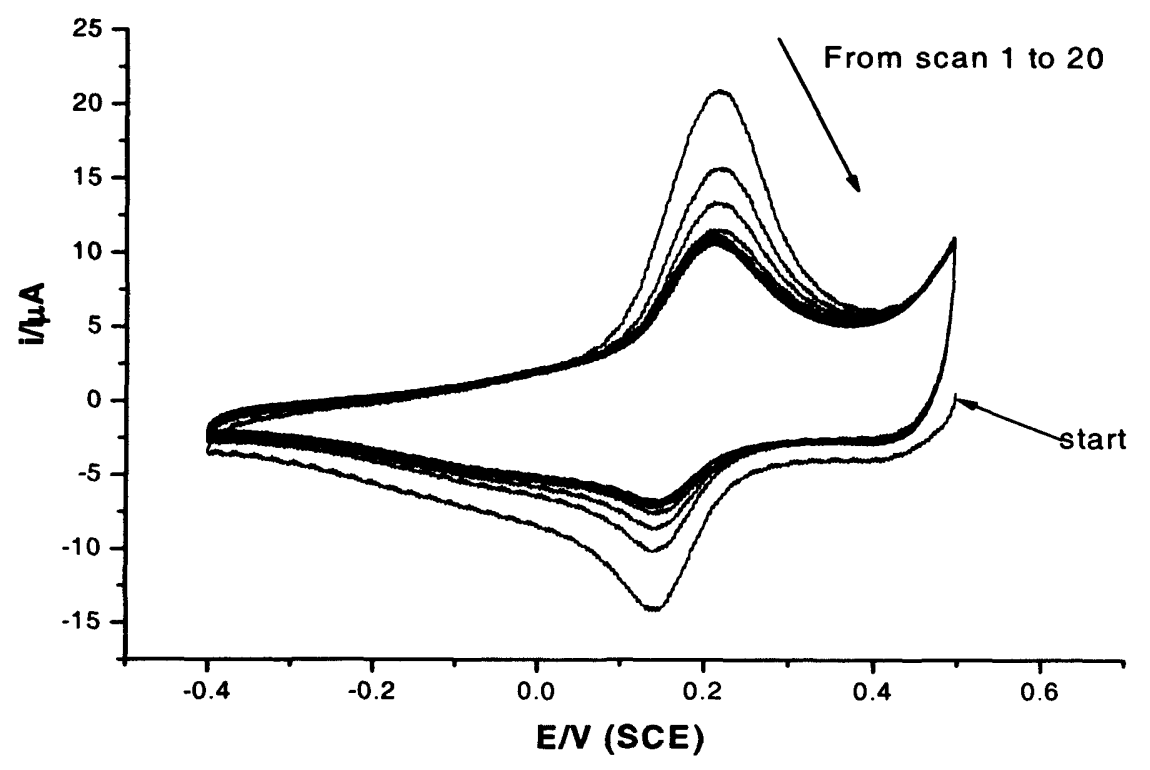

Figure 6.2: Repetitive cycling voltammograms of TDP-modified Au SAM electrode, after adsorbing $\mathrm{Cu}(\mathrm{II})_{(\mathrm{aq})}$ ions, in copper-free $0.1 \mathrm{M} \mathrm{KNO}_{3(\mathrm{aq})}(\mathrm{pH} 5.5)$ using unpolished crystal. From $1^{\text {st }}$ to $20^{\text {th }}$ cycles, scan rate $100 \mathrm{mV} \mathrm{s}^{-1}$.

We noticed that the voltammograms on unpolished crystals remained unchanged from the fourth to twentieth cycles, but on polished crystals after the first cycle very stable voltammograms were obtained with no change in peak currents or potentials. During the course of the first cycle voltammetric cycle some rearrangement of the copper coordination in the film may take place, but thereafter a stable response was recorded upon repetitively cycling in a copper-free electrolyte solution. No change in current peak is observed until the fortieth cycle, after that the anodic and cathodic peaks disappear, indicating that the TDP/Au modified electrode was relatively stable. These results indicate that copper ions remain strongly adsorbed in both oxidation states within the potential range of the experiment.

The stability of the cyclic voltammogramms was obtained at different sweep rates, from 10 to $100 \mathrm{mV} \mathrm{s}^{-1}$, as shown in Figure 6.3. The peak current increased linearly with increasing the scan rate for both the oxidation and reduction (see the inset of Figure 6.3), indicating a surface redox reaction [ 3 ]. 


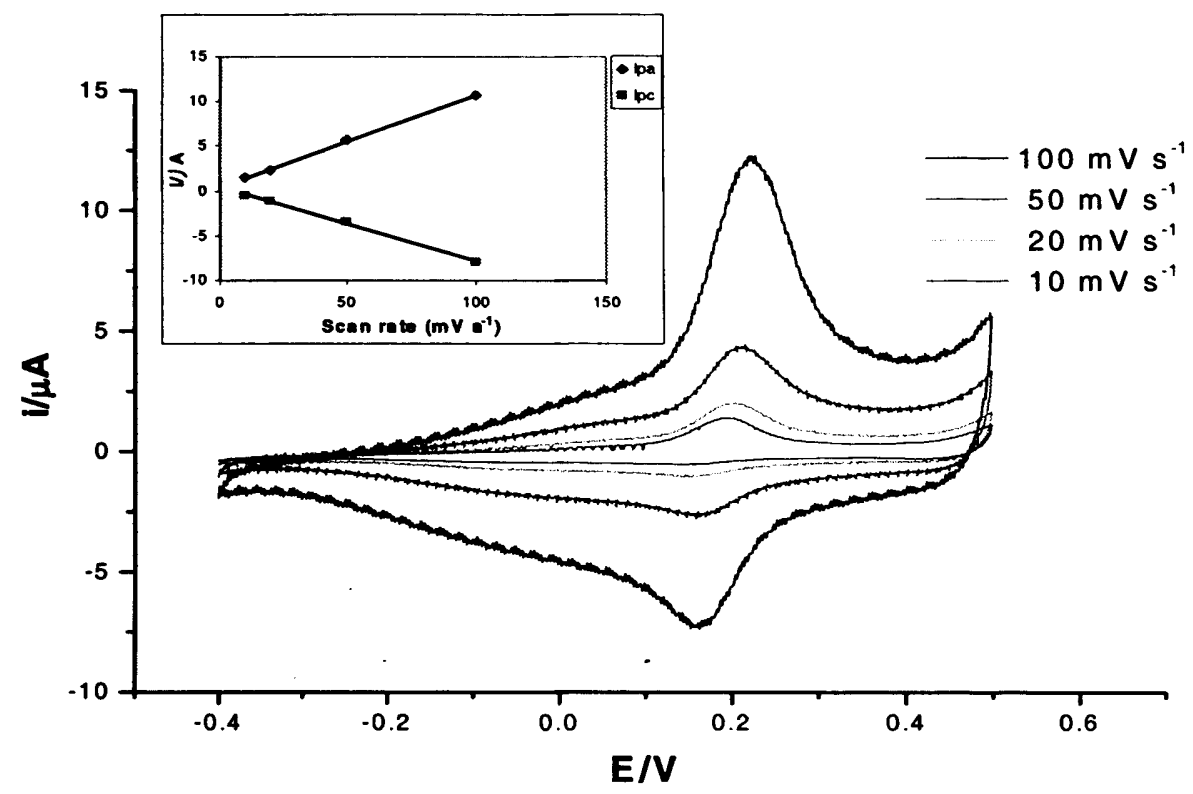

Figure 6.3: The influence of different potential scan rates (from 10 to $100 \mathrm{mV} \mathrm{s}^{-1}$ ) on the $\mathrm{Cu}(I I)$ peak current. For conditions see Fig.6.1. Inset represents the variation of anodic $\left(I_{\mathrm{pa}}\right)$ and cathodic $\left(I_{\mathrm{pc}}\right)$ peak currents vs. scan rate.

\subsubsection{Effect of pH of preconcentration solution}

The two terminal carboxylic acid groups move freely into the solution side at the interface and dissociate in solutions with pH above 4-5 [4, 5]. The acid dissociation constants, $\mathrm{pK}_{1}$ and $\mathrm{pK}_{2}$ are 4.2 and 5.2 respectively [6]. The $\mathrm{pH}$ of the supporting electrolyte (pure $0.1 \mathrm{M} \mathrm{KNO}_{3}+1 \mathrm{mM} \mathrm{Cu}\left(\mathrm{NO}_{3}\right)_{2}$ ) is 5.5 and the $\mathrm{pH}$ of the pure $0.1 \mathrm{M}$ $\mathrm{KNO}_{3}$ is 6 . Since these are higher than $\mathrm{pK}_{1}$ and $\mathrm{pK}_{2}$, the electrode surface becomes negatively charged. To test whether the $\mathrm{pH}$ would have any effect on the determination of copper species during the preconcentration processes, the TDP electrode was immersed in a (pure $\left.0.1 \mathrm{M} \mathrm{KNO} \mathrm{KN}_{3}+1 \mathrm{mM} \mathrm{Cu}\left(\mathrm{NO}_{3}\right)_{2}\right)$ solution at different $\mathrm{pH}(3,4,5.5$ and 7$)$. Solutions of different $\mathrm{pH}$ were adjusted to the appropriate $\mathrm{pH}$ with $0.1 \mathrm{M} \mathrm{HNO}_{3}$ and $0.1 \mathrm{M} \mathrm{KOH}$. Figure 6.4: shows the influence of electrolyte solution $\mathrm{pH}$ on the electrochemistry of complexed $\mathrm{Cu}^{2+}$. Clearly, at $\mathrm{pH} 3$ and 4 a poor response was obtained, as seen by low currents and a large separation in peak potential and the peaks exhibited irregular shape indicating that the $\mathrm{pH}$ had a strong effect on the preconcentration processes. 


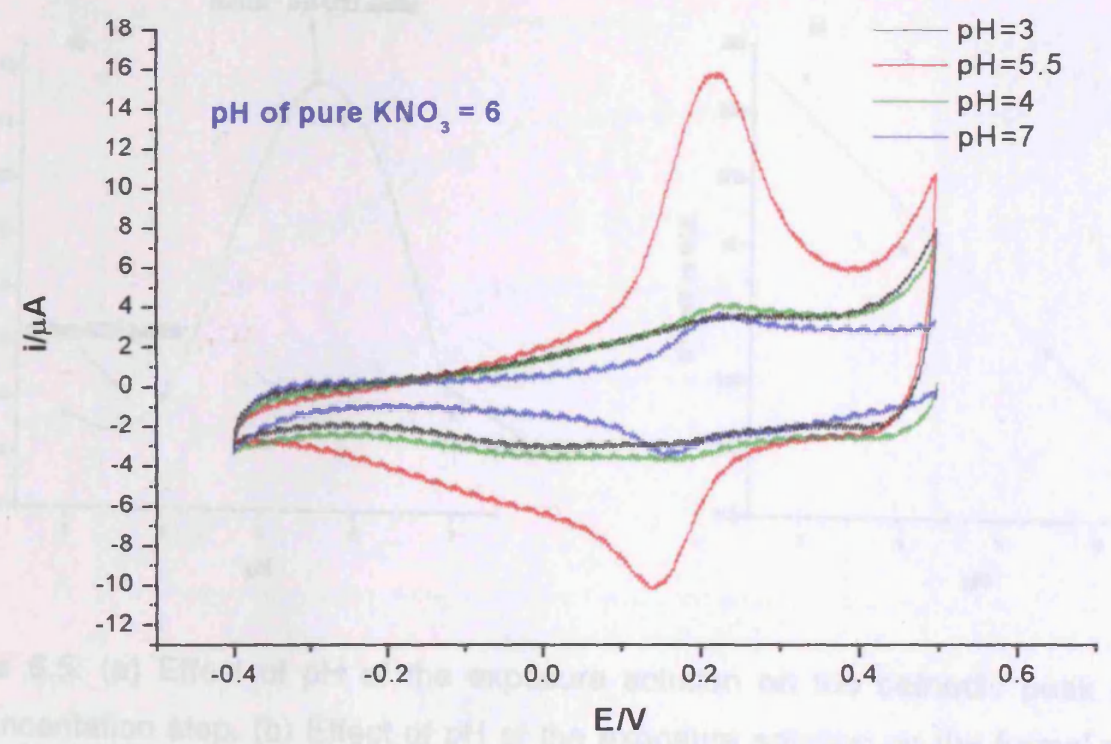

Figure 6.4: Effect of the electrolyte $\mathrm{pH}$ on the peak current for the complexed of $\mathrm{Cu}^{2+}$ with (TDP) coated gold electrode. Cyclic voltammetry in copper-free $0.1 \mathrm{M} \mathrm{KNO}{ }_{3}$ at the $\mathrm{pH}$ values indicated; sweep rate $100 \mathrm{mV} \mathrm{s}^{-1}$; preconcentration time $=20 \mathrm{~min}$; open circuit.

After the third cycle the copper was completely removed from the surface. This was due to the acid induced dissociation of the surface bound complex. The maximum copper incorporation to the ligand was observed for an exposure $\mathrm{pH}$ of 5.5 . Interestingly, the peak current also decreased with increasing and decreasing $\mathrm{pH}$ to either side of 5.5 as can be seen from Figure $6.5 \mathrm{a}$. At higher $\mathrm{pH}$, the lower response is attributed to copper ion hydrolysis to produce insoluble hydroxide species [7]. Judging by the small $\mathrm{K}_{\mathrm{sp}}$ of $\mathrm{Cu}(\mathrm{OH})_{2}$, which is $2.2 \times 10^{-20} \mathrm{M}^{3}$, it is not likely for neutral $\mathrm{Cu}(\mathrm{OH})_{2}$ or negatively charged $\mathrm{Cu}(\mathrm{II})$-hydroxide complexes to be taken up by the negatively charged carboxylates on the TDP SAM. It is reasonable to believe that the decrease in current signal was due to the small amount of free $\mathrm{Cu}$ (II) ions present. This behaviour is in agreement with the carboxylic acid becoming deprotonated at high $\mathrm{pH}$ and thus more available for copper complexation chemistry. Figure $6.5 \mathrm{~b}$ shows the effect of solution $\mathrm{pH}$ on the formal potential $\left(E^{0}\right)$ of copper complexed in a TDP monolayer. It was found that a linear shift to lower potential can be observed as the $\mathrm{pH}$ of the solution increases. This is indicative that the protonation steps are involved in the overall redox process. The slope of the curve $E^{0}$ versus $\mathrm{pH}$ showed a value of $28.4 \mathrm{mV}$, which can indicate that two protons per one electron are involved in the complexation between $\mathrm{Cu}^{2+}$ and TDP. 

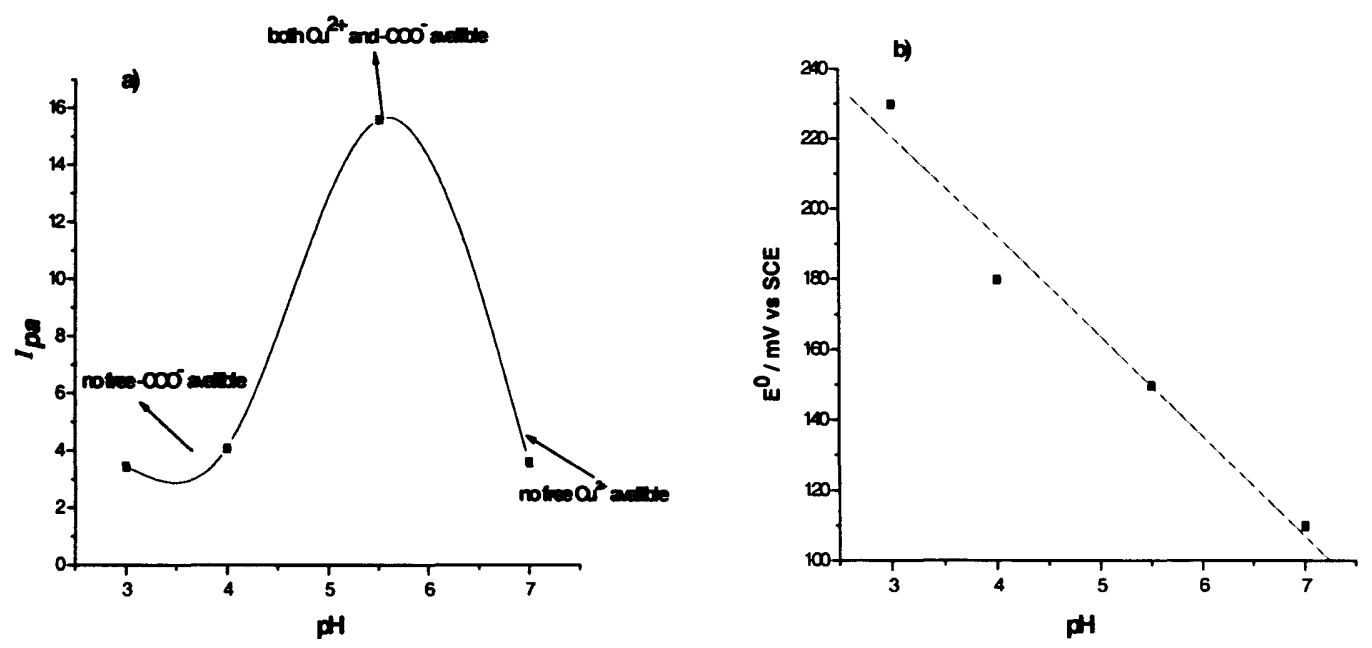

Figure 6.5: (a) Effect of $\mathrm{pH}$ of the exposure solution on the cathodic peak current after preconcentation step. (b) Effect of $\mathrm{pH}$ of the exposure solution on the formal potential $\left(\mathrm{E}^{0}\right)$ of the copper complexed on a TDP SAM electrode after preconcentration step. Conditions: cyclic voltammetry in copper-free $0.1 \mathrm{M} \mathrm{KNO}_{3}$ at the $\mathrm{pH}$ values indicated; sweep rate 100 $\mathrm{mV} \mathrm{s}^{-1}$; preconcentration time $=20 \mathrm{~min}$; open circuit; temperature $25^{\circ} \mathrm{C}$.

\subsubsection{Effect of the preconcentration time}

The accumulation time is a decisive factor in any preconcentration step. The dependence of integrated charge for the incorporated copper species in TDP SAM on the preconcentration time was studied for three different concentrations of $\mathrm{Cu}$ (II) under carefully controlled conditions (see Figure 6.6). The cathodic peak charge increased with increasing accumulation time up to a plateau region around $20 \mathrm{~min}$, thus the occurrence of this plateau region value for the charge of the peak at longer accumulation periods could originate from attainment of complexation equilibrium between $\mathrm{Cu}(\mathrm{II})$ in solution and TDP at surface or saturation of the binding sites at high concentration of $\mathrm{Cu}(\mathrm{II})$. For each of the preconcentration times employed the steady state peak charge value was different; larger values being obtained for higher concentrations of $\mathrm{Cu}$ (II) (a saturation value of $60 \mu \mathrm{C}$ for $1 \mathrm{mM} \mathrm{Cu}^{2+}$ was obtained). Thus, a preconcentration time of $20 \mathrm{~min}$ was chosen for the concentration from $1 \mathrm{mM} \mathrm{Cu}$ (II). 


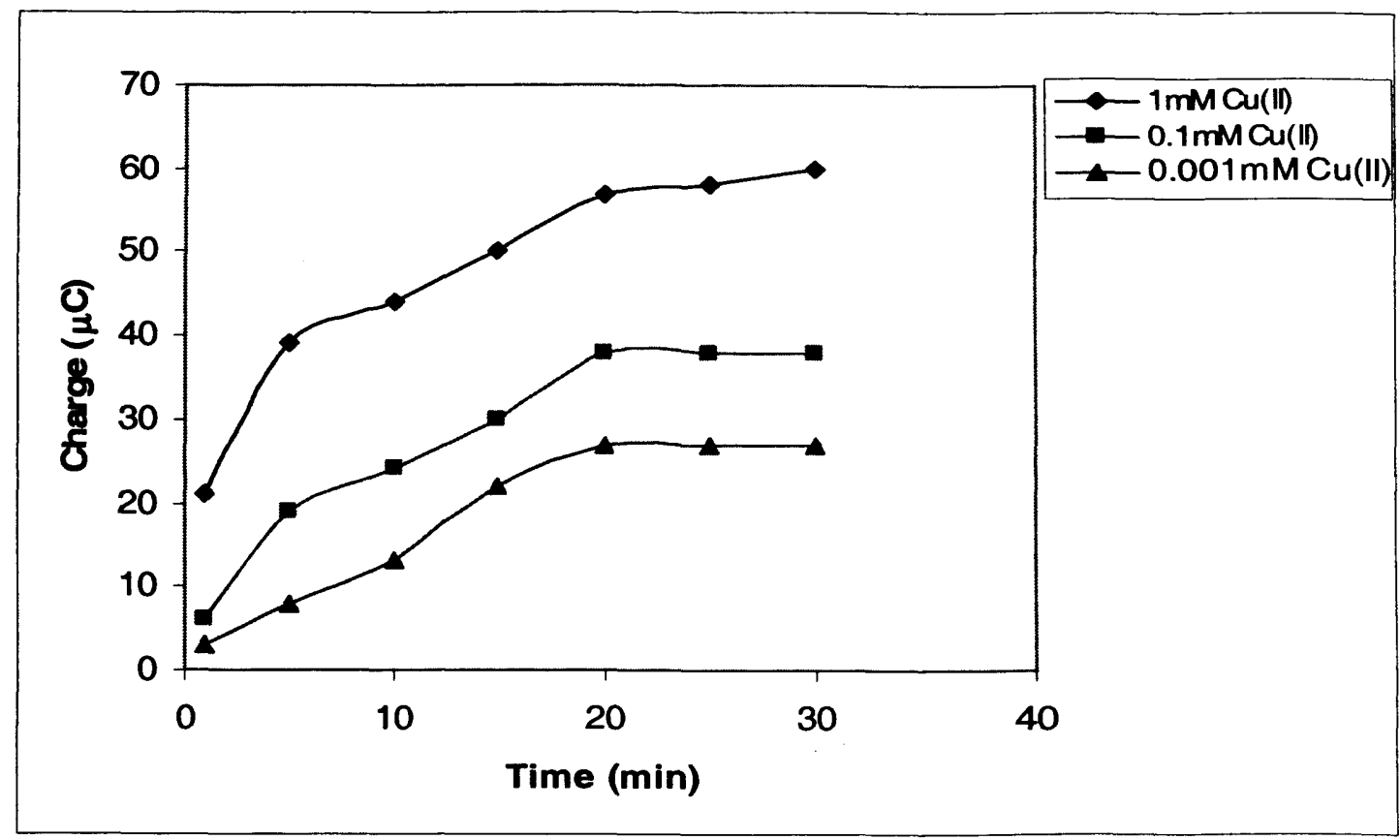

Figure 6.6: Effect of preconcentration time on the CV charge for the binding $\mathrm{Cu}$ (II) to TDP SAM modified electrode. Solution conditions: Cyclic voltammetry in $\mathrm{Cu}(\mathrm{II})$-free $\mathrm{KNO}_{3}(\mathrm{aq})$ having been exposed to $(0.001,0.1$ and $1 \mathrm{mM})$ of $\mathrm{Cu}(\mathrm{II})_{(\mathrm{aq})}$. Scan rate $100 \mathrm{mV} \mathrm{s}^{-1}$.

\subsubsection{Concentration dependence of the characteristic peak charge}

Using the optimum experimental conditions and the procedures described above, the relation between the $\mathrm{CV}$ peak current and the $\mathrm{Cu}(\mathrm{II})$ concentration was studied. Figure 6.7 shows the CVs obtained with a TDP/Au modified electrode for different $\mathrm{Cu}$ (II) concentrations (from $1 \times 10^{-8}$ to $1 \times 10^{-3} \mathrm{M}$ ). It is clear that the peak current increases with increasing concentration of $\mathrm{Cu}(\mathrm{II})$. Figure 6.8 shows calibration curves for the determination of copper ions at two different surface coverages of TDP on polished and unpolished crystals. The cathodic peak charge reached a limiting value of 63 and $69 \mu \mathrm{C}$, corresponding to a copper coverage 1.64 and 1.70 $\mathrm{nmol} \mathrm{cm}^{-2}$, on polished and unpolished crystals, respectively, when the copper concentration was $1 \mathrm{mM}$. The lowest copper concentration that could be measured was $0.01 \mu \mathrm{M}$ (see the inset in Figure 6.8). 


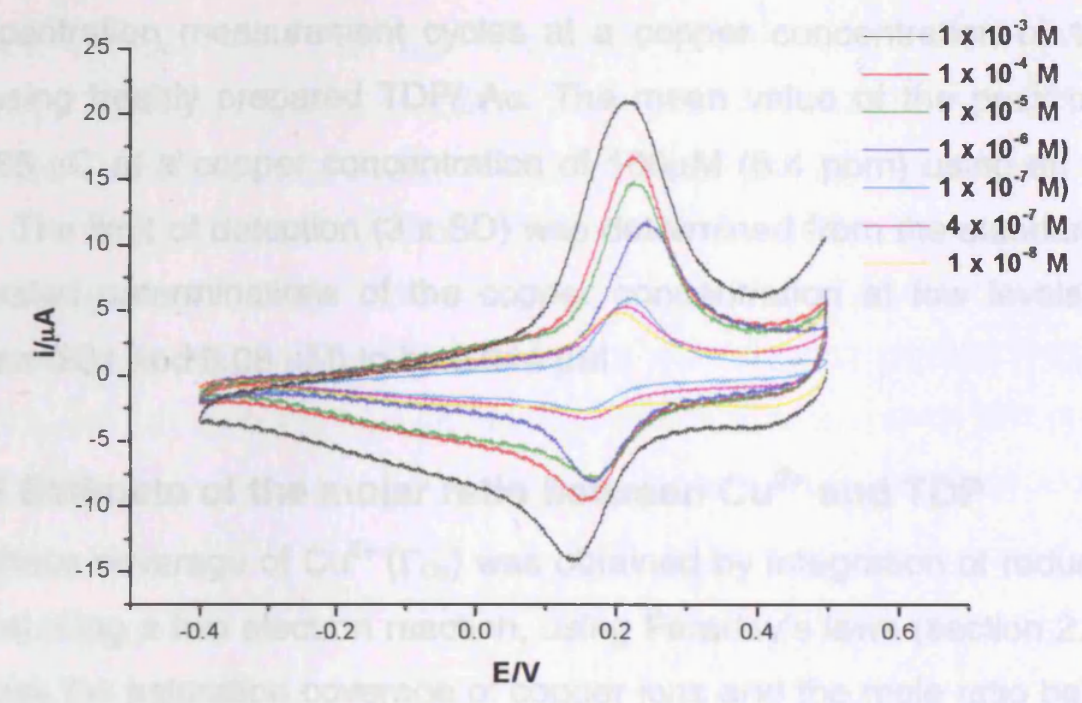

Figure 6.7: Cyclic voltammograms in $\mathrm{Cu}^{2+}$-free $\mathrm{KNO}_{3}(\mathrm{aq})$ using 20min open circuit preconcentration at a TDP SAM modified gold electrode exposed to $1 \times 10^{-8}, 4 \times 10^{-7}, 1 \mathrm{x}$ $10^{-7}, 1 \times 10^{-6}, 1 \times 10^{-5}, 1 \times 10^{-4}$ and $1 \times 10^{-3} \mathrm{M} \mathrm{Cu}^{2+}$, scan rate $100 \mathrm{mV} \mathrm{s}^{-1}$.

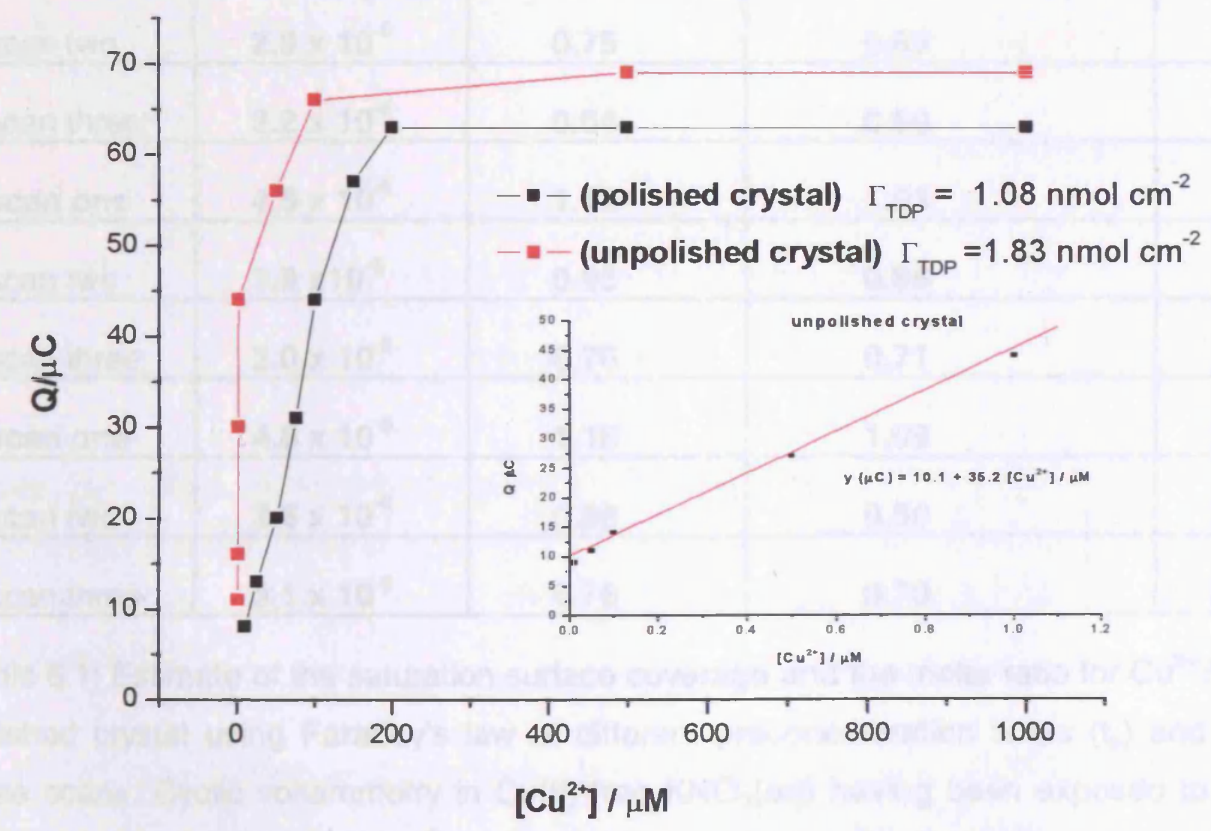

Figure 6.8: Cathodic peak charge response of incorporated copper species as a function of copper concentration at two different surface coverage of TDP on polished and unpolished crystals. Common conditions: Cyclic voltammetry in $\mathrm{Cu}(\mathrm{II})$-free $\mathrm{KNO}_{3}(\mathrm{aq})$ having been exposed to different concentrations (from 0.01 to $1000 \mu \mathrm{M}$ ) of $\mathrm{Cu}(\mathrm{III})_{\text {(aq) }}$. Scan rate $100 \mathrm{mV} \mathrm{s}^{-1}$, preconcentration time $20 \mathrm{~min}$. 
The variability was found to be less than 5\% R.S.D for six replicate preconcentration measurement cycles at a copper concentration of $100 \mu \mathrm{M}(6.4$ ppm) using freshly prepared TDP/ Au. The mean value of the peak charge was about $65 \mu \mathrm{C}$ at a copper concentration of $100 \mu \mathrm{M}(6.4 \mathrm{ppm})$ using an unpolished crystal. The limit of detection ( $3 \times$ SD) was determined from the standard deviation of repeated determinations of the copper concentration at low levels of copper (between 0.01 and $0.08 \mu \mathrm{M}$ ) to be $0.014 \mu \mathrm{M}$.

\subsubsection{Estimate of the molar ratio between $\mathrm{Cu}^{2+}$ and TDP}

The surface coverage of $\mathrm{Cu}^{2+}\left(\Gamma_{\mathrm{Cu}}\right)$ was obtained by integration of reduction peaks and, assuming a two electron reaction, using Faraday's laws (section 2.7.2). Table 6.1 shows the saturation coverage of copper ions and the mole ratio between $\mathrm{Cu}^{2+}$ and TDP at different preconcentration times.

\begin{tabular}{|c|c|c|c|c|}
\hline Scans & $\mathrm{Q} / \mu \mathrm{C}$ & $\Gamma_{\mathrm{Cu}} \mathrm{nmol} \mathrm{cm}{ }^{-2}$ & Molar ratio $\left(\mathrm{Cu}^{2+}: \mathrm{TDP}\right)$ & $\mathrm{t}_{\mathrm{p}}$ \\
\hline scan one & $3.3 \times 10^{-6}$ & 0.80 & 0.74 & $8 \mathrm{~min}$ \\
\hline scan two & $2.9 \times 10^{-6}$ & 0.75 & 0.65 & $8 \mathrm{~min}$ \\
\hline scan three & $2.2 \times 10^{-6}$ & 0.54 & 0.50 & $8 \mathrm{~min}$ \\
\hline scan one & $4.5 \times 10^{-6}$ & 1.12 & 1.03 & $14 \mathrm{~min}$ \\
\hline scan two & $3.8 \times 10^{-6}$ & 0.93 & 0.86 & $14 \mathrm{~min}$ \\
\hline scan three & $3.0 \times 10^{-6}$ & 0.76 & 0.71 & $14 \mathrm{~min}$ \\
\hline scan one & $4.8 \times 10^{-6}$ & 1.18 & 1.09 & $20 \mathrm{~min}$ \\
\hline scan two & $3.6 \times 10^{-6}$ & 0.88 & 0.80 & $20 \mathrm{~min}$ \\
\hline scan three & $3.1 \times 10^{-6}$ & 0.76 & 0.70 & $20 \mathrm{~min}$ \\
\hline
\end{tabular}

Table 6.1: Estimate of the saturation surface coverage and the molar ratio for $\mathrm{Cu}^{2+} /$ TDP on polished crystal using Faraday's law at different preconcentration times $\left(t_{p}\right)$ and the first three scans. Cyclic voltammetry in $\mathrm{Cu}(11)$-free $\mathrm{KNO}_{3}(\mathrm{aq})$ having been exposed to $1 \mathrm{mM}$ of $\mathrm{Cu}(\mathrm{II})_{(\mathrm{aq})}$. Scan rate $100 \mathrm{mV} \mathrm{s}^{-1}$. The surface coverage of the ligand was estimated using QCM frequency measurements and equal to $1.08 \mathrm{nmol} \mathrm{cm}^{-2}$.

The saturation surface coverage of copper on a polished crystal obtained from $1 \mathrm{mM} \mathrm{Cu}^{2+}$ solution (see table 6.1 ), is $1.31 \mathrm{nmol} \mathrm{cm}^{-2}$. It was found that the ratio of the saturation coverage of $\mathrm{Cu}^{2+}$ to TDP was about 1:1. The best results for the mole ratio between $\mathrm{Cu}^{2+}$ and TDP were obtained at 20 min preconcentration time. 
There is a good agreement for the mole ratio between TDP / $\mathrm{Cu}^{2+}$ on polished and unpolished crystals with the QCM frequency measurements, of chapter five; comparison between QCM and EQCM data will be made in separate section. Figure 6.9 shows the change in the mole ratio between the copper ions and TDP with number of scans at a scan rate of $100 \mathrm{mV} \mathrm{s}^{-1}$. From the first until the sixteenth cycle, the mean value of the mole ratio between $\mathrm{Cu}^{2+}$ and TDP is $0.94 \pm 0.1$, which indicates that the incorporated $\mathrm{Cu}^{2+}$ ions are not released quickly from the TDP modified electrode on potential cycling. Figure 6.9 also suggests that one layer of copper is firstly bound and 0.3-0.4 of a layer is loosely bound and lost upon cycling.

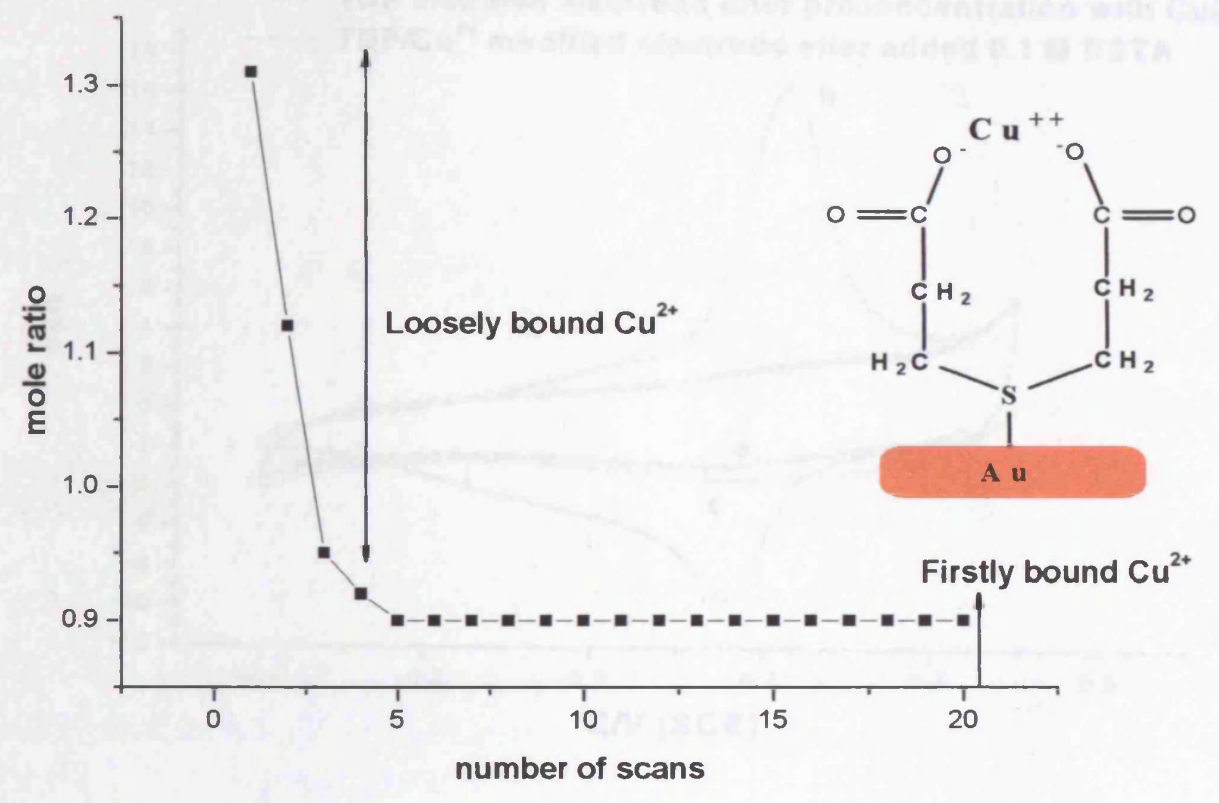

Figure 6.9: Effect of number of scans on the mole ratio between $\mathrm{Cu}^{2+}$ and TDP SAM. Solution conditions: Cyclic voltammetry in $\mathrm{Cu}(\mathrm{II})$-free $\mathrm{KNO}_{3}(\mathrm{aq})$ (having been exposed to $1 \mathrm{mM})$ of $\mathrm{Cu}(\mathrm{II})_{\text {(aq) }}$. Scan rate $100 \mathrm{mV} \mathrm{s}^{-1}$. Preconcentration time $=20 \mathrm{~min}$; open circuit; temperature $25 \mathrm{C}^{\circ}$.

The TDP SAM modified electrodes could easily be regenerated for repeated use. The incorporated copper species were readily removed from the TDP SAM modified electrode by simple immersion of the TDP/ $\mathrm{Cu}^{2+}$ in $0.1 \mathrm{M}$ EDTA solutions for a few minutes and washing with ultra pure water. A cyclic voltammogram almost identical to figure $6.1 \mathrm{~b}$ was obtained after addition of EDTA, indicating that the incorporated $\mathrm{Cu}^{2+}$ had been completely removed from the TDP modified electrode (see Figure 6.10). Then the regenerated TDP could be applied for repeated copper determinations without appreciable deterioration in the modified electrode 
response. QCM measurements were applied to estimate the frequency shift for the TDP modified electrode before and after TDP modified electrode regeneration. The regenerated TDP modified electrode was used for one experiment because the integrated charge for incorporated $\mathrm{Cu}^{2+}$ decreased with the number of repetitive cycles. A decrease of about $30 \%$ in peak charge was obtained after five successive preconcentration measurements. The decrease in the integrated charge resulting from the copper ion incorporated in the TDP SAM was possibly caused by a decrease of SAM coverage after repeating the cycling.

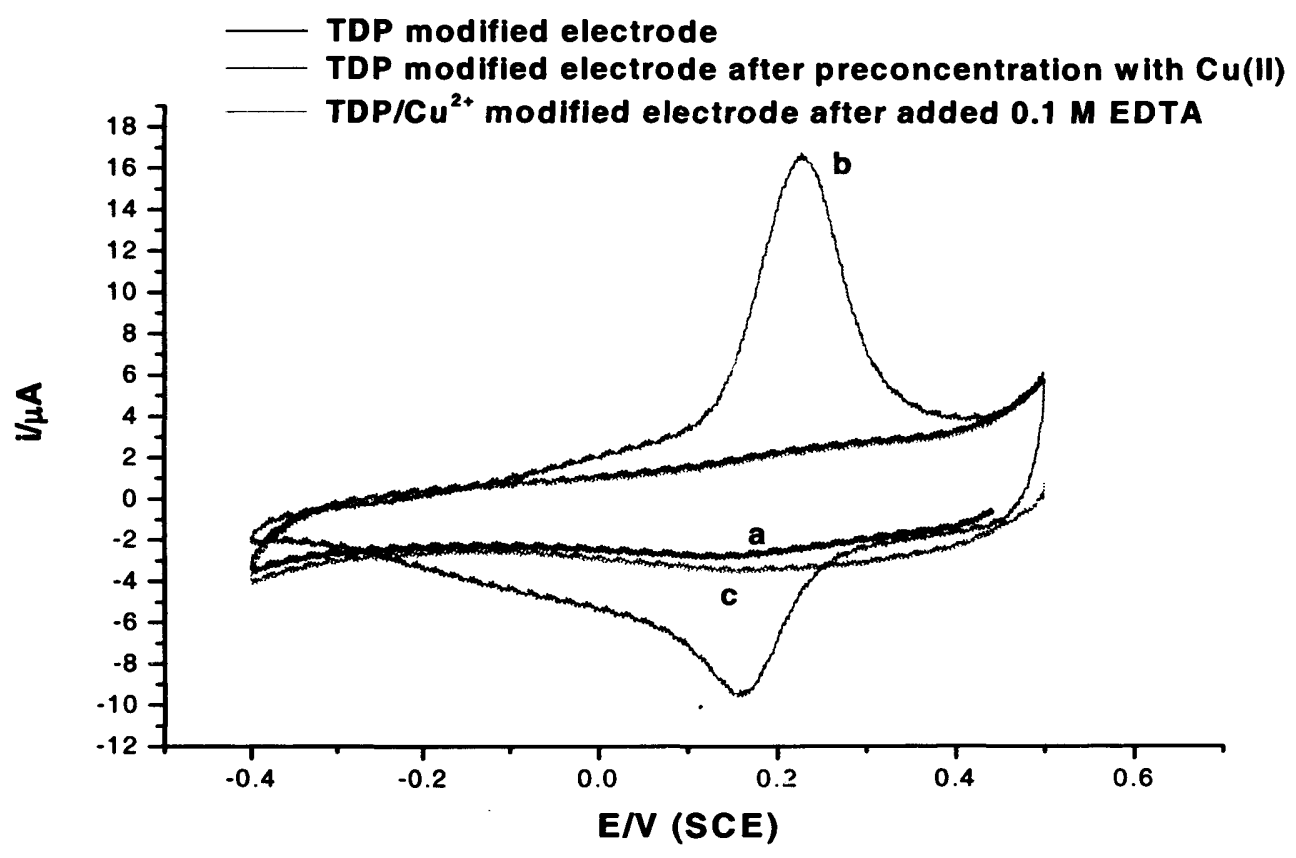

Figure 6.10: Cyclic voltammogram of (TDP) modified Au SAM electrode (a) TDP on Au in $\mathrm{Cu}(\mathrm{II})$-free $\mathrm{KNO}_{3(\mathrm{aq})}$, (b) SAM on $\mathrm{Au}$ in $\mathrm{Cu}(\mathrm{II})$-free $\mathrm{KNO}_{3}(\mathrm{aq})$ having been exposed to $1 \mathrm{mM}$ $\mathrm{Cu}(\mathrm{II})_{(\mathrm{aq})}$ (c) after added $0.1 \mathrm{M}$ EDTA to TDP/Cu $\mathrm{Cu}^{2+}$ modified electrode. Scan rate $100 \mathrm{mV} \mathrm{s}^{-1}$.

\subsubsection{Incorporation mechanism of copper ions on TDP SAM electrode}

The results discussed above show that the preconcentration of copper ions at a TDP SAM modified Au electrode was directly related to the TDP itself rather than the Au bare electrode. The mechanism of the TDP modified Au electrode and $\mathrm{Cu}^{2+}$ reactions is attributed to the following steps: firstly, the complexation of $\mathrm{Cu}^{2+}$ from aqueous solutions to the TDP on the surface of the gold via modification of the surface with TDP SAM at open circuit as can be seen in figure (6.11). Secondly, chemically accumulated $\mathrm{Cu}^{2+}$ ions are reduced to $\mathrm{Cu}^{0}$ at $-0.4 \mathrm{~V}$ as can be seen in figure (6.12). 


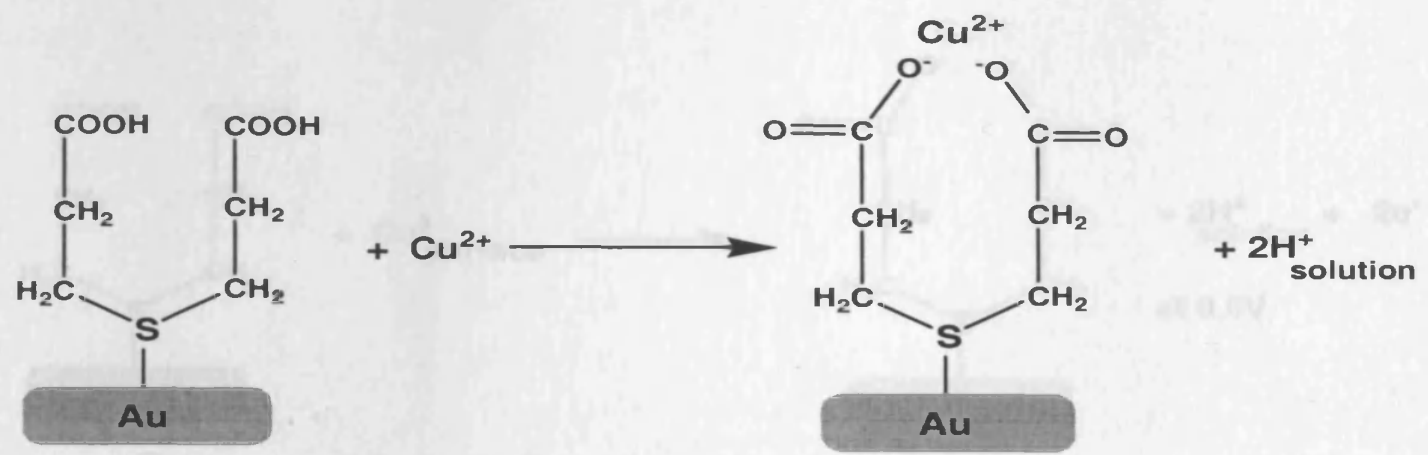

Figure 6.11: Preconcentration step at open circuit for the accumulation $\mathrm{Cu}^{2+}$ from the aqueous solution to the surface of TDP SAM modified gold electrode at pH 5.5.

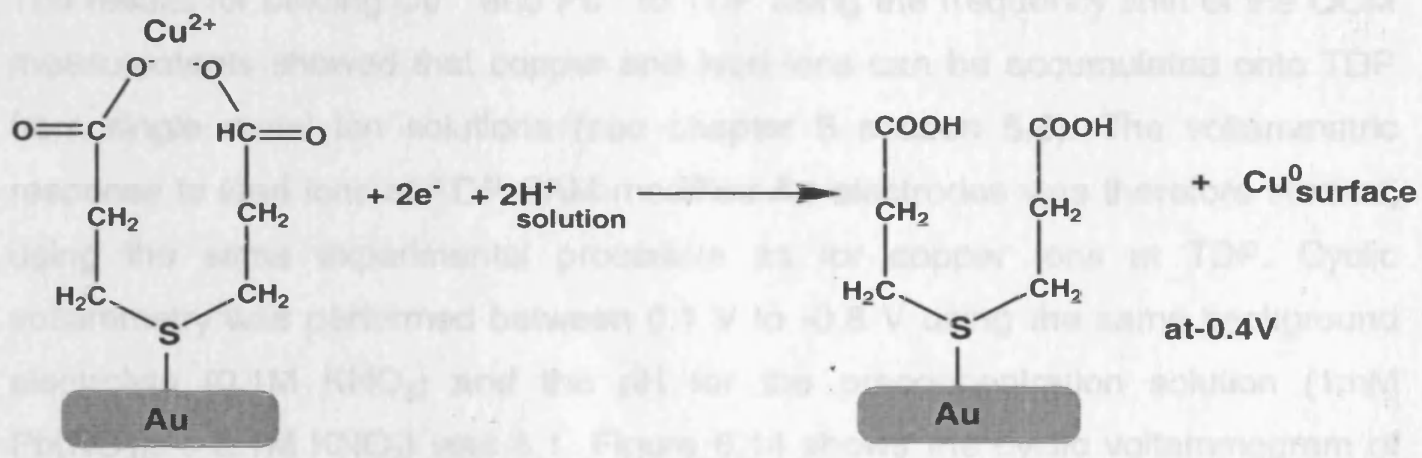

Figure 6.12: Reduction of accumulated $\mathrm{Cu}^{2+}$ ions on the TDP SAM to $\mathrm{Cu}^{0}$ at $-0.4 \mathrm{~V}$ during the initial potential scan. The location of the $\mathrm{Cu}^{0}$ (as clusters, islands, etc) is not known.

In the final step the reduced $\mathrm{Cu}^{0}$ species were oxidized to $\mathrm{Cu}^{2+}$ at $0.5 \mathrm{~V}$ which was accumulated and recomplexed by the TDP carboxylate groups as can be seen in figure 6.13. The anodic peak current corresponds to the oxidation of copper metal to $\mathrm{Cu}^{2+}$ and recomplexed with the TDP modified electrode. 


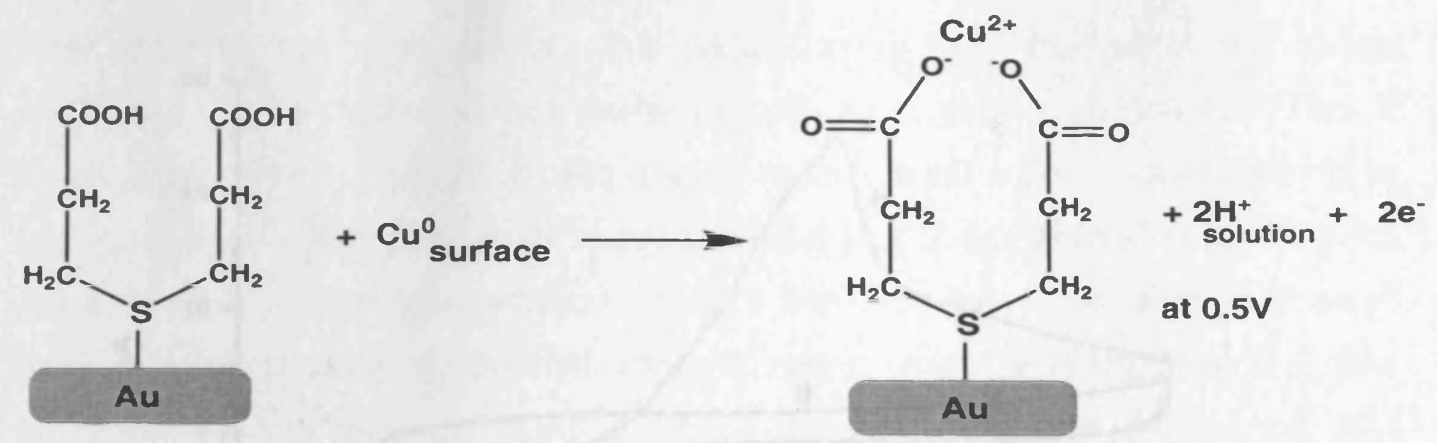

Figure 6.13: Oxidation of the surface bound copper (which was chemically accumulated as $\mathrm{Cu}(\mathrm{II}) / \mathrm{TDP}$ complex on Au electrode surface during the final potential scan (0.5 V).

\subsubsection{Voltametric response of the Au/TDP electrode to $\mathrm{Pb}(\mathrm{II})$ ions}

The results for binding $\mathrm{Cu}^{2+}$ and $\mathrm{Pb}^{2+}$ to TDP using the frequency shift of the $\mathrm{QCM}$ measurements showed that copper and lead ions can be accumulated onto TDP from single metal ion solutions (see chapter 5 section 5.6 ). The voltammetric response to lead ions at TDP SAM modified Au electrodes was therefore studied, using the same experimental procedure as for copper ions at TDP. Cyclic yoltammetry was performed between $0.1 \mathrm{~V}$ to $-0.8 \mathrm{~V}$ using the same background electrolyte $\left(0.1 \mathrm{M} \mathrm{KNO}_{3}\right)$ and the $\mathrm{pH}$ for the preconcentration solution (1mM $\left.\mathrm{Pb}\left(\mathrm{NO}_{3}\right)_{2}+0.1 \mathrm{M} \mathrm{KNO}_{3}\right)$ was 5.1. Figure 6.14 shows the cyclic voltammogram of TDP SAM modified Au electrode (a) before and (b) after accumulation in $1 \mathrm{mM} \mathrm{Pb}^{2+}$ in $0.1 \mathrm{M} \mathrm{KNO}_{3}(\mathrm{pH} 5.1)$ for $30 \mathrm{~min}$ in lead-free nitrate solution ( $\mathrm{pH} 5.5$ ).

In the absence of $\mathrm{Pb}^{2+}$ in the accumulation solution, the electrode was inactive between 0.1 to $-0.8 \mathrm{~V}$. After a preconcentration of $\mathrm{Pb}(\mathrm{II})$ for $30 \mathrm{~min}$, well defined peaks were observed (see Figure 6.14b), at $E_{p, a}=-0.250 \mathrm{~V}$ and $E_{p, c}=-0.375 \mathrm{~V}$. The average of these two values is very close to $E^{0}=-0.31 \mathrm{~V}$ for the $\mathrm{Pb}^{2+} / \mathrm{Pb}^{0}$ couple[8]. Successful binding of $\mathrm{Pb}(\mathrm{II})$ to the TDP modified electrode surface is clearly evidenced by the presence of a well defined lead reduction peak. The anodic peak was sharper than the cathodic peak. In the same experiment, repeated cyclic scans between 0.1 and $-0.8 \mathrm{~V}$ at $300 \mathrm{mV} \mathrm{s}^{-1}$ showed the anodic and cathodic peaks did not disappear after twenty cycles, (see Figure 6.15). These results indicate that lead remains strongly adsorbed in both oxidation states $\left(\mathrm{Pb}^{2+}\right.$ and $\mathrm{Pb}^{0}$ ) within the potential range of the experiment. 


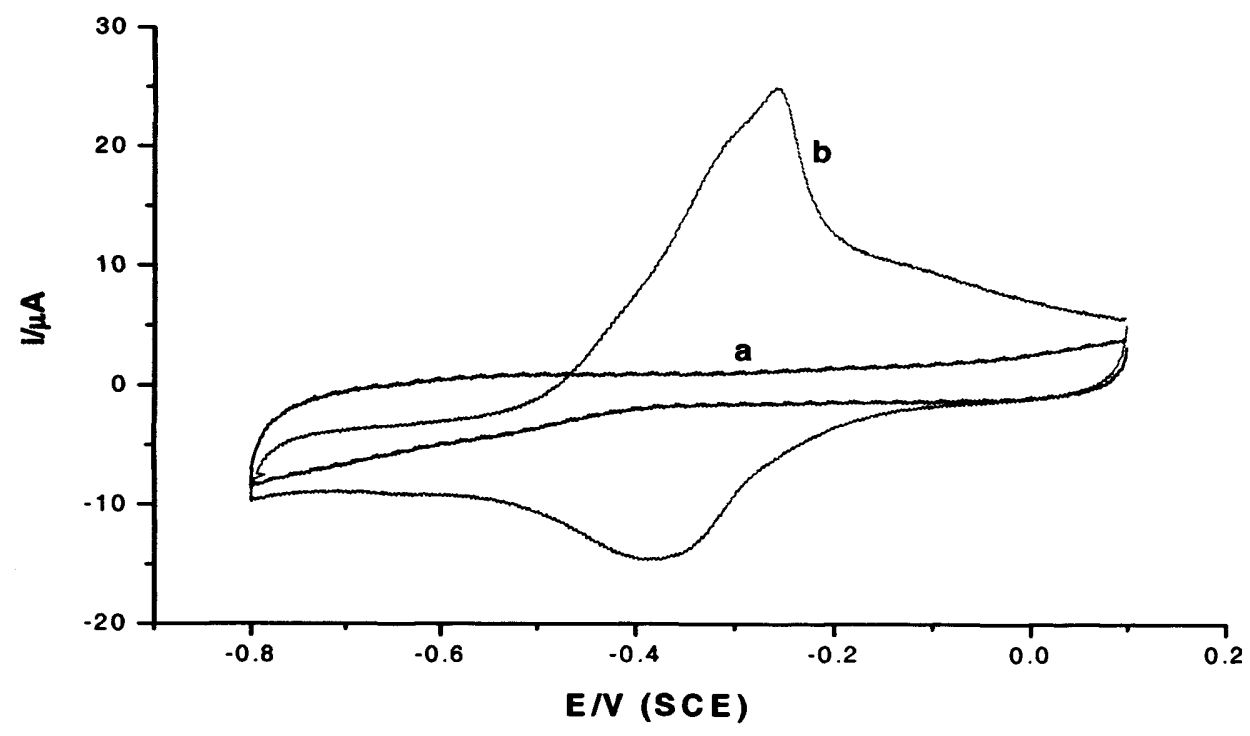

Figure 6.14: Cyclic voltammograms of (TDP) modified Au SAM electrode for the detection of $\mathrm{Pb}$ (II) ions measured in $0.1 \mathrm{M} \mathrm{KNO}_{3}$ lead-free nitrate ( $\mathrm{pH}$ 5.5). (a) SAM on Au before accumulated of $\mathrm{Pb}$ (II) and (b) after accumulation in $1 \mathrm{mM}$ of $\mathrm{Pb}$ (II) for $30 \mathrm{~min}$ preconcentration time. Scan rate $100 \mathrm{mV} \mathrm{s}^{-1}$.

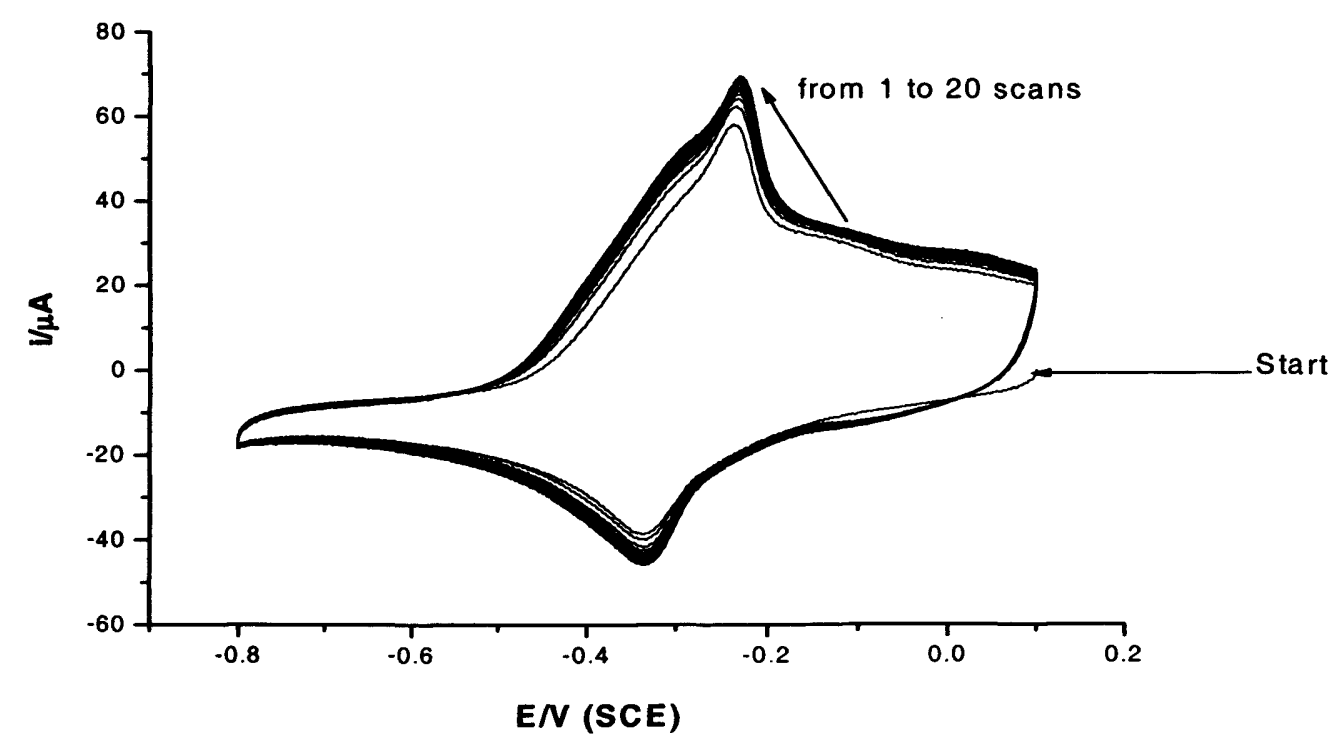

Figure 6.15: Repetitive sweep cyclic voltammograms of TDP modified Au SAM electrode after accumulating $\mathrm{Pb}(\mathrm{II})_{(\mathrm{aq})}$ ions in lead-free $0.1 \mathrm{M} \mathrm{KNO}_{3(\mathrm{aq})}(\mathrm{pH} 5.5)$ using unpolished crystal. From $1^{\text {st }}$ to $20^{\text {th }}$ cycles at $300 \mathrm{mV} \mathrm{s}^{-1}$, preconcentration time $30 \mathrm{~min}$ in $\left(1 \mathrm{mM} \mathrm{Pb}\left(\mathrm{NO}_{3}\right)_{2}\right.$ $+0.1 \mathrm{M} \mathrm{KNO}_{3}$ ). 
The effect of the scan rate on the peak current was studied in the range $5-900 \mathrm{mV} \mathrm{s}^{-1}$. The peak current increased with increasing scan rate (see Figure 6.16). The peak separation in this Figure could result from a quasi-reversible surface reaction. However, even in this case the plot of anodic and cathodic peak currents versus scan rate yielded a straight line with the same slope and small peak separations between the cathodic and anodic peaks (see the inset of Figure 6.16 ), indicating a reversible surface redox reaction of adsorbed species and fast electron transfer to redox active species on the TDP SAM modified Au electrode. Continuous cycling of the redox couples at slower scan rates from 5 to $70 \mathrm{mV} \mathrm{s}^{-1}$ results in the formation of additional waves. This is evidence of metal ion speciation at TDP SAM modified Au electrode. Figure 6.17 shows the effect of lower scan rates on the anodic and cathodic peaks for the redox couple and the mass change $(\Delta \mathrm{m})$ during the cycling. It was found that at scan rates of 70 and100 $\mathrm{mV} \mathrm{s}^{-1}$, two peaks appeared. The mass change during the oxidation and reduction of the $\mathrm{Pb}(\mathrm{II})$ at TDP SAM is 207 and $155 \mathrm{ng}$ for the same surface coverage of TDP $140 \mathrm{ng}$ at scan rates 70 and $100 \mathrm{mV} \mathrm{s}^{-1}$, respectively. This fact might be due to the incorporation between $\mathrm{Pb}^{2+}$ and negatively charged carboxylate groups for TDP when the reduction and oxidation steps are occurred.

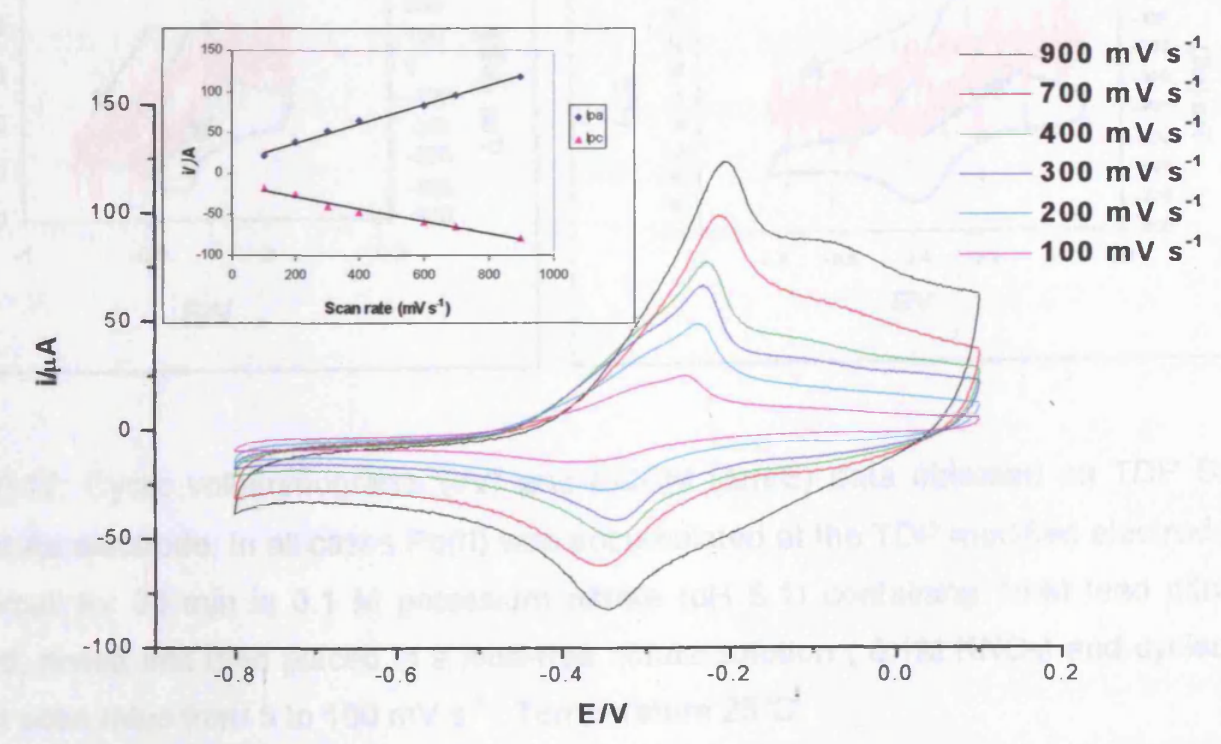

Figure 6.16: The effect of the variation sweep rate from 100 to $900 \mathrm{mV} \mathrm{s}^{-1}$ on the $\mathrm{Pb}$ (II) peak current using the same conditions in Figure 6.14. Inset: the dependence of the anodic and cathodic peak current on sweep rate. 

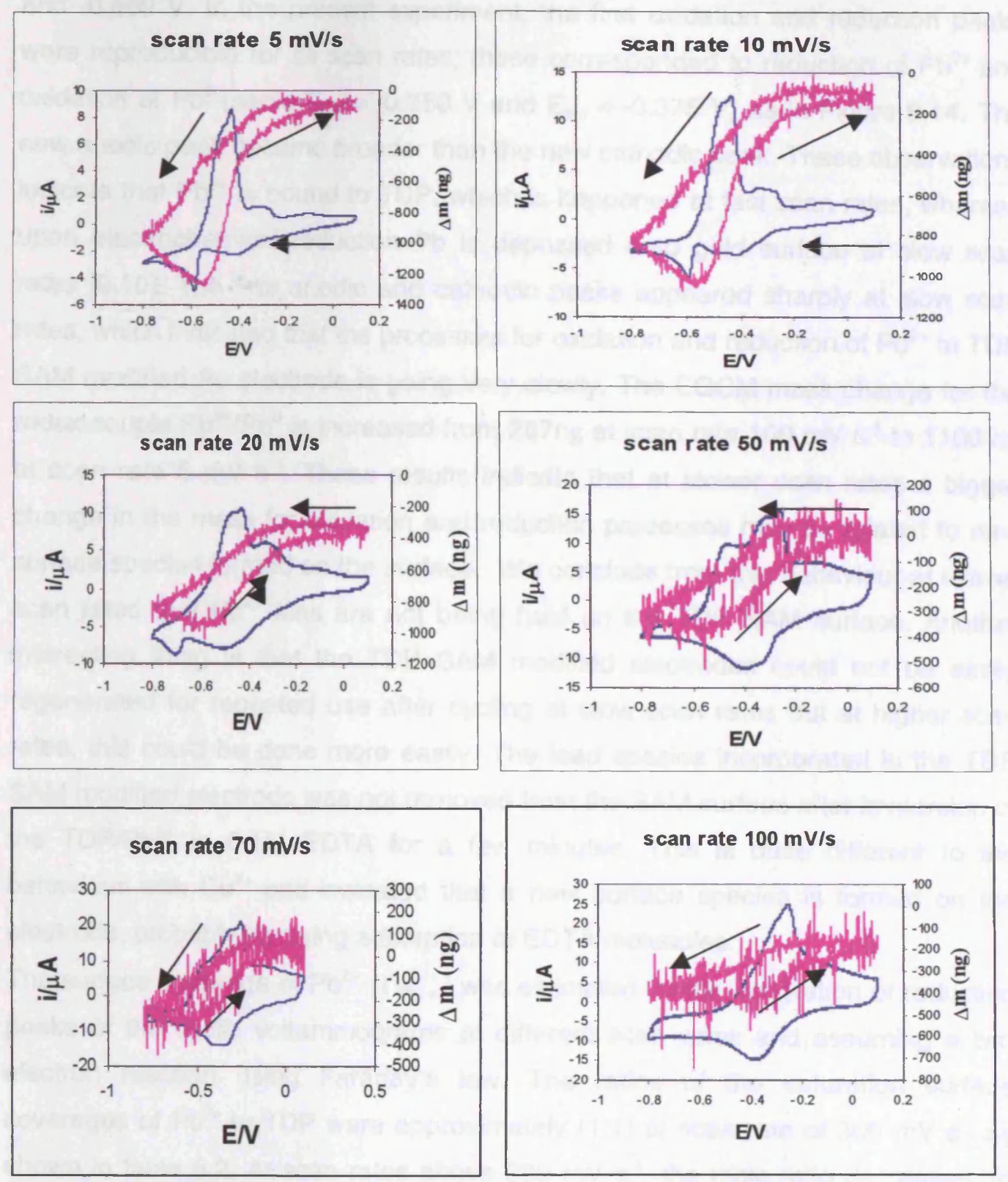

Figure 6.17: Cyclic voltammograms (i $N)$ and $\operatorname{EQCM}(\Delta \mathrm{m} / \mathrm{E})$ data obtained on TDP SAM modified $\mathrm{Au}$ electrode. In all cases $\mathrm{Pb}$ (II) was accumulated at the TDP modified electrode at open circuit for $30 \mathrm{~min}$ in $0.1 \mathrm{M}$ potassium nitrate $(\mathrm{pH} 5.1)$ containing $1 \mathrm{mM}$ lead nitrate, removed, rinsed and then placed in a lead-free nitrate solution $\left(0.1 \mathrm{M} \mathrm{KNO}_{3}\right)$ and cycled at different scan rates from 5 to $100 \mathrm{mV} \mathrm{s}^{-1}$. Temperature $25^{\circ} \mathrm{C}$.

Upon further decreasing the scan rate to $50 \mathrm{mV} \mathrm{s}^{-1}$, there was a dramatic change in the voltammogram shape. Two anodic peaks appeared (at $-0.255 \mathrm{~V}$ and $-0.440 \mathrm{~V}$ ) with one cathodic peak at $-0.375 \mathrm{~V}$. At 5,10 and $20 \mathrm{mV} \mathrm{s}^{-1}$ two anodic peaks appeared, at $-0.255 \mathrm{~V}$ and $-0.440 \mathrm{~V}$ and two cathodic peaks appeared at $-0.375 \mathrm{~V}$ 
and $-0.589 \mathrm{~V}$. In the present experiment, the first oxidation and reduction peaks were reproducible for all scan rates; these corresponded to reduction of $\mathrm{Pb}^{2+}$ and oxidation of $\mathrm{Pb}^{0}$ (same $\mathrm{E}_{\mathrm{p}, \mathrm{a}}=-0.250 \mathrm{~V}$ and $\mathrm{E}_{\mathrm{p}, \mathrm{c}}=-0.375 \mathrm{~V}$ ) as in Figure 6.14. The new anodic peak became broader than the new cathodic peak. These observations indicate that $\mathrm{Pb}^{2+}$ is bound to TDP, which is happened at fast scan rates, whereas upon electrochemical reduction $\mathrm{Pb}$ is deposited onto gold surface at slow scan rates $[9,10]$. The first anodic and cathodic peaks appeared sharply at slow scan rates, which indicated that the processes for oxidation and reduction of $\mathrm{Pb}^{2+}$ at TDP SAM modified Au electrode is going very slowly. The EQCM mass change for the redox couple $\mathrm{Pb}^{2+} / \mathrm{Pb}^{0}$ is increased from $207 \mathrm{ng}$ at scan rate $100 \mathrm{mV} \mathrm{s}^{-1}$ to $1100 \mathrm{ng}$ at scan rate $5 \mathrm{mV} \mathrm{s} \mathrm{s}^{-1}$. These results indicate that at slower scan rates a bigger change in the mass for oxidation and reduction processes may be related to new surface species formed on the surface. We conclude from this behaviour at slower scan rates that $\mathrm{Pb}^{2+}$ ions are not being held on the TDP SAM surface. Another interesting thing is that the TDP SAM modified electrodes could not be easily regenerated for repeated use after cycling at slow scan rates but at higher scan rates, this could be done more easily. The lead species incorporated in the TDP SAM modified electrode was not removed from the SAM surface after immersion of the $\mathrm{TDP} / \mathrm{Pb}^{2+}$ in $0.1 \mathrm{M}$ EDTA for a few minutes. This is quite different to the behaviour with $\mathrm{Cu}^{2+}$ and indicated that a new surface species is formed on the electrode, probably involving adsorption of EDTA molecules.

The surface coverage of $\mathrm{Pb}^{2+}\left(\Gamma_{\mathrm{Pb}++}\right)$ was estimated by the integration of reduction peaks of the cyclic voltammograms at different scan rates and assuming a two electron reaction using Faraday's law. The ratios of the saturation surface coverages of $\mathrm{Pb}^{2+}$ to TDP were approximately (1:1) at scan rate of $300 \mathrm{mV} \mathrm{s}^{-1}$ as shown in table 6.2. At scan rates above $300 \mathrm{mV} \mathrm{s}^{-1}$, the mole ratio decreases, to 0.6 at $900 \mathrm{mV} \mathrm{s}^{-1}$. The comparison between the variation of the mole ratio between copper and lead ions with TDP SAM are shown in figure 6.17. One can see that the mole ratio between copper ions and TDP equal to about one independent of scan rate. The behaviour for lead ions at TDP is somewhat different.

The cyclic voltammogram of an electrode modified with TDP SAM measured in cadmium-free nitrate $\left(0.1 \mathrm{M} \mathrm{KNO}_{3}\right)$. After accumulation of $\mathrm{Cd}^{2+}(1 \mathrm{mM})$ for $30 \mathrm{~min}$ no distinct peaks for $\mathrm{Cd}^{2+} / \mathrm{Cd}^{0}$ redox chemistry were obtained. Both QCM measurements and cyclic voltammetry show that the ligand has a weak selectivity to cadmium ions. 


\begin{tabular}{|c|c|c|c|c|}
\hline Scan rate & $\mathbf{Q} / \mu \mathrm{C}$ & $\Gamma_{\mathrm{Pb}} / \mathbf{n m o l ~ \mathbf { ~ m } ^ { - 2 }}$ & $\Gamma_{\mathrm{TDP}} / \mathbf{\mathrm { nmol } \mathrm { cm }} \mathbf{c}^{-2}$ & $\begin{array}{l}\text { Mole ratio } \\
\mathbf{P b}: \text { TDP }\end{array}$ \\
\hline $300 \mathrm{mV} / \mathrm{s}$ & 78 & 1.92 & 1.82 & 1.05 \\
\hline $200 \mathrm{mV} / \mathrm{s}$ & 72 & 1.70 & 1.82 & 0.93 \\
\hline $100 \mathrm{mV} / \mathrm{s}$ & 63 & 1.55 & 1.82 & 0.85 \\
\hline
\end{tabular}

Table 6.2: Estimate of the saturation surface coverage and the molar ratio for $\mathrm{Pb}^{2+} / \mathrm{TDP}$ on unpolished crystal using Faraday's law at different sweep rates. Cyclic voltammetry in $\mathrm{Pb}(\mathrm{II})$ - free $\mathrm{KNO}_{3}(\mathrm{aq})$ having been exposed to $1 \mathrm{mM}$ of $\mathrm{Pb}(\mathrm{II})_{(\text {aq) }}$.

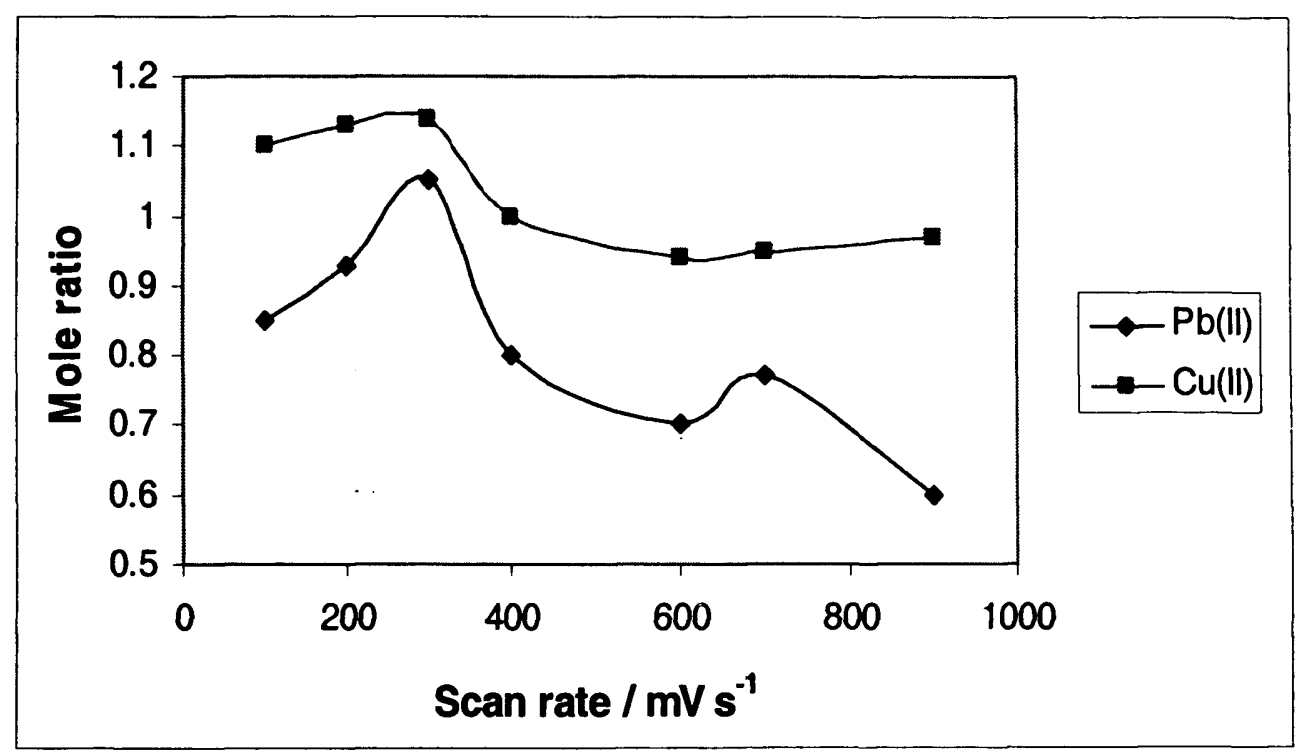

Figure 6.18: Comparison between the variation of the mole ratio $\mathrm{Cu}(\mathrm{II})$ and $\mathrm{Pb}(\mathrm{II})$ to TDP after adsorbing $\mathrm{Cu}(\mathrm{II})$ and $\mathrm{Pb}$ (II) from single metal ion solutions in $\mathrm{Pb}$ (II)-free $\mathrm{KNO}_{3}(0.1 \mathrm{M}$ $\mathrm{KNO}_{3}$ ) at different scan rates from 100 to $900 \mathrm{mV} \mathrm{s}^{-1}$. Common conditions: preconcentration time $20 \mathrm{~min}$ for $\mathrm{Cu}(\mathrm{II})$ and $30 \mathrm{~min}$ for $\mathrm{Pb}(\mathrm{II}), \mathrm{pH}$ 5.5, E vs SCE.; open circuit; temperature $25^{\circ} \mathrm{C}$.

\subsubsection{Interference effects on $\mathrm{Cu}(\mathrm{II})$ responses in binary mixtures}

Cyclic voltammetry shows that both $\mathrm{Cu}^{2+}$ and $\mathrm{Pb}^{2+}$ species are adsorbed at TDP SAMs and they undergo subsequent electroreduction. The interferences were examined by introducing other metal ions into the preconcentration medium under open circuit conditions. The interferences of copper incorporation at TDP SAM modified $\mathrm{Au}$ electrodes from zinc and cadmium ions in binary mixtures have been 
investigated under the optimal conditions previously described. In the present experiment, the peak current for copper ions of a given concentration was studied. Then, 10-fold excess of other metal ions $(\mathrm{Zn}(\mathrm{II})$ and $\mathrm{Cd}(\mathrm{II}))$ were added to the preconcentration solution of copper ions and the changes of current were recorded. Interference studies were conducted by exposing the TDP SAM modified Au electrode in a solution containing $0.08 \mathrm{mM}$ copper nitrate and $1 \mathrm{mM}$ zinc nitrate or $1 \mathrm{mM}$ cadmium nitrate in the presence of $0.1 \mathrm{M} \mathrm{KNO}_{3}$ for $20 \mathrm{~min}$ at open circuit. Voltammetric responses resulting from the presence of $\mathrm{Zn}(\mathrm{II})$ or $\mathrm{Cd}(\mathrm{II})$ in binary mixtures were then compared with that obtained for copper ion solution. Experimental results are shown in figure 6.19. For the pure copper nitrate single solution test, the reduction peak charge was $27 \mu \mathrm{C}$. In the presence of cadmium or zinc at $1 \mathrm{mM}$, the peak charge was 25.5 and $26.7 \mu \mathrm{C}$, respectively. The deceases in the peak charge responses for copper ions in the presence of 10-fold excess of cadmium and zinc ions were $5.6 \%$ and $1.1 \%$ respectively. No additional voltammetric peak from these interferants was observed in binary mixtures.

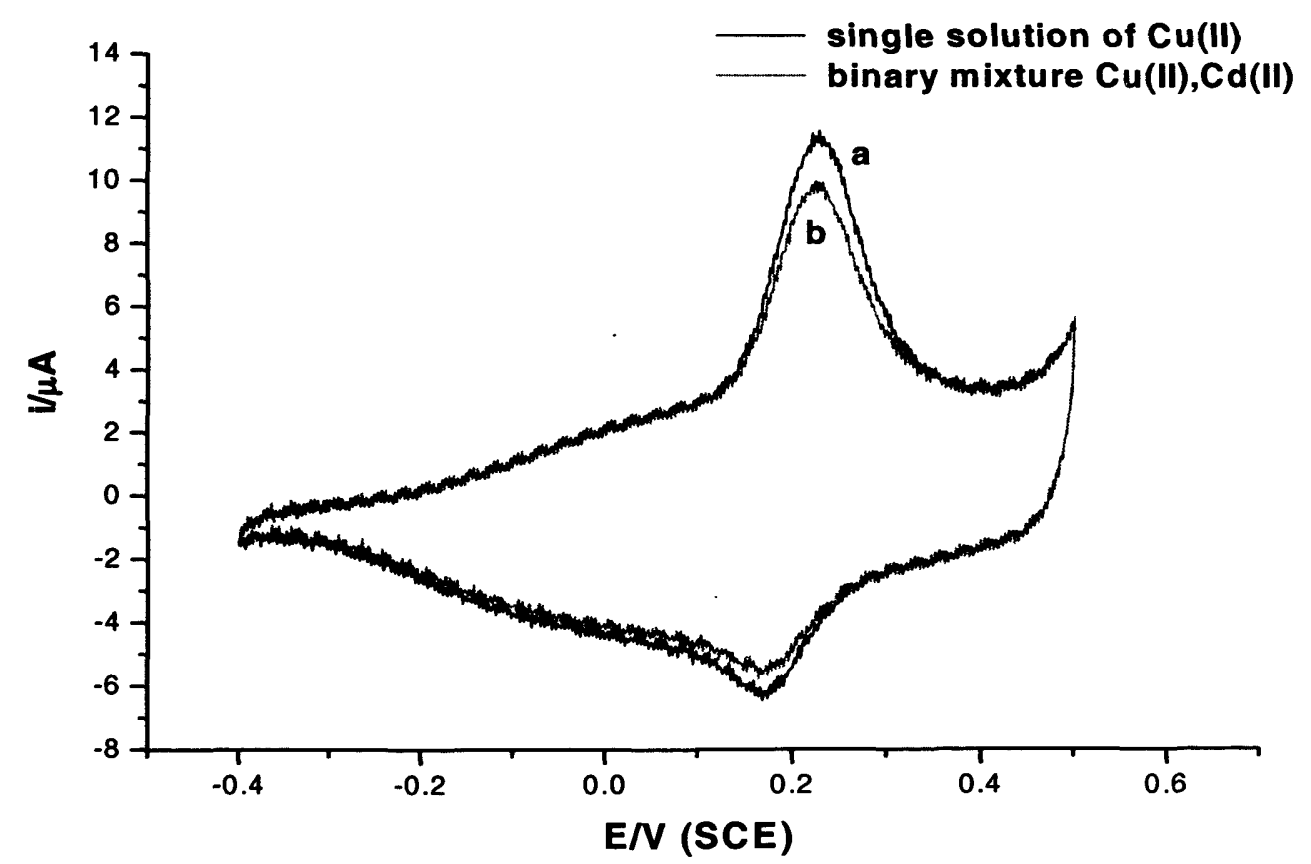

Figure 6.19: Cyclic voltammograms of the TDP SAM modified Au electrode in $0.1 \mathrm{M} \mathrm{KNO}_{3}$ (a) after adsorbing copper ions from $0.08 \mathrm{mM}$ copper nitrate (b) after exposure to a solution containing both $0.08 \mathrm{mM} \mathrm{Cu}(\mathrm{II})$ and $1 \mathrm{mM} \mathrm{Cd}(\mathrm{II})$. Common conditions: preconcentration time $20 \mathrm{~min}$; $\mathrm{pH}$ 5.5; open circuit; scan rate $100 \mathrm{mV} \mathrm{s}^{-1}$.

The fact that no significant interference was observed from $\mathrm{Zn}^{2+}$ and $\mathrm{Cd}^{2+}$ provides a strong indication that $\mathrm{Cu}^{2+}$ binds more strongly to the TDP, with a higher affinity 
constant than $\mathrm{Zn}^{2+}$ or $\mathrm{Cd}^{2+}$. This is consistent with the binding constants were extracted using QCM measurements in section (5.6) of chapter five.

\subsection{Complexation between 3-thiodisuccinic acid and $\mathrm{Cd}^{2+}$}

The complexation between cadmium, lead or copper ions with 3-thiodisuccinic acid was studied previously in chapter five using the QCM. In this section the electrochemical behaviour of cadmium, lead and copper ions at 3-thiodisuccinic acid (TDS) modified electrodes will be studied. When the fresh TDS modified Au electrode is immersed in a freshly prepared mixture of three metal ions, a competition involving co-existing species occur as described in chapter five. On one hand, TDS can adsorb on the gold surface spontaneously due to strong chemical interactions between the sulphur and gold. On the other hand, the four free terminal carboxlate groups can coordinate with $\mathrm{Cd}^{2+}$. However, one question can be answered with electrochemical measurements for TDS/Cd $\mathrm{d}^{2+}$, the stability of coordination of $\mathrm{Cd}^{2+}, \mathrm{Pb}^{2+}$ and $\mathrm{Cu}^{2+}$ with free terminal carboxylate groups. The ratio of $\mathrm{Cd}^{2+}$ : TDS is also important in determining the assembling of the TDS and the formation of recognition cavities.

\subsubsection{Experimental}

The experimental procedures were described in section 6.2.1: the preconcentration solution contained $1 \mathrm{mM} \mathrm{Cd}\left(\mathrm{NO}_{3}\right)_{2}+0.1 \mathrm{M} \mathrm{KNO}_{3} \mathrm{pH} 6.5$, the preconcentration time was $10 \mathrm{~min}$ at open circuit, and cyclic voltammograms (CVs) were recorded between 0.1 to $-0.6 \mathrm{~V}$, commencing at $0.1 \mathrm{~V}$; at scan rates from 10 to $800 \mathrm{mV} \mathrm{s}^{-1}$.

\subsubsection{Results and discussions}

The TDS SAM modified Au electrodes were tested for uptake of $\mathrm{Cd}^{2+}$ in solution using open circuit accumulation in electrolyte containing the desired concentration of cadmium ion, followed by placing the electrode in a metal ion-free electrolyte solution and measuring the response by cyclic voltammetry. Figure 6.20 shows typical cyclic voltammograms for the detection of $\mathrm{Cd}^{2+}$ following open circuit accumulation onto a TDS SAM modified Au electrode. Two peaks appeared in the $\mathrm{CV}$, due to the cathodic process $(-0.317 \mathrm{~V})$ and an anodic process $(-0.190 \mathrm{~V})$ with an average formal potential of $-0.253 \mathrm{~V}$. The peak separation $\left(\Delta E=E_{p, a}-E_{p c}\right)$ was $0.127 \mathrm{~V}$ at a scan rate of $100 \mathrm{mV} \mathrm{s}^{-1}$. The redox peaks were ascribed to the redox couple $\mathrm{Cd}^{2+} / \mathrm{Cd}^{0}[11]$. In the absence of $\mathrm{Cd}^{2+}$ in the accumulation solution $(0.1 \mathrm{M}$ $\mathrm{KNO}_{3}$ ), the electrode is inactive between 0.1 to $-0.4 \mathrm{~V}$ as can be seen in Figure 
6.22. The bare gold electrode was used for the alternative stripping analysis of $\mathrm{Cd}(\mathrm{II})$ but no peak current was observed with the bare gold electrode under the above conditions. This indicates that the chemical preconcentration of $\mathrm{Cd}$ (II) does not take place at a bare gold surface.

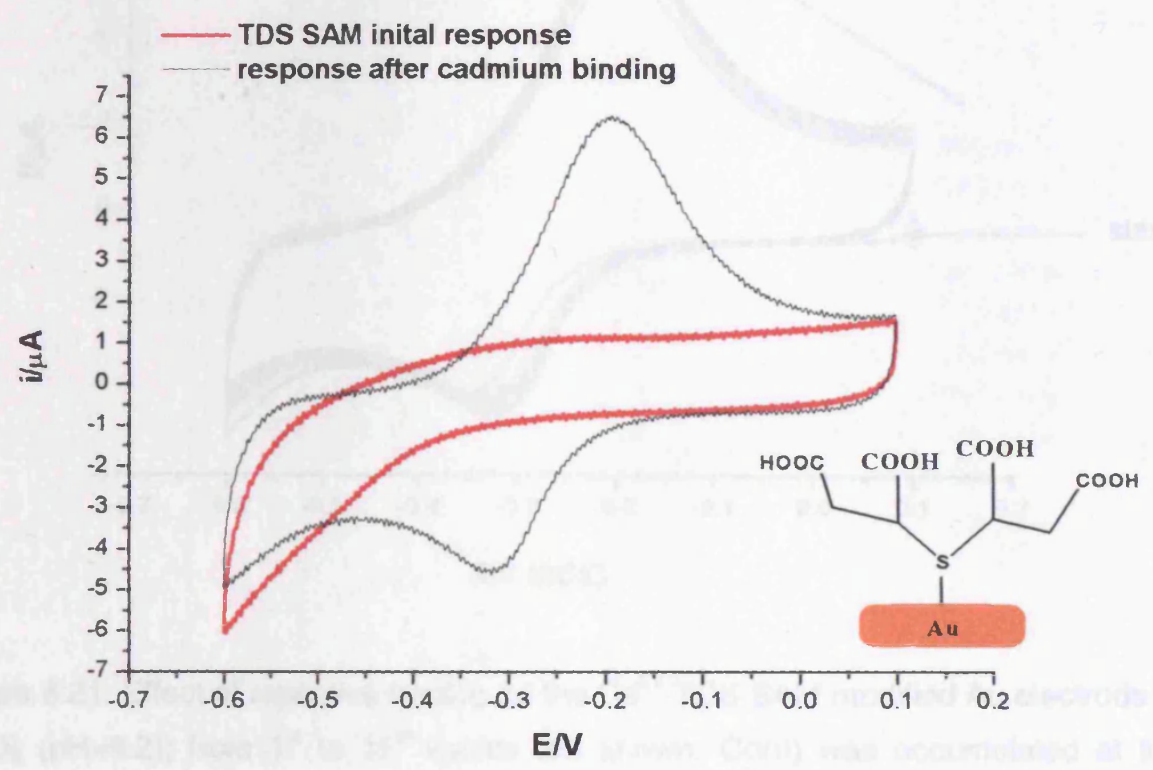

Figure 6.20: Cyclic voltammogram of TDS SAM modified electrode before and after exposure to cadmium in $0.1 \mathrm{M}$ potassium nitrate solution $(\mathrm{pH} 6.5$ ). In all cases $\mathrm{Cd}$ (II) was accumulated at the TDS modified electrode at open circuit for $10 \mathrm{~min}$ in a $0.1 \mathrm{M}$ potassium nitrate solution $(\mathrm{pH} 6.2)$ containing $1 \mathrm{mM}$ cadmium nitrate, removed, rinsed and then placed in a cadmium- free potassium nitrate solution. Sweep rate $100 \mathrm{mV} \mathrm{s}^{-1}$.

\subsubsection{Stability and effect of the sweep rate}

The electrochemistry for the redox couple $\mathrm{Cd}^{2+} / \mathrm{Cd}^{0}$ is reversible and the current is reasonably stable, (see figure 6.21). This figure shows the repetitive cyclic voltammograms from first to fifteenth cycles at scan rate of $100 \mathrm{mV} \mathrm{s}^{-1}$ on an unpolished crystal. However there is a shift in a peak current and the base line after the first cycle. The cathodic peak was shifted from $-0.353 \mathrm{~V}$ to $-0.317 \mathrm{~V}$ in the second cycle and stayed at $-0.317 \mathrm{~V}$ until the fifteenth cycle. During the course of the first voltammetric cycle some rearrangement of the cadmium coordination in the TDS SAM may take place, but thereafter a stable response is recorded upon repetitive cycling in a cadmium-free electrolyte solution. 


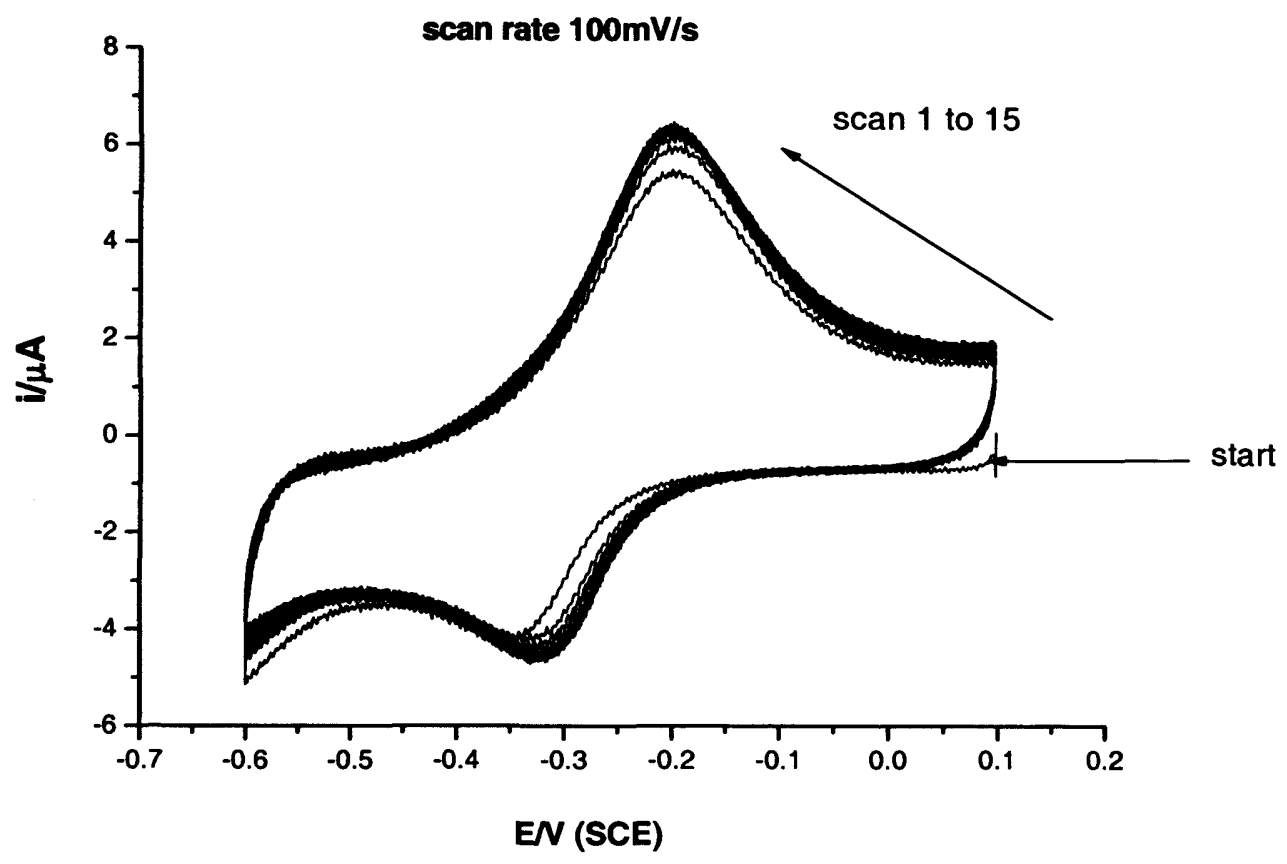

Figure 6.21: Effect of repetitive cycling on the $\mathrm{Cd}^{2+}$-TDS SAM modified Au electrode in 0.1M $\mathrm{KNO}_{3}(\mathrm{pH}=6.2)$; from $1^{\text {st }}$ to $15^{\text {th }}$ cycles are shown. $\mathrm{Cd}(\mathrm{II})$ was accumulated at the TDS modified electrode at open circuit for $10 \mathrm{~min}$ in a $0.1 \mathrm{M}$ potassium nitrate solution $(\mathrm{pH}$ 6.2) containing $1 \mathrm{mM}$ cadmium nitrate, removed, rinsed and then placed in a cadmium-free potassium nitrate solution. Scan rate $100 \mathrm{mV} \mathrm{s}^{-1}$.

The above experimental results show that the TDS SAM modified electrode forms strong complexes with cadmium ions. The stability of the response also indicates that on the time scale of these experiments, the binding of $\mathrm{Cd}^{2+}$ to the TDS is irreversible. To further verify that the voltammetric response was a result of surface immobilized species and not freely diffusing solution species, the peak current for TDS-Cd(II) was determined at various scan rates. The variation of the anodic and cathodic peak currents with the sweep rate from 10 to $800 \mathrm{mV} \mathrm{s}^{-1}$ is shown in Figure 6.22. It was found that the peak current increased linearly with increasing the scan rate. The ratio of $\mathrm{I}_{\mathrm{pa}} / \mathrm{I}_{\mathrm{pc}}$ remains close to one, as expected for surfacetype behaviour [12]. The linear relation is consistent with the following two equations: $I_{p, a}(\mu A)=26.6+2.33 v\left(m ~ s^{-1}\right)$ and $I_{p c}(\mu A)=22.6+2.11 v\left(m^{2} s^{-1}\right)$. The values of $R^{2}$ for two equations are 0.989 and 0.987 respectively. 
This indicated that the surface-bound controlled process of cadmium ions on the TDS modified electrodes. Most part of current was due to surface bound cadmium ions. Since there is no diffusion process, and thus no possibility of different transport rates of the two species being involved, we see the expected ratio $i_{p, a} / i_{p, c} \approx 1$.

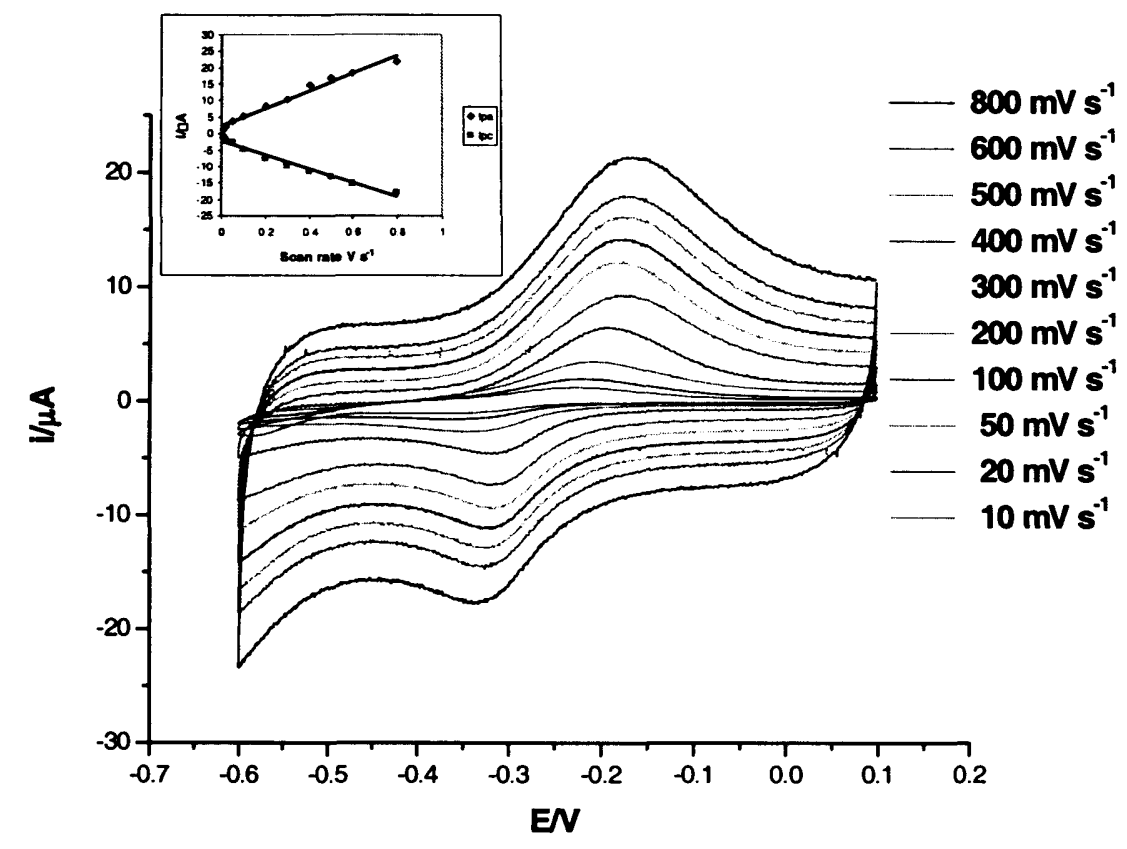

Figure 6.22: Effect of the variation of the sweep rate $\left(10\right.$ to $\left.800 \mathrm{mV} \mathrm{s}^{-1}\right)$ on the anodic and cathodic peak currents. In all cases $\mathrm{Cd}$ (II) was accumulated at the TDS modified electrode at open circuit for $10 \mathrm{~min}$ in a $0.1 \mathrm{M}$ potassium nitrate solution $(\mathrm{pH} 6.2)$ containing $1 \mathrm{mM}$ cadmium nitrate, removed, rinsed and then placed in a cadmium-free potassium nitrate solution. Inset: the dependence of the anodic and cathodic peak current on sweep rate.

\subsubsection{Effect of $\mathrm{pH}$ of preconcentration solution}

Throughout the process of accumulation the $\mathrm{pH}$ of the solution plays a crucial role because of its direct effect on both ligand and metal ion chemistry. The effect of the $\mathrm{pH}$ on the oxidation and reduction currents was studied. The $\mathrm{pH}$ of the supporting electrolyte (pure $0.1 \mathrm{M} \mathrm{KNO}_{3}+1 \mathrm{mM} \mathrm{Cd}\left(\mathrm{NO}_{3}\right)_{2}$ ) is 6.5 and the $\mathrm{pH}$ of the pure $0.1 \mathrm{M}$ $\mathrm{KNO}_{3}$ is 6.2 , which is higher than the ligand $\mathrm{pK}_{\mathrm{a}}$ making the modified electrode surface negatively charged. The dependence of the $\mathrm{Cd}(\mathrm{II})$ accumulation and voltammetric response on the $\mathrm{pH}$ of the supporting electrolyte solution over the range of $\mathrm{pH} 4-6.5$ is shown in Figure 6.23. 


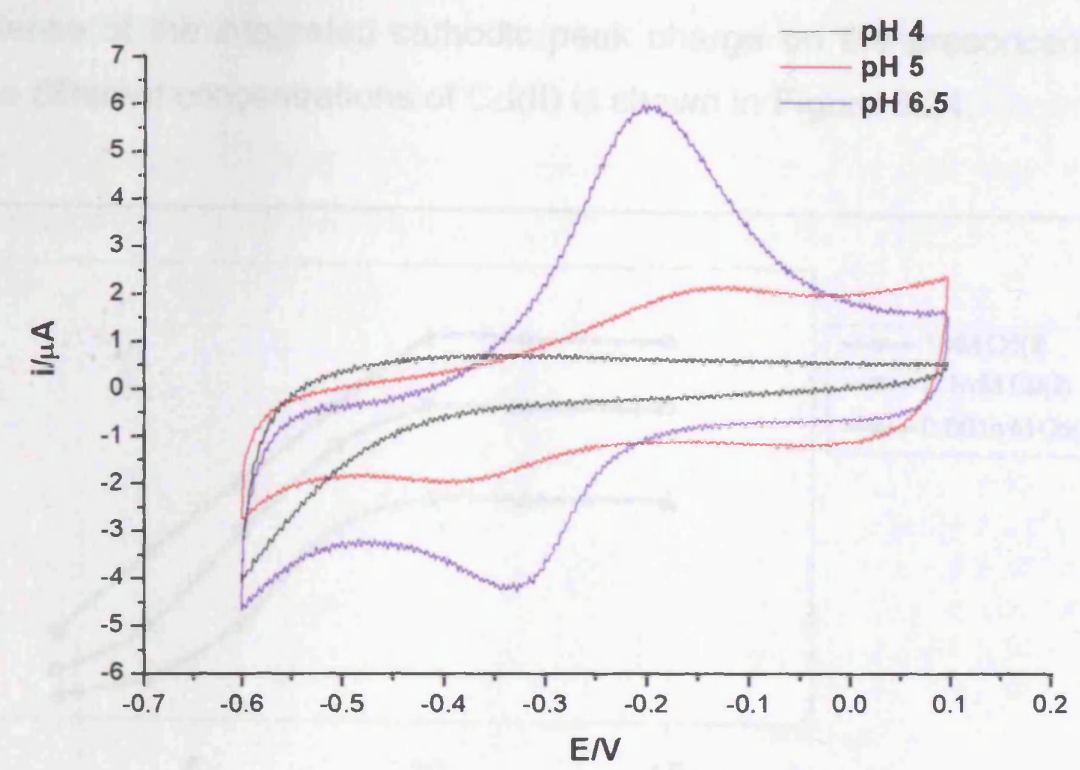

Figure 6.23: Effect of the preconcentration electrolyte $\mathrm{pH}$ on the peak current intensity for the complexed of $\mathrm{Cd}^{2+}$ with (TDS) coated gold electrode. Cyclic voltammetry in cadmiumfree $0.1 \mathrm{M} \mathrm{KNO}_{3}(\mathrm{pH} 6.2)$; sweep rate $100 \mathrm{mV} \mathrm{s}^{-1}$; preconcentration time $=10 \mathrm{~min}$; open circuit.

Initial studies were performed using $0.1 \mathrm{M} \mathrm{KNO}_{3}$ with $\left.1 \mathrm{mM} \mathrm{Cd}\left(\mathrm{NO}_{3}\right)_{2}\right)$ at $\mathrm{pH} 6.5$ using 10 minute open circuit accumulation. However, maximum oxidation and reduction peak currents were observed at $\mathrm{pH} 6.5$. This can readily be explained in terms of the strong complex formation of cadmium ions with TDS SAM at the Au surface at $\mathrm{pH} 6.5$. Further $\mathrm{pH}$ investigation was undertaken using $0.1 \mathrm{M} \mathrm{HNO}_{3}$ or $0.1 \mathrm{M} \mathrm{KOH}$ alone to adjust the preconcentration solution to the desired $\mathrm{pH}$. Clearly, at low $\mathrm{pH}$, no response was found, which can be ascribed to the protonated carboxylate groups in TDS SAM modified Au electrode. There are two evident reasons for the non linear dependence of $\mathrm{Cd}(\mathrm{II})$ accumulation on the $\mathrm{pH}$ of the preconcentration solution: first, at low $\mathrm{pH}$ values $(\mathrm{pH}<6.5)$, all carboxylic acid terminal groups of SAM should be fully protonated. Secondly, at high $\mathrm{pH}$ values $(\mathrm{pH}>6.5), \mathrm{Cd}(\mathrm{II})$ tends to be hydrolysed and produce insoluble hydroxide $\mathrm{Cd}(\mathrm{OH})_{2}$, $\mathrm{K}_{\mathrm{sp}}$ of $\mathrm{Cd}(\mathrm{OH})_{2}$ is $5.0 \times 10^{-15} \mathrm{M}^{3}$. It is not likely for the neutral $\mathrm{Cd}(\mathrm{OH})_{2}$ to be taken up by the negatively charged carboxylates on the TDP SAM. Because of this behaviour, a preconcentration solution of $\mathrm{pH} 6.5$ was chosen as the most sensitive for the preconcentration of $\mathrm{Cd}(\mathrm{II})$ at the TDS modified $\mathrm{Au}$ electrode under open circuit conditions. 


\subsubsection{Effect of the preconcentration time}

The dependence of the integrated cathodic peak charge on the preconcentration time for three different concentrations of $\mathrm{Cd}(\mathrm{II})$ is shown in Figure 6.24.

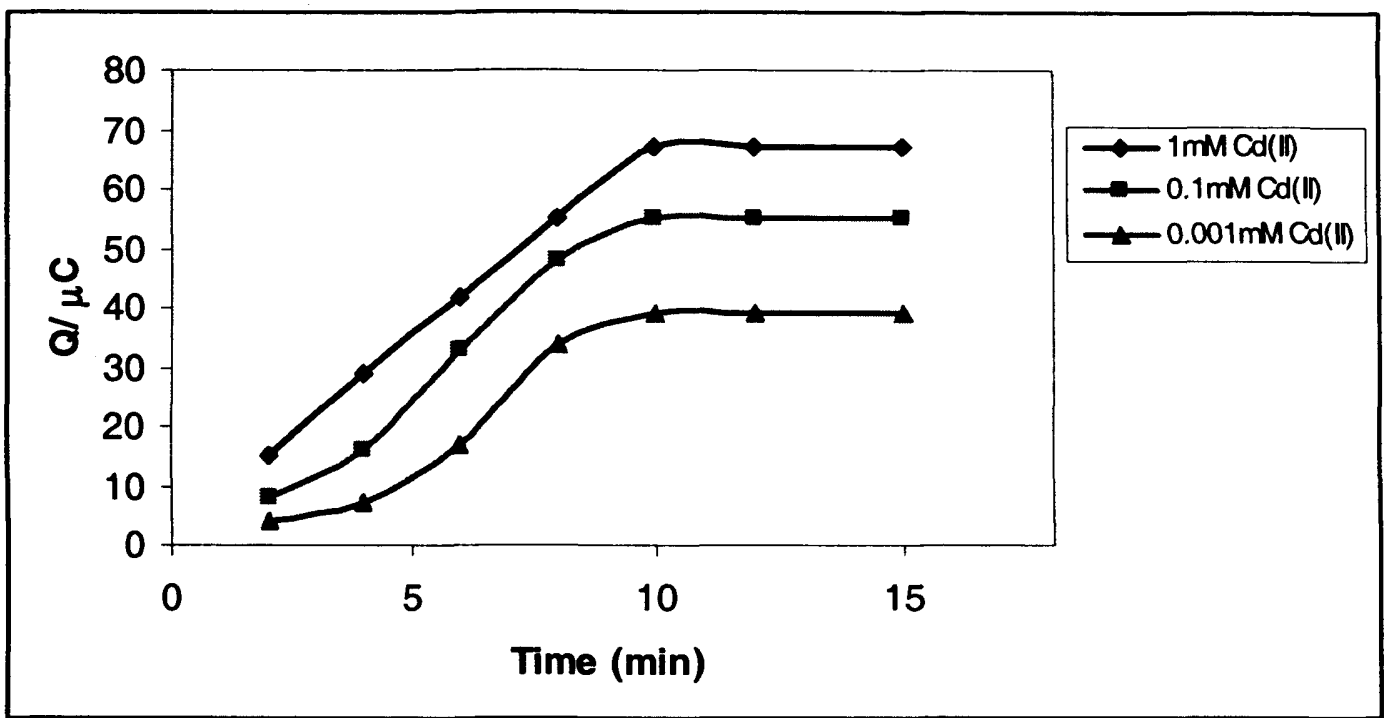

Figure 6.24: Effect of preconcentration time on the integrated cathodic peak charge of $\mathrm{Cd}^{2+}$ ions with TDS SAM modified Au electrode. Cyclic voltammetry in $\mathrm{Cd}(\mathrm{II})$-free $\mathrm{KNO}_{3(\mathrm{aq})}$ having been exposed to $0.001,0.1$ and $1 \mathrm{mM} \mathrm{Cd(II)})_{(\text {aq) }}$. Scan rate $100 \mathrm{mV} \mathrm{s}^{-1}$.

The above experimental results show that the integrated cathodic peak charge increases with increasing preconcentration time, indicating an enhancement of Cd(II) ion concentration at TDS surface. When the preconcentration time was 10 min, the integrated peak charge became constant. For the three different concentrations of $\mathrm{Cd}(\mathrm{II}), 10 \mathrm{~min}$ preconcentration time yielded a rapid increase in the integrated charge. Beyond $10 \mathrm{~min}$ preconcentration time, the curves levelled off. The occurrence of this limiting plateau of the integrated peak charge at longer preconcentration periods could originate from attainment of complexation equilibrium between carboxylate groups of TDS and $\mathrm{Cd}(\mathrm{II})$ in solution or saturation of the binding sites. For each of the concentrations employed, the plateau state integrated peak charge value was different, larger values were being obtained for higher concentrations of $\mathrm{Cd}(\mathrm{II})$. This is due to the maximum amount of the $\mathrm{Cd}(\mathrm{II})$ TDS complex that can be preconcentrated in such a prolonged preconcentration time is determined by the complexation equilibrium constant. For all subsequent measurements a preconcentration time of 10 min was employed. 


\subsubsection{Concentration dependence of the characteristic peak charge}

Following the procedures described above, cyclic voltammograms at different concentrations of $\mathrm{Cd}(\mathrm{II})$ are shown in Figure 6.25. The relationship between the peak currents and concentration of $\mathrm{Cd}^{2+}$ in the accumulation solution was studied. The peak currents increased with increase the concentration of $\mathrm{Cd}^{2+}$ from $1 \times 10^{-8}$ to $1 \times 10^{-4} \mathrm{M}$.

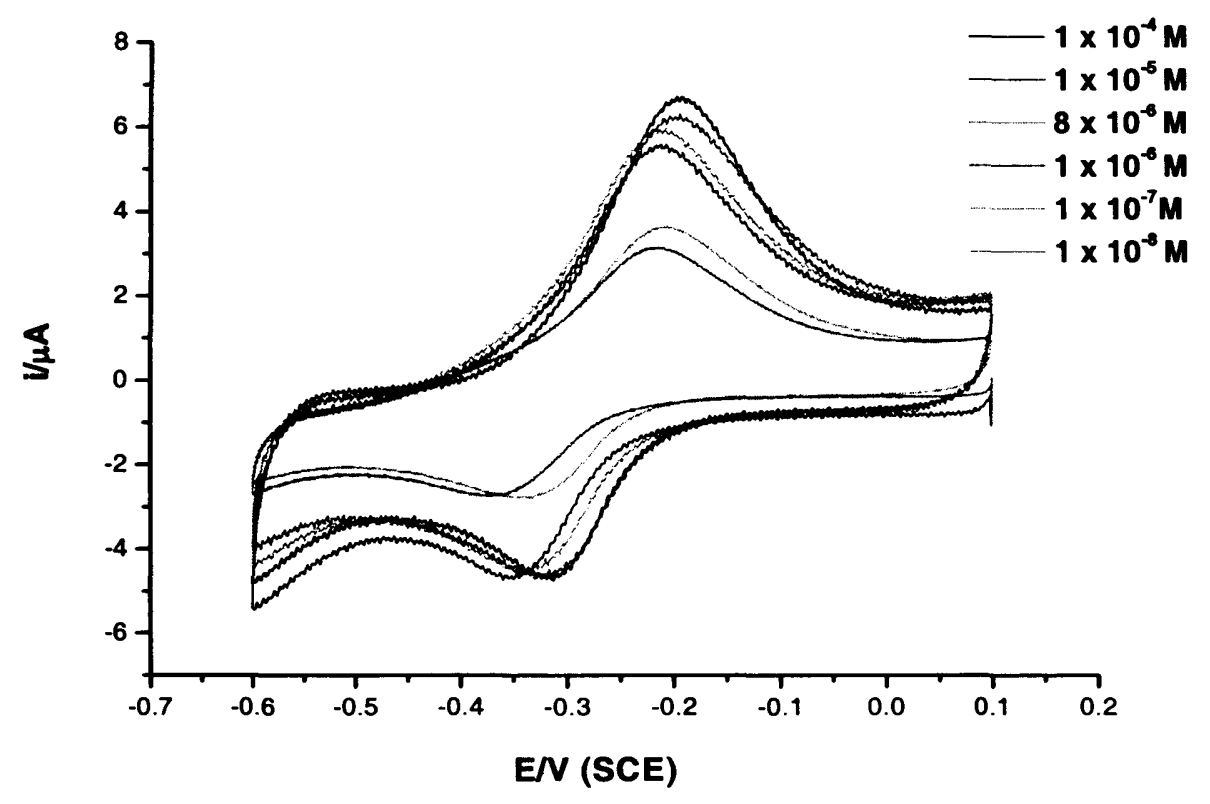

Figure 6.25: Cyclic voltammograms of complexed $\mathrm{Cd}^{2+}$ at a TDS SAM modified electrode after preconcentration time of $10 \mathrm{~min}$ in $0.1 \mathrm{M} \mathrm{KNO}_{3}$ solution $\left(\mathrm{pH} \mathrm{6.2)} \mathrm{containing} 1 \times 10^{-4}, 1\right.$ $\times 10^{-5}, 1 \times 10^{-6}, 5 \times 10^{-6}, 1 \times 10^{-7}$ and $1 \times 10^{-8} \mathrm{M}$ of $\mathrm{Cd}(\mathrm{II})$. Scan rate $100 \mathrm{mV} \mathrm{s}^{-1}$.

The dependence of the cathodic peak charge on the $\mathrm{Cd}^{2+}$ concentration in solution is shown in Figure 6.26. The integrated peak charge for cadmium ion reduction increased steeply with increasing the concentration up to ca. $15 \mu \mathrm{M}$. The cathodic peak charge reached a constant value of $56 \pm 1.5 \mu \mathrm{C}(n=5)$ at a $\mathrm{Cd}^{2+}$ concentration of $100 \mu \mathrm{M}(11.2 \mathrm{ppm})$, which corresponds to a saturation surface coverage $\Gamma_{\mathrm{Cd} 2+}=$ $1.41 \pm 0.04 \mathrm{nmol} \mathrm{cm}^{-2}(\mathrm{n}=5)$. The TDS modified electrode showed a linear calibration curve by systematically increasing the concentration of $\mathrm{Cd}^{2+}$ from 0.01 to $1 \mu \mathrm{M}$ using a $10 \mathrm{~min}$ preconcentration time. The curve is represented by the equation $y(\mu \mathrm{C})=7.1+37 \times\left(\mu \mathrm{mol} \mathrm{L} L^{-1}\right)$ with a regression coefficient of 0.977 . The coefficient of variation, determined on fresh TDS SAM modified Au electrode at a concentration of $1 \times 10^{-5} \mathrm{M} \mathrm{Cd}^{2+}$, was calculated to be $4.0 \%(n=6)$. 


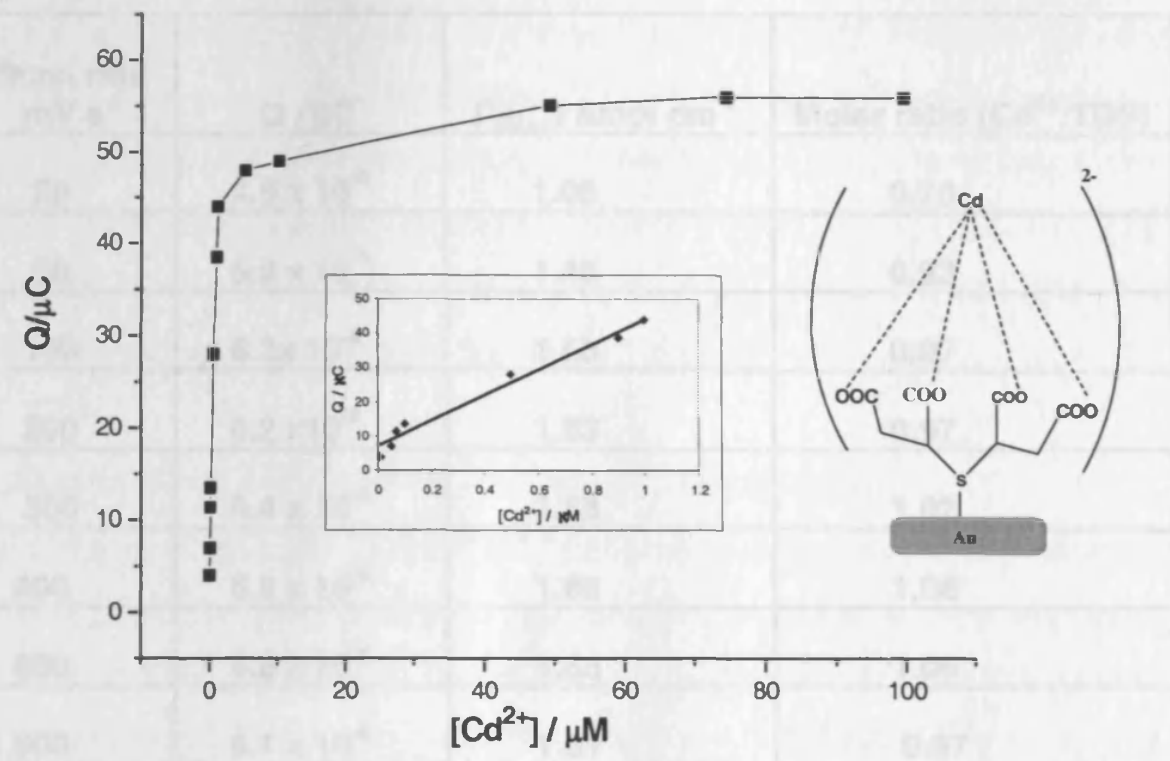

Figure 6.26: Influence of cadmium concentrations in solution on the cadmium integrated peak charge. Common conditions: Cyclic voltammetry in $\mathrm{Cd}(\mathrm{II})$-free $\mathrm{KNO}_{3}(\mathrm{aq})$ having been exposed to different concentrations (from 0.01 to $100 \mu \mathrm{M}$ ) of $\mathrm{Cd}(\mathrm{II})_{(\text {aq) }}$. Scan rate $100 \mathrm{mV} \mathrm{s}^{-1}$, preconcentration time $10 \mathrm{~min}$. Point represent average of five measurements on same surface coverage of TDS SAM modified Au electrode $\left(\Gamma_{\text {TDS }}=1.57 \pm\right.$ $0.05 \mathrm{nmol} \mathrm{cm}^{-2}$ ).

\subsubsection{Estimate of the mole ratio between $\mathrm{Cd}^{2+}$ and TDS}

The surface coverage of $\mathrm{Cd}^{2+}\left(\Gamma_{\mathrm{Cd}++}\right)$ was obtained by integration of reduction peaks and assuming a two electron reaction using Faraday's law (section 2.7.2). The ratio between $\mathrm{Cd}^{2+}$ and TDS modified electrode is presented in table 6.3. It was found that the ratio of the saturation coverage of $\mathrm{Cd}^{2+}$ to TDS is equal to one at

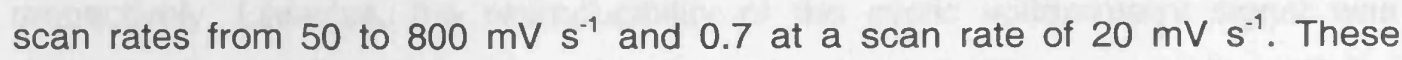
experimental results indicated that the complexation process (adsorption) between TDS SAM modified Au electrode and cadmium ions is strong and stable. 


\begin{tabular}{|c|c|c|c|}
\hline $\begin{array}{c}\text { Scan rate } \\
\mathrm{mV} \mathrm{s}^{-1}\end{array}$ & $\mathrm{Q} / \mu \mathrm{C}$ & $\Gamma_{\mathrm{Cd}++} / \mathrm{nmol} \mathrm{cm}^{-2}$ & Molar ratio $\left(\mathrm{Cd}^{2+}: \mathrm{TDS}\right)$ \\
\hline 20 & $4.5 \times 10^{-6}$ & 1.09 & 0.70 \\
\hline 50 & $5.9 \times 10^{-6}$ & 1.46 & 0.93 \\
\hline 100 & $6.2 \times 10^{-6}$ & 1.53 & 0.97 \\
\hline 200 & $6.2 \times 10^{-6}$ & 1.53 & 0.97 \\
\hline 300 & $6.4 \times 10^{-6}$ & 1.58 & 1.02 \\
\hline 400 & $6.8 \times 10^{-6}$ & 1.68 & 1.06 \\
\hline 600 & $6.8 \times 10^{-6}$ & 1.68 & 1.06 \\
\hline 800 & $6.1 \times 10^{-6}$ & 1.51 & 0.97 \\
\hline
\end{tabular}

Table 6.3: Estimate of the surface coverage and the mole ratio of $\mathrm{Cd}^{2+} /$ TDS on an unpolished crystal using Faraday's law at different scan rates: cyclic voltammetric experiments in $\mathrm{Cd}(\mathrm{II})$-free $\mathrm{KNO}_{3}(\mathrm{aq})$ having been exposed to $1 \mathrm{mM} \mathrm{Cd}(\mathrm{II})_{(\mathrm{aq})}$. Preconcentration time $10 \mathrm{~min}$, scan rate $100 \mathrm{mV} \mathrm{s}^{-1}$. The surface coverage of the ligand was $1.57 \pm 0.05 \mathrm{nmol} \mathrm{cm}^{-2}(\mathrm{n}=5)$.

The reproducibility of successive cyclic voltammetry measurements with the same electrode surface was tested (adsorb the metal ion, perform cyclic voltammetry, remove the metal with EDTA, readsorb and perform cyclic voltammetry). Sets of five successive measurements of two $C d(I I)$ concentrations levels $\left(5.0 \times 10^{-5}\right.$ and $5.0 \times 10^{-6} \mathrm{M}$ ) yielded a relative standard deviation for the $\Delta \mathrm{Q}$ of 5.5 and $4.9 \%$ respectively. Likewise, the reproducibility of the cyclic voltammetry signal was checked. Sets of five measurements of two levels of Cd(II) concentrations $(5.0 \mathrm{x}$ $10^{-5}$ and $5.0 \times 10^{-6} \mathrm{M}$ ) were carried out for five different surface coverages of TDS SAM. The R.S.D values obtained were $7.3 \%$ and $7.6 \%$ respectively. These results suggest that the fabrication procedure of the TDS SAM modified Au electrode is reliable.

The limit of detection ( $3 \times$ SD) was determined from the standard deviation of ten repeated determinations of the cadmium concentration at low levels of cadmium (0.01-1 $\mu \mathrm{M})$. The limit of detection in this condition was found to be 20 $\mathrm{nM}$. There is very good agreement between the mole ratio estimated here and that estimated from frequency shifts of QCM measurements (see chapter five). On the 
other hand, the detection limit was estimated here is lower than estimated from acoustic wave sensors by a factor of three. The TDS SAM modified Au electrode performance can be compared with other $\mathrm{Cd}^{2+}$ selective electrodes. A carbon paste electrode modified with $\mathrm{N}$-p-chlorophenyl-cinnamohydroxamic acid has a detection limit of $9.8 \mathrm{nM}$ when measured by anodic stripping voltammetry [13]. Gooding et al. [14] reported a detection limit of $5 \mathrm{nM}$ using glutathione monolayer modified electrode after a preconcentration step for $10 \mathrm{~min}$.

\subsubsection{Renewal of TDS SAM modified Au electrode}

To utilize the electrode for multiple and long term operation, the regeneration of the TDS modified Au electrode in a reproducible manner is very important. The incorporated cadmium species were readily removed by exposing the TDS modified electrode to 0.1M EDTA solution for a few minutes. Moreover, there was no decrease in the electrode response after several successive runs. The TDS SAM modified electrode can be used for multiple analytical determinations for about two weeks. The electrode with a fresh TDS surface gave signals with approximately the same degree of precision (about 10\%) as that obtained with the electrode with a "used" surface. The complex formation between TDS SAM and cadmium ions is stable for two days: after that the oxidation and reduction peak currents decreased gradually with increasing number of experimental runs.

\subsubsection{Complexation between 3-thiodisuccinic acid and $\mathrm{Cu}^{2+}$}

The complexation between TDS and other metal ions such as $\mathrm{Cu}^{2+}$ was studied, in order to compare the voltammetric responses with the QCM frequency shifts presented in chapter five. Figure 6.27 shows cyclic voltammograms of a gold electrode covered with TDS SAM obtained in copper-free nitrate solution before and after a preconcentration step in $1 \mathrm{mM} \mathrm{Cu}^{2+}$ solution for 20 minutes at open circuit. Chemical reversibility behaviour was seen after the preconcentration step. The reduction and oxidation of $\mathrm{Cu}(\mathrm{II})$ occurred at $\mathrm{E}_{\mathrm{p}, \mathrm{a}}=+0.359$ and $\mathrm{E}_{\mathrm{pc}}=-0.057 \mathrm{~V}$. $A$ bare gold electrode showed no peaks under the same conditions. This indicates that the chemical preconcentration of $\mathrm{Cu}(\mathrm{II})$ can not take a place on a bare gold electrode. Thus, the voltammetric response of the TDS/Au electrode to $\mathrm{Cu}^{2+}$ is attributed to the interaction between TDS SAM and copper ion.

The stability of the TDS- $\mathrm{Cu}^{2+}$ layer was examined by repeated cyclic voltammetric scans, as shown in the inset of Figure 6.27. The results show that the anodic and cathodic peak currents decrease gradually with repeated cyclic scans. The 
cathodic peak charge decreases rapidly from $57\left(1^{\text {st }}\right)$ to $7 \mu \mathrm{C}\left(20^{\text {th }}\right)$ at a scan rate of $100 \mathrm{mV} \mathrm{s}^{-1}$. These results indicate weak complexation between copper ions and TDS. These results are opposite to those for TDS/Cd $d^{2+}$.

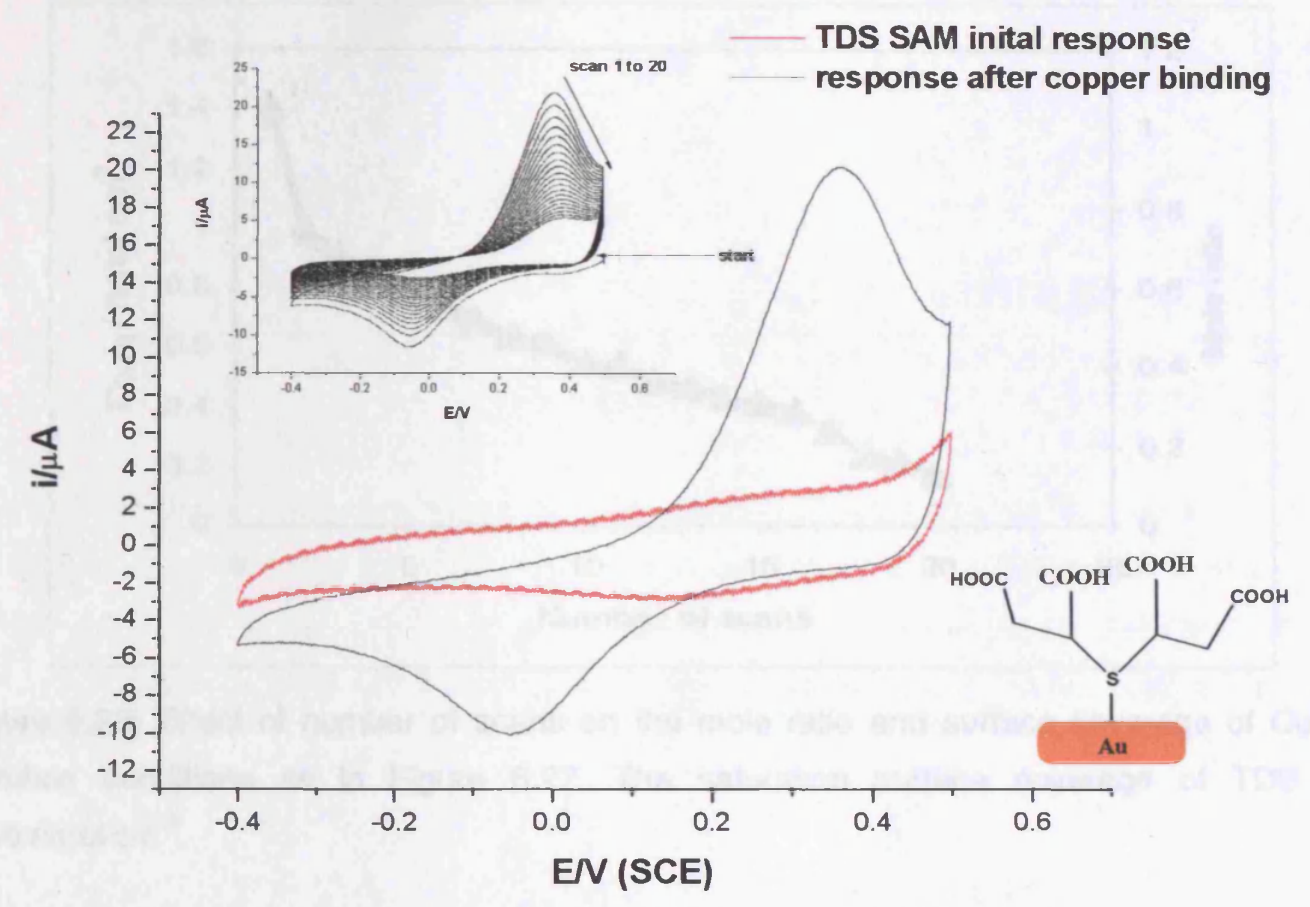

Figure 6.27: Cyclic voltammograms of TDS SAM modified electrode before and after exposure to copper in $0.1 \mathrm{M}$ potassium nitrate solution $(\mathrm{pH} 6.0$ ). In all cases $\mathrm{Cu}(\mathrm{II})$ was accumulated at the TDS modified electrode at open circuit for $20 \mathrm{~min}$ in a $0.1 \mathrm{M}$ potassium nitrate solution containing $1 \mathrm{mM}$ copper nitrate $(\mathrm{pH} 5.5)$, removed, rinsed and then placed in a copper-free potassium nitrate solution. Sweep rate $100 \mathrm{mV} \mathrm{s}^{-1}$. Inset: effect of repetitive cycling on the $\mathrm{Cu}^{2+}-$ TDS SAM modified Au electrode.

We did not study the effect of the scan rates on the complexation between TDS and $\mathrm{Cu}^{2+}$ because the weak stability of the TDS- $\mathrm{Cu}^{2+}$ layer.

\subsubsection{Estimate of the mole ratio between $\mathrm{Cu}^{2+}$ and TDS}

The surface coverage of $\mathrm{Cu}^{2+}\left(\Gamma_{\mathrm{Cu} 2+}\right)$ was estimated by the integration of reduction peaks of the cyclic voltammograms and assuming two electron reaction at different repeated cyclic voltammetric scans using Faraday's law. The saturation surface coverage of $1 \mathrm{mM} \mathrm{Cu}^{2+}$ obtained for the first cyclic voltammetric scan was $1.85 \pm 0.08 \mathrm{nmol} \mathrm{cm}{ }^{-2}$ ( number of samples, $\mathrm{n}=4$ ) and the saturation coverage of 
TDS was $1.40 \pm 0.03 \mathrm{nmol} \mathrm{cm} \mathrm{cm}^{-2}(n=4)$, which gives the ratio of the saturation coverage of $\mathrm{Cu}^{2+}$ to TDP was about (1:1). In replicate experiments, the mole ratio decreased from 1.4 to 0.17 with cyclic scans (see Figure 6.28). This result shows complexation between copper ions and TDS SAM is weak.

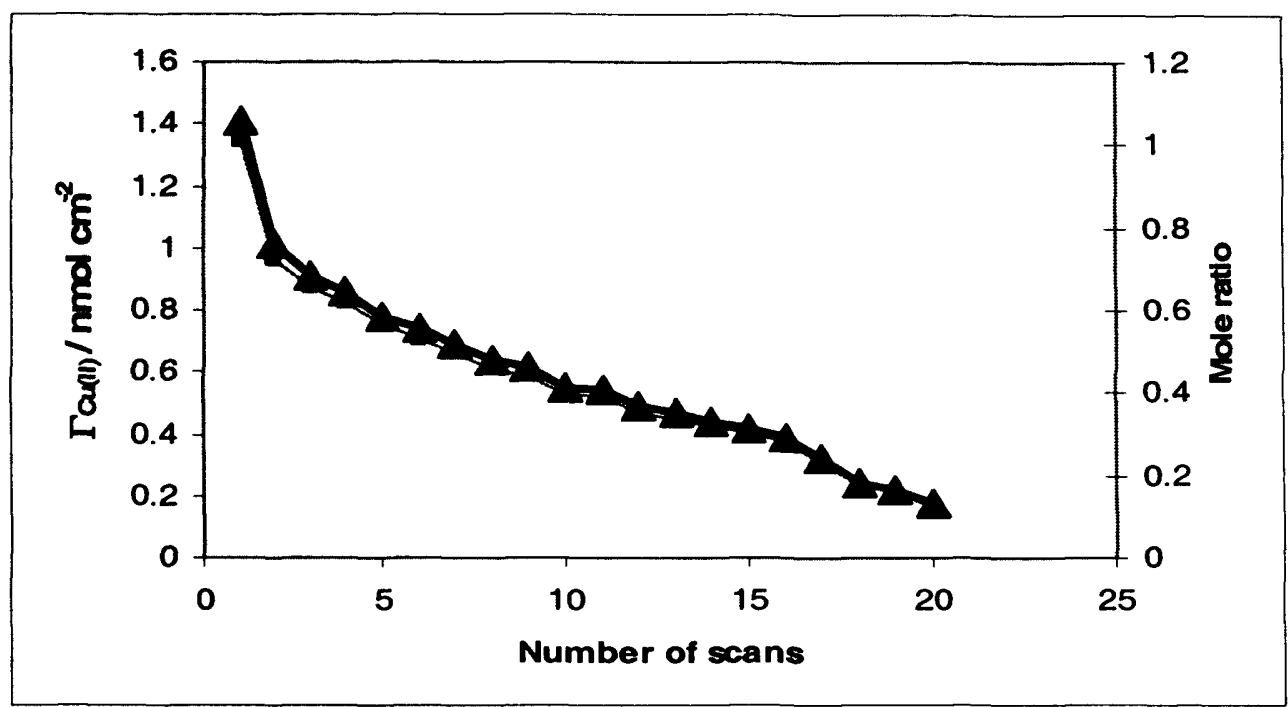

Figure 6.28: Effect of number of scans on the mole ratio and surface coverage of $\mathrm{Cu}^{2+}$. Solution conditions as in Figure 6.27. The saturation surface coverage of TDS is $1.40 \mathrm{nmol} \mathrm{cm}^{-2}$.

\subsubsection{Complexation between 3-thiodisuccinic acid and $\mathrm{Pb}^{2+}$}

The ability of TDS SAM to bind lead ions from aqueous solutions was examined using the same experimental conditions as above. Typical cyclic voltammograms of a TDS SAM modified Au electrode in lead-free nitrate solution $\left(0.1 \mathrm{M} \mathrm{KNO}_{3}\right)$ after accumulation of lead ions from $1 \mathrm{mM} \mathrm{Pb}\left(\mathrm{NO}_{3}\right)_{2}$ are shown in Figure 6.29. The observed redox chemistry after the preconcentration step with $E_{p, a}=-0.177 \mathrm{~V}, E_{c}=$ $-0.376 \mathrm{~V}$ and $\mathrm{E}^{0}=-0.276 \mathrm{~V}$, is due to the $\mathrm{Pb}^{2+} / \mathrm{Pb}^{0}$ couple. The fact that the oxidation peak appeared broad rather than sharp is indicative that the process is not a stripping peak where the lead is removed from the TDS SAM-modified electrode. It is postulated that some $\mathrm{Pb}^{2+}$ is recaptured by TDS when $\mathrm{Pb}^{0}$ is reoxidised. Unfortunately, no redox activity was observed after the third cycle; the lead ions were lost from the electrode. We can not do the effect of the scan rate on the complexation between $\mathrm{Pb}^{2+}$ and TDS if the stability is so low. 


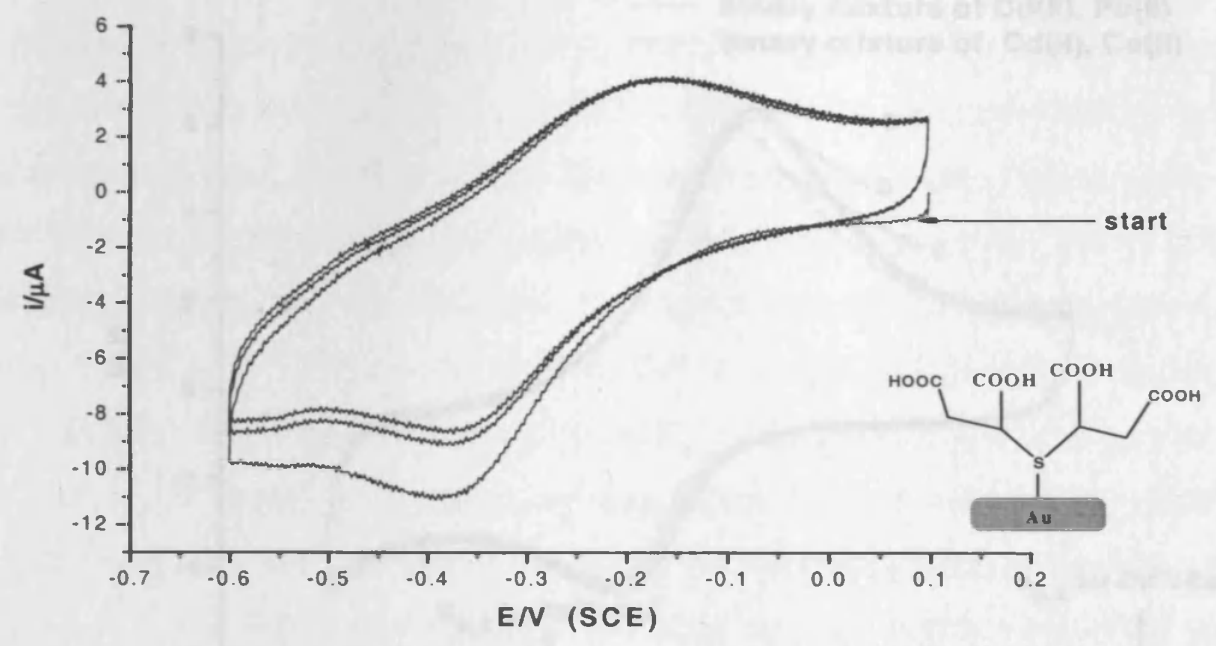

Figure 6.29: Cyclic voltammograms of TDS SAM-modified electrode in lead-free $0.1 \mathrm{M}$ potassium nitrate solution $(\mathrm{pH} 6.0)$. $\mathrm{Pb}$ (II) was accumulated at the TDS modified electrode at open circuit for $30 \mathrm{~min}$ in a $0.1 \mathrm{M}$ potassium nitrate solution containing $1 \mathrm{mM}$ lead nitrate $(\mathrm{pH}=6)$, removed, rinsed and then cycled in a lead- free potassium nitrate solution. Sweep rate $100 \mathrm{mV} \mathrm{s}^{-1}$.

\subsubsection{Interference effects on $\mathrm{Cd}$ (II) responses in binary mixtures}

The interferences were examined by introducing other metal ions into the preconcentration medium under open circuit conditions. The interfence effect of $\mathrm{Pb}^{2+}$ and $\mathrm{Cu}^{2+}$ with the $\mathrm{Cd}^{2+}$ signal in binary mixtures was addressed for the TDS modified electrode using the same experimental conditions in section 6.3.4. Figure 6.30 shows the comparisons between cyclic voltamograms of single cadmium ion solution and binary mixtures of $\mathrm{Cd}$ (II) with $\mathrm{Cu}$ (II) and $\mathrm{Pb}$ (II) using $10 \mathrm{~min}$ preconcentration time. It was found that from cadmium nitrate solution, the reduction peak current obtained was $4.7 \mu \mathrm{A}$. In the presence of lead and copper at $1 \mathrm{mM}$, the peak currents were 4.6 and $4.5 \mu \mathrm{A}$, respectively. No additional voltammetric peaks from these interferents was observed either. The $E_{p, a}$ and $E_{p, c}$ for $\mathrm{Cd}^{2+} / \mathrm{Cd}^{0}$ redox couple did not change in these binary mixtures. The fact that no interference was observed from copper and lead provides a strong indication that $\mathrm{Cd}^{2+}$ binds more strongly to the TDS modified electrode, with significantly higher affinity constant than $\mathrm{Cu}^{2+}$ and $\mathrm{Pb}^{2+}$. 


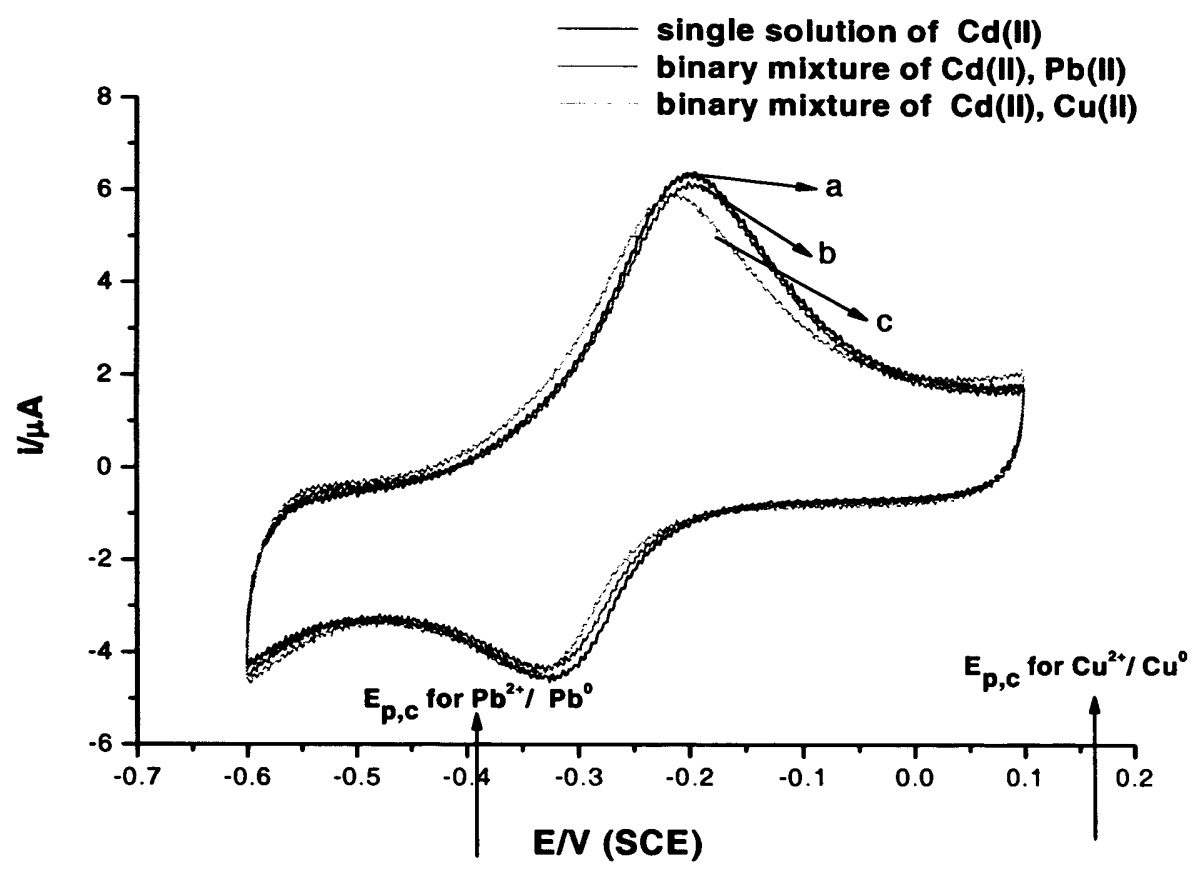

Figure 6.30: Cyclic voltammograms of the TDS SAM-modified Au electrode in $0.1 \mathrm{M} \mathrm{KNO}_{3}$ (a) after adsorbing cadmium ions from $0.1 \mathrm{mM}$ cadmium nitrate (b) after exposure to a solution containing both $0.1 \mathrm{mM} \mathrm{Cd}$ (II) and $1 \mathrm{mM} \mathrm{Pb}$ (II) (c) after exposure to a solution containing both $0.1 \mathrm{mM} \mathrm{Cd}(\mathrm{II})$ and $1 \mathrm{mM} \mathrm{Cu}(\mathrm{II})$. Common conditions: preconcentration time $10 \mathrm{~min}$; pH 5.5; open circuit; scan rate $100 \mathrm{mV} \mathrm{s}^{-1}$.

\subsection{Complexation between meso-2,3-dimercaptosuccinic acid and $\mathrm{Pb}^{2+}$}

QCM frequency shift data show that 2,3-dimercaptosuccinic acid (DMSA) self assembled monolayer modified electrodes have a high selectivity to lead ions in solution. This section describes the electrochemical responses of $\mathrm{DMS}$ to $\mathrm{Pb}^{2+}$ in solution using the same experimental procedures as for copper and cadmium. DMSA SAM contains two thiol groups attached to the Au surface, leaving two carboxylate groups free to adsorb lead ions from aqueous solutions.

\subsubsection{Experimental}

The experimental procedures were as described in section 6.2.1: preconcentration from $1 \mathrm{mM} \mathrm{Pb}\left(\mathrm{NO}_{3}\right)_{2}+0.1 \mathrm{M} \mathrm{KNO} 3(\mathrm{pH} \mathrm{5.5)}$, for $15 \mathrm{~min}$ at open circuit. Cyclic voltammograms were recorded from +0.1 to $-0.6 \mathrm{~V}$ at scan rates from 10 to $500 \mathrm{mV} \mathrm{s}^{-1}$. 


\subsubsection{Results and discussion}

Cyclic voltammetry was used to investigate the possibility of accumulating $\mathrm{Pb}^{2+}$ at the DMSA SAM-modified electrode. Figure 6.31 shows a cyclic voltammogram at a sweep rate of $100 \mathrm{mV} \mathrm{s}^{-1}$ of the DMSA modified Au electrode before and after incubation in a lead nitrate solution. The electrochemistry due to reduction of $\mathrm{Pb}^{2+}$ to $\mathrm{Pb}^{0}$ and oxidation back to $\mathrm{Pb}^{2+}$ suggests the lead ions are bound to DMSA via carboxylate groups at the interface after preconcentration. The observed redox chemistry of the $\mathrm{Pb}^{2+} / \mathrm{Pb}^{0}$ couple occurred at $\mathrm{E}_{p, \mathrm{a}}=-0.300 \mathrm{~V}$ and $\mathrm{E}_{p, \mathrm{c}}=-0.420 \mathrm{~V}$ with $E^{0}=-0.360 \mathrm{~V}$. The peak separation $\left(\Delta E=E_{p, a}-E_{p, c}\right)$ was $0.120 \mathrm{~V}$ at a scan rate of $100 \mathrm{mV} \mathrm{s}^{-1}$. A bare gold electrode was used for the alternative voltammetric analysis of $\mathrm{Pb}(\mathrm{II})$, by immersing the bare $\mathrm{Au}$ electrode into a solution of $1 \mathrm{mM}$ $\mathrm{Pb}\left(\mathrm{NO}_{3}\right)_{2}+0.1 \mathrm{M} \mathrm{KNO}_{3}, \mathrm{pH} 5.5$, for 15 min but no peak current was observed.

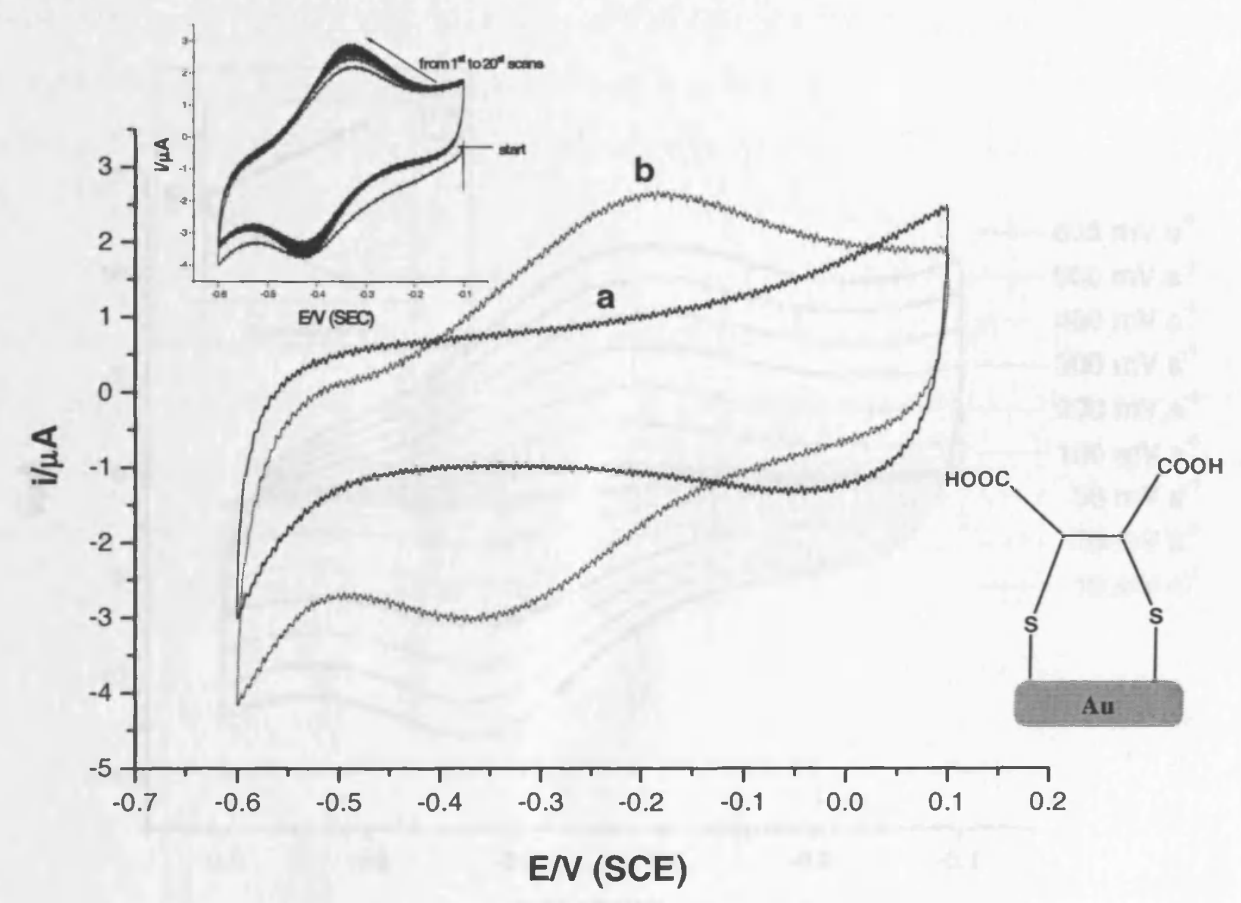

Figure 6.31: Cyclic voltammogram of DMSA SAM modified electrode before (a) and after (b) exposure to lead in $0.1 \mathrm{M}$ potassium nitrate solution $(\mathrm{pH} 6.0)$. $\mathrm{Pb}$ (II) was accumulated at the DMSA modified electrode at open circuit for $15 \mathrm{~min}$ in a $0.1 \mathrm{M}$ potassium nitrate solution containing $1 \mathrm{mM}$ lead nitrate $(\mathrm{pH} 5.5)$, removed, rinsed and then placed in a lead-free potassium nitrate solution. Sweep rate $100 \mathrm{mV} \mathrm{s}^{-1}$. Inset: effect of repetitive cyclic scans on the $\mathrm{Pb}^{2+}$-DMSA SAM-modified Au electrode. 


\subsubsection{Stability and effect of the sweep rate}

The influence of repeated cyclic scans $\left(20^{\text {th }}\right.$ cycles $)$ at scan rate of $100 \mathrm{mV} \mathrm{s}^{-1}$ on the complexation between DMSA SAM at the surface and lead ions in aqueous solutions is shown in the inset of Figure 6.31. The results show that lead is strongly complexed at the surface of Au electrode. There is no shift of the redox potential, $\mathrm{E}^{0}$ and the potential peak separation remains constant, ca. $\Delta \mathrm{E}_{\text {peak }}=120 \mathrm{mV}$. The underpotential for lead in these conditions did not happen because the underpotential of lead has been observed at bare gold electrode at approximately $0.2 \mathrm{~V}$ [15]. The stability of the cyclic voltammograms obtained here allowed the study of this system at different sweep rates. Figure 6.32 represents the effect of variation of the scan rate from 10 to $500 \mathrm{mV} \mathrm{s}^{-1}$ on the behaviour of cyclic voltammograms.

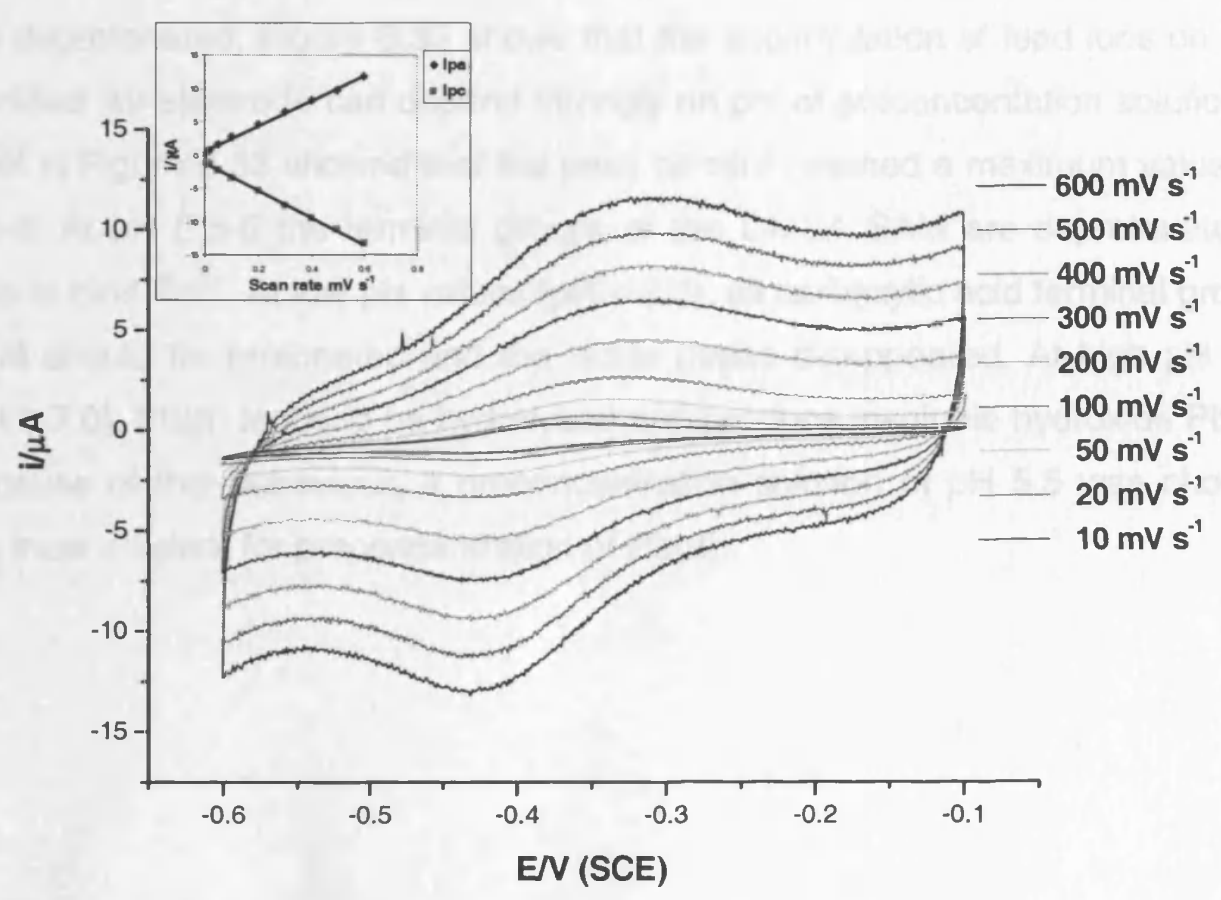

Figure 6.32: Effect of varying scan rate on the $\mathrm{Pb}^{2+}$-DMSA SAM modified $\mathrm{Au}$ electrode response. In all cases $\mathrm{Pb}(\mathrm{II})$ was accumulated at the DMSA modified electrode at open circuit for $15 \mathrm{~min}$ in a $0.1 \mathrm{M}$ potassium nitrate solution containing $1 \mathrm{mM}$ lead nitrate $(\mathrm{pH}$ 5.5), removed, rinsed and then placed in lead-free potassium nitrate solution. Inset: the dependence of the anodic and cathodic peak currents on sweep rate. 
The results from Figure 6.32 show that that the peak current increased and the peak became broader with increasing scan rate. The peak currents of the voltammograms are linearly proportional to the scan rate between $10-600 \mathrm{mV} \mathrm{s}^{-1}$ as can be seen in the inset of figure 6.32. The ratio of $I_{p a} / I_{p c}$ remains close to unity at all scan rates. This also indication that the anodic and cathodic peak currents are associated with surface-bound lead ions on DMSA SAM modified electrode. The linear regression equations for anodic and cathodic peak currents are $I_{p a}(\mu A)=$ $18.9+0.54 V(\mathrm{mV} / \mathrm{s})$ and $\mathrm{I}_{p c}(\mu \mathrm{A})=20.3+0.58 \mathrm{~V}(\mathrm{mV} / \mathrm{s})$. The values of correlation coefficients $\left(R^{2}\right)$ for two equations are 0.996 and 0.998 respectively.

\subsubsection{Effect of pH of preconcentration solution}

The effect of the $\mathrm{pH}$ on the reduction and oxidation currents was studied in the $\mathrm{pH}$ range 4-7.5. The $\mathrm{pH}$ of $0.1 \mathrm{M} \mathrm{KNO}_{3}+0.1 \mathrm{mM} \mathrm{Pb}\left(\mathrm{NO}_{3}\right)_{2}$ is 5.5. The $\mathrm{pK}_{\mathrm{a}}$ values of two carboxylic acid groups in DMSA are $2.7 \pm 0.2$ and $3.43 \pm 0.2$, respectively $[16,17]$. The $\mathrm{pH}$ value of $0.1 \mathrm{M} \mathrm{KNO}_{3}$ was 6.2 , which means the two carboxylic acid groups are deprotonated. Figure 6.33 shows that the accumulation of lead ions on DMSA modified Au electrode can depend strongly on $\mathrm{pH}$ of preconcentation solution. The inset in Figure 6.33 showed that the peak current reached a maximum value at $\mathrm{pH}$ 5.5-6. At pH 5.5-6 the terminal groups of the DMSA SAM are deprotonated and able to bind $\mathrm{Pb}^{2+}$. At low $\mathrm{pH}$ values $(\mathrm{pH} \leq 3.0)$, all carboxylic acid terminal groups of SAM should be protonated and the redox peaks disappeared. At high $\mathrm{pH}$ values $(\mathrm{pH} \geq 7.0), \mathrm{Pb}(\mathrm{II})$ tends to be hydrolysed and produce insoluble hydroxide $\mathrm{Pb}(\mathrm{OH})_{2}$. Because of this behaviour, a preconcentration solution of $\mathrm{pH} 5.5$ was chosen as the most efficient for preconcentration of $\mathrm{Pb}(\mathrm{II})$. 


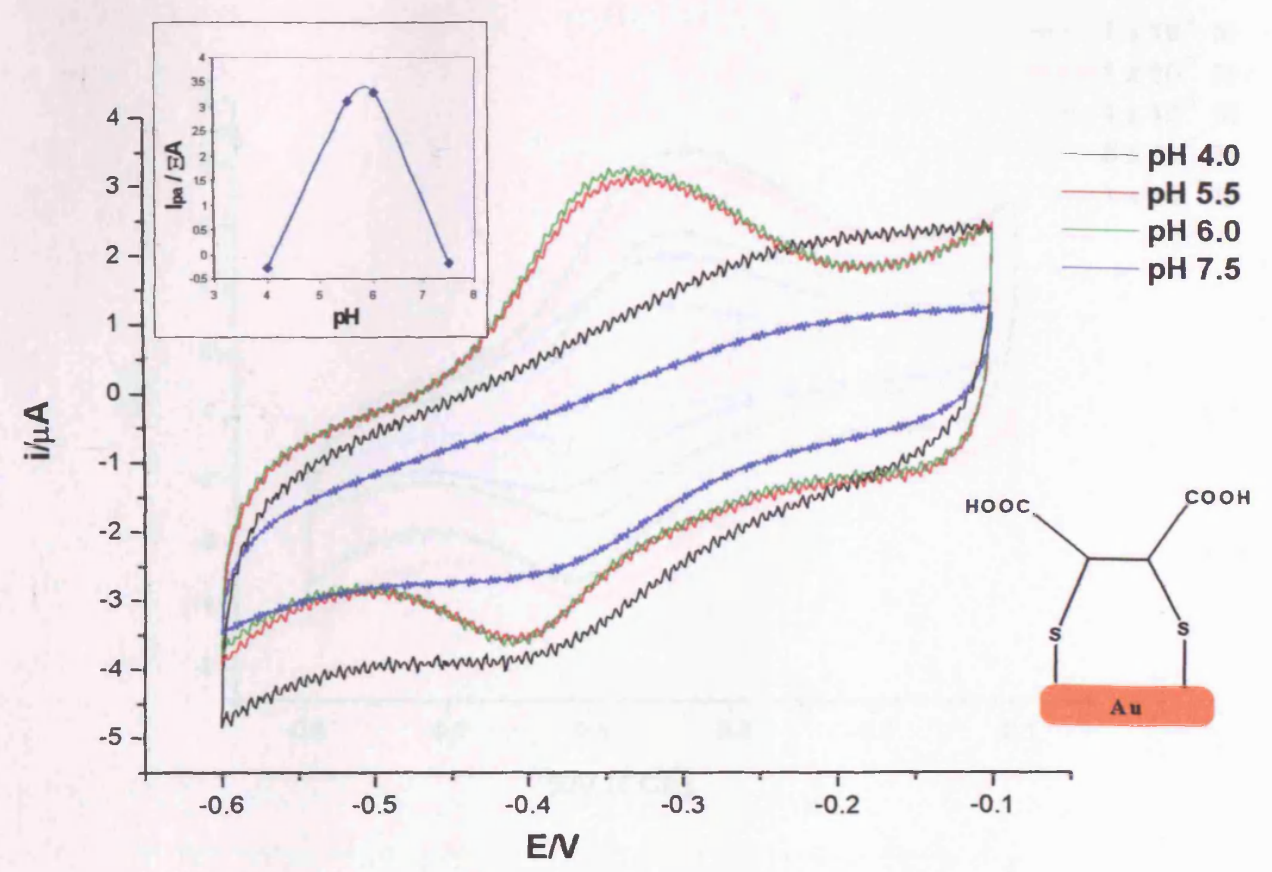

6.33: Effect of the preconcentration electrolyte $\mathrm{pH}$ on the peak current intensity for the complexed of $\mathrm{Pb}^{2+}$ with (DMSA) coated gold electrode. Inset: Plot of cathodic peak currents against $\mathrm{pH}$ after preconcentration. Cyclic voltammetry in lead-free $0.1 \mathrm{M} \mathrm{KNO} \mathrm{KN}_{3} \mathrm{pH} 6.2$; sweep rate $100 \mathrm{mV} \mathrm{s}^{-1}$; preconcentration time $=15 \mathrm{~min}$; open circuit.

\subsubsection{Concentration dependence of the characteristic peak charge}

The uptake ability of DMSA modified Au electrodes was investigated using different concentrations of lead ions. Cyclic voltammograms at different concentrations of $\mathrm{Pb}(\mathrm{II})$ are displayed in Figure 6.34. The cathodic peak current increased rapidly with increase in $\mathrm{Pb}(\mathrm{II})$ concentration to $1 \times 10^{-4} \mathrm{M}$. 


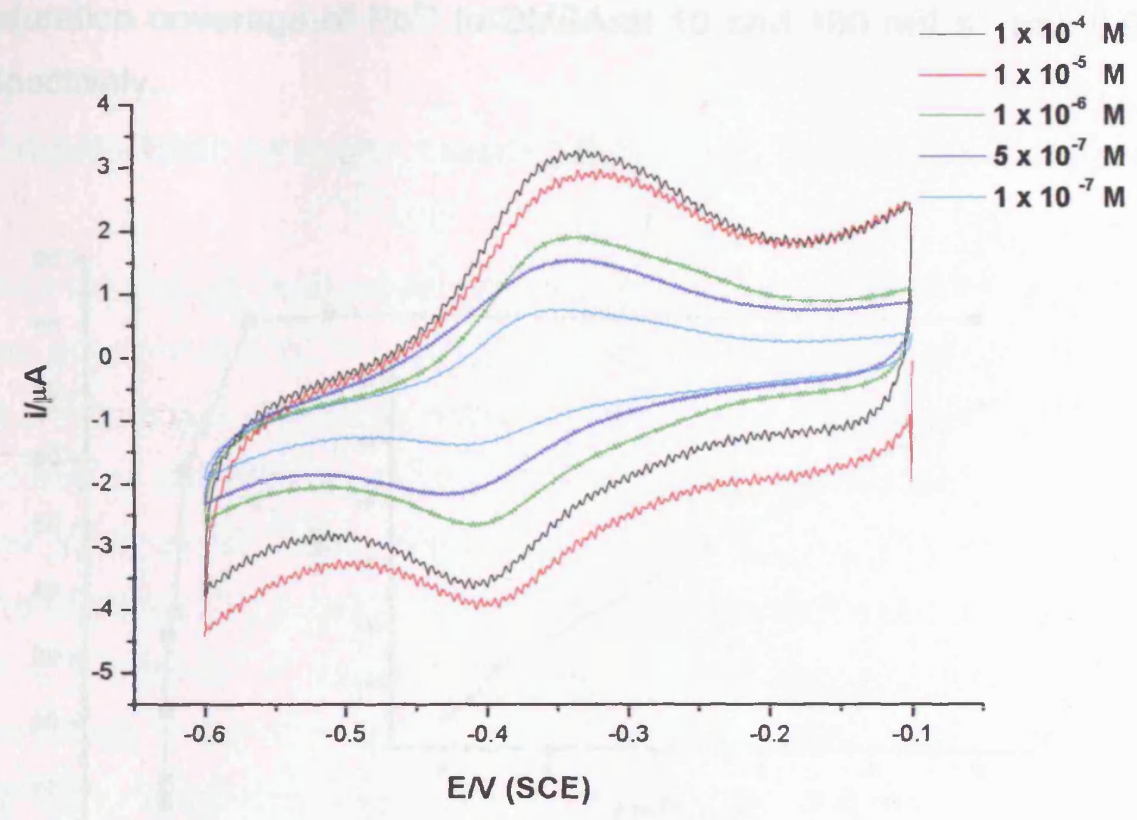

Figure 6.34: Cyclic voltammograms of complexed $\mathrm{Pb}^{2+}$ at a DMSA SAM modified $\mathrm{Au}$ electrode modified in lead-free nitrate $\left(0.1 \mathrm{M} \mathrm{KNO}_{3} \mathrm{pH} 6.2\right)$ after preconcentration time of 15 min in $0.1 \mathrm{M} \mathrm{KNO}_{3}$ solution ( $\mathrm{pH} 5.5$ ) containing $1 \times 10^{-4}, 1 \times 10^{-5}, 1 \times 10^{-6}, 5 \times 10^{-6}, 1 \times 10^{-7}$ and $5 \times 10^{-7} \mathrm{M}$ of $\mathrm{Pb}(\mathrm{II})$. Scan rate $100 \mathrm{mV} \mathrm{s}^{-1}$.

The dependence of the cathodic peak charge on the $\mathrm{Pb}^{2+}$ concentration in solution is shown in Figure 6.35. The cathodic peak charge increased rapidly with increase in the concentration of lead ions to a plateau level, indicative of a saturated DMSA SAM. The cathodic peak charge reached a constant value of $82 \pm 1.1 \mu \mathrm{C}(n=5$ on the same SAM) at concentration of $5 \mathrm{mM}(1000 \mathrm{ppm})$ of $\mathrm{Pb}^{2+}$ ions, which corresponds to a saturation surface coverage of lead ions $2.05 \pm 0.07 \mathrm{nmol} \mathrm{cm}$ $(n=5$ on the same SAM). A linear relationship between CV peak charge and concentrations of lead ions from 0.1 to $10 \mu \mathrm{M}$ was observed with the slope and correlation coefficient of $2.57 \mu \mathrm{A} / \mu \mathrm{M}$ and 0.995 , respectively, as shown in the inset graph in Figure 6.35. The reproducibility for cyclic voltammograms on the same surface coverage of DMSA was evaluated via the relative standard deviations $(\mathrm{n}=8)$ for the determination of 10 and $100 \mu \mathrm{M}$ of $\mathrm{Pb}^{2+}$ concentration. The R.S.D for the $\Delta \mathrm{Q}$ was $2.67 \%$ and $3.33 \%$, respectively.

The surface coverage of $\mathrm{Pb}^{2+}\left(\Gamma_{\mathrm{Pb}++}\right)$ was obtained by integration of oxidation peaks and assuming two electron reaction using Faraday's laws (section 2.7.2). The estimated surface coverage of lead ions at two different scan rates (10 and 100 $\left.\mathrm{mV} \mathrm{s}{ }^{-1}\right)$ was $2.55 \pm 0.07$ and $2.34 \pm 0.05 \mathrm{nmol} \mathrm{cm}(\mathrm{n}=7)$ at $1 \mathrm{mM}$ of $\mathrm{Pb}^{2+}$. The 
saturated surface coverage of the DMSA after $24 \mathrm{~h}$ was $2.64 \mathrm{nmol} \mathrm{cm}{ }^{-2}$. The ratio of the saturation coverage of $\mathrm{Pb}^{2+}$ to DMSA at 10 and $100 \mathrm{mV} \mathrm{s}^{-1}$ was 0.96 and 0.87 , respectively.

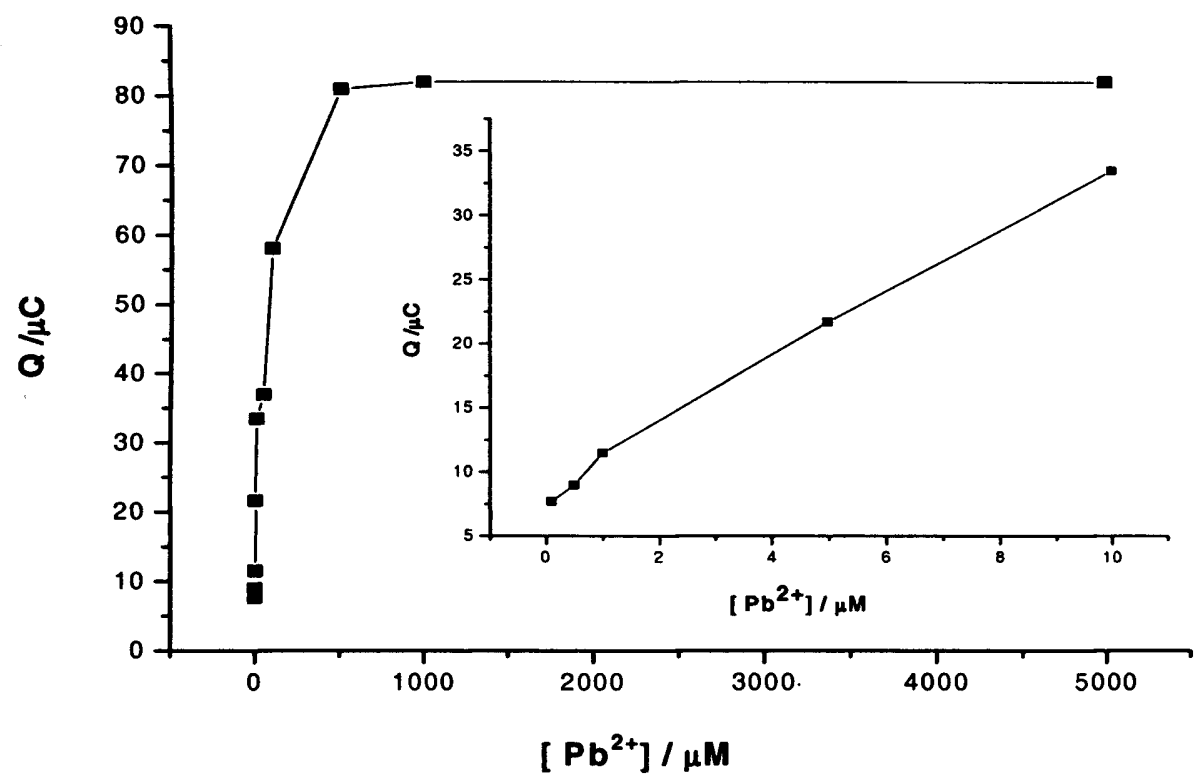

Figure 6.35: Influence of lead concentration in solution on the integrated peak charge. Cyclic voltammetry in lead-free $0.1 \mathrm{M} \mathrm{KNO} \mathrm{KN}_{3}(\mathrm{aq})$ having been exposed to different concentrations (from 0.1 to $5000 \mu \mathrm{M}$ ) $\mathrm{Pb}(\mathrm{II})_{(\mathrm{aq})}$. Scan rate $100 \mathrm{mV} \mathrm{s}^{-1}$, time $15 \mathrm{~min}$.

The above results show that the maximum uptake of lead ions in preconcentration solutions occurs at concentrations above $1 \mathrm{mM}$. This can be explained due to the formation of a 1:1 complex (metal to ligand) at higher concentrations of lead ions while at low concentrations of lead ions, there was low uptake and the saturation of the ligand with lead ions did not occur.

The effective reproducible preconcentration was illustrated by seven preconcentration experiments with $1.0 \times 10^{-5}$ and $5.0 \times 10^{-6} \mathrm{M}$ using two different surface coverage of DMSA. The results show that the modified electrode has good reproducibility with average anodic peak charges of 45.4 and $33.1 \mu \mathrm{C}$ and relative standard deviations of $9.6 \%$ and $8.2 \%$, respectively.

The limit of detection ( $3 \times \mathrm{SD}$ ) was found to be $0.08 \mu \mathrm{M}$. ' When the DMSA modified electrode is immersed into a $0.1 \mathrm{M}$ EDTA solution after voltammetric experiments in lead free nitrate solution, the responses due to $\mathrm{Pb}^{2+}$ ion completely disappear. This means the original surface of the uncomplexed of DMSA is successfully restored, and the modified electrode is ready for exposure to metal ions. 
Furthermore, the same voltammetric behaviour in Figure 6.31a is observed with EDTA experiment for five days without any deterioration.

\subsubsection{Complexation between meso-2,3 dimercaptosuccinic acid and $\mathrm{Cu}^{2+}$}

The Frequency shift of QCM measurements shows that the DMSA has little affinity for copper ions in solution. Figure 6.36 shows the cyclic voltammogram of a gold electrode covered with DMSA monolayer obtained in a copper-free nitrate solution without (curve a) and after a preconcentration step in $0.1 \mathrm{mM} \mathrm{Cu}^{2+}$ solution for 15 $\min$ (curve b). A pair of redox couples appeared at $E_{p, a}=0.292 \mathrm{~V}, E_{p, c}=0.190 \mathrm{~V}$ and formal potential $E^{\circ}=0.242 \mathrm{~V}$. The cathodic and anodic peaks correspond to the reduction and oxidation of copper complexed by the DMSA modified Au electrode, they undoubtedly result from the $\mathrm{Cu}^{2+} / \mathrm{Cu}^{0}$ redox transition. Control experiments, under the same conditions, demonstrated that copper ions do not adsorb onto a naked gold electrode surface. Thus, the voltammetric response of the DMSA modified $\mathrm{Au}$ electrode to $\mathrm{Cu}^{2+}$ should be attributed to the interaction between the DMSA SAM on the surface and the metal ion in solution.

The voltammetric characterisation of the Cu(II)-DMSA SAM modified Au electrode at subsequent scans (from $1^{\text {st }}$ to $20^{\text {th }}$ cyclic scans) is shown in the inset of Figure 6.36. The cyclic voltammograms were not stable. The anodic and cathodic peak currents decreased over 20 cycles. The cathodic peak current decreased rapidly from $2.49 \mu \mathrm{A}\left(1^{\text {st }}\right)$ to $0.70 \mu \mathrm{A}\left(20^{\text {st }}\right)$. These results indicate weak complexation between copper ions and DMSA. To further investigative the characteristics of the in-site complexation between copper species and DMSA modified electrode, the effect of scan rate on the voltammetric behaviour of $\mathrm{Cu}$ (II)/DMSA electrode was difficult to study because the Cu(II)/DMSA layer was not stable. 


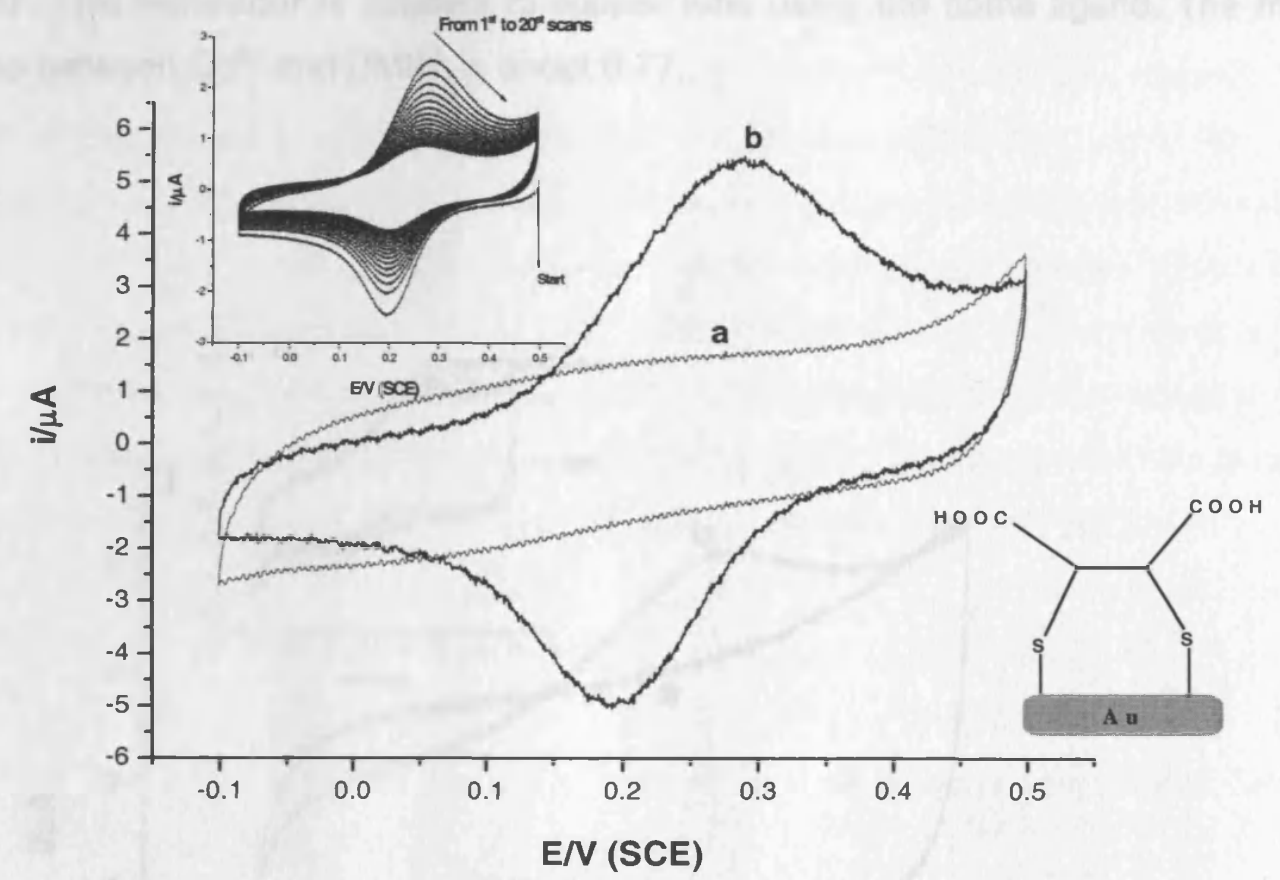

Figure 6.36: Cyclic voltammograms (CV) of DMSA/Au modified electrode in $0.1 \mathrm{M} \mathrm{KNO}_{3}$ solution, $\mathrm{pH} 6.2$ before (curve a) and after a preconcentration step at open circuit in a 0.1 $\mathrm{mM}$ copper nitrate solution $\mathrm{pH} 5.5$ for $15 \mathrm{~min}$. Scan rate $100 \mathrm{mV} \mathrm{s}^{-1}$ Inset: effect of repetitive cycling on the $\mathrm{Cu}^{2+}$-DMSA SAM modified Au electrode.

The saturation surface coverage of $\mathrm{Cu}^{2+}$ obtained in the first cyclic voltammetric scan was $1.90 \pm 0.2 \mathrm{nmol} \mathrm{cm} \mathrm{cm}^{-2}(n=5)$ and the saturation coverage of DMSA SAM was $2.64 \mathrm{nmol} \mathrm{cm}^{-2}$ (same surface DMSA coverage was used for binding lead ions) and $3.19 \mathrm{nmol} \mathrm{cm}^{-2}$ (fresh DMSA SAM) which gives the ratio of the saturation coverage of $\mathrm{Cu}^{2+}$ to DMSA as $0.7: 1$ and $0.6: 1$, respectively.

\subsubsection{Complexation between meso-2,3 dimercaptosuccinic acid and $\mathrm{Cd}^{2+}$}

The ability of DMSA SAM to bind cadmium ions from single aqueous solutions were examined using the same experimental condition was used above. Figure 6.37 shows cyclic voltammograms in $0.1 \mathrm{M}$ cadmium-free nitrate solution for DMSA SAM modified Au electrode before and after adsorption of cadmium ions $(0.1 \mathrm{mM})$ from preconcentration solution for $15 \mathrm{~min}$. Two small redox peaks appeared at approximately $E_{p, a}=-0.150 \mathrm{~V}$ and $E_{p, c}=-0.380 \mathrm{~V}$, (formal potential $E^{\circ}=-0.265 \mathrm{~V}$ ). 
These could be attributed from the oxidation and reduction processes for $\mathrm{Cd}^{2+} / \mathrm{Cd}^{0}$. The good stability to potential cycling at $100 \mathrm{mV} \mathrm{s}^{-1}$ is shown in the inset of Figure 6.37. This behaviour is different to copper ions using the same ligand. The mole ratio between $\mathrm{Cd}^{2+}$ and DMSA is about 0.77 .

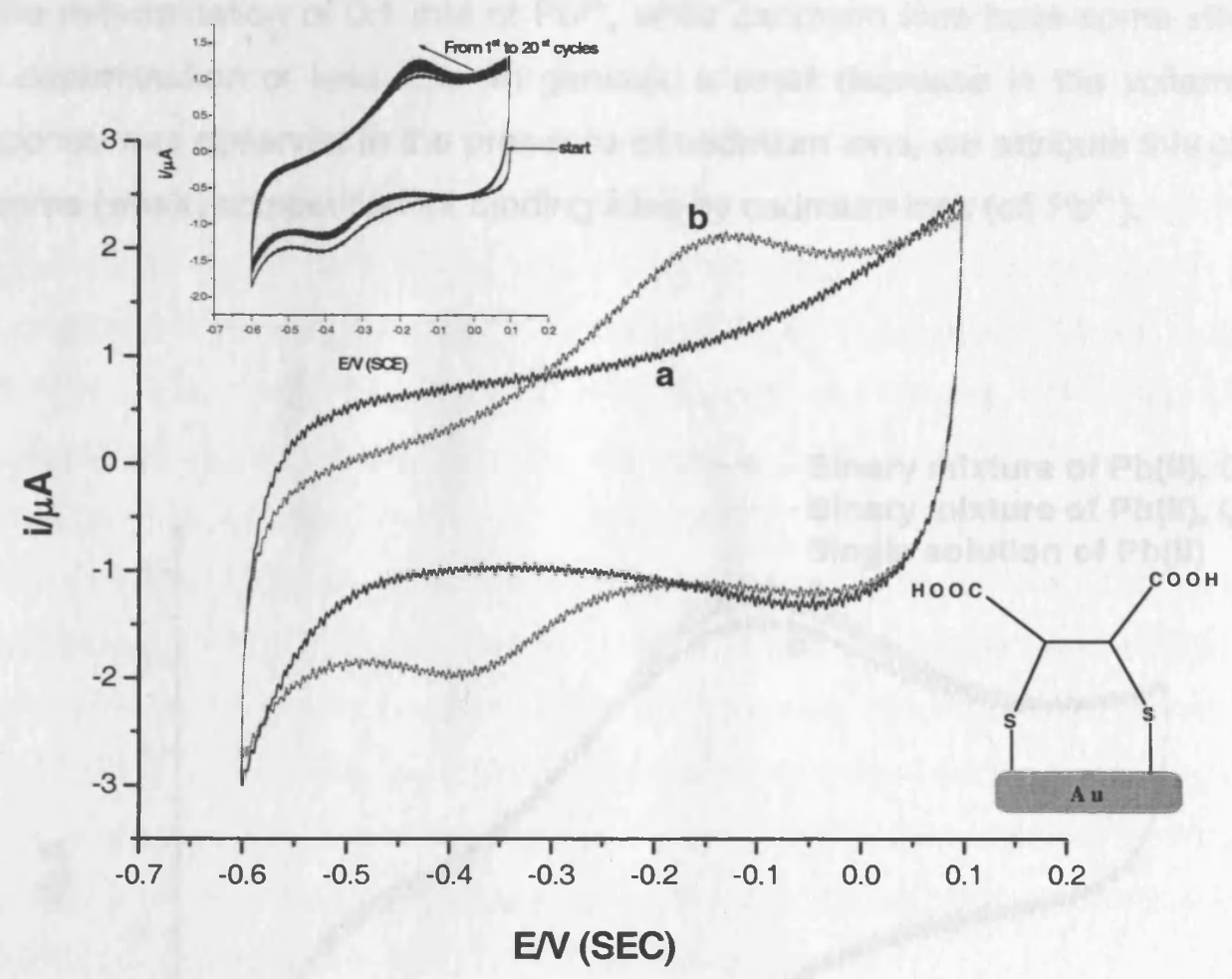

Figure 6.37: Cyclic voltammograms of DMSAAu modified electrode in $0.1 \mathrm{M} \mathrm{KNO}_{3}$ solution, $\mathrm{pH} 6.2$ before (curve a) and after (curve b) a preconcentration step at open circuit in a 0.1 $\mathrm{mM}$ cadmium nitrate solution, $\mathrm{pH} 5.5$, for $15 \mathrm{~min}$. Inset: effect of repetitive potential cycling on the $\mathrm{Cd}^{2+}$-DMSA SAM modified Au electrode from $1^{\text {st }}$ to $20^{\text {th }}$ cycles. Scan rate $100 \mathrm{mV} \mathrm{s}^{-1}$.

\subsubsection{Interference effects on $\mathrm{Pb}(\mathrm{II})$ responses in binary mixtures}

The selectivity of the DMSA modified Au electrodes were examined by introducing $\mathrm{Cu}^{2+}$ and $\mathrm{Cd}^{2+}$, up to 10 fold molar excess, into the preconcentration medium. The experimental results were nearly the same as in section 6.3.4. The voltammetic responses resulting from the presence of interfering ions were then compared with that obtained for single lead ion incorporation. The influence of $\mathrm{Cu}^{2+}$ and $\mathrm{Cd}^{2+}$ 
presence in the preconcentration solution on peak current response of $\mathrm{Pb}^{2+}$ is shown in Figure 6.38. It was found that from lead nitrate single solution, the reduction peak current obtained was $3.8 \mu \mathrm{A}$. In the presence of copper and cadmium ions at $1 \mathrm{mM}$, the peak currents were $3.6 \mu \mathrm{A}$ and $3.5 \mu \mathrm{A}$, respectively. The cathodic peak currents were changed by less than $6.5 \%$ for copper ions and $7.8 \%$ for cadmium ions when the concentration of copper and cadmium ions were fixed at $1 \mathrm{mM}$. These results clearly demonstrate that copper ions did not interfere in the determination of $0.1 \mathrm{mM}$ of $\mathrm{Pb}^{2+}$, while cadmium ions have some effect on the determination of lead ions. In general, a small decrease in the voltammetric response was observed in the presence of cadmium ions, we attribute this change to some (weak) competition for binding sites by cadmium ions (cf. $\mathrm{Pb}^{2+}$ ).

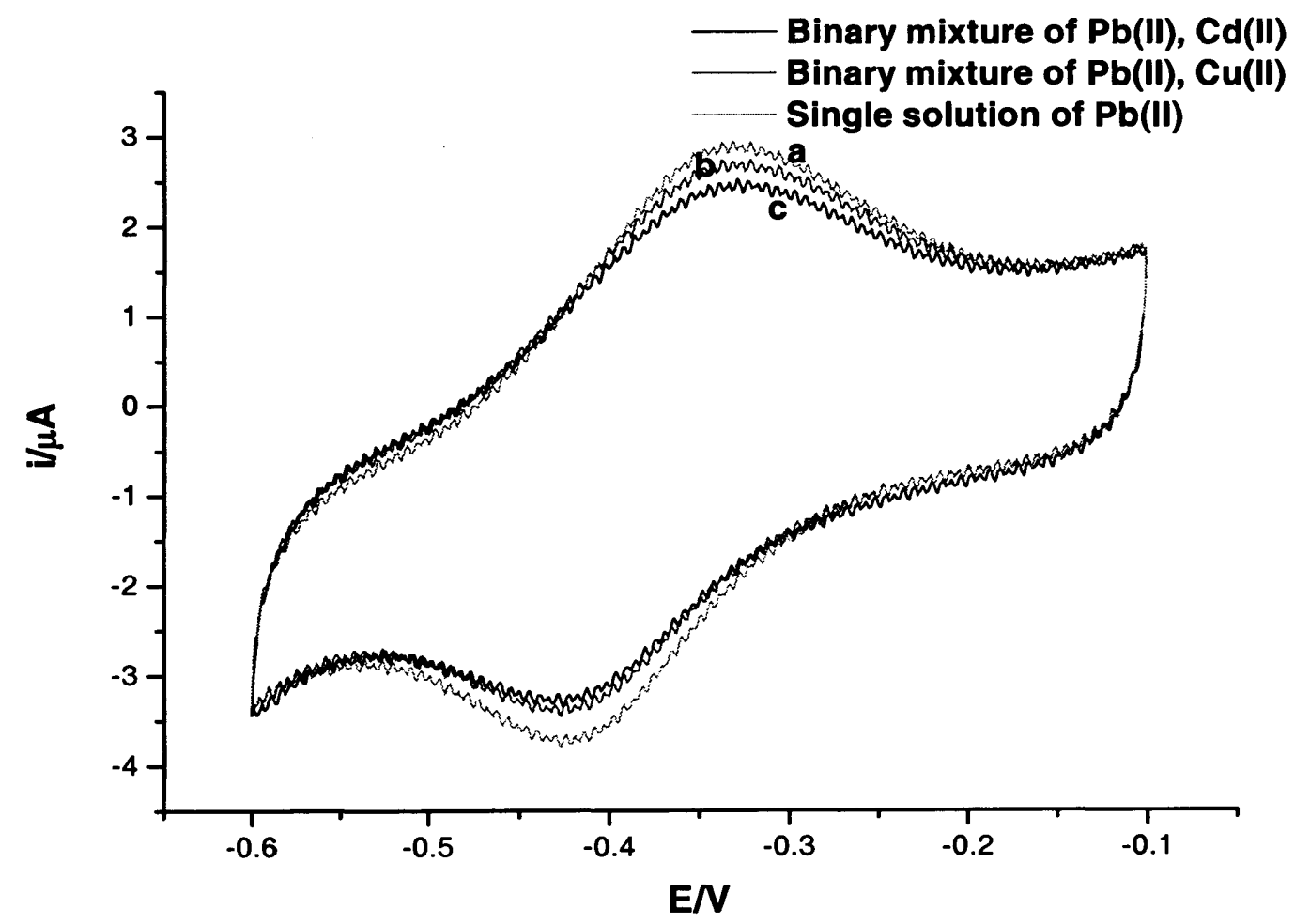

Figure 6.38: Cyclic voltammograms of the DMSA SAM modified Au electrode in $0.1 \mathrm{M}$ $\mathrm{KNO}_{3}(\mathrm{a})$ after adsorbing lead ions from $0.1 \mathrm{mM}$ lead nitrate (b) after exposure to a solution containing both $0.1 \mathrm{mM} \mathrm{Pb}$ (II) and $1 \mathrm{mM} \mathrm{Cu}$ (II) (c) after exposure to a solution containing both $0.1 \mathrm{mM} \mathrm{Pb}(\mathrm{II})$ and $1 \mathrm{mM} \mathrm{Cd}(\mathrm{II})$. Preconcentration time $15 \mathrm{~min}$; $\mathrm{pH}$ 5.5; open circuit; scan rate $100 \mathrm{mV} \mathrm{s}^{-1}$. 


\subsection{Complexation between 1,4-benzenedimethanethiol and $\mathrm{Hg}^{2+}$}

All of the SAMs studied in previous sections contained terminal free carboxylate groups. These kinds of SAMs are not suitable for binding mercury ions because $\mathrm{Hg}$ (II) can penetrate the monolayers and form a $\mathrm{Hg} / \mathrm{Au}$ amalgam, which was explained in detail for binding $\mathrm{Hg}(\mathrm{II})$ to 1,6-hexanedithiol using the QCM frequency shift in chapter five. The complexation between 1,4-benzenedimethanethiol (BDMT) SAM-modified Au electrode and mercury ions will be studied. The electrochemical characterisation of the BDMT SAM was studied previously in chapter four. It was found that the BDMT SAM coated Au surface exhibits better blocking properties compared with 1,6-hexanedithiol. Murty et al. [16] have found excellent thermal stability of BDMT SAM on gold compared to aliphatic $n$ alkanethiol SAMs in their SERS studies. They attributed this to the strong lateral van der Waals interactions among the phenyl rings. As a result, BDMT molecules can form better organized monolayers on gold compared with aliphatic 1,6hexanedithiol molecules. In fact, the monolayer formed by BDMT is much more organized on Au surface than even 1,6-hexanedithiol monolayers. Previous studies on BDMT using X-ray photoelectron spectroscopy (XPS) and X-Ray Auger electron spectroscopy (XAES) [17] confirmed that the BDMT molecules formed SAMs with only one thiol end group attached to the gold surface, leaving the other thiol group free, which it used in this experiment to adsorb $\mathrm{Hg}$ (II) ions from aqueous solutions. The electrochemical responses for the binding $\mathrm{Hg}$ (II) from solution to the BMDT modified Au electrode will be studied using the same experimental procedures was used for binding copper ions to TDP SAM in section 6.2.

\subsubsection{Experimental}

The experimental procedures were described in section 6.2.1: the preconcentration solution contained $1 \mathrm{mM}\left(\mathrm{Hg}\left(\mathrm{NO}_{3}\right)_{2}+0.1 \mathrm{M} \mathrm{KNO}_{3}\right), \mathrm{pH} 5.0$, the preconcentration time was $20 \mathrm{~min}$ at open circuit, and cyclic voltammograms were recorded between 0.8 to $-0.4 \mathrm{~V}$, versus SCE: at scan rates from 10 to $100 \mathrm{mV} \mathrm{s}^{-1}$.

\subsubsection{Results and Discussion}

Figure 6.39 shows representative cyclic voltammograms recorded for the BDMT SAM modified Au electrode in $0.1 \mathrm{M}$ potassium nitrate (pH 5.5) after "preconcentration" in mercury-free $0.1 \mathrm{M} \mathrm{KNO}_{3}$ (curve a) and after (curve b) "preconcentration" in $1.0 \times 10^{-4} \mathrm{M} \mathrm{Hg}^{2+}$ solution, respectively. As shown in Figure 6.39a, the BDMT SAM modified Au electrode showed no electroactivity in the 
potential range between $0.8 \mathrm{~V}$ and $-0.4 \mathrm{~V}$ before the preconcentration step. However, the cyclic voltammogram (Figure 6.39b) of BDMT after the preconcentration of $\mathrm{Hg}^{2+}$ ions had taken place for $20 \mathrm{~min}$, showed a well defined anodic peak at $+0.65 \mathrm{~V}$ and two cathodic peaks at $+0.594 \mathrm{~V}$ and $+0.194 \mathrm{~V}$. The standard reduction potentials of $\mathrm{Hg}_{2}{ }^{2+}$ and $\mathrm{Hg}^{2+}$ to $\mathrm{Hg}^{0}$ are $+0.548 \mathrm{~V}$ and $+0.610 \mathrm{~V}$ (vs. SCE), respectively. Thus, the first small cathodic peak (+0.594 V) corresponds to the reduction of $\mathrm{Hg}^{2+}$ to $\mathrm{Hg}_{2}{ }^{2+}$ species. The second cathodic peak $(+0.194 \mathrm{~V})$ corresponds to the reduction of $\mathrm{Hg}_{2}{ }^{2+}$ to $\mathrm{Hg}^{\circ}$ at the surface of BDMT. The well defined anodic peak ( $+0.65 \mathrm{~V}$ ) corresponds to the oxidation of $\mathrm{Hg}^{0}$ to $\mathrm{Hg}^{2+}$ ions. Moreover, the cathodic peak current observed at $+0.194 \mathrm{~V}$ was directly proportional to the preconcentration time and concentration of $\mathrm{Hg}^{2+}$ ions. Thus, we used this cathodic peak current as the analytical signal for further analytical investigations. These results indicate that the preconcentration of $\mathrm{Hg}^{2+}$ ions is occurring on the BDMT SAM modified Au electrode surface.

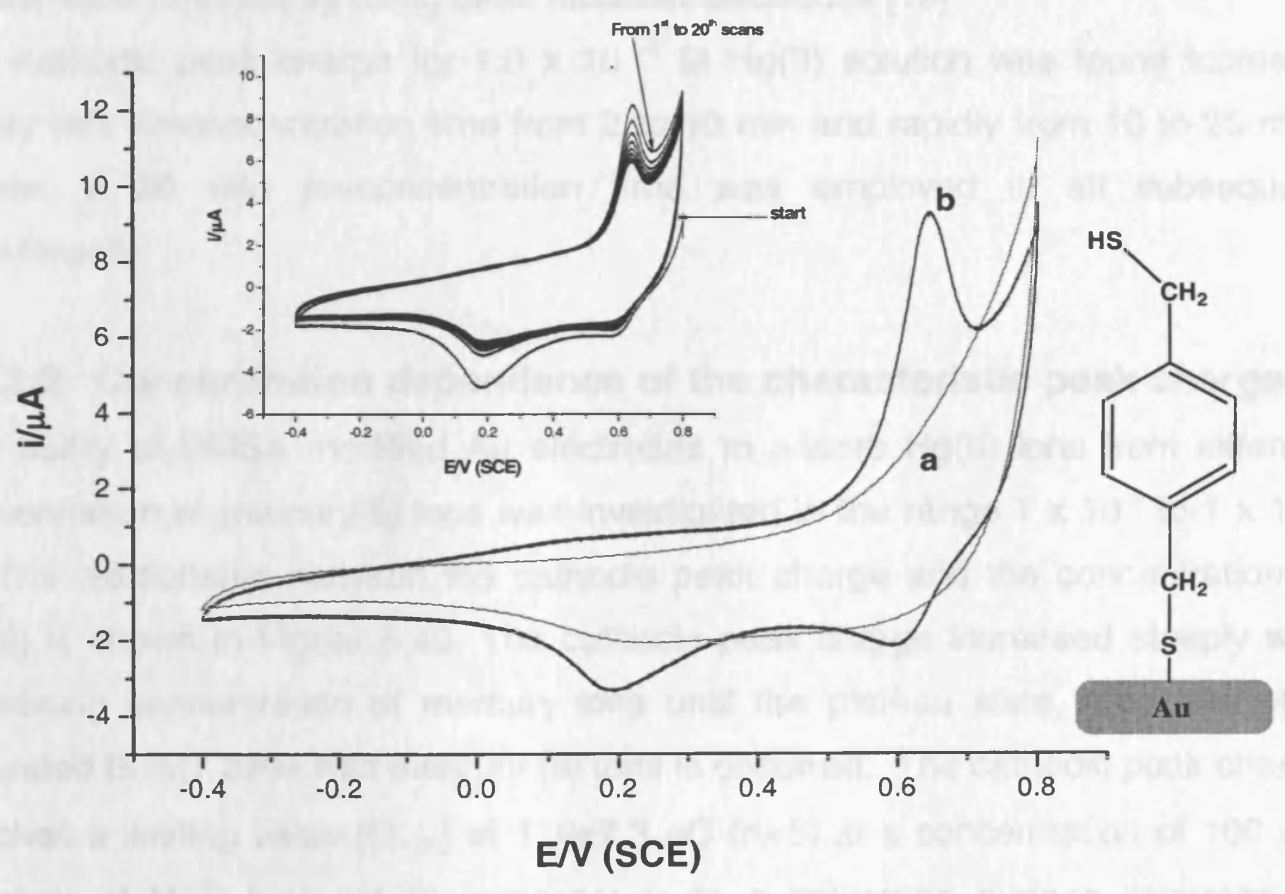

Figure 6.39: Cyclic voltammograms for the BDMT SAM modified Au electrode in mercuryfree $0.1 \mathrm{M} \mathrm{KNO}_{3}$ ( $\mathrm{pH} 6.0$ ); (a) before preconcentration step and (b) after dipping the BDMT SAM modified Au electrode in a $1.0 \times 10^{-4} \mathrm{M}$ of mercury nitrate solution for $20 \mathrm{~min}$ ( $\mathrm{pH} 5.0$ ). BDMT modified Au electrode was removed rinsed and then cycled in a mercury-free nitrate solution. Sweep rate $50 \mathrm{mV} \mathrm{s}^{-1}$. Inset: effect of repetitive cyclic scans on the $\mathrm{Hg}^{2+}$-BDMT SAM-modified Au electrode at scan rate $70 \mathrm{mV} \mathrm{s}^{-1}$. 


\subsubsection{Stability and effect of the sweep rate}

The voltammetric characterisation of the $\mathrm{Hg}$ (II)-BDMT SAM modified Au electrode over 20 subsequent scans at a sweep rate of $70 \mathrm{mV} \mathrm{s}^{-1}$ is shown in the inset of Figure 6.39. The results show a diminishing anodic peak current with two cathodic peak currents over five successive cycles and stability thereafter, with no shift in the anodic and cathodic peak potentials. The peaks are better resolved as the scan rate increase from 10 to $100 \mathrm{mV} \mathrm{s}^{-1}$. Furthermore, the cathodic peak current was linear with the scan rate in this range. This indicates that all adsorbed $\mathrm{Hg}(\mathrm{II})$ ions are retained on the SAM surface and the electrode reactions are controlled by adsorption.

The variation of cathodic peak current according to the $\mathrm{pH}$ of the preconcentration solution between 4 and 7.5 was studied. The $\mathrm{pH}$ of the preconcentration solution was controlled by using $0.1 \mathrm{M} \mathrm{KOH}$ and $0.1 \mathrm{M} \mathrm{HNO}_{3}$. Maximum peak height was achieved when the preconcentration was done at $\mathrm{pH}$ 5.0. At $\mathrm{pH}<5.0$ the ligand undergoes protonation. Around the natural $\mathrm{pH}(7-8)$, no voltammetric peaks are observed because $\mathrm{Hg}$ (II) could be changed to produce $\mathrm{Hg}(\mathrm{OH})_{2}$ [18]. Similar results were reported by using other modified electrodes [19].

The cathodic peak charge for $1.0 \times 10^{-4} \mathrm{M} \mathrm{Hg}(\mathrm{II})$ solution was found increase slowly with preconcentration time from 2 to $10 \mathrm{~min}$ and rapidly from 10 to $25 \mathrm{~min}$. Hence, a $20 \mathrm{~min}$ preconcentration time was employed in all subsequent experiments.

\subsubsection{Concentration dependence of the characteristic peak charge}

The ability of DMSA modified Au electrodes to adsorb $\mathrm{Hg}$ (II) ions from different concentration of mercury(II) ions was investigated in the range $1 \times 10^{-8}$ to $1 \times 10^{-4}$ $M$. The relationship between the cathodic peak charge and the concentration of $\mathrm{Hg}(\mathrm{II})$ is shown in Figure 6.40. The cathodic peak charge increased steeply with increasing concentration of mercury ions until the plateau state, indicative of a saturated BDMT SAM with mercury (II) ions is occurred. The cathodic peak charge reached a limiting value $\left(Q_{\max }\right)$ of $119 \pm 7.3 \mu \mathrm{C}(n=5)$ at a concentration of $100 \mu \mathrm{M}$ (40ppm) of $\mathrm{Hg}^{2+}$ ions, which corresponds to a saturation surface coverage of mercury ions of $2.93 \pm 0.03 \mathrm{nmol} \mathrm{cm}{ }^{-2}(n=5)$ on the same surface coverage of the ligand. The variation of cathodic peak charge with concentration of mercury ions was linear between 0.1 and $1 \mu \mathrm{M}$; least squares analysis gave $\mathrm{Q}(\mu \mathrm{C})=17.3(\mu \mathrm{C})$ $+65.3 \mathrm{c}(\mu \mathrm{M})$, with a correlation coefficient of 0.990 (see the inset graph of Figure $6.40)$. The reproducibility for cyclic voltamogramms on the same surface coverage 
of BDMT was evaluated by relative standard deviation $(n=6)$ for the determination of 10 and $100 \mu \mathrm{M}$ of $\mathrm{Hg}^{2+}$ concentration. The R.S.D for the $\Delta \mathrm{Q}$ was $3.51 \%$ and $3.93 \%$, respectively. The limit of detection ( $3 \times \mathrm{SD})$ was determined from the standard deviation of repeated determinations of the lead concentration at low levels of mercury (between 0.1 and $1 \mu \mathrm{M}$. The limit of detection was found to be $0.05 \mu \mathrm{M}$ (10 ppb).

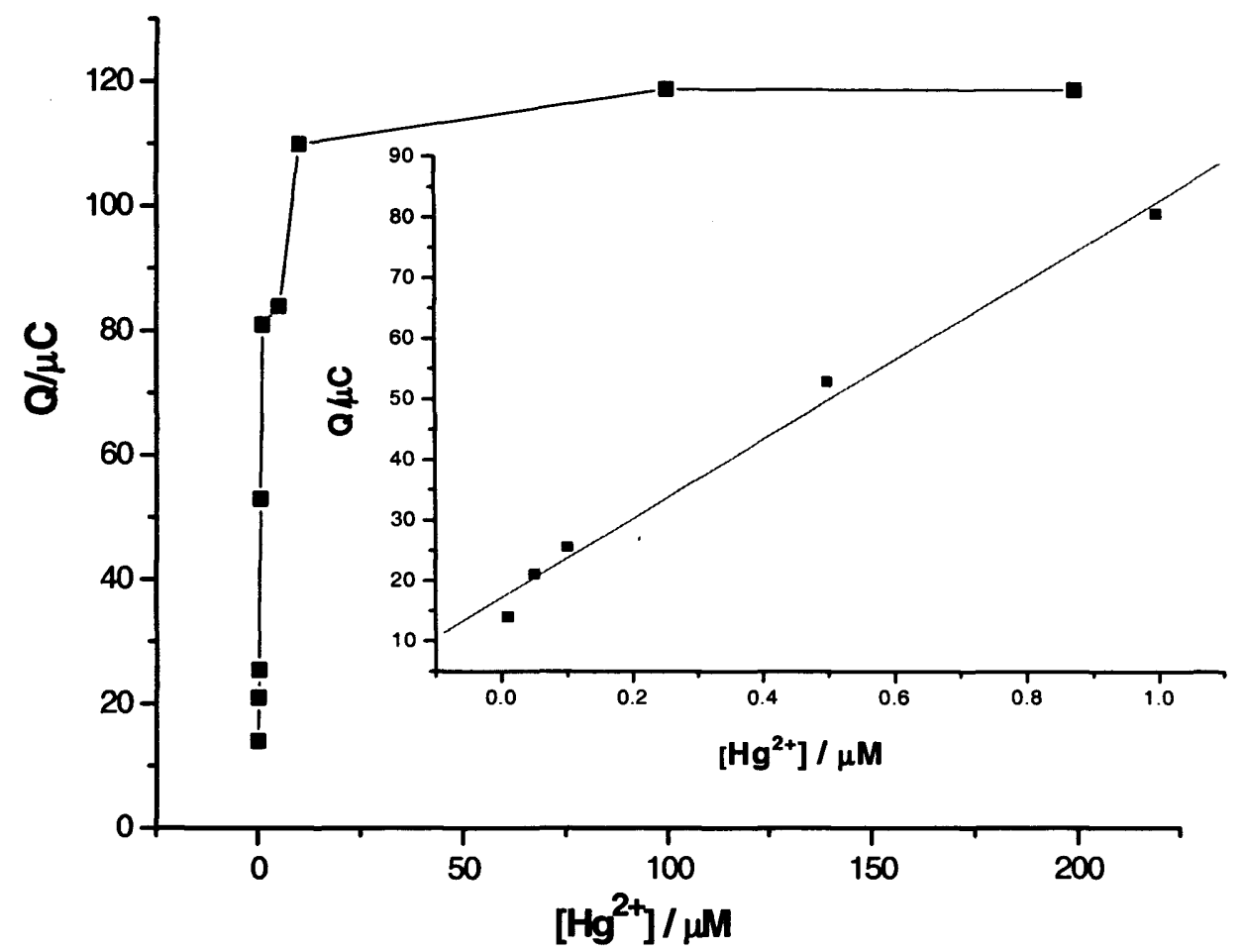

Figure 6.40 Variation of cathodic peak charge with concentration of $\mathrm{Hg}(\mathrm{II})$ ions. Cyclic voltammetry in mercury-free $0.1 \mathrm{M} \mathrm{KNO}_{3}(\mathrm{aq})$ having been exposed to different concentrations (from 0.1 to $200 \mu \mathrm{M}$ ) $\mathrm{Hg}(\mathrm{II})_{(\text {(aq) }}$. Scan rate $70 \mathrm{mV} \mathrm{s}^{-1}$, preconcentration time $20 \mathrm{~min}$.

The surface coverage of $\mathrm{Hg}^{2+}\left(\Gamma_{\mathrm{Hg++}}\right)$ was obtained by integration of reduction peaks and assuming a two electron reaction using Faraday's laws (section 2.7.2). The estimated surface coverage of mercury ions at scan rate $70 \mathrm{mV} \mathrm{s}^{-1}$ was $3.08 \pm 0.04 \mathrm{nmol} \mathrm{cm}^{-2}(\mathrm{n}=7)$ at $100 \mu \mathrm{M}$ of $\mathrm{Hg}^{2+}$. The saturated surface coverage of the ligand was $3.22 \pm 0.14 \mathrm{nmol} \mathrm{cm}^{-2}(n=4)$. The ratio of the saturation coverage of $\mathrm{Hg}^{2+}$ to BDMT was 0.955 . The BDMT SAM modified Au electrode demonstrated good reproducibility for the $\mathrm{Hg}(\mathrm{II})$ detections, For example, for two different fresh 
BDMT SAM surfaces with surface concentrations, 3.22 and $2.88 \mathrm{nmol} \mathrm{cm}$, the relative standard deviations (RSD) for the determinations of $200 \mu \mathrm{M}$ of $\mathrm{Hg}(\mathrm{II})(\mathrm{n}=6)$ were $8.5 \%$ and $9.33 \%$ respectively. The regeneration of the BDMT modified electrode was difficult and poor, when a fresh solution of 0.1M EDTA was used. The selectivity of the BDMT modified Au electrodes towards $\mathrm{Hg}(\mathrm{II})$ were examined by introducing $\mathrm{Cu}^{2+}$, up to 10 fold molar excess, into the preconcentration medium. No effect on the voltammeric response of BMDT to $\mathrm{Hg}$ (II) was observed and the same voltammogram in Figure $6.39 \mathrm{~b}$ was obtained.

\subsection{Complexation between 2-mercaptobenzimidizole and ${\mathbf{~} g^{2+}}^{2+}$}

The ligand 2-mercaptobenzimidazole is a well known analytical reagent for mercury. It has been used as a precipitating agent in gravimetric determination or as a spectrophotometric reagent [20]. $\mathrm{MBI}$ is a heterocyclic thiol containing an aromatic ring. The $\mathrm{MBI} S A M$ on $\mathrm{Au}$ is formed through the $\mathrm{Au}$ thiolate linkage and $\mathrm{MBI}$ is adsorbed in the thione form leaving the nitrogen centres free for complexation with mercury ions from aqueous solutions, which was developed by Berchmans et al using anodic stripping voltammetry [21]. In this section we will examine the ability of this ligand to adsorb $\mathrm{Hg}(\mathrm{II})$ ions from aqueous solutions using MBI SAM.

\subsubsection{Experimental}

The experimental procedures were described in section 6.2.1: the preconcentration solution contained $1 \mathrm{mM}\left(\mathrm{Hg}\left(\mathrm{NO}_{3}\right)_{2}+0.1 \mathrm{M} \mathrm{KNO}_{3}\right) \mathrm{pH} 5.0$, the preconcentration time was $15 \mathrm{~min}$ at open circuit, and cyclic voltammograms were recorded between 0.7 to $-0.1 \mathrm{~V}$, versus SCE, at scan rates from 10 to $300 \mathrm{mV} \mathrm{s}^{-1}$.

\subsubsection{Results and Discussion}

Figure 6.41 shows typical cyclic voltammograms obtained in mercury-free nitrate $\left(0.1 \mathrm{M} \mathrm{KNO}_{3}\right.$ ) before the preconcentration step (curve a) and after the preconcentration step (curve b). Two well-defined anodic and cathodic peaks were observed, corresponding to the redox chemistry of $\mathrm{Hg}(\mathrm{II})$. The anodic peak was obtained at $+0.65 \mathrm{~V}$, related to oxidation of mercury after the measurement and the cathodic peak was obtained at $+0.580 \mathrm{~V}$, related to reduction of $\mathrm{Hg}(\mathrm{II})$ after the preconcentration step. These results indicate that the preconcentration of $\mathrm{Hg}^{2+}$ ions is occurring on the BMI SAM modified Au electrode surface. The chemical preconcentration procedure, as described in section 6.7 .1 , was carried out on a 
bare Au electrode. The anodic and cathodic peak potentials are totally absent under the experimental conditions. This shows that the chemical preconcentration of $\mathrm{Hg}(\mathrm{II})$ does not take a place on a bare Au surface. Maximum anodic and cathodic peak currents were observed at $\mathrm{pH}$ 5. At low $\mathrm{pH}$ values, the complexation reaction between $\mathrm{Hg}(\mathrm{II})$ ions and BMI SAM modified $\mathrm{Au}$ electrode is inhibited because the protonation of two nitrogen centres of MBI SAM modified $A u$ electrode. The best $\mathrm{pH}$ for the deprotonation of the two nitrogen centres is around five.

\subsubsection{Stability and effect of the sweep rate}

The anodic and cathodic peak currents (see the inset of Figure 6.41) decrease slightly with increasing the number of cycles until the ninth one, after which the anodic and cathodic peak currents stabilized. No shift in the anodic and cathodic peak potentials was observed during fifteen cycles. The effect of scan rate from 10 to $300 \mathrm{mV} \mathrm{s}^{-1}$ on the stability of the complexation between $\mathrm{Hg}(\mathrm{II})$ and MBI SAM modified Au electrode was also examined. The anodic and cathodic peak currents are linear with the scan rate in the range 10 to $70 \mathrm{mV} \mathrm{s}^{-1}$, indicating a typical surface process. At scan rates bigger than $70 \mathrm{mV} \mathrm{s}^{-1}$, the relation between anodic and cathodic peak currents with scan became non-linear; this may be a kinetic effect. 


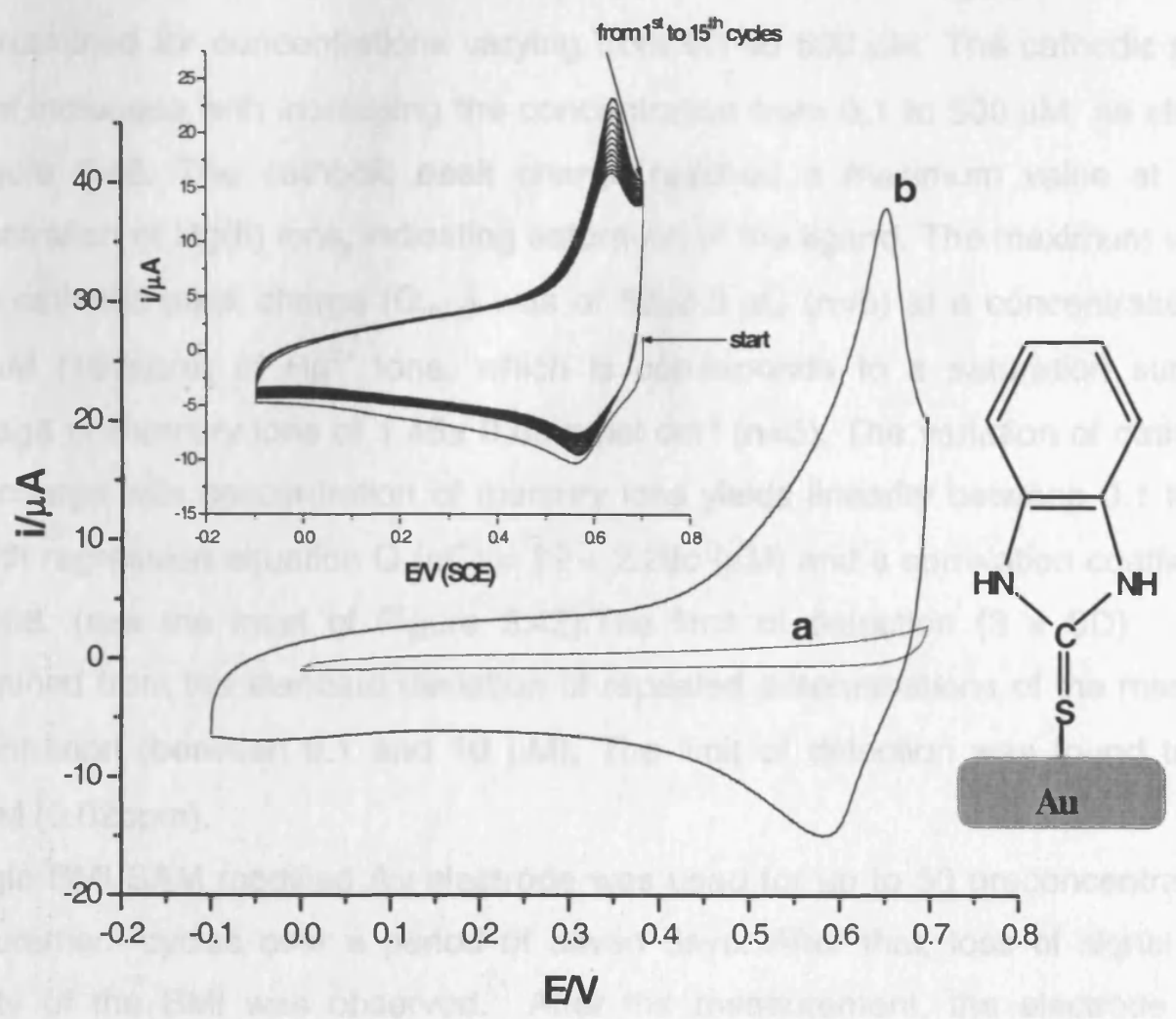

Figure 6.41: Cyclic Voltammograms for the BMI SAM modified Au electrode in mercury-free $0.1 \mathrm{M} \mathrm{KNO}_{3}(\mathrm{pH} 6.0)$; (a) before preconcentration step (b) after dipping the BMI SAM modified Au electrode in a $1.0 \times 10^{-4} \mathrm{M}$ of mercury nitrate solution for $15 \mathrm{~min}(\mathrm{pH} 5.0)$. In all cases the BMI modified Au electrode was removed, rinsed and then cycled in a mercuryfree nitrate solution. Inset: effect of repetitive cyclic scans on the $\mathrm{Hg}^{2+}-\mathrm{MBI}$ modified $\mathrm{Au}$ electrode. Sweep rate $100 \mathrm{mV} \mathrm{s}^{-1}$.

The surface coverage of $\mathrm{Hg}^{2+}\left(\Gamma_{\mathrm{Hg++}}\right)$ was obtained by integration of the reduction peak and assuming a two electron reaction using Faraday's laws (section 2.7.2). The estimated surface coverage of mercury ions at scan rate $100 \mathrm{mV} \mathrm{s}^{-1}$ was $1.50 \pm 0.08 \mathrm{nmol} \mathrm{cm}^{-2}(\mathrm{n}=6)$ at $500 \mu \mathrm{M}$ of $\mathrm{Hg}^{2+}$. The saturated surface coverage of BMI SAM-modified Au electrode was $3.24 \mathrm{nmol} \mathrm{cm}^{-2}$. The ratio of the saturation coverage of $\mathrm{Hg}^{2+}$ to BMI SAM was 0.500 . These results showed that one mercury ion complexes with two neighboring $\mathrm{MBI}$ molecules. 


\subsubsection{Concentration dependence of the characteristic peak charge}

The relationship of oxidation and reduction peak currents with $\mathrm{Hg}(\mathrm{II})$ concentration was examined for concentrations varying from 0.1 to $500 \mu \mathrm{M}$. The cathodic peak current increases with increasing the concentration from 0.1 to $500 \mu \mathrm{M}$, as shown in Figure 6.42. The cathodic peak charge reached a maximum value at high concentration of $\mathrm{Hg}(\mathrm{II})$ ions, indicating saturation of the ligand. The maximum value of the cathodic peak charge $\left(Q_{\max }\right)$ was of $59 \pm 3.3 \mu \mathrm{C}(n=5)$ at a concentration of $500 \mu \mathrm{M}$ (100ppm) of $\mathrm{Hg}^{2+}$ ions, which is corresponds to a saturation surface coverage of mercury ions of $1.45 \pm 0.03 \mathrm{nmol} \mathrm{cm}^{-2}(n=5)$. The variation of cathodic peak charge with concentration of mercury ions yields linearity between 0.1 to 10 $\mu \mathrm{M}$ with regression equation $\mathrm{Q}(\mu \mathrm{C})=19+2.28 \mathrm{c}(\mu \mathrm{M})$ and a correlation coefficient of 0.988 . (see the inset of Figure 6.42). The limit of detection ( $3 \times$ SD) was determined from the standard deviation of repeated determinations of the mercury concentration (between 0.1 and $10 \mu \mathrm{M}$ ). The limit of detection was found to be $0.09 \mu \mathrm{M}(0.02 \mathrm{ppm})$.

A single BMI SAM modified Au electrode was used for up to 50 preconcentration/ measurement cycles over a period of seven days. After that, loss of signal and stability of the BMI was observed. After the measurement, the electrode was cycled in 0.1M EDTA solution to ensure that the $\mathrm{Hg}$ (II) could be completely removed from the electrode surface. The surface coverage of BMI was nearly same before regeneration step. 


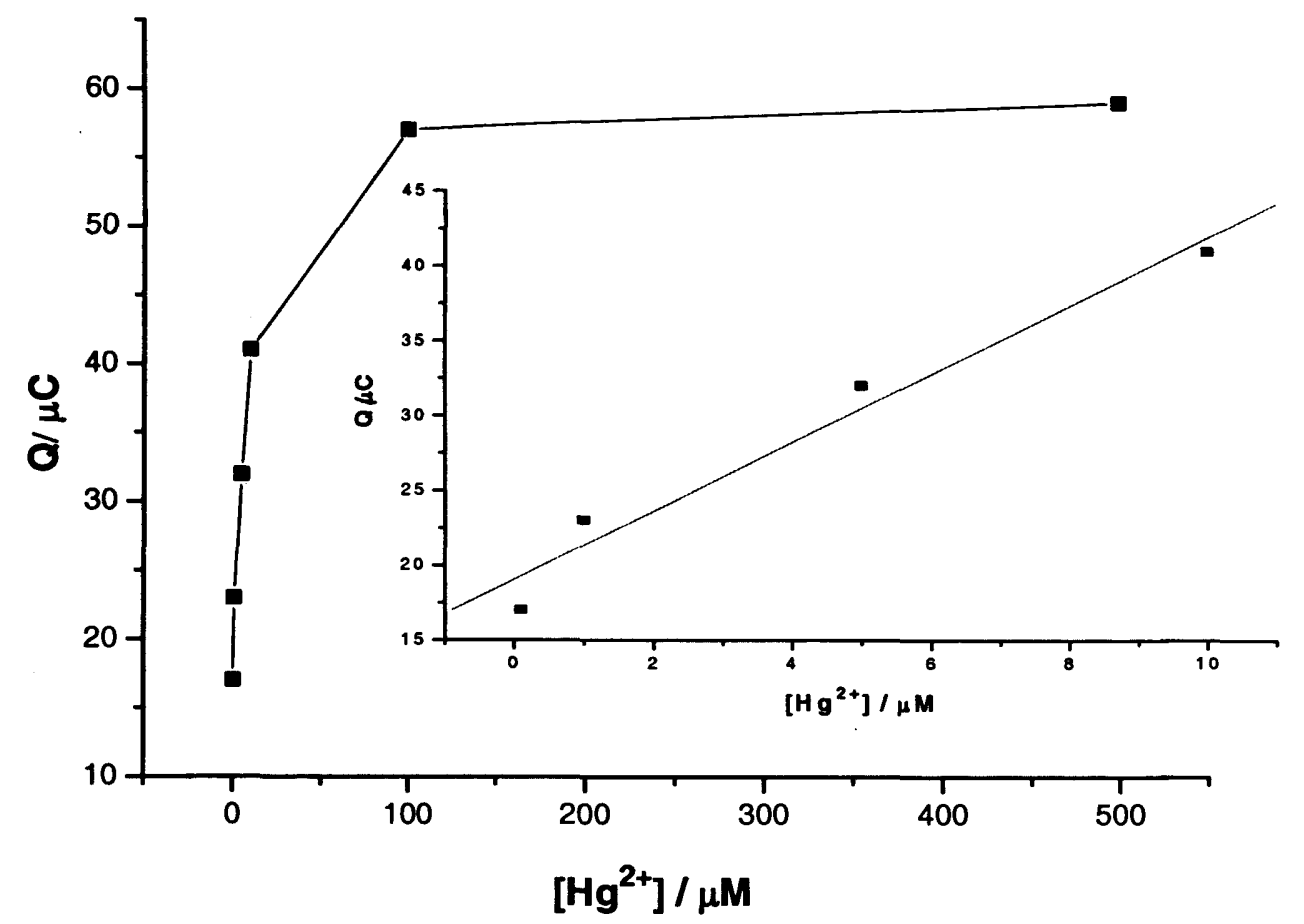

Figure 6.42: Variation of cathodic peak charge with concentration of $\mathrm{Hg}(\mathrm{II})$ ions. Cyclic voltammetry in mercury-free $0.1 \mathrm{M} \mathrm{KNO}_{3}$ (aq) having been exposed to different concentrations (from 0.1 to $500 \square \mathrm{M}$ ) $\mathrm{Hg}(\mathrm{II})_{(\mathrm{aq})}$. Conditions as in Figure 6.41.

\subsection{General Conclusions}

The experiments discussed in this chapter have shown successful electrochemical responses of different trace metal ions bound to different SAMs using EQCM as an electrochemical probe of the SAM interface. The results indicate that the complexation can preconcentrate target metal ions onto the SAM modified $\mathrm{Au}$ surface. Voltammetric responses after the complexation between SAMs modified Au surface and trace metal ions in aqueous solutions depend on several factors such as scan rate, repetitive cycling, preconcentration time, $\mathrm{pH}, \mathrm{pK}_{\mathrm{a}}$, modification time for the SAM and the concentration of target metal ions. Cyclic voltammetry of the redox couple of metal ions allows determination of metal ion concentration. The results show the peak charge after complexation with metal ions depends on the concentration of metal ions used in the preconcentration step, which can be used to detect the concentration of trace metal ions from micromolar to nanomolar level. The saturation surface coverage of target metal ions was estimated at high concentrations of metal ions from the integrated cathodic peak charge and the mole ratio between metal ion:SAM was found to be close to 1:1. The voltammetric 
peaks were extracted have excellent selectivity for target metal ions in presence of foreign ions in binary mixtures for example TDS SAM modified Au electrode has a high selectivity to $\mathrm{Cd}^{2+}$ in the presence of lead or copper at 10-fold excess, the cathodic peak currents were changed by less than 10\%. DMSA SAM- modified Au electrode also has selectivity to $\mathrm{Pb}^{2+}$ ions in presence of $\mathrm{Cd}^{2+}$ or $\mathrm{Cu}^{2+}$, no additional voltammetric peaks from these interferents was observed in the two cases and the cathodic peak current for the reduction of $\mathrm{Pb}^{2+}$ were decreased less than $11 \%$. BDMT SAM-modified Au electrode has a high affinity to $\mathrm{Hg}(\mathrm{II})$ in presence of $\mathrm{Cu}(\mathrm{II})$ and the cathodic peak current decreased less than $4 \%$.

There is a very good agreement between the results were extracted here for the selectivity of SAMs towards target metal ions, mole ratio and maximum surface coverage of target metal ions and that extracted from the QCM frequency measurements in chapter five.

The detection limit was estimated here three times magnitude lower than estimated using QCM as a gravimetric probe. The EQCM experiments gave more details about the nature of complexation between target metal ions and SAMs than QCM frequency measurements.

\subsection{References}

1- R.Rolf, Angewandte Chemie., 29, (1990), 329-343.

2- P.Norouzi, M.Namazian and A.Badiei, Analytical Sciences., 20, (2004), 519.

3- E. Laviron, J.Electroanal. Chem., (1979), 101,19.

4- J.Zhao, L.Luo, X.Yang, E. Wang and S.Dong, Electroanalysis., 11, (1999), 1108.

5- H. Munakata and S. Kuwabata, Chem. Commun., (2001), 1338.

6- S. Bharathi, V.Yegnaraman and G.P. Rao, Langmuir., 11, (1995), 666.

7- J.A.Dean, Lange's Handbook of Chemistry, McGraw-Hill, 1999.

8- E. Chow, D.B.Hibbert and J.J.Gooding, Anal.Chim. Acta., 543, (2005), 167.

9- S.Steinberg, Y.Tor, A.Shanzer and I.Rubinstein, Thin Films., 20, (1995), 183.

10- D.W.M.Arrigan, L.L.Bihan and M.J.Pickup, The Analyst., 124, (1999), 1797.

11- E.Chow, D.B. Hibbert and J.J.Gooding, Electrochem. Commun., 7, (2005), 101.

12- A.J.Bard, L.R. Faulkner, Electrochemical Methods: Fundamentals and Applications; Wiely: New York 2001, p. 307.

13- K.Fanata and B.S. Chandravanshi, Electroanalysis., 13, (2001), 484.

14- E.Chow, D.B. Hibbert and J.J.Gooding, TheAnalyst., 130 (2005), 831. 
15- D.Oyamastu, S.Kuwabata and H.Yoneyama, J.Electroanal.Chem., 473, (1999), 59.

16- U.K.Sur, R.Subramanian and V.Lakshminarayanan, J.Colloid Interface. Sci, 266, (2003), 175.

17- K.V.G.K.Murty, M.Venkataramanan and T.Pradeep, Langmuir., 14, (1998), 5446.

18- M.S.Won, D.W.Moon and Y.B.Shim, Electroanalysis., 7, (1995), 1171.

19- M.S.Won, Y.J.Bae, S.S.Lee and Y.B.Shim, Electroanalysis., 13, (2001), 1003.

20- A.K. Majumdar and M.M.Chakarabarty, Fresenius J. anal. Chem., 162, (1958), 101.

21- S.Berchmans, S.Arivulkkodi and V.Yegnaraman, Electrochem. Commun., 2, (2000), 226. 


\section{Chapter 7: XPS study of the complexation between trace}

\section{metal ions and SAMs}

\subsection{Introduction}

X-Ray photoelectron spectroscopy (XPS) is a well-established tool for the characterization of the molecular structure of organic films. XPS survey spectra can be used to confirm the presence of desired elements in the film and to evaluate its atomic composition [1,2]. Peak positions can give information about the local environment and degree of packing of the chains in the film. Atomic ratios have to be interpreted with great care since photoelectrons from atoms near the substrate are attenuated by overlaying material. The measured atomic composition is thus sensitive to the elemental distribution perpendicular to the surface in addition to the actual composition of the film. High resolution X-ray photoelectron spectroscopy has often been used to identify the interaction of a metal ion with the surface chemical groups on an adsorbent material during adsorption because the creation of a chemical bond between a metal ion and an atom on surface of the adsorbent changes the distribution of the electrons around the corresponding atoms. The electron donating ligand can lower the binding energy (BE) of the core level electrons while electron-withdrawing ligands can increase the BE $[3,4,5]$. The XPS technique will allow us to explore both the Au/SAM and SAM/analyte interfaces, providing insight into adsorption stoichiometry and metal ion speciation.

\subsection{Aims and Objectives}

When SAM surfaces are exposed to solutions of trace metal ions a preconcentration equilibrium is set up at electrode solution interface. We need to understand the nature of the interfacial binding process, which controls the interfacial composition, and thereby sensor performance. Using the QCM in gravimemtric mode we have determined binding constants of different metal species e.g. $\mathrm{Cu}^{2+}, \mathrm{Pb}^{2+}, \mathrm{Cd}^{2+}$ and $\mathrm{Hg}^{2+}$, in single and mixed ion solutions. This is a thermodynamic probe of the interface. Similarly we have used EQCM in coulometric mode (by integrating the voltammetric responses of surface-bound complexed metal ions) to determine metal ion coverage. However, neither of these population-based techniques provides an insight into the nature of the metal species and ligand within the complex. We aim to use high resolution XPS in order to acquire quantitative correlations of surface composition and structure at the interface. Thus, we will use XPS to probe the nature of adsorbed metal ion; it's 
coordination environment, oxidation state, and that of the underlying SAM as a function of electrode potential. We hope to learn more about the preconcentration equilibria (particularly under competitive situations) and metal speciation at SAMs exposed to dilute aqueous solutions of single and multiple metal ions. The important questions to be answered using XPS are the mole ratio between the metal ion and SAM ligand (S:M) (different free terminal groups) for all metal ions and the chemical changes occurring in the oxidation states of sulphur, carbon, oxygen and nitrogen before and after complexation with metal ions. Finally we seek to use ex situ high resolution X-ray photoelectron spectroscopy (XPS) to calibrate the results that were extracted from QCM-EQCM measurements and to compare the adsorption stoichiometry results from the three techniques.

The same ligands in chapter five and six will be examined here for selectivity towards $\mathrm{Cu}^{2+}, \mathrm{Cd}^{2+}, \mathrm{Pb}^{2+}$ and $\mathrm{Hg}^{2+}$ ions. The ligands used are 3,3-thiodipropionic acid (TDP), 3-thiodisuccinic acid (TDS), meso-2,3-dimercaptosuccinic acid (DMSA), 4-mercaptobenzoic acid (MBA), 11-mercaptoundecanoic acid (MUD), 2-

mercaptobenzimidizole (MBI), 1,6-hexanedithiol (HDT) and 1,4benzenedimethanethiol (BDMT). The free terminal groups are $(-\mathrm{COOH}),(-\mathrm{NH})$ and (-SH).

\subsection{Experimental}

The experimental instrumentation and procedures for preparation of SAMs are described in chapter three. For XPS experiments, all SAMs were immobilized onto thin film gold electrodes that were fabricated by metal gold evaporation onto glass microscope slides. The adsorption procedure for each metal ion onto SAMmodified Au electrode was described in chapters five and six. The concentrations within mixtures of metal ions were fixed at $1 \mathrm{mM}$ for both target and interfering ions. In order to confirm the immobilization of the organic layer and adsorption of metal ion onto the SAM-modified Au electrode, XPS spectra of the following sample surfaces were collected: SAM (surface one), SAM/M ${ }^{2+}$ (surface two) and SAM/mixture of metal ions (surface three). The SAM samples were introduced into the spectrometer immediately after washing in absolute ethanol and deionized water. The SAM/M $\mathrm{M}^{2+}$ samples were introduced into the spectrometer immediately after preconcentration of each metal ion and washing with deionized water. For each sample, an initial survey was performed followed by high resolution scans of the $A u\left(4 f_{7 / 2}\right), S\left(2 p_{3 / 2}\right), C(1 s), N(1 s), O(1 s), C u\left(2 p_{3 / 2}\right), P b\left(4 d_{5 / 2}\right), C d\left(3 d_{5 / 2}\right)$ and $\mathrm{Hg}\left(4 \mathrm{f}_{7 / 2}\right)$ regions. Due to the charging of the sample, there was a binding energy shift about $3 \mathrm{eV}$ for all the regions, so the binding energy $(\mathrm{BE})$ corrections were 
made by referencing all spectra to the $\mathrm{Au}\left(4 \mathrm{f}_{7 / 2}\right)$ peak at $84.0 \mathrm{eV}$ [6]. Analysis of the resulting spectra (i.e., smoothing, background subtraction and curve fitting) was undertaken using Scienta Win ESCA software, Microsoft Office Excel 2003 and Microcal Origin 7.0 according to procedures reported in the literature [7]. Assignment of spectral peaks was made using the UKSAF, NIST database and literature references $[8,9,10]$.

\subsection{Results and Discussions}

The results of the XPS experiments for SAMs are divided into three parts: firstly, XPS elemental composition for SAMs; secondly, peak fitting analysis for $S\left(2 p_{3 / 2}\right)$, $C_{1 s}, O_{1 s}$ and $N_{1 s}$; and finally, peak fitting analysis for $\mathrm{Cu}\left(2 p_{3 / 2}\right), \operatorname{Pb}\left(4 d_{5 / 2}\right), C d\left(3 d_{5 / 2}\right)$ and $\mathrm{Hg}\left(4 \mathrm{f}_{7 / 2}\right)$.

\subsubsection{Elemental composition for SAM and SAM/M ${ }^{2+}$}

Preliminary survey spectra scans of the various SAM-modified Au samples indicated the presence of all the expected elements (viz., C, S, O, Au and also target metal ions except 2-mercaptobenzimidazole $\mathrm{MBI}$ which contains $\mathrm{N}$ ) but no extraneous elements were observed. These results confirmed that the gold surfaces we used for the monolayer preparations and for monolayers with metal ions were of high purity and did not have any significant amount of contamination. The results of the survey spectra and high resolution spectra for the elements in the SAMs of meso-2,3-dimercaptosuccinic acid DMSA, 3-thiodisuccinic acid TDS, 3,3-thiodipropionic acid TDP, 1,6-hexanedithiol HDT and 2-mercaptobenzimidazole $\mathrm{MBI}$ before and after adsorbed $\mathrm{Pb}^{2+}, \mathrm{Cd}^{2+}, \mathrm{Cu}^{2+}$ and $\mathrm{Hg}^{2+}$, respectively, are shown in Figures 7.1, 7.2, 7.3 and 7.4. The XPS spectra in these figures showed characteristic peaks corresponding to $\mathrm{C}(1 \mathrm{~s})(285 \mathrm{eV}), \mathrm{O}(1 \mathrm{~s})(532 \mathrm{eV}), \mathrm{S}\left(2 \mathrm{p}_{3 / 2}\right)(162$ $\mathrm{eV}), \mathrm{Cu}\left(2 \mathrm{p}_{3 / 2}\right)(933 \mathrm{eV}), \mathrm{Pb}\left(4 \mathrm{~d}_{5 / 2}\right)(413 \mathrm{eV}), \mathrm{Cd}\left(3 \mathrm{~d}_{5 / 2}\right)(405 \mathrm{eV}), \mathrm{Hg}\left(4 \mathrm{f}_{7 / 2}\right)(101 \mathrm{eV})$ and $\mathrm{N}(1 \mathrm{~s})$ for $\mathrm{MBI}$ at $(398 \mathrm{eV})$. Analysis of core binding energy of the different environments will be discussed in the next section. 
Elemental quantification for all SAMs was studied from the high resolution XPS spectra for each element present in SAMs. The atom percent of carbon, oxygen, sulphur, nitrogen and target metal ions were calculated from the high resolution spectra collected at normal emission by integration of the areas of the $S\left(2 p_{3 / 2}\right), C_{1 s}$, $\mathrm{O}_{1 \mathrm{~s}}, \mathrm{~N}_{1 \mathrm{~s}}, \mathrm{Cu}\left(2 \mathrm{p}_{3 / 2}\right), \mathrm{Pb}\left(4 \mathrm{~d}_{5 / 2}\right), \mathrm{Cd}\left(3 \mathrm{~d}_{5 / 2}\right)$ and $\mathrm{Hg}\left(4 \mathrm{f}_{7 / 2}\right)$ peaks after application of a linear background subtraction [11] over a suitable range encompassing the peaks, and correction using the atomic sensitivity factors. The atomic sensitivity factors employed in this study for comparison of relative atomic concentration in SAMs were provided by the instrumental manufacturer and are as follows: $S\left(2 p_{3 / 2}\right)=2.08$, $\mathrm{C}_{1 \mathrm{~s}}=1.00, \mathrm{O}_{1 \mathrm{~s}}=2.80, \mathrm{~N}_{1 \mathrm{~s}}=1.73, \mathrm{Cu}\left(2 \mathrm{p}_{3 / 2}\right)=16.73, \mathrm{~Pb}\left(4 \mathrm{~d}_{5 / 2}\right)=13.02, \mathrm{Cd}\left(3 \mathrm{~d}_{5 / 2}\right)=$ 11.95 and $\mathrm{Hg}\left(4 \mathrm{f}_{7 / 2}\right)=10.57$. The results of the elemental analysis are summarized in tables 7.1 to 7.5 . 

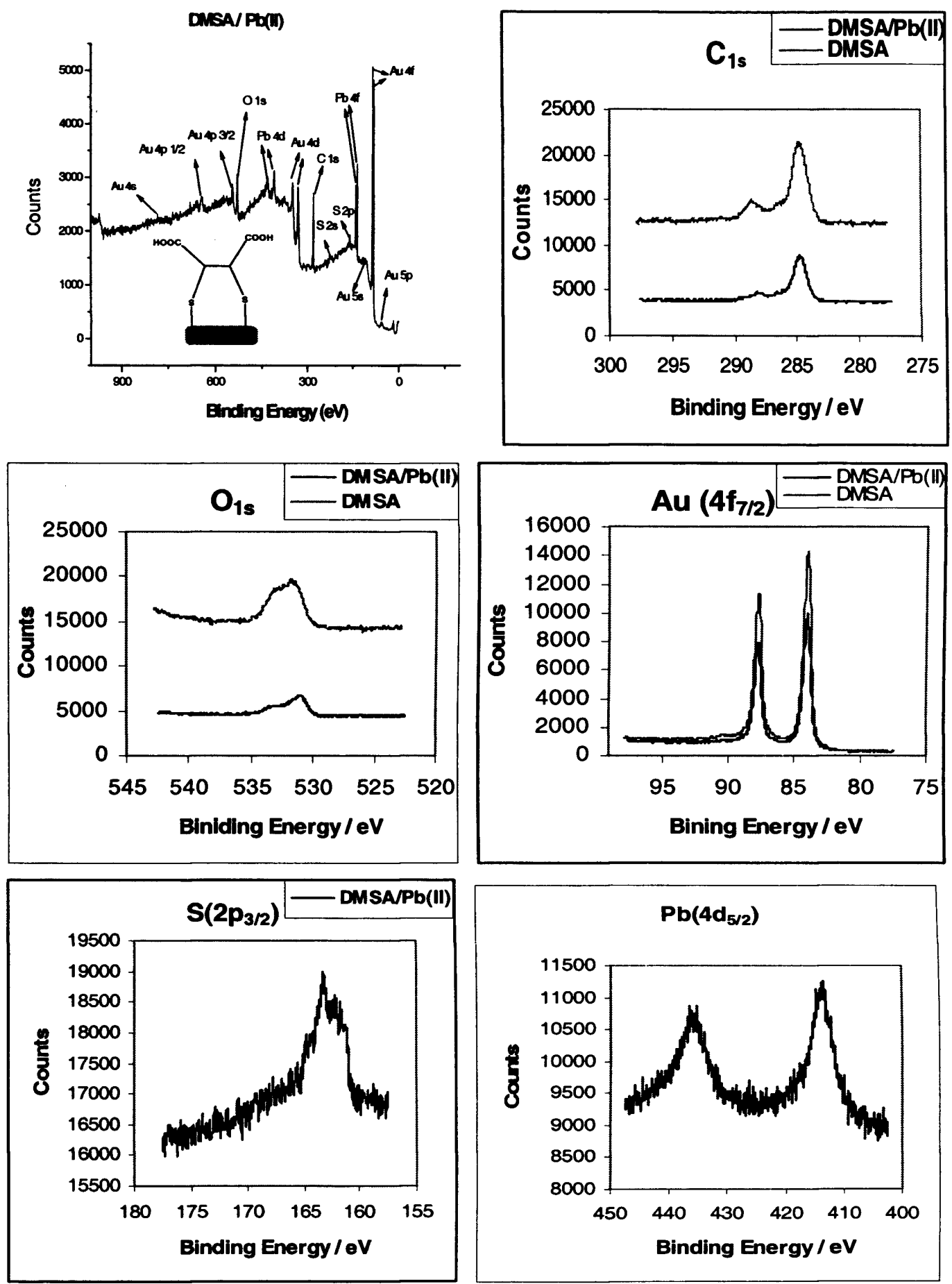

Figure 7.1: XPS wide scan spectra of a SAM of meso-2,3-dimercaptosuccinic acid before and after a preconcentration step in $1 \mathrm{mM}$ lead nitrate solution (pH 5.5) for $15 \mathrm{~min}$ followed by high resolution XPS scans for $C_{1 s}, O_{1 s}, A u\left(4 f_{7 / 2}\right), S\left(2 p_{3 / 2}\right)$ and $P b\left(4 d_{5 / 2}\right)$. 

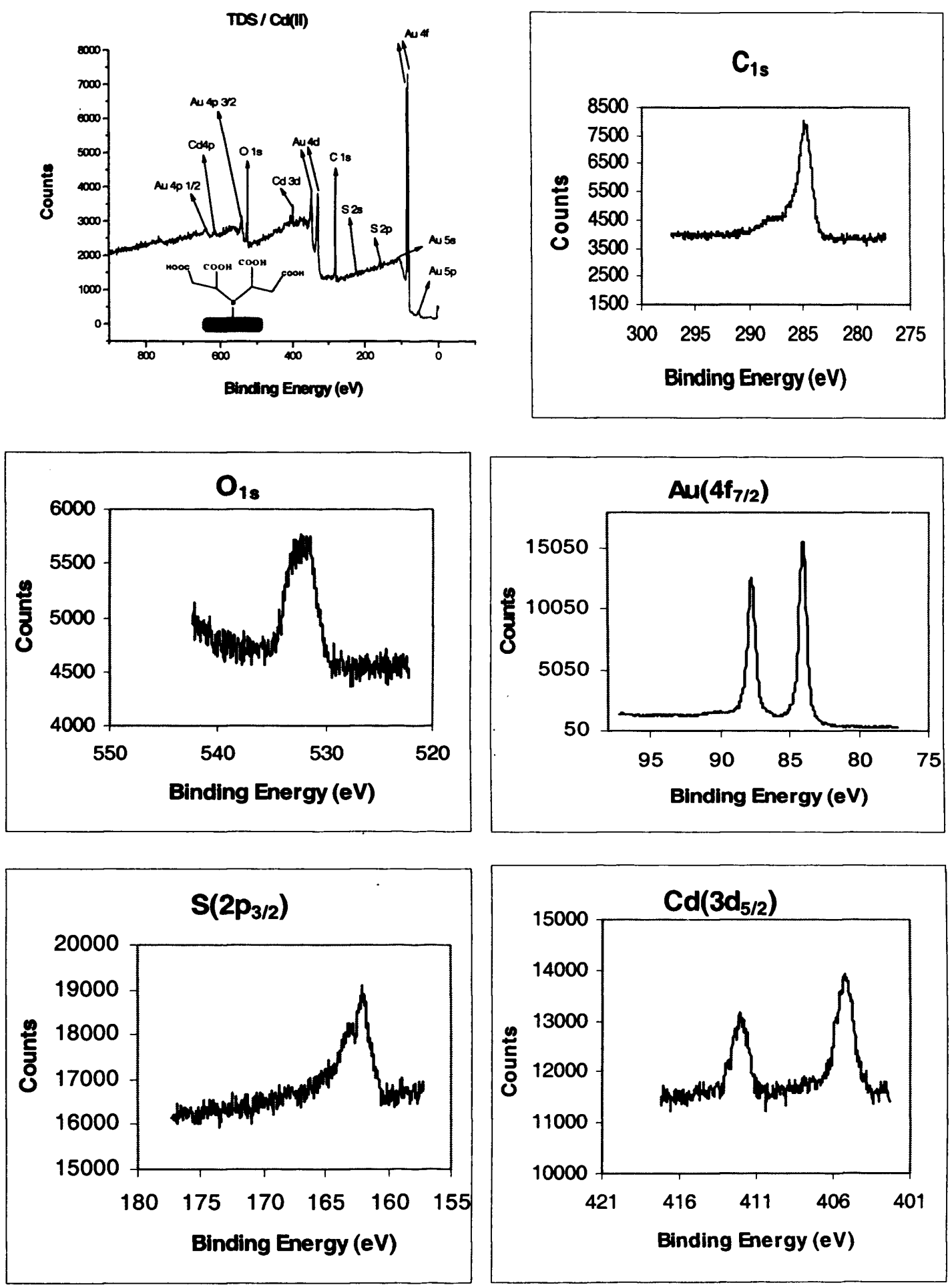

Figure 7.2: XPS wide scan spectra of a SAM of 3-thiodisuccinic acid after a preconcentration step in $1 \mathrm{mM}$ cadmium nitrate solution $(\mathrm{pH} \mathrm{6.2)}$ for $10 \mathrm{~min}$ followed by high resolution XPS scans for $C_{1 s}, O_{1 s}, A u\left(4 f_{7 / 2}\right), S\left(2 p_{3 / 2}\right)$ and $C d\left(3 d_{5 / 2}\right)$. 

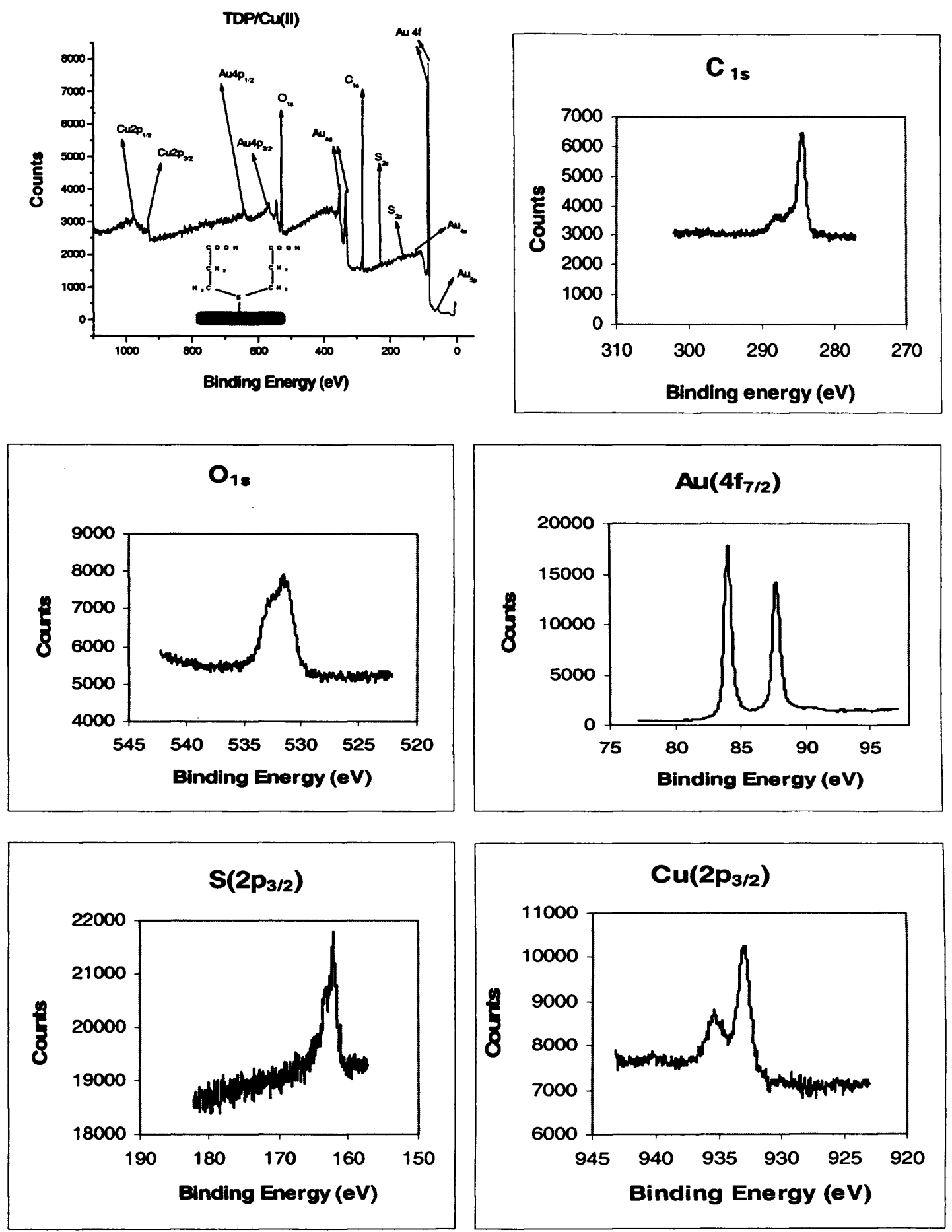

Figure 7.3: XPS wide scan spectra of a SAM of 3,3-thiodipropionic acid after a preconcentration step in $1 \mathrm{mM}$ copper nitrate solution $(\mathrm{pH} \mathrm{5.5)}$ for $20 \mathrm{~min}$ followed by high resolution XPS scans for $\mathrm{C}_{1 \mathrm{~s}}, \mathrm{O}_{1 \mathrm{~s}}, \mathrm{Au}\left(4 \mathrm{f}_{7 / 2}\right), \mathrm{S}\left(2 \mathrm{p}_{3 / 2}\right)$ and $\mathrm{Cu}\left(2 \mathrm{p}_{3 / 2}\right)$. 

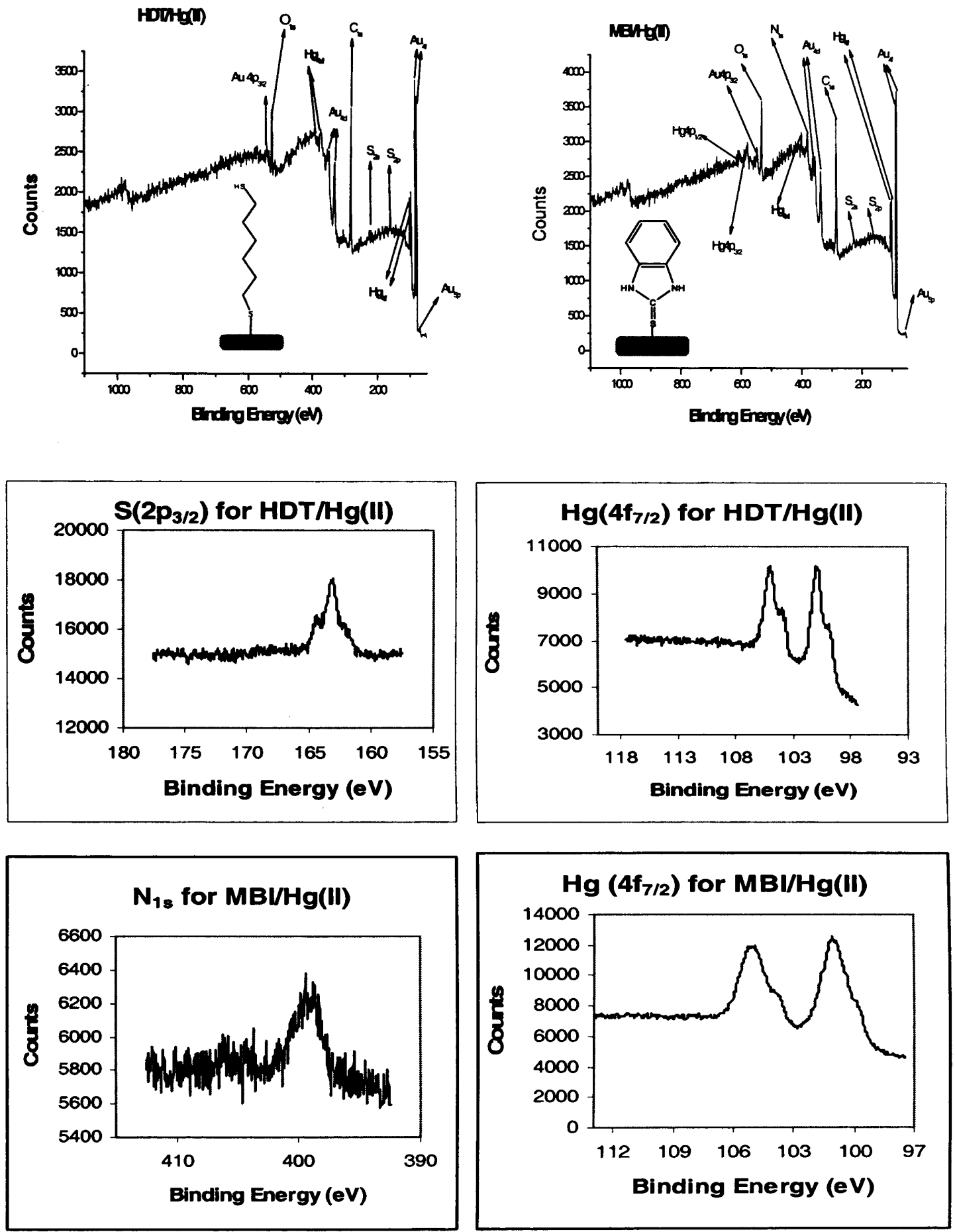

Figure 7.4: XPS wide scan spectra of a SAM of 1,6-hexanedithiol HDT and 2mercaptobenzimidazole $\mathrm{MBI}$ after a preconcentration step in $1 \mathrm{mM}$ mercury nitrate solution (pH 5.0) for $20 \mathrm{~min}$ followed by high resolution XPS scans for $S\left(2 p_{3 / 2}\right), N_{1 s}(M B I)$ and $\mathrm{Hg}\left(4 \mathrm{f}_{7 / 2}\right)$. 


\begin{tabular}{|c|c|c|c|c|c|}
\hline SAMs / M & Name & $\begin{array}{c}\text { Area } \\
\text { (cps) }\end{array}$ & Sensitivity & Atom. (\%) & M:S \\
\hline \multirow[t]{4}{*}{ DMSA / Pb(II) } & $\mathrm{C}$ 1s & 2894 & 1.00 & 65.87 & \\
\hline & $01 \mathrm{~s}$ & 2584 & 2.80 & 22.83 & \\
\hline & S 2p3/2 & 470 & 2.08 & 5.59 & \\
\hline & $\mathrm{Pb} 4 \mathrm{~d} 5 / 2$ & 3010 & 13.02 & 5.71 & 1.02 \\
\hline \multirow[t]{4}{*}{ DMSA / Cu(II) } & $\mathrm{C} 1 \mathrm{~s}$ & 2359 & 1.00 & 62.55 & M:S \\
\hline & $01 \mathrm{~s}$ & 2380 & 2.80 & 22.19 & \\
\hline & $S 2 p 3 / 2$ & 812 & 2.08 & 10.19 & \\
\hline & $\mathrm{Cu} 2 \mathrm{p} 3 / 2$ & 3246 & 16.73 & 5.07 & 0.50 \\
\hline \multirow[t]{4}{*}{ DMSA / Cd(II) } & C 1s & 3289 & 1.00 & 64.53 & M:S \\
\hline & $01 \mathrm{~s}$ & 3501 & 2.80 & 24.53 & \\
\hline & $S 2 p 3 / 2$ & 669 & 2.08 & 6.31 & \\
\hline & $\mathrm{Cd} 3 d 5 / 2$ & 2818 & 11.95 & 4.70 & 0.75 \\
\hline \multirow[t]{6}{*}{ DMSA / mixtures } & C 1s & 2352 & 1.00 & 63.16 & M:S \\
\hline & $01 \mathrm{~s}$ & 2556 & 2.80 & 24.54 & \\
\hline & S 2p 3/2 & 544 & 2.08 & 7.03 & \\
\hline & $\mathrm{Cd} 3 d 5 / 2$ & nill & nill & nill & nill \\
\hline & $\mathrm{Pb} 4 \mathrm{~d} 5 / 2$ & 2477 & 13.02 & 5.20 & 0.73 \\
\hline & $\mathrm{Cu} \mathrm{2p3/2}$ & 42 & 16.73 & 0.07 & 0.003 \\
\hline
\end{tabular}

Table 7.1: Elemental composition of SAM of meso-2,3-dimercaptosuccinic acid DMSA (obtained from high resolution XPS spectra) after adsorbing $\mathrm{Pb}^{2+}, \mathrm{Cd}^{2+}$ and $\mathrm{Cu}^{2+}$ from single metal ion solutions and triple mixture of $\left(\mathrm{Pb}^{2+}, \mathrm{Cu}^{2+}, \mathrm{Cd}^{2+}\right)$, the concentration of the three metal ions was $1 \mathrm{mM}$. 


\begin{tabular}{|c|c|c|c|c|c|}
\hline SAMs / M & Name & $\begin{array}{c}\text { Area } \\
\text { (cps) }\end{array}$ & Sensitivity & Atom. (\%) & M:S \\
\hline \multirow[t]{4}{*}{ TDS/Cu(II) } & $\mathrm{C}$ 1s & 2929 & 1.00 & 78.40 & \\
\hline & $01 \mathrm{~s}$ & 1792 & 2.80 & 17.14 & \\
\hline & $S 2 p 3 / 2$ & 207 & 2.08 & 2.67 & \\
\hline & $\mathrm{Cu} 2 \mathrm{p} 3 / 2$ & 1123 & 16.73 & 1.80 & 0.67 \\
\hline \multirow[t]{4}{*}{ TDS/Pb(II) } & $\mathrm{C} 1 \mathrm{~s}$ & 1601 & 1.00 & 72.36 & M:S \\
\hline & $01 \mathrm{~s}$ & 1223 & 2.80 & 19.74 & \\
\hline & $S 2 p 3 / 2$ & 225 & 2.08 & 4.89 & \\
\hline & $\mathrm{Pb} 5 \mathrm{~d} 5 / 2$ & 869 & 13.02 & 3.42 & 0.70 \\
\hline \multirow[t]{4}{*}{ TDS/Cd(II) } & $C$ is & 2498 & 1.00 & 76.37 & M:S \\
\hline & $01 \mathrm{~s}$ & 1649 & 2.80 & 17.34 & \\
\hline & $S 2 p 3 / 2$ & 112 & 2.08 & 2.18 & \\
\hline & $\mathrm{Cd} 3 d / 2$ & 1350 & 11.95 & 4.43 & 2.03 \\
\hline \multirow[t]{6}{*}{ TDS / mixtures } & C 1s & 2161 & 1.00 & 73.80 & M:S \\
\hline & $01 \mathrm{~s}$ & 1506 & 2.80 & 18.37 & \\
\hline & $S 2 p 3 / 2$ & 152 & 2.08 & 4.40 & \\
\hline & $\mathrm{Cd} 3 d \mathrm{~d} / 2$ & 1550 & 11.95 & 7.69 & 1.74 \\
\hline & $\mathrm{Pb} 4 \mathrm{~d} 5 / 2$ & nill & nill & nill & nill \\
\hline & $\mathrm{Cu} 2 \mathrm{p} 3 / 2$ & 225 & 16.73 & 0.46 & 0.08 \\
\hline
\end{tabular}

Table 7.2: Elemental composition of SAM of 3-thiodisuccinic acid TDS (obtained from high resolution XPS spectra) after adsorbing $\mathrm{Cd}^{2+}, \mathrm{Pb}^{2+}$ and $\mathrm{Cu}^{2+}$ from single metal ion solutions and triple mixture of $\left(\mathrm{Cd}^{2+}, \mathrm{Pb}^{2+}, \mathrm{Cu}^{2+}\right)$, the concentration of the three metal ions was $1 \mathrm{mM}$. 


\begin{tabular}{|c|c|c|c|c|c|}
\hline SAMs / M & Name & $\begin{array}{l}\text { Area } \\
\text { (cps) }\end{array}$ & Sensitivity & Atom. (\%) & M:S \\
\hline \multirow[t]{4}{*}{ TDP / Cu(II) } & C 1s & 3588 & 1.00 & 69.65 & \\
\hline & $01 \mathrm{~s}$ & 3430 & 2.80 & 23.53 & \\
\hline & $S 2 p 3 / 2$ & 420 & 2.08 & 4.08 & \\
\hline & $\mathrm{Cu} 2 \mathrm{p} 3 / 2$ & 2389 & 16.73 & 2.74 & 0.71 \\
\hline \multirow[t]{4}{*}{ TDP / Pb(II) } & C 1s & 3056 & 1.00 & 73.37 & M:S \\
\hline & $01 \mathrm{~s}$ & 2307 & 2.80 & 19.79 & \\
\hline & $S 2 p 3 / 2$ & 243 & 2.08 & 2.90 & \\
\hline & $\mathrm{Pb} 4 \mathrm{~d} 5 / 2$ & 2189 & 13.02 & 4.04 & 1.39 \\
\hline \multirow[t]{4}{*}{ TDP / Cd(II) } & C 1s & 3355 & 1.00 & 61.71 & M:S \\
\hline & $01 \mathrm{~s}$ & 4122 & 2.80 & 27.07 & \\
\hline & $S 2 p 3 / 2$ & 551 & 2.08 & 4.88 & \\
\hline & $\mathrm{Cd} 3 d \mathrm{~d} / 2$ & 4124 & 11.95 & 6.35 & 1.30 \\
\hline \multirow[t]{6}{*}{ TDP/mixtures } & C 1s & 3451 & 1.00 & 75.36 & M:S \\
\hline & $01 \mathrm{~s}$ & 1957 & 2.80 & 15.26 & \\
\hline & $S 2 p 3 / 2$ & 410 & 2.08 & 4.30 & \\
\hline & $\mathrm{Cd} 3 d 5 / 2$ & 122 & 11.95 & 0.22 & 0.05 \\
\hline & $\mathrm{Pb} 4 \mathrm{~d} 5 / 2$ & 1185 & 13.02 & 1,99 & 0.46 \\
\hline & $\mathrm{Cu} 2 \mathrm{p} 3 / 2$ & 2195 & 16.73 & 2.86 & 0.66 \\
\hline
\end{tabular}

Table 7.3: Elemental composition of SAM of 3,3-thiodipropionic acid TDP (obtained from high resolution XPS spectra) after adsorbing $\mathrm{Cu}^{2+}, \mathrm{Pb}^{2+}$ and $\mathrm{Cd}^{2+}$ from single metal ion solutions and triple mixture of $\left(\mathrm{Cd}^{2+}, \mathrm{Pb}^{2+}, \mathrm{Cu}^{2+}\right)$, the concentration of the three metal ions was $1 \mathrm{mM}$. 


\begin{tabular}{|c|c|c|c|c|c|}
\hline SAMs / M & Name & $\begin{array}{l}\text { Area } \\
\text { (cps) }\end{array}$ & Sensitivity & Atom. (\%) & M:S \\
\hline \multirow[t]{4}{*}{ MBA / Cd(II) } & C 1s & 3352 & 1.00 & 71.61 & \\
\hline & $01 \mathrm{~s}$ & 2759 & 2.80 & 21.05 & \\
\hline & $s 2 p 3 / 2$ & 343 & 2.08 & 3.53 & \\
\hline & $\mathrm{Cd} 3 d 5 / 2$ & 2137 & 11.95 & 3.82 & 1.08 \\
\hline \multirow[t]{4}{*}{ MBA Pb(II) } & C 1s & 2970 & 1.00 & 63.88 & M:S \\
\hline & 0 is & 3380 & 2.80 & 25.97 & \\
\hline & S 2p 3/2 & 340 & 2.08 & 3.52 & \\
\hline & $\mathrm{Pb} \mathrm{4d5/2}$ & 4012 & 13.02 & 6.63 & 1.88 \\
\hline \multirow[t]{4}{*}{ MBA / Cu(II) } & C 1s & 4147 & 1.00 & 79.40 & M:S \\
\hline & O 1s & 2045 & 2.80 & 13.98 & \\
\hline & $S 2 p 3 / 2$ & 513 & 2.08 & 4.72 & \\
\hline & $\mathrm{Cu} 2 \mathrm{p} 3 / 2$ & 1649 & 16.73 & 1.89 & 0.40 \\
\hline \multirow[t]{6}{*}{ MBAmixtures } & C 1s & 3940 & 1.00 & 70.28 & M:S \\
\hline & $01 \mathrm{~s}$ & 3778 & 2.80 & 24.07 & \\
\hline & $S 2 p 3 / 2$ & 210 & 2.08 & 1.80 & \\
\hline & $\mathrm{Cd} \mathrm{3d} \mathrm{5/2}$ & 747 & 11.95 & 1.12 & 0.63 \\
\hline & $\mathrm{Pb} 4 \mathrm{~d} 5 / 2$ & 1402 & 13.02 & 1.92 & 1.06 \\
\hline & $\mathrm{Cu} 2 \mathrm{p} 3 / 2$ & 762 & 16.73 & 0.81 & 0.45 \\
\hline \multirow[t]{4}{*}{ HDT / Hg(II) } & C 1s & 3679 & 1.00 & 77.79 & M:S \\
\hline & $01 \mathrm{~s}$ & 1862 & 2.80 & 14.06 & \\
\hline & $\mathrm{S} 2 \mathrm{p} 3 / 2$ & 534 & 2.08 & 5.42 & \\
\hline & $\mathrm{Hg} \mathrm{4f7/2}$ & 1364 & 10.57 & 2.73 & 0.50 \\
\hline
\end{tabular}

Table 7.4: Elemental composition of SAM of 4-mercaptobenzoic acid MBA (obtained from high resolution XPS spectra) after adsorbing $\mathrm{Cd}^{2+}, \mathrm{Pb}^{2+}$ and $\mathrm{Cu}^{2+}$ from single metal ion solutions and triple mixture of $\left(\mathrm{Cd}^{2+}, \mathrm{Pb}^{2+}, \mathrm{Cu}^{2+}\right)$, the concentration of the three metal ions was $1 \mathrm{mM}$. Table also shows elemental composition of SAM of 1,6-hexanedithiol HDT after adsorbing $\mathrm{Hg}^{2+}$ from single ion solution. 


\begin{tabular}{|c|c|c|c|c|c|}
\hline SAMs / M & Name & $\begin{array}{l}\text { Area } \\
\text { (cps) }\end{array}$ & Sensitivity & Atom. (\%) & M:S \\
\hline \multirow[t]{4}{*}{ BDMT / Hg(II) } & $\mathrm{C}$ 1s & 2854 & 1.00 & 65.49 & \\
\hline & $01 \mathrm{~s}$ & 2526 & 2.80 & 20.70 & \\
\hline & $S 2 p 3 / 2$ & 581 & 2.08 & 6.40 & \\
\hline & $\mathrm{Hg} 4\lceil 7 / 2$ & 3413 & 10.57 & 7.41 & 1.15 \\
\hline \multirow[t]{4}{*}{ BDMT / Cu(II) } & C 1s & 4539 & 1.00 & 80.03 & M:S \\
\hline & $01 \mathrm{~s}$ & 1701 & 2.80 & 10.71 & \\
\hline & $\mathrm{s} 2 \mathrm{p} 3 / 2$ & 1044 & 2.08 & 8.85 & \\
\hline & $\mathrm{Cu} 2 \mathrm{p} 3 / 2$ & 387 & 16.73 & $0 . .50$ & 0.05 \\
\hline \multirow[t]{5}{*}{ BDMT / mixture } & C 1s & 3322 & 1.00 & 72.24 & M:S \\
\hline & $01 \mathrm{~s}$ & 2214 & 2.80 & 17.20 & \\
\hline & $S 2 p 3 / 2$ & 424 & 2.08 & 4.44 & \\
\hline & $\mathrm{Hg} 4 f 7 / 2$ & 2978 & 10.57 & 6.13 & 1.38 \\
\hline & $\mathrm{Cu} 2 \mathrm{p} 3 / 2$ & nill & nill & nill & nill \\
\hline \multirow[t]{5}{*}{ MBI / Hg(II) } & $\mathrm{C} 1 \mathrm{~s}$ & 4410 & 1.00 & 72.22 & M:S \\
\hline & $01 \mathrm{~s}$ & 3239 & 2.80 & 18.46 & \\
\hline & $\mathrm{N}$ is & 668 & 1.73 & 6.16 & \\
\hline & $S 2 p 3 / 2$ & 254 & 2.08 & 1.95 & \\
\hline & $\mathrm{Hg} 4 \mathrm{f7} / 2$ & 800 & 10.57 & 1.21 & 0.60 \\
\hline \multirow[t]{5}{*}{$\mathrm{MBI} / \mathrm{Cu}(\mathrm{II})$} & C $1 \mathrm{~s}$ & 4568 & 1.00 & 72.51 & M:S \\
\hline & O ts & 2355 & 2.80 & 13.35 & \\
\hline & $\mathrm{N} 1 \mathrm{~s}$ & 900 & 1.73 & 8.26 & \\
\hline & $\mathrm{S} 2 \mathrm{p} 3 / 2$ & 552 & 2.08 & 4.22 & \\
\hline & Cu $2 p 3 / 2$ & 1749 & 16.73 & 1.66 & 0.40 \\
\hline
\end{tabular}

Table 7.5: Elemental composition of SAM of 1,4-benzenedimethanethiol BDMT (obtained from high resolution XPS spectra) after adsorbing $\mathrm{Hg}^{2+}$ and $\mathrm{Cu}^{2+}$ from single metal ion solutions and binary mixture of $\left(\mathrm{Hg}^{2+}, \mathrm{Cu}^{2+}\right)$, the concentration of the two metal ions was $1 \mathrm{mM}$. Table also presents elemental composition of SAM of 2-mercaptobenzimidazole MBI after adsorbing $\mathrm{Hg}^{2+}$ and $\mathrm{Cu}^{2+}$ from single ion solutions and binary mixture of $\left(\mathrm{Hg}^{2+}, \mathrm{Cu}^{2+}\right)$. 
The relative concentration of the detected elements was used to determine the metal ion to sulphur ratio are presented in tables 7.1 to 7.5 . The mole ratios were estimated after exposure to high concentrations of metal ion, as in the QCM and EQCM experiments. The mole ratios extracted from the XPS analyse are in agreement with the mole ratios extracted from QCM and EQCM measurements except 3-thiodisuccinic acid and 1,6-hexanedithiol, which are different. The comparison between the mole ratios extracted from QCM, EQCM and XPS are presented in table 7.6.

\begin{tabular}{|c|c|c|c|}
\hline $\mathbf{M}^{2+} / \mathrm{SAM}$ & $\mathrm{QCM}$ & $\mathrm{EQCM}$ & $\mathrm{XPS}$ \\
\hline $\mathrm{Pb}^{2+} / \mathrm{DMSA}$ & $0.94: 1.0$ & $0.96: 1.0$ & $0.98: 1.0$ \\
\hline $\mathrm{Cu}^{2+} / \mathrm{DMSA}$ & $0.7: 1.0$ & $0.7: 1.0$ & $0.8: 1.0$ \\
\hline $\mathrm{Cd}^{2+} / \mathrm{DMSA}$ & $0.75: 1.0$ & $0.71: 1.0$ & $0.75: 1.0$ \\
\hline $\mathrm{Cd}^{2+} / \mathrm{TDS}$ & $1: 1$ and $2: 1$ & $0.94: 1.0$ & $2.03: 1.0$ \\
\hline $\mathrm{Cu}^{2+} / \mathrm{TDP}$ & $0.99: 1.0$ & $1.09: 1.0$ & $0.98: 1.0$ \\
\hline $\mathrm{Pb}^{2+} / \mathrm{TDP}$ & $0.93: 1.0$ & $0.85: 1.0$ & $1.1: 1.0$ \\
\hline $\mathrm{Cd}^{2+} / \mathrm{MBA}$ & $0.96: 1.0$ & $0.91: 1.0$ & $1.1: 1.0$ \\
\hline $\mathrm{Pb}^{2+} / \mathrm{MBA}$ & $0.97: 1.0$ & $1.05: 1.0$ & $1.8: 1.0$ \\
\hline $\mathrm{Cu}^{2+} / \mathrm{MBA}$ & $0.98: 2.0$ & $1.1: 2.0$ & $1: 2$ \\
\hline $\mathrm{Hg}^{2+} / \mathrm{HDT}$ & $1.2: 1$ & - & $1: 2$ \\
\hline $\mathrm{Hg}^{2+} / \mathrm{BDMT}$ & $0.94: 1$ & - & $1.1: 1.0$ \\
\hline $\mathrm{Hg}^{2+} / \mathrm{MBI}$ & $0.1: 2.0$ & - & $1.0: 1.66$ \\
\hline
\end{tabular}

Table: 7.6: Comparison between the mole ratio between the metal ions and SAM from the QCM, EQCM and XPS results using the same experimental conditions. The mole ratio was estimated at $1 \mathrm{mM}$ of metal ions from single ion solutions.

The results of analysing mixtures of metal ions using elemental analysis from XPS show that the DMSA, TDS, TDP, HDT, BDMT and MBI have a high selectivity 
towards target metal ions. These results are agreement with QCM and EQCM experiments in chapters five and six. The ligand 4-mercaptobenzoic acid MBA has not any selectivity towards $\mathrm{Cd}^{2+}$.

\subsubsection{XPS peak fitting}

The peaks in elemental core-level spectra were fitted using the Scienta Win ESCA software. A convolution of Lorentzian and Gaussian line shapes was used to fit the individual peaks. A linear function was used to model the background, with the corresponding coefficients fit simultaneously with the peaks. In most cases, the full widths at half maximum (Fwhm) and background parameters converged to consistent values throughout the series of fitting to the same SAM. The number of species in the fitting was established considering the analysis conditions and organic functionalities of the SAMs on the Au surfaces.

\subsubsection{Analysis using S(2p) XPS spectra}

For the thiol SAMs used in our experiments, the sulphur signal in XPS is more important because it provides information about the degree to which the SAM is immobilized and gives more information about $S-A u$ bond. The $S(2 p)$ signal in different SAMs before adsorption of metal ions from aqueous solutions is shown in Figure 7.5 .

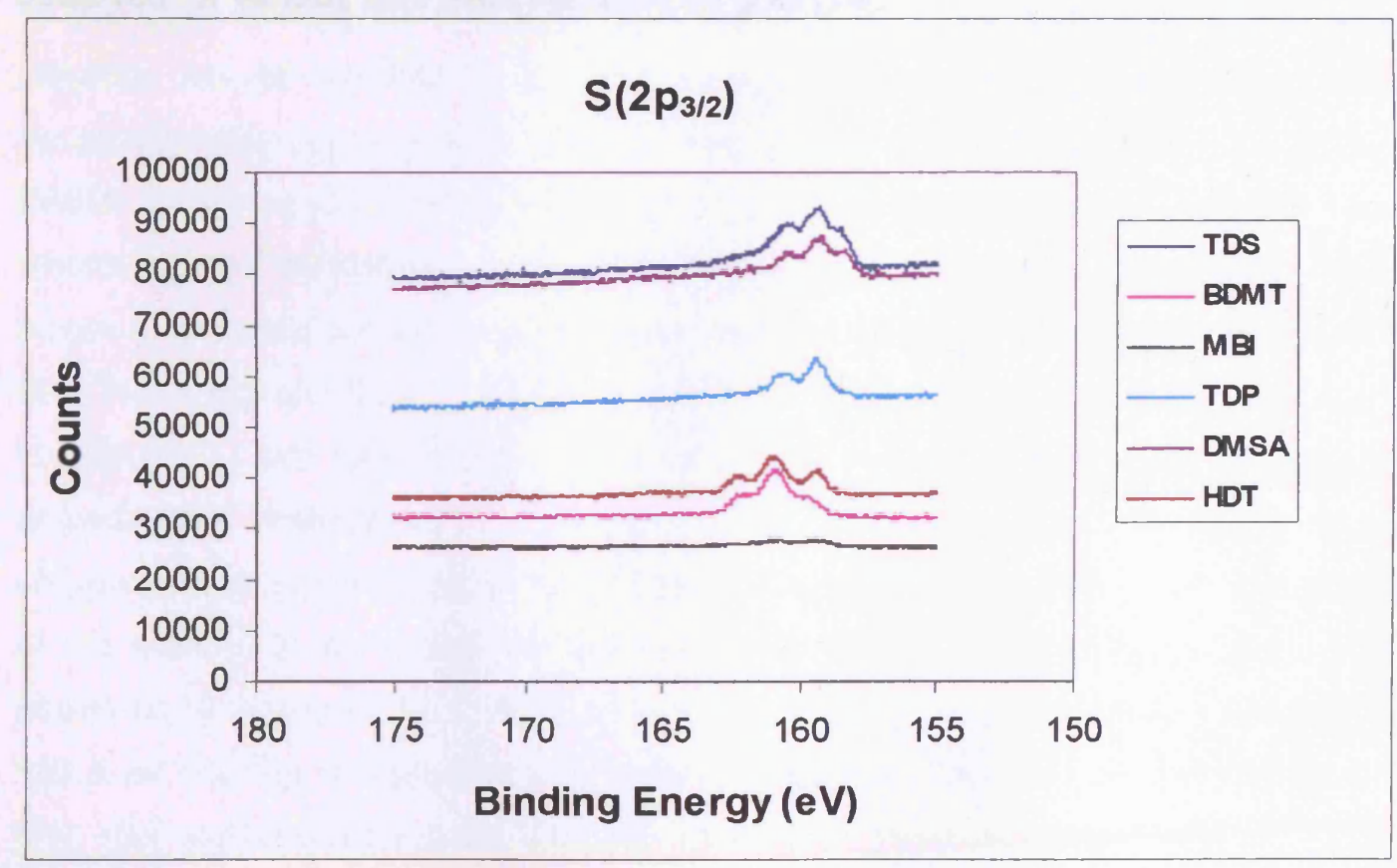

Figure 7.5: S 2p photoemission spectra of various thiol SAMs on Au. All the SAMs were prepared by immersion for $24 \mathrm{~h}$ into $1 \mathrm{mM}$ solution. 
The results show that SAMs of 3-thiodisuccinic acid TDS have a stronger S2p signal than other SAMs and 2-mercaptobenzimidazole (MBI) has a weaker signal than SAMs of TDS, DMSA, TDP, BDMT and HDT. This can be explained from the sulfur binding energy is mainly determined by the electronegativity difference between sulphur and carbon, and the interaction between sulphur and the $\mathrm{Au}$ surface [12]. Interestingly, since sulfur and carbon have almost the same electronegativity [13], the C-S bond should be electrically neutral. This same electronegativity could well explain the fact that the $S(2 p)$ binding energy of thiols and disulphides and monosulfides are almost the same (see the Figure 7.5). The experimental spectra for all SAMs are best fit with two $2 p_{3 / 2}-2 p_{1 / 2}$ spin-orbit doublets which are composed of two Gaussian shaped components separated by $1.5 \mathrm{eV}$ with an intensity ratio of $2: 1$, as theoretically determined from the spin-orbit splitting effect. After fitting the sum of the two curves matches the experimental data well. Within each measurement series the absolute signal intensities showed good reproducibility. These distinct doublets are labelled as A and B. Figures 7.6a and 7.6b show four peaks attributed to different sulfur species for meso-2,3-dimercapto succinic acid before and after adsorbed $\mathrm{Pb}$ (II) from a singe metal ion solution. Main high binding peaks (B species) were observed for DMSA alone at $162.11 \mathrm{eV}$ $\left(S 2 p_{3 / 2}\right)$ and $163.5 \mathrm{eV}\left(S 2 p_{1 / 2}\right)$. Low binding peaks (A species) were also observed at $161.2 \mathrm{eV}\left(S 2 p_{3 / 2}\right)$ and $162.9 \mathrm{eV}\left(S 2 p_{1 / 2}\right)$. Both sulfur species $A$ and $B$ can be assigned to the bound sulphur because such similar binding peaks are usually observed for various thiol SAM samples on gold [14]. 

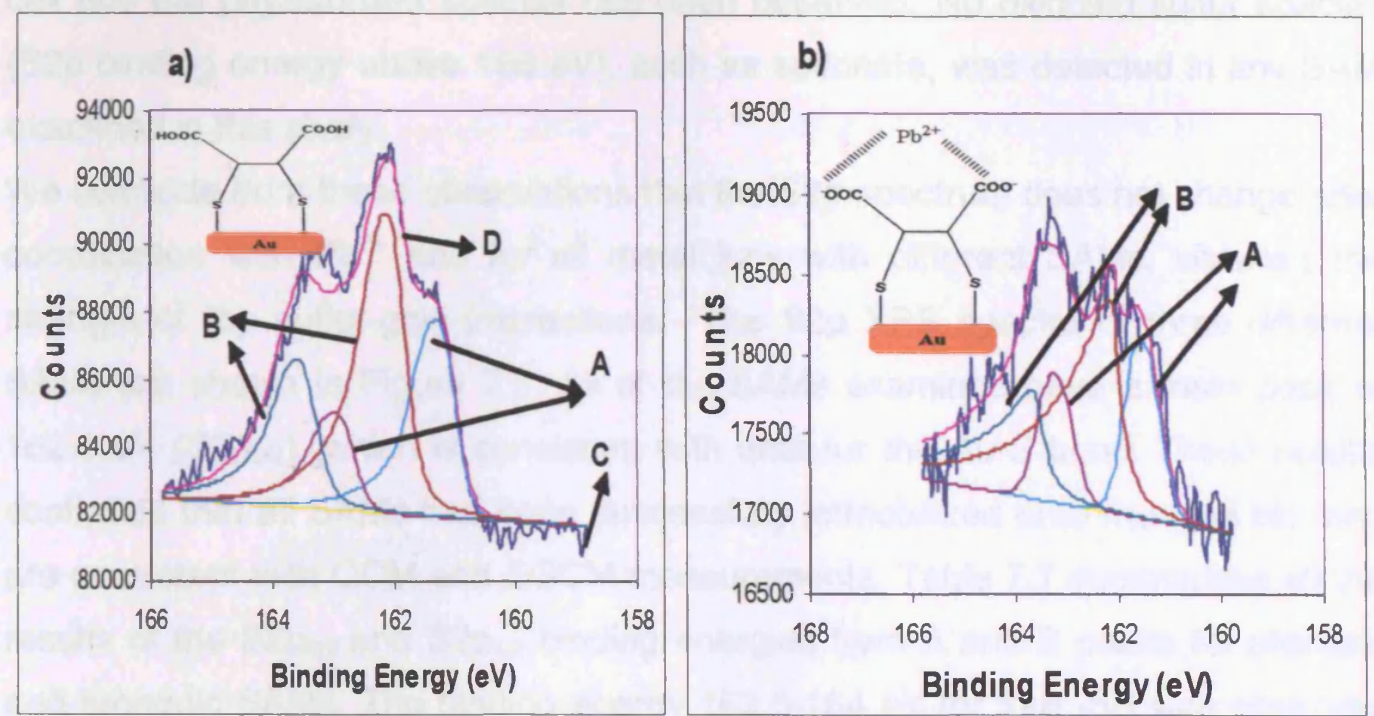

Figure 7.6: XPS spectra in the S2p region of meso-2,3-dimercaptosuccinic acid DMSA on $\mathrm{Au}$ before adsorption of $\mathrm{Pb}(\mathrm{II})$ ions (a) and after adsorption of $\mathrm{Pb}(\mathrm{II})$ ions (b). To properly fit the experimental SAM spectrum, two $S(2 p)$ doublets with 2:1 areas ratio and splitting 1.5 $\mathrm{eV}$ were required. (C) data as acquired, (D) linear combination of peaks.

Low binding peaks (A species) were also observed at $161.5 \mathrm{eV}\left(\mathrm{S} 2 \mathrm{p}_{3 / 2}\right)$ and 163.2 $\mathrm{eV}\left(\mathrm{S}_{2} \mathrm{p}_{1 / 2}\right)$ for DMSA after adsorption of $\mathrm{Pb}(\mathrm{II})$ from aqueous solutions. The high binding energy peaks (B species) were also observed at $162.15 \mathrm{eV}\left(\mathrm{S} 2 \mathrm{p}_{3 / 2}\right)$ and $163.4 \mathrm{eV}\left(\mathrm{S} 2 \mathrm{p}_{1 / 2}\right)$. The binding energy of the $\mathrm{S} 2 \mathrm{p}_{3 / 2}$ peak was $162.11 \mathrm{eV}(\mathrm{DMSA})$ and $162.15\left(\mathrm{DMSA} / \mathrm{Pb}^{2+}\right)$, consistent with the sulfur atoms being chemically bound to the gold surface as a thiolate species. This is in excellent agreement with previous results reported by several research groups $[15,16]$. In particular, no detectable intensity is present in the binding energy region above $163.4 \mathrm{eV}$ for the DMSA before or after adsorption of lead ions. This means that no unbound thiol molecules are detected by XPS and the two thiol groups are bound to the Au, which is a consistent with the proposal from the QCM measurements in chapter five. The observed binding energy of $161.2 \mathrm{eV}\left(\mathrm{S} 2 \mathrm{p}_{3 / 2}\right)(\mathrm{DMSA})$ and $161.5\left(\mathrm{~S} 2 \mathrm{p}_{3 / 2}\right)$ $\left(\mathrm{DMSA} / \mathrm{Pb}^{2+}\right.$ ) are attributed to adsorbed atomic sulfur [15] and differently bound thiolate [17], respectively. Differently bound thiolate has been attributed to a change in the hybridization of the thiolate sulfur atom from $\mathrm{sp}^{3}$ to $\mathrm{sp}$ or adsorption of the thiolate to a Au surface site other than that of a 3-fold hollow [18]. The observed binding energy of 163.3 and $162.9 \mathrm{eV}\left(\mathrm{S} 2 \mathrm{p}_{1 / 2}\right)$ for DMSA and 163.2 and $163.4 \mathrm{eV}\left(\mathrm{S} 2 \mathrm{p}_{1 / 2}\right)$ is attributed to physisorbed DMSA molecules on the monolayer [18]. Our samples have been carefully rinsed with absolute ethanol and ultrapure water to ensure a complete removal of physisorbed species before XPS measurements. Castener [19] rinsed his samples with solvent in an ultrasonicated 
cell and the physisorbed species has been observed. No oxidized sulfur species (S2p binding energy above $166 \mathrm{eV}$ ), such as sulfonate, was detected in any SAM examined in this study.

We conclude from these observations that the S2p spectrum does not change after coordination with $\mathrm{Pb}^{2+}$ and for all metal ions with different SAMs, showing the strength of the sulfur-gold interactions. The S2p XPS spectra of three different SAMs are shown in Figure 7.7. All of the SAMs examined have a main peak at $162.0 \mathrm{eV}\left(\mathrm{S}_{2} \mathrm{p}_{3 / 2}\right)$, which is consistent with data for the Au-S bond. These results confirmed that all SAMs had been successfully immobilized onto the gold film and are consistent with QCM and EQCM measurements. Table 7.7 summarizes all the results of the $S 2 p_{3 / 2}$ and $S 2 p_{1 / 2}$ binding energies from $A$ and $B$ peaks for aliphatic and aromatic SAMs. The binding energy 163.6-164 eV for free -SH was observed only for 1,4-benzenedimethanethiol BDMT and 1,6-hexanedithiol HDT (see Figures 7.6e, 7,6f and table 7.7) before adsorption of $\mathrm{Hg}(\mathrm{II})$ ions and disappeared after adsorption of $\mathrm{Hg}$ (II) from aqueous solutions. Based on the peak area ratios, ratio of the two chemical states of sulfur, XPS data suggest that one-ended dithiol molecules (HDT and BDMT) attached to the Au substrate to form AuSR and the other ended thiol groups were left as free $-\mathrm{SH}$, which is consistent with previous studies for 1-5-pentanedithiol and 1,9-nonanedithiol on Au electrodes (see table 7.7). 

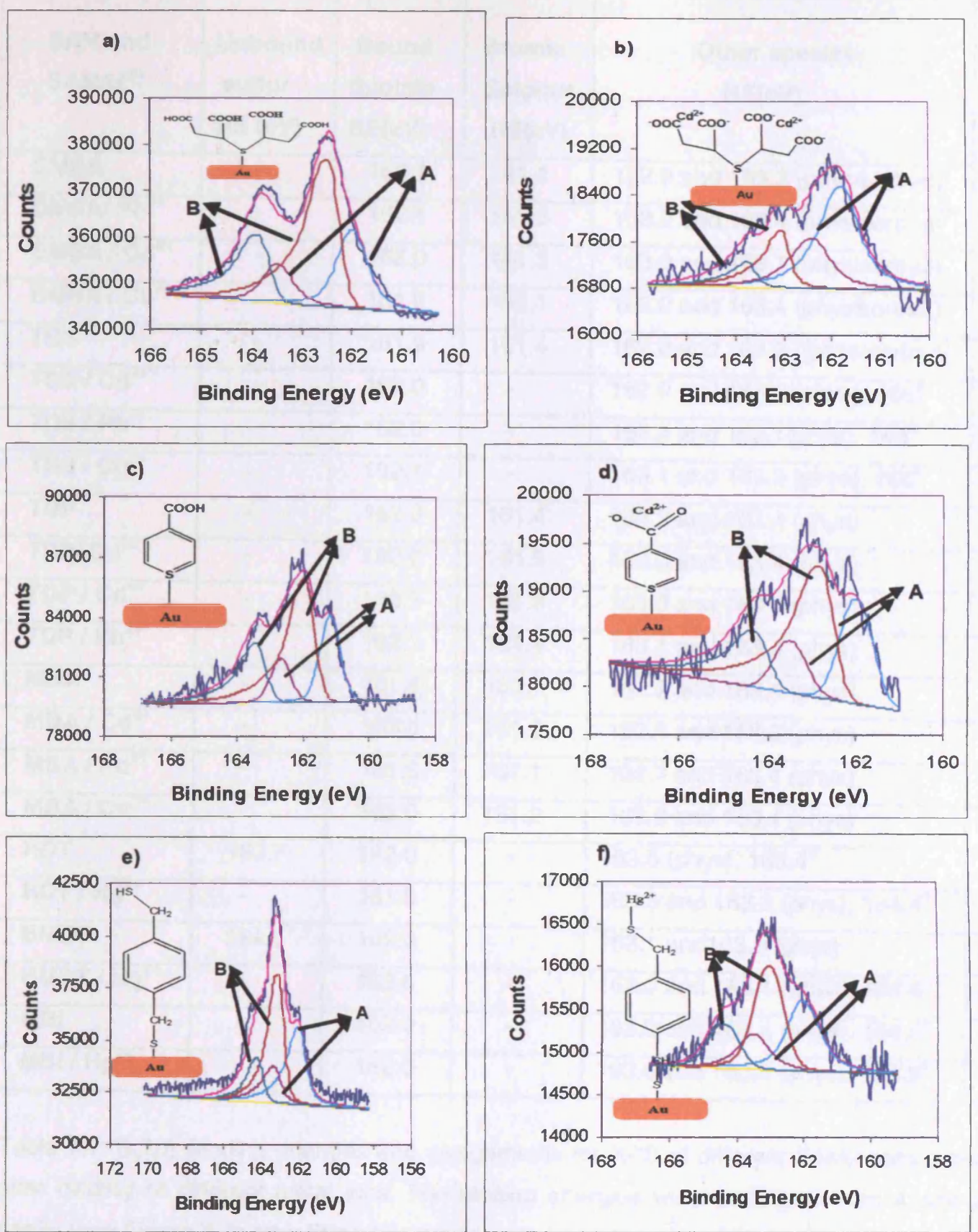

Figure 7.7: XPS spectra in $S(2 p)$ region of aliphatic and aromatic thiol SAMs of (a) TDS; (b) TDS/Cd(II); (c) MBA; (d) MBACC(II); (e) BMDT and (f) BDMT/Hg(II). All the SAMs were prepared by immersion for $24 \mathrm{~h}$ into $1 \mathrm{mM}$ solution. Two $52 \mathrm{p}$ doublets, both with a $2: 1$ area ratio and a splitting of $1.5 \mathrm{eV}$, were used to peak fit the experimental spectrum. 


\begin{tabular}{|c|c|c|c|c|c|}
\hline $\begin{array}{l}\text { SAM and } \\
\text { SAM } / M^{2+}\end{array}$ & $\begin{array}{l}\text { Unbound } \\
\text { sulfur } \\
\text { BE (eV) }\end{array}$ & $\begin{array}{l}\text { Bound } \\
\text { thiolate } \\
\text { BE(eV) }\end{array}$ & $\begin{array}{l}\text { Atomic } \\
\text { Sulphur } \\
\text { BE(eV) }\end{array}$ & $\begin{array}{l}\text { Other species } \\
\text { BE(eV) }\end{array}$ & Ref \\
\hline DMSA & - & 162.1 & 161.1 & 162.9 and 163.3 (physisorbed) & 15,16 \\
\hline DMSA Pb ${ }^{2+}$ & - & 162.1 & 161.5 & 163.2 and 163.4 (physisorbed) & 15,16 \\
\hline DMSA / Cd & - & 162.0 & 161.3 & 163.0 and 163.1 (physisorbed) & 15,16 \\
\hline DMSA / $\mathrm{Cu}^{2+}$ & - & 161.9 & 161.1 & 163.2 and 163.4 (physisorbed) & 15,16 \\
\hline TDS & - & 161.9 & 161.4 & 162.9 and 163.3 ((physisorbed) & 20,21 \\
\hline TDS $/ \mathrm{Cd}^{2+}$ & - & 162.0 & - & 162.9 and 163.2 (phys), $165^{8}$ & 20,21 \\
\hline TDS / $\mathrm{Pb}^{2+}$ & - & 162.0 & - & 162.8 and 163.1 (phys), $165^{\mathrm{a}}$ & 20,21 \\
\hline TDS $/ \mathrm{Cu}^{2+}$ & - & 162.1 & - & 163.1 and 163.3 (phys), $165^{\mathrm{a}}$ & 20,21 \\
\hline TDP & - & 162.0 & 161.4 & 163.2 and 163.4 (phys) & 20,21 \\
\hline TDP/Cu ${ }^{2+}$ & - & 162.0 & 161.5 & 163.0 and 163.4 (phys) & 20,21 \\
\hline TDP $/ \mathrm{Cd}^{2+}$ & - & 162.1 & 161.3 & 163.3 and 163.4 (phys) & 20,21 \\
\hline TDP / $\mathrm{Pb}^{2+}$ & - & 162.1 & 161.4 & 163.1 and 163.4 (phys) & 20,21 \\
\hline MBA & - & 161.9 & 161.1 & 162.7 and 163.4 (phys) & 20,21 \\
\hline MBA / $\mathrm{Cd}^{2+}$ & - & 162.0 & 161.3 & 162.8 and 163.3 (phys) & 22,23 \\
\hline MBA / $\mathbf{P b}^{2+}$ & - & 161.9 & 161.1 & 162.7 and 163.4 (phys) & 22,23 \\
\hline $\mathrm{MBA} / \mathrm{Cu}^{2+}$ & - & 162.0 & 161.2 & 162.8 and 163.4 (phys) & 22,23 \\
\hline HDT & 163.7 & 162.0 & - & 163.5 (phys), $165.4^{\circ}$ & 24,25 \\
\hline HDT / $\mathrm{Hg}^{2+}$ & - & 161.9 & - & 163.0 and 163.3 (phys), $164.4^{c}$ & 24,25 \\
\hline BMDT & 164.0 & 162.0 & - & 163.1 and163.3 (phys) & 24,25 \\
\hline BMDT / $\mathrm{Hg}^{2+}$ & - & 162.0 & - & 163.0 and 163.4 (phys), $164.6^{d}$ & 24,25 \\
\hline MBI & - & 162.0 & - & 163.0 and 163.4 (phys), $164.5^{e}$ & 26 \\
\hline $\mathrm{MBI} / \mathrm{Hg}^{2+}$ & - & 162.0 & - & 163.0 and 163.4 (phys), $164.5^{e}$ & 26 \\
\hline
\end{tabular}

Table 7.7: S(2p) Binding energies and assignments for XPS of different SAMs before and after binding of different metal ions. The binding energies were estimated from $A$ and $B$ peaks (see Figure 7.7) after fitting the experimental spectrum. (a) $165 \mathrm{eV}$ and (b) $165.4 \mathrm{eV}$ are attributed to un-bound thiol or disulfide species within or on top of the thiolate layer [27], (c) $164.4 \mathrm{eV}$ and (d) 164.6 not clear, (e) 164.5 is attributed to thioketo form [28].

\subsubsection{Analysis using C(1s) XPS spectra}

The spectra were fitted by single symmetric Voigt curves with a linear type background. The curve-fitted high resolution XPS spectra obtained for the (C1s) binding energy region for the DMSA, TDP, TDS and MBA before and after adsorption of metal ions is shown in Figure 7.8. The curve-fitted high resolution XPS spectra obtained for the $C(1 s)$ binding energy region present four peaks in the 
290.0 to $284.5 \mathrm{eV}$ energy range for the terminal free carboxylate SAMs, three peaks in the 286 to 284.5 energy range for the terminal free $-\mathrm{SH}$, and $-\mathrm{NH}$. The peaks at 288.5, 286.4, 285 and $284.5 \mathrm{eV}$, for the DMSA Au surface (see Figure 7.8a), are assigned from literature references to the $-\mathrm{COOH}, \mathrm{C}-\mathrm{S}, \mathrm{C}-\mathrm{C}$ and $-\mathrm{CH}_{2}$ functional groups $[29,30,31,32]$.
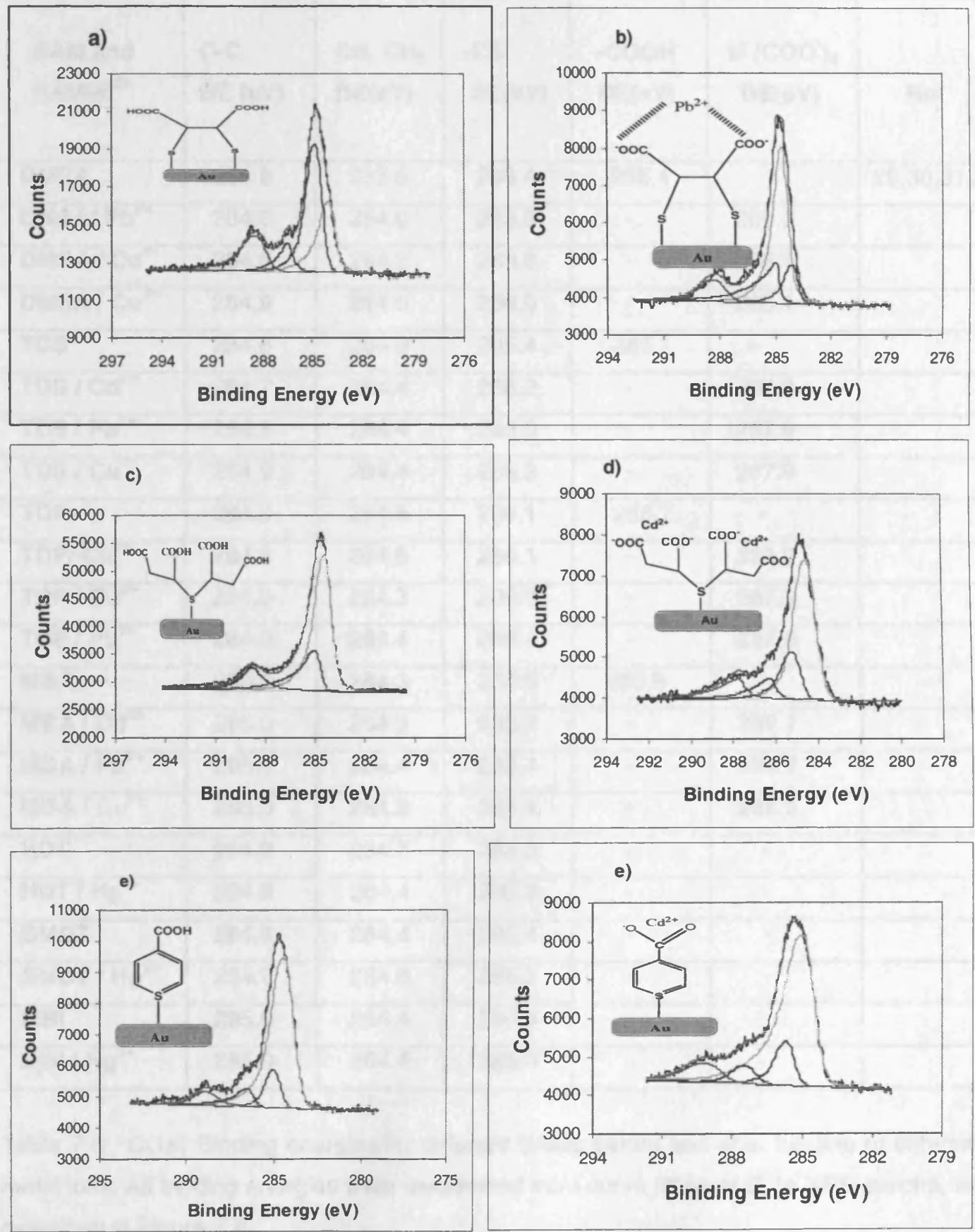

Figure 7.8: Curves-fitted high resolution XPS spectra fro the $C(1 \mathrm{~s})$ region of (a) DMSA before adsorption of lead ions, (b) DMSA / $\mathrm{Pb}^{2+}$, (c) TDS, (d) TDS / $\mathrm{Cd}^{2+}$, (e) MBA and (f) $\mathrm{MBA} / \mathrm{Cd}^{2+}$. All the SAMs were prepared by immersion for $24 \mathrm{~h}$ into $1 \mathrm{mM}$ solution. 
The results of the assignment of the peaks for different SAMs before and after adsorption of metal ions are summarized in table 7.8. It was found that in all cases the $\mathrm{C}(1 \mathrm{~s})$ binding energy for metal ion carboxylate is smaller than free carboxlate groups. XPS results for $\mathrm{C}(1 \mathrm{~s})$ peak further confirm that all the gold surfaces were modified with SAMs.

\begin{tabular}{|c|c|c|c|c|c|c|}
\hline $\begin{array}{l}\text { SAM and } \\
\text { SAM } / M^{2+}\end{array}$ & $\begin{array}{l}\text { C-C } \\
\text { BE (eV) }\end{array}$ & $\begin{array}{l}\mathrm{CH}, \mathrm{CH}_{2} \\
\mathrm{BE}(\mathrm{eV})\end{array}$ & $\begin{array}{l}-\mathrm{CS} \\
\mathrm{BE}(\mathrm{eV})\end{array}$ & $\begin{array}{l}-\mathrm{COOH} \\
\mathrm{BE}(\mathrm{eV})\end{array}$ & $\begin{array}{c}M(\mathrm{COO})_{2} \\
\mathrm{BE}(\mathrm{eV})\end{array}$ & Ref \\
\hline DMSA & 284.8 & 283.9 & 286.4 & 288.4 & - & $29,30,31,32$ \\
\hline DMSA Pb $^{2+}$ & 284.6 & 284.0 & 285.5 & - & 288.0 & \\
\hline DMSA / Cd ${ }^{2+}$ & 284.8 & 284.2 & 285.6 & - & 288.1 & \\
\hline DMSA / Cu & 284.9 & 284.0 & 286.0 & - & 288.1 & \\
\hline TDS & 284.6 & 284.0 & 285.4 & 288.1 & - & \\
\hline TDS / Cd & 284.7 & 284.4 & 286.2 & - & 287.8 & \\
\hline TDS / $\mathrm{Pb}^{2+}$ & 284.8 & 284.4 & 286.0 & - & 287.9 & \\
\hline TDS $/ \mathrm{Cu}^{2+}$ & 284.9 & 284.4 & 286.3 & - & 287.9 & \\
\hline TDP & 284.9 & 284.5 & 286.1 & 288.7 & - & \\
\hline TDP/ $\mathrm{Cu}^{2+}$ & 284.8 & 284.5 & 286.1 & - & 288.0 & \\
\hline TDP / Cd $d^{2+}$ & 284.9 & 284.3 & 286.3 & - & 287.9 & \\
\hline TDP / $\mathrm{Pb}^{2+}$ & 284.9 & 284.4 & 286.4 & - & 287.8 & \\
\hline MBA & 285.0 & 284.3 & 286.5 & 289.9 & - & \\
\hline MBA $/ \mathrm{Cd}^{2+}$ & 285.0 & 284.3 & 285.7 & - & 289.1 & \\
\hline MBA / $\mathrm{Pb}^{2+}$ & 285.0 & 284.4 & 285.4 & - & 288.7 & \\
\hline $\mathrm{MBA} / \mathrm{Cu}^{2+}$ & 285.0 & 284.3 & 286.1 & - & 288.9 & \\
\hline HDT & 284.9 & 284.3 & 286.3 & - & - & \\
\hline HDT $/ \mathrm{Hg}^{2+}$ & 284.9 & 284.4 & 286.3 & - & - & \\
\hline BMDT & 284.8 & 284.4 & 285.4 & - & - & \\
\hline BMDT $/ \mathrm{Hg}^{2+}$ & 284.7 & 284.0 & 285.2 & - & - & \\
\hline MBI & 285.0 & 284.4 & 286.0 & - & - & \\
\hline $\mathrm{MBI} / \mathrm{Hg}^{2+}$ & 285.0 & 284.4 & 285.8 & - & - & \\
\hline
\end{tabular}

Table 7.8: $C(1 \mathrm{~s})$ Binding energies for different SAMs before and after binding of different metal ions. All binding energies were determined from curve fitting of $C$ is XPS spectra, as described in Figure 7.8. 


\subsubsection{Analysis using O(1s) XPS spectra}

The spectra were fitted by two single symmetric Voigt curves with a linear type background, as for the $C(1 s)$ data. The peak fitting analysis for the $O(1 \mathrm{~s})$ peaks is shown in Figure 7.9.
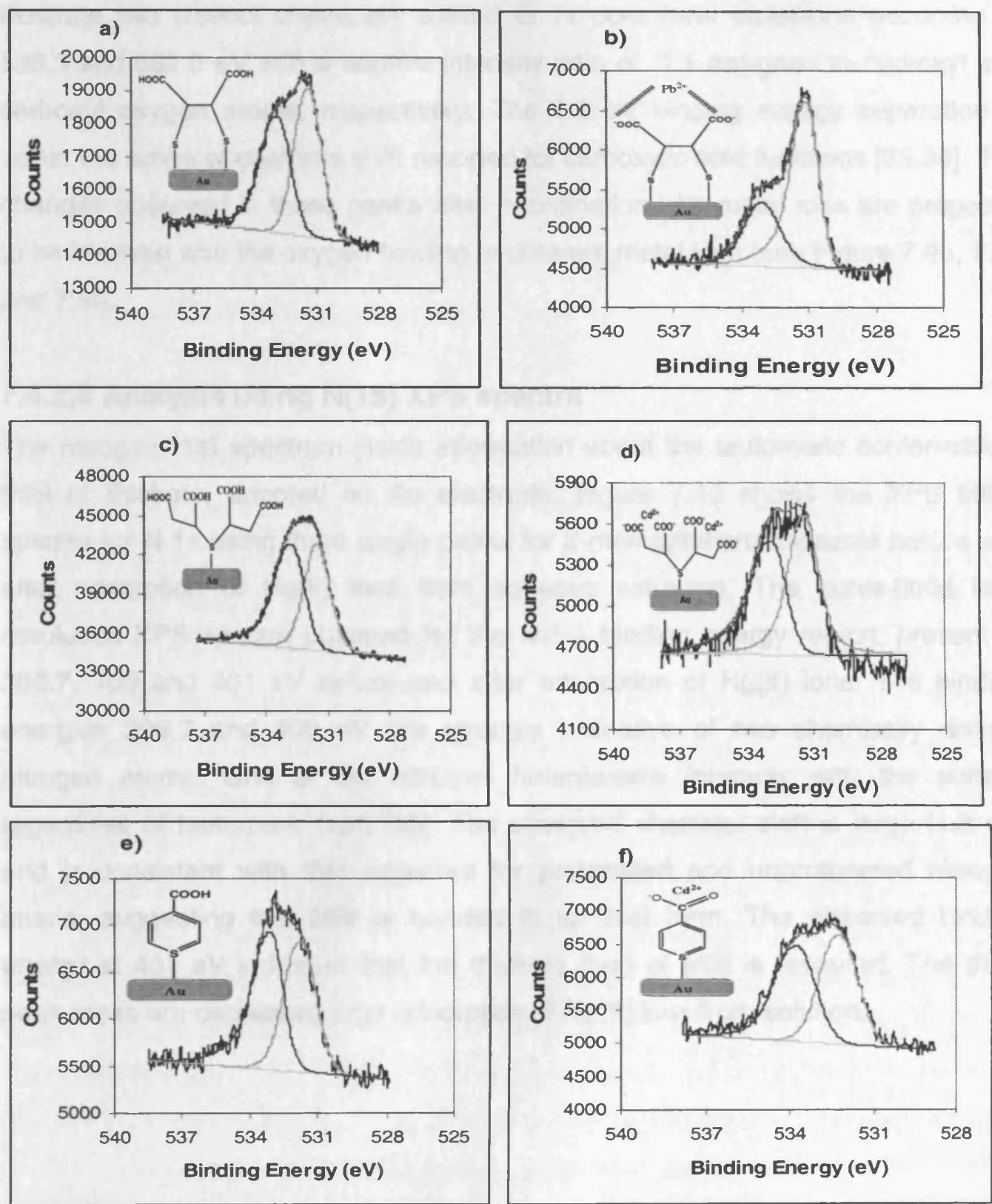

Figure 7.9: Curves-fitted high resolution XPS spectra fro the $O(1 \mathrm{~s})$ region of (a) DMSA, (b) DMSA / $\mathrm{Pb}^{2+}$, (c) TDS, (d) TDS / $\mathrm{Cd}^{2+}$, (e) MBA and (f) MBA / $\mathrm{Cd}^{2+}$. The same XPS instrumental analysis conditions were used. The minimum number of peak components with a linear background was chosen to produce random residuals. 
The oxygen in SAMs comes from the carboxylate groups $(-\mathrm{COOH})$ before complexation and metal ion carboxylate after the complexation because all the SAMs did not show any sulfur oxide in the wide scan XPS spectra or the high resolution XPS spectra for the $S\left(2 p_{3 / 2}\right)$. The results of peak fitting for $O$ is illustrate two distinct chemically shifted $O$ is core level emissions occurring at 533.3 and $532.0 \mathrm{eV}$ with a relative intensity ratio of $1: 1$ assigned to hydroxyl and carbonyl oxygen atoms, respectively. The $1.3 \mathrm{eV}$ binding energy separation is within the range of chemical shift reported for carboxylic acid functions [33,34]. The changes observed in these peaks after coordination with metal ions are proposed to be involved with the oxygen binding to different metal ions (see Figure 7.9b, 7.9d and 7.9f).

\subsubsection{Analysis using N(1s) XPS spectra}

The nitrogen (1s) spectrum yields information about the tautomeric conformation, thiol or thioketo, adopted on Au electrode. Figure 7.10 shows the XPS fitting spectra for $\mathrm{N}$ is using three single peaks for 2-mercaptobenzimidazole before and after adsorption of $\mathrm{Hg}(\mathrm{II})$ ions from aqueous solutions. The curve-fitted high resolution XPS spectra obtained for the $N(1 \mathrm{~s})$ binding energy region, present at 398.7, 400 and $401 \mathrm{eV}$ before and after adsorption of $\mathrm{Hg}(\mathrm{II})$ ions. The binding energies 398.7 and $400 \mathrm{eV}$ are strongly indicative of two chemically distinct nitrogen atoms. One of the nitrogen heteroatoms interacts with the surface regardless of tautomeric form [35]. The observed chemical shift is large $(1.3 \mathrm{eV})$ and is consistent with that expected for protonated and unprotonated nitrogen atoms, suggesting that $\mathrm{MBI}$ is bonded in its thiol form. The observed binding energy at $401 \mathrm{eV}$ indicated that the thioketo form of $\mathrm{MBI}$ is favoured. The three peak areas are decreased after adsorption of $\mathrm{Hg}(\mathrm{II})$ ions from solution. 

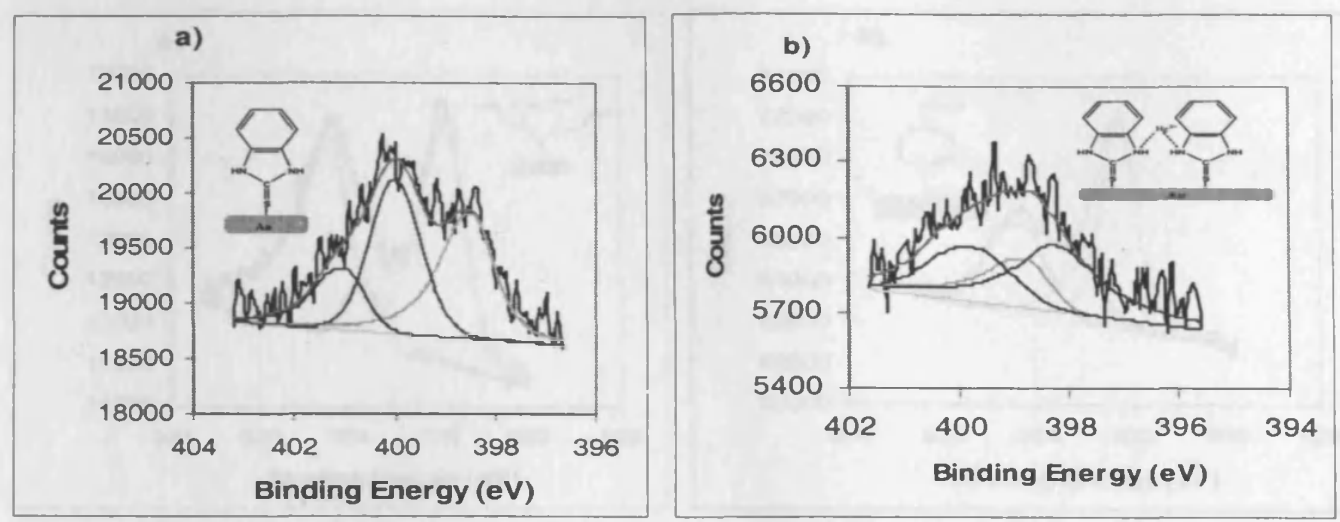

Figure 7.10: High resolution XPS data for $N$ 1s binding energy regions, showing curvefitting components for (a) $\mathrm{MBI}$ and (b) $\mathrm{MBI} / \mathrm{Hg}(\mathrm{II})$. The minimum number of peak components with a linear background was chosen to produce random residuals.

\subsubsection{Analysis using $\mathrm{Cu}(2 \mathrm{p})$ spectra}

The nature of the chemical interaction between the $\mathrm{Cu}(\mathrm{II})$ and carboxylate groups of the different SAMs can be seen in the $\mathrm{Cu}\left(2 \mathrm{p}_{3 / 2}\right)$ XPS region. The $\mathrm{Cu}\left(2 \mathrm{p}_{3 / 2}\right)$ spectrum was fitted to three single peaks using the same methodology for fitting $C$ 1s and N 1s peaks. Figure 7.11 shows the XPS fitting data for copper ions before and after binding to different SAMs. The results for binding of $\mathrm{Cu}$ (II) to different SAMs show three different environments: $\mathrm{Cu}(\mathrm{II})$ alone, $\mathrm{Cu}(\mathrm{I})$ alone and both $\mathrm{Cu}(\mathrm{II})$ and $\mathrm{Cu}(\mathrm{I})$. Figures $7.11 \mathrm{a}$ and $7.11 \mathrm{~b}$ show that the first peak at $933.0 \mathrm{eV}$ is characterized as the alginate-bound $\mathrm{Cu}(\mathrm{II})$ and the second peak at $934.8 \mathrm{Cu}(\mathrm{II})$ originated from $\mathrm{Cu}(\mathrm{II})$ located in the coordination form (copper carboxylate) $[36,37]$. The $\mathrm{Cu}(\mathrm{II})$ is about $88 \%$ available for complexation with TDS and MBA. The comparison between the XPS regions for copper acetate solution (blank) and copper acetate after complexation with TDS and MBA is shown in Figure 7.12. The copper XPS shows both $\mathrm{Cu}(\mathrm{II})$ and $\mathrm{Cu}(\mathrm{I})$ peaks have appeared (see Figures 9.11C and $9.11 \mathrm{~d}$ ). The $\mathrm{Cu} 2 \mathrm{p}_{3 / 2}$ peaks at a binding energy $932-932.4 \mathrm{eV}$ can be attributed to $\mathrm{Cu}(\mathrm{I})$. The copper XPS fitting spectra for BDMT and MBI SAMs shows three different environments; $\mathrm{Cu}(\mathrm{I}), \mathrm{Cu}(0)$ and $\mathrm{CuS}$. $\mathrm{Cu}(\mathrm{I})$ is more available than $\mathrm{Cu}(\mathrm{II})$ and the peak at 932.5 can be attributed to copper sulphide [38]. No copper hydroxide or copper oxide was observed in any sample. 

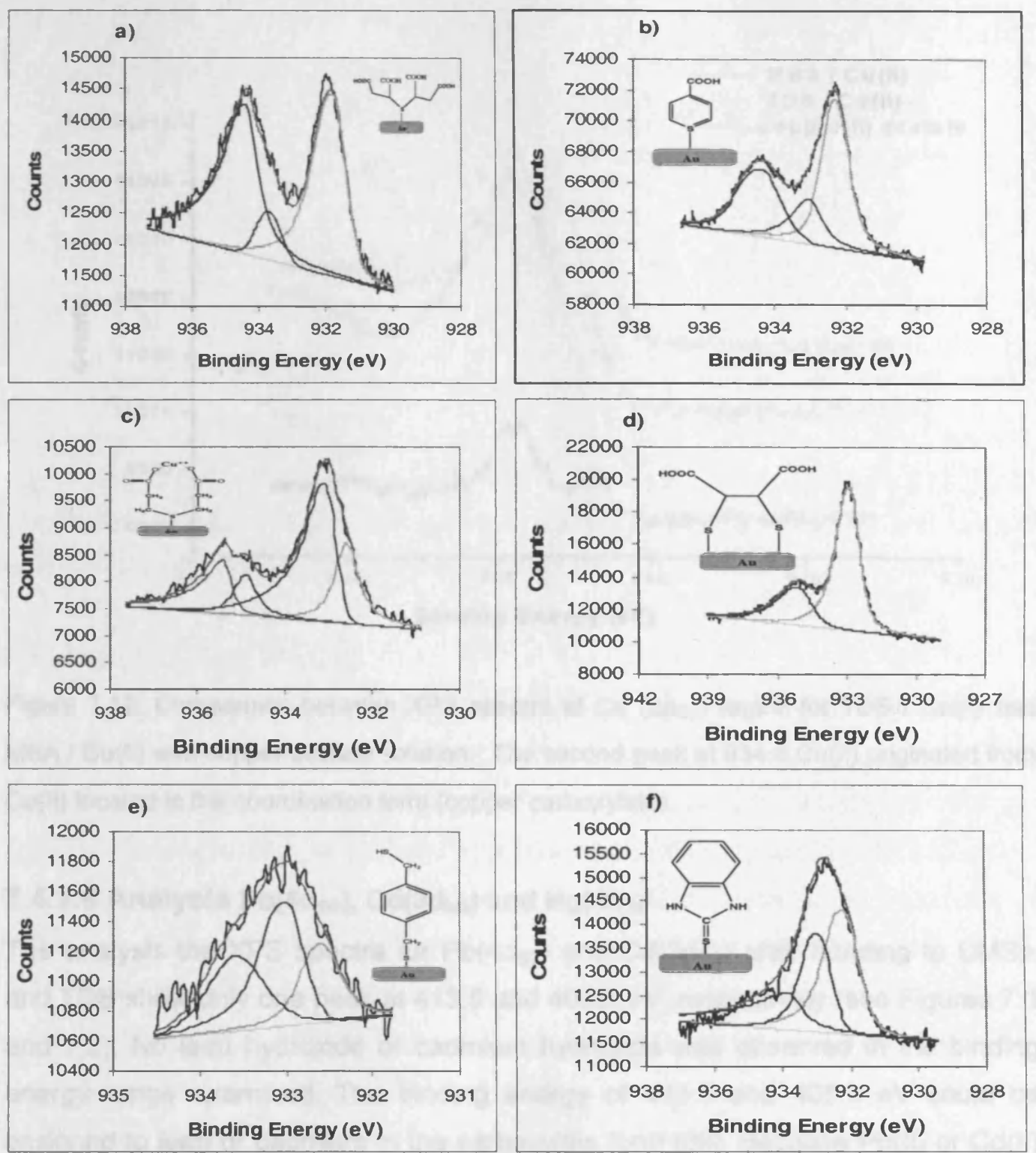

Figure 7.11: Curved-fitted high resolution XPS spectra fro the $\mathrm{Cu}\left(2 \mathrm{p}_{3 / 2}\right)$ region of (a) TDS / $\mathrm{Cu}^{2+}$, (b) $\mathrm{MBA} / \mathrm{Cu}^{2+}$, (c) TDP $/ \mathrm{Cu}^{2+}$, (d) $D M S A / \mathrm{Cu}^{2+}$, (e) $\mathrm{BDMT} / \mathrm{Cu}^{2+}$ and (f) $\mathrm{MBI} / \mathrm{Cu}^{2+}$. The same XPS instrumental analysis conditions were used. The minimum number of peak components with a linear background was chosen to produce random residuals. 


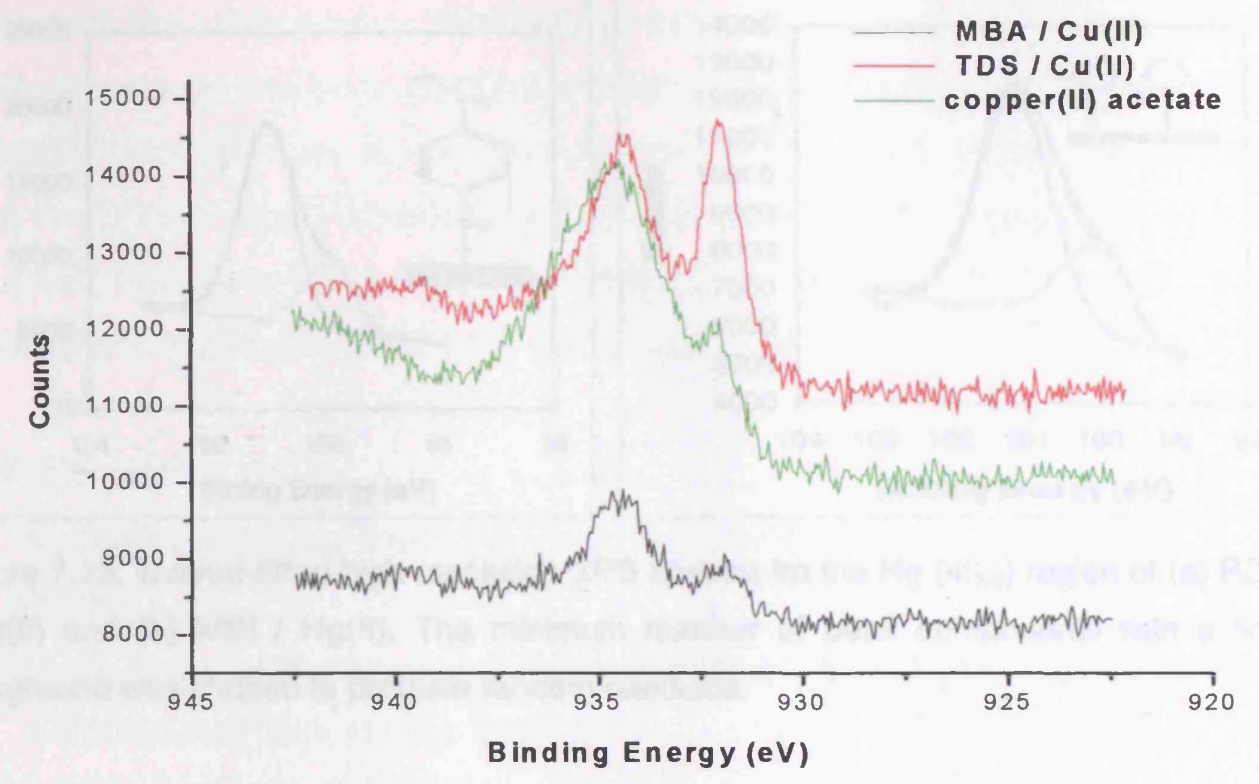

Figure 7.12: Comparison between XPS spectra of $\mathrm{Cu}\left(2 \mathrm{p}_{3 / 2}\right)$ region for TDS / Cu(II) and MBA / Cu(II) with copper acetate solution. The second peak at $934.8 \mathrm{Cu}$ (II) originated from $\mathrm{Cu}$ (II) located in the coordination form (copper carboxylate).

\subsubsection{Analysis $\mathrm{Pb}\left(4 \mathrm{~d}_{5 / 2}\right), \mathrm{Cd}\left(3 \mathrm{~d}_{5 / 2}\right)$ and $\mathrm{Hg}\left(4 \mathrm{f}_{7 / 2}\right)$}

The analysis the XPS spectra for $\mathrm{Pb}\left(4 \mathrm{~d}_{5 / 2}\right)$ and $\mathrm{Cd}\left(3 \mathrm{~d}_{5 / 2}\right)$ after bonding to DMSA and TDS show only one peak at 413.5 and $405.3 \mathrm{eV}$, respectively (see Figures 7.1 and 7.2). No lead hydroxide or cadmium hydroxide was observed in the binding energy range examined. The binding energy of 413.5 and $405.3 \mathrm{eV}$ could be assigned to lead or cadmium in the carboxylate form [39]. Because $\mathrm{Pb}$ (II) or $\mathrm{Cd}$ (II) ions have completely filled d subshells, their adsorption was through ionic binding with carboxylate groups of these SAMs on Au surfaces. The $\mathrm{Hg}(4 \mathrm{f} / 2)$ spectra for $\mathrm{BDMT} / \mathrm{Hg}(\mathrm{II})$ and $\mathrm{MBI} / \mathrm{Hg}(\mathrm{II})$ were fitted with two peaks with a binding energy of 99.9 and $100.8 \mathrm{eV}$ for BDMT / Hg(II) and 99.9 and $101.1 \mathrm{eV}$ for $\mathrm{MBI} / \mathrm{Hg}$ (II). The results of peak fitting for both systems are shown in Figure 7.13. The binding energy of $99.9 \mathrm{eV}(15 \%)$ is attributed to metallic mercury and the binding energy of $100.8 \mathrm{eV}(85 \%)$ and $101.1 \mathrm{eV}(85 \%)$ is attributed to $\mathrm{Hg}(\mathrm{II})$ bound to sulphur and nitrogen of terminal free groups of BDMT and MBI SAMs. 

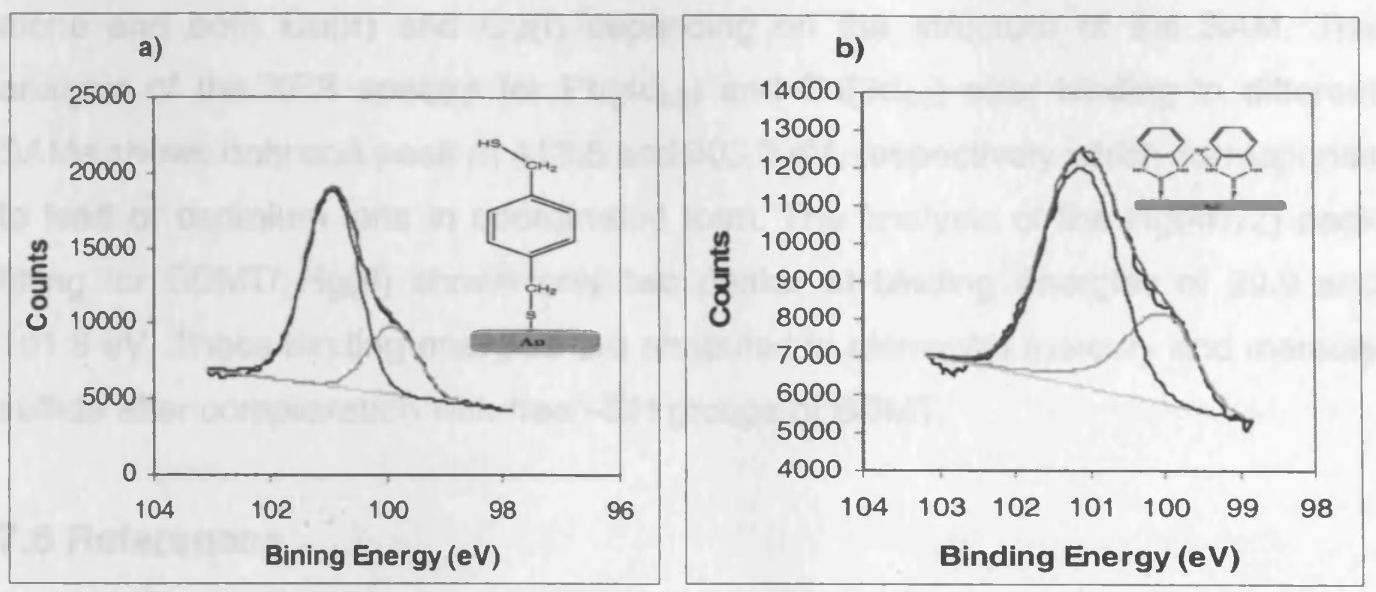

Figure 7.13: Curved-fitted high resolution XPS spectra fro the $\mathrm{Hg}\left(4 \mathrm{f}_{7 / 2}\right)$ region of (a) BDMT $/ \mathrm{Hg}(\mathrm{II})$ and (b) $\mathrm{MBI} / \mathrm{Hg}(\mathrm{II})$. The minimum number of peak components with a linear background was chosen to produce random residuals.

\subsection{General Conclusions}

The results presented for high resolution XPS elemental composition for SAM and $\mathrm{SAM} / \mathrm{M}^{2+}$ show the presence of sulfur, carbon, oxygen, nitrogen, gold and target metal ions. These results also confirmed that SAMs are successfully immobilized on Au surfaces and can bind different metal ions from aqueous solutions with some selectivity. These results also demonstrated the presence of terminal free groups of SAMs for example; - $\mathrm{COOH},-\mathrm{SH},-\mathrm{NH}$.

The information on stoichiometry provided by XPS shows that a metal ion is coordinated by the SAMs. The M:S ratio was in good agreement with the stoichiometry extracted from QCM and EQCM measurements. The S(2p) signal of SAMs / Au interfaces does not change after complexation with metal ions. The results of the peak fitting analysis from this study along with previous results from the literature indicate only one bound thiolate species is detectable by XPS after adsorption of SAM molecules onto gold surfaces. The $S\left(2 p_{3 / 2}\right)$ binding energy of this bound thiolate species is $162 \mathrm{eV}$ for all SAMs. The results of peak fitting analysis for $\mathrm{C}(1 \mathrm{~s})$ for SAMs with free carboxylate show four peaks at binding energies of $288.5,286.4,285$ and $284.5 \mathrm{eV}$, which are assigned to $-\mathrm{COOH}, \mathrm{C}-\mathrm{S}$, $\mathrm{C}-\mathrm{C}$ and $-\mathrm{CH}_{2}$ or $-\mathrm{CH}$, respectively. The results of peak fitting for $\mathrm{O}$ 1s illustrate two distinct chemically shifted $O 1$ s core level emissions occurring at 533.3 and $532.0 \mathrm{eV}$ with a relative intensity ratio of $1: 1$ assigned to hydroxyl and carbonyl oxygen atoms, respectively. XPS peak fitting also demonstrates the metal ions are successfully bound to the different SAMs. The results of peak fitting for binding of $\mathrm{Cu}(\mathrm{II})$ to different SAMs show three different environments: $\mathrm{Cu}(\mathrm{II})$ alone, $\mathrm{Cu}(\mathrm{I})$ 
alone and both $\mathrm{Cu}(\mathrm{II})$ and $\mathrm{Cu}(\mathrm{I})$ depending on the structure of the SAM. The analysis of the XPS spectra for $\mathrm{Pb}\left(4 \mathrm{~d}_{5 / 2}\right)$ and $\mathrm{Cd}\left(3 \mathrm{~d}_{5 / 2}\right)$ after binding to different SAMs shows only one peak at 413.5 and $405.3 \mathrm{eV}$, respectively which corresponds to lead or cadmium ions in coordinated form. The analysis of the $\mathrm{Hg}(4 \mathrm{f} 7 / 2)$ peak fitting for BDMT/ $\mathrm{Hg}(\mathrm{II})$ shows only two peaks, at binding energies of 99.9 and $101.8 \mathrm{eV}$. These binding energies are attributed to elemental mercury and mercury sulfide after complexation with free -SH groups of BDMT.

\subsection{References}

1- H.L.Zhang, S.D.Evans and K.Critchley, J. Chem. Physics., 122, (2005), 224707.

2- K.D.Weldige, M.Rohwerder, E.Vago, H.Viefhaus and M.Stratmann, Fresenius J Anal Chem., 353, (1995), 329.

3- X.Zhang and R.B.Bai, J.Mater. Chem, 12, (2002), 2733.

4- M.S.Polo and J.R.Utrilla, Environ.Sci. Technol., 36, (2002), 3850.

5- A.S.Duwez, J.Electr.Spectrosc.Relat. Phenom., 134, (2004), 97.

6- T.Ishida, M.Hara, I.Kojima, S.Tsuneda, N.Nishida, H.Sasabe and W.Knoll, Langmiur., 14, (1998), 2092.

7- J.M.Cooper, A.Glidle, A.R.Hillman, M.D.Ingram,C.Ryder and K.S.Ryder, Phys. Chem. Chem Phys., 6, (2004), 2403.

8- J.F.Modulder, W.F.Stickle, P.E.Sobol and K.D.Bomben, Handbook of X-ray Photoelectron Spectroscopy, Perkin-Elmer, Physical electronics Divison, Eden Prairie, MN 55344, (1992).

9- D.Briggs and M.P.Seah, Practical Surface Analysis by Auger and X-ray Photoelectron Spectroscopy, John Wiley\& Sons, Chichester, (1990).

10- NIST Standard Reference Database 20, Version 3.3, Web version, (http://srdata.nist.gov/xps/.

11- K.L.Lin, Y.K.Cadwell and N.L.Abbott, Adv. Mater., 15, (2003), 1819.

12- J.Noh, E.Ito, K.Nakajima, J.Kim, H.Lee and M.Hara, J.Phys. Chem B., 106, (2002), 7139.

13- T.Ishida and N.Choi, Langmuir., 15, (1999), 6799.

14- D.G.Castner, Langmuir., 12, (1996), 5083.

15- V.Tai, A.Shaporenko, H.T.Rong, M.Buck, W.Eck, M.Grunze and M.Zharnikov, J.Phys. Chem B., 108, (2004), 16806.

16- D.L.Pugmire, M.J.Tarlov and R.D.V.Zee, Langmuir., 19, (2003), 3720.

17- D.R.Mullins and P.F.Lyman, j.Phys.Chem., 97, (1993), 9226.

18- D.G.Castner, K.Hinds and D.W.Grainger, Langmuir., 12, (1996), 5083. 
19- M.Wirde, L.Gelius and L.Nyholm, Langmuir., 15, (1999), 6370.

20- O.Cavalleri, G.Gonella, S.Terreni, M.Vignolo, P.Pelori, L.Florenano,

A.Morgante, M.Canepa and R.Rolandi, J.Phys.Condens. Matter., 16, (2004), S2477.

21- F.Bensebaa, Y.Zhou, Y.Deslandes, E.Kruus and T.H.Ellis, Surf. Sci, 405, (1998), L472.

22- E.Ito, K.Konno, J.Noh, K.Kanai, Y.Ouchi, K.Seki and M.Hara, Applied Surface Science., 244, (2005), 584.

23- O.Cavalleri, L.Oliveri, A.Dacca, R.Parodi and R.Rolandi, Applied Surface Science., 175-176, (2001), 357.

24- W.Deng, D.Fujita, L.Yang, H.Nejo and C.Bai, Jpn.J.Appl.Phys., 39, (2000), L751.

25- W.Deng, L.Yang, D.Fujita, H.Nejoh and C.Bai, Appl.Phys.A., 71, (2000), 639.

26- B.Heinz, H.Morgner, Surf.Sci., 372, (1997), 100.

27- Y.Joseph, I.Besnard, M.Rosenberger, B.Guse, H.G.Nothofer, J.M.Wessels, U.wild, A.K.Cericke, D.Su, R.Schlogl, A.Yasuda and T.Vossmeyer, J.Phys. Chem.B., 107, (2003), 7406.

28- C. M.Whelan, M.R.Smyth, C.J.Barnes, N.M.D.Brown and C.A.Anderson, Applied Surface Science., 134, (1998), 144.

29- T.Ishida, M.Hara, I.Kojima, S.Tsuneda, N.Nishida, H.Sasabe and W.Knoll, Langmiur., 14, (1998), 2092.

30- R.Brito, R.Tremont, O.Feliciano and C.R.Cabrera, J.Electroanal.Chem., 540, (2003), 53.

31- K.V.G.K.Murty, M.Venkataramanan and T.Pradeep, Langmuir., 14, (1998), 5446.

32- S.Fleith, A.Ponche, R.Bareille, J.Amedee and M.Nardin, Colloids.Surfaces. B: Biointerfaces., 44, (2005), 15.

33- C.M.Whelan, J.Ghijsen, J.J.Pireaux and K.Maex, Thin Solid Films., 464-465, (2004), 388.

34- D.r.Jung, A.W.Czanderna and G.C.Herdt, J.Vac.Sci.Technol., 14, (1996), 1779.

35- C. M.Whelan, M.R.Smyth, C.J.Barnes, N.M.D.Brown and C.A.Anderson, Applied Surface Science., 134, (1998), 144.

36- S.Deng and Y.P.Ting, Langmuir., 21, (2005), 5948.

37- J.P.chen, L.Hong,S.Wu and L.Wang, Langmuir., 18, (2202),9413.

38- E.L.Smith, C.A.Alves, J.W.Anderegg and M.D.Porter, Langmiur., 8, (1992), 2707.

39- A.V.Nabok and T.Richardson, Langmiur., 13, 1997, 3198. 


\section{Chapter 8: General conclusions and scope for future work}

\subsection{Functionalization of the QCM with SAMs}

Self-assembled monolayers of different thiols, some aliphatic and others aromatic, were immobilised on the gold electrode of a QCM. The first aim of the work was successful immobilisation of these monolayers on gold, using the QCM and cyclic voltammetry measurements to monitor the extent and integrity of this process. QCM frequency shift measurements allowed study of these monolayers during immobilization via the mass change at the solid / liquid interface. The mass change of the SAMs from the modification solutions were determined on dry (emersed) and wet (immersed) crystals before and after modification (24h). The relation between frequency shift on the emersed crystals $\left(\Delta f_{\text {dry }}\right)$ and of immersed crystals $\left(\Delta f_{\text {wet }}\right)$ for all SAMs was studied. The observations indicate this relation is linear for most SAMs and the frequency shifts for aromatic SAMs are bigger than those for aliphatic SAMs. it was found that the amount of adsorption of the SAMs on to gold electrode depended on the structure, temperature and concentration of solution species.

A series of cyclic voltammetry experiments were carried out for the redox couple $\left[\mathrm{Fe}(\mathrm{CN})_{6}\right]^{3-14-}$ at clean gold electrodes and gold electrodes modified with SAMs. The oxidation-reduction reactions were influenced by the modification of the surface with different kinds of SAMs. Cyclic voltammetry allows one to judge the quality of the SAM, particularly regarding pinhole defects. It is obvious from the voltammograms that the suppression of the current strongly depends on the thickness of the monolayer, the greatest decrease in the current occurring for the long chain carbon thiols and aromatic thiols. The relation between the electron transfer rate constant for $\left[\mathrm{Fe}(\mathrm{CN})_{6}\right]^{3-14-}$ at SAMs and $\mathrm{QCM}$ frequency shifts on emersed and immersed crystals showed that SAMs containing m-electron rich aromatic constituents have similar electron transfer constants and bigger frequency shifts than aliphatic SAMs (such as 4-mercaptobenzoic acid (MBI), 2mercaptobenzimidazole (MBI) and 4-acetomidothiophenol (AMTP)).

\subsection{Binding of trace metal ions to SAMs using the QCM as a gravimetric probe}

The recorded frequency change observed for the binding of $\mathrm{Cd}^{2+}, \mathrm{Cu}^{2+}, \mathrm{Pb}^{2+}$ and $\mathrm{Hg}^{2+}$ from single metal ion solutions to different monolayer structures was tested on emersed and immersed crystals. The binding of different concentrations of these 
metal ions to different kinds of SAMs was studied; 200ppm and 800ppm concentrations of selected metal ions from the solution were bound to all monolayers. Frequency measurements allowed estimation of the amount of metal ion bound to the SAM (using the Sauerbrey Equation). From this it was possible to determine the mole ratio between the metal ion and SAM. Based on concentrations studies, the best ligands were selected: 3-thiodisuccunic acid and 4mercaptobenzoic for $\mathrm{Cd}^{2+}$ ions, meso-2,3-dimercaptosuccinic acid for $\mathrm{Pb}^{2+}$ ions, 3,3-thiodipropionic acid and 4-acetomidothiophenol for $\mathrm{Cu}^{2+}$ ions and finally 1,6hexanedithiol and 1,4-benzenedimethanethiol for $\mathrm{Hg}^{2+}$.

\subsubsection{Estimation of the equilibrium binding constant between trace metal ions and aliphatic-aromatic SAMs}

Aliphatic self-assembled monolayer 3-thiodisuccinic acid and aromatic selfassembled monolayer 4-mercaptobenzoic acid were studied for binding a range of $\mathrm{Cd}^{2+}$ concentrations $(10 \mu \mathrm{M}$ to $10 \mathrm{mM})$ from aqueous solution at $\mathrm{pH}=6.3$ using QCM frequency shift measurements. The equilibrium binding constant was estimated for the binding of $\mathrm{Cd}^{2+}$ to the SAM from the relation between the frequency shifts for binding cadmium ions to SAM on emersed and immersed crystals with concentration of cadmium ions in solution. The experimental adsorption isotherms were fitted to Langmuir, Temkin, Freundlich, Frumkin, ElAwady and Flory-Huggins isotherms. There is a very good fit of the experimental results (relation between frequency shifts and concentration of binding $\mathrm{Cd}^{2+}$ to SAM) to the Temkin isotherm. From linear regression of the Temkin isotherm, the binding constant was estimated (see chapter 5 section 5.5 ). This is a thermodynamic probe of the interface. Thermodynamic parameters such as the Gibbs free energy $\left(\Delta \mathrm{G}^{\circ}\right)$ and interaction constant can also be deduced from these adsorption isotherms. The negative values of $\Delta \mathrm{G}^{\circ}$ indicate that the adsorption of cadmium ions by the ligand was spontaneous under the experimental conditions. It was found that the stoichiometry between $\mathrm{Cd}^{2+}$ and 3-thiodisuccinic acid is $1: 1$ at low concentrations of $\mathrm{Cd}^{2+}(10 \mu \mathrm{M}$ to $500 \mu \mathrm{M})$ and $(2: 1)$ at high concentrations $(600 \mu \mathrm{M}$ to $10 \mathrm{mM})$. The equilibrium-binding constant between $\mathrm{Cd}^{2+}$ to 4 mercaptobenzoic acid was estimated from the Frumkin isotherm. The stoichiometry between $\mathrm{Cd}^{2+}$ and 4-mercaptobenzoic acid is 1:1 indicating that the chemical interaction of $\mathrm{Cd}^{2+}$ ions with the carboxylic acid groups is related to the bondingcomplex interaction between SAM and cadmium ions. The detection of $\mathrm{Cd}^{2+}$ ions by complexation from solution to the surface 3-thiodisuccinic acid and 4- 
mercaptobenzoic acid films on gold electrode was possible and stable at $\mathrm{pH}=6.3$. The pK for the 3-thiodisuccinic acid is about 4.2 and for 4-mercaptobenzoic acid is about 4.95, in the two cases; the $\mathrm{pH}$ of the cadmium ion solution is above the $\mathrm{pK}_{\mathrm{a}}$ of the ligand which indicates that terminal groups of the two monolayers are deprotonated and negatively charged. The selectivity of the 3-thiodisuccinic acid and 4-mercaptobenzoic acid towards $\mathrm{Cd}^{2+}, \mathrm{Pb}^{2+}$ and $\mathrm{Cu}^{2+}$ was studied in single metal ion solutions by estimating the binding constant for each metal ion to the ligand. The results show that 3-thiodisuccinic acid has a very high affinity to cadmium ions and 4-mercaptobenzoic acid has a similar selectivity to $\mathrm{Cd}^{2+}$ and $\mathrm{Pb}^{2+}$.

The binding constant between meso-2,3-dimercaptosuccinic acid and $\mathrm{Pb}^{2+}$ was estimated from the slope and intercept of linear regression of the Frumkin isotherm for both emersed and immersed crystals. The binding constants were also estimated for $\mathrm{Cd}^{2+}$ and $\mathrm{Cu}^{2+}$ to the same ligand to examine the selectivity of the ligand towards these single metal ion solutions. The selectivity of the ligand towards these metal ion solutions followed the sequence: $\mathrm{Pb}^{2+}>\mathrm{Cu}^{2+}>\mathrm{Cd}^{+2}$. SAM of 3.3-thiodipropionic acid modified Au electrodes shows similar selectivity to copper and lead ion solutions. SAM 1,6-hexanedithiol modified Au electrodes have a high selectivity to $\mathrm{Hg}(\mathrm{II})$ over other metal ions. The order of the binding constants is $\mathrm{Hg}(\mathrm{II})>\mathrm{Zn}$ (II) $>\mathrm{Cd}(\mathrm{II})>\mathrm{Pb}(\mathrm{II})>\mathrm{Ni}(\mathrm{II})>\mathrm{Cu}(\mathrm{II})>\mathrm{Ag}^{+}$.

The selectivity of selected ligands towards $\mathrm{Cd}^{2+}, \mathrm{Cu}^{2+}, \mathrm{Pb}^{2+}$ and $\mathrm{Hg}^{2+}$ in binary mixtures was examined using two procedures. In the first one, the concentration of target metal ion in binary mixtures was fixed and the concentration of interfering metal ion was varied. It was found that the frequency shifts for target metal ions in binary mixtures are similar to those reported in single metal ion solutions. In the second one, the concentration of target metal ions in binary mixtures was varied using same methodology for single metal ion solutions and the concentration of interfering metal ion was fixed. The relation between the concentrations of target metal ions in binary mixtures with the frequency shifts was also studied. After fitting of the experimental data to different isotherms one can estimate the binding constants for target metal ions in binary mixtures. The results also indicate that the second metal ion has no significant influence on the detection of target metal ions. The detection limit of target metal ions at selected SAM modified Au electrodes was in the range $(0.9-7 \mu \mathrm{M})$. 


\subsection{Binding of trace metal ions to SAMs using the EQCM as an}

\section{electrochemical probe}

The results for binding different target metal ions to different SAMs show that in some cases the EQCM can be used as an electrochemical probe of the SAM interface. Cyclic voltammetry of surface complexed $\mathrm{Cu}^{2+}$ or $\mathrm{Pb}^{2+}$ ions in metal-free nitrate solution shows that both metal ions are adsorbed at the 3,3-thiodipropionic acid SAM modified Au electrodes and that they undergo subsequent quasireversible electroreductions. The cathodic peak position identifies the metal ion. Interestingly, upon re-oxidation, the oxidised species are not quickly released from the surface (retained on the surface) in solutions that are otherwise free from metal ion. The voltammetric responses after the binding of target metal ions to selected SAMs depended on several factors such as scan rate, repetitive cycling, preconcentration time, $\mathrm{pH}, \mathrm{pK}_{\mathrm{a}}$, modification time for the SAM and the concentration of target metal ions. The integrated cathodic peak area charge formed after metal ion complexation with SAM allows one to estimate the surface coverage of metal ion bound to the SAM, using Faraday's law. The voltammetric responses for binding of cadmium ions to 3-thiodisuccinic acid and lead ions to meso-2,3-dimercaptosuccinic acid showed high selectivity to cadmium and lead ions, respectively, in the presence of interfering ions in binary mixtures. SAMs of 1,4-benzenedimethanethiol modified $\mathrm{Au}$ electrodes have a high selectivity to mercury ions in the presence of copper ions in binary mixtures. The EQCM experiments gave more details about the nature of complexation between target metal ions and the surface ligand. Voltammetry of the redox couple of the target metal ions allows determination of metal ion concentration. The detection limit of target metal ions at selected SAM modified Au electrodes was in range (0.01-0.08 $\mu \mathrm{M}$ ) and was three times lower than using QCM measurements.

\subsection{XPS study of the complexation between trace metal ions and SAMs}

X-Ray photoelectron spectroscopy (XPS) has been used successfully to study the complexation of trace metal ions to SAMs. Analyses of the high resolution XPS elemental composition for SAM and SAM/ $\mathrm{M}^{2+}$ show the presence of sulfur, carbon, oxygen, nitrogen, gold and the target metal ion. These results also demonstrated the presence of terminal free groups of SAMs for example; $-\mathrm{COOH},-\mathrm{SH},-\mathrm{NH}$. The results of this study, along with results from the literature, show only one bound thiolate species is detectable by XPS after adsorption of SAM molecules onto gold surfaces. The $S(2 p)$ signals of $S A M / A u$ interfaces do not change after 
complexation with metal ions. No oxidized sulfur species (S2p binding energy above $166 \mathrm{eV}$ ), such as sulfonate, was detected in any SAM examined in this study. This confirms the SAMs are very stable to the complexation processes. The results of peak fitting for $\mathrm{C}(1 \mathrm{~s})$ show four peaks, with binding energies at 288.5 , $286.4,285$ and $284.5 \mathrm{eV}$, which are assigned to $-\mathrm{COOH},-\mathrm{CS}, \mathrm{C}-\mathrm{C}$ and $-\mathrm{CH}$ or $\mathrm{CH}_{2}$, respectively. The results of the peak fitting for $\mathrm{O}(1 \mathrm{~s})$ show the presence of terminal free carboxylate groups. XPS gives excellent resolution of different target metal ions: XPS peak fitting shows the presence of three different environments of copper ( $\mathrm{Cu}(\mathrm{II})$ alone, $\mathrm{Cu}(\mathrm{I})$ alone, $\mathrm{Cu}(\mathrm{II})$ and $\mathrm{Cu}(\mathrm{I})$ ) and two different environments of mercury $(\mathrm{Hg}(\mathrm{I})$ and $\mathrm{Hg}(\mathrm{II}))$. The analysis of the XPS peak fitting for $\mathrm{Pb}(4 \mathrm{~d} 5 / 2)$ and $\mathrm{Cd}(3 \mathrm{~d} 5 / 2)$ each shows only one peak at 413.5 and $405.3 \mathrm{eV}$, respectively. The M:S ratio was in good agreement with the stiochiometry extracted from QCM and EQCM measurements.

\subsection{Future Prospects}

I will plan to separate the future work into three parts. The first stage involves experiments for binding other trace metal ions with different oxidation states such as $\mathrm{Al}(\mathrm{III}), \mathrm{Cr}(\mathrm{III}), \mathrm{Cr}(\mathrm{VI}), \mathrm{Sn}(\mathrm{II}), \mathrm{Sn}(\mathrm{IV}), \mathrm{Fe}(\mathrm{II}), \mathrm{Fe}(\mathrm{III})$ and $\mathrm{As}$ (III) to other SAMs containing different terminal functional groups. In the second stage, the goal is to explore the capability of different structure polymer films and self-assembled multilayers containing different terminal free groups at the solid/solution interface to function as pre-concentration steps. The ability of the attached different ligands to the polymer film or SAM will be studied. Detection will involve a combination of electrochemical and acoustic wave measurements. It is based upon a preconcentration strategy involving SAMs multilayers-attached ligands and polymeric surface-immobilised ligands. Characterization of the composition and dynamics of these surface layers will be explored using thickness shear mode acoustic resonators, in gravimetric and viscoelastic modes. Overall interfacial performance will be determined coulometrically or voltammetrically, as appropriate. In the latter case, an important question to be answered in two cases is the extent to which the response is gravimetrically or viscoelastically controlled. This will require the determination of film shear modulus data for thin polymer films before and after adsorption of trace metal ions. The characterization of these systems using atomic force microscopy (AFM) and X-ray photoelectron spectroscopy (XPS) will be studied. The comparisons between self-assembled multilayers, polymeric films with the results we have in this thesis will be made. The comparisons include the heterogeneous binding constant, mole ratio and surface coverage of metal 
ions. The final stage involves application of these systems to real environmental samples. 


\section{APPENDIX I}
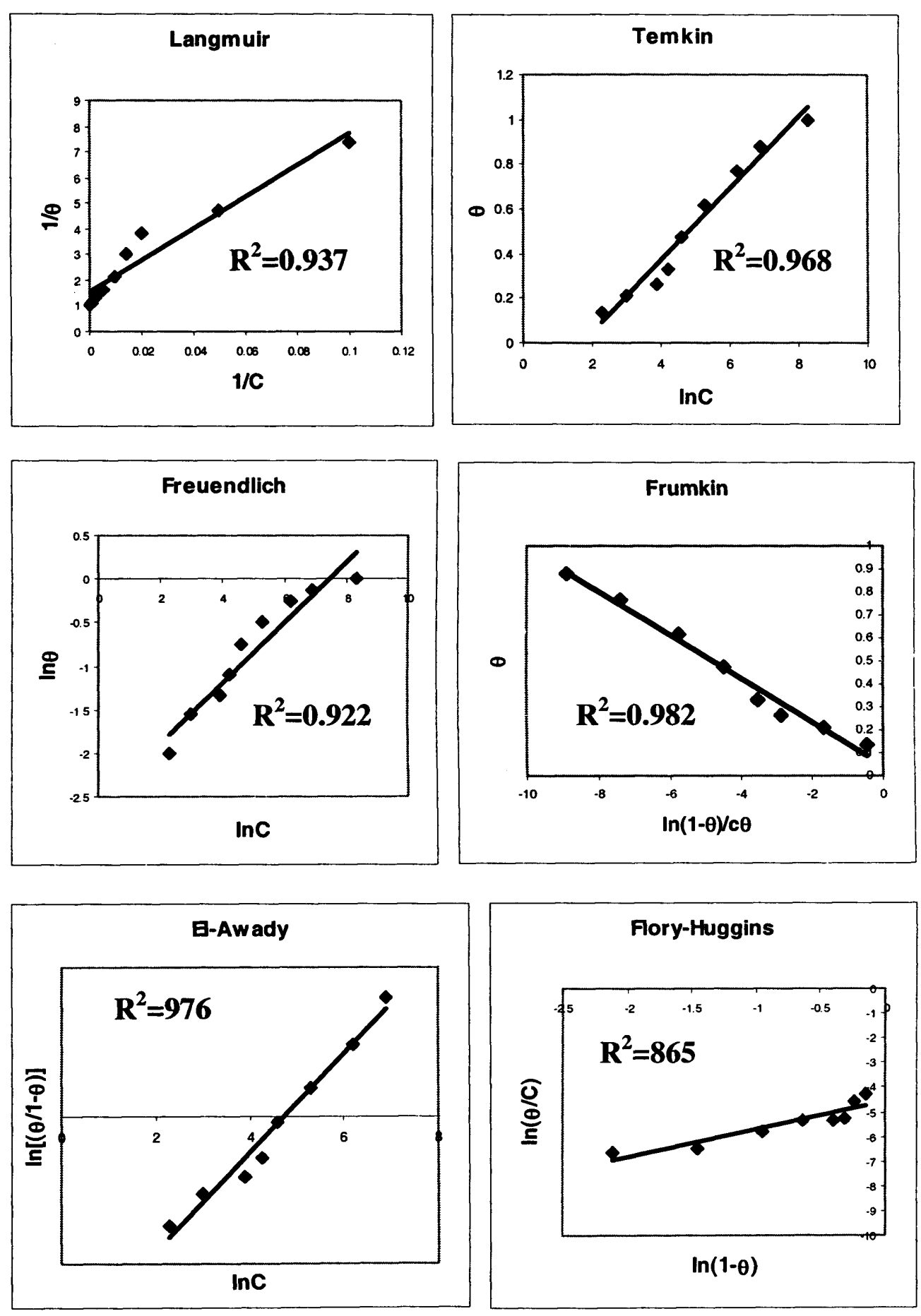

Figure AI. 1a: Plot of Langmuir, Temkin, Freunlich, Frumkin, El-Awady and Flory-Huggins isotherms to estimate the binding constant between $\mathrm{Pb}^{2+}$ and meso-2,3-dimercaptosuccinic acid on dry (emersed) crystal. The line represents the linear least squares fit to the experimental data (points). 

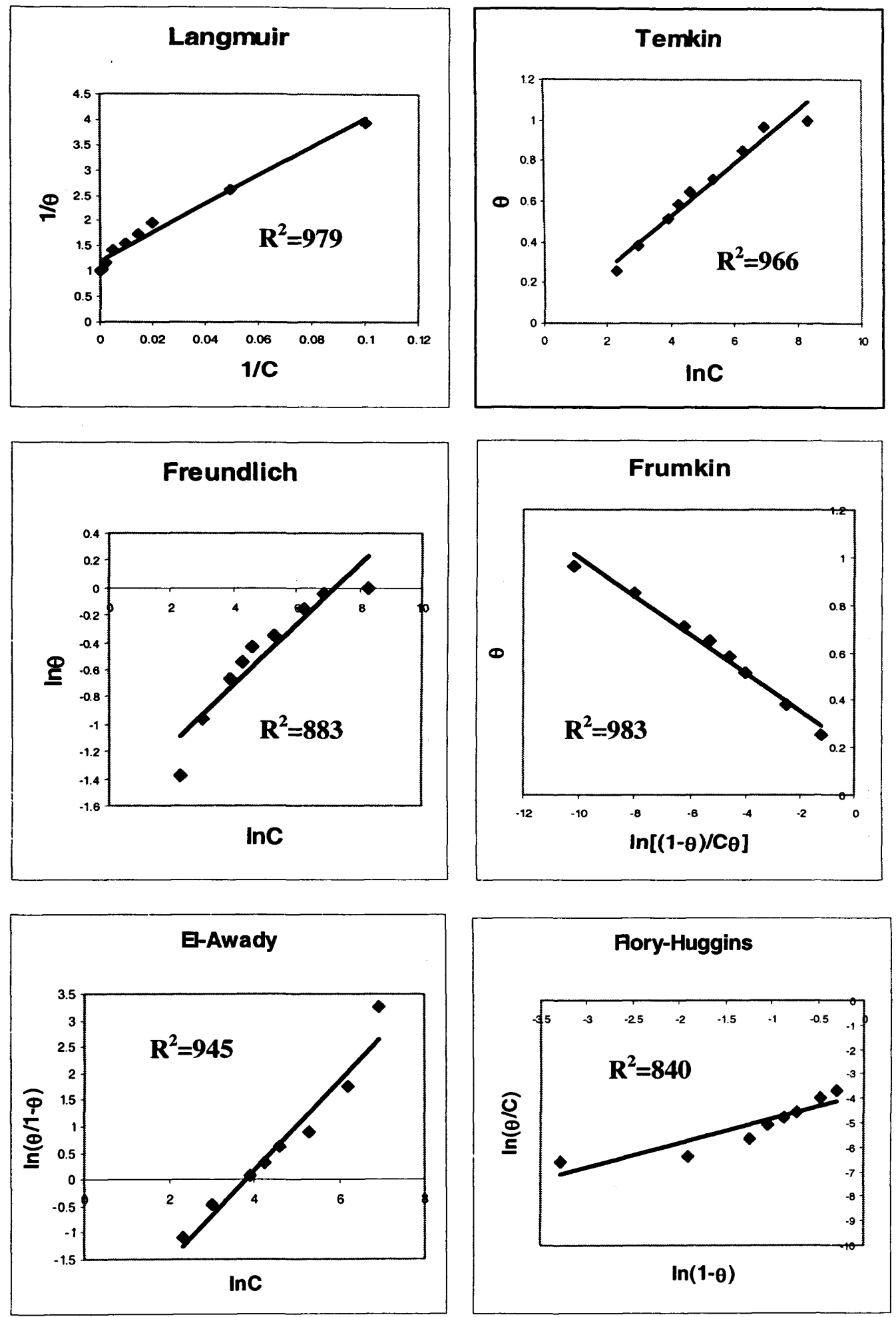

Figure Al. 1b: Plot of Langmuir, Temkin, Freunlich, Frumkin, El-Awady and Flory-Huggins isotherms to estimate the binding constant between $\mathrm{Pb}^{2+}$ and meso-2,3-dimercaptosuccinic acid on wet (immersed) crystal. The line represents the linear least squares fit to the experimental data (points). 

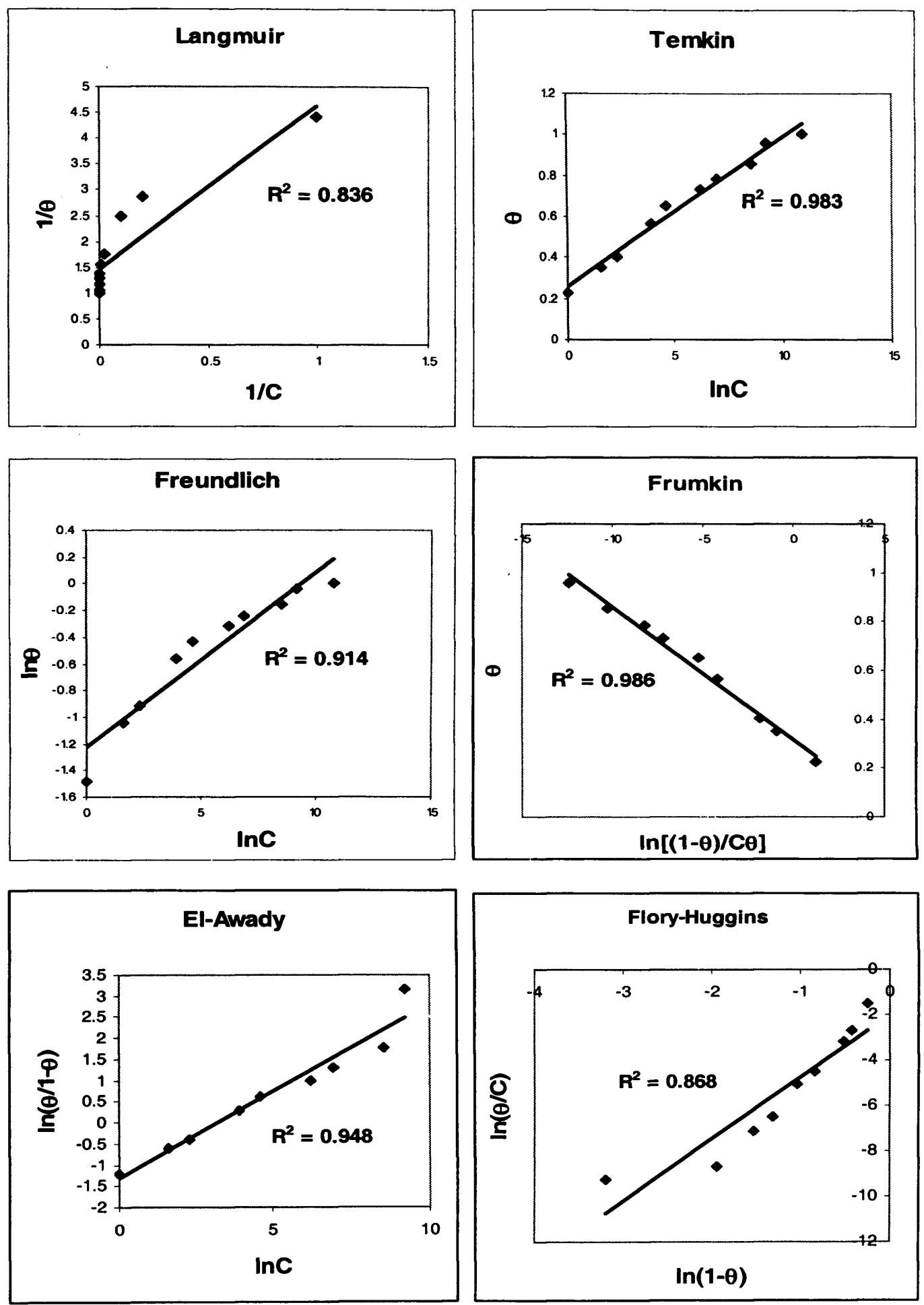

Figure Al. 2a: Plot of Langmuir, Temkin, Freundlich, Frumkin, El-Awady and Flory-Huggins isotherms to estimate the binding constant between $\mathrm{Hg}^{2+}$ and 1,6-hexanedithol on dry (emersed) crystal. The line represents the linear least squares fit to the experimental data (points). 

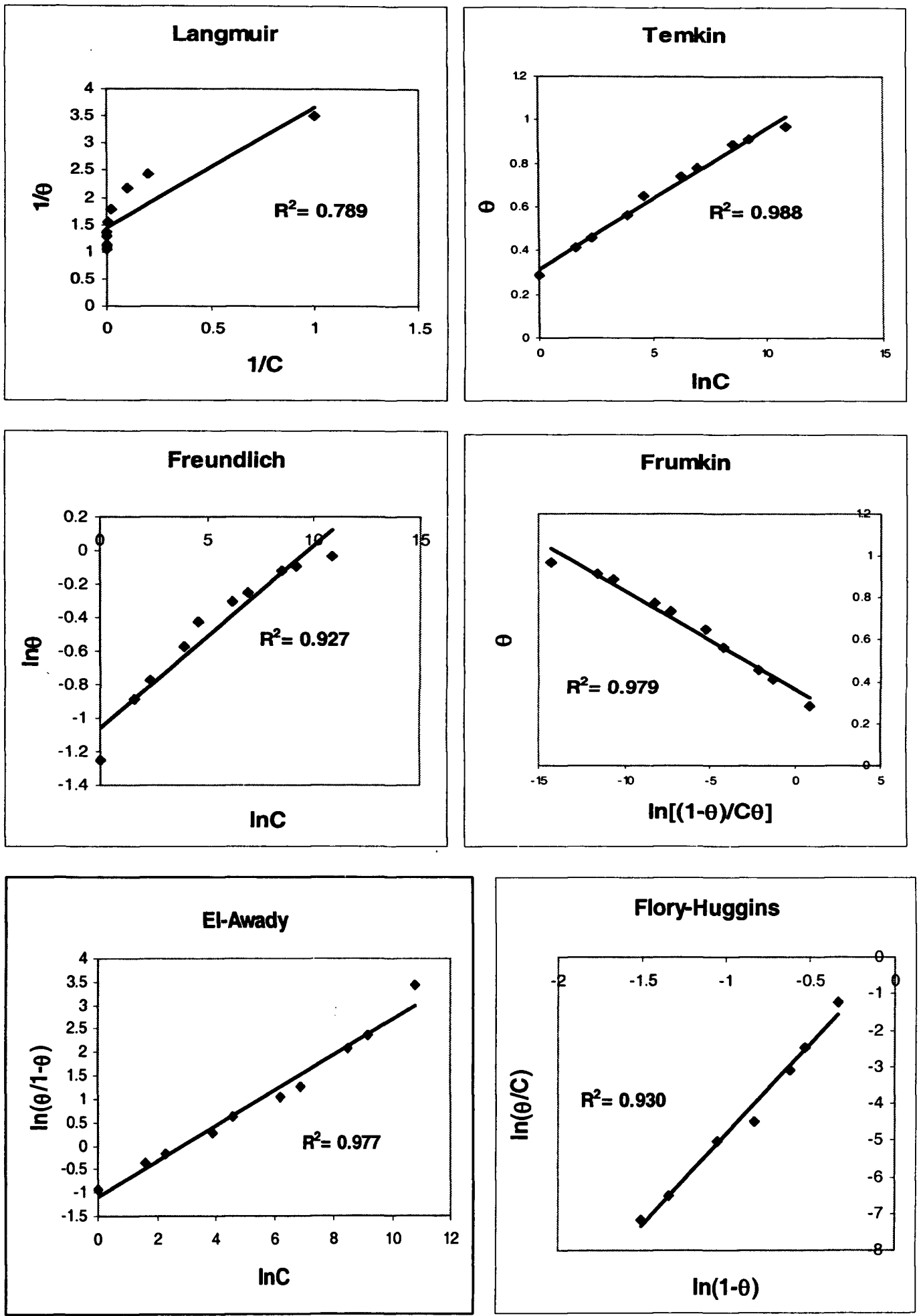

Figure Al. 2b: Plot of Langmuir, Temkin, Freundlich, Frumkin, El-Awady and Flory-Huggins isotherms to estimate the binding constant between $\mathrm{Hg}^{2+}$ and 1,6-hexanedithol on wet (immersed) crystal. The line represents the linear least squares fit to the experimental data (points). 

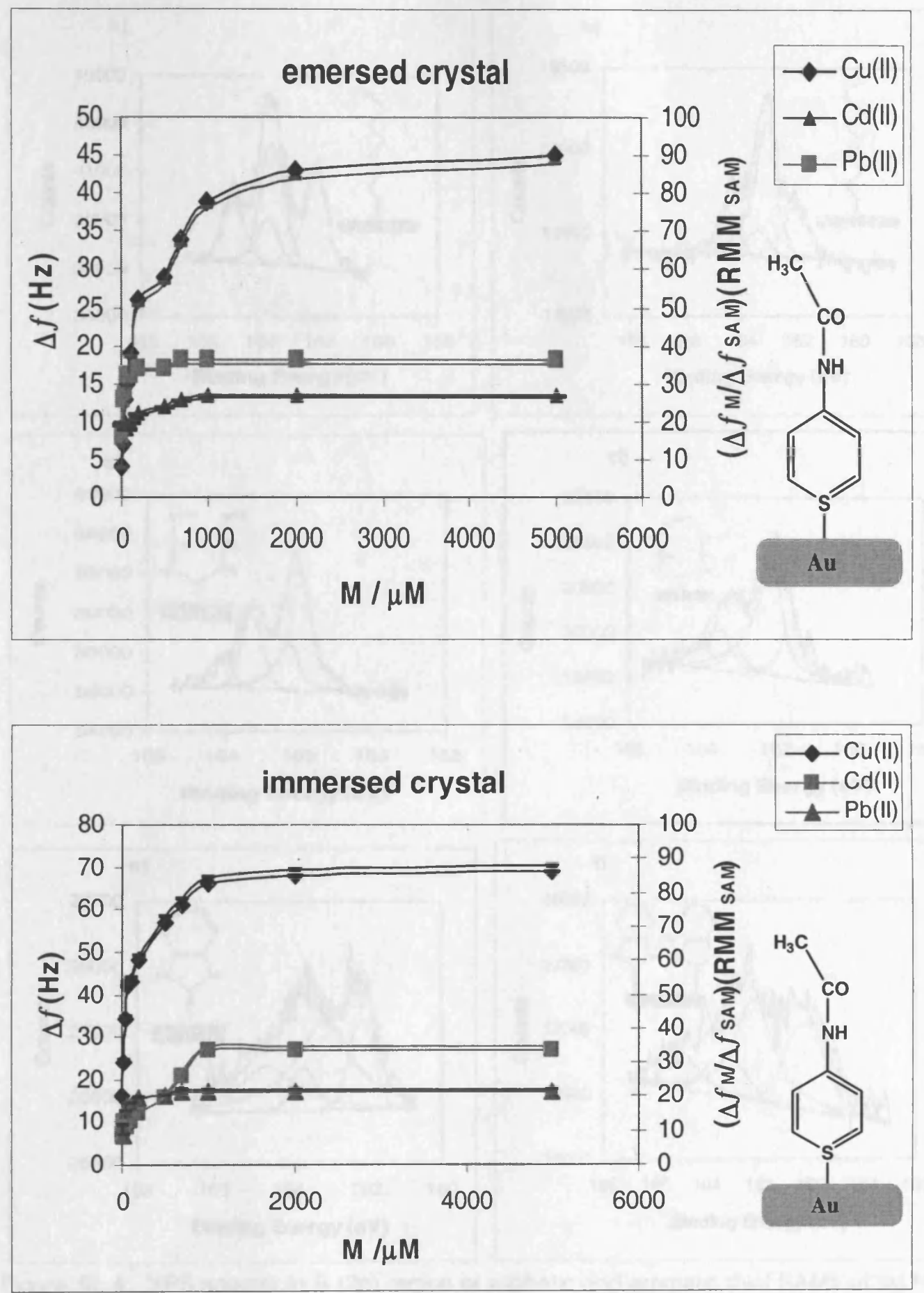

Figure Al. 3. The binding isotherms for the binding of $\mathrm{Cu}^{2+}, \mathrm{Cd}^{2+}$ and $\mathrm{Pb}^{2+}$ to acetomidothiophenol on emersed and immersed crystals. The surface coverage of the monolayer was 2.17 and $2.40 \mathrm{nmol} \mathrm{cm}^{-2}$, respectively. 

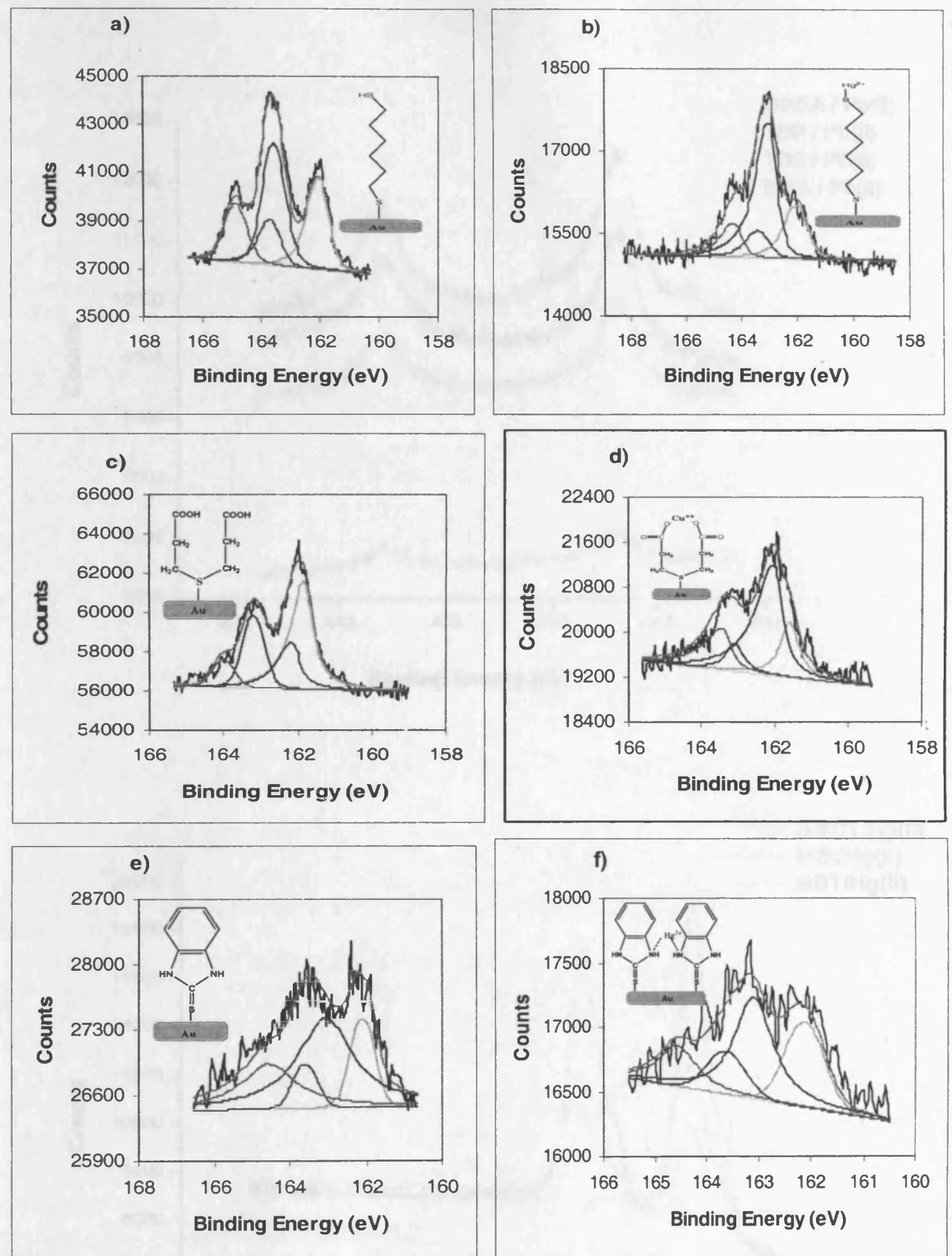

Figure Al. 4: XPS spectra in S (2p) region of aliphatic and aromatic thiol SAMs of (a) HDT; (b) HDT/Hg(II); (c) TDP; (d) TDP/Cu(II); (e) MBI and (f) $\mathrm{MBI} / \mathrm{Hg}(\mathrm{II})$. All the SAMs were prepared by immersion for $24 \mathrm{~h}$ into $1 \mathrm{mM}$ solution. Two S2p doublets, both with a 2:1 area ratio and a splitting of $1.5 \mathrm{eV}$, were used to peak fit the experimental spectrum. 

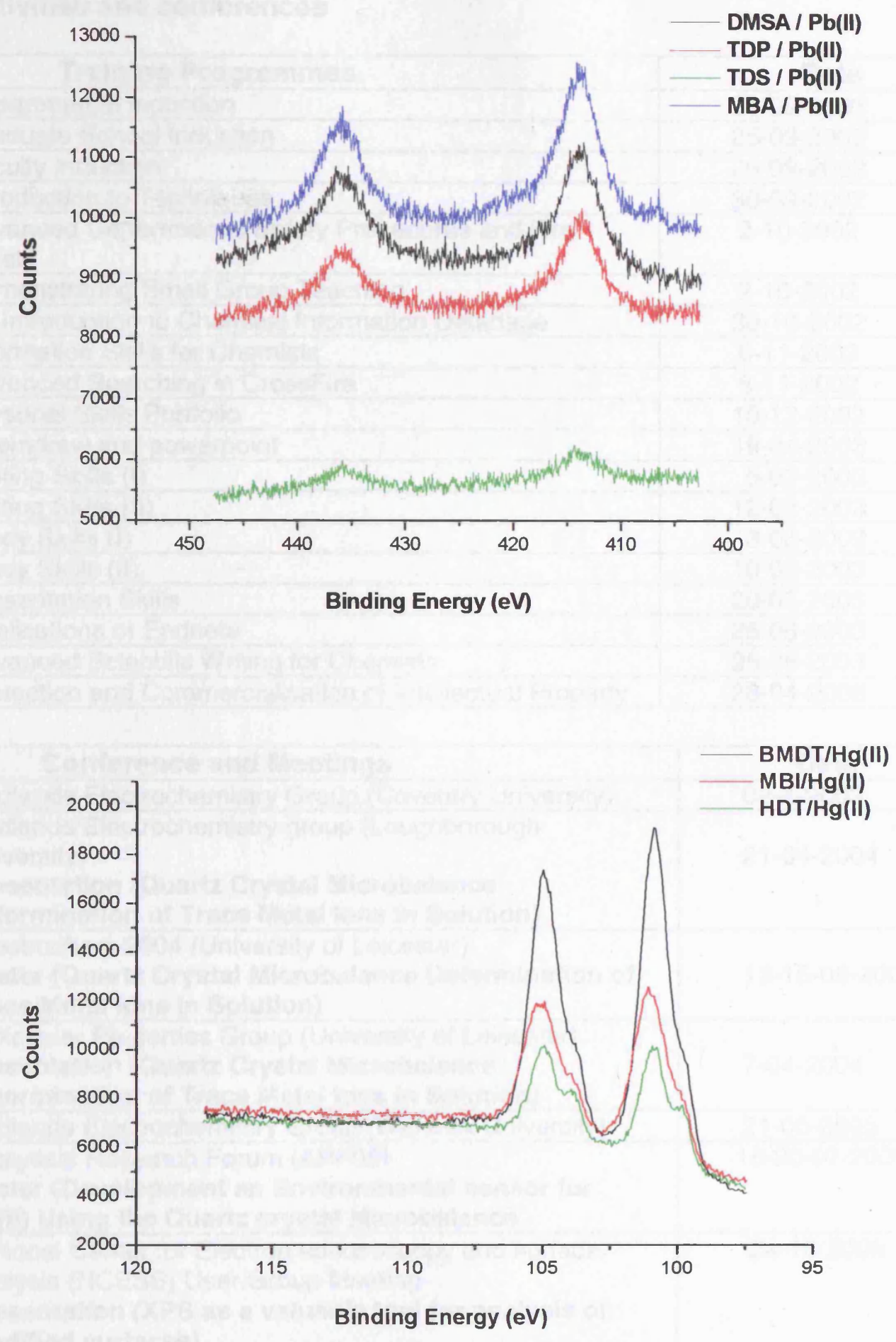

Figure Al. 5: High resolution XPS spectra of $\mathrm{Pb}\left(4 \mathrm{~d}_{5 / 2}\right)$ and $\mathrm{Hg}\left(4 \mathrm{f}_{7 / 2}\right)$ regions for different SAMs after adsorption of $\mathrm{Pb}(\mathrm{II})$ and $\mathrm{Hg}$ (II) from single metal ion solutions. 


\section{Activities and conferences}

\begin{tabular}{|l|c|}
\hline \multicolumn{1}{|c|}{ Training Programmes } & Date \\
\hline Departmental Induction & $24-09-2002$ \\
\hline Graduate School Induction & $25-09-2002$ \\
\hline Faculty Induction & $26-09-2002$ \\
\hline Introduction to Techniques & $30-09-2002$ \\
\hline $\begin{array}{l}\text { Advanced Departmental Safety Procedures and Fire } \\
\text { Safety }\end{array}$ & $2-10-2002$ \\
\hline Demonstrating Small Group Teaching & $2-10-2002$ \\
\hline An Introduction to Chemical Information Database & $30-10-2002$ \\
\hline Information Skills for Chemists & $6-11-2002$ \\
\hline Advanced Searching in CrossFire & $6-11-2002$ \\
\hline Personal Skills Portfolio & $10-12-2002$ \\
\hline Chemdraw and powerpoint & $19-04-2003$ \\
\hline Writing Skills (I) & $5-02-2003$ \\
\hline Writing Skills (II) & $12-02-2003$ \\
\hline Study Skills (I) & $3-03-2003$ \\
\hline Study Skills (II) & $10-03-2003$ \\
\hline Presentation Skills & $20-03-2003$ \\
\hline Applications of Endnote & $25-06-2003$ \\
\hline Advanced Scientific Writing for Chemists & $25-06-2003$ \\
\hline Protection and Commercialisation of Intellectual Property & $28-04-2003$ \\
\hline
\end{tabular}

\begin{tabular}{|l|c|}
\hline \multicolumn{1}{|c|}{ Conference and Meetings } & Date \\
\hline Midlands Electrochemistry Group (Coventry University) & $09-4-2003$ \\
\hline $\begin{array}{l}\text { Midlands Electrochemistry group (Loughborough } \\
\text { University) } \\
\text { Presentation (Quartz Crystal Microbalance } \\
\text { Determination of Trace Metal lons in Solution) }\end{array}$ & $21-04-2004$ \\
\hline $\begin{array}{l}\text { Electrochem 2004 (University of Leicester) } \\
\text { Poster (Quartz Crystal Microbalance Determination of } \\
\text { Trace Metal lons in Solution) }\end{array}$ & $13-15-09-2004$ \\
\hline $\begin{array}{l}\text { Molecular Properties Group (University of Leicester) } \\
\text { Presentation (Quartz Crystal Microbalance } \\
\text { Determination of Trace Metal lons in Solution) }\end{array}$ & $7-04-2004$ \\
\hline Midlands Electrochemistry Group (Warwick University) & $21-05-2005$ \\
\hline $\begin{array}{l}\text { Analytical Research Forum (ARF05) } \\
\text { Poster (Development an Environmental sensor for } \\
\text { Hg(II) Using the Quartz crystal Microbalance }\end{array}$ & $18-20-07-2005$ \\
\hline $\begin{array}{l}\text { National Center for Electron spectroscopy and surface } \\
\text { analysis (NCESS) User Group Meeting } \\
\text { Presentation (XPS as a valuable tool for analysis of } \\
\text { modified surfaces) }\end{array}$ & $24-10-2005$ \\
\hline Electrochemistry at the Nanoscale & $16-11-2005$ \\
\hline
\end{tabular}

\begin{tabular}{|c|c|}
\hline Courses & \\
\hline Analytical Chemistry CH3020 & $66 \%$ \\
\hline Interfacial Structures CH4007 & $80 \%$ \\
\hline
\end{tabular}

